\title{
TOWARDS NOVEL BODIPY COMPOUNDS FOR MATERIAL \& MEDICAL APPLICATIONS
}

\author{
by \\ Burhan Ahmed Hussein, \\ BSc. Ryerson, 2014 \\ A thesis \\ presented to Ryerson University \\ in partial fulfillment of the \\ requirements for the degree of \\ Master of Science \\ in the Program of \\ Molecular Science \\ Toronto, Ontario, Canada, 2017 \\ (C) Burhan Ahmed Hussein 2017
}




\section{Declaration}

I hereby declare that I am the sole author of this thesis. This is a true copy of the thesis, including any required final revisions, as accepted by my examiners. I authorize Ryerson University to lend this thesis to other institutions or individuals for the purpose of scholarly research. I further authorize Ryerson University to reproduce this thesis by photocopying or by other means, in total or in part, at the request of other institutions or individuals for the purpose of scholarly research. I understand that my thesis may be made electronically available to the public 


\begin{abstract}
TOWARDS NOVEL BODIPY COMPOUNDS FOR MATERIAL \& MEDICAL APPLICATIONS

Burhan Ahmed Hussein

Master of Science, Molecular Science, Ryerson University , 2017
\end{abstract}

This thesis describes the synthesis of a novel family of 4,4-difluoro-4-bora-3a-4a-diaza-s-indacenes for material and education applications (BODIPYs).This thesis also presents the exploration of their optical and electrochemical properties, as well as potential for application in dye-sensitised solar cells, and synthetic laboratories. Chapter 1 gives an overview of the photophysical of chromophores of large organic electronics. BODIPYs are introduced as suitable building blocks for such applications, and synthetic approaches for extending the conjugation of these fluorophores are presented. Chapter 2 gives an overview the development of an engaging senior undergraduate laboratory course based on BODIPY that reinforces traditionally important technical skills and explore property-directed synthesis \& research. Chapter 3 describes the use of the redox robust ferrocene as electron donor in the synthesis of ferrocene-BODIPY dyes for DSSC applications. Chapter 4 explores the effects of confined intramolecular $\pi-$ stacking on the physicochemical properties of $\pi-\pi$ BODIPY dimers in cyclophane architectures through the synthesis of a novel cyclophane consisting of BODIPY and anthracene moieties 


\section{Acknowledgements}

First and foremost, I like to thank Dr. Bryan Koivisto for all the opportunities he has given me as well as the freedom to explore my curiosities in new areas of chemistry, particularly in synthetic work. I am really grateful to him for allowing me to have a part of the creation of the CHY 399 and CHY 307 and allowing me to take a leadership role in that endeavor. Many thanks to Dr. Bryan Koivisto for helping me grow as person and a professional over the last five years, for all the support, good times, and encouragement and understanding during the not so great times, and expanding my horizons. I am truly lucky to have had you as a supervisor and to be able to call you my friend, I will cherish the time I've had working for you.

Many thanks to my fellow graduate colleague and conference/coffee buddy Muhammed Yousaf as well as Jennifer Huynh, Ben Fischer, Sahana Sritharan, Devin Machin, Maryam Abdinejad, and Omar Abdi for all the advice and being wonderful colleagues. I would also like to thank past and present undergraduate labmates for making the KHN 211 a great lab environment. I also want to thank Jeffery Pau, and Nande Wright for being great chemistry lab neighbors. I would also like to thank Malek El-Aooiti for helping me with my chemistry. You have made my time at Ryerson memorable. I like to thank Dr. Russell Viirre, Dr. Daniel Foucher, Dr. Stephen Wylie, Dr. Robert Gossage and Dr. Andrew McWilliams for all their help with my thesis and being great mentors.

I thank all the students who have been a part of the development of synergetic CHY399 and CHY307. Especially Ben Fischer, Malek El-Alooiti, Sahana Sritharan, Jasjit Singh, Jeffery Pau, Jeanette Adjei, Jasveer Dhindsa and David Lau whose work is shown in Chapter 2.

I thank Dr. Alan Lough for the crystallography work for my thesis. I thank Dr. Eduardo Schott for the density functional theory calculations for my cyclophanes work, it is much appreciated. Many thanks to Dr. Curtis Berlinguette for the device fabrication opportunity as well.

Finally I would like to thank my family and friends, especially my parents, Aisha Mohammed and Ahmed. Your support, love and sacrifice gotten me this far; I hope I have made you proud. Many thanks to my friends, especially Agnes Klimoski, Chris Helmeste, Laura Acevedo, Adrian Junor for being there for me. Special shout to SRK. 


\section{TABLE OF CONTENTS}

Declaration

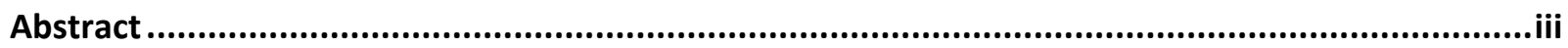

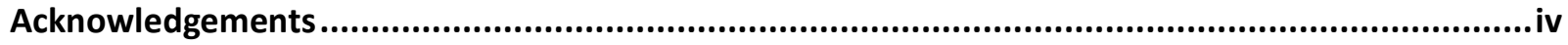

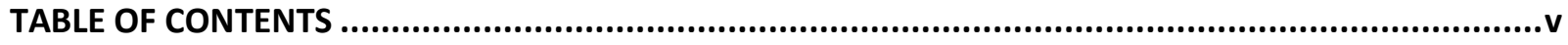

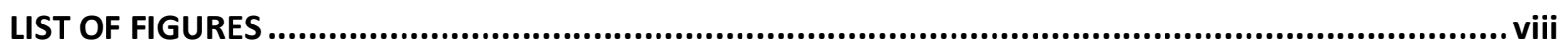

LIST OF SCHEMES

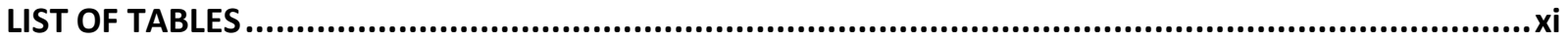

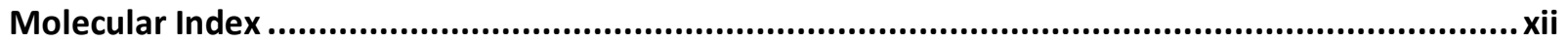

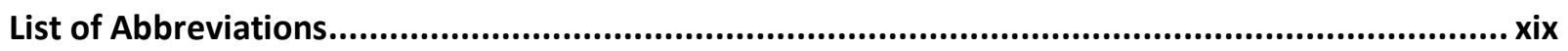

Chapter 1: Introduction........................................................................

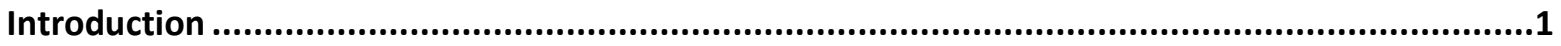

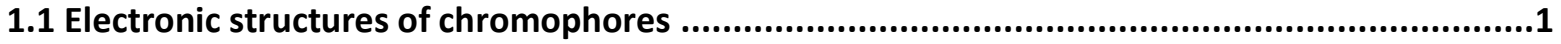

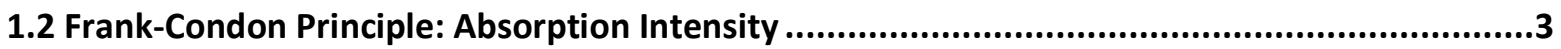

1.3 Kasha's Rule and Stokes Shift: After Absorption ........................................................5

1.4 Photoinduced energy and electron transfer processes ....................................................6

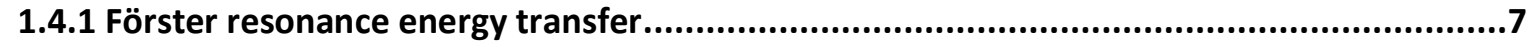

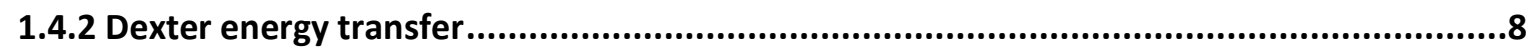

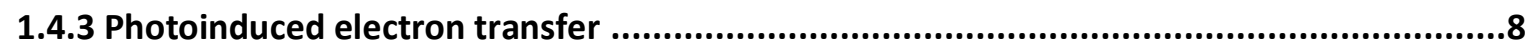

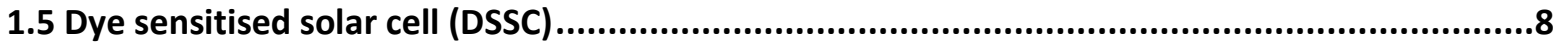

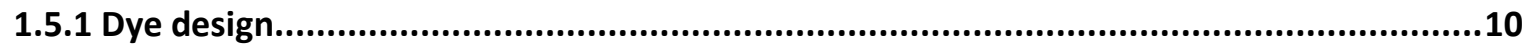

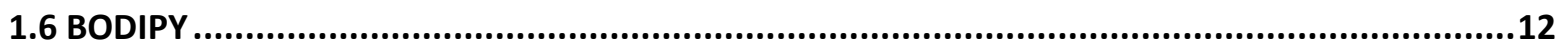

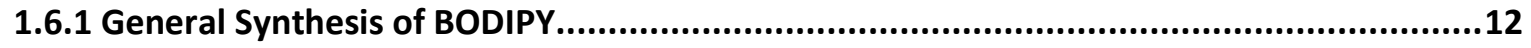

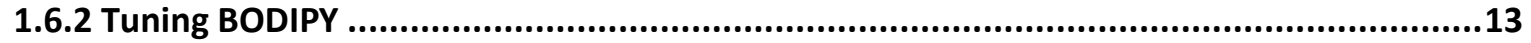

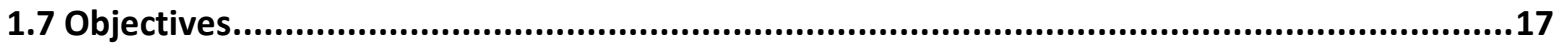

Chapter 2: Exploring BODIPY Dyes in Synergistic Undergraduate Laboratories for Enhanced Experiential Learning and Research Output ...................................18

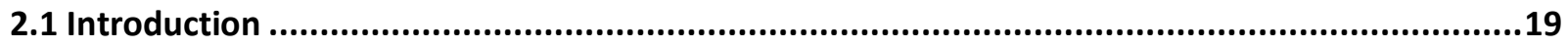

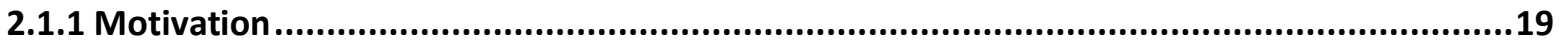

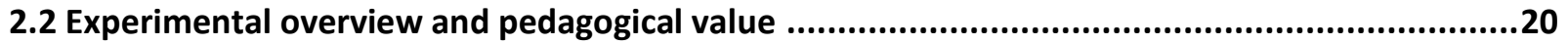

2.3 Stage 1: Developing labs skills \& building blocks ...............................................................20

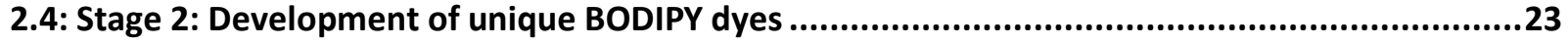




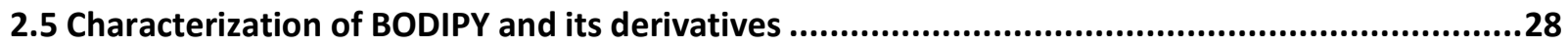

2.6 Conclusions

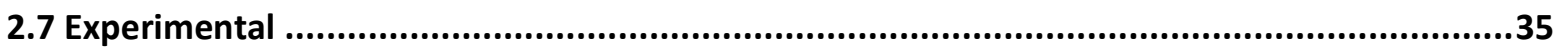

Chapter 3: Ferrocene D- $\pi$-A BODIPY Dyes .....................................................48

3.1 Literature review of ferrocene-BODIPY dyes .....................................................................48

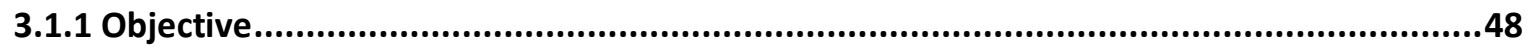

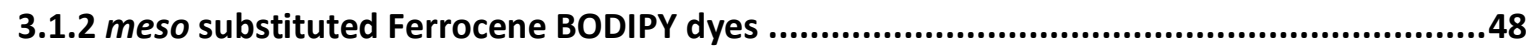

3.1.3 $\alpha$ substituted ferrocene BODIPY dyes..................................................................54

3.1.4 $\beta$ substituted ferrocene- BODIPY adducts ............................................................58

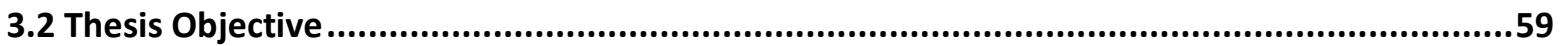

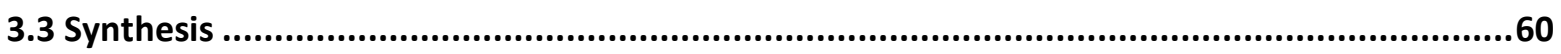

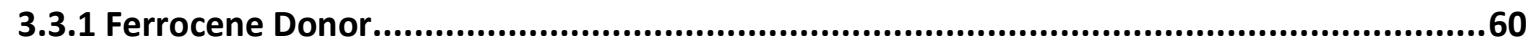

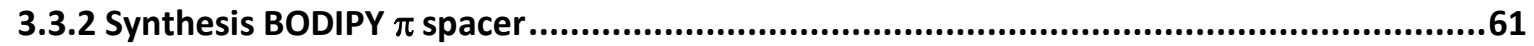

3.3.3 Installation of the acceptor units...........................................................................62

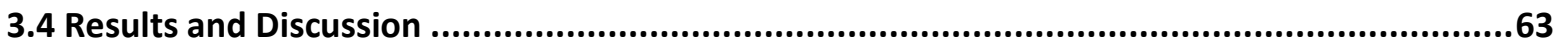

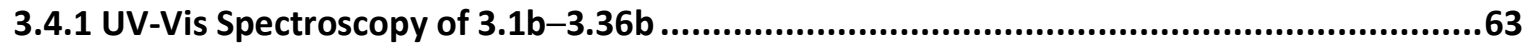

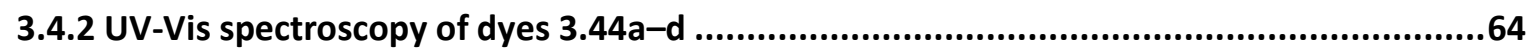

3.4.3 Preliminary testing of device...................................................................................65

3.4.4 Electrochemistry of Ferrocene D- $\pi$-A BODIPY dyads..................................................66

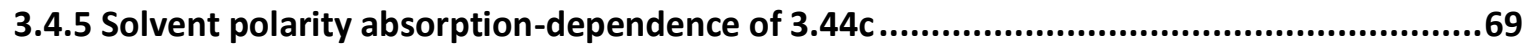

3.4.6 Spectroelectrochemical studies of Ferrocene $D-\pi$-A dyes ..........................................70

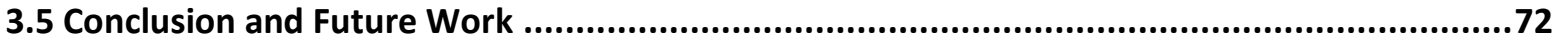

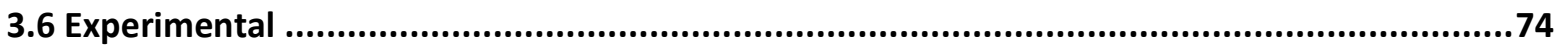

\section{Chapter 4: Towards BODIPY-Anthracene Cyclophanes: Tuneable HOMO-LUMO}

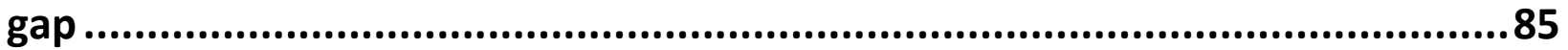

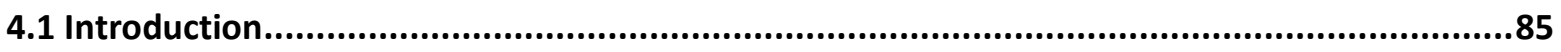

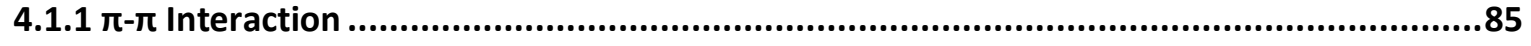

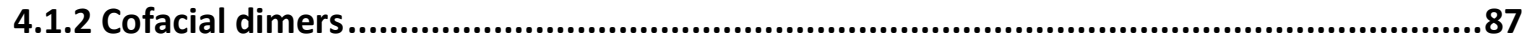

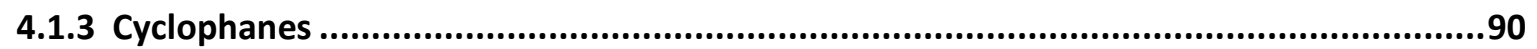

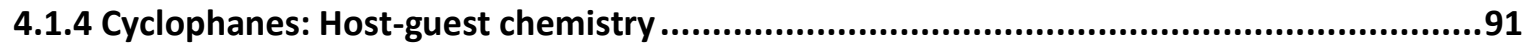

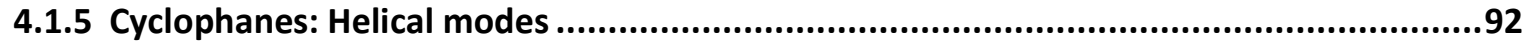


4.1.6 Cyclophanes: Subporphyrin cages cyclophanes......................................................94

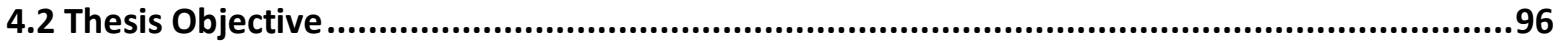

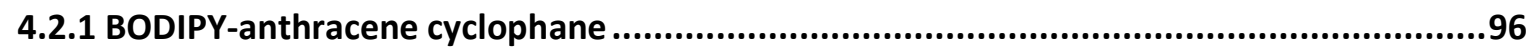

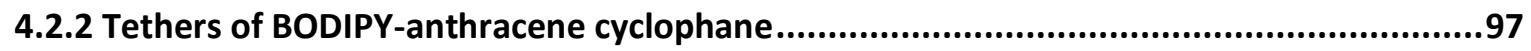

4.3 Theoretical Calculations ...................................................................................................97

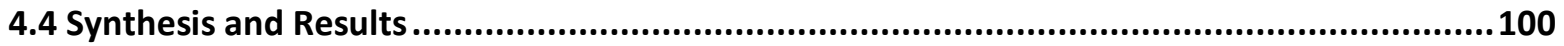

4.4.1 Synthetic route for BODIPY-anthracene cyclophanes ...........................................100

4.4.2 Synthesis of Anthracene Building Blocks..................................................................102

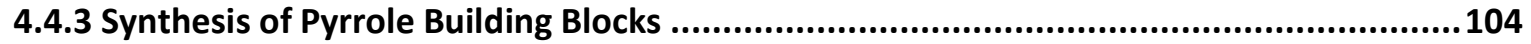

4.4.4 Tethering of Pyrrole and Anthracene for BODIPY-Anthracene Cyclophane 4.5 ..............106

4.4.5 Tethering of Pyrrole and Anthracene for BODIPY-Anthracene Cyclophane 4.6 .............107

4.4.6 Tethering of Pyrrole and Anthracene for BODIPY-Anthracene Cyclophane 4.4 .............109

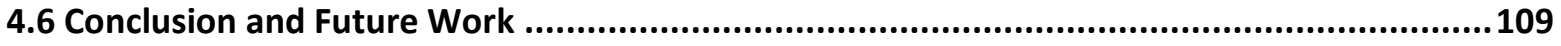

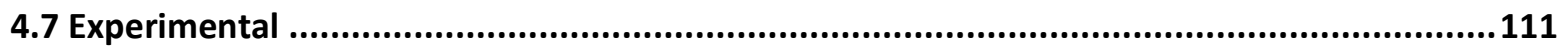

Conclusions and Future work .............................................................. 119

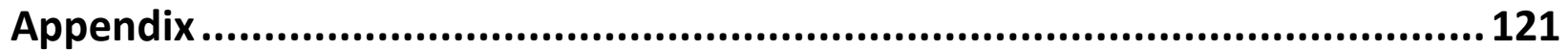

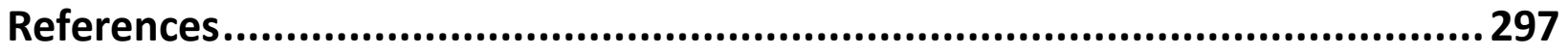




\section{LIST OF FIGURES}

Figure 1.1: Molecular orbital diagram

Figure 1.2: Schematic molecular orbital diagram to show the effect of increasing the number of conjugated $\pi$-bonds.

Figure 1.3: Potential energy curves for the ground state and an excited state ..................................... 4

Figure 1.4: Jablonski diagram descripting unimolecular excitation/relaxation pathways.......................... 5

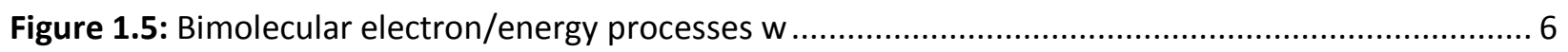

Figure 1.6: Pictorial representation of energy transfer through FRET and Dexter electron exchange ....... 7

Figure 1.7: Bimolecular photoinduced electron transfer ................................................................ 8

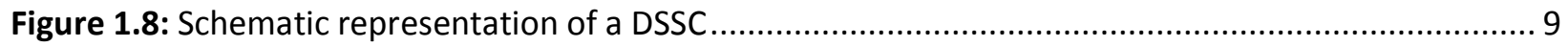

Figure 1.9: Competing thermodynamics pathways in the DSSC ........................................................ 9

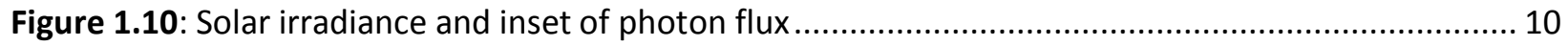

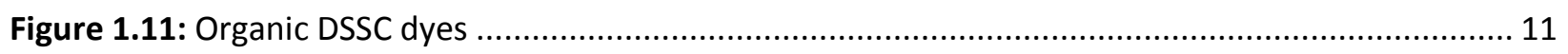

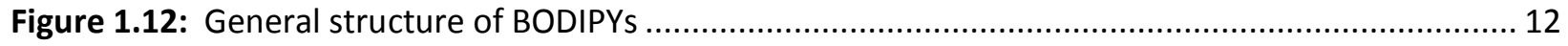

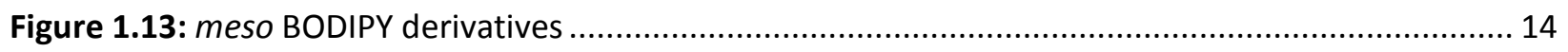

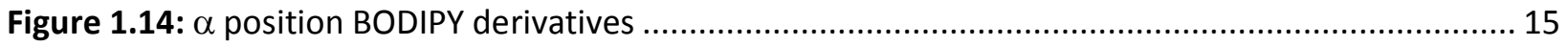

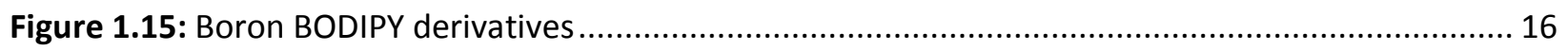

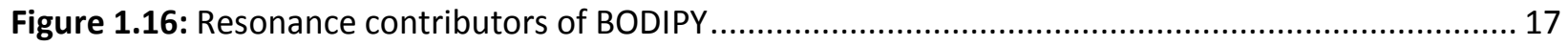

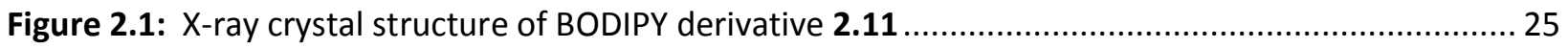

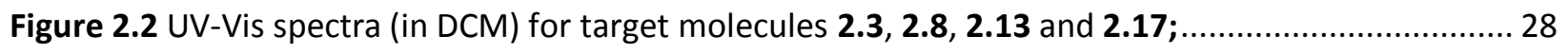

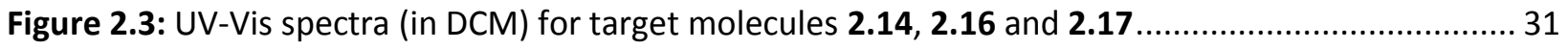

Figure 2.4: UV-Vis spectra (in DCM) for target molecules 2.4, 2.14 and 2.19.................................... 32

Figure 2.5: UV-Vis spectra (in DCM) for target molecules 2.3, 2.5, 2.24 and 2.25a............................. 33

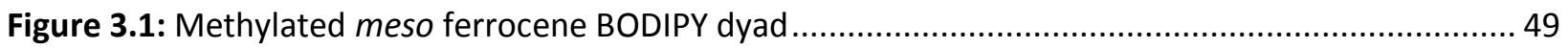

Figure 3.2: meso triazole non-conjugated ferrocene BODIPY dyad. ..................................................... 50

Figure 3.3: PET process of meso substituted ferrocene methylated BODIPY dyes, ............................... 51

Figure 3.4: Novel meso ferrocene un-substituted BODIPY dyes ....................................................... 53

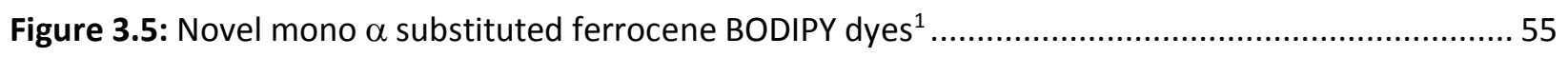

Figure 3.6: Novel diferrocene $\alpha$ substituted BODIPY dyads................................................................ 57

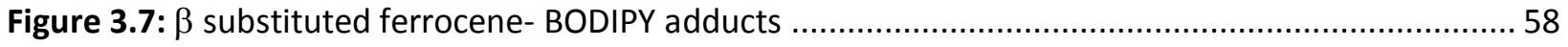

Figure 3.8: Family of target ferrocene BODIPY dyes ........................................................................ 59

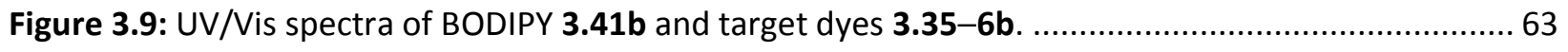

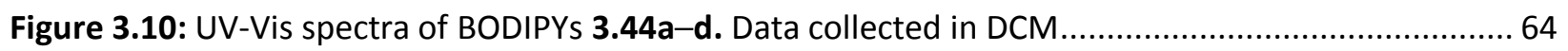

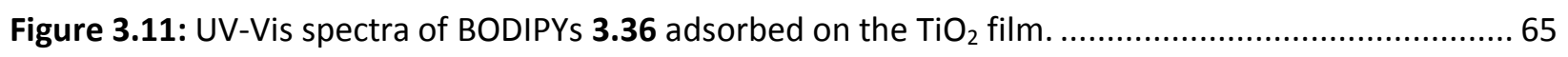

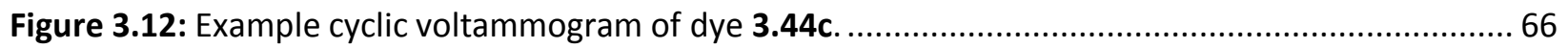

Figure 3.13: UV-Vis spectra of BODIPYs 3.44c in DCM, MeCN, toluene, MeOH ..................................... 69

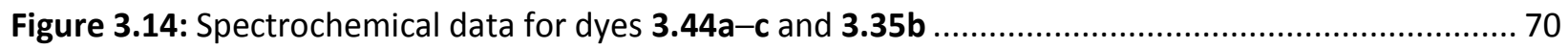

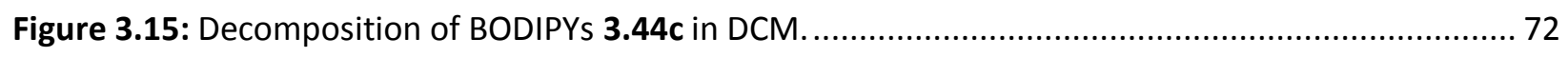

Figure 3.16: Proposed ferrocene BODIPY for raising LUMO energy level. ............................................ 72 
Figure 4.1: Electrostatic view of aromatic-aromatic interaction....................................................... 86

Figure 4.2: Electrostatic view of electron rich/electron deficient aromatic-aromatic interaction ............ 87

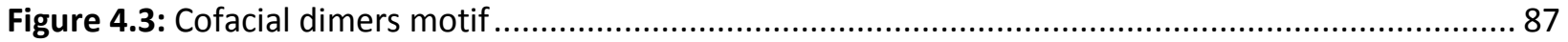

Figure 4.4: Porphyrin cofacial dimers motifs and the different spacers and the reference porphyrin

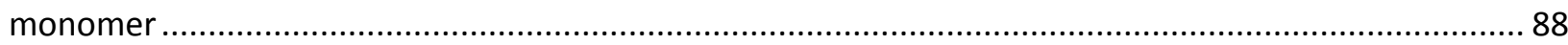

Figure 4.5: Porphyrin cofacial dimers oxidation cause the formation of exciton................................... 88

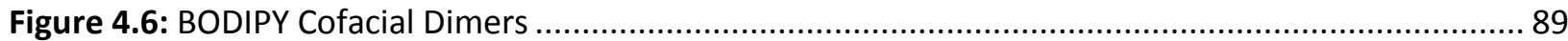

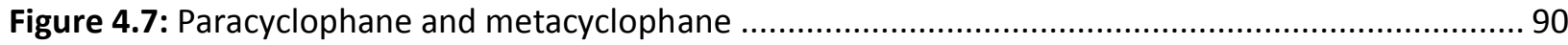

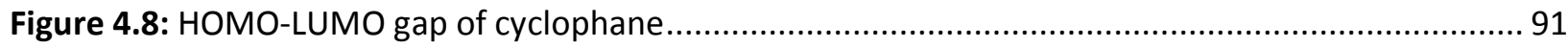

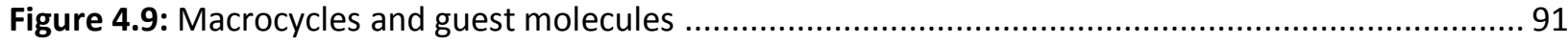

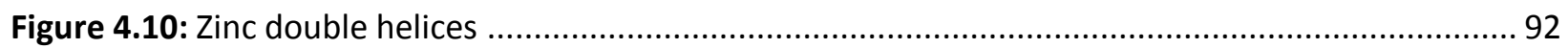

Figure 4.11: Short $(S)$ and long (L) modes of the Zn-dipyrrin double helices .................................... 93

Figure 4.12: Electronic structure of Zn-dipyrrin double helices ...................................................... 93

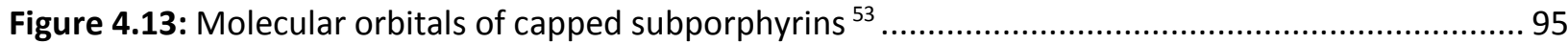

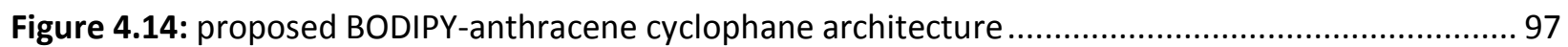

Figure 4.15: DFT: B3LYP 3-21G of BODIPY-anthracene and predicted molecular orbitals of HOMO-LUMO

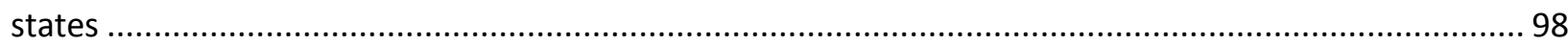

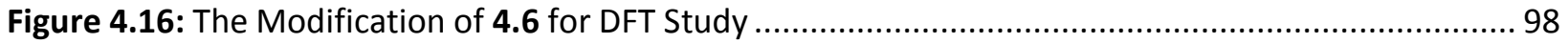

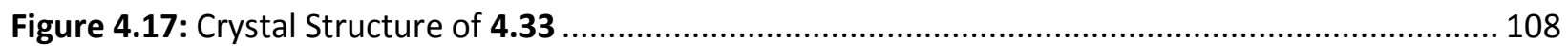

Figure 4.18: BODIPY-anthracene cyclophane architectures ............................................................ 109 


\section{LIST OF SCHEMES}

Scheme 1.1: General synthetic approach to BODIPY using acid chlorides ......................................... 13

Scheme 1.2: General synthetic approach to BODIPY using aldehydes ............................................... 13

Scheme 1.3: General synthetic approach to 8-Cl-BODIPY .................................................................. 13

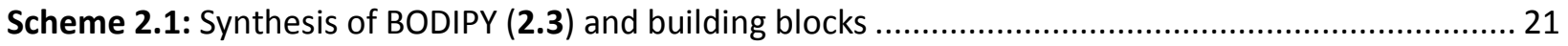

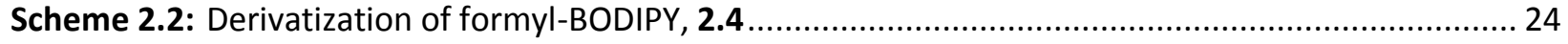

Scheme 2.3: Synthetic routes of BODIPY derivatives (2.14-22) from formyl BODIPY, 2.4 ..................... 26

Scheme 2.4: BODIPY derivatization possibilities starting from iodo-BODIPY, 2.5 ................................. 27

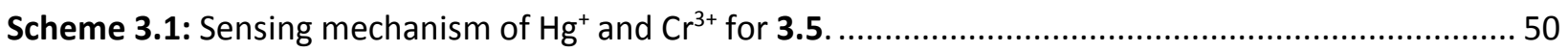

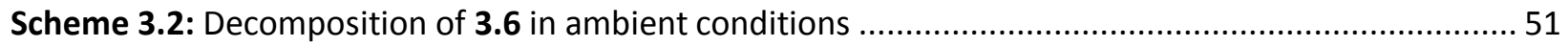

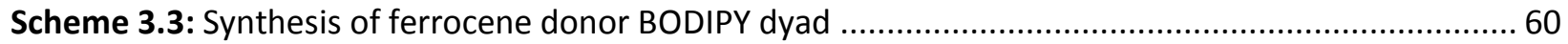

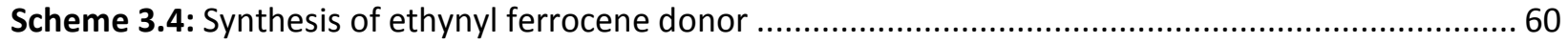

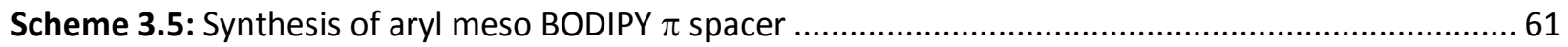

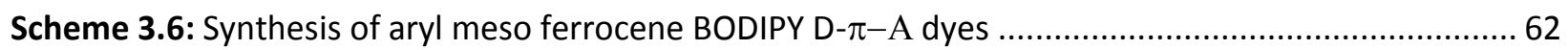

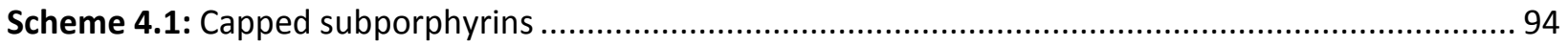

Scheme 4.2: retrosynthetic approach to synthesis cyclophanes 4.4, 4.5, 4.6 ................................... 101

Scheme 4.3: Synthesis of 1,8 diacetoxyanthracene through the reduction of anthraquinone using $\mathrm{Zn} .102$

Scheme 4.4: Improved synthesis of 1,8 diacetoxyanthracene through the reduction of anthraquinone

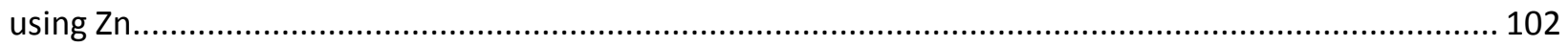

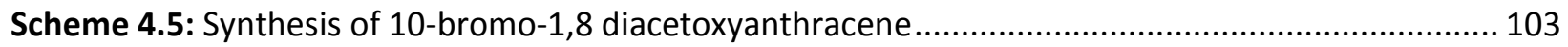

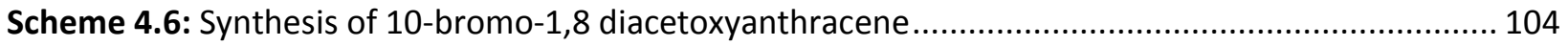

Scheme 4.7: Original synthetic towards the pyrrole building blocks …............................................. 104

Scheme 4.8: Synthesis towards the methyl pyrrole carboxyaldehyde............................................... 105

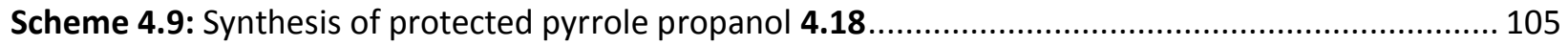

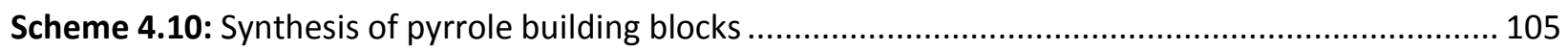

Scheme 4.11: Synthesis of anthracene-pyrrole building block for 4.5 ........................................... 106

Scheme 4.12: Williamson ether synthesis of anthracene/anthraquinone ........................................... 107

Scheme 4.13: Synthesis of anthracene-pyrrole building block for 4.6 ............................................... 108

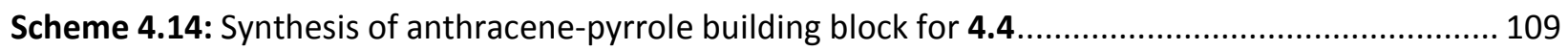




\section{LIST OF TABLES}

Table 2.1: Physicochemical characterization of BODIPY (2.3) and its derivatives (2.4-7) ................... 23

Table 2.2: Physicochemical characterization of BODIPY (2.4) and its derivatives (2.14-22). ................. 30

Table 2.3: Physicochemical characterization of molecules derived from iodo-BODIPY,2.5 ................... 33

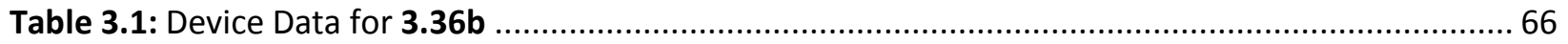

Table 3.2: Physicochemical properties for dyes 3.41a-d, 3.42a-d, 3.44a-d, 3.35bd, 3.36b ................... 68

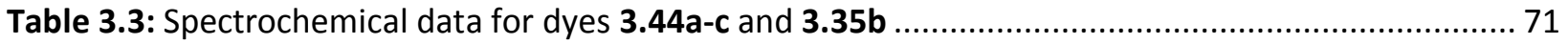

Table 4.1: Optical properties of capped subporphyrins.................................................................. 96

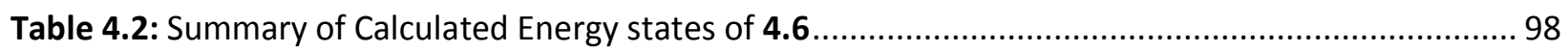




\section{Molecular Index}
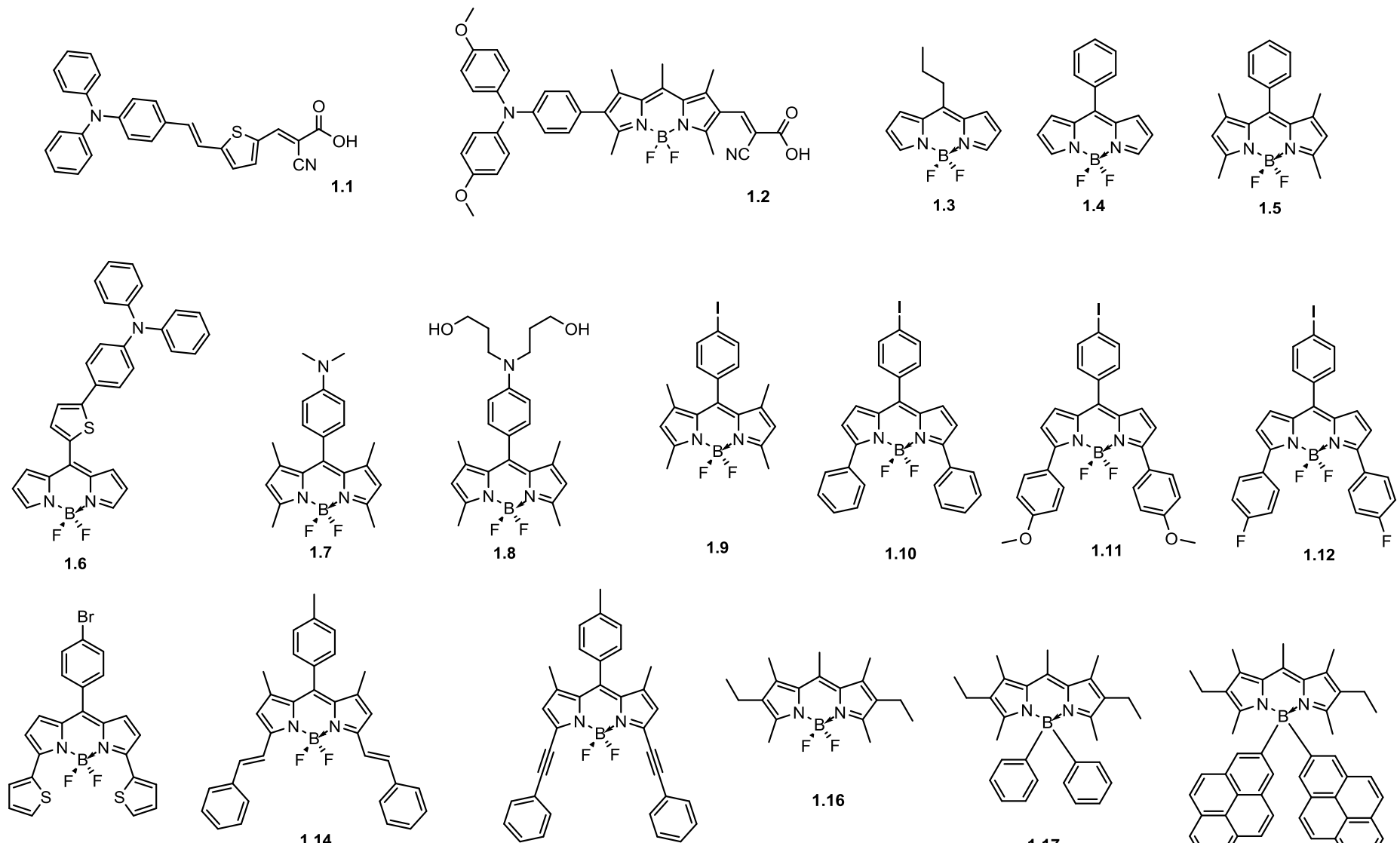

1.13
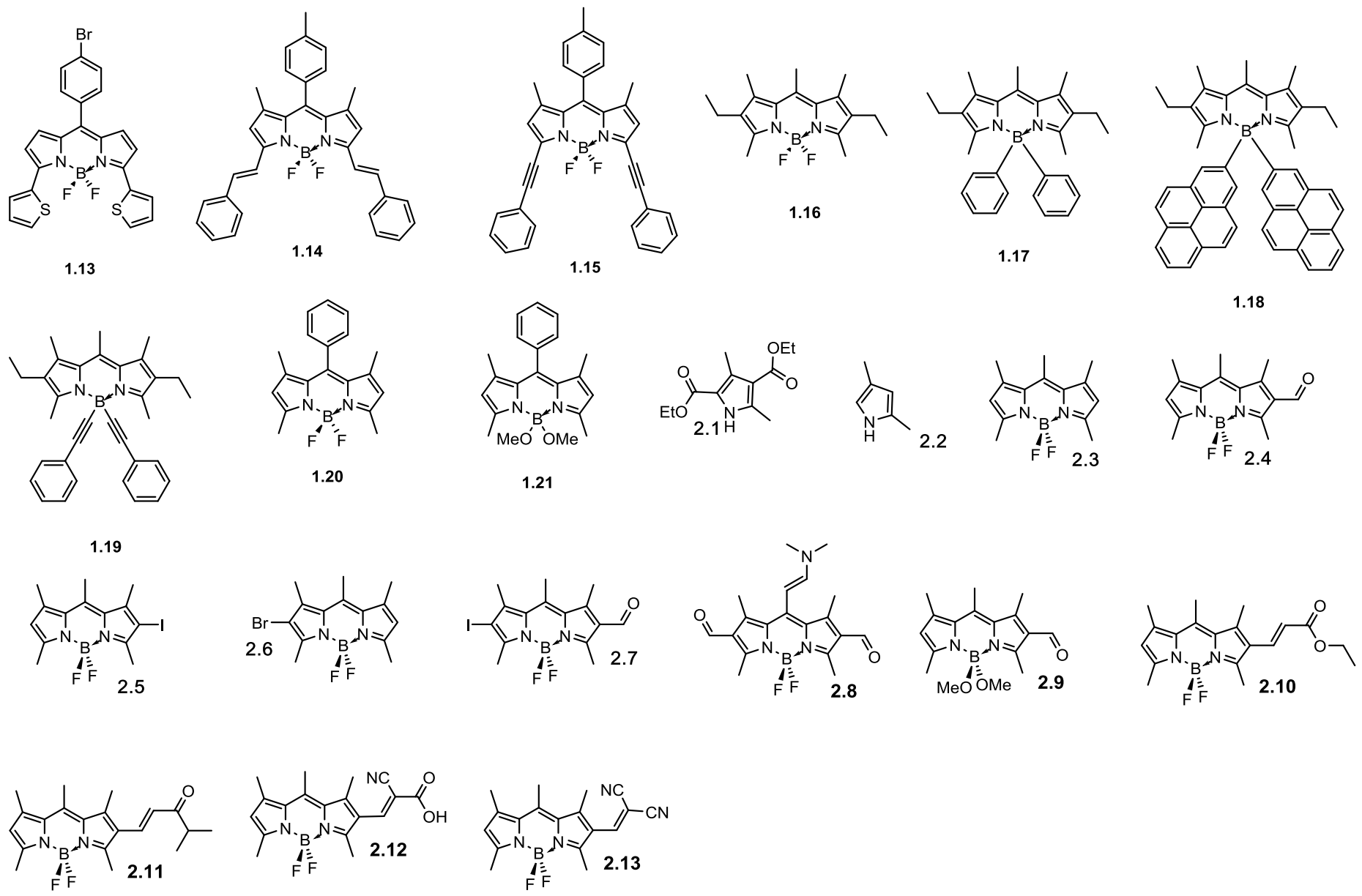
Fot

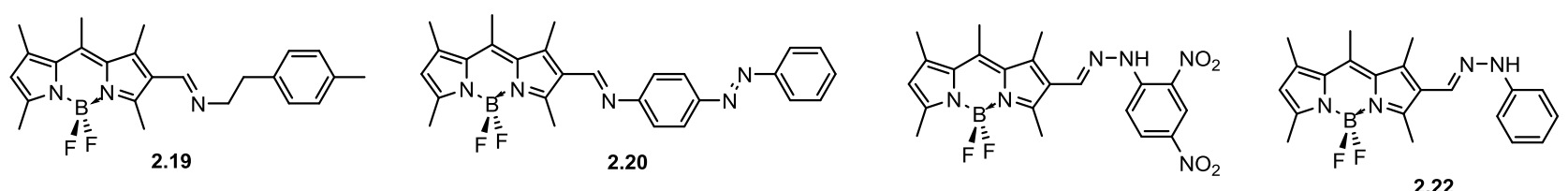

2.

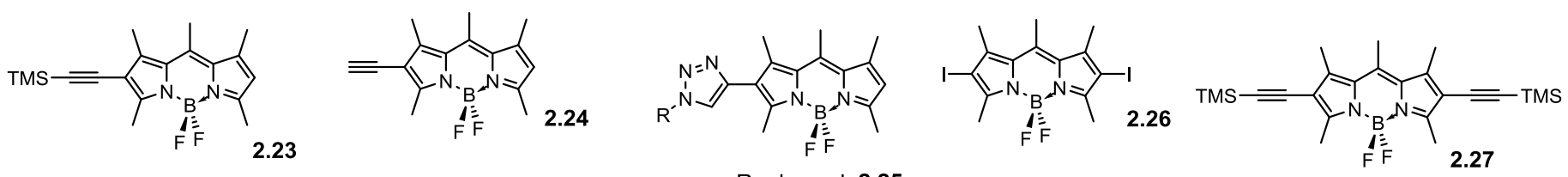

$\mathrm{R}=$ benzyl; 2.25a

= phenyl; 2.25b

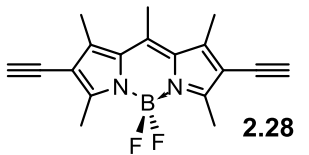

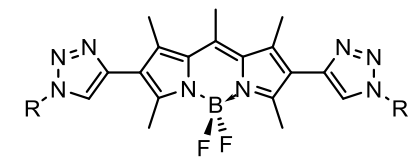

$\mathrm{R}=$ benzyl; 2.29a

$=$ phenyl; $2.29 \mathrm{~b}$

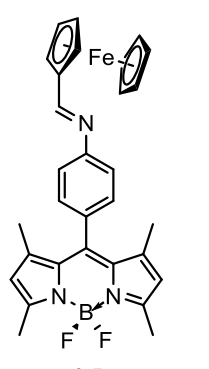

3.5

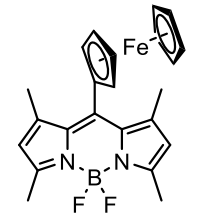

3.6

(9)

3.12

3.13

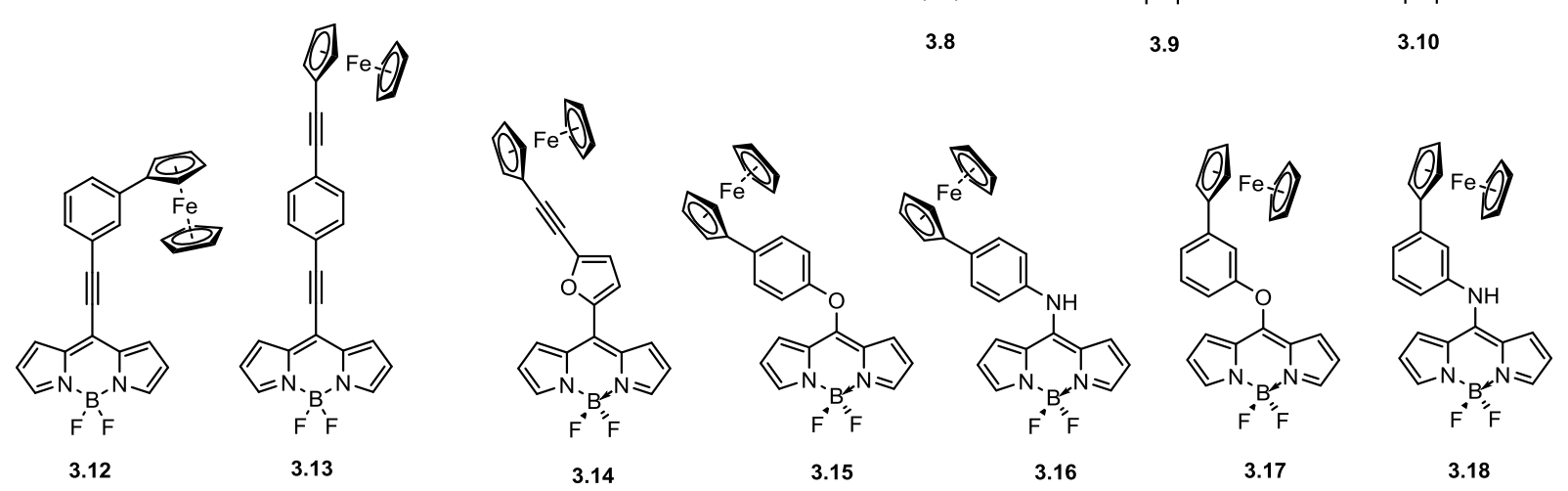

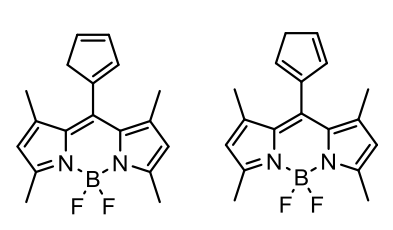

$3.7 \mathrm{a}$

3.7b

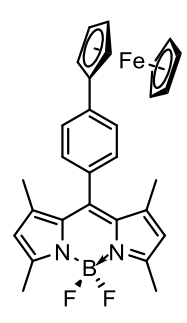

3.1

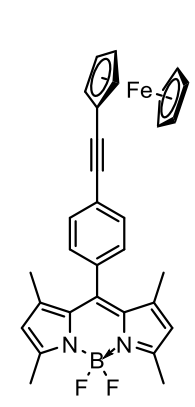

3.2

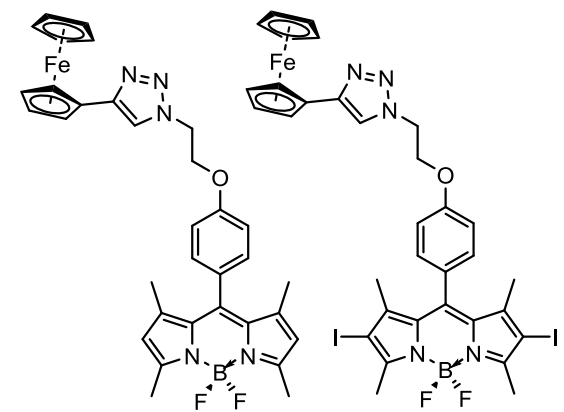

3.3

3.4

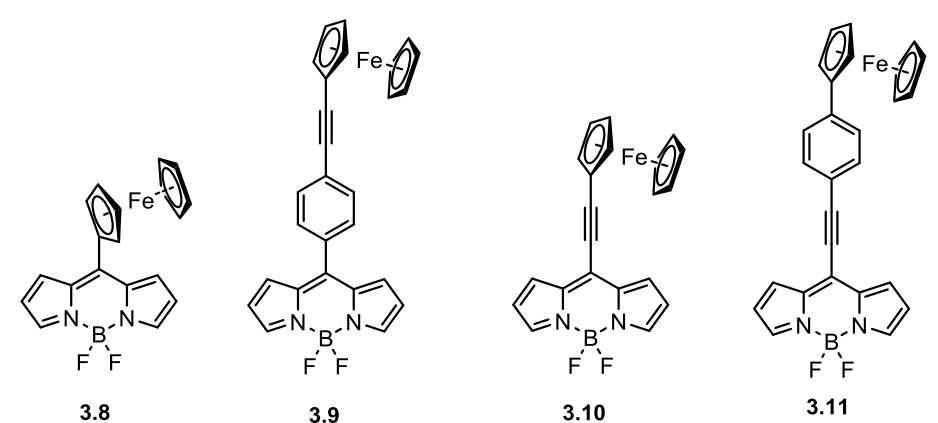




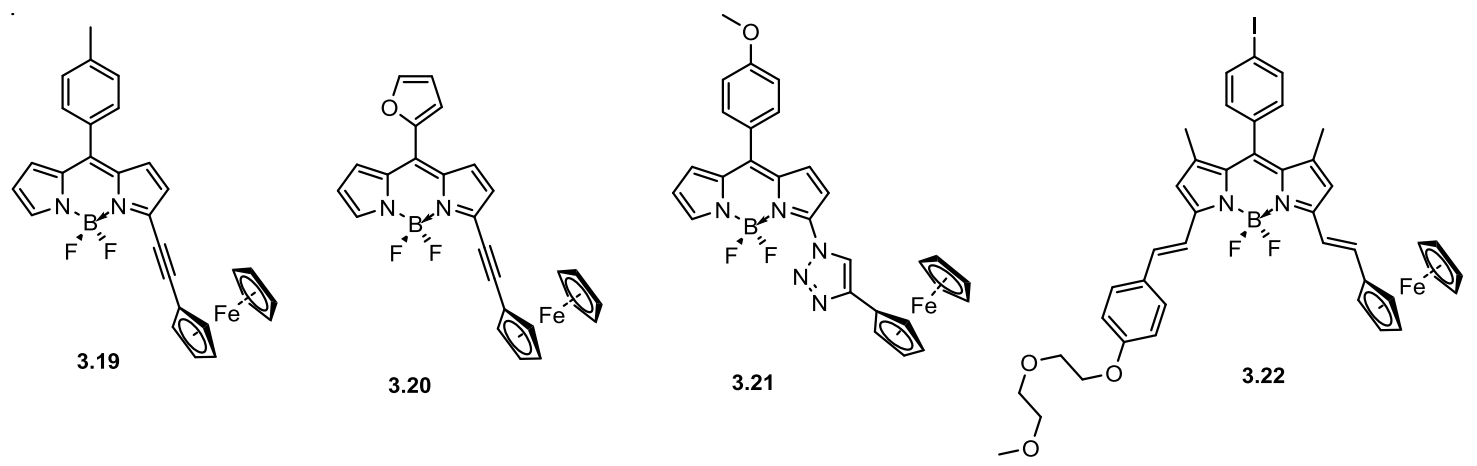

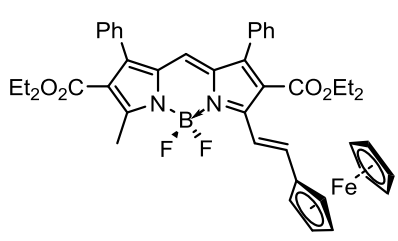

3.23

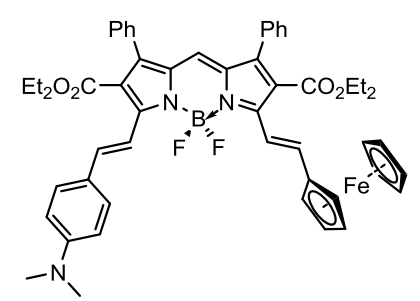

3.24

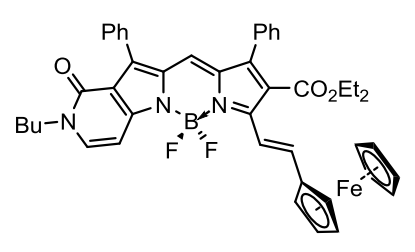

3.25
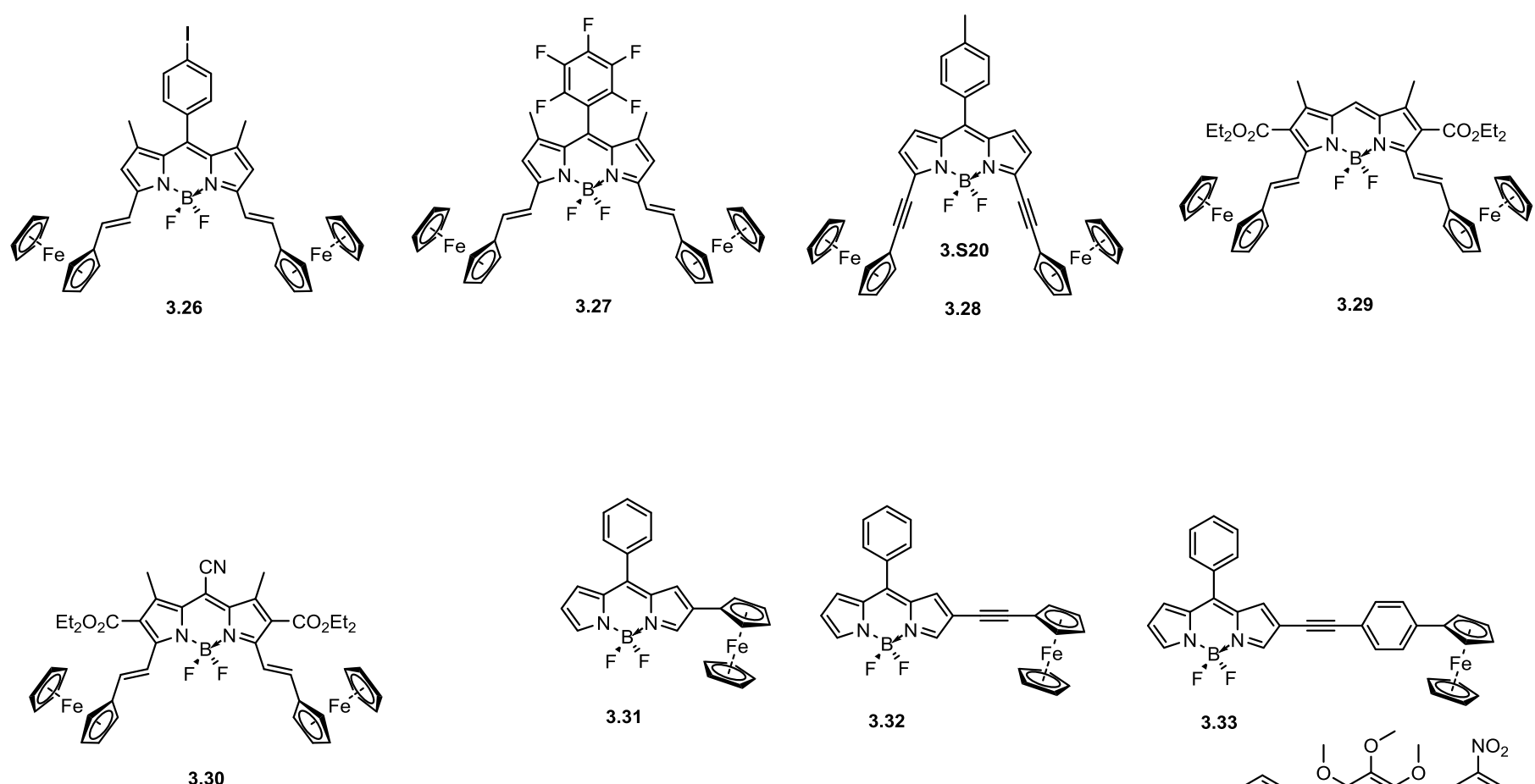

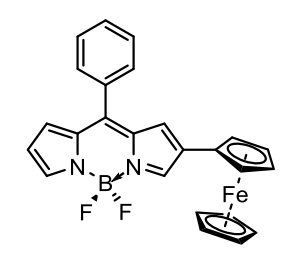

3.31

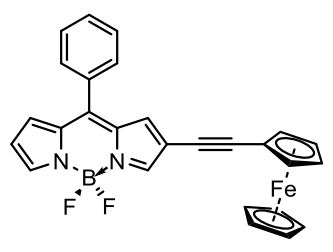

3.32
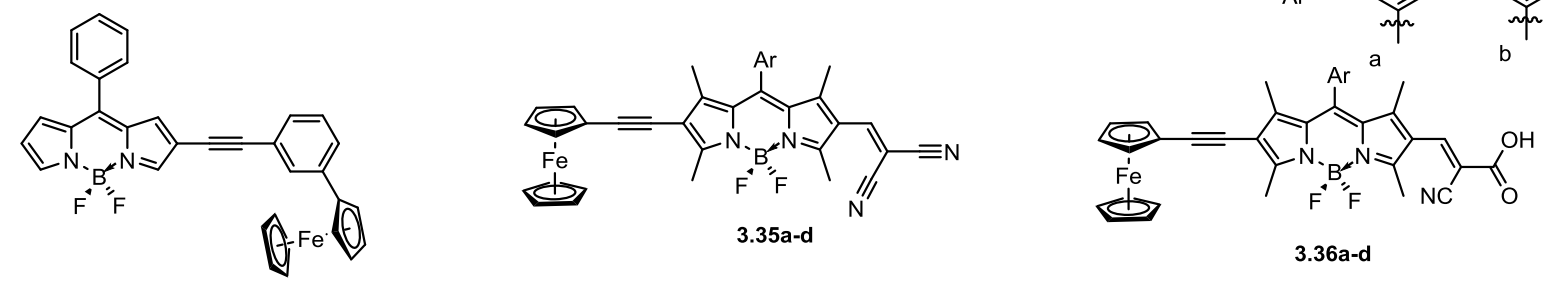

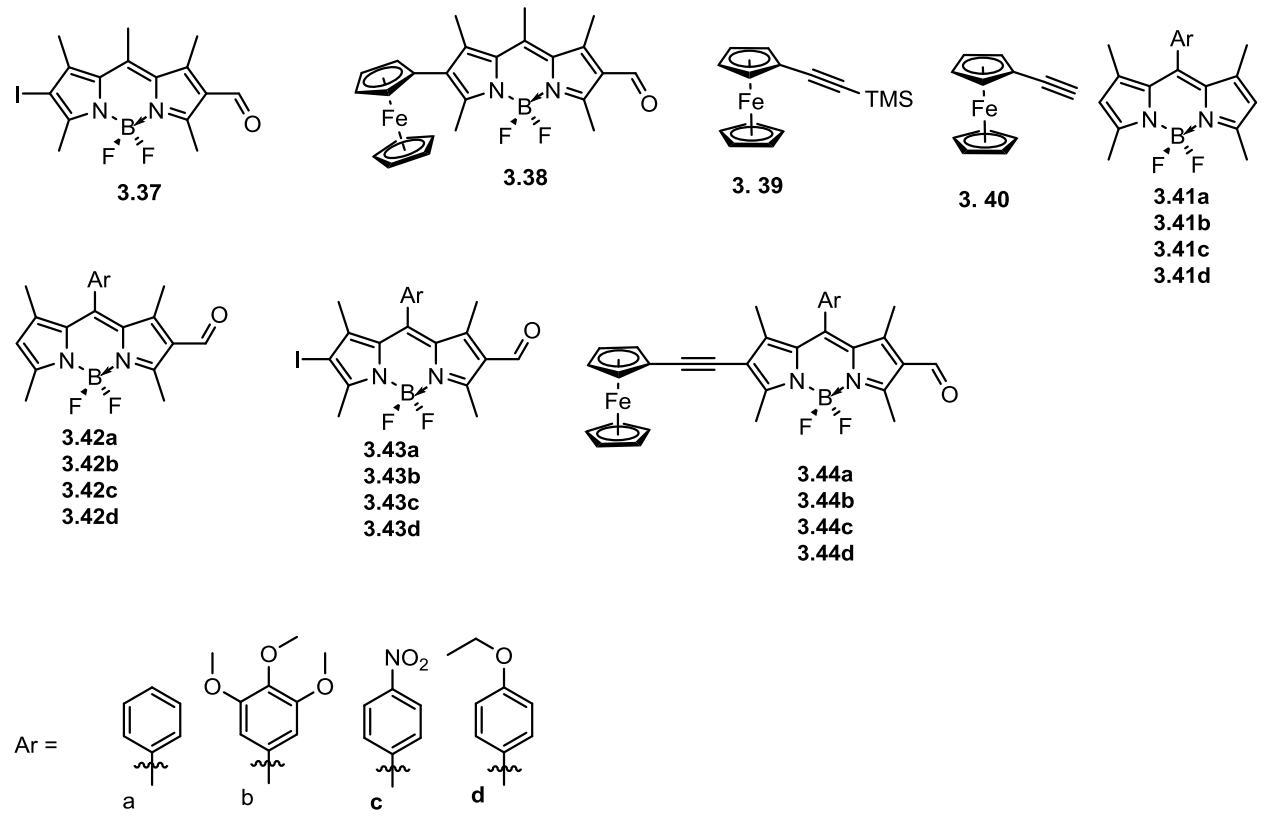


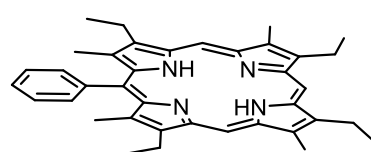

$\mathrm{Zn}\left(\mathrm{Et}_{4} \mathrm{Me}_{4} \mathrm{PhP}\right)$

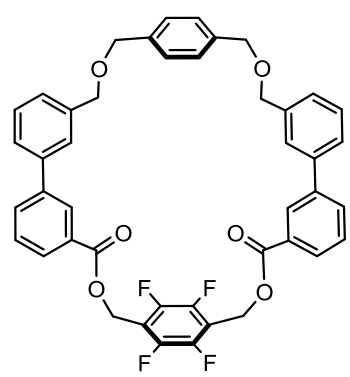

4.1

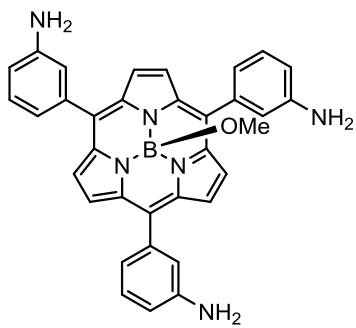

S1

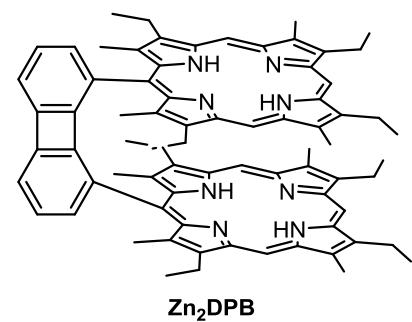

$\mathrm{Zn}_{2}$ DPB

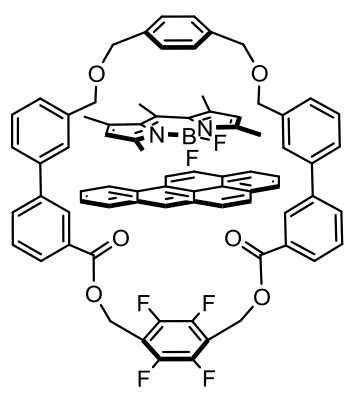

4.1- $G_{1} G_{2}$

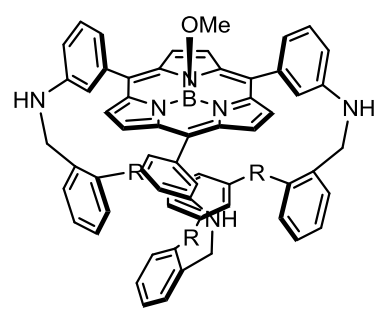

4.3

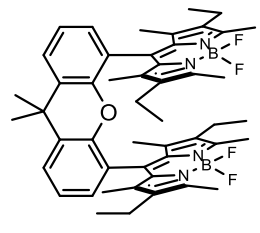

4S1

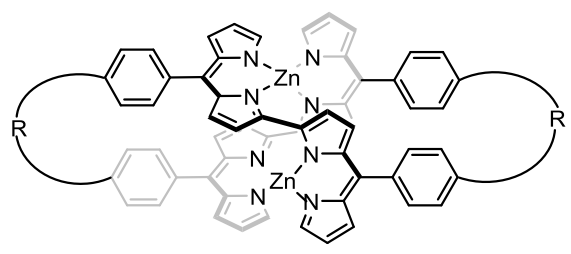

a $\mathrm{R}=\mathrm{O}\left(\mathrm{CH}_{2}\right) \mathrm{CH}=\mathrm{CH}\left(\mathrm{CH}_{2}\right) \mathrm{O}$ b R= $\mathrm{O}\left(\mathrm{CH}_{2}\right)_{2} \mathrm{CH}=\mathrm{CH}\left(\mathrm{CH}_{2}\right)_{2} \mathrm{O}$ c R $=\mathrm{O}\left(\mathrm{CH}_{2}\right)_{3} \mathrm{CH}=\mathrm{CH}\left(\mathrm{CH}_{2}\right)_{3} \mathrm{O}$ d R $=\mathrm{O}\left(\mathrm{CH}_{2}\right)_{4} \mathrm{CH}=\mathrm{CH}\left(\mathrm{CH}_{2}\right)_{4} \mathrm{O}$

4.2

a $\mathrm{R}=\frac{3}{3} \mathrm{O} \frac{4}{3}$

b $\mathrm{R}=\mathrm{s}^{\mathrm{s}} \prod_{\mathrm{O}}^{3 / 4}$

c $\mathrm{R}=\xi_{\xi} \mathrm{O} \sim \mathrm{O}^{\frac{\xi}{5}}$

d $\mathrm{R}=\xi_{3} \mathrm{O}+\mathrm{T}_{2} \mathrm{O} \frac{\xi}{\mathrm{s}}$

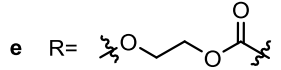




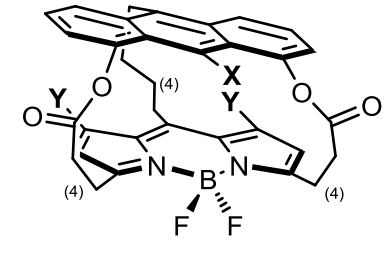

4.4

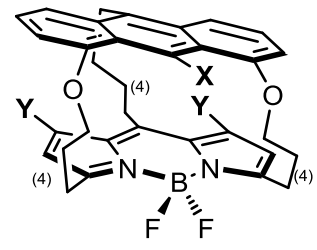

4.5

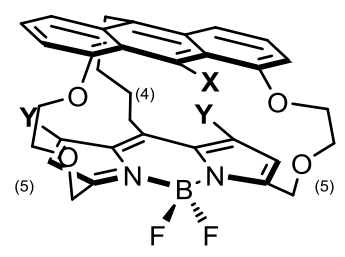

4.6<smiles>CC(=O)Oc1cccc2c1C(=O)c1c(OC(C)=O)cccc1C2=O</smiles>

4.7<smiles>CC(=O)Oc1cccc2cc3c(Br)ccc(OC(C)=O)c3cc12</smiles>

4.11<smiles>Cc1c[nH]c(C=O)c1</smiles>

4.16<smiles>Oc1cccc2c(Br)c3cccc(O)c3cc12</smiles>

4.12<smiles>O=Cc1cccn1[13S]</smiles>

4.17<smiles>CC(=O)Oc1cccc2cc3cccc(OC(C)=O)c3cc12</smiles>

4.8<smiles>O=Cc1cc(Br)c[nH]1</smiles>

4.14<smiles>CN(C)C1c2cc(Br)cn2C(N(C)C)c2cc(Br)cn21</smiles>

4.15<smiles>COC(=O)CCc1ccc[nH]1</smiles>

4.21<smiles>CCOC(=O)CCc1cccn1S</smiles>

4.22<smiles>Brc1c2cccc(OCCCc3ccc[nH]3)c2cc2c(OCCCc3ccc[nH]3)cccc12</smiles>

4.26

4.27<smiles>O=C(O)CCc1cccn1[As]</smiles>

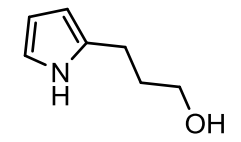

4.23<smiles>[Se-]OCCCc1ccc[nH]1</smiles>

4.24

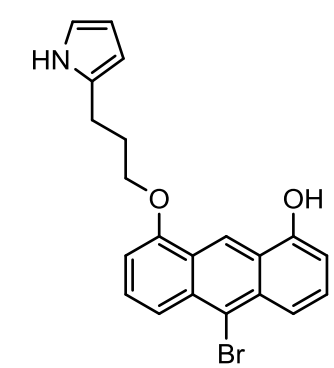

4.28 


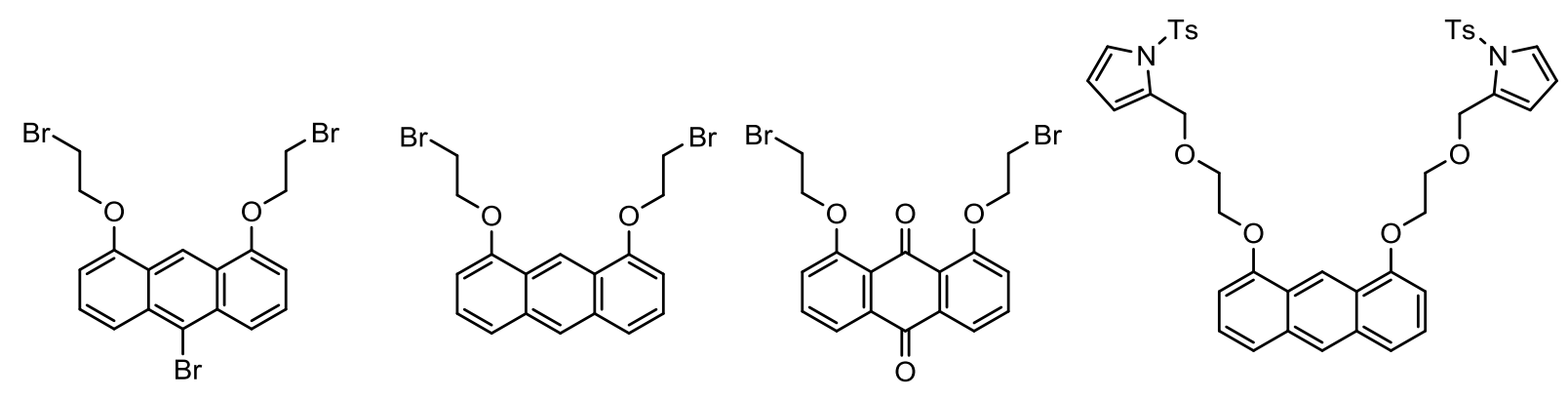

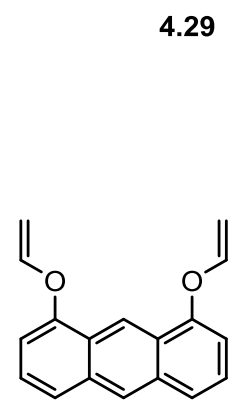

4.33

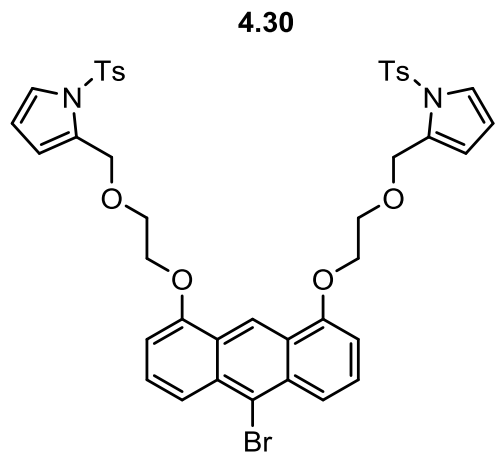

4.34

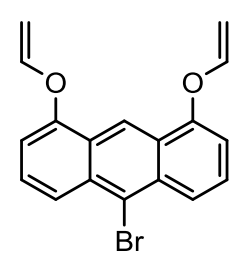

4.35
4.32<smiles>O=C(CCc1cccn1[As])Oc1cccc2cc3cccc(OC(=O)CCc4cccn4[As])c3cc12</smiles>

4.36 


\section{List of Abbreviations}

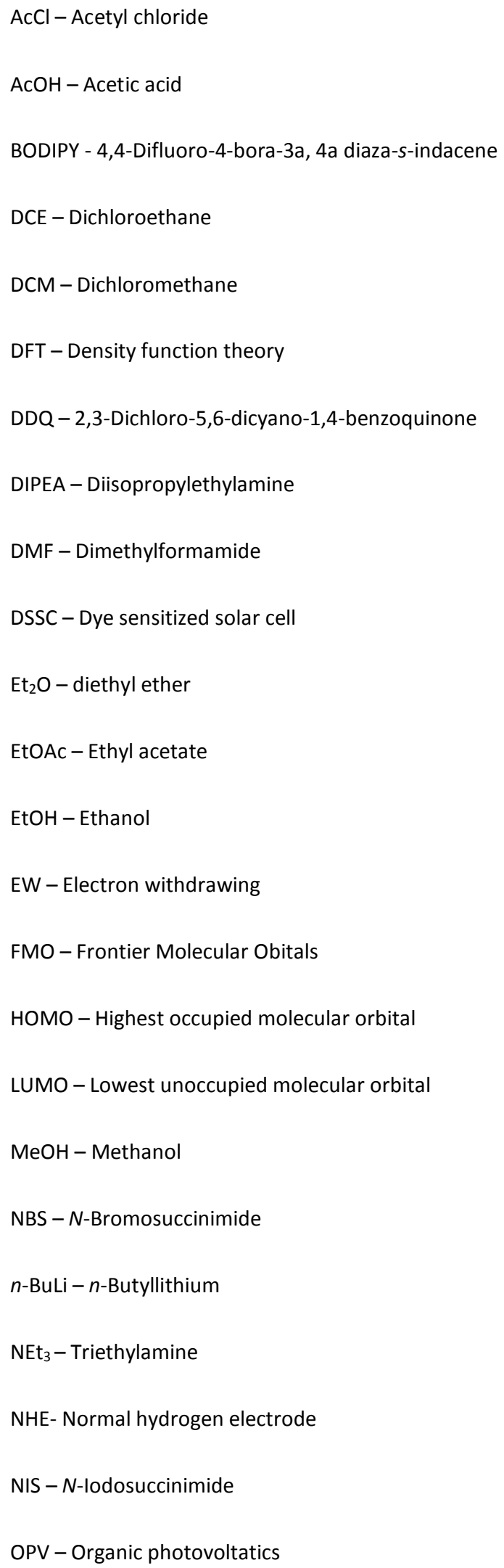


ORTEP - Oak Ridge thermal ellipsoid plot

PCC - Pyridinium chlorochromate

$\mathrm{Pd} / \mathrm{C}-$ Palladium on carbon

1, 10 Phen $-1,10$ Phenanthroline

PV - Photovoltaic

TD-DFT - Time dependent density functional theory

TFA - Trifluoroacetic acid

THF - Tetrahydrofuran

TPA - Triphenylamine

$p$-TsOH $-p$-Toluenesulfonic acid 


\section{Chapter 1: Introduction}

\section{Introduction}

As the demand for technological advances in optoelectronics rises, there has been a concurrent push towards novel organic materials. In order to develop new organic materials for optoelectronic devices, it is important to understand photophysical behaviours relating to electronic structure of chromophores such as light absorption or emission and charge transport within these materials. This introduction chapter aims to review the underlying photophysical behaviour in chromophores and fluorophores and how these properties are applied to make materials suitable for applications as near-IR dyes, photovoltaics and medical imaging. This review will provide a platform from which to better appreciate the physical properties in the chapters that follow. The exploration of synthetic routes towards such novel light harvesting compounds will be discussed in each chapter.

\subsection{Electronic structures of chromophores}

In order to understand underlying photophysical behaviour in light absorbing chromphores, one must understand frontier molecular orbital theory. The simplest molecule, hydrogen, can be used as an example to demonstrate frontier molecular orbital theory. Referring to Figure 1.1a, when two hydrogen nuclei form a covalent $\sigma$ bond, two molecular orbitals are produced. The highest occupied molecular orbital (HOMO) contains two electrons between the two nuclei forming the bonding molecular orbital, and it is more stable than the $1 \mathrm{~s}$ atomic orbitals of the hydrogen atom. The lowest unoccupied molecular orbital (LUMO) forms the anti-bonding molecular orbital which contains a node in the electronic wave function and has no electrons shared between the two hydrogen nuclei in the ground state. The LUMO is more destabilized compared to the $1 \mathrm{~s}$ atomic orbital, which in turn creates an energy gap between the two molecular orbitals. 


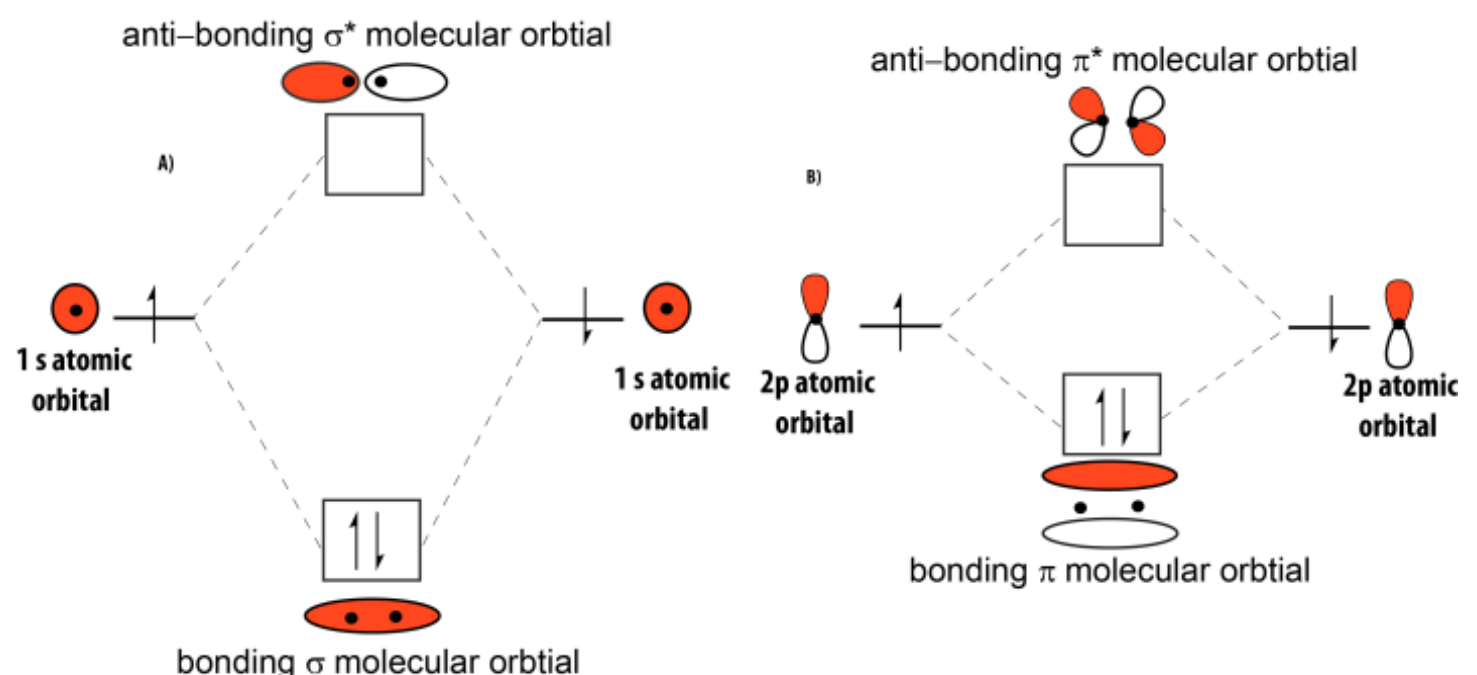

c)

Figure 1.1: Molecular orbital diagram representing a) the formation of $\sigma$-bonding and anti-bonding molecular orbitals from two $1 \mathrm{~s}$ atomic orbitals $\mathrm{b}$ ) the formation of $\pi$-bonding and anti-bonding molecular orbitals from two $1 \mathrm{~s}$ atomic orbitals $\mathrm{c}$ ) formation of a $\pi$-bond in ethylene

In larger molecules that contain $\pi$ bonds such as ethylene (Figure 1.1b), the energy gap between the HOMO and LUMO is smaller due to the introduction of a $\pi$ bond (Figure 1.1c). The formation of the $\pi$ bond creates new frontier molecular orbitals (FMOs), and that are closer together. The $\pi$ electrons in extended molecular orbitals through conjugation obey the "particle in the box" model and when molecules are conjugated, the electrons can delocalize throughout this extended $\pi$ system (the box). Lengthening the "box" increases the number of FMO energy levels (Figure 1.2), and decreases the energy gap between the HOMO and LUMO levels. Example of a larger $\mathrm{p}$ systems is ferrocene (Figure 1.2) in which there is a large amount electrons within the molecule (access to $d$ orbitals). However, the HOMO is in the p orbitals (contribution from both Fe $4 p$ orbitals and ligand $p$ orbital) and the energy gap is much smaller compared to ethylene. 


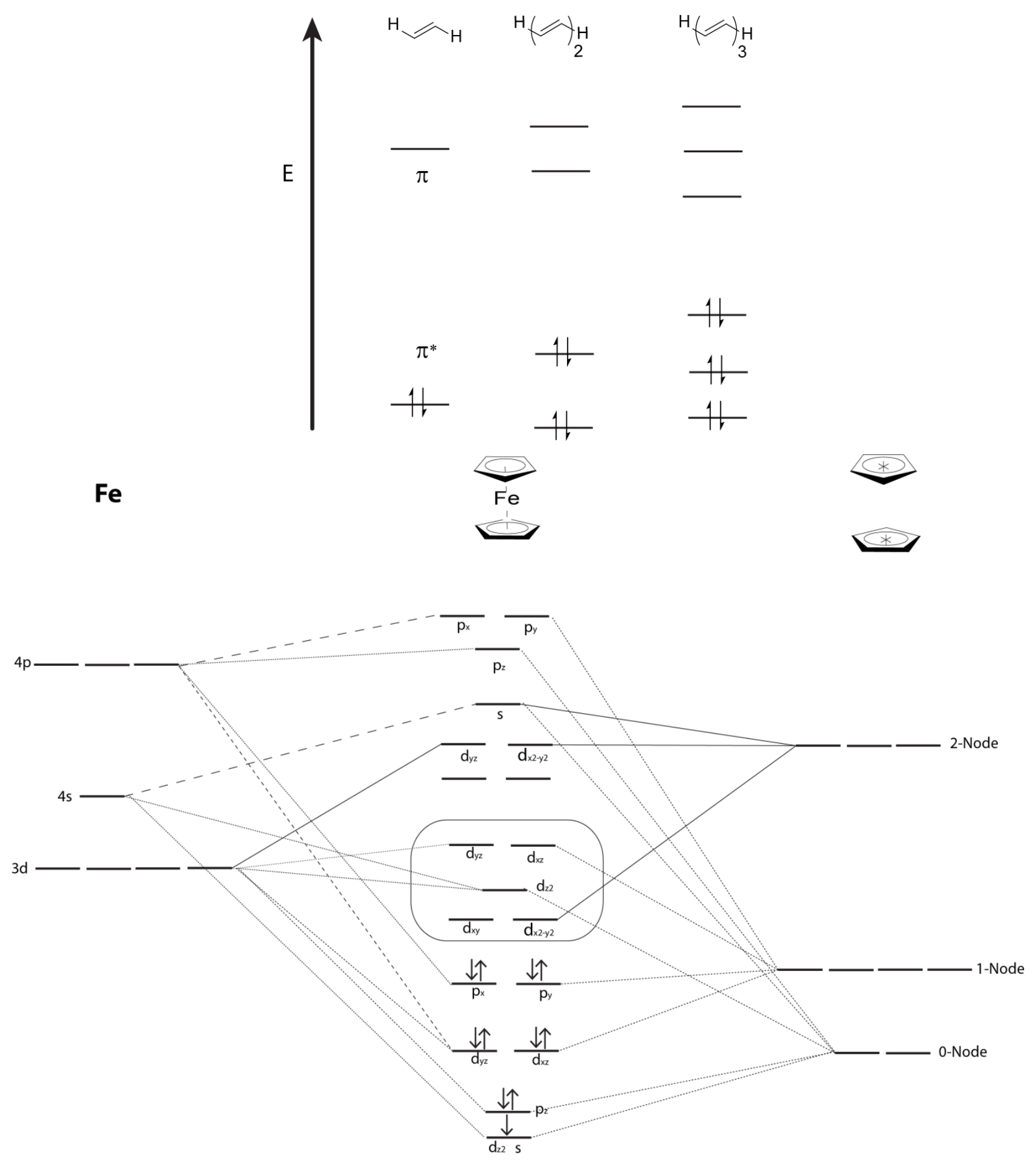

Figure 1.2: Molecular orbital diagrams showing the frontier orbitals of ferrocene and the effect of increasing the number of conjugated $\pi$ - bonds

\subsection{Frank-Condon Principle: Absorption Intensity}

In order for an organic chromophore to absorb a photon of light effectively (with a high molar extinction coefficient), at the photo cross-section of the molecule, there needs to be significant molecular orbital 
overlap between the ground and excited state level. This is best described using the consequences of the Frank-Condon Principle described below.

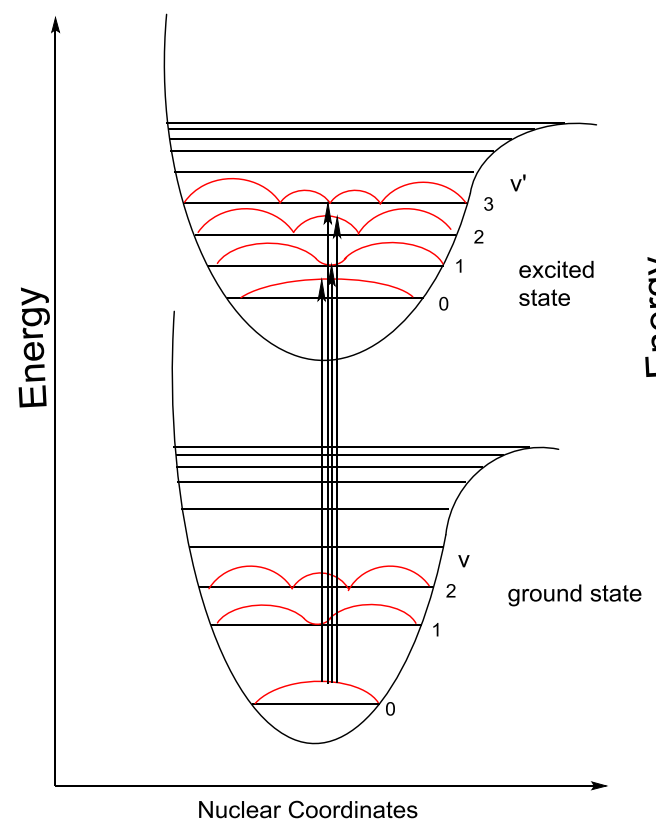

A

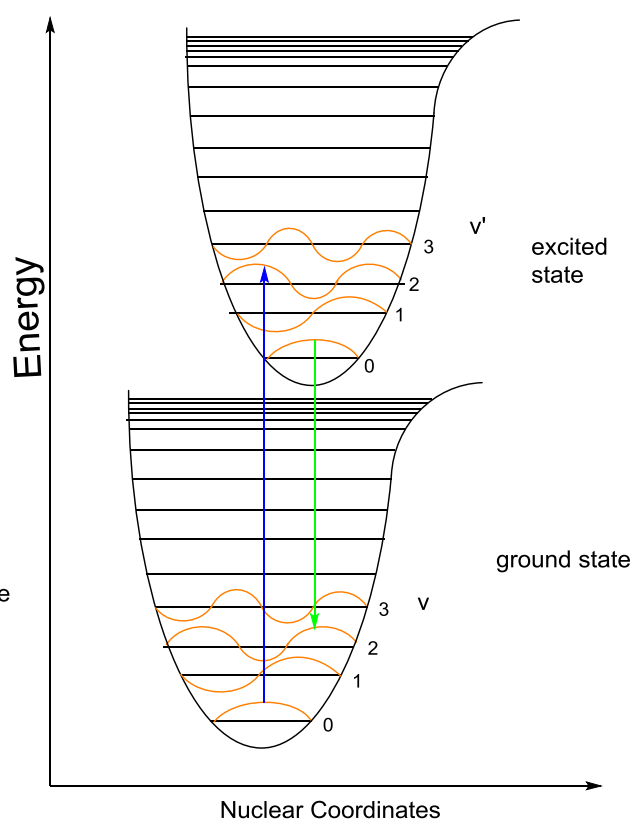

B

Figure 1.3: A) Potential energy curves for the ground state and an excited state of a homo-diatomic molecule B) Potential energy curves for the ground state and an excited state of a hetero-diatomic molecule

The energy gained through the absorption of a photon can cause vibrational, rotational and electronic transitions within a chromophore. For electronic transitions, each energy level is accompanied by a subset of vibrational quantum levels. Figure 1.3 illustrates the potential energy curves of homo-diatomic (a) and hetero-diatomic molecules (b). In both cases, there are two profiles; one is the ground state (bottom) and the other represents the excited state (top). These potential energy curves consist of a number of vibrational sublevels according to an anharmonic oscillator approximation. Considering that nuclei are heavier than electrons, when a photon is absorbed by a chromophore the excitation of an electron is much faster than nuclear rearrangement. Therefore, when light strikes a molecule, an electron is excited before any other process occurs. In order to have an intense absorption of light, there has to be significant overlap of the vibration wave functions in the ground and excited state and the dominant electronic transition occurs between states with the most significant overlap. Therefore, as highlighted in Figure 1.3a the most intense absorption would be from $v=0$ to to $v^{\prime}=0$. In Figure $1.3 b$, the most intense absorption 
would be $v=0$ to $v^{\prime}=2$. This principle also applies to fluorescence (reverse of absorption); however, there is a bathochromic shift for the fluorescence for hetero diatomic molecules (b) due to Kasha's rule. ${ }^{1}$

\subsection{Kasha's Rule and Stokes Shift: After Absorption}

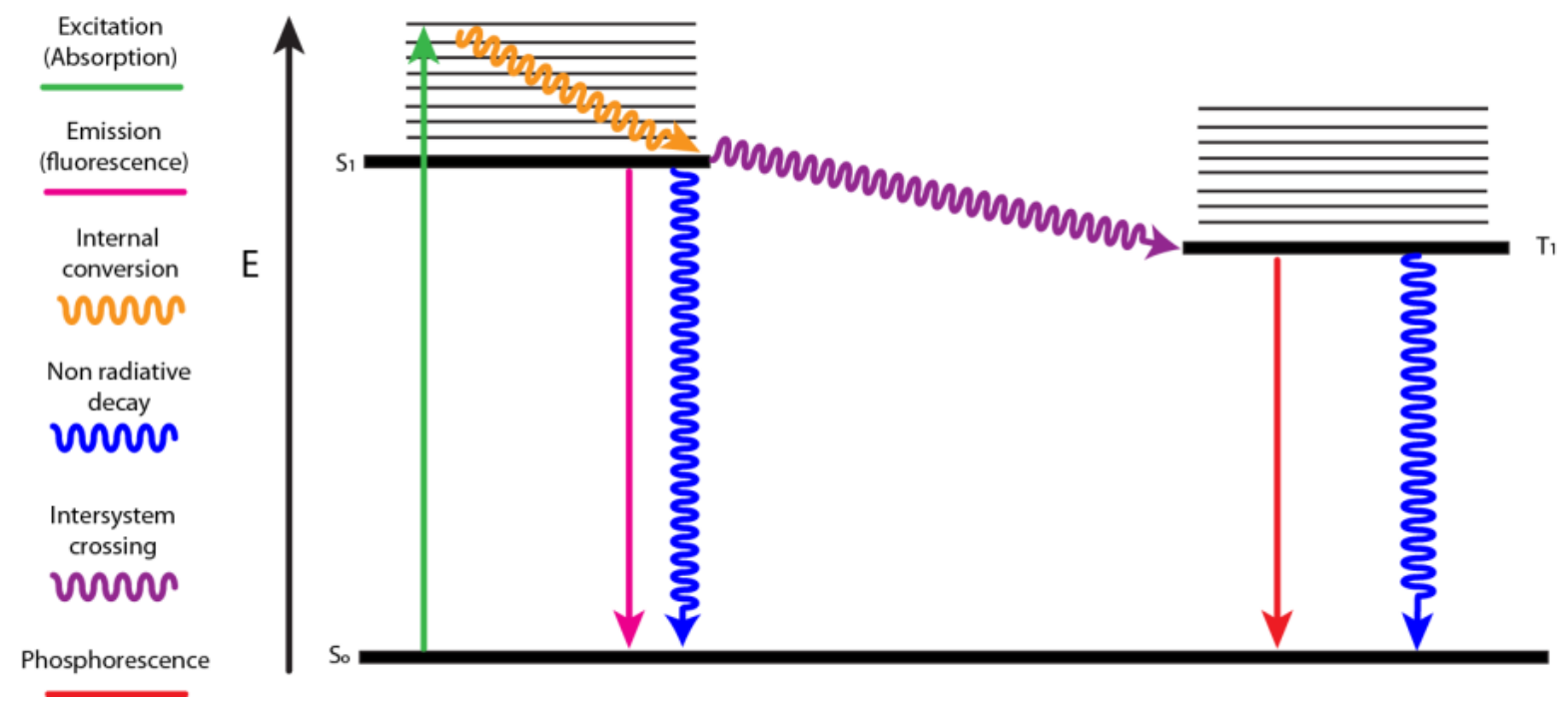

Figure 1.4: Jablonski diagram descripting unimolecular excitation/relaxation pathways.

Kasha's rule states that emission of a photon will occur from the lowest excited ground state (which is usually the LUMO) because internal conversion (vibrational relaxation) occurs faster than emission. ${ }^{1}$ The Jablonski diagram (Figure 1.4) illustrates that after a chromophore absorbs a photon, an electron is transferred from a ground $\left(S_{0}\right)$ to excited state $\left(S_{1}\right)$. This electron will eventually come back down to the ground state through radiative (fluorescence) or non-radiative decay. If a photon is emitted (radiative), it will have a longer wavelength than the photon that was absorbed due to the rapid loss of vibrational energy (internal conversion) in the excited state. The difference in energy between absorption and emission is known as the Stokes shift. Phosphorescence is yet another possibility if intersystem crossing can occur. Intersystem crossing is most commonly observed in molecules that contain heavy atoms, due to the introduction of more vibration energy levels, and the degree of spin and orbital interactions (spin orbit coupling) increases. This spin orbital coupling causes the inversion of spin to become more favorable, which in turn favours intersystem crossing. The Jablonski diagram describes unimolecular excitation/relaxation pathways that can occur, however, energy transfer pathways also can occur between two or more molecules as well. 


\subsection{Photoinduced energy and electron transfer processes}

Referring to Figure 1.5, if the lifetime of a chromophore (A-donor) in the excited state is sufficient, it can interact with another chromophore (B-acceptor) in the environment and undergo different electron/energy transfer processes if certain criteria are satisfied. Bimolecular processes like these are important because it can determine the photophysical properties of large $\pi$ systems such as fluorescence quenching, reactivity, and chemical changes to other molecules in the environment.

$$
\begin{aligned}
A+B+h v \longrightarrow & A^{*}+B \quad \text { photoexcitation } \\
A^{*}+B & A^{+}+B^{-} \text {oxidative electron transfer } \\
A^{*}+B & \longrightarrow A^{-}+B^{+} \text {reductive electron transfer } \\
A^{*}+B & \longrightarrow A+B^{*} \text { energy transfer }
\end{aligned}
$$

Figure 1.5 Bimolecular electron/energy processes with a light absorbing molecule unit $(A)$ and the other molecular (B)

Bimolecular energy transfer can occur when molecules are in close proximity, particularly in large $\pi$ systems. Energy transfer can be viewed as non-radiative transition between two excited states of two different molecules. Bimolecular energy transfer occurs from excited chromophore (donor) to accepting chromophore (acceptor). There can be two modes of energy transfer (Figure 1.6): 1) Förster resonance energy transfer (FRET); 2) Dexter energy exchange. 


\section{Förster resonance energy transfer}
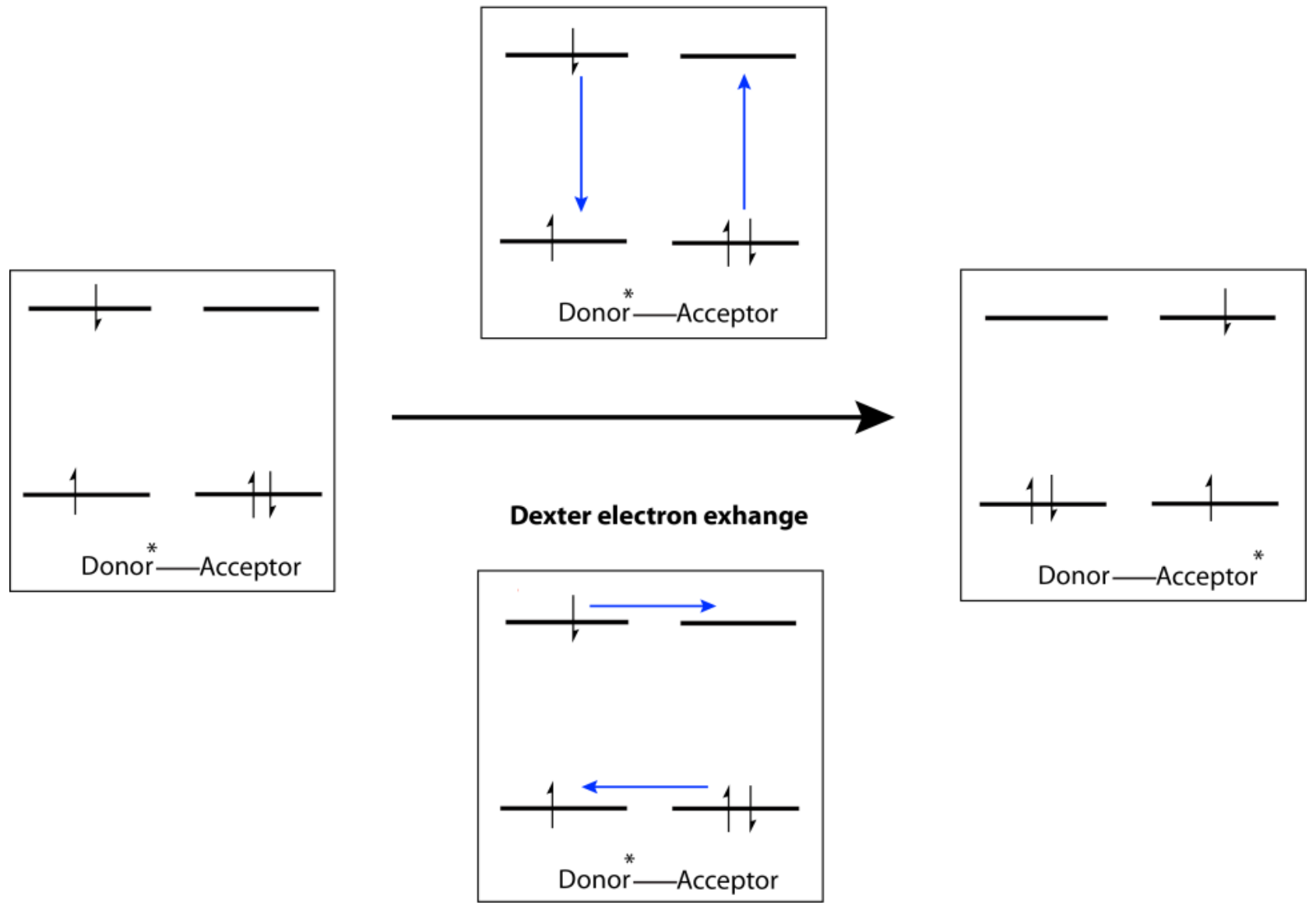

Figure 1.6: Pictorial representation of energy transfer through FRET and Dexter electron exchange

\subsubsection{Förster resonance energy transfer}

Resonance energy transfer occurs through long range dipole-dipole coupling (through space) (Figure 1.6). Under the right conditions (vide infra), an excited donor chromophore can act as an oscillating dipole that can undergo an energy exchange with a second dipole (acceptor) that has a similar resonance frequency. FRET dipole-dipole coupling is analogous to the classic pair of tuning forks vibrating at the same frequency. A consequence of this type of energy transfer is the decrease or quenching of the emission from the donor and can be accompanied by an increased intensity in fluorescence of the acceptor. However, there are criteria that must be met in order for FRET to occur. These criteria are: 1) the emission of the donor must have sufficient overlap with the absorption profile of the acceptor chromophore. 2) The donor and acceptor must be in close proximity (10-100 ̊). 3) The excited state lifetime of the donor must live long enough for FRET to occur. 4) The orientation of the donor dipole must be parallel to the acceptor dipole. $2-3$ 


\subsubsection{Dexter energy transfer}

Dexter electron exchange can be viewed as a double electron processes that results in energy transfer from one molecule to another (Figure 1.6). The excited electron from the LUMO of the donor travels to the LUMO of the acceptor, and the other electron travels from the HOMO of the acceptor to the LUMO of the donor (hole transfer). In order for Dexter electron exchange to occur, significant $\pi$ orbital lap is required. As a consequence the distance between the two chromophores for Dexter energy transfer to occurs is small (10 $\AA$ ) compared to FRET. Quenching of the emission from the donor also occurs in this type of energy transfer. Also solvation of the chromophore can affect Dexter energy transfer due to the movement of electron. This solvent-dependent property is not seen in the FRET due to the lack of electron transfer.

\subsubsection{Photoinduced electron transfer}

Unlike Dexter electron transfer, photoinduced electron transfer is a one electron process from an electron donating molecule to an acceptor that results in a charge transfer state(or vice versa) (Figure 1.7). This charge separated state contains both radical cation (donor) and anion (acceptor) that completes with other excitation/relaxation pathways of the two chromophores. Photoinduced electron transfer also quenches emission from the donor and decreases the excited state.

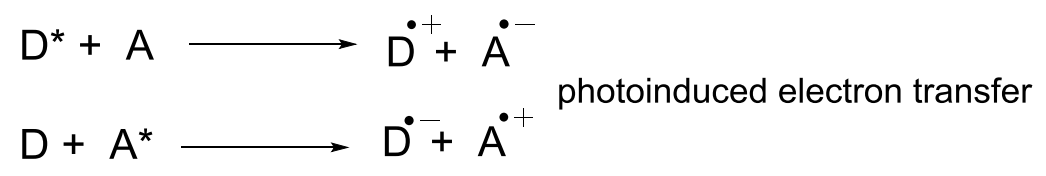

Figure 1.7: Bimolecular photoinduced electron transfer with a light absorbing molecule unit (D) and the accepting molecular (A)

The importance of photoinduced electron transfer cannot be overstated, as it is common in multiple applications such as photosynthesis, photovoltaics ${ }^{4,5}$, even photography. ${ }^{3}$

\subsection{Dye sensitised solar cell (DSSC)}

The Koivisto Research group has applied their efforts towards the preparation of novel organic materials for light harvesting and photovoltaics, with the primary focus on the dye sensitized solar cell (DSSC) as a promising next generation photovoltaic technology. ${ }^{6-8}$ The design principles of dye-sensitised solar cell (DSSC) is shown in Figure 1.7 where dye molecules are adsorbed onto the surface of a nanoparticle mesoporous metal oxide semiconductor film (titanium dioxide anode, $\mathrm{TiO}_{2}$ ). This dye/nanoparticle anode 
is supported by an indium-doped tin oxide conducting glass (Figure 1.7). The device is then sandwiched with an ITO cathode that has a thin layer of platinum to catalyze the regeneration of the electrolyte.
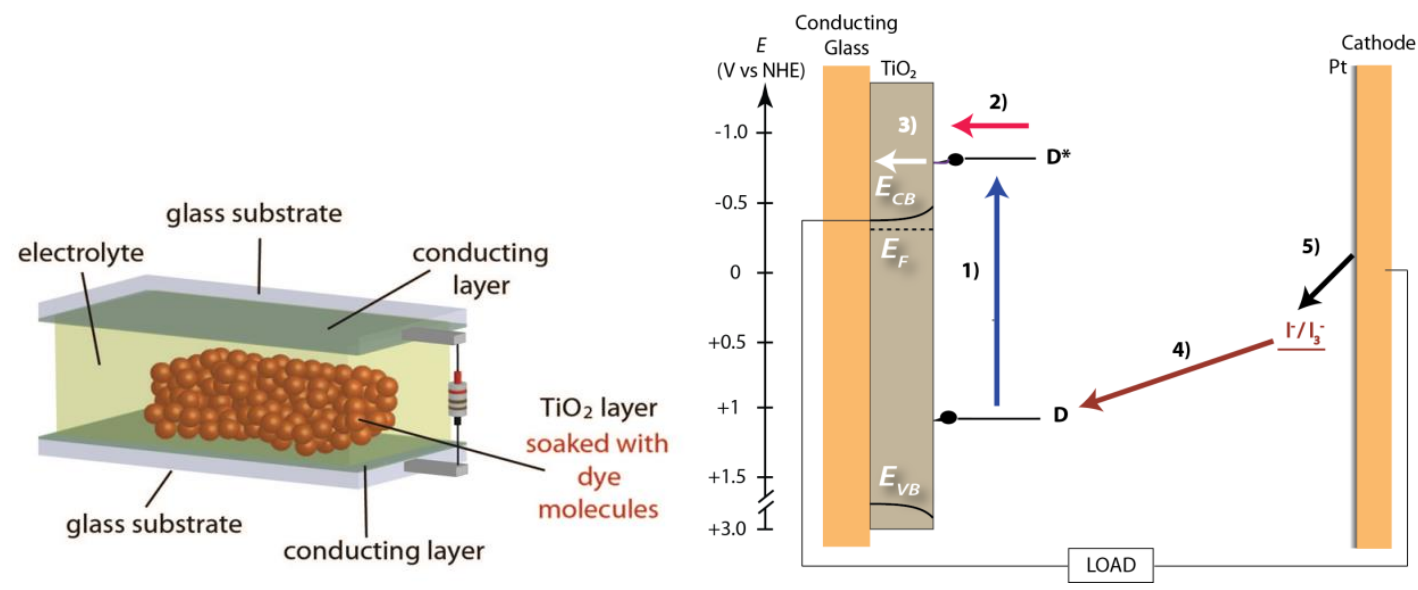

Figure 1.8: Schematic representation of a DSSC

Figure 1.8 shows the working principle of a DSSC. When light strikes the dye: 1) the absorption of a photon by the chromophore generates an excited state which undergoes electron transfer; 2) an electron from the dye excited state is injected into the conducting band of $\mathrm{TiO}_{2}$, and it is important that this excited state is higher in energy than the titania conduction band; 3 ) The electrons percolates through the $\mathrm{TiO}_{2}$ layer to the conducting class; 4) The dye is regenerated by electron transfer from a redox active electrolyte (such as the $\mathrm{I}^{-} / \mathrm{I}_{3}^{-}$redox couple); 5) The electrolyte is regenerated by the platinized cathode to complete the circuit. This represents the ideal thermodynamic pathway for the operation of the DSSC. However, there are many competing processes which may act to reduce the overall cell performance. Figure 1.8 highlights competing processes that hinder DSSC performance.

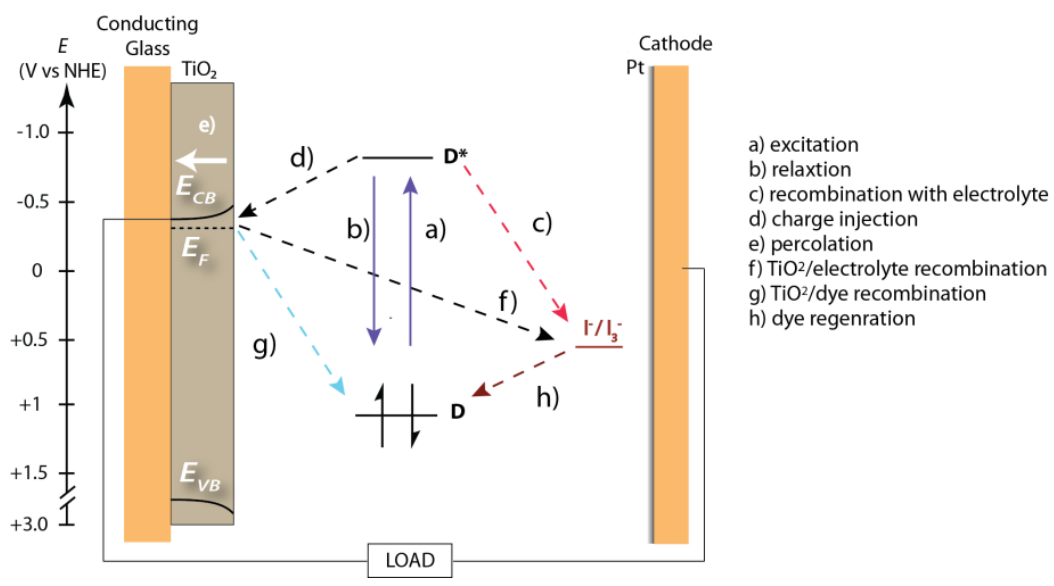

Figure 1.9: Competing thermodynamics pathways in the DSSC 
After photo-excitation (a) of the dye sensitiser (D) generates an excited state ( $\left.D^{*}\right)$ which may either undergo the desired charge injection (d) or relax back to the ground state either by emission or by nonradiative decay (b). Also the device can short circuit by transfer of the excited electron to electrolyte solution (c). In order for charge injection to be successful, the rate of electron injection must be faster than the rate of emission/non-radiative decay. Once the charge injection occurs into the conduction band of $\mathrm{TiO}_{2}$, it may also undergo several pathways. One of the pathways is the desired percolation of the electron through $\mathrm{TiO}_{2}$ (e). ${ }^{9}$ However, charge recombination can occur with dye radical cation (g) or oxidized electrolyte (f). If the desired pathways are followed, then the dye should be regenerated by the electrolyte (h). All components of the DSSC can be modified to maximize the efficiency of these devices. In the Koivisto group, the interest focus is the dye molecule, which acts as the device's power house and is responsible for light absorption.

\subsubsection{Dye design}

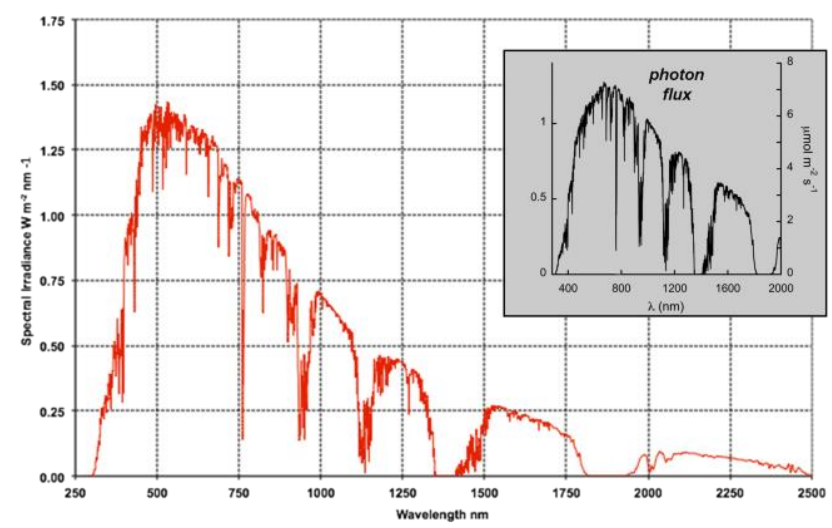

Figure 1.10: Solar irradiance and inset of photon flux

The power house of DSSC is the dye sensitiser, therefore, theses chromphores have to harvest as much solar energy as possible. The solar spectrum and photon flux diagrams are shown in Figure 1.9 illustrates the terrestrial electromagnetic spectrum. Little energy can be harnessed in the UV region of the electromagnetic spectrum due to the ozone layer. Due to this photon flux issue, dyes for DSSC applications must be have a panchromatic absorption profile, particularly in the near infrared (NIR) region. 

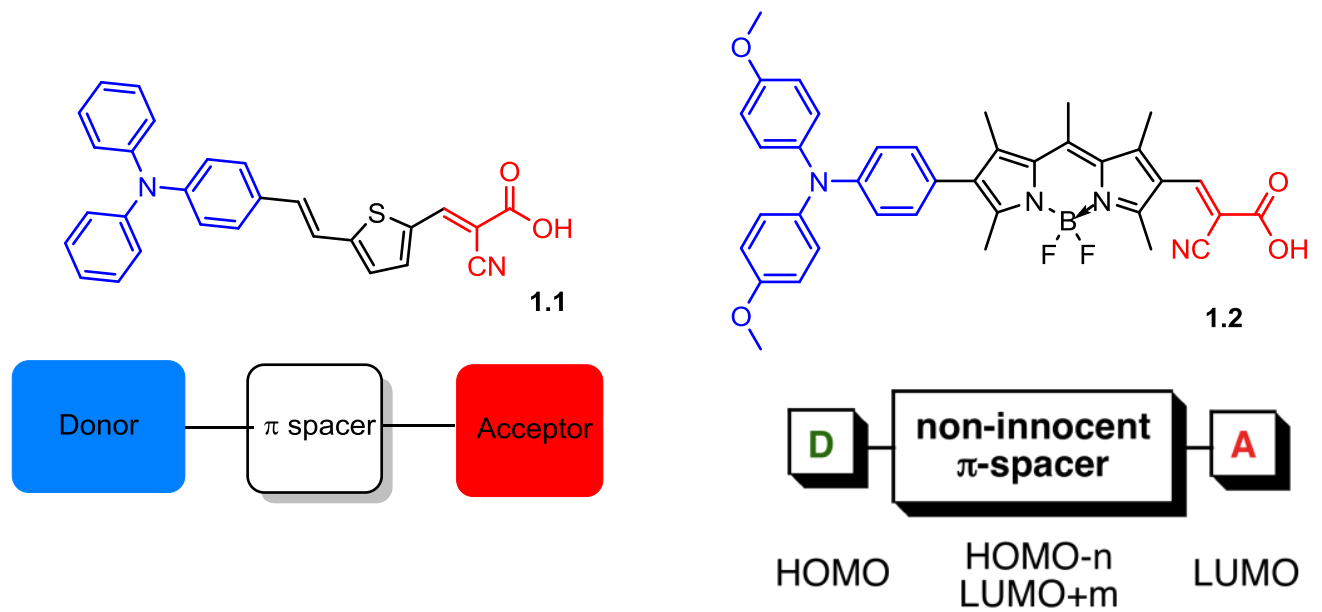

Figure 1.11: Organic DSSC dyes ${ }^{7-10}$

In order to achieve such panchromatic absorption profiles, organic DSSC dyes utilize three major components: redox active electron rich donor, a $\pi$-spacer, and strong electron acceptor are needed (Figure 1.10). There are however, some energetic design principles that must be considered regarding the electronic energy levels of HOMO and LUMO in the DSSC dye: 1) The LUMO of the dye must be higher in energy than the conduction band of $\mathrm{TiO}_{2} ; 2$ ) $\mathrm{HOMO}$ must be above the valence band of $\mathrm{TiO}_{2} ; 3$ ) The energy gap between HOMO and LUMO has to be small as possible in order to redshift absorption profile into the NIR region.

\subsubsection{The donor}

The electron donor is responsible for the electron in the photoinduced electrons transfer of a DSSC dye. Criteria for desirable characteristics for electron donor are as followed: 1) must be redox active in order for electron transfer; 2) electron rich in nature so the HOMO level can be as high as possible 3) stable in the oxidized state. Electron donor also should be non-planar to avoid $\pi$ stacks leading to dye aggregation. An example of common electron donor is triphenylamine (TPA); the heteroatom is the source of the redox active component of the donor. The three phenyl rings are non-planar due steric strain which hinders dye aggregation. TPA is also quite stable in the oxidized state as well.

\subsubsection{The non-innocent $\pi$-spacer}


The $\pi$-spacer is the conjugated aromatic bridge connecting the donor and acceptor. The length of $\pi-$ spacer can be relevant in two ways. 1) Lengthening the $\pi$ spacer where the electron density can delocalize further, therefore decreasing the HOMO-LUMO gap. 2) However $\pi$-spacer is too long, then molecular overlap between ground and excited state will be minimized, leading to decrease in absorption intensity. The introduction of non-innocent $\pi$-spacer (redox active conjugated bridge) can aid to electron transfer of the dye and increase orbital overlap which in turns increases the absorption intensity. Non-innocent $\pi$ spacer also can redshift absorption profiles. In the Kovisito group, we synthesized a series of novel BODIPY dyes based on molecule 1.2. ${ }^{11}$ The acceptor moiety of the dye (red, Figure 1.10) must be capable of binding $\mathrm{TiO}_{2}$. Electron deficient anchor groups like carbocyclic acidic, sulphate are acceptor groups seen in literature, however cyanoacetic acid groups seems to be the best option. ${ }^{7}$

\subsection{BODIPY}

4,4-Difluoro-4-bora-3a, 4a diaza-s-indacenes (BODIPY) (Figure 1.12) have been used in a variety of advanced material applications due to their highly desirable properties such as high absorption coefficients, sharp emission peaks exhibiting high quantum yields, and chemical stability under wide range of conditions. As such, BODIPY that can used for chemosensers, labeling proteins and DNA for intracellular imaging labeling reagents ${ }^{11}$, fluorescent switches ${ }^{12}$, laser dyes ${ }^{12-14}$, dye sensitised solar cells ${ }^{7}$ and OPVs (organic photovoltaic) ${ }^{15}$.

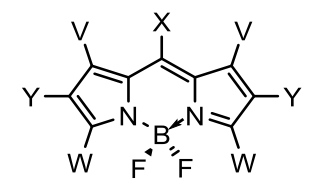

Figure 1.12: General structure of BODIPY: $Z, Y=\beta$ or 2,6 positions, $X=$ meso position, $W=\alpha$ or 3,5 positions, $V=1,7$ positions or other $\beta$ positions

\subsubsection{General Synthesis of BODIPY}

BODIPY can be prepared using two main synthetic routes: 1) the pyrrole is reacted with an acid chloride through a double electrophilic aromatic substitution and condensation to make a dipyrrin core which then (Scheme 1.1) reacts with $\mathrm{BF}_{3} \cdot \mathrm{OEt}_{2}$ and a base to form BODIPY. 


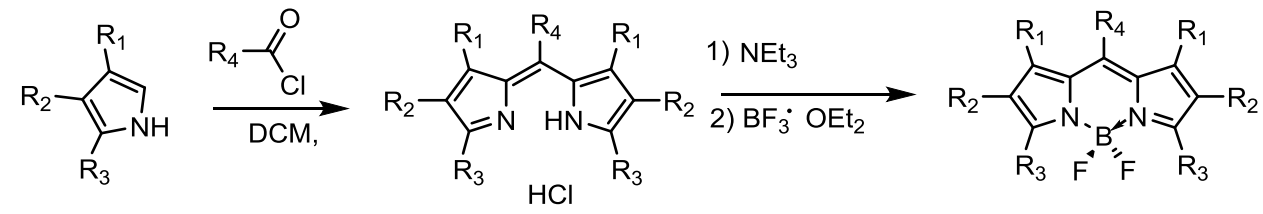

Scheme 1.1: General synthetic approach to BODIPY using acid chlorides

2) The uses of aldehydes (Scheme 1.2) as the electrophilic centre to create a dipyrrolemethane. This intermediate is oxidized using DDQ or $p$-chlornail, and reacted with an organic base and $\mathrm{BF}_{3} \cdot \mathrm{OEt}_{2}$ to form BODIPY.

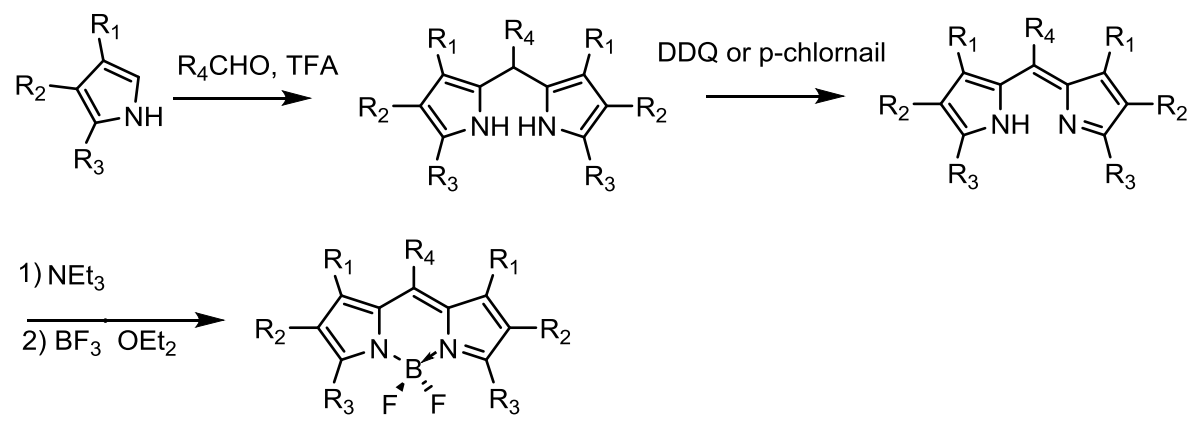

Scheme 1.2: General synthetic approach to BODIPY using aldehydes

A more recent method to make BODIPY is the production of dipyrrolyl-methanone through oxidation of pyrrole using phosgene or reacting hydrogen peroxide with dipyrrylthioketone. ${ }^{16}$ Dipyrrolyl-methanone can react with $\mathrm{POCl}_{3}$ to produce 8 halogenated dipyrrin followed by depronation $\left(\mathrm{NEt}_{3}\right)$ and complexation $\left(\mathrm{BF}_{3} \cdot \mathrm{OEt}_{2}\right)$ to make 8-Cl-BODIPY.

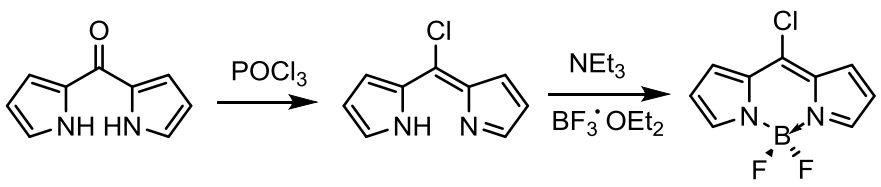

Scheme 1.3: General synthetic approach to 8-Cl-BODIPY

\subsubsection{Tuning BODIPY}

Owing to the symmetry and myriad of R groups' possible, BODIPYs tunability and small modifications to their structure can result in significant changes in their physicals properties. Synthetic modifications to the BODIPY core have been studied in depth at certain positions, in particular the $\alpha, \beta$, and meso (X) positions (Figure 1.12). Each of these positions have important considerations for functional applications. ${ }^{7}$ 


\subsubsection{The meso position}

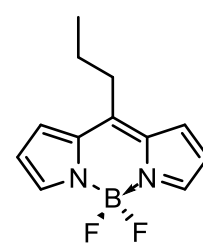

1.3

$\lambda_{\mathrm{abs}}=497 \mathrm{~nm}$

$\lambda_{\mathrm{ems}}=505 \mathrm{~nm}$

$\Phi_{\mathrm{f}}=0.96$

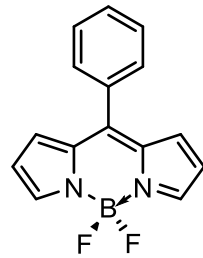

1.4

$\lambda_{\mathrm{abs}}=455 \mathrm{~nm}$

$\lambda_{\text {ems }}=550 \mathrm{~nm}$

$\Phi_{\mathrm{f}}=0.05$

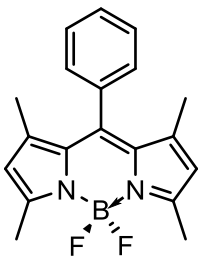

1.5

$\lambda_{\mathrm{abs}}=498 \mathrm{~nm}$

$\lambda_{\mathrm{ems}}=508 \mathrm{~nm}$

$\Phi_{\mathrm{f}}=0.65$

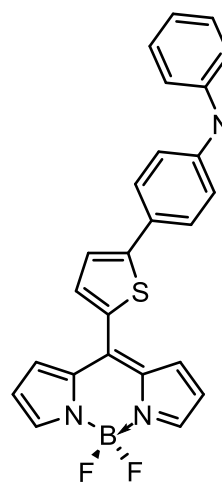

1.6

$\lambda_{\mathrm{abs}}=520 \mathrm{~nm}$

$\lambda_{\mathrm{ems}}=600 \mathrm{~nm}$

$\Phi_{\mathrm{f}}=0.35$<smiles>Cc1cc(C)n(P(F)F)c1-c1c(C)cc(C)n1P(F)P</smiles>

1.7

1.8

Figure 1.13: meso BODIPY derivatives ${ }^{7}$

The meso position (or $\mathrm{X}$ position) sits on the mirror plane of BODIPY, This position can be modified using different aldehydes and acid chlorides derivatives as describe above. The meso position can also be modified post synthesis of the meso halogenated BODIPY. Alkyl substituents on the meso position of BODIPY (1.3) have no significant effect on the absorption and emission properties. Aromatic substituents however, can be used to affect the photo-optics of BODIPY. The addition of the aromatic group to meso position (1.4) hinders fluorescence due to its rotation in the excited state. However, the addition of methyl groups on the 1, 7 positions (1.5) will stop the free rotation, restoring fluorescence. However, the addition of the methyl groups decreases the effect of the phenyl absorption properties due to the co-planarity. The presence of the electron donating conjugated substituent such as $\mathbf{1 . 6}$ has a bathochromic effect on absorption compared to 1.3. Also, there is the presence of intramolecular charge transfer in absorption profile, evidence of electron transfer (BODIPY is the electron acceptor). Simple modification of the meso substituent allows for chemosenser such as pH probes (1.7) metal-chelators (1.8).

\subsubsection{The $\alpha$ positions}



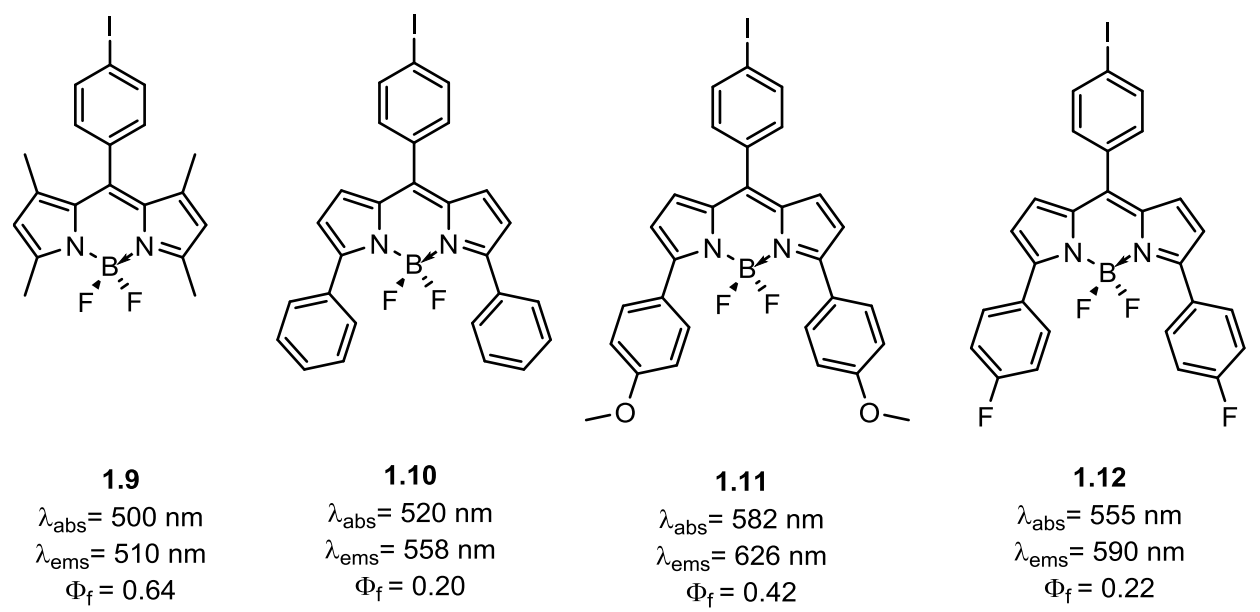

1.11

1.12

$\lambda_{\mathrm{abs}}=555 \mathrm{~nm}$

$\lambda_{\mathrm{ems}}=590 \mathrm{~nm}$

$\lambda_{\mathrm{ems}}=626 \mathrm{~nm}$

$\Phi_{\mathrm{f}}=0.42$

$\Phi_{\mathrm{f}}=0.22$
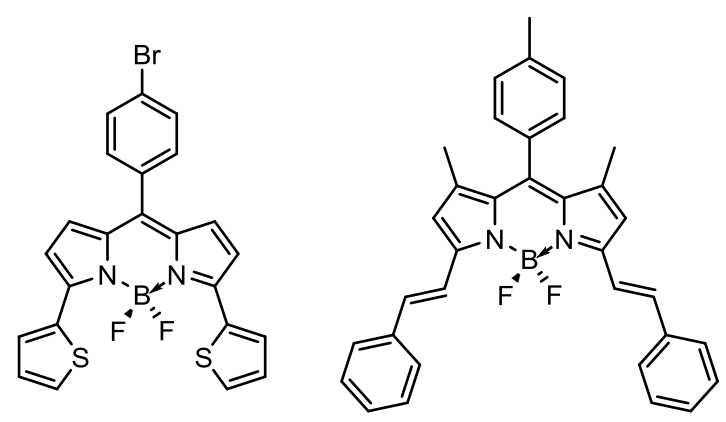

1.13

$\lambda_{\text {abs }}=622 \mathrm{~nm}$

$\lambda_{\text {ems }}=643 \mathrm{~nm}$

$\Phi_{\mathrm{f}}=0.82$

$$
\begin{gathered}
1.14 \\
\lambda_{\text {abs }}=626 \mathrm{~nm} \\
\lambda_{\text {ems }}=639 \mathrm{~nm} \\
\Phi_{\mathrm{f}}=0.92
\end{gathered}
$$

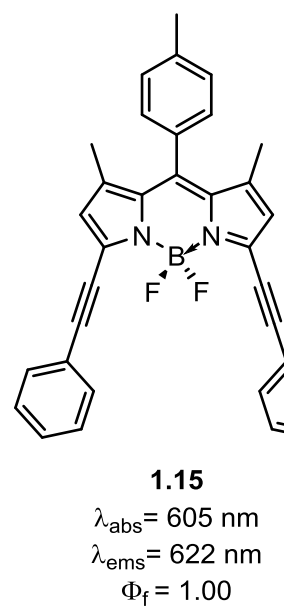

Figure 1. 14: $\alpha$ position BODIPY derivatives, ${ }^{18}$

The 3, 5 or $\alpha$ positions on BODIPY are used introduce aromatic units at the 3,5-positions ( $\alpha$-position). Direct installing phenyl substituents at these two positions (1.10) can extend the conjugation system, red shifting $\lambda_{\max } \sim 20 \mathrm{~nm}$, the quantum yield decreases due to free rotation of the phenyl rings like meso phenyl, 1.4. However introducing electron donating groups to the phenyl like 1.11, causes a $\sim 60 \mathrm{~nm}$ red shift in the absorption profile and slightly restores the quantum yield. The electron withdrawing fluorine derivative (1.12) still red shifts the absorption but not to the degree of a methoxy substituent. Heterocyclic aromatic rings such as thiophene at the 3,5-positions could induce more significant red shift $(\sim 100 \mathrm{~nm})$ and increase the quantum yield. The 3, 5 methyl groups are acidic in nature, so Knoevenagel reactions can be used to make styrl BODIPY dyes like 1.14. The red shift is more significant compared to other 3,5 position manipulation and significantly increases the quantum yield of the BODIPY core. 
Palladium cross coupling is also possible using 3,5 halogenated BODIPYs, like the Sonogashira product 1.15. The ethynyl derivative gave the highest quantum yield within the series.

\subsubsection{Boron centre}

The fluoride atoms on the boron centre can be displaced via basic conditions. Aryl groups can be introduced to the boron centre through the use of aryl Grignards or aryl lithium to displace the fluoride atoms $(1.17,1.18)$. The use of bulky aryl groups will increase the quantum yield. ${ }^{2,} 7$ B-aryl BODIPY compounds seem to have a slight red shift in both absorption and fluorescence when in the presence of a polar solvent. Alkyne groups can also be added by using an acetylene anion to displace fluoride (1.19). ${ }^{2}$
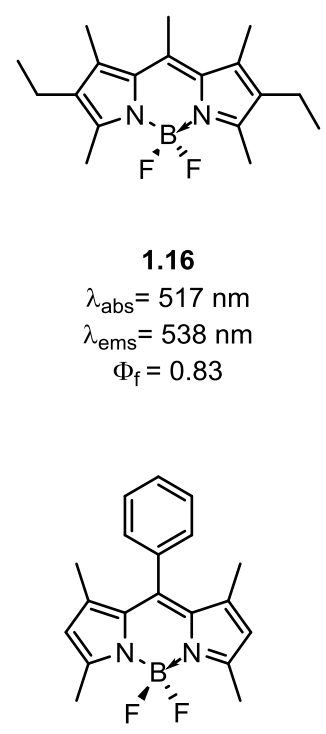

1.20

$\lambda_{\text {abs }}=497 \mathrm{~nm}$

$\lambda_{\mathrm{ems}}=507 \mathrm{~nm}$ $\Phi_{\mathrm{f}}=0.46$

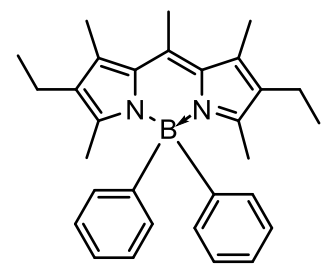

$$
\begin{gathered}
1.17 \\
\lambda_{\text {abs }}=513 \mathrm{~nm} \\
\lambda_{\text {ems }}=548 \mathrm{~nm} \\
\Phi_{\mathrm{f}}=0.91
\end{gathered}
$$

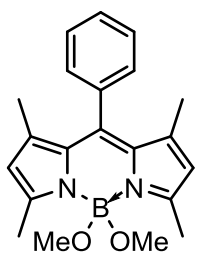

$$
\begin{gathered}
1.21 \\
\lambda_{\mathrm{abs}}=498 \mathrm{~nm} \\
\lambda_{\mathrm{ems}}=509 \mathrm{~nm} \\
\Phi_{\mathrm{f}}=0.49
\end{gathered}
$$

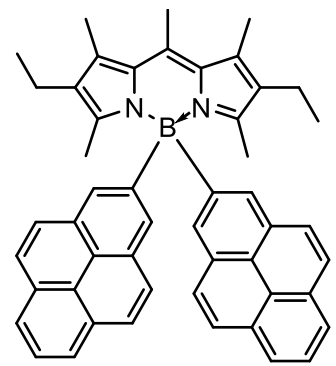

$$
1.18
$$$$
\lambda_{\text {abs }}=526 \mathrm{~nm}
$$$$
\begin{aligned}
\lambda_{\mathrm{ems}} & =562 \mathrm{~nm} \\
\Phi_{\mathrm{f}} & =0.19
\end{aligned}
$$

$\Phi_{\mathrm{f}}=0.19$

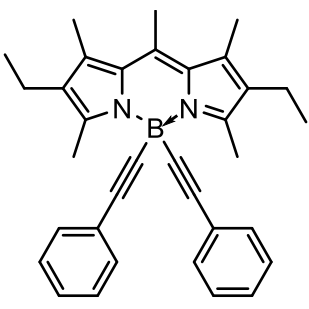

1.19

$\lambda_{\mathrm{abs}}=516 \mathrm{~nm}$

$\lambda_{\text {ems }}=537 \mathrm{~nm}$

$\Phi_{\mathrm{f}}=0.95$

Figure 1. 15: Boron BODIPY derivatives ${ }^{7}$

Adding aryl or alkyne groups to the boron centre does not add to the conjugation of the quasi-aromatic BODIPY. This means that there are no significant changes in the UV-Vis absorption and emission spectra. The only effect this modification can have is on the quantum yield through sterics. Alkoxide substituents can be introduced through aluminum trichloride in the desired alcohol (1.20). These derivatives seem to 
be slightly more water soluble then fluorinated BODIPYs. However, these derivatives also have no significant change to the electronics properties of BODIPY.

\subsubsection{The $\beta$ positions}

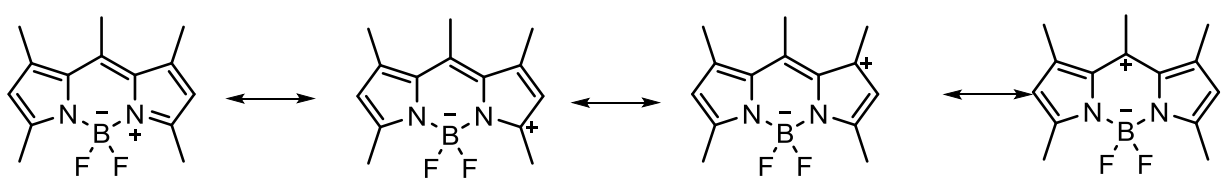

Figure 1. 16: Resonance contributors of BODIPY

2,6 or $\beta$ positions of BODIPY are the most electronegative positions. Referring to Figure 1.16 , throughout all resonance contributors, the positive charge of the nitrogen is never on the 2 or 6 positions. This demonstrates that the 2, 6 positions are the most nucleophilic carbons in BODIPY. These positions have been modified extensively using electrophilic substitution reactions. ${ }^{19}$ An example of electrophilic aromatic substitution that can be used is the Vilsmeier-Haack formylation to install aldehydes and functionalizes those aldehydes using a myriad of reactions (such as Grignard reaction, Knoevenagel condensation and oxidation of the aldehydes, Schiff bases). Another important electrophilic substitution reaction that is done on the 2,6 positions are halogenations. This is important for cross coupling reactions which from $\mathrm{C}-\mathrm{C}$ bonds. The cross coupling reactions that can be done on BODIPY include Suzuki, Sonogashira, Stille, and Heck couplings. These cross coupling reaction can be utilized to extend conjugation throughout the BODIPY.

\subsection{Objectives}

To design unique BODIPY dyes for material applications, creative architectures and an in depth study of the synthetic methodologies of BODIPYs is needed. This thesis aims to tune BODIPY's physicochemical properties with unique synthetic strategies and architectures. This includes the introduction of more stable electron donors (ferrocene) and using different methods to tune the HOMO - LUMO gap through intramolecular interactions. To this end, this thesis has been broken into 3 distinct stories.

1) The development of modern synergistic synthetic chemistry laboratories that develop important BODIPY precursors. These laboratories provide undergraduates with research experience \& 
assisting research programs by building valuable feedstock and elucidating structure-property relationship en masse.

2) To stabilize the photoxidized BODIPY spacer for DSSC applications with a redox robust electrophore. To this end, synthesis towards ferrocene BODIPY dyes and dyads towards elucidating their viability and physicochemical properties. As a side note, it would be the first case of adding Fe (a cheap transition metal) effectively into a DSSC dye.

3) As a means to explore tunable $\pi-\pi$ interactions in organic supramolecular material applications ( $\pi$-spacer, DSSC, OPVs, photoacoustic imaging, etc.), a BODIPY cyclophane is proposed. To this end, an interest in the tunability of the HOMO-LUMO gap in BODIPY cyclophanes using the confined intramolecular $\pi$ stacking. Synthesis towards BODIPY - anthracene cyclophane is discussed

\section{Chapter 2: Exploring BODIPY Dyes in Synergistic Undergraduate Laboratories}




\section{for Enhanced Experiential Learning and Research Output}

\subsection{Introduction}

\subsubsection{Motivation}

Most traditional synthetic undergraduate laboratories focus on reaction sequences that develop basic laboratory techniques using well-studied organic reactions (e.g. Fischer esterification); however, the products of these reactions are seldom studied further. While the synthesis of these compounds offer unique teaching and learning opportunities, the physicochemical properties are not typically examined. As a result, important structure-property relationships are omitted from practical undergraduate curricula. As a result, these products do not have inherent value outside of technical training; at the end of these traditional labs, these products are seldom used again, creating waste that ultimately has a negative impact on the environment. Owing to the expense of basic research, the development of modern synthetic chemistry laboratories, that develop important precursors or building blocks, represents a synergistic opportunity for research and undergraduate education. ${ }^{20}$

BODIPY (4,4'-difluoro-4-bora-3a,4a-diaza-s-indacene)-based dyes have garnered considerable attention owing to their highly desirable properties (absorption, fluorescence and photo-stability). ${ }^{17,11}$ As such, BODIPY derivatives have been explored in a myriad of applications including bio-imaging \& ion-sensing, ${ }^{18}$ and more recently as non-innocent (redox-active) $\pi$-spacers in next-generation photovoltaic materials. ${ }^{5-10}$ The Koivisto group, have shown that BODIPYs are synthetically versatile and can be readily tuned using a variety of synthetic techniques. ${ }^{11-13}$

Herein, the development of an engaging senior undergraduate laboratory course that reinforces traditionally important technical training is discussed. Using this colorful and robust BODIPY platform the learner is provided with the opportunity to explore property-directed synthesis \& research and contribute to the greater scientific community by preparing novel molecules. The derivatives prepared by the two original cohorts are also discussed. 


\subsection{Experimental overview and pedagogical value}

The impetus to explore this particular platform was to design a property-directed laboratory where the target molecules would have significant pedagogical value when it comes to training undergraduates with advanced synthetic, purification and characterization techniques. The first stage of this laboratory is the synthesis of the BODIPY derivatives based on pentamethyl-BODIPY $(\mathbf{2 . 3}$, Scheme 1$) .{ }^{14}$ The synthetic route described in Scheme 1 compliments the technical level of an undergraduate and serves to train the students for the discovery-based learning in Stage 2.

Upon successful completion of the targets in Scheme 2.1, physical properties are evaluated and structureproperty relationships are gleaned. Complementing optical (UV-Vis) and redox data (cyclic voltammetry) are theoretical time dependent-density functional theory (TD-DFT) calculations ${ }^{15}$ of the frontier molecular orbitals (FMOs) that significantly contribute to the optical transitions. Together (Table 2.1) these physicochemical methods produce a table of relevant benchmarks when comparing to the derivatives produced in Stage 2.

\subsection{Stage 1: Developing labs skills \& building blocks}

Nearly half of the semester is dedicated to the synthesis, purification and characterization of BODIPY 2.3 and its derivatives $\mathbf{2 . 4}$ and 2.5. Both 2.6, and $\mathbf{2 . 7}$ have been included here, because of their synthetic value to our research program, but they were not explored further. Forty-six students have completed stage 1. While it is conceded that the synthetic route is more complementary for senior undergraduate chemistry students, the diverse student demographic ranging in experience and background (see Table A1), demonstrates this laboratory is easily accessible for most undergraduate science students. 


\subsubsection{Synthesis of BODIPY and building block}

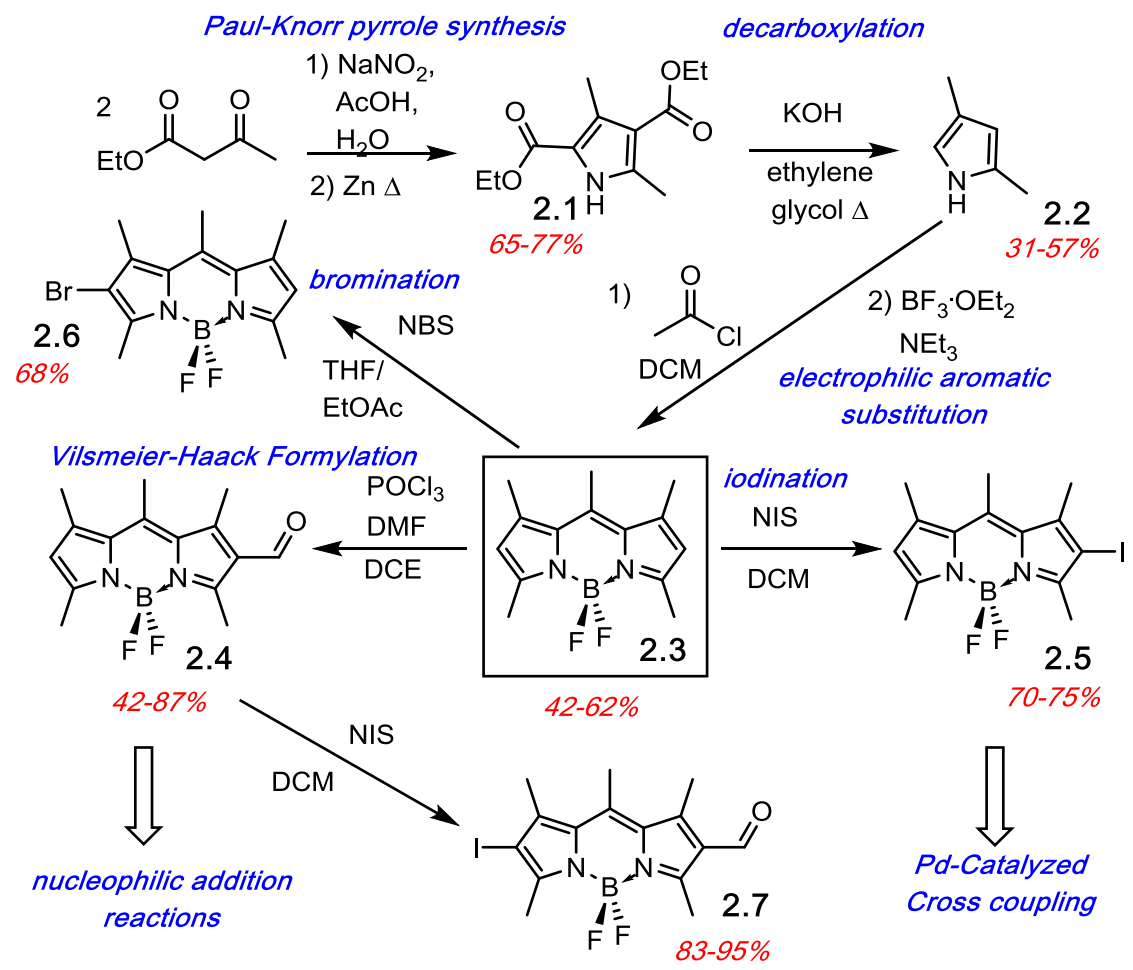

Scheme 2.1: Stage 1 Synthesis of BODIPY (2.3) and building blocks

The syntheses of BODIPY and simple derivatives are presented in Scheme 2.1, and a detailed synthetic protocol is provided in the experimental (section 2.6). En route to the preparation of BODIPY, diverse synthetic manipulations are followed. For example, in the Paul-Knorr pyrrole synthesis to make 2.1, ${ }^{21}$ students are exposed to nitration, reduction, and ring condensation; providing wonderful opportunities to appreciate mechanistic organic chemistry. After recrystallization, students typically obtain 15 grams of pyrrole product (2.1), starting from inexpensive starting materials. The decarboxylation of the pyrrole ester, followed by an in situ high-vacuum distillation provides the pyrrole product, 2.2, (as an modestly air and light-sensitive oil). ${ }^{22}$ Synthesis of 2.2, provides the opportunity for students to appreciate air-sensitive techniques, but without the strict need for them. Finally the sequential one-pot electrophilic aromatic substitution reactions, condensation, and boron-chelation results in BODIPY, $\mathbf{2 . 3}$.

Typically this sequence to prepare BODIPY is done in student pairs, but this step has also been done individually with equal success. Depending on the proficiency of the students, the yield of BODIPY, $\mathbf{2 . 3}$ after the first three steps is $1-2$ grams. This is far more than is needed to complete the remainder of the experimentation, resulting in the production of a constant supply of a precious building block for our research program. Furthermore, BODIPY 2.3, is readily modified to explore a myriad of structure-property 
relationships. In one possibility, the Vilsmeier-Haack formylation leads to aldehyde, $\mathbf{2 . 4}{ }^{23}$ that can be modified further with nucleophilic addition reactions. Conversely, halogens (iodine or bromine) can be installed using the respective succinimide halogen reagent to generate iodo-BODIPY, 2.5, 2.7 or bromoBODIPY, 2.6, respectively. ${ }^{19}$ These halogenated BODIPY derivatives can be further modified using a variety of cross-coupling reactions. The further modifications of $\mathbf{2 . 4}$ and $\mathbf{2 . 6}$ represent the essence of this pedagogical venture, and is discussed in future sections (Stage 2).

\subsection{2: Purification and characterization of BODIPY and its derivatives}

Owing to their intense colour and fluorescence, BODIPY and derivatives are ideal candidates to appreciate chromatographic techniques. While other methods are possible, the purification of BODIPY derivatives is effectively accomplished using chromatography. In the case of compounds 2.3-2.7, chromatography was used exclusively for the isolation of the products. Despite only subtle changes in structure, it remains facile to separate these byproducts using the methods described (see supporting information). For example, in the purification of $\mathbf{2 . 5}$, both the di-iodo derivative, and BODIPY starting material can be readily separated and isolated visually using chromatography.

In our study, students used NMR spectroscopy $\left({ }^{1} \mathrm{H}(400 \mathrm{MHz}),{ }^{13} \mathrm{C},{ }^{19} \mathrm{~F}\right.$ and $\left.{ }^{11} \mathrm{~B}\right)$ as the main method for characterization. Owing to the multiple NMR-active nuclei, it was possible to appreciate a number of NMR features, including heteronuclear coupling (B-F), and the coupling effect of higher order spin systems ( $I_{\text {boron }}$ $=3 / 2$ ). For students whose exposure was limited to characterization in second year organic chemistry, spectroscopy (in particular coupling) is a concept that can be overwhelming with large molecules. However, the high symmetry and trivial multiplicity patterns in the ${ }^{1} \mathrm{H}-\mathrm{NMR}$ of the pentamethyl-BODIPY derivatives facilitated the use of this technique for structural elucidation, and reinforced previous understanding of symmetry and chemical environments.

Physical characterization of compounds 2.3-2.7 has been collated in Table 2.1. In addition to the optical properties (UV-Vis absorbance), these pentamethyl-BODIPY derivatives possess rich redox behaviour (i.e., reversible oxidation and reduction) that has been quantified using electrochemistry (cyclic voltammetry). While cyclic voltammetry is a technique seldom used in undergraduate curricula, it is ubiquitous in materials-based chemistry and provides another method to probe structure-property relationships (especially when referenced to NHE using an internal reference like ferrocene). As seen in Table 2.1, the installation of electron withdrawing (EW) groups stabilize both the HOMO and LUMO levels, with the aldehyde having the largest EW effect. Modest shifts in the absorption maxima are also observed within this series, with the larger halogen atoms red-shifting the absorption maxima more significantly. These 
subtle changes in visual properties upon modification, manifest in the ability to readily separate product mixtures using column chromatography.

Table 2.1: Physicochemical characterization of BODIPY (2.3) and its derivatives (2.4-2.7).

\begin{tabular}{|c|c|c|c|c|}
\hline \multirow{2}{*}{ Compound } & \multicolumn{2}{|c|}{$E_{1 / 2}(V v s N H E)^{a}$} & \multirow{2}{*}{$\begin{array}{l}U V \text {-vis }^{b} \lambda_{\max }(\varepsilon) \\
n m\left(/ 10^{4} M^{-1} \mathrm{~cm}^{-1}\right)\end{array}$} & \multirow{2}{*}{$\begin{array}{ll}\text { Dominant } & F M O \\
\text { transition } & \text { from } \\
\text { TD-DFT } & \end{array}$} \\
\hline & Ered & $E_{o x}$ & & \\
\hline 2.3 & -1.05 & 1.50 & 497 (8.3) & HOMO to LUMO \\
\hline 2.4 & -0.87 & 1.77 & $493(1.5)$ & HOMO to LUMO \\
\hline 2.5 & -0.92 & 1.60 & $510(7.5)$ & HOMO to LUMO \\
\hline 2.6 & - & 1.61 & $508(3.9)$ & HOMO to LUMO \\
\hline 2.7 & -0.71 & 1.83 & $513(13.0)$ & HOMO to LUMO \\
\hline \multicolumn{5}{|c|}{$\begin{array}{l}{ }^{a} \text { Data collected using } 0.1 \mathrm{M} \mathrm{NBu}_{4} \mathrm{PF}_{6} \mathrm{DCM} \text { solutions at } 100 \mathrm{mVs}^{-1} \text { and referenced } \\
\text { to a }[\mathrm{Fc}] /[\mathrm{Fc}]+\text { internal standard followed by conversion to } \mathrm{NHE} ;[\mathrm{Fc}] /[\mathrm{Fc}+]= \\
+765 \mathrm{mV} \text { vs. NHE in } \mathrm{DCM} ;{ }^{b} \text { low energy or visible transitions from UV-Vis of } \\
\text { BODIPY family in DCM. }\end{array}$} \\
\hline
\end{tabular}

Complementing the optical and redox data are theoretical time dependent-density functional theory (TDDFT) calculations ${ }^{24}$ of the frontier molecular orbitals (FMOs) that significantly contribute to the understanding of the optical transitions. While DFT is typically outside the scope of most undergraduate curricula, it provides students with valuable insight into the electronic structure of the FMOs and can be readily appreciated by undergraduate students at this level. In this particular series, the dominant optical transitions occur between the HOMO and LUMO levels. In addition to this described level of physical characterization, it is also possible to study fluorescence behavior with these systems but that was outside the scope of our examination.

\section{4: Stage 2: Development of unique BODIPY dyes}

The exposure to a myriad of techniques in Stage 1 provides the learner with the basic training necessary to undertake the second discovery-based learning stage; the design and synthesis of a unique target molecule. While the target selection for each student is carefully done with the guidance of the teaching assistant, the learner is responsible for the synthesis and full characterization of the new target. After 
sharing data with other students, it is expected that a short scientific manuscript will be written comparing the structure-property relationships.

\subsubsection{Nucleophilic Additions to BODIPY Derivative 2.4}

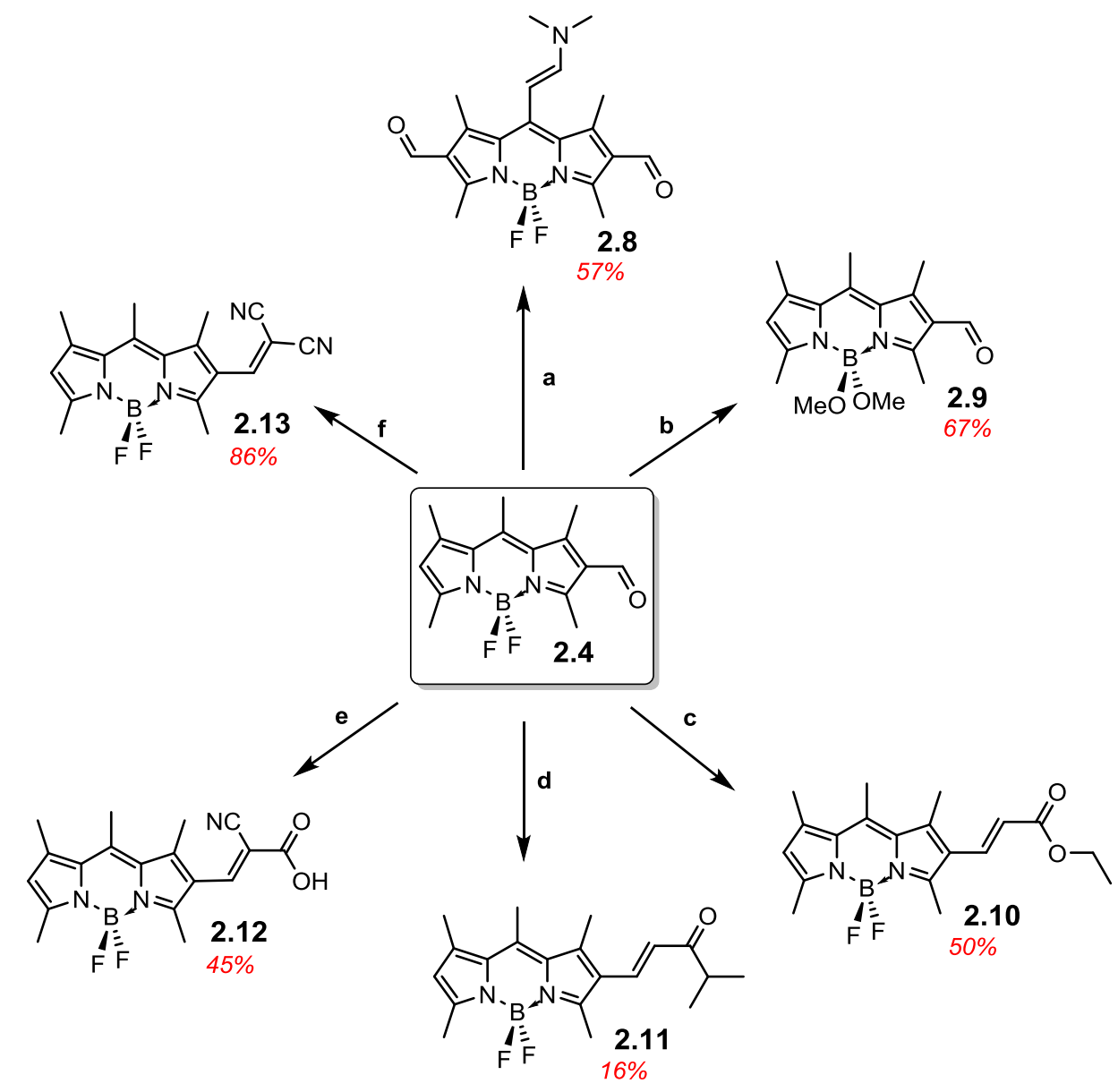

Scheme 2.2: Derivatization of formyl-BODIPY, 2.4:a) $\mathrm{AlCl}_{3}, \mathrm{DCM}$, reflux, $5 \mathrm{~min}, 67 \%$; b) DMF, $\mathrm{POCl}_{3}, 57 \%$; c) (carboxymethylene)triphenylphosphorane, dry DCM, $12 \mathrm{~h}, \mathrm{rt}, 50 \% \mathrm{~d}$ ) $\mathrm{EtOH}$, acetone, $p-\mathrm{TsOH}$ (cat.), $\mathrm{MgSO}_{4}$, reflux, $80^{\circ} \mathrm{C}, 24 \mathrm{~h}, 16 \%$; e) cyanoacetic acid, $\mathrm{CHCl}_{3}$, piperidine, reflux, $12 \mathrm{~h}, 45 \%$.; f) malononitrile, $\mathrm{NEt}_{3}, \mathrm{CHCl}_{3}$, reflux, $16 \mathrm{~h}, 86 \%$;

Showcasing the variety of nucleophilic addition reactions possible, a number of derivatives have been prepared starting with 2.4. Formyl-BODIPY, 2.4 has been a popular building block in our group and its efficient synthesis has been simplified and optimized for study in the undergraduate laboratory. Scheme 2.2 highlights the nucleophilic addition reactions of $\mathbf{2 . 4}$ students completed including previous work done 
in the group. The misfit previous synthesized Vilsmeier-Haack derivative, $\mathbf{2 . 8}$ was not expected. The desired product was not the bis-aldehyde as previously reported. ${ }^{25}$ The second example is a nucleophilic substitution where the fluorines have been replaced with methoxy moieties to yield derivative 2.9. Scheme 1 also depicts the successful nucleophilic addition reactions to install conjugated carbonyl derivatives via Wittig (2.10), aldol (2.11) and Knoevenagel condensations (2.12 \& 2.13). The Wittig reaction went smoothly so long as a stabilized phosphonium ylide was prepared and isolated in advance. Product 2.11 was not planned and remained a puzzling curiosity until it was identified using x-ray crystallography (Figure 2.1). An aldol condensation with small (but significant) amounts of acetone led to product 2.11. Finally, strong electron withdrawing groups can also be installed using Knoevenagel condensations to yield $\mathbf{2 . 1 2}$ and $\mathbf{2 . 1 3}$.

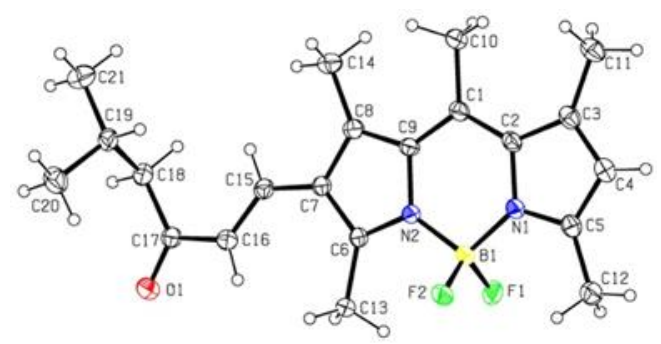

Figure 2.1: X-ray crystal structure of BODIPY derivative 2.11 


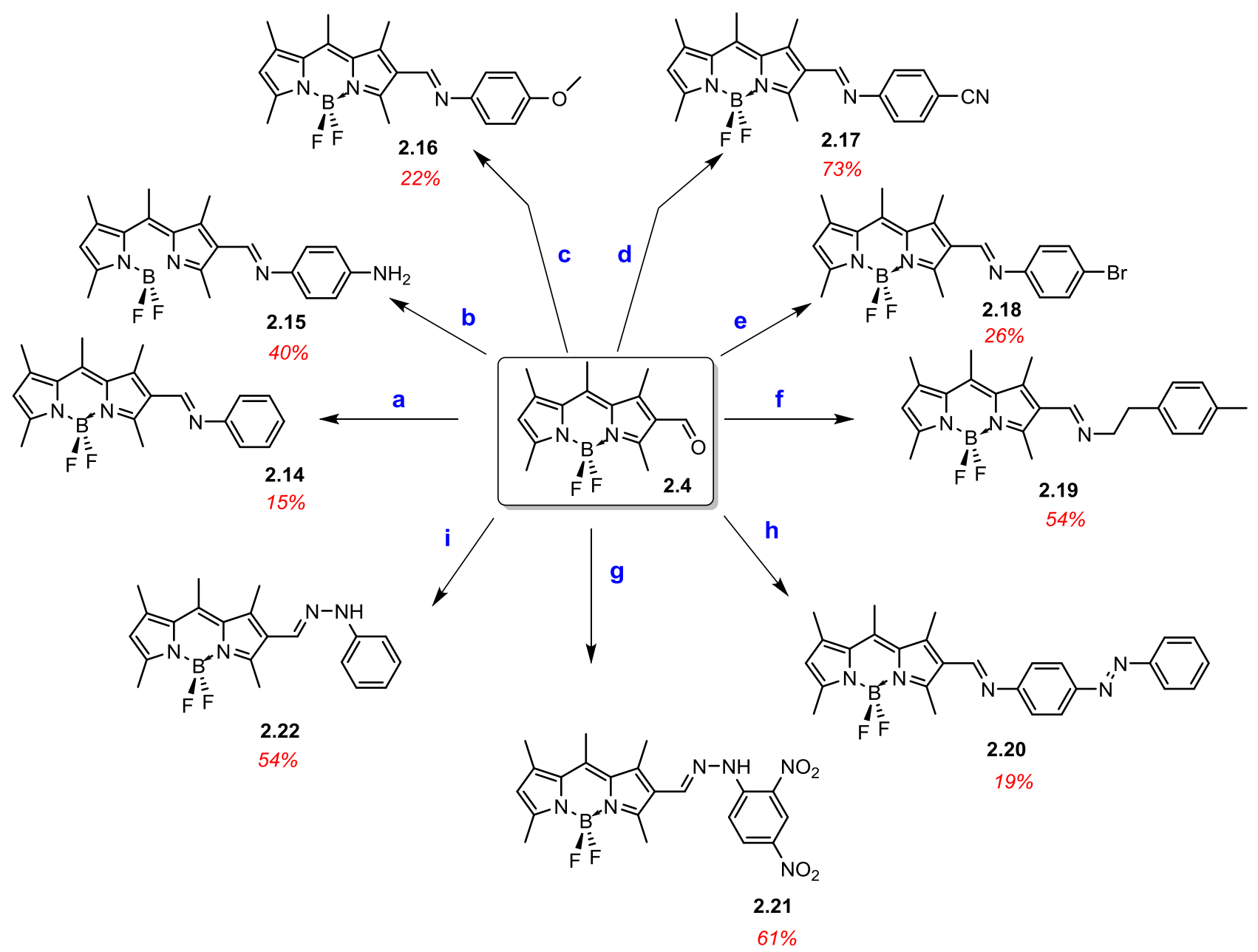

Scheme 2.3: Synthetic routes of BODIPY derivatives (2.14-2.22) from formyl BODIPY, 2.4: a) aniline, $p-$ $\mathrm{TsOH}$ (cat.), $\mathrm{MgSO}_{4}, \mathrm{EtOH}$, reflux, 4 h, 15\%; b) p-phenylenediamine, $p$ - TsOH (cat.), $\mathrm{MgSO}_{4}$, EtOH, reflux, $6 \mathrm{~h}, 40 \%$; c) $p$-anisidine, $p-\mathrm{TsOH}$ (cat.), $\mathrm{Na}_{2} \mathrm{SO}_{4}$, $\mathrm{EtOH}$, reflux, 1 h, 22\%; d) molecular sieves, toluene, 4aminobenzonitrile, $4.5 \mathrm{~h}, 110^{\circ} \mathrm{C}, 73 \%$; e) 4-bromoaniline, dry toluene, $4 \mathrm{~h}, 110^{\circ} \mathrm{C}, 26 \%$; f) $2-(p$ tolyl)ethylamine, $p$ - TsOH (cat.) , $\mathrm{Na}_{2} \mathrm{SO}_{4}$, EtOH, reflux, $1 \mathrm{~h}, 54 \%$; g) (E)-4-(phenyldiazenyl)aniline, $\mathrm{MgSO}_{4}$, EtOH, reflux 24 h, 19\%; h) 2,4-dinitrophenylhydrzine, DCM, 15 mins, 61\%; i) phenylhydrazine, DCM, 2 h, rt, $54 \%$.

Scheme 2.3 highlights the derivatization of 2.4, into a variety of Schiff-bases ${ }^{26}(\mathbf{2 . 1 4}-\mathbf{2 . 2 0})$ and hydrazones (2.21 \& 2.22) using nucleophilic addition reactions. Within the Schiff-base family of BODIPY dyes, the intent was to explore the effect of adding electron donating or withdrawing groups in order to tune the optical properties of the dye (2.14-2.17). Similarly, the bromide (2.18) was prepared to showcase the possibility of further modification using Pd-catalyzed cross coupling. Imine (2.19) was prepared to assess the impact of breaking conjugation with the BODIPY 
core, while $\mathbf{2 . 2 0}$ sought to marry the unique properties of azo-compounds with the BODIPY core. Depending on the electronic nature of the amine, the synthesis required slightly different conditions. In our hands, electron donating amines reacted smoothly, while less electron donating groups required more strenuous conditions in order to shift the equilibrium towards the imine. As a result, these reactions typically resulted in lower yields (owing to BODIPY decomposition) and disappointingly, only a minimal amount of imine 2.17 was isolated.

\subsubsection{Pd-Catalyzed cross-coupling and Click reactions starting from BODIPY Derivative, 2.5}

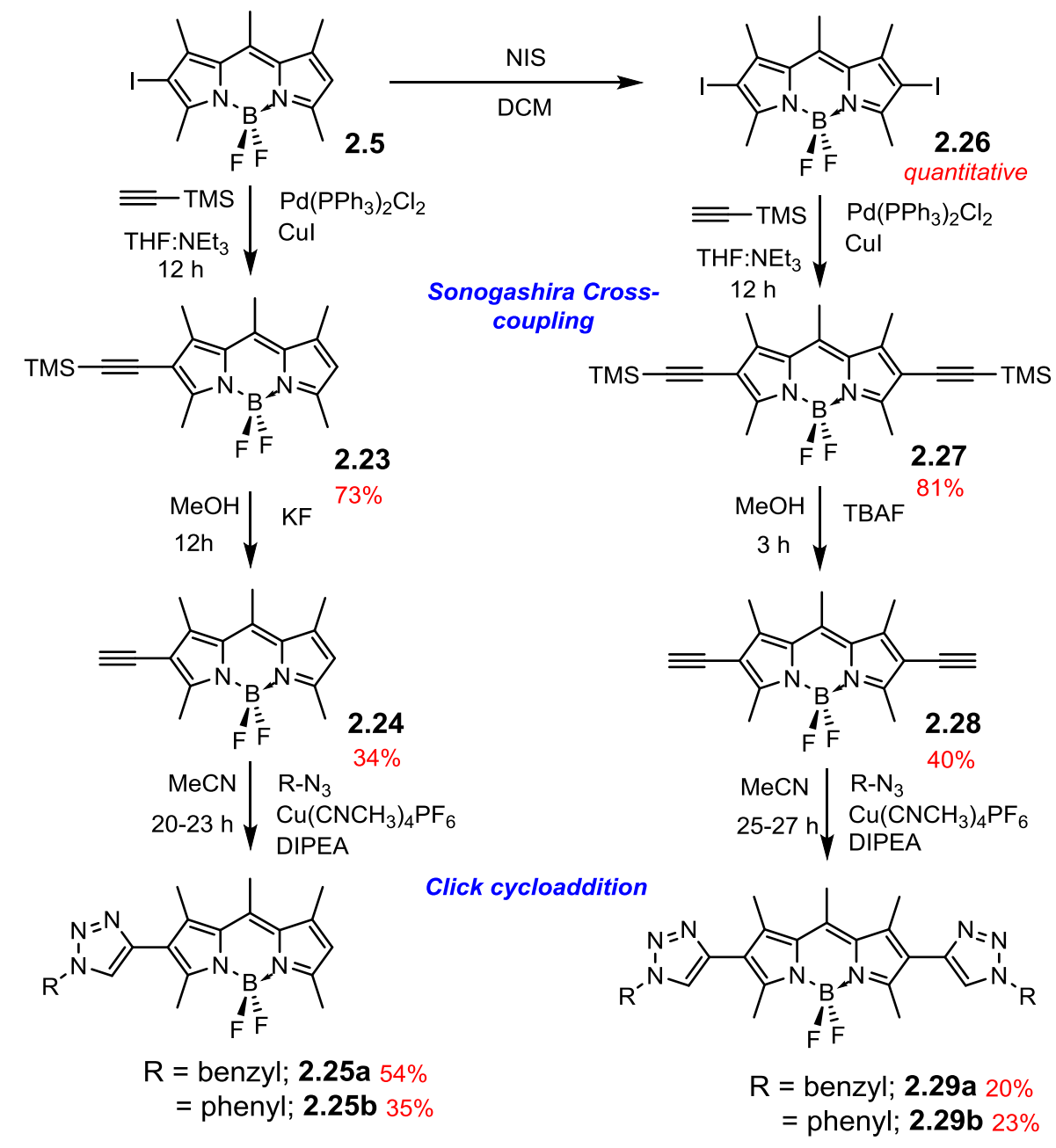

Scheme 2.4: BODIPY derivatization possibilities starting from iodo-BODIPY, 2.5 . 
The synthesis of a number of mono- and di-substituted derivatives starting from 2.5 and 2.26, respectfully, are displayed in Scheme 2.4. The Sonogashira reaction was employed to prepare $\mathbf{2 . 2 3}$ and $\mathbf{2 . 2 7}$ from $\mathbf{2 . 5}$ and 2.26, respectively. In this instance, deprotection of the TMS alkynes was poor owing to decomposition of the BODIPY core and the Click reaction had mixed success, but did result in mono- and di-substituted (both benzyl a, and phenyl b) triazole derivatives 2.25, and 2.29. Despite its limitations, this alkyne/click methodology creates a protocol that has received minimal attention previously, ${ }^{12,22-25}$ and provides access to a wide range of unique 2, 6-substituted BODIPY derivatives.

\subsection{Characterization of BODIPY and its derivatives}

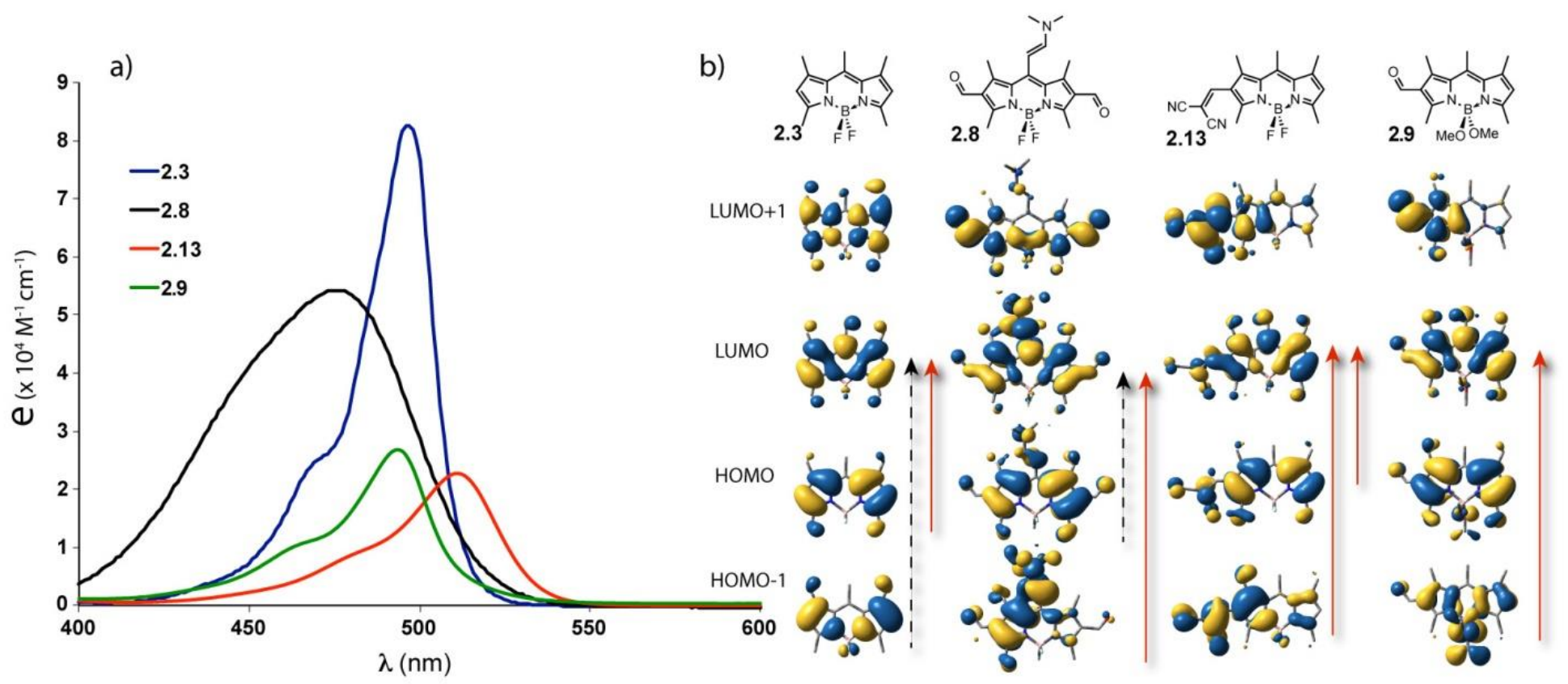

Figure 2.2 UV-Vis spectra (in DCM) for target molecules 2.3, 2.8, 2.13 and 2.17; comparing the effect of electronic withdrawing character after nucleophilic addition reactions with formyl BODIPY, 2.4

The physicochemical properties for compounds 2.4-2.29 and the physical characterization is described herein (Figure 2.2-2.5 and Table 2.2-5). UV-vis (in DCM) and TD-DFT data of the derivatization of formyl-BODIPY is collated in Figure 2.2. The absorption profiles shown in Figure 2.2 showcase the structure-property relationships upon functional group modification. When comparing the absorption to BODIPY $\mathbf{2 . 3}$, derivative $\mathbf{2 . 8}$ is significantly blue-shifted as a consequence of a significant HOMO-1 to LUMO contribution. Despite the dominant transition being HOMO-1 to LUMO, the profile for $\mathbf{2 . 9}$ is nearly identical to 2.3. In this instance, the LUMO is stabilized by the aldehyde, and the HOMO-1 orbital is destabilized owing to the electron-donating 
(ED) methoxy substituents. Conversely, derivative $\mathbf{2 . 1 2}$ and $\mathbf{2 . 1 3}$ are significantly red-shifted owing to the LUMO being stabilized by the strong malonitrile/cyanoacetic acid electron-withdrawing (EW) group, and are expected to be significantly stronger electron withdrawing groups to enones 2.10-11 and aldehyde 2.4. This trend is consistent with the observation that the oxidation of 2.12 and 2.13 occurs closer to the solvent decomposition window, while the oxidation potential of $\mathbf{2 . 4}$ rests approximately $80 \mathrm{mV}$ below 2.13. When comparing the effect of electron withdrawing groups on the optical properties, red-shifting of the absorption profile is observed for derivatives 2.10-13 by $24,25,16$, and $14 \mathrm{~nm}$, respectively, compared to the formyl-BODIPY 2.4. This is not surprising considering the TD-DFT calculations, where the dominant transitions (denoted by the solid arrow) remain the same for enone 2.10, malonitrile $\mathbf{2 . 1 3}$ and cyanoacetic acid 2.12; however, the electron density shifts more towards the BODIPY core for enone $\mathbf{2 . 1 0}$ when comparing the HOMO-1 to LUMO transition. While the optical properties of formyl-BODIPY, 2.4, are different from 2.10-2.13, the electron withdrawing nature of the enone 2.10-11 and malonitrile/cyanoacetic acid 2.12-13 are only modestly different from each other. 
Table 2.2: Physicochemical characterization of BODIPY (2.4) and its derivatives

$(2.5-2.22)$.

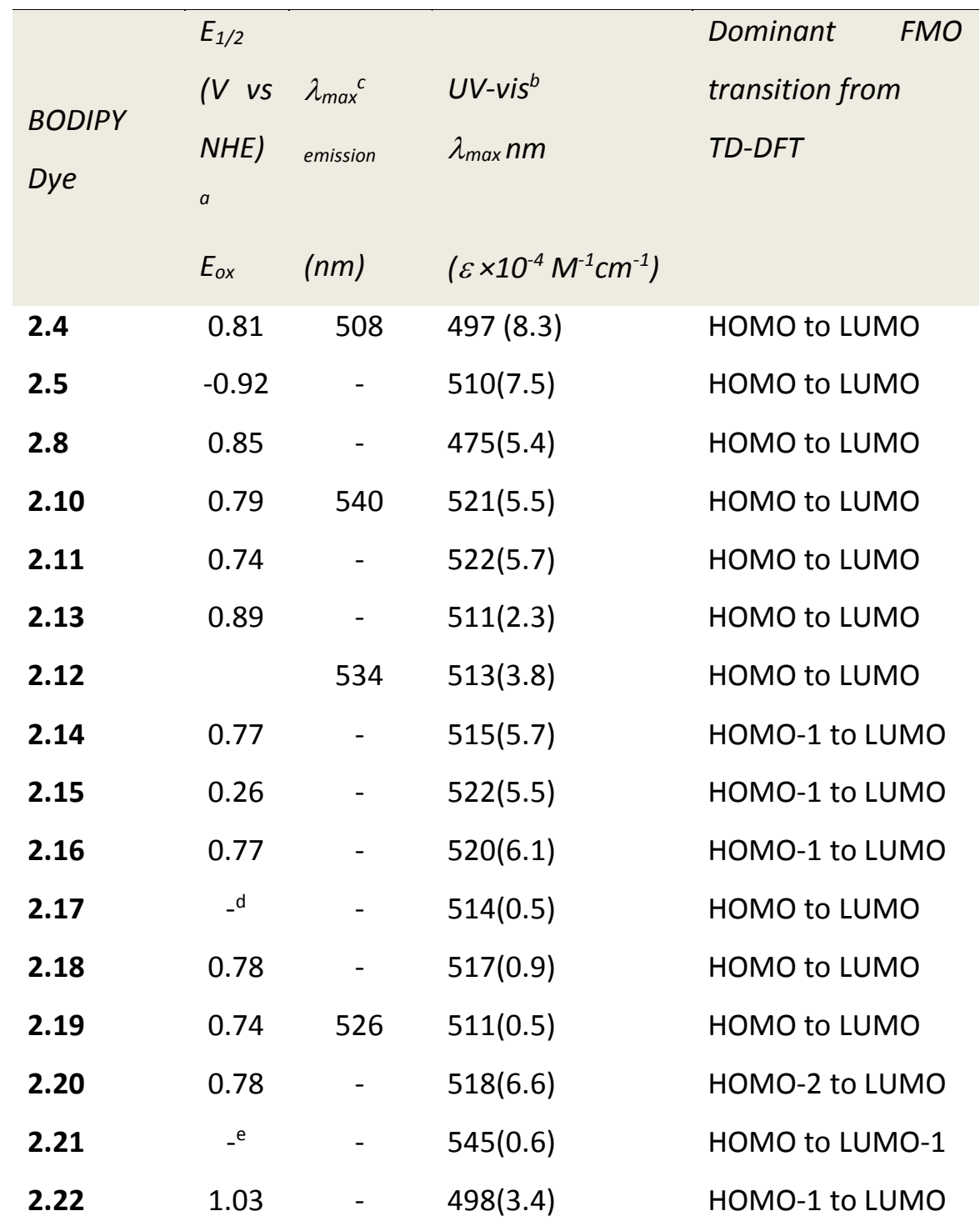

\footnotetext{
a Data collected using $0.1 \mathrm{M} \mathrm{NBu}_{4} \mathrm{PF}_{6} \mathrm{DCM}$ solutions at $100 \mathrm{mVs}^{-1}$ and referenced to a $[\mathrm{Fc}] /[\mathrm{Fc}]+$ internal standard followed by conversion to $\mathrm{NHE} ;[\mathrm{Fc}] /[\mathrm{Fc}+]=+765 \mathrm{mV}$ vs. NHE in DCM; ${ }^{b}$ low energy transitions from UV-Vis of BODIPY family in DCM; ${ }^{c}$ emission in DCM solutions corresponding to excitation at absorption maxima; ${ }^{d}$ not synthesized in sufficient quantities; ${ }^{e}$ insoluble in DCM
}

When exploring the effect of varying the electronic nature of the Schiff base, little change is observed in both the optical and electronic properties when varying the para-substituent. While this para-substituent seems to have a significant effect on the reactivity, comparing the effect of 
electron donating and withdrawing groups attached to the BODIPY they are nearly at parity when considering oxidation potential. Moreover, only a modest red-shift in the absorption profile is observed for $\mathbf{2 . 1 6}$ and a slight blue-shift is observed in the absorption profile of $\mathbf{2 . 1 7}$, consistent with perturbation of the frontier orbitals (Figure 2.3). When comparing the TD-DFT calculations, the dominant transitions (denoted by the dashed arrow) differ throughout the series. When comparing the effect of extended versus broken conjugation in Schiff-base BODIPY derivatives 2.14 and 2.19, a modest hypsochromic shift in the absorption profile is observed for $\mathbf{2 . 1 9}$ (Figure 2.4). When aldehyde $\mathbf{2 . 4}$ is appended with azo- or hydrazine derivatives (2.20 and 2.21, respectively), more substantial changes to the absorption properties are observed. When comparing the optical properties of the Schiff-base BODIPY derivatives, red-shifting of the absorption profile is observed for both derivatives 2.20 and 2.21 by 21 and $48 \mathrm{~nm}$, respectively,

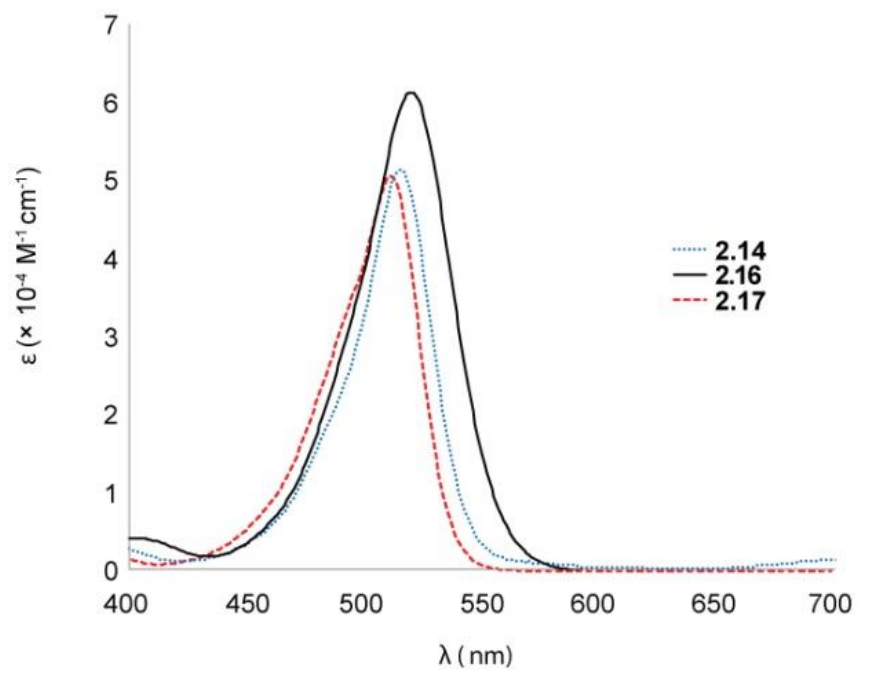

b)

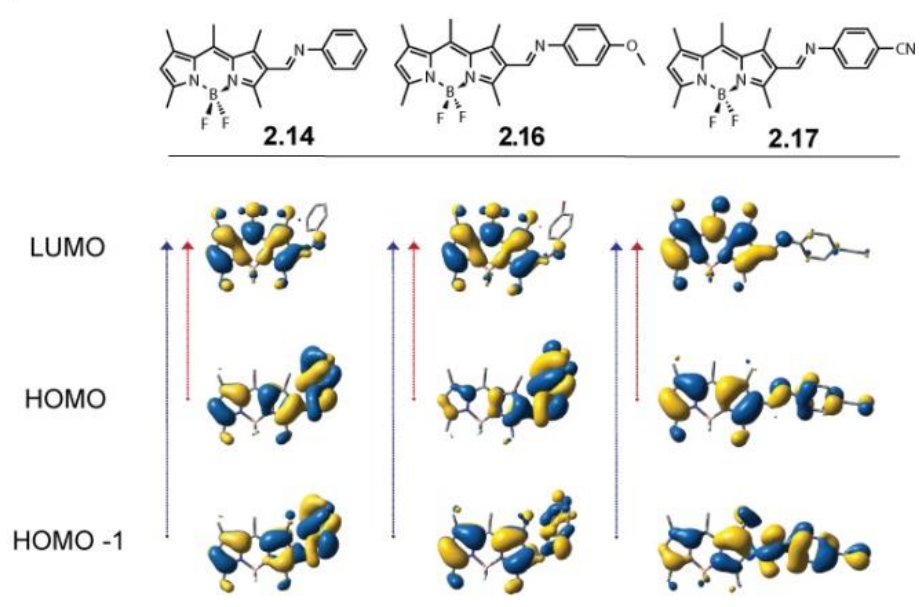

Figure 2.3: UV-Vis spectra (in DCM) for target molecules 2.14, 2.16 and 2.17; comparing the effect of electronic withdrawing character after nucleophilic addition reactions with formyl BODIPY, $\mathbf{2 . 4}$

compared to the formyl-BODIPY 2.4, but poor solubility of $\mathbf{2 . 2 1}$ in DCM (or anything) leads to anomalous extinction coefficients. Based on the TD-DFT calculations, and owing to the structural diversity in this series, the dominant transitions (denoted by the red arrow) differ throughout the series. When comparing the electrochemical properties, oxidation potentials are also varied and a second oxidation corresponding to the $\mathrm{N}=\mathrm{N}$ bond is observed for $\mathbf{2 . 2 0}$ (not listed in table). 
a)

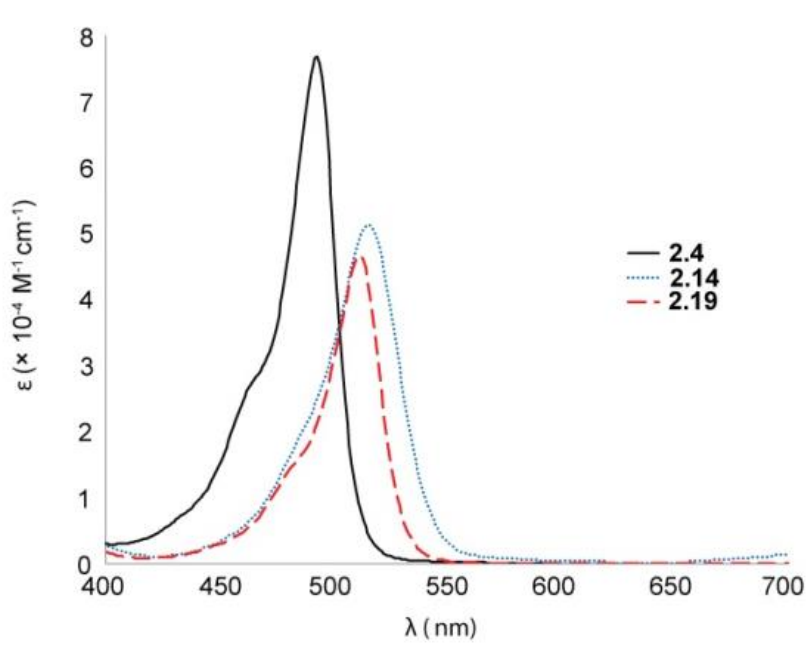

b)
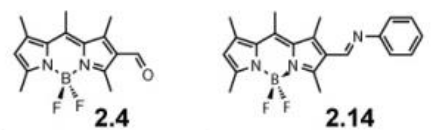

2.14

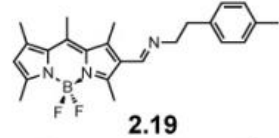

LUMO

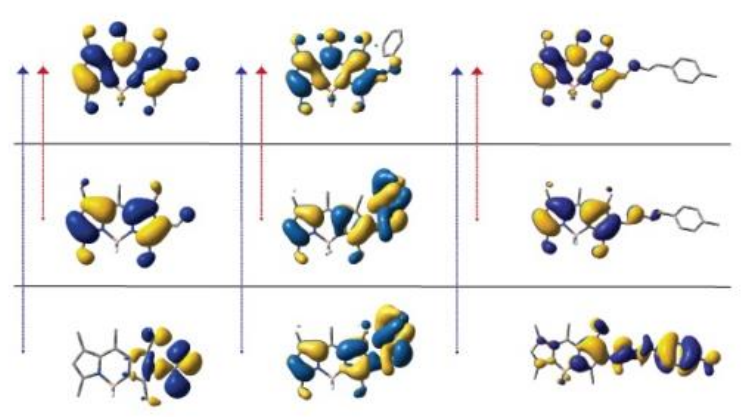

Figure 2.4: UV-Vis spectra (in DCM) for target molecules 2.4, 2.14 and 2.19; comparing the effect of Schiff - base formation on a family of BODIPY dyes

UV-vis (in DCM) and TD-DFT data for select mono-substituted iodinated derivatives have been collated in Figure 2.5. Unlike the previous series presented in Figure 2, this series has less variation in the absorption spectra. While all derivatives are red-shifted compared to BODIPY 2.3, there are only slight variations between 2.5, 2.23, and 2.25a, with alkyne 2.23 having the longest wavelength absorption. This result is likely due to a lack of planarity \& conjugation between the triazole ring and the BODIPY portion owning to steric reasons. This is also consistent with the TD-DFT calculations, showing that nearly all the dyes have the similar orbital transitions leading to the absorption (HOMO to LUMO dominates, but significant HOMO-1 to LUMO character contributes). Furthermore, in the optimized geometry, the TMS portion of $\mathbf{2 . 2 3}$ adopts a coplanar geometry relative to the BODIPY, while triazole coplanarity cannot be accommodated in the ground state. 


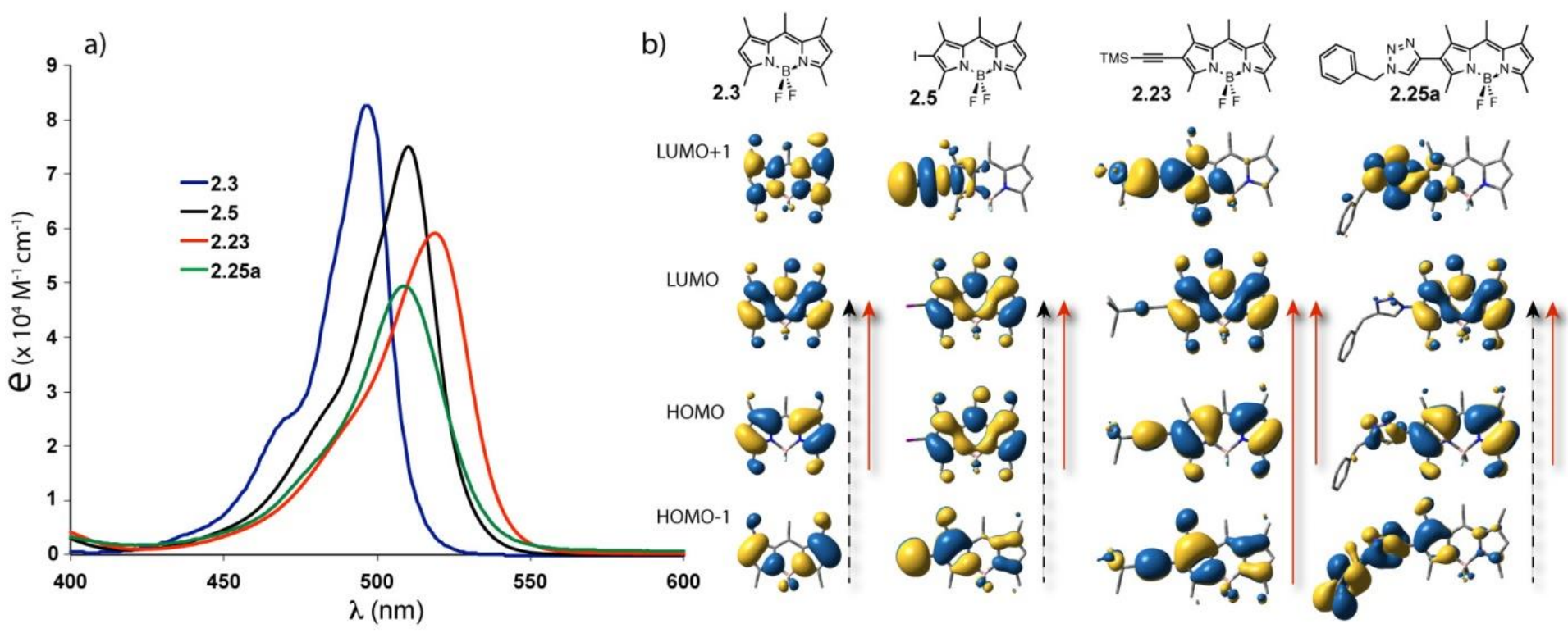

Figure 2.5: UV-Vis spectra (in DCM) for target molecules 2.3, 2.5, 2.24 and 2.25a. b) Calculated TD-DFT transitions (dominant contributions in red, significant contributions represented as dashed arrow) between the corresponding frontier molecular orbitals

Table 2.3 Physicochemical characterization of molecules derived from iodo-BODIPY, 2.5 .

\begin{tabular}{|c|c|c|c|c|}
\hline \multirow{3}{*}{ Compound } & \multicolumn{2}{|c|}{$\begin{array}{l}E_{1 / 2} \quad\left(\begin{array}{ll}V & v s \\
N H E)^{a}\end{array}\right.\end{array}$} & $U V$-vis $^{b} \lambda_{\max }(\varepsilon)$ & \multirow{3}{*}{$\begin{array}{l}\text { Dominant } F M O \\
\text { transition from } \\
T D-D F T\end{array}$} \\
\hline & $E_{\text {red }}$ & $E_{o x}$ & $\mathrm{~nm} \quad\left(\times 10^{4} \quad M^{-}\right.$ & \\
\hline & & & $\left.{ }^{1} \mathrm{~cm}^{-1}\right)$ & \\
\hline 2.3 & -1.05 & 1.50 & $497(8.3)$ & HOMO to LUMO \\
\hline 2.5 & -1.03 & 1.67 & $520(5.9)$ & HOMO to LUMO \\
\hline 2.24 & -0.88 & 1.67 & $513(9.2)$ & HOMO to LUMO \\
\hline $2.25 a$ & - & 1.42 & $510(4.9)$ & HOMO to LUMO \\
\hline $2.25 b$ & -0.94 & 1.46 & $507(6.3)$ & HOMO to LUMO \\
\hline 2.26 & -0.83 & 1.65 & $528(9.2)$ & HOMO to LUMO \\
\hline 2.27 & -1.06 & 1.72 & $546(5.4)$ & HOMO to LUMO \\
\hline 2.28 & -1.21 & 1.69 & $533(8.2)$ & HOMO to LUMO \\
\hline $2.29 a$ & - & 1.36 & $522(6.2)$ & HOMO to LUMO \\
\hline $2.29 b$ & - & 1.50 & $522(1.0)$ & HOMO to LUMO \\
\hline
\end{tabular}




\footnotetext{
a Data collected using $0.1 \mathrm{M} \mathrm{NBu}_{4} \mathrm{PF}_{6} \mathrm{DCM}$ solutions at $100 \mathrm{mVs}^{-1}$ and referenced to a $[\mathrm{Fc}] /[\mathrm{FC}]+$ internal standard followed by conversion to $\mathrm{NHE}$; $\mathrm{FC}] /[\mathrm{FC}+]=+765 \mathrm{mV}$ vs. $\mathrm{NHE}$ in $\mathrm{DCM} ;{ }^{b}$ low energy or visible transitions from UV-Vis of BODIPY family in DCM.
}

These observations are also supported by electrochemical data and all physical data for this series is collated in Table 2. 3. The strongly inductive EW effect of the alkyne (2.23, 2.24, 2.27 and 2.28) manifests as higher oxidation potentials relative to $\mathbf{2 . 3}$ owing to the stabilization of the HOMO orbital. Despite being a heteroaromatic, even the $6 \pi$ triazole serves as an ED group, as evidenced by the destabilization of the HOMO orbital making it easier to oxidize $\mathbf{2 . 2 5}$ and 2.29. Varying between benzyl and phenyl substitutes, results in the benzyl derivatives being easier to oxidize than the phenyl derivatives owing to the additional benzylic ED effect. Unfortunately owing to the instability of the triazole and alkyne to reduction, reversible electrochemical behaviour was not observed for many of the compounds in this series.

\subsection{Conclusions}

Using BODIPY as a scaffold, undergraduate students have prepared and examined the structure-property relationships of 18 novel derivatives. In addition, this robust platform has the modularity and tunability to explore a multitude of structural variation and property relationships. From a pedagogical exercise, the development of novel derivatives is an incredibly valuable opportunity for students to appreciate \& contribute to research, while reinforcing \& applying their knowledge from undergraduate curricula. It was found, that even more conceptually challenging physical characterization (e.g., DFT, electrochemistry, etc.) could be introduced and appreciated when directly related to the derivatives prepared. This is the way that most graduate students learn advanced techniques, and this early exposure also benefits undergraduate learning.

In addition to the practical knowledge acquired, the manuscript created by the learner (and peers), provides the student with the opportunity to assemble \& draft a scientific manuscript contrasting \& appreciating the structure-property relationships. Exposure to this level of scientific writing provides the learner with a high sense of accomplishment as they have contributed to both discovery-based research and the greater scientific community.

From the first course trials, one of the most significant challenges for a student is to assemble their work into a logical scientific document. Students had difficulty collecting good data from the BODIPY derivatives because the lack of prerequisite analytical skills. 


\subsection{Experimental}

\section{General Considerations}

All reagents were purchased from Aldrich except $\mathrm{PdCl}_{2}\left(\mathrm{PPh}_{3}\right)_{2}$ and ferrocene (Pressure Chemical Co., Pittsburg, PA). Purification by column chromatography was carried out using silica (Silicycle: ultrapure flash silica). Analytical thin-layer chromatography was performed on aluminum-backed sheets precoated with silica $60 \mathrm{~F} 254$ adsorbent (0.25 mm thick; Silicycle) and visualized under UV light. Absorbance in solution (usually DCM) was measured in a $1 \mathrm{~cm}$ cuvette using an Agilent Cary Series UV-Vis-NIR Spectrophotometer between the ranges of 200-2000 nm. Electrochemcial data was acquired with a 3electrode cell (Pt working, Pt counter, Ag pseudo reference) using a MetrOhm aAutolab II potentiostat/galvanostat. Cyclic voltammetry was acquired in DCM $(100 \mathrm{mV} / \mathrm{s})$, and ferrocene was added as an internal calibrant (referenced to $0.765 \mathrm{~V}$ vs NHE). Routine ${ }^{1} \mathrm{H},{ }^{13} \mathrm{C}\{1 \mathrm{H}\},{ }^{11} \mathrm{~B}\{1 \mathrm{H}\}$ and ${ }^{19} \mathrm{~F} \mathrm{NMR}$ spectra were recorded at 400,100, 128 and $376 \mathrm{MHz}$, respectively, on a Bruker AV 400 instrument at ambient temperature. Chemical shifts $(\delta)$ are reported in parts per million (ppm) from low to high field and

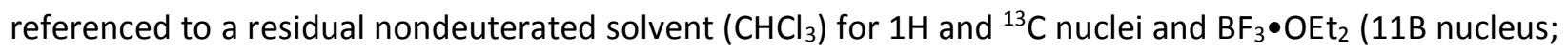
? $=0 \mathrm{ppm}$ ) C6F6 (19F nucleus; $\delta=0 \mathrm{ppm}$ ). Standard abbreviations indicating multiplicity are used as follows: $s$ = singlet; $d=$ doublet; $m=$ multiplet; $b r=$ broad.

\section{Theoretical Calculations}

All DFT and TD-DFT calculation was done use Gaussian 09M version C software. All structure were optimized at ground state default spin using the B3LYP exchange correlation function. These calclations were done in the gas phase (no solvent). For TD-DFT calculations were done at TD-SCF using the B3LYP exchange correlation function. All calculation were optimized using $3.21 \mathrm{G}$ basis set $(6.31 \mathrm{G}$ basis set when mentioned).

\section{Experimental Details}<smiles>CCOC(=O)c1[nH]c(C)c(C(=O)OCC)c1C</smiles>

\section{1}

2.1: Ethyl acetoacetate $(19.5 \mathrm{~mL}, 154 \mathrm{mmol})$ was diluted in glacial $\mathrm{AcOH}(70 \mathrm{~mL})$ and the solution was cooled in an ice bath. In another flask, a solution of $\mathrm{NaNO}_{2}(12.2 \mathrm{~g}, 177 \mathrm{mmol})$ and $\mathrm{H}_{2} \mathrm{O}(20 \mathrm{~mL})$ was cooled in an ice bath and added dropwise to the first mixture, maintaining an internal temperature between 6 $10^{\circ} \mathrm{C}$. The resulting yellow solution was stirred at room temperature overnight and ethyl acetoacetate (22.5 $\mathrm{mL}, 178 \mathrm{mmol}$ ) was added to the mixture. Zinc powder $(22.6 \mathrm{~g}, 344 \mathrm{mmol})$ was added in small portions to increase the internal temperature of the mixture to $65^{\circ} \mathrm{C}$. The mixture was then cooled to 0 ${ }^{\circ} \mathrm{C}$ and the ice bath was removed. Zinc powder was added in small portions to increase the internal 
temperature of the mixture to $70^{\circ} \mathrm{C}$ and the remaining was added, maintaining an internal temperature between $60-80^{\circ} \mathrm{C}$. After the last addition of zinc powder, the mixture was refluxed $24 \mathrm{~h}$ and poured onto ice. The solids were filtered and washed with $\mathrm{H}_{2} \mathrm{O}$. The crude material was recrystallized from hot EtOH, affording the desired product as a peach coloured solid $(15.48 \mathrm{~g}, 84 \%) .{ }^{1} \mathrm{H} \mathrm{NMR}\left(\mathrm{CDCl}_{3}\right): \delta=9.09(\mathrm{~s}, 1 \mathrm{H}$, $\mathrm{Ha}), 4.30\left(\mathrm{q},{ }^{3} \mathrm{~J}=7.1 \mathrm{~Hz}, 6 \mathrm{H}, \mathrm{H}_{\mathrm{Et}}\right), 2.56\left(\mathrm{~s}, 3 \mathrm{H}, \mathrm{H}_{\mathrm{c}}\right), 2.51\left(\mathrm{~s}, 3 \mathrm{H}, \mathrm{H}_{\mathrm{b}}\right), 1.36\left(\mathrm{t},{ }^{3} \mathrm{~J}=7.1 \mathrm{~Hz}, 4 \mathrm{H}, \mathrm{H}_{\mathrm{d}}\right)$. The remainder of the characterization was also consistent with previously reported literature. ${ }^{22}$

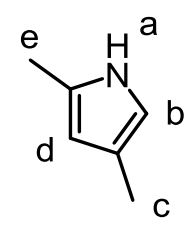

\section{2}

2.2: In a $250 \mathrm{~mL}$ Schlenk flask, under an atmosphere of $\mathrm{N}_{2}$, diethyl-3,5-dimethyl-1H-pyrrole-2,4dicarboxylate $(15.0 \mathrm{~g}, 63 \mathrm{mmol})$ was dissolved in ethylene glycol $(75 \mathrm{~mL})$ and $\mathrm{KOH}(11.7 \mathrm{~g}, 157 \mathrm{mmol})$ was slowly added. The mixture was heated to $160^{\circ} \mathrm{C}$ for $3 \mathrm{~h}$. After being cooled to room temperature, the product was distilled off under vacuum, affording the desired product as an oxygen sensitive dark oil (3.75 g, 63\%). ${ }^{1} \mathrm{HNMR}\left(\mathrm{CDCl}_{3}\right): \delta=7.62\left(\mathrm{~s}, 1 \mathrm{H}, \mathrm{H}_{\mathrm{a}}\right), 6.41\left(\mathrm{~s}, 1 \mathrm{H}, \mathrm{H}_{\mathrm{b}}\right), 5.76\left(\mathrm{~s}, 1 \mathrm{H}, \mathrm{H}_{\mathrm{d}}\right), 2.24\left(\mathrm{~s}, 3 \mathrm{H}, \mathrm{H}_{\mathrm{e}}\right), 2.09\left(\mathrm{~s}, 3 \mathrm{H}, \mathrm{H}_{\mathrm{d}}\right)$. The remainder of the characterization was also consistent with previously reported literature. ${ }^{16}$

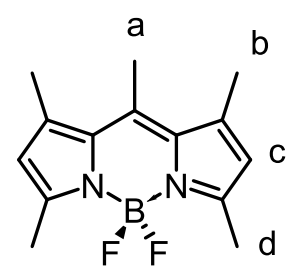

2.3

2.3: In a $250 \mathrm{~mL}$ Schlenk flask, under an atmosphere of $\mathrm{N}_{2}$, acetyl chloride ( $4.35 \mathrm{~mL}, 60.70 \mathrm{mmol}$ ), was added dropwise at room temperature over $30 \mathrm{~min}$ to a solution of 2,4-dimethyl-pyrrole ( $2.5 \mathrm{~g}, 26 \mathrm{mmol})$, in dry DCM $(10 \mathrm{~mL})$. The mixture was refluxed for $1 \mathrm{~h}$ and after cooling to room temperature, hexanes (50 $\mathrm{mL}$ ) was added to the mixture. Volatiles (azeotroped hexanes and excess acetyl chloride) were removed in vacuo and dry DCM $(120 \mathrm{~mL})$ was added. $\mathrm{NEt}_{3}(10.4 \mathrm{~mL}, 75 \mathrm{mmol})$ was added and the mixture stirred for $10 \mathrm{~min} . \mathrm{BF}_{3}(13.6 \mathrm{~mL}, 113 \mathrm{mmol}$ ) was added dropwise and stirred for $1 \mathrm{~h}$ at room temperature. The mixture was washed with aqueous saturated $\mathrm{NaHCO}_{3}$ and the organic layer was dried over $\mathrm{MgSO}_{4}$, filtered and volatiles were removed in vacuo. The oily residue was chromatographed using $D C M\left(R_{f}=0.92\right)$ and the fluorescent yellow band collected. After removing the volatiles the resulting residue was recrystallized from EtOH (2.65 g, 77\%). ${ }^{1} \mathrm{H} \mathrm{NMR}\left(\mathrm{CDCl}_{3}\right): \delta=6.05\left(\mathrm{~s}, 2 \mathrm{H}, \mathrm{H}_{\mathrm{c}}\right), 2.57\left(\mathrm{~s}, 3 \mathrm{H}, \mathrm{H}_{\mathrm{a}}\right), 2.51\left(\mathrm{~s}, 6 \mathrm{H}, \mathrm{H}_{\mathrm{d}}\right), 2.41(\mathrm{~s}, 6 \mathrm{H}$, $\left.\mathrm{H}_{\mathrm{b}}\right)$. The remainder of the characterization was also consistent with previously reported literature. ${ }^{27}$ 


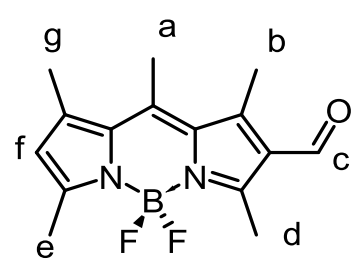

2.4

2.4: An oven dried Schlenk flask was charged with DMF $(10 \mathrm{~mL})$, and DCE $(30 \mathrm{~mL})$. The solution was cooled to $0{ }^{\circ} \mathrm{C}$ and $\mathrm{POCl}_{3}(12 \mathrm{~mL})$ was added over $5 \mathrm{~min}$. The solution was stirred an additional $5 \mathrm{~min}$ before being warmed to room temperature. After $30 \mathrm{~min}$ stirring at room temperature, a solution of $2.3(0.26 \mathrm{~g}, 1$ $\mathrm{mmol})$ in DCE $(30 \mathrm{~mL})$ was added in one portion. The reaction was stirred for $3 \mathrm{~h}$ at room temperature and monitored by TLC for consumption of starting material 2.3. The solution was slowly poured onto saturated aqueous $\mathrm{NaHCO}_{3}(400 \mathrm{~mL})$ at $0{ }^{\circ} \mathrm{C}$ then warmed to room temperature overnight. The organic layer was extracted with DCM and solvents were removed in vacuo. The residue was purified by column

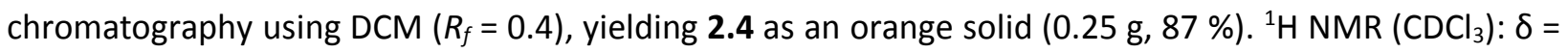
$10.10\left(\mathrm{~s}, 1 \mathrm{H}, \mathrm{H}_{\mathrm{c}}\right), 6.24\left(\mathrm{~s}, 1 \mathrm{H}, \mathrm{H}_{\mathrm{f}}\right), 2.77\left(\mathrm{~s}, 3 \mathrm{H}, \mathrm{H}_{\mathrm{d}}\right), 2.74\left(\mathrm{~s}, 3 \mathrm{H}, \mathrm{H}_{\mathrm{b}}\right), 2.69\left(\mathrm{~s}, 3 \mathrm{H}, \mathrm{H}_{\mathrm{a}}\right), 2.58\left(\mathrm{~s}, 3 \mathrm{H}, \mathrm{H}_{\mathrm{e}}\right), 2.48(\mathrm{~s}$, $\left.3 \mathrm{H}, \mathrm{H}_{\mathrm{g}}\right)$. The remainder of the characterization was also consistent with previously reported literature. ${ }^{23}$

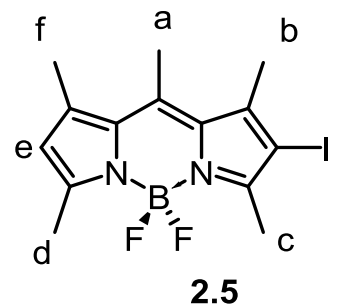

2.5: 2.3 ( $1.0 \mathrm{~g}, 3.8 \mathrm{mmol})$ was dissolved in a minimum amount of DCM and NIS $(0.86 \mathrm{~g}, 3.8 \mathrm{mmol})$ was added in one portion. The mixture was stirred $12 \mathrm{~h}$ in a round bottom flask covered with Al foil. Volatiles were removed in vacuo and the crude material was purified via column chromatography using hexanes:DCM (1:1) as the eluent, affording the desired compound as an orange solid $(1.13 \mathrm{~g}, 77 \%)$, In addition, starting material 2.3 (52 mg, 5\%) was recovered and diiodo derivative 2.26 (112 $\mathrm{mg}, 6 \%)$ was also obtained. ${ }^{1} \mathrm{H} \mathrm{NMR}\left(\mathrm{CDCl}_{3}, 400 \mathrm{MHz}\right): \delta=6.12\left(\mathrm{~s}, 1 \mathrm{H}, \mathrm{H}_{\mathrm{e}}\right), 2.61\left(\mathrm{~s}, 3 \mathrm{H}, \mathrm{H}_{\mathrm{a}}\right), 2.60\left(\mathrm{~s}, 3 \mathrm{H}, \mathrm{H}_{\mathrm{c}}\right), 2.53(\mathrm{~s}, 3 \mathrm{H}$, $\left.\mathrm{H}_{\mathrm{d}}\right), 2.46\left(\mathrm{~s}, 3 \mathrm{H}, \mathrm{H}_{\mathrm{b}}\right), 2.43\left(\mathrm{~s}, 3 \mathrm{H}, \mathrm{H}_{\mathrm{f}}\right)$. The remainder of the characterization was also consistent with previously reported literature. ${ }^{19}$

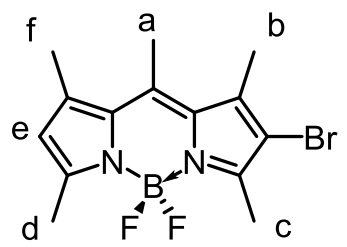

2.6 
2.6: In a $250 \mathrm{~mL} \operatorname{RBF}(\mathbf{2 . 3})$ (500 mg, $1.91 \mathrm{mmol}$ ), and NBS (373 mg, $2.10 \mathrm{mmol}$ ), were dissolved in DCM $(100 \mathrm{~mL})$. The flask was covered in Al foil and stirred at room temperature, under $\mathrm{N}_{2}$ overnight. The solvent was removed in vacuo and the residue was purified through column chromatography in DCM:hexanes (1:1) $\left(R_{f}=0.5\right)$, to yield a bright orange powder (440 mg, 68\%). ${ }^{1} \mathrm{H} \mathrm{NMR}\left(400 \mathrm{MHz}, \mathrm{CDCl}_{3}\right): \delta$ $=6.11\left(\mathrm{~s}, 1 \mathrm{H}, \mathrm{H}_{\mathrm{e}}\right), 2.61\left(\mathrm{~s}, 3 \mathrm{H}, \mathrm{H}_{\mathrm{a}}\right), 2.56\left(\mathrm{~s}, 3 \mathrm{H}, \mathrm{H}_{\mathrm{d}}\right), 2.53\left(\mathrm{~s}, 3 \mathrm{H}, \mathrm{H}_{\mathrm{c}}\right), 2.43\left(\mathrm{~s}, 6 \mathrm{H}, \mathrm{H}_{\mathrm{b}}, \mathrm{H}_{\mathrm{f}}\right) .{ }^{13} \mathrm{C}\left\{{ }^{1} \mathrm{H}\right\} \mathrm{NMR}\left(\mathrm{CDCl}_{3}\right.$, $100 \mathrm{MHz}): \delta=156.1,149.6,143.0,141.6,136.4,132.7,130.6,122.3,110.4,17.5,16.8,16.1,14.6,13.4$. $\left.{ }^{11} \mathrm{~B}\left\{{ }^{1} \mathrm{H}\right\} \mathrm{NMR}\left(\mathrm{CDCl}_{3}, 128 \mathrm{MHz}\right): \delta=0.46\left(\mathrm{t},{ }^{1} J_{\mathrm{BF}}=32 \mathrm{~Hz},\right)\right) \cdot{ }^{19} \mathrm{~F} \mathrm{NMR}\left(\mathrm{CDCl}_{3}, 376 \mathrm{MHz}\right): \delta=-146.4(\mathrm{q}) . \mathrm{HRMS}$ (EI): $m / z 340.0570\left([\mathrm{M}+\mathrm{H}]^{+}\right)$, calculated for $\left[{ }^{12} \mathrm{C}_{14}{ }^{1} \mathrm{H}_{16}{ }^{11} \mathrm{~B}^{79} \mathrm{Br}^{19} \mathrm{~F}_{2}{ }^{14} \mathrm{~N}_{2}\right]^{+}: m / z 340.055$

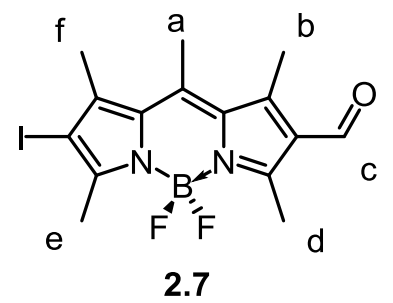

2.7: 2.4 (100 mg, $0.34 \mathrm{mmol}$ ) was dissolved in DCM ( $35 \mathrm{~mL}$ ) and NIS ( $81 \mathrm{mg}, 0.36 \mathrm{mmol}$ ) was added. The mixture was stirred at room temperature $12 \mathrm{~h}$ with the flask covered with Al foil. Volatiles were removed in vacuo and the crude material was purified via column chromatography using DCM as the eluent, affording the desired product as an orange solid (125 mg, 88\%). Characterization is consistent with previously reported. ${ }^{4}{ }^{1} \mathrm{H} N M R\left(\mathrm{CDCl}_{3}\right): \delta 10.12\left(\mathrm{~s}, 1 \mathrm{H}, \mathrm{H}_{\mathrm{c}}\right), 2.79\left(\mathrm{~s}, 3 \mathrm{H}, \mathrm{H}_{\mathrm{d}}\right), 2.75\left(\mathrm{~s}, 3 \mathrm{H}, \mathrm{H}_{\mathrm{b}}\right), 2.73\left(\mathrm{~s}, 3 \mathrm{H}, \mathrm{H}_{\mathrm{a}}\right)$, $2.67\left(\mathrm{~s}, 3 \mathrm{H}, \mathrm{H}_{\mathrm{e}}\right), 2.53\left(\mathrm{~s}, 3 \mathrm{H}, \mathrm{H}_{\mathrm{f}}\right)$. The remainder of the characterization was also consistent with previously reported literature. ${ }^{11}$

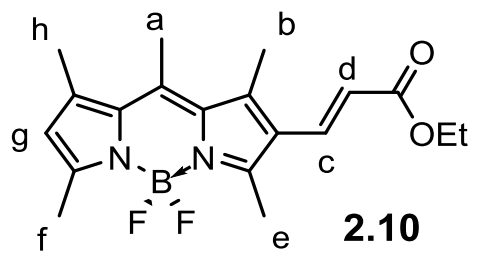

2.10: In a $250 \mathrm{~mL}$ round bottom flask, formyl BODIPY $(100 \mathrm{mg}, 0.34 \mathrm{mmol})$ and (carboxymethylene)triphenylphosphorane $(259 \mathrm{mg}, 0.776 \mathrm{mmol}$ ) were dissolved in a mixture of $20 \mathrm{~mL}$ dry DCM and was stirred overnight at room temperature in an inert atmosphere $\left(\mathrm{N}_{2}\right)$. Volatiles were removed in vacuo and the crude material was purified via column chromatography using 3:1 hexanes:EtOAc as the eluent affording the desired product as a orange solid (60 mg, 50\%). ${ }^{1} \mathrm{H} \mathrm{NMR}\left(\mathrm{CDCl}_{3}\right): \delta=7.70\left(\mathrm{~d},{ }^{3} \mathrm{~J}=16.22\right.$ $\left.\mathrm{Hz}, 1 \mathrm{H}, \mathrm{H}_{\mathrm{d}}\right), 6.13\left(\mathrm{~s}, 1 \mathrm{H}, \mathrm{H}_{\mathrm{g}}\right), 6.94\left(\mathrm{~d},{ }^{3} \mathrm{~J}=16.23 \mathrm{~Hz}, 1 \mathrm{H}, \mathrm{H}_{\mathrm{c}}\right), 4.26\left(\mathrm{q},{ }^{3} \mathrm{~J}=7.1 \mathrm{~Hz}, 2 \mathrm{H}, \mathrm{H}_{\mathrm{Et}}\right), 2.66\left(\mathrm{~s}, 3 \mathrm{H}, \mathrm{H}_{\mathrm{e}}\right)$, $2.63\left(\mathrm{~s}, 3 \mathrm{H}, \mathrm{H}_{\mathrm{b}}\right), 2.54\left(\mathrm{~s}, 3 \mathrm{H}, \mathrm{H}_{\mathrm{a}}\right), 2.48\left(\mathrm{~s}, 3 \mathrm{H}, \mathrm{H}_{\mathrm{h}}\right), 2.43\left(\mathrm{~s}, 3 \mathrm{H}, \mathrm{H}_{\mathrm{f}}\right), 1.34\left(\mathrm{t},{ }^{3} \mathrm{~J}=7.1 \mathrm{~Hz}, 3 \mathrm{H}, \mathrm{H}_{\mathrm{Et}}\right) .{ }^{13} \mathrm{C}\left\{{ }^{1} \mathrm{H}\right\} \mathrm{NMR}$ $\left(\mathrm{CDCl}_{3}, 100 \mathrm{MHz}\right): \delta=167.77,156.82,156.77,152.69,143.36,143.30,142.35,138.22,136.06,133.58$, $131.72,124.88,124.85,122.92,118.04,60.53,17.78,17.26,15.11,14.79,14.53,14.45 .{ }^{11} \mathrm{~B}\left\{{ }^{1} \mathrm{H}\right\} \mathrm{NMR}$ $\left(\mathrm{CDCl}_{3}, 128 \mathrm{MHz}\right): \delta=0.60$ (t). ${ }^{19} \mathrm{~F} \mathrm{NMR}\left(\mathrm{CDCl}_{3}, 376 \mathrm{MHz}\right): \delta=-145.27$ (q). HRMS (EI): $\mathrm{m} / z$ 360.1829 ([M] $]^{+}$), calculated for $\left[{ }^{12} \mathrm{C}_{19}{ }^{1} \mathrm{H}_{23}{ }^{11} \mathrm{~B}^{19} \mathrm{~F}_{2} \mathrm{~N}_{2}{ }^{16} \mathrm{O}_{2}\right]^{+}: \mathrm{m} / \mathrm{z} 360.1821$. 


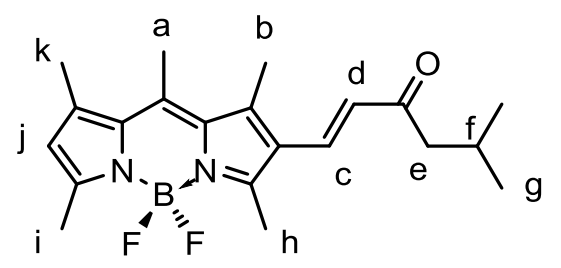

2.11

2.11: Into a $250 \mathrm{~mL}$ round bottom flask charged with EtOH $(40 \mathrm{~mL})$, formyl BODIPY (100 mg, 0.34 $\mathrm{mmol}$ ), $p-\mathrm{TsOH}\left(10\right.$ mole \%), acetone and $\mathrm{MgSO}_{4}$ were added. The reaction was brought to reflux at $80^{\circ} \mathrm{C}$ for $24 \mathrm{~h}$. The solution was dried under vacuo and further extracted with DCM. The organic layer was dried with $\mathrm{MgSO}_{4}$ and the solvent was removed in vacuo. The product was purified through an aluminium oxide column with a 7:3 (DCM/hexanes) solvent mixture $\left(R_{f}=0.53\right)$, and the fluorescent orange band was collected. After removing the volatiles, a dark red powder of product was obtained (20.1 mg, 16\%). ${ }^{1} \mathrm{H}$ NMR $\left(\mathrm{CDCl}_{3}\right): \delta=7.60\left(\mathrm{~d},{ }^{3} \mathrm{~J}=16 \mathrm{~Hz}, 1 \mathrm{H}, \mathrm{H}_{\mathrm{c}}\right), 6.44\left(\mathrm{~d}, 3^{3} \mathrm{~J}\right.$ $\left.=16 \mathrm{~Hz}, 1 \mathrm{H}, \mathrm{H}_{\mathrm{d}}\right), 6.15\left(\mathrm{~s}, 1 \mathrm{H}, \mathrm{H}_{\mathrm{j}}\right), 2.66\left(\mathrm{~s}, 6 \mathrm{H}, \mathrm{H}_{\mathrm{e}}, \mathrm{H}_{\mathrm{h}}\right), 2.55\left(\mathrm{~s}, 3 \mathrm{H}, \mathrm{H}_{\mathrm{a}}\right), 2.51\left(\mathrm{~s}, 3 \mathrm{H}, \mathrm{H}_{\mathrm{i}}\right), 2.49\left(\mathrm{~s}, 3 \mathrm{H}, \mathrm{H}_{\mathrm{k}}\right)$, $2.45\left(\mathrm{~s}, 3 \mathrm{H}, \mathrm{H}_{\mathrm{e}}\right), 2.22\left(\mathrm{dt},{ }^{3} \mathrm{~J}=13.7,6.8 \mathrm{~Hz}, 1 \mathrm{H}, \mathrm{H}_{\mathrm{j}}\right), 1.00-0.98\left(\mathrm{~d},{ }^{3} \mathrm{~J}=6.6 \mathrm{~Hz}, 6 \mathrm{H}, \mathrm{H}_{\mathrm{g}}\right) .{ }^{13} \mathrm{C}\left\{{ }^{1} \mathrm{H}\right\} \mathrm{NMR}$ $\left(\mathrm{CDCl}_{3}, 100 \mathrm{MHz}\right): \delta=200.3,157.1,142.3,133.9,126.4,123.1,53.6,34.8,31.7,29.2,25.6,25.4,22.8,18.9$, 18.6, 17.8, 17.3, 15.2, 14.3, 11.6. ${ }^{11} \mathrm{~B}\{\mathrm{H}\} \mathrm{NMR}\left(\mathrm{CDCl}_{3}, 128 \mathrm{MHz}\right): \delta=0.58\left(\mathrm{t},{ }^{1} J_{\mathrm{BF}}=30.9 \mathrm{~Hz}\right) .{ }^{19} \mathrm{~F} \mathrm{NMR}$ $\left(\mathrm{CDCl}_{3}, 376 \mathrm{MHz}\right): \delta=-145.35$ (q). HRMS (EI): $\mathrm{m} / z \quad 373.2259$ [(M) $\left.{ }^{+}\right]$, calculated for $\left[{ }^{12} \mathrm{C}_{21}{ }^{1} \mathrm{H}_{27}{ }^{11} \mathrm{~B}^{19} \mathrm{~F}_{2} \mathrm{~N}_{2}{ }^{16} \mathrm{O}\right]^{+}: m / z 372.2668$.

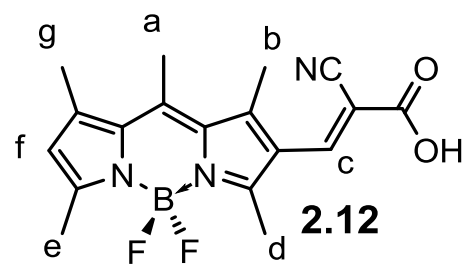

2.12: Formyl BODIPY ( $100 \mathrm{mg}, 0.35 \mathrm{mmol}$ ) was dissolved in minimal amount of $\mathrm{CHCl}_{3}$ and cyanoacetic acid ( $56 \mathrm{mg}, 0.66 \mathrm{mmol}$ ) and a drop of piperidine were added. The mixture was refluxed overnight and after being cooled to room temperature, the organic layer was washed with $1 \mathrm{M} \mathrm{HCl}$. Volatiles were removed in vacuo. The crude was recrystallized using DCM/Hexanes (3:2) to obtain a red solid (56 mg, 45\%). ${ }^{1} \mathrm{H}$ $\operatorname{NMR}\left(\mathrm{CDCl}_{3}\right): \delta=8.30\left(\mathrm{~s}, 1 \mathrm{H}, \mathrm{H}_{\mathrm{c}}\right), 6.23\left(\mathrm{~s}, 1 \mathrm{H}, \mathrm{H}_{\mathrm{f}}\right), 2.68\left(\mathrm{~s}, 1 \mathrm{H}, \mathrm{H}_{\mathrm{d}}\right), 2.62\left(\mathrm{~s}, 1 \mathrm{H}, \mathrm{H}_{\mathrm{b}}\right), 2.577\left(\mathrm{~s}, 1 \mathrm{H}, \mathrm{H}_{\mathrm{a}}\right), 2.51(\mathrm{~s}$, $\left.1 \mathrm{H}, \mathrm{H}_{\mathrm{e}}\right), 2.47\left(\mathrm{~s}, 1 \mathrm{H}, \mathrm{H}_{\mathrm{g}}\right) .{ }^{13} \mathrm{C}\left\{{ }^{1} \mathrm{H}\right\} \mathrm{NMR}\left(\mathrm{CDCl}_{3}, 100 \mathrm{MHz}\right): \delta=192.4,158.9,151.9,148.8,144.9,142.8,137.9$, $134.4,132.2,123.8,123.6,116.4,45.1,17.1,14.8,14.0 .{ }^{11} \mathrm{~B}\{\mathrm{H}\} \mathrm{NMR}\left(\mathrm{CDCl}_{3}, 128 \mathrm{MHz}\right): \delta=0.855 .{ }^{19} \mathrm{~F} \mathrm{NMR}$ $\left(\mathrm{CDCl}_{3}, 376 \mathrm{MHz}\right): \delta=-145.9$ (q). HRMS (HREI): $\mathrm{m} / \mathrm{z} 380.13523\left([\mathrm{M}+\mathrm{Na}]^{+}\right)$, calculated for $\left[{ }^{12} \mathrm{C}_{18}{ }^{1} \mathrm{H}_{18}{ }^{11} \mathrm{~B}^{19} \mathrm{~F}_{2} \mathrm{~N}_{3}{ }^{16} \mathrm{O}_{2}\right]^{+}: \mathrm{m} / \mathrm{z} 357.1460$. 


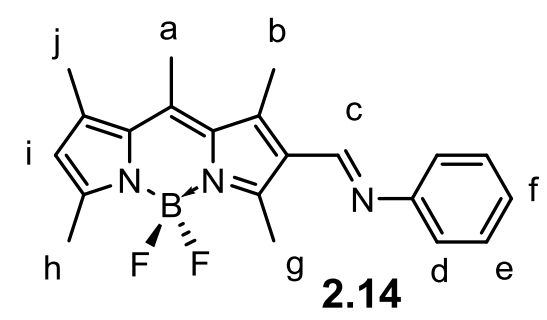

2.14: Formyl BODIPY ( $100 \mathrm{mg}, 0.34 \mathrm{mmol}$ ), aniline $(64 \mathrm{mg}, 0.68 \mathrm{mmol}), p-\mathrm{TsOH}$ (cat.) and $\mathrm{MgSO}_{4}$ in anhydrous EtOH was stirred under reflux for $4 \mathrm{~h}$. The residue was purified by column chromatography using basic alumina and a starting eluent of 1:1 DCM:hexanes to a gradient of 9:1 DCM:EtOAc $\left(R_{f}, 1: 1\right.$ DCM:hexanes $=0.44)$, yielding 2.14 as a red solid $(18 \mathrm{mg}, 15 \%) .{ }^{1} \mathrm{H} \mathrm{NMR}(400 \mathrm{MHz}, \mathrm{CDCl} 3): \delta=8.52(\mathrm{~s}, 1 \mathrm{H}$, $\left.\mathrm{H}_{\mathrm{c}}\right), 7.39\left(\mathrm{t},{ }^{3} \mathrm{~J}=7.5 \mathrm{~Hz}, 2 \mathrm{H}, \mathrm{H}_{\mathrm{d}}\right), 7.14-7.20\left(\mathrm{~m}, 3 \mathrm{H}, \mathrm{H}_{\mathrm{e}}, \mathrm{H}_{\mathrm{f}}\right) 6.16\left(\mathrm{~s}, 1 \mathrm{H}, \mathrm{H}_{\mathrm{i}}\right), 2.82\left(\mathrm{~s}, 3 \mathrm{H}, \mathrm{H}_{\mathrm{g}}\right), 2.76\left(\mathrm{~s}, 3 \mathrm{H}, \mathrm{H}_{\mathrm{b}}\right)$, $2.69\left(\mathrm{~s}, 3 \mathrm{H}, \mathrm{H}_{\mathrm{a}}\right), 2.57\left(\mathrm{~s}, 3 \mathrm{H}, \mathrm{H}_{\mathrm{h}}\right), 2.46\left(\mathrm{~s}, 3 \mathrm{H}, \mathrm{H}_{\mathrm{j}}\right) .{ }^{13} \mathrm{C}\left\{{ }^{1} \mathrm{H}\right\} \mathrm{NMR}\left(100 \mathrm{MHz}, \mathrm{CDCl}_{3}\right): \delta=157.00,154.62,154.38$, 153.50, 142.83, 139.61, 133.77, 131.66, 125.45, 123.06, 32.07, 31.07, 29.85, 29.81, 29.51, 22.84, 17.85, $17.23,14.85,14.27,13.63 .{ }^{11} \mathrm{~B}\left\{{ }^{1} \mathrm{H}\right\} \mathrm{NMR}\left(\mathrm{CDCl}_{3}, 128 \mathrm{MHz}\right) \delta=0.63\left(\mathrm{t},{ }^{1} \mathrm{~J}_{\mathrm{BF}}=32.3 \mathrm{~Hz}\right) .{ }^{19} \mathrm{~F} \mathrm{NMR}\left(\mathrm{CDCl}_{3}, 376\right.$ $\mathrm{MHz}) \delta=-145.51$ (q). HRMS (El): $m / z 365.1871\left([\mathrm{M}]^{+}\right)$, calculated for $\left[{ }^{12} \mathrm{C}_{21}{ }^{1} \mathrm{H}_{22}{ }^{11} \mathrm{~B}^{19} \mathrm{~F}_{2} \mathrm{~N}_{3}\right]^{+}: \mathrm{m} / z 365.1875$.

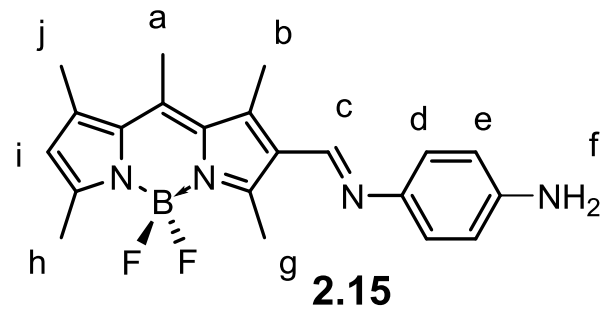

2.15: Formyl BODIPY ( $290 \mathrm{mg}, 1 \mathrm{mmol}$ ), dianiline ( $1 \mathrm{mmol}), p-\mathrm{TsOH}$ (cat.) and $\mathrm{MgSO}_{4}$ were added to a round bottom flask with EtOH. The mixture was stirred and refluxed for $6 \mathrm{~h}$ and then diluted with EtOAc and washed with water and saturated aqueous $\mathrm{NaHCO}_{3}$. The organic layer was extracted and dried with $\mathrm{Na}_{2} \mathrm{SO}_{4}$ and filtered. The residue was precipitated with $\mathrm{DCM}$ and $\mathrm{MeOH}$. The resultant product was washed with $\mathrm{MeOH} .{ }^{1} \mathrm{H} \mathrm{NMR}\left(400 \mathrm{MHz}, \mathrm{CDCl}_{3}\right): \delta=8.54\left(\mathrm{~s}, 1 \mathrm{H}, \mathrm{H}_{\mathrm{c}}\right), 7.09$ $\left(\mathrm{d},{ }^{3} \mathrm{~J}=8.6 \mathrm{~Hz} 2 \mathrm{H}, \mathrm{H}_{\mathrm{d}}\right), 6.72\left(\mathrm{~d},{ }^{3} \mathrm{~J}=8.6 \mathrm{~Hz} 2 \mathrm{H}, \mathrm{H}_{\mathrm{e}}\right) 6.14\left(\mathrm{~s}, 1 \mathrm{H}, \mathrm{H}_{\mathrm{i}}\right), 3.67\left(\mathrm{~s}, 2 \mathrm{H}, \mathrm{H}_{\mathrm{f}}\right), 2.82\left(\mathrm{~s}, 3 \mathrm{H}, \mathrm{H}_{\mathrm{g}}\right)$, $2.74\left(\mathrm{~s}, 3 \mathrm{H}, \mathrm{H}_{\mathrm{b}}\right), 2.68\left(\mathrm{~s}, 3 \mathrm{H}, \mathrm{H}_{\mathrm{a}}\right), 2.56\left(\mathrm{~s}, 3 \mathrm{H}, \mathrm{H}_{\mathrm{h}}\right), 2.46\left(\mathrm{~s}, 3 \mathrm{H}, \mathrm{H}_{\mathrm{j}}\right) .{ }^{11} \mathrm{~B}\left\{{ }^{1} \mathrm{H}\right\} \mathrm{NMR}\left(\mathrm{CDCl}_{3}, 128 \mathrm{MHz}\right) \delta=$ $0.56\left(\mathrm{t},{ }^{1} J_{\mathrm{BF}}=31.55 \mathrm{~Hz}\right) .{ }^{19} \mathrm{~F} \mathrm{NMR}\left(\mathrm{CDCl}_{3}, 376 \mathrm{MHz}\right) \delta=-145.53$ (q). HRMS (El): $\mathrm{m} / z 380.1981\left([\mathrm{M}]^{+}\right)$, calculated for $\left[{ }^{12} \mathrm{C}_{21}{ }^{1} \mathrm{H}_{23}{ }^{11} \mathrm{~B}^{19} \mathrm{~F}_{2} \mathrm{~N}_{4}\right]^{+}: \mathrm{m} / z 380.1984$.

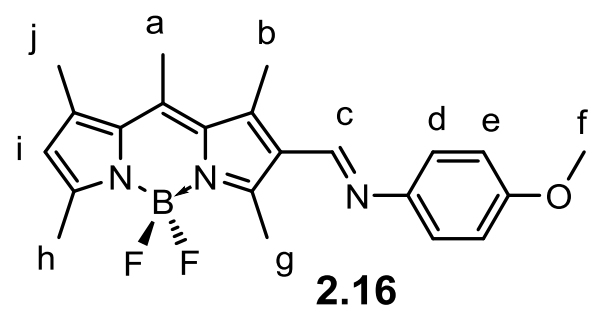

2.16: Formyl BODIPY ( $100 \mathrm{mg}, 0.34 \mathrm{mmol}), p$ - anisidine $(85 \mathrm{mg}, 0.69 \mathrm{mmol}), p-\mathrm{TsOH}$ (cat.) and anhydrous $\mathrm{Na}_{2} \mathrm{SO}_{4}$ in $\mathrm{EtOH}$ was stirred under reflux for $1 \mathrm{~h}$. The residue was purified by column chromatography using 
basic alumina and 4:1 hexane:EtOAc, yielding compound 2.16 as a dark red-brown solid $(0.03 \mathrm{~g}, 22 \%) .{ }^{1} \mathrm{H}$ $\operatorname{NMR}\left(400 \mathrm{MHz}, \mathrm{CDCl}_{3}\right): \delta=8.53\left(\mathrm{~s}, 1 \mathrm{H}, \mathrm{H}_{\mathrm{c}}\right), 7.16\left(\mathrm{~d},{ }^{3} \mathrm{~J}=8.0 \mathrm{~Hz}, 2 \mathrm{H}, \mathrm{H}_{\mathrm{d}}\right), 6.92\left(\mathrm{~d},{ }^{3} \mathrm{~J}=8.0 \mathrm{~Hz}, \mathrm{H}_{\mathrm{e}}\right), 6.15(\mathrm{~s}, 1 \mathrm{H}$, $\left.\mathrm{H}_{\mathrm{i}}\right), 3.83\left(\mathrm{~s}, 3 \mathrm{H}, \mathrm{H}_{\mathrm{f}}\right), 2.81\left(\mathrm{~s}, 3 \mathrm{H}, \mathrm{H}_{\mathrm{g}}\right), 2.75\left(\mathrm{~s}, 3 \mathrm{H}, \mathrm{H}_{\mathrm{b}}\right), 2.69\left(\mathrm{~s}, 3 \mathrm{H}, \mathrm{H}_{\mathrm{a}}\right), 2.56\left(\mathrm{~s}, 3 \mathrm{H}, \mathrm{H}_{\mathrm{h}}\right), 2.46\left(\mathrm{~s}, 3 \mathrm{H}, \mathrm{H}_{\mathrm{j}}\right) .{ }^{13} \mathrm{C}$ $\mathrm{NMR}\left(\mathrm{CDCl}_{3}\right): \delta=157.96,156.51,154.58,152.56,146.43,143.18,142.73,125.55,122.83,121.97,114.50$, $55.66,29.84,17.78,17.14,14.79,13.68 .{ }^{11} \mathrm{~B}\left\{{ }^{1} \mathrm{H}\right\} \mathrm{NMR}\left(\mathrm{CDCl}_{3}, 128 \mathrm{MHz}\right) \delta=0.63\left(\mathrm{t},{ }^{1} \mathrm{~J}_{\mathrm{BF}}=32.2 \mathrm{~Hz}\right) .{ }^{19} \mathrm{~F} \mathrm{NMR}$ ( $\mathrm{CDCl}_{3}, 376 \mathrm{MHz}$ ): -145.46 (q). HRMS (EI): $\mathrm{m} / \mathrm{z} 395.1979$ ([M] $\left.]^{+}\right)$, calculated for $\left[{ }^{12} \mathrm{C}_{22}{ }^{1} \mathrm{H}_{24}{ }^{11} \mathrm{~B}^{19} \mathrm{~F}_{2} \mathrm{~N}_{3}{ }^{16} \mathrm{O}\right]^{+}: \mathrm{m} / z$ 395.1980

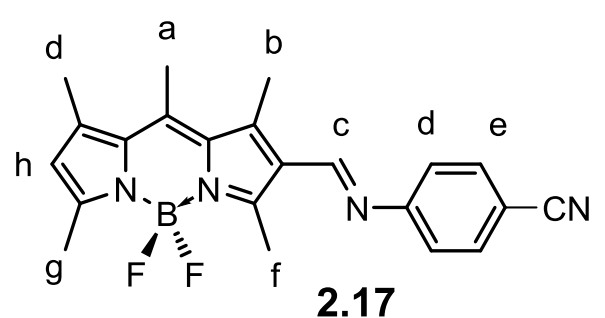

2.17: $20 \mathrm{~mL}$ microwave vial with a handful of molecular sieves was sparged with $\mathrm{N}_{2}$ for 10 minutes, and then flame dried. Formyl BODIPY ( $100 \mathrm{mg}, 0.34 \mathrm{mmol}$ ) was added to $5 \mathrm{~mL}$ of toluene and sparged for 5 minutes with $\mathrm{N}_{2}$. 4-aminobenzonitrile $(63 \mathrm{mg}, 0.53 \mathrm{mmol}$ ) was added to the solution and sparged again with $\mathrm{N}_{2}$ for 5 minutes. After capping the microwave vial, the mixture was stirred for $4 \frac{1}{2}$ hours in an oil bath set to $108^{\circ} \mathrm{C}$. After cooling to room temperature, the residue was purified by column chromatography using basic alumina and a starting eluent of 5:1 DCM:hexane $\left(R_{f}, 1: 1 \mathrm{DCM}\right.$ :hexanes = 0.53), yielding 2.17 as a red solid (93 mg, 73\%). $\left.{ }^{1} \mathrm{H} \mathrm{NMR} \mathrm{(400} \mathrm{MHz,CDCl}\right): \delta=8.46\left(\mathrm{~s}, 1 \mathrm{H}, \mathrm{H}_{\mathrm{c}}\right), 7.66\left(\mathrm{~d},{ }^{3} \mathrm{~J}=\right.$ $\left.8.5 \mathrm{~Hz}, 2 \mathrm{H}, \mathrm{H}_{\mathrm{e}}\right), 7.18\left(\mathrm{~d},{ }^{3} \mathrm{~J}=8.5 \mathrm{~Hz}, 2 \mathrm{H}, \mathrm{H}_{\mathrm{d}}\right), 6.20\left(\mathrm{~s}, 2 \mathrm{H}, \mathrm{H}_{\mathrm{h}}\right), 2.81\left(\mathrm{~s}, 3 \mathrm{H}, \mathrm{H}_{\mathrm{f}}\right), 2.75\left(\mathrm{~s}, 3 \mathrm{H}, \mathrm{H}_{\mathrm{b}}\right), 2.70(\mathrm{~s}, 3 \mathrm{H}$, $\left.\mathrm{H}_{\mathrm{a}}\right), 2.58\left(\mathrm{~s}, 3 \mathrm{H}, \mathrm{H}_{\mathrm{g}}\right), 2.48\left(\mathrm{~s}, 3 \mathrm{H}, \mathrm{H}_{\mathrm{d}}\right) .{ }^{13} \mathrm{C} \mathrm{NMR}\left(\mathrm{CDCl}_{3}\right): \delta=167.66,156.82,156.77,152.69,143.36,143.30$, $142.35,138.22$, 136.06, .133.58, 131.72, 124.88, 122.92, 118.04, 60.53, 17.78, 17.26, 15.11, 14.79, 14.53, 14.45. ${ }^{11} \mathrm{~B}\left\{{ }^{1} \mathrm{H}\right\} \mathrm{NMR}\left(\mathrm{CDCl}_{3}, 128 \mathrm{MHz}\right) \delta=0.60$ (t). ${ }^{19} \mathrm{~F} \mathrm{NMR}\left(\mathrm{CDCl}_{3}, 376 \mathrm{MHz}\right):-145.27$ (q). HRMS (EI): $\mathrm{m} / \mathrm{z}$ $390.183\left([\mathrm{M}]^{+}\right)$, calculated for $\left[{ }^{12} \mathrm{C}_{22}{ }^{1} \mathrm{H}_{21}{ }^{11} \mathrm{~B}^{19} \mathrm{~F}_{2} \mathrm{~N}_{4}\right]^{+}: \mathrm{m} / z$ 390.1827.

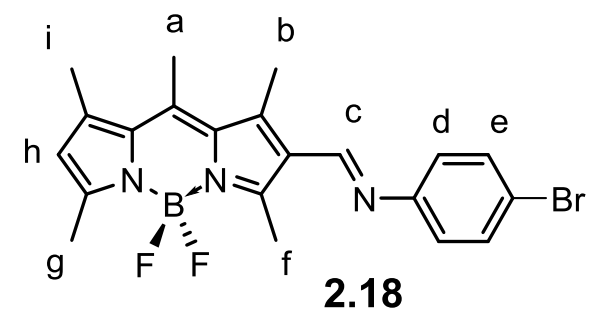

2.18: A microwave vial was flame dried under nitrogen environment. After being cooled to room temperature, formyl BODIPY (100 mg, $0.34 \mathrm{mmol}$ ) and $5 \mathrm{~mL}$ of dry toluene were added to the vial. The solution was stirred and 4-bromoaniline $(237 \mathrm{mg}, 1.38 \mathrm{mmol}$ ) was added. The reaction mixture was then left to stir for $4 \mathrm{~h}$ in an oil bath at $110^{\circ} \mathrm{C}$, and monitored by TLC for consumption of starting material. The solvent was removed in vacuo, and the residue was purified by column chromatography using basic alumina and a starting eluent of $6: 4$ hexanes:DCM $\left(R_{f}, 1: 1\right.$ DCM:hexanes $\left.=0.22\right)$, yielding 2.18 as a pink- 
red solid (46 mg, 26\%). ${ }^{1} \mathrm{H}$ NMR (400 MHz, CDCl $)$ ): $\delta=8.48\left(\mathrm{~s}, 1 \mathrm{H}, \mathrm{H}_{\mathrm{c}}\right.$ ), $7.47\left(\mathrm{~d},{ }^{3} \mathrm{~J}=8.7 \mathrm{~Hz}, 2 \mathrm{H}, \mathrm{H}_{\mathrm{d}}\right) .7 .02(\mathrm{~d}$, $\left.{ }^{3} \mathrm{~J}=8.7 \mathrm{~Hz}, 2 \mathrm{H}, \mathrm{H}_{\mathrm{e}}\right), 6.17\left(\mathrm{~s}, 1 \mathrm{H}, \mathrm{H}_{\mathrm{i}}\right), 2.81\left(\mathrm{~s}, 3 \mathrm{H}, \mathrm{H}_{\mathrm{f}}\right), 2.75\left(\mathrm{~s}, 3 \mathrm{H}, \mathrm{H}_{\mathrm{b}}\right), 2.70\left(\mathrm{~s}, 3 \mathrm{H}, \mathrm{H}_{\mathrm{a}}\right), 2.57\left(\mathrm{~s}, 3 \mathrm{H}, \mathrm{H}_{\mathrm{g}}\right), 2.47$ $\left(\mathrm{s}, 3 \mathrm{H}, \mathrm{H}_{\mathrm{i}}\right) .{ }^{11} \mathrm{~B}\left\{{ }^{1} \mathrm{H}\right\} \mathrm{NMR}\left(\mathrm{CDCl}_{3}, 128 \mathrm{MHz}\right) \delta=0.62$ (t). ${ }^{19} \mathrm{~F} \mathrm{NMR}\left(\mathrm{CDCl}_{3}, 376 \mathrm{MHz}\right):-145.52$ (q). HRMS (EI): $\mathrm{m} / \mathrm{z} 443.0981\left([\mathrm{M}]^{+}\right)$, calculated for $\left[{ }^{12} \mathrm{C}_{21}{ }^{1} \mathrm{H}_{21}{ }^{11} \mathrm{~B}^{19} \mathrm{~F}_{2} \mathrm{~N}_{3}{ }^{79} \mathrm{Br}\right]^{+}: \mathrm{m} / \mathrm{z} 443.0980$.

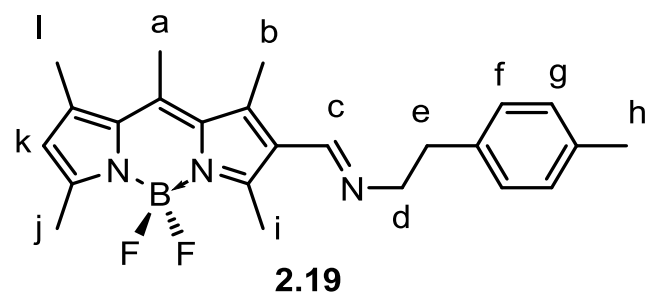

2.19: Formyl BODIPY ( $100 \mathrm{mg}, 0.34 \mathrm{mmol}$ ), 2-(p-Tolyl)ethylamine ( $70 \mathrm{mg}, 0.52 \mathrm{mmol}), p$ - TsOH (cat.) and anhydrous $\mathrm{Na}_{2} \mathrm{SO}_{4}$ in EtOH was stirred under reflux for $1 \mathrm{~h}$. The solution was then dried under vacuo and triturated in EtOH, yielding 2.19 (80 mg, 54\%). $\left.{ }^{1} \mathrm{H} \mathrm{NMR} \mathrm{(400} \mathrm{MHz,} \mathrm{CDCl}_{3}\right): \delta=8.20\left(\mathrm{~s}, 1 \mathrm{H}, \mathrm{H}_{\mathrm{c}}\right), 7.11(\mathrm{~m}, 4 \mathrm{H}$, $\left.\mathrm{H}_{\mathrm{g}}, \mathrm{H}_{\mathrm{h}}\right), 6.11\left(\mathrm{~s}, 1 \mathrm{H}, \mathrm{H}_{\mathrm{k}}\right), 3.79\left(\mathrm{t},{ }^{3} \mathrm{~J}=7.2 \mathrm{~Hz}, 2 \mathrm{H}, \mathrm{H}_{\mathrm{e}}\right), 2.95\left(\mathrm{t},{ }^{3} \mathrm{~J}=7.2 \mathrm{~Hz}, 2 \mathrm{H}, \mathrm{H}_{\mathrm{d}}\right), 2.66\left(\mathrm{~s}, 3 \mathrm{H}, \mathrm{H}_{\mathrm{h}}\right), 2.64(\mathrm{~s}, 3 \mathrm{H}$, $\left.\mathrm{H}_{\mathrm{i}}\right) 2.60\left(\mathrm{~s}, 3 \mathrm{H}, \mathrm{H}_{\mathrm{b}}\right), 2.54\left(\mathrm{~s}, 3 \mathrm{H}, \mathrm{H}_{\mathrm{a}}\right), 2.43\left(\mathrm{~s}, 3 \mathrm{H}, \mathrm{H}_{\mathrm{j}}\right), 2.32\left(\mathrm{~s}, 3 \mathrm{H}, \mathrm{H}_{\mathrm{l}}\right) \cdot{ }^{13} \mathrm{C}\left\{{ }^{1} \mathrm{H}\right\} \mathrm{NMR}\left(\mathrm{CDCl}_{3}, 100 \mathrm{MHz}\right): \delta=$ $155.15,142.45,139.17,137.03,135.48,128.95,64.59,53.41,37.42,21.01,14.59,13.24 .{ }^{11} \mathrm{~B}\left\{{ }^{1} \mathrm{H}\right\} \mathrm{NMR}$ $\left(\mathrm{CDCl}_{3}, 128 \mathrm{MHz}\right): \delta=0.81$ (t). ${ }^{19} \mathrm{~F} \mathrm{NMR}\left(\mathrm{CDCl}_{3}, 376 \mathrm{MHz}\right): \delta=-145.8$ (q). HRMS (El): $\mathrm{m} / z$ 407.2341 ([M] $]^{+}$), calculated for $\left[{ }^{12} \mathrm{C}_{24}{ }^{1} \mathrm{H}_{28}{ }^{11} \mathrm{~B}^{19} \mathrm{~F}_{2} \mathrm{~N}_{3}\right]^{+}: \mathrm{m} / z$ 407.2344.

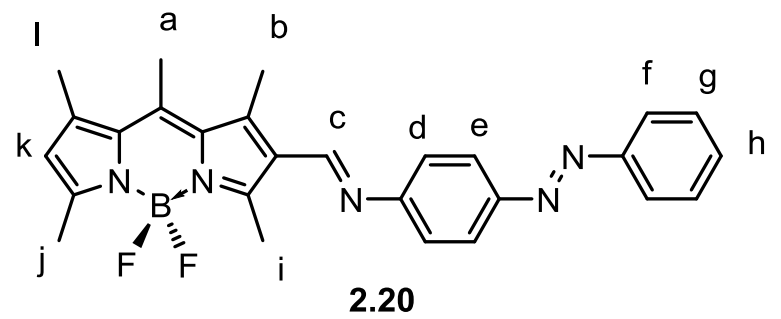

2.20: Formyl BODIPY (100 mg, $0.34 \mathrm{mmol}$ ), 4-diazoaniline (70 mg, $0.34 \mathrm{mmol}), p$-Toluenesulfonic acid (10 mole \%), and $\mathrm{MgSO}_{4}$ were added into a $250 \mathrm{~mL}$ round bottom flask charged with $\mathrm{EtOH}(\sim 40 \mathrm{~mL}$ ). The reaction was brought to reflux at $80^{\circ} \mathrm{C}$ for $24 \mathrm{~h}$. The solution was then dried under vacuo and further extracted with $\mathrm{DCM}$. The organic layer was kept and dried with $\mathrm{MgSO}_{4}$ and brought to vacuo. The product was purified through an aluminium oxide column 4:1 (hexanes/EtOAc) $\left(R_{f}=0.81\right.$ ), and a pink band was collected. After removing the volatiles, the resulting residue was dissolved in hexanes and stored in a $36^{\circ} \mathrm{C}$ fridge to crystalize the pure derivative 2.20 and to remove trace amounts of formyl BODIPY. The crystals were filtered through a Buchner funnel, and dark red crystals $(19 \%, 30.1 \mathrm{mg})$ were obtained. ${ }^{1} \mathrm{H}$ $\operatorname{NMR}\left(\mathrm{CDCl}_{3}\right): \delta=8.58\left(\mathrm{~s}, 1 \mathrm{H}, \mathrm{H}_{\mathrm{c}}\right), 7.98\left(\mathrm{dd},{ }^{3} \mathrm{~J}=8.7 \mathrm{~Hz}, 2 \mathrm{H}, \mathrm{H}_{\mathrm{f}}\right), 7.93-7.91\left(\mathrm{dd},{ }^{3} \mathrm{~J}=7.0 \mathrm{~Hz}, 2 \mathrm{H}, \mathrm{H}_{\mathrm{e}}\right), 7.52$ $\left(\mathrm{t},{ }^{3} \mathrm{~J}=7.3 \mathrm{~Hz}, 2 \mathrm{H}, \mathrm{H}_{\mathrm{g}}\right), 7.47\left(\mathrm{~d},{ }^{3} \mathrm{~J}=7.2 \mathrm{~Hz} 1 \mathrm{H}, \mathrm{H}_{\mathrm{h}}\right), 7.27\left(\mathrm{dd},{ }^{3} \mathrm{~J}=8 \mathrm{~Hz}, 2 \mathrm{H}, \mathrm{H}_{\mathrm{d}}\right), 6.18\left(\mathrm{~s}, 1 \mathrm{H}, \mathrm{H}_{\mathrm{k}}\right), 2.85$ $\left(\mathrm{s}, 3 \mathrm{H}, \mathrm{H}_{\mathrm{i}}\right), 2.79\left(\mathrm{~s}, 3 \mathrm{H}, \mathrm{H}_{\mathrm{b}}\right), 2.71\left(\mathrm{~s}, 3 \mathrm{H}, \mathrm{H}_{\mathrm{a}}\right), 2.58\left(\mathrm{~s}, 3 \mathrm{H}, \mathrm{H}_{\mathrm{j}}\right), 2.48\left(\mathrm{~s}, 3 \mathrm{H}, \mathrm{H}_{\mathrm{l}}\right) .{ }^{13} \mathrm{C}\left\{{ }^{1} \mathrm{H}\right\} \mathrm{NMR}\left(\mathrm{CDCl}_{3}, 100\right.$ $\mathrm{MHz}): \delta=186.3,155.9,154.8,153.0,143.9,130.8,129.2,128.2,127.7,124.3,122.9,122.6,121.6,121.5$, $121.2,120.4,18.0,17.9,17.3,14.9,14.2 .{ }^{11} \mathrm{~B}\{\mathrm{H}\} \mathrm{NMR}\left(\mathrm{CDCl}_{3}, 128 \mathrm{MHz}\right): \delta=0.63\left(\mathrm{t},{ }^{1} J_{\mathrm{BF}}=30.9 \mathrm{~Hz}\right) .{ }^{19} \mathrm{~F}$ $\mathrm{NMR}\left(\mathrm{CDCl}_{3}, 376 \mathrm{MHz}\right): \delta=-145.53$ (q). HRMS (EI): $\mathrm{m} / z$ 469.2248 [(M) ${ }^{+}$, calculated for $\left[{ }^{12} \mathrm{C}_{27}{ }^{1} \mathrm{H}_{26}{ }^{11} \mathrm{~B}^{19} \mathrm{~F}_{2}{ }^{14} \mathrm{~N}_{5}\right]^{+}: \mathrm{m} / z 469.2249$. 


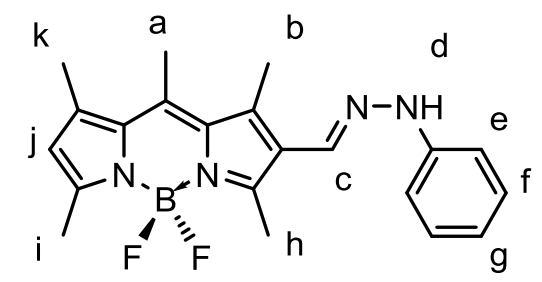

2.22

2.22: A $50 \mathrm{~mL}$ round bottom flask was charged with formyl BODIPY ( $100 \mathrm{mg}, 0.34 \mathrm{mmol})$ in DCM ( $20 \mathrm{~mL})$. Phenyl hydrazine $(0.5 \mathrm{~mL})$ was added in one portion and the reaction was stirred at room temperature for $2 \mathrm{~h}$ while being monitored by TLC for consumption of starting material. The solution was purified by column chromatography using DCM $\left(\mathrm{R}_{f}=0.4\right)$, yielding 2.22 as a black solid $(0.071 \mathrm{~g}, 55 \%) .{ }^{1} \mathrm{H} \mathrm{NMR}$ $\left((C D 3)_{2} C O\right): \delta=9.26\left(\mathrm{~s}, 1 \mathrm{H}, \mathrm{H}_{\mathrm{d}}\right), 8.01\left(\mathrm{~s}, 1 \mathrm{H}, \mathrm{H}_{\mathrm{c}}\right), 7.22\left(\mathrm{t},{ }^{3} \mathrm{~J}=7.7 \mathrm{~Hz}, 2 \mathrm{H}, \mathrm{H}_{\mathrm{f}}\right), 7.07\left(\mathrm{~d},{ }^{3} \mathrm{~J}=8.0 \mathrm{~Hz}, 2 \mathrm{H}, \mathrm{H}_{\mathrm{e}}\right), 6.75$ $\left(\mathrm{t},{ }^{3} \mathrm{~J}=7.2 \mathrm{~Hz}, 1 \mathrm{H}, \mathrm{H}_{\mathrm{g}}\right), 6.21\left(\mathrm{~s}, 1 \mathrm{H}, \mathrm{H}_{\mathrm{j}}\right), 2.74\left(\mathrm{~s}, 3 \mathrm{H}, \mathrm{H}_{\mathrm{h}}\right), 2.71\left(\mathrm{~s}, 3 \mathrm{H}, \mathrm{H}_{\mathrm{b}}\right), 2.63\left(\mathrm{~s}, 3 \mathrm{H}, \mathrm{H}_{\mathrm{a}}\right), 2.48-2.46\left(\mathrm{~s}, 6 \mathrm{H}, \mathrm{H}_{\mathrm{i}}\right.$, $\left.\mathrm{H}_{\mathrm{k}}\right) \cdot{ }^{11} \mathrm{~B}\left\{{ }^{1} \mathrm{H}\right\} \mathrm{NMR}\left(\mathrm{CDCl}_{3}, 128 \mathrm{MHz}\right): \delta=0.60$ (t). ${ }^{19} \mathrm{~F} \mathrm{NMR}\left(\mathrm{CDCl}_{3}, 376 \mathrm{MHz}\right): \delta=-145.2$ (q). HRMS (EI): $\mathrm{m} / z$ $380.1981\left([\mathrm{M}]^{+}\right)$, calculated for $\left[{ }^{12} \mathrm{C}_{21}{ }^{1} \mathrm{H}_{23}{ }^{11} \mathrm{~B}^{19} \mathrm{~F}_{2} \mathrm{~N}_{4}\right]^{+}: \mathrm{m} / z 380.1984$.

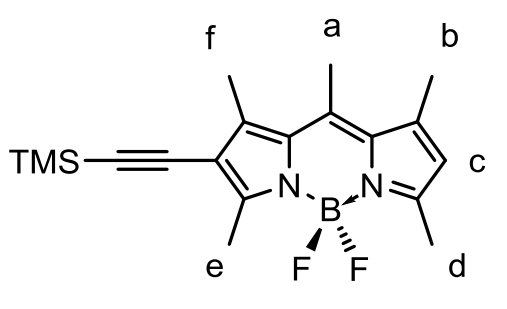

2.23: 2.5 ( $0.400 \mathrm{~g}, 1.031 \mathrm{mmol}), \mathrm{PdCl}_{2}\left(\mathrm{PPh}_{3}\right)_{2},(0.054 \mathrm{~g}, 0.077 \mathrm{mmol})$ and $\mathrm{Cul}(0.029 \mathrm{~g}, 0.155 \mathrm{mmol})$ were added to a $100 \mathrm{~mL}$ Schlenk flask under $\mathrm{N}_{2}$. After adding anhydrous THF (20 ml) and $\mathrm{NEt}_{3}\left(10 \mathrm{ml}^{2}\right.$, the mixture was sparged for $15 \mathrm{~min}$ with $\mathrm{N}_{2}$. Ethynyltrimethylsilane $(1.013 \mathrm{~g}, 10.309 \mathrm{mmol}$ ) was added and the mixture was left at room temperature stirring overnight. After the solvent was removed in vacuo, DCM was added to the mixture and it was washed with water. The organic layer was collected and dried with $\mathrm{MgSO}_{4}$. The dark red solid was then run through a silica column using DCM:hexanes $(2: 1)\left(R_{f}=0.79\right)$ as the eluent to get an orange solid. (251.4 mg, 68\%). ${ }^{1} \mathrm{H} \mathrm{NMR}\left(\mathrm{CDCl}_{3}\right): \delta=6.10\left(\mathrm{~s}, 1 \mathrm{H}, \mathrm{H}_{\mathrm{c}}\right), 2.60\left(\mathrm{~s}, 3 \mathrm{H}, \mathrm{H}_{\mathrm{d}}\right)$, $2.59\left(\mathrm{~s}, 3 \mathrm{H}, \mathrm{H}_{\mathrm{b}}\right), 2.53\left(\mathrm{~s}, 3 \mathrm{H}, \mathrm{H}_{\mathrm{a}}\right), 2.49\left(\mathrm{~s}, 3 \mathrm{H}, \mathrm{H}_{\mathrm{e}}\right), 2.43\left(\mathrm{~s}, 3 \mathrm{H}, \mathrm{H}_{\mathrm{f}}\right), 0.26\left(\mathrm{~s}, 9 \mathrm{H}, \mathrm{H}_{\mathrm{TMS}}\right) .{ }^{13} \mathrm{C}\left\{{ }^{1} \mathrm{H}\right\} \mathrm{NMR}\left(\mathrm{CDCl}_{3}, 100\right.$ $\mathrm{MHz}): \delta=155.9,155.2,142.6,141.9,141.0,122.2,100.7,97.8,17.5,16.7,15.8,14.6,13.3,0.2 .{ }^{11} \mathrm{~B}\left\{{ }^{1} \mathrm{H}\right\}$ $\mathrm{NMR}\left(\mathrm{CDCl}_{3}, 128 \mathrm{MHz}\right): \delta=0.48\left(\mathrm{t},{ }^{1} J_{\mathrm{BF}}=32.14 \mathrm{~Hz}\right) \cdot{ }^{19} \mathrm{~F} \mathrm{NMR}\left(\mathrm{CDCl}_{3}, 376 \mathrm{MHz}\right): \delta=-146.78$ (q). HRMS (EI): $\mathrm{m} / \mathrm{z} 358.1861\left([\mathrm{M}+\mathrm{H}]^{+}\right)$, calculated for $\left[{ }^{12} \mathrm{C}_{\mathrm{x}}{ }^{1} \mathrm{H}_{\mathrm{x}}{ }^{11} \mathrm{~B}^{19} \mathrm{~F}_{\mathrm{x}}{ }^{14} \mathrm{~N}_{\mathrm{x}}\right]^{+}: \mathrm{m} / \mathrm{z} 358.1848$. 


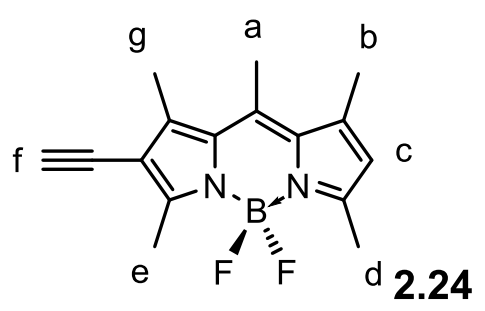

2.14: 2.23 (0.698 mmol, $0.250 \mathrm{~g}$ ) was dissolved in $\mathrm{MeOH}(25 \mathrm{~mL})$ and THF $(25 \mathrm{~mL})$ and then sparged with $\mathrm{N}_{2}$ for $10 \mathrm{~min}$. The reaction was then stirred overnight at room temperature with excess KF $(0.122 \mathrm{~g}, 3$ eq). The solvent was then removed in vacuo and solubilized with EtOAc $(50-100 \mathrm{~mL})$. The organic phase was washed with water $(3 \times 100 \mathrm{~mL})$, to remove any excess $\mathrm{KF}$. The EtOAc phase was then dried with $\mathrm{MgSO}_{4}$, gravity filtered and the solvent removed in vacuo. The residue was then subjected to TLC and column chromatography using DCM as the eluent $\left(R_{f}=0.69\right)(68.3 \mathrm{mg}, 34 \%) .{ }^{1} \mathrm{H} \mathrm{NMR}\left(\mathrm{CDCl}_{3}\right): \delta 6.11(\mathrm{~s}, 1 \mathrm{H}$, $\left.\mathrm{H}_{\mathrm{c}}\right), 3.33\left(\mathrm{~s}, 1 \mathrm{H}, \mathrm{H}_{\mathrm{f}}\right), 2.60\left(\mathrm{~s}, 3 \mathrm{H}, \mathrm{H}_{\mathrm{d}}\right), 2.59\left(\mathrm{~s}, 3 \mathrm{H}, \mathrm{H}_{\mathrm{b}}\right), 2.53\left(\mathrm{~s}, 3 \mathrm{H}, \mathrm{H}_{\mathrm{a}}\right), 2.49\left(\mathrm{~s}, 3 \mathrm{H}, \mathrm{H}_{\mathrm{e}}\right), 2.42\left(\mathrm{~s}, 3 \mathrm{H}, \mathrm{H}_{\mathrm{g}}\right) .{ }^{13} \mathrm{C}\left\{{ }^{1} \mathrm{H}\right\}$ $\operatorname{NMR}\left(\mathrm{CDCl}_{3}, 100 \mathrm{MHz}\right): \delta=207.0,156.3,154.8,143.1,142.1,141.3,133.2,130.5,122.4,113.6,83.2,77.2$, 17.5, 16.6, 15.7, 14.6, 13.2. ${ }^{11} \mathrm{~B}\left\{{ }^{1} \mathrm{H}\right\}$ NMR $\left(\mathrm{CDCl}_{3}, 128 \mathrm{MHz}\right): \delta=0.46\left(\mathrm{t},{ }^{1} \mathrm{~J}_{\mathrm{BF}}=32.07 \mathrm{~Hz}\right) .{ }^{19} \mathrm{~F} \mathrm{NMR}\left(\mathrm{CDCl}_{3}, 376\right.$ $\mathrm{MHz}): \delta=-146.51$ (q). HRMS (El): $m / z 286.1462\left([\mathrm{M}+\mathrm{H}]^{+}\right)$, calculated for $\left[{ }^{12} \mathrm{C}_{16}{ }^{1} \mathrm{H}_{17}{ }^{11} \mathrm{~B}^{19} \mathrm{~F}_{2}{ }^{14} \mathrm{~N}_{2}\right]^{+}: m / z$ 286.1453.

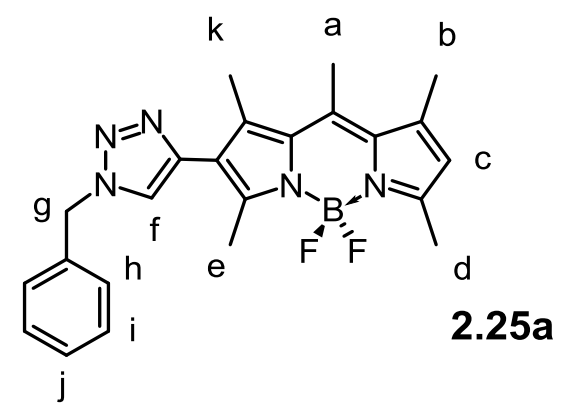

2.25a: To a solution of benzyl azide (0.018 mL, $0.140 \mathrm{mmol}), 2.24(0.050 \mathrm{~g}, 0.175 \mathrm{mmol})$ and tetrakis(acetonitrile)copper(I) hexafluorophosphate $(0.007 \mathrm{~g}, 0.017 \mathrm{mmol})$ in acetonitrile $(10 \mathrm{ml})$, were added. The resulting mixture was sparged with $\mathrm{N}_{2}$ for $10 \mathrm{~min}$ followed by the addition of $N, N$ diisopropylethylamine $(0.016 \mathrm{~g}, 0.140 \mathrm{mmol})$. The solution was stirred for $18 \mathrm{~h}$ while being monitored by TLC. The solvent was removed in vacuo and the residue subjected to column chromatography in hexanes:EtOAc (3:1) $\left(R_{f}=0.13\right)$. The desired product was isolated as an orange fluorescent band. (30.8 $\mathrm{mg}$, 54\%). ${ }^{1} \mathrm{H} \mathrm{NMR}\left(\mathrm{CDCl}_{3}\right): \delta$ 7.42-7.44-7.37 (m, $\left.4 \mathrm{H}, \mathrm{H}_{\mathrm{j}}, \mathrm{H}_{\mathrm{h}}, \mathrm{H}_{\mathrm{f}}\right), 7.32\left(\mathrm{dd},{ }^{3} \mathrm{~J}=7.9 \mathrm{~Hz},{ }^{4} \mathrm{~J}=2.0 \mathrm{~Hz}, \mathrm{H}_{\mathrm{i}}\right), 6.09(\mathrm{~s}, 1 \mathrm{H}$, $\left.\mathrm{H}_{\mathrm{c}}\right), 5.61\left(\mathrm{~s}, 2 \mathrm{H}, \mathrm{H}_{\mathrm{g}}\right), 2.64\left(\mathrm{~s}, 3 \mathrm{H}, \mathrm{H}_{\mathrm{d}}\right), 2.54\left(\mathrm{~s}, 3 \mathrm{H}, \mathrm{H}_{\mathrm{b}}\right), 2.53\left(\mathrm{~s}, 3 \mathrm{H}, \mathrm{H}_{\mathrm{a}}\right), 2.46\left(\mathrm{~s}, 3 \mathrm{H}, \mathrm{H}_{\mathrm{e}}\right), 2.43\left(\mathrm{~s}, 3 \mathrm{H}, \mathrm{H}_{\mathrm{k}}\right) .{ }^{13} \mathrm{C}\left\{{ }^{1} \mathrm{H}\right\}$ NMR $\left(\mathrm{CDCl}_{3}, 100 \mathrm{MHz}\right): \delta=154.8,151.8,142.1,142.0,141.2,137.8,134.8,132.7,131.7,129.1,128.7$, 128.0, 122.2, 121.9, 77.3, 54.1, 34.6, 25.2, 17.4, 16.8, 15.4. ${ }^{11} \mathrm{~B}\left\{{ }^{1} \mathrm{H}\right\} \mathrm{NMR}\left(\mathrm{CDCl}_{3}, 128 \mathrm{MHz}\right): \delta=0.66\left(\mathrm{t},{ }^{1} J_{\mathrm{BF}}\right.$ $=32.4 \mathrm{~Hz}) \cdot{ }^{19} \mathrm{~F} \mathrm{NMR}\left(\mathrm{CDCl}_{3}, 376 \mathrm{MHz}\right): \delta=-146.24$ (q). HRMS (EI): $m / z 419.2097\left([\mathrm{M}+\mathrm{H}]^{+}\right)$, calculated for $\left[{ }^{12} \mathrm{C}_{23}{ }^{1} \mathrm{H}_{24}{ }^{11} \mathrm{~B}^{19} \mathrm{~F}_{2}{ }^{14} \mathrm{~N}_{5}\right]^{+}: \mathrm{m} / \mathrm{z} 419.2093$. 


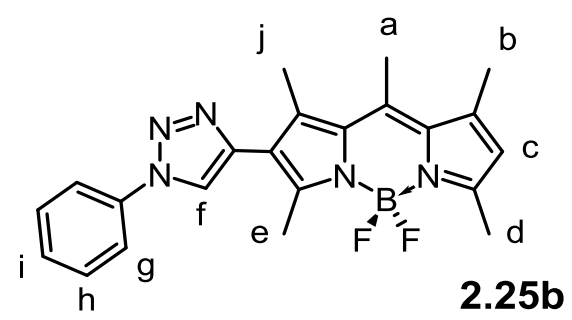

2.26b: To a solution of azidobenzene $(0.031 \mathrm{~g}, 0.210 \mathrm{mmol}), 2.24(0.060 \mathrm{~g}, 0.210 \mathrm{mmol})$ and tetrakis(acetonitrile)copper(I) hexafluorophosphate $(0.009 \mathrm{~g}, 0.025 \mathrm{mmol})$ in acetonitrile $(10 \mathrm{ml})$, were added. The resulting mixture was sparged with $\mathrm{N}_{2}$ for $10 \mathrm{~min}$ followed by the addition of $N, N$ diisopropylethylamine $(0.024 \mathrm{~g}, 0.210 \mathrm{mmol})$. The solution was stirred for $18 \mathrm{~h}$ while being monitored by TLC. The solvent was removed in vacuo and the residue subjected to column chromatography in hexanes:EtOAc (3:1) $\left(R_{f}=0.22\right)$. The desired product was isolated as an orange fluorescent band. (24.4 mg, 43\%). ${ }^{1} \mathrm{H}$ NMR $\left(\mathrm{CDCl}_{3}\right): \delta 7.94\left(\mathrm{~s}, 1 \mathrm{H}, \mathrm{H}_{\mathrm{f}}\right), 7.82\left(\mathrm{~d},{ }^{3} \mathrm{~J}=8.7 \mathrm{~Hz}, 2 \mathrm{H}, \mathrm{H}_{\mathrm{g}}\right), 7.57\left(\mathrm{t},{ }^{3} \mathrm{~J}=7.4 \mathrm{~Hz}, 2 \mathrm{H}, \mathrm{H}_{\mathrm{h}}\right), 7.47$ (t, $\left.{ }^{3} \mathrm{~J}=7.7 \mathrm{~Hz}, 1 \mathrm{H}, \mathrm{H}_{\mathrm{i}}\right), 6.13\left(\mathrm{~s}, 1 \mathrm{H}, \mathrm{H}_{\mathrm{c}}\right), 2.69\left(\mathrm{~s}, 3 \mathrm{H}, \mathrm{H}_{\mathrm{d}}\right), 2.64\left(\mathrm{~s}, 3 \mathrm{H}, \mathrm{H}_{\mathrm{b}}\right), 2.56,\left(\mathrm{~s}, 3 \mathrm{H}, \mathrm{H}_{\mathrm{a}}\right), 2.55\left(\mathrm{~s}, 3 \mathrm{H}, \mathrm{H}_{\mathrm{e}}\right), 2.46(\mathrm{~s}$, $\left.3 \mathrm{H}, \mathrm{H}_{\mathrm{j}}\right) .{ }^{13} \mathrm{C}\left\{{ }^{1} \mathrm{H}\right\}$ NMR $\left(\mathrm{CDCl}_{3}, 100 \mathrm{MHz}\right): \delta=155.3,151.7,142.2,142.2,141.6,137.9,137.0,132.9,131.8$, $129.8,128.8,122.1,120.5,120.2,29.7,17.6,16.9,15.5,14.6,13.5 .{ }^{11} \mathrm{~B}\left\{{ }^{1} \mathrm{H}\right\} \mathrm{NMR}\left(\mathrm{CDCl}_{3}, 128 \mathrm{MHz}\right): \delta=0.71$ $\left(\mathrm{t},{ }^{1} J_{\mathrm{BF}}=32.05 \mathrm{~Hz}\right) .{ }^{19} \mathrm{~F} \mathrm{NMR}\left(\mathrm{CDCl}_{3}, 376 \mathrm{MHz}\right): \delta=-146.13$ (q). HRMS (EI): $m / z 405.1950\left([\mathrm{M}+\mathrm{H}]^{+}\right)$, calculated for $\left[{ }^{12} \mathrm{C}_{\mathrm{x}}{ }^{1} \mathrm{H}_{\mathrm{x}}{ }^{11} \mathrm{~B}^{19} \mathrm{~F}_{\mathrm{x}}{ }^{14} \mathrm{~N}_{\mathrm{x}}\right]^{+}: \mathrm{m} / \mathrm{z} 405.1936$.

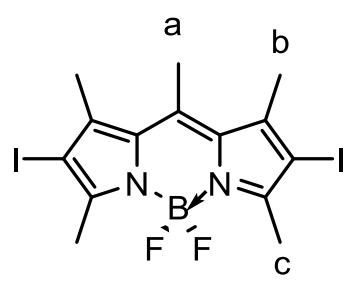

\subsection{6}

2.26: NIS ( $680 \mathrm{mg}, 3.04 \mathrm{mmol}$ ) was added to a solution of 2.3 (199 mg, $0.76 \mathrm{mmol}$ ) in DCM ( $35 \mathrm{~mL})$. The mixture was stirred $12 \mathrm{~h}$ at room temperature and volatiles were removed in vacuo. The crude material was purified through column chromatography using DCM as the eluent to afford the product as an orange solid (quantitative). ${ }^{1} \mathrm{H} \mathrm{NMR}\left(\mathrm{CDCl}_{3}\right): \delta 2.64\left(\mathrm{~s}, 3 \mathrm{H}, \mathrm{H}_{\mathrm{a}}\right), 2.61\left(\mathrm{~s}, 6 \mathrm{H}, \mathrm{H}_{\mathrm{b}}\right), 2.47\left(\mathrm{~s}, 6 \mathrm{H}, \mathrm{H}_{\mathrm{c}}\right)$. The remainder of the characterization was also consistent with previously reported literature. ${ }^{11}$

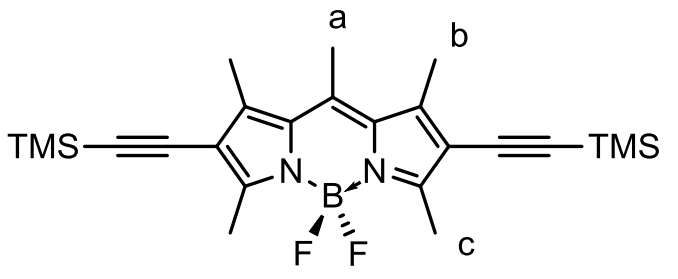

2.27

2.27: 2.26 (0.400 g, $0.778 \mathrm{mmol}), \mathrm{PdCl}_{2}\left(\mathrm{PPh}_{3}\right)_{2}(0.041 \mathrm{~g}, 0.058 \mathrm{mmol})$, and Cul $(0.022 \mathrm{~g}, 0.117 \mathrm{mmol})$ were added to a $100 \mathrm{~mL}$ Schlenk flask under $\mathrm{N}_{2}$. After adding anhydrous THF (20 ml) and $\mathrm{NEt}_{3}(10 \mathrm{ml})$, the mixture was sparged for $15 \mathrm{~min}$ with $\mathrm{N}_{2}$. Ethynyltrimethylsilane $(0.765 \mathrm{~g}, 7.784 \mathrm{mmol})$ was added and the 
mixture was stirred at room temperature overnight. After the solvent was removed in vacuo, DCM was added and the mixture was washed with water. The organic layer was collected and dried with $\mathrm{MgSO}_{4}$. The dark red solid was then run through a silica column using DCM:hexanes (2:1) $\left(R_{f}=0.77\right)$ yielding an orange solid (288.3 mg, 81\%). ${ }^{1} \mathrm{H}$ NMR $\left(\mathrm{CDCl}_{3}\right): \delta 2.63\left(\mathrm{~s}, 3 \mathrm{H}, \mathrm{H}_{\mathrm{a}}\right), 2.60\left(\mathrm{~s}, 6 \mathrm{H}, \mathrm{H}_{\mathrm{b}}\right), 2.50\left(\mathrm{~s}, 6 \mathrm{H}, \mathrm{H}_{\mathrm{c}}\right), 0.26(\mathrm{~s}$, $\left.18 \mathrm{H}, \mathrm{H}_{\text {TMS }}\right)$. Characterization is consistent with previously reported. ${ }^{28}$

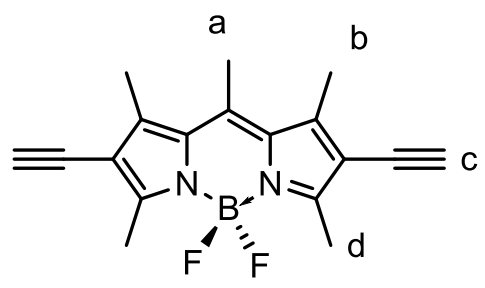

\subsection{8}

2.28: 2.27 ( $0.274 \mathrm{~g}, 0.603 \mathrm{mmol}$ ) was solubilized in $\mathrm{MeOH}(5 \mathrm{~mL})$ and THF $\left(5 \mathrm{~mL}\right.$ ) and sparged with $\mathrm{N}_{2}$ for $10 \mathrm{~min}$. The reaction was then stirred overnight at room temperature with excess KF $(0.175 \mathrm{~g}, 3.015$ $\mathrm{mmol})$. Solvents were removed in vacuo and the residue dissolved in EtOAc $(50-100 \mathrm{~mL})$. The organic phase was washed with water $(3 \times 100 \mathrm{~mL})$ to remove any excess $\mathrm{KF}$. The ethyl acetate phase was then dried with $\mathrm{MgSO}_{4}$, gravity filtered and the solvent removed in vacuo. The residue was then subjected to TLC and column chromatography using DCM $\left(R_{f}=0.86\right)$. The desired product was isolated as a dark orange solid. (75.2 mg, 40\%) ${ }^{1} \mathrm{H}$ NMR $\left(\mathrm{CDCl}_{3}\right): \delta 3.37\left(\mathrm{~s}, 2 \mathrm{H}, \mathrm{H}_{\mathrm{c}}\right), 2.64\left(\mathrm{~s}, 3 \mathrm{H}, \mathrm{H}_{\mathrm{a}}\right), 2.61\left(\mathrm{~s}, 6 \mathrm{H}, \mathrm{H}_{\mathrm{b}}\right), 2.52\left(\mathrm{~s}, 6 \mathrm{H}, \mathrm{H}_{\mathrm{d}}\right)$. Characterization is consistent with previously reported. ${ }^{7}$

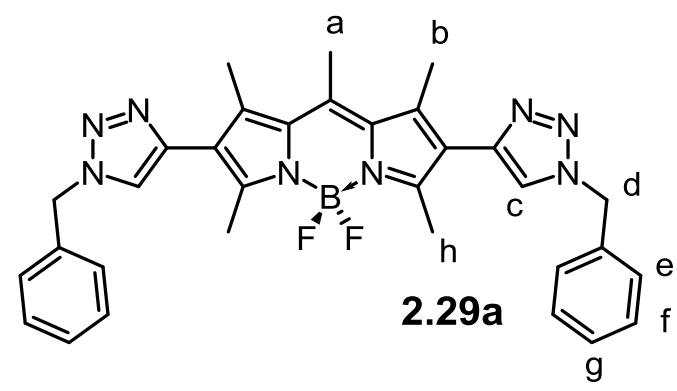

2.29a: To a solution of benzyl azide $(0.037 \mathrm{~g}, 0.280 \mathrm{mmol}), 2.28(0.040 \mathrm{~g}, 0.130 \mathrm{mmol})$ and tetrakis(acetonitrile)copper(l) hexafluorophosphate $(0.010 \mathrm{~g}, 0.028 \mathrm{mmol})$ in acetonitrile $(10 \mathrm{ml})$, were added. The resulting mixture was sparged with $\mathrm{N}_{2}$ for $10 \mathrm{~min}$ followed by the addition of $N, N$ diisopropylethylamine $(0.032 \mathrm{~g}, 0.280 \mathrm{mmol})$. The solution was stirred for $18 \mathrm{~h}$ and monitored by TLC. The solvents were removed in vacuo and the residue subjected to column chromatography in hexanes:EtOAc (1:1) $\left(R_{f}=0.17\right)$. The desired product was isolated as an orange fluorescent band. (15.2 mg, 20\%). ${ }^{1} \mathrm{H}$ NMR $\left(\mathrm{CDCl}_{3}\right): \delta=7.42\left(\mathrm{~s}, 2 \mathrm{H}, \mathrm{H}_{\mathrm{c}}\right), 7.42-7.37\left(\mathrm{~m}, 6 \mathrm{H}, \mathrm{H}_{\mathrm{g}}, \mathrm{H}_{\mathrm{f}}\right), 7.32\left(\mathrm{~d},{ }^{3} \mathrm{~J}=8.0 \mathrm{~Hz}, 4 \mathrm{H}, \mathrm{H}_{\mathrm{e}}\right), 5.61\left(\mathrm{~s}, 4 \mathrm{H}, \mathrm{H}_{\mathrm{d}}\right), 2.71(\mathrm{~s}$, $\left.3 \mathrm{H}, \mathrm{H}_{\mathrm{a}}\right), 2.56\left(\mathrm{~s}, 6 \mathrm{H}, \mathrm{H}_{\mathrm{b}}\right), 2.48\left(\mathrm{~s}, 6 \mathrm{H}, \mathrm{H}_{\mathrm{h}}\right) .{ }^{13} \mathrm{C}\left\{{ }^{1} \mathrm{H}\right\} \mathrm{NMR}\left(\mathrm{CDCl}_{3}, 100 \mathrm{MHz}\right): \delta=153.3,141.1,138.7,134.7$, $129.2,128.8,128.0,122.2,77.2,54.3,29.7,29.4,17.4,15.7,13.5 .{ }^{11} \mathrm{~B}\left\{{ }^{1} \mathrm{H}\right\} \mathrm{NMR}\left(\mathrm{CDCl}_{3}, 128 \mathrm{MHz}\right): \delta=0.71$ $\left(\mathrm{t},{ }^{1} \mathrm{~J}_{\mathrm{BF}}=32.07 \mathrm{~Hz}\right) .{ }^{19} \mathrm{~F} \mathrm{NMR}\left(\mathrm{CDCl}_{3}, 376 \mathrm{MHz}\right): \delta=-145.74$ (q). HRMS (EI): $\mathrm{m} / \mathrm{z} 576.2730\left([\mathrm{M}+\mathrm{H}]^{+}\right)$, calculated for $\left[{ }^{12} \mathrm{C}_{32}{ }^{1} \mathrm{H}_{31}{ }^{11} \mathrm{~B}^{19} \mathrm{~F}_{2}{ }^{14} \mathrm{~N}_{8}\right]^{+}: m / z 576.2733$ 


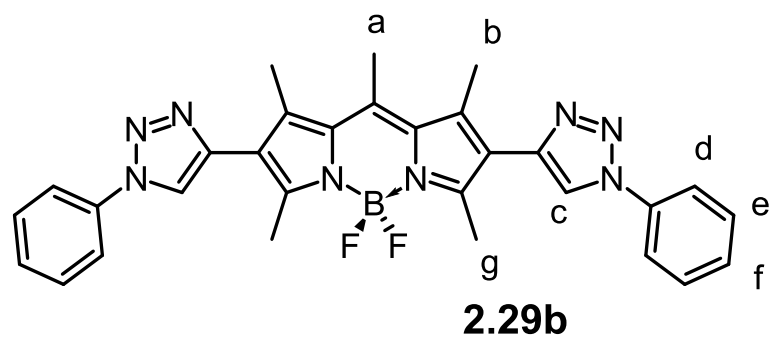

2.29b: To a solution of azidobenzene $(0.033 \mathrm{~g}, 0.280 \mathrm{mmol}), 2.28(0.040 \mathrm{~g}, 0.130 \mathrm{mmol})$ and tetrakis(acetonitrile)copper(I) hexafluorophosphate $(0.011 \mathrm{~g}, 0.030 \mathrm{mmol})$ in acetonitrile $(10 \mathrm{ml})$, were added. The resulting mixture was sparged with $\mathrm{N}_{2}$ for $10 \mathrm{~min}$ followed by the addition of $\mathrm{N}, \mathrm{N}-$ diisopropylethylamine $(0.032 \mathrm{~g}, 0.280 \mathrm{mmol})$. The solution was stirred for $18 \mathrm{~h}$ and monitored by TLC. The solvent was removed in vacuo and the residue subjected to column chromatography using hexanes:EtOAc (1:1) $\left(R_{\mathrm{f}}=0.15\right)$. The desired product was isolated as an orange fluorescent band. (16.2 mg, 23\%). ${ }^{1} \mathrm{H}$ NMR $\left(\mathrm{CDCl}_{3}\right): \delta 7.97\left(\mathrm{~s}, 2 \mathrm{H}, \mathrm{H}_{\mathrm{c}}\right), 7.83\left(\mathrm{~d},{ }^{3} \mathrm{~J}=8.7 \mathrm{~Hz}, 4 \mathrm{H}, \mathrm{H}_{\mathrm{d}}\right), 7.58\left(\mathrm{t},{ }^{3} \mathrm{~J}=7.4 \mathrm{~Hz}, 4 \mathrm{H}, \mathrm{H}_{\mathrm{e}}\right), 7.48\left(\mathrm{t},{ }^{3} \mathrm{~J}=7.7 \mathrm{~Hz}, 2 \mathrm{H}, \mathrm{H}_{\mathrm{f}}\right)$, $2.80\left(\mathrm{~s}, 3 \mathrm{H}, \mathrm{H}_{\mathrm{a}}\right), 2.69\left(\mathrm{~s}, 6 \mathrm{H}, \mathrm{H}_{\mathrm{b}}\right), 2.59,\left(\mathrm{~s}, 6 \mathrm{H}, \mathrm{H}_{\mathrm{g}}\right) .{ }^{13} \mathrm{C}\left\{{ }^{1} \mathrm{H}\right\} \mathrm{NMR}\left(\mathrm{CDCl}_{3}, 100 \mathrm{MHz}\right): \delta=132.1,129.9,128.9$, $120.5,120.3,100.0,77.2,31.9,29.7,29.7,29.4,22.7,15.8,14.1 .{ }^{11} \mathrm{~B}\left\{{ }^{1} \mathrm{H}\right\} \mathrm{NMR}\left(\mathrm{CDCl}_{3}, 128 \mathrm{MHz}\right): \delta=0.79$ $\left(\mathrm{t},{ }^{1} \mathrm{~J}_{\mathrm{BF}}=28.67 \mathrm{~Hz}\right) \cdot{ }^{19} \mathrm{~F} \mathrm{NMR}\left(\mathrm{CDCl}_{3}, 376 \mathrm{MHz}\right): \delta=-145.61$. HRMS $(\mathrm{El}): \mathrm{m} / \mathrm{z} 549.2504\left([\mathrm{M}+\mathrm{H}]^{+}\right)$, calculated for $\left[{ }^{12} \mathrm{C}_{30}{ }^{1} \mathrm{H}_{27}{ }^{11} \mathrm{~B}^{19} \mathrm{~F}_{2}{ }^{14} \mathrm{~N}_{8}\right]^{+}: \mathrm{m} / \mathrm{z} 548.2420$. 


\section{Chapter 3: Ferrocene D- $\pi$-A BODIPY Dyes}

\subsection{Literature review of ferrocene-BODIPY dyes}

\subsubsection{Objective}

Ferrocene and its derivatives are ubiquitous in organometallic chemistry, owing to desirable redox robustness. As such there has been significant effort to incorporate ferrocene into organic chromophores to prevent undesirable photo-oxidation in the excited state. To this end, ferrocene and its electron transfer behaviour, shows potential in dye-sensitized solar cell applications, chemosensing and within modern electrolytes. One subset, and our predominant interest is the design of Ferrocene BODIPY adducts, and the combination of the redox robustness of ferrocene and intense absorption and high quantum yield fluorescence of BODIPY should lead to interesting compounds with unique photo-physical properties. The classes of ferrocene-BODIPY adducts are explored herein with a discussion of physicochemical behaviour where appropriate.

\subsection{2 meso substituted Ferrocene BODIPY dyes}

The most common way of making ferrocene BODIPY dyads is achieved by substituting ferrocene on the meso position of BODIPY. Synthetic approach towards meso substituted ferrocene BODIPY adducts have been done three ways: 1) the use of ferrocene aldehyde as the aryl aldehyde for BODIPY synthesis 2) use of palladium cross coupling 3) nucleophilic aromatic substitution of $8-\mathrm{Cl}$ BODIPY. Presented below is a variety of meso ferrocene BODIPY adducts and their unique physicochemical properties for methyl substituted and non-methylated BODIPY cores. 


\subsubsection{1 meso ferrocene methylated BODIPY dyes}

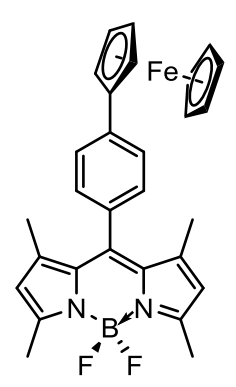

3.1

$\lambda_{\max }=498 \mathrm{~nm}$

$\varepsilon\left(10^{4} \mathrm{M}^{-1} \mathrm{~cm}^{-1}\right)=$

N/A

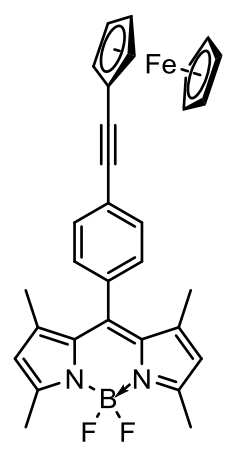

3.2

$\lambda_{\max }=502 \mathrm{~nm}$

$\varepsilon\left(10^{4} \mathrm{M}^{-1} \mathrm{~cm}^{-1}\right)=\mathrm{N} / \mathrm{A}$

Figure 3.1: Methylated meso ferrocene BODIPY dyad ${ }^{29}$

One of the first examples of meso ferrocene BODIPY was ferrocene-BODIPY derivative $\mathbf{3 . 1}$ and $\mathbf{3 . 2}$ by Yin and coworkers. ${ }^{29} 3.1$ is meso phenyl $p$-ferrocene-BODIPY that has weak conjugation to BODIPY core due to steric constrains breaking planarity. $\mathbf{3 . 2}$ has an ethynyl bridge spacer between the phenyl and ferrocene to extend to conjugation. The absorption profiles reveal the $\lambda_{\max }$ for both $\mathbf{3 . 1}$ and $\mathbf{3 . 2}$ is predominantly the BODIPY $\pi-\pi^{*}$ transition ( $490-502 \mathrm{~nm}$ ) which is not a significant change to the parent BODIPY due the weak conjugation and long distance between ferrocene and BODIPY. There was no observed charge transfer bands in the absorption profile for both 3.1 and 3.2 to indicate MLCT. Interestingly, the emission profile 3.2 was extremely dependent on the solvent. ${ }^{29}$ Both 3.1 and 3.2 were weakly emissive in hexanes and $\mathrm{DCM}\left(\Phi_{\mathrm{F}}<0.01\right)$ due ferrocene quenching fluorescence through photo-induced electron transfer (PET). But 3.2 fluoresces more intensely in polar solvents such as $\operatorname{DMF}\left(\Phi_{\mathrm{F}}=0.02\right), 1,4$ dioxanes $\left(\Phi_{\mathrm{F}}=0.04\right)$ and DMSO $\left(\Phi_{\mathrm{F}}=0.08\right)$. Spectroelectrochemical studies of $\mathbf{3 . 2}$ in DMSO reveal that if ferrocene is oxidized, there is no change in fluorescence meaning electron transfer is not playing a role in quenching emission. This fluorescence property of $\mathbf{3 . 2}$ was due to the viscosity of solvents caused by the molecular rotor motion of $\mathbf{3 . 2}$ making it an efficient viscosity probe. ${ }^{29}$ 


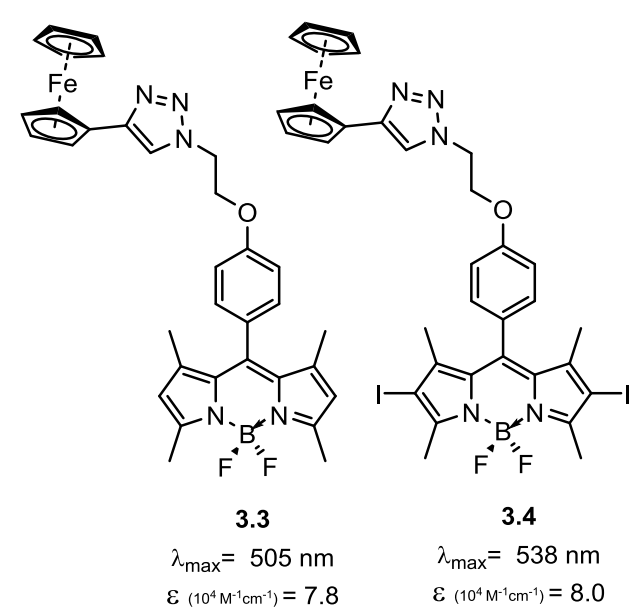

Figure 3. 2: meso triazole non-conjugated ferrocene BODIPY dyad. ${ }^{30}$

Wu, X. et al. made the first non-conjugated meso ferrocene BODIPY $\mathbf{3 . 3}$ and $\mathbf{3 . 4}$ through click chemistry. ${ }^{30}$ Both 3.3 and $\mathbf{3 . 4}$ have little to no electronic communication between ferrocene and BODIPY in the ground state due to the non-conjugation. However these dyes are more emissive compared to conjugated counterparts (3.1 and 3.2) $\left(\Phi_{\mathrm{F}}=0.04-0.09\right)$. A degree of quenching is still occurring, and this quenching process via PET is similar to the previous ferrocene BODIPY dyads, however the fluorescence can be turned on more intensely by the oxidation of the ferrocene. However, 3.4 also has a triplet state excited state (owing to the heavy atom effect) that is quenched through triplet-triplet electron transfer. ${ }^{30}$

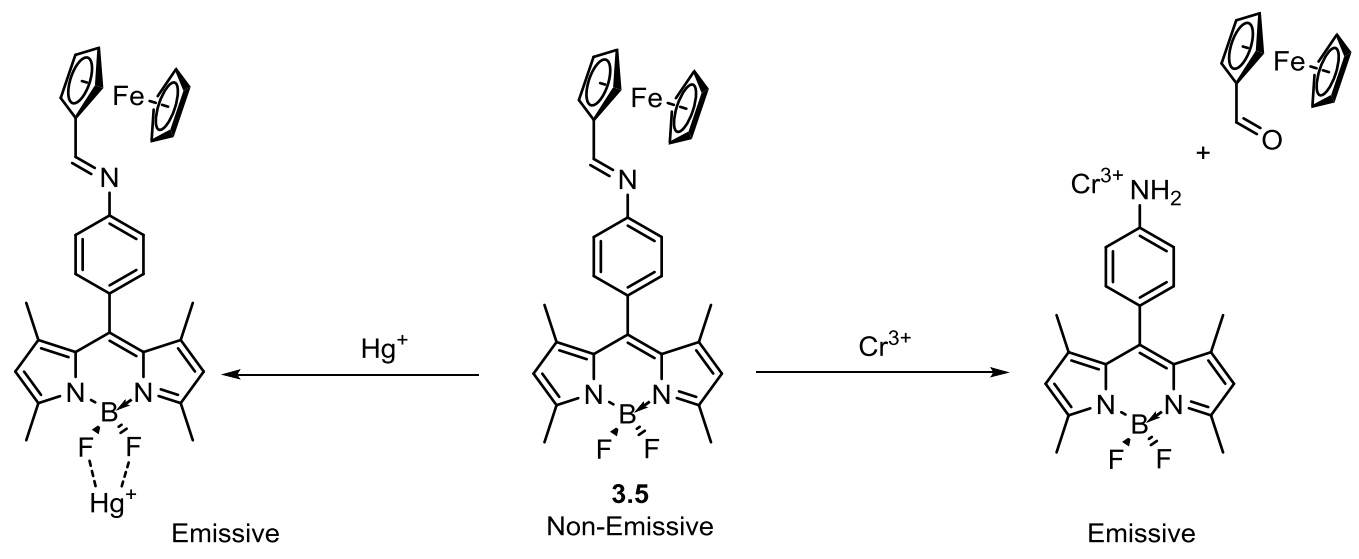

Scheme 3.1: Sensing mechanism of $\mathrm{Hg}^{+}$and $\mathrm{Cr}^{3+}$ for $3.5 .^{31}$

Navdeep et al. designed the first Schiff base ferrocene BODIPY dyad 3.5 that can detect $\mathrm{Hg}+$ and $\mathrm{Cr}+$ using fluorescence. ${ }^{31}$ While no charge transfer properties could be determined through absorption spectroscopy between ferrocene and BODIPY due the weak conjugation, the emission was quenched in the dyad owing to photo-induced electron transfer. However in the presence of $\mathrm{Hg}^{2+}$ ions, results in an 
adduct being formed that enhances the emission. Conversely the presence of $\mathrm{Cr}^{3+}$ ions, results in the hydrolysis of the imine bond that also increased emission. ${ }^{31}$ This on-off fluorescence systems makes 3.5 a good chemosenser for $\mathrm{Hg}^{2+}$ and chemodosimeter for $\mathrm{Cr}^{3+}$.

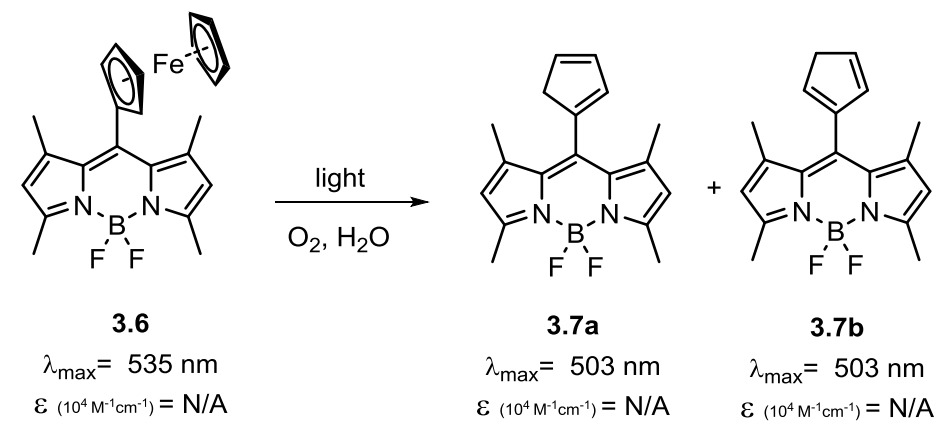

Scheme 3.2: Decomposition of 3.6 in ambient conditions. ${ }^{32}$

Sumig et al. was the first group to substitute ferrocene directly to the BODIPY at the meso position of methylated BODIPY core using ferrocene carboxyaldehyde (3.6). ${ }^{32}$ Molecule $\mathbf{3 . 6}$ however, was not stable and the first example of a ferrocene-BODIPY dyad decomposing under ambient condition in the literature. ${ }^{32}$ In the presence of water and light, the ferrocene decomposes to make isomers of cyclopentadienes derivatives $3.7 \mathbf{a}, \mathbf{b}$ through the production of cyclopentadiene radicals.
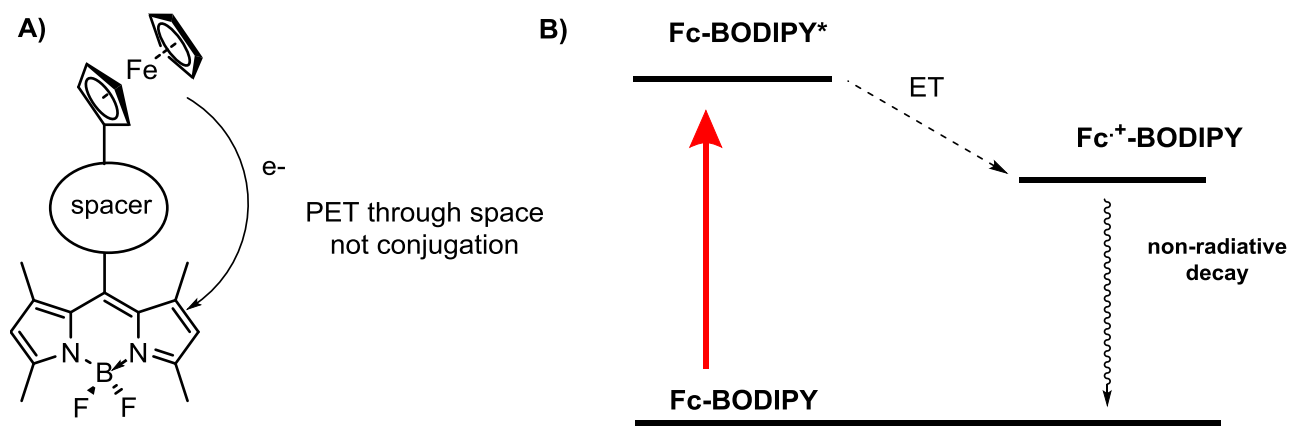

Figure 3. 3: PET process of meso substituted ferrocene methylated BODIPY dyes, b) energy diagram for the quenching mechanism for ferrocene-BODIPY adducts 
In general, meso substituted ferrocene methylated BODIPY dyes seem to have poor electron communication between ferrocene and BODIPY in the ground state due to the steric strains for both conjugated and non-conjugated. Shorting the distance between ferrocene and BODIPY helps increase the electron coupling of ferrocene and BODIPY. However, this shorting can result in decomposition of ferrocene (3.2). However, all meso ferrocene methylated BODIPY derivatives mentioned are quenched through a photo-induced electron transfer (Figure 3.3b) through space by the proximity of the ferrocene. This photo induced transfer process of quenching fluorescence will be relevant in ferrocene BODIPY dyads discussed further.

\subsubsection{2 meso Ferrocene non-methylated BODIPY}

When a BODIPY core is not methylated, it typically becomes less stable. However, not having methyl groups present does alter the frontier molecular orbital energies, and often can alter PET-based processes and even shut them down completely. Furthermore, for an unsubstituted BODIPY core, the addition of ferrocene to the meso position allows for better electronic communication compared to methylated BODIPY due to the absence of steric constraint. This reintroduction of planarity increases conjugation, which results in the presence of charge transfer bands in the absorption profiles. This means ferrocene in these motifs acts an electron donor more efficiently. This intramolecular charge transfer will be relevant in ferrocene BODIPY dyads discussed further. 

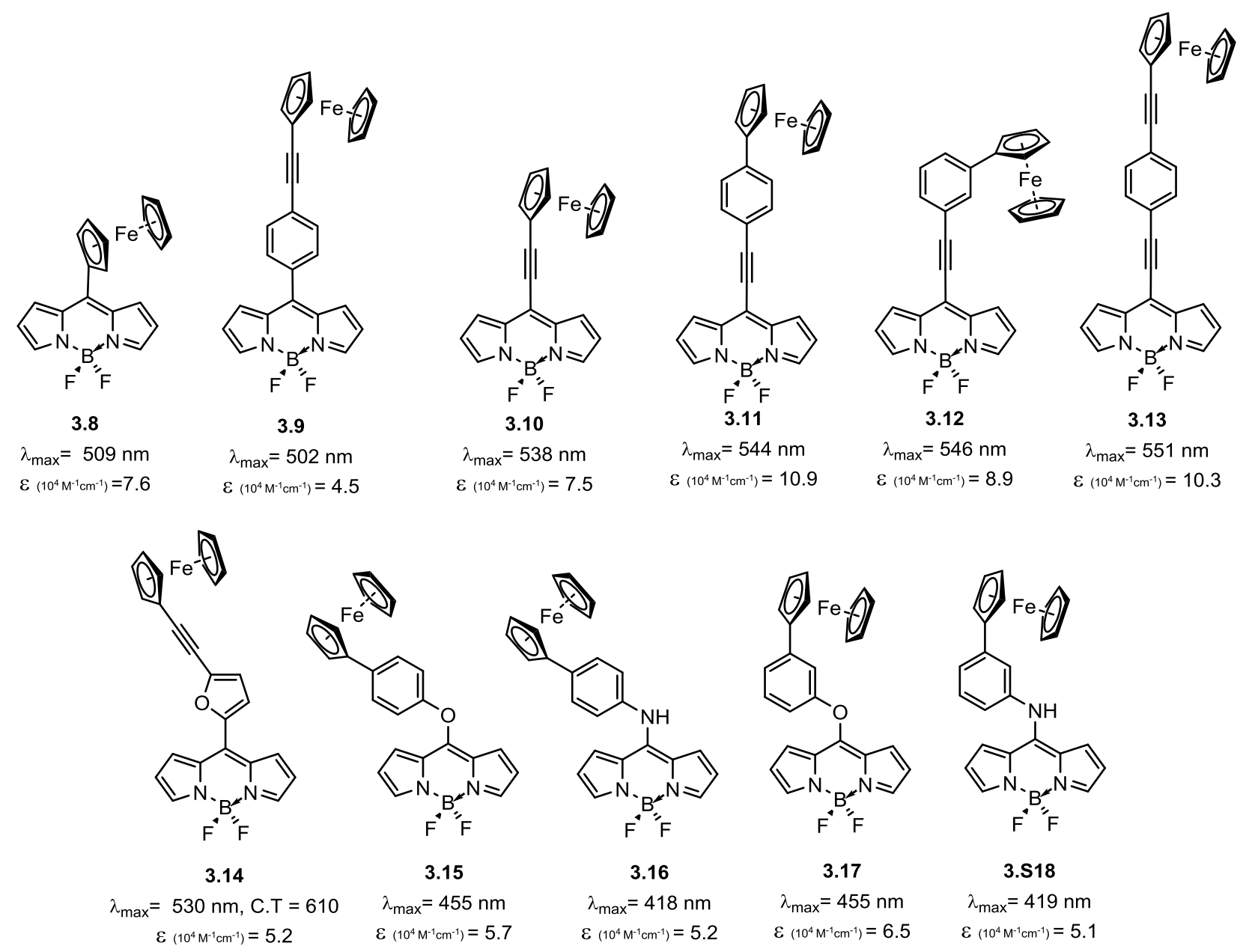

Figure 3.4: Novel meso ferrocene un-substituted BODIPY dyes $\mathrm{s}^{33-34}$

Figure 3.4 contains meso ferrocene un-substituted BODIPY dyes that will be discussed in this section. Mirsa et al. synthesised mesophenyl derivatives $\mathbf{3 . 8}$ and $\mathbf{3 . 9}$ which are counterparts to $\mathbf{3 . 6}$ and $\mathbf{3 . 2}$ respectively. ${ }^{33}$ The $\lambda_{\max }$ for $\mathbf{3 . 8}$ is more blue-shifted compared to the methylated versions by $26 \mathrm{~nm}$ revealing that ferrocene is not effectively communicating with BODIPY core, but most likely the dominate transition is not the HOMO to LUMO but a higher energy transition. Unfortunately DFT calculations were not done by the authors. Compound $\mathbf{3 . 8}$ however, did have a weak charge transfer band due to MLCT at $656 \mathrm{~nm}$. Mirsa et al installed ethynyl spacers between ferrocene and BODIPY directly to the meso position to avoid the steric strain hindering conjugation (3.10-3.13). ${ }^{35}$ BODIPYs 3.10-3.13 have intense absorption bands with $\lambda_{\max }$ ranging $538-551 \mathrm{~nm}$ attributed to the HOMO to LUMO transition (BODIPY $\pi-\pi^{*}$ ). BODIPYs 3.10-13 $\lambda_{\max }$ are significantly red shifted compared meso phenyl 3.1-3.2 counterparts to due to enhanced electronic communication between aromatics substituents on the meso position. Dyes 3.10-3.13 experience charge transfer bands between $600-650 \mathrm{~nm}$ indicating there is significant electron coupling 
between ferrocene and BODIPY. BODIPY 3.10 had the most defined broad charge transfer band, likely due the proximity of the ferrocene and BODIPY. According to DFT studies for dyes 3.11-3.13, ferrocene acts as electron donor to BODIPY, however, this was opposite for 3.10. ${ }^{35}$ Khan et al. installed furan in the meso position (3.14) as a spacer between ferrocene and BODIPY along with an ethynyl bridge. ${ }^{36}$ Comparing 3.14 to phenyl spacer 3.2, there is a red shift of $30 \mathrm{~nm}$ due to extension of the $\pi$ localization within the molecule compared to 3.2. However $\mathbf{3 . 1 4}$ has poor electronic communication between ferrocene and BODIPY due to the distance. ${ }^{36}$ Mirsa et al. also reported the synthesis of meso heteroatom bridge ferrocene BODIPY dyads 3.15-3.18. ${ }^{34}$ In compounds 3.15-3.16, the ferrocene is para substituted to the oxygen and nitrogen atoms meaning the presence of conjugation to the BODIPY core. ${ }^{34}$ However, compounds 3.17-3.18 are meta substituted to oxygen and nitrogen atoms, which mean it is cross-conjugated to the BODIPY core. Dyes 3.15-3.18 are significantly blue-shifted compared to the traditional ferrocene BODIPY dyad so far discussed due to the dominant HOMO-2 to LUMO and HOMO-3 to LUMO transitions. The hypsochromic shift in the absorption profiles are also attributed to the delocalization of the lone pair electrons of the meso-substituted heteroatom into the electron-deficient BODIPY to form cross-conjugated hemicyanine resonance structures. ${ }^{34}$ The meso heteroatom donors destabilizes the LUMO energy level, and the ferrocene destabilizes the HOMO, HOMO-1, and HOMO-2 widening the energy gap. ${ }^{34}$

\subsection{3 $\alpha$ substituted ferrocene BODIPY dyes}

Another common method for installing ferrocene to BODIPY is through the $\alpha$ or 3,5 position of BODIPY. This can be achieved using palladium cross couplings or Knoevenagel condensations. $\alpha$ positions have more of an effect towards the electronic structure of BODIPY compared to the meso position and therefore facilitate stronger electron coupling between ferrocene and BODIPY. Presented below is the ferrocene BODIPY dyes using the $\alpha$ position and their physicochemical properties and applications

\subsubsection{Mono $\alpha$ substituted ferrocene BODIPY dyes}




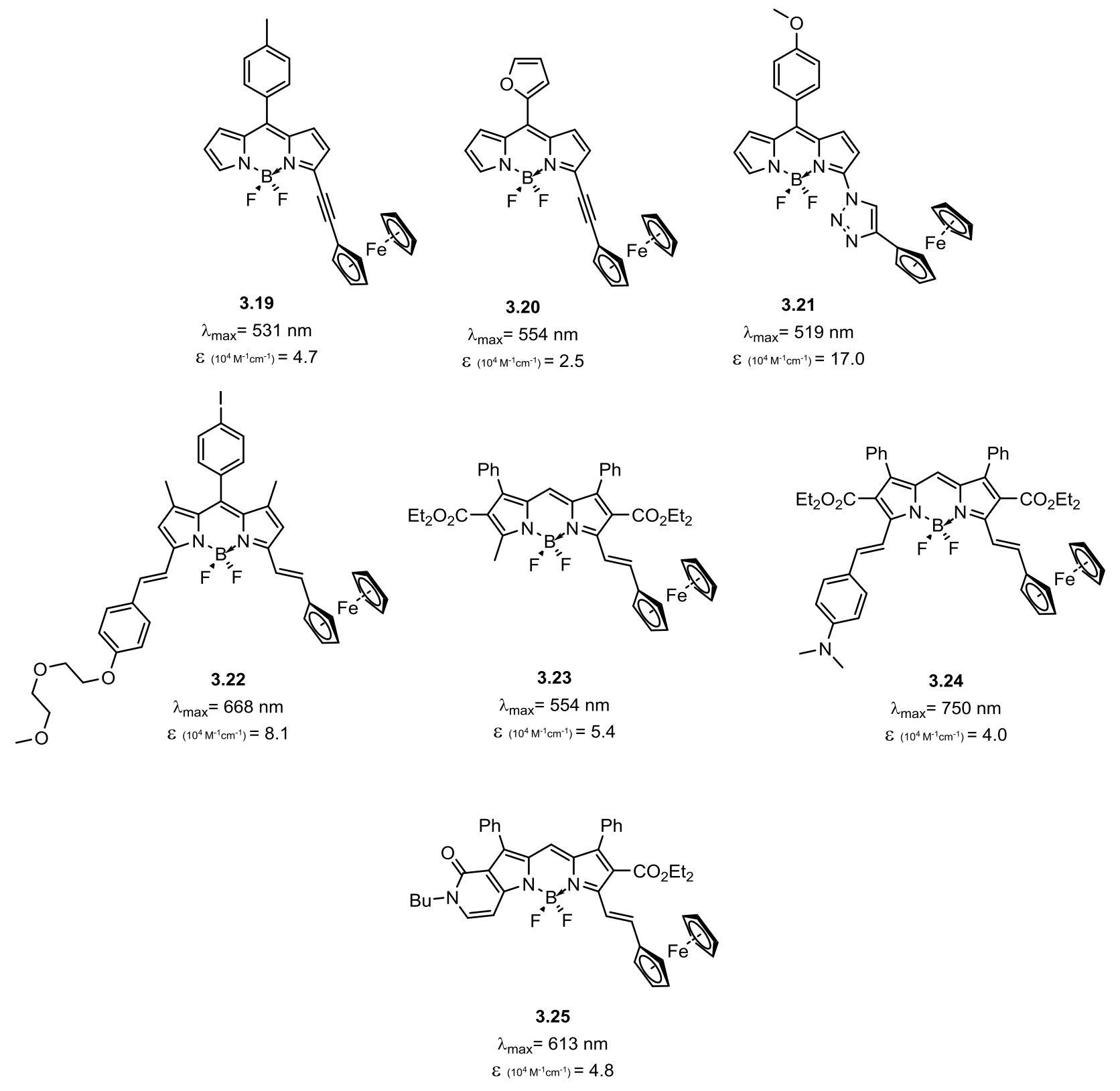

Figure 3.5: Novel mono $\alpha$ substituted ferrocene BODIPY dyes ${ }^{33}, 36,37-38$

Rajeswara et al. were the first to report the synthesis of mono $\alpha$ substituted ferrocene BODIPY dyad 3.19 through Sonogashira cross-coupling at the $\alpha$ position. ${ }^{33}$ The dominant transition of absorption profile for 3.19 is HOMO to LUMO $\left(\pi-\pi^{*}\right)$, which is similar to 3.10 , most likely due to the same proximity of the ferrocene to BODIPY and the same ethynyl bridge. The fluorescence of 3.19 is quenched through PET like other ferrocene BODIPYs. However, $\mathbf{3 . 1 9}$ has a more intense charge transfer band at $613 \mathrm{~nm}$ compared to 3.10, meaning there is better electronic communication between ferrocene and BODIPY at the $\alpha$ position compared to the meso position. The same comparison can be made for dye $\mathbf{3 . 2 0}$ to meso 
derivative 3.14. ${ }^{36}$ The triazole bridge derivative 3.21 does has poor electronic communication between ferrocene and BODIPY compared to the ethynyl bridged 3.19-3.20, ${ }^{37}$ but electron transfer properties were not studied further. Bura et al. made an intense ferrocene NIR absorption dye which used a vinyl linker at the $\alpha$ position. ${ }^{39}$ The addition of the (2-(2-methoxyethoxy)ethoxy) phenyl moiety (3.22) at the other $\alpha$ position red shifts the absorption profile significantly. However, the addition of (2-(2methoxyethoxy)ethoxy) phenyl moiety however does not restore the fluorescence. Zasikha et al. were first to report true $D-\pi$-A dyads $(3.23$ - 3.25) with ferrocene as electron donor and esters as acceptors in the $\beta$ position. ${ }^{38}$ Molecules $\mathbf{3 . 2 3}$ - $\mathbf{3 . 2 5}$ are all NIR absorbers that have fluorescence quenched due to presence of ferrocene (PET) with well-defined charge transfer bands $(613 \mathrm{~nm}, 750 \mathrm{~nm}, 715 \mathrm{~nm}$ respectively). However for $\mathbf{3 . 2 4}$ the dominant absorption was $\pi-\pi^{*}$ within BODIPY and was lower in energy than the MLCT. Through transient absorption experiments, electron charge transfer was determined and the electron transfer from BODIPY to ferrocene was 136 ps for $\mathbf{3 . 2 3}$ and 260 ps for $\mathbf{3 . 2 4} .^{38}$ This difference in rate can be related to the introduction of an electron donor dimethylamino phenyl moiety slowing the electron transfer BODIPY to ferrocene. The same observations are seen for 3.25 (260 ps) which can be attributed to additional electron withdrawing attached to the BODIPY core. ${ }^{38}$ It was also noted that the dissymmetry of the electron density within the excite state within molecules $\mathbf{3 . 2 4}$ and $\mathbf{3 . 2 5}$ slows down electron transfer rate. The mono $\alpha$ substituted ferrocene BODIPY dyes that have been presented have shown that $\alpha$ position can allow for ferrocene to have a greater effect on the BODIPY physicochemical properties compared to meso position with more intense charge transfer bands and red-shifting absorption profiles. 


\subsubsection{Diferrocene $\alpha$ substituted BODIPY dyads}
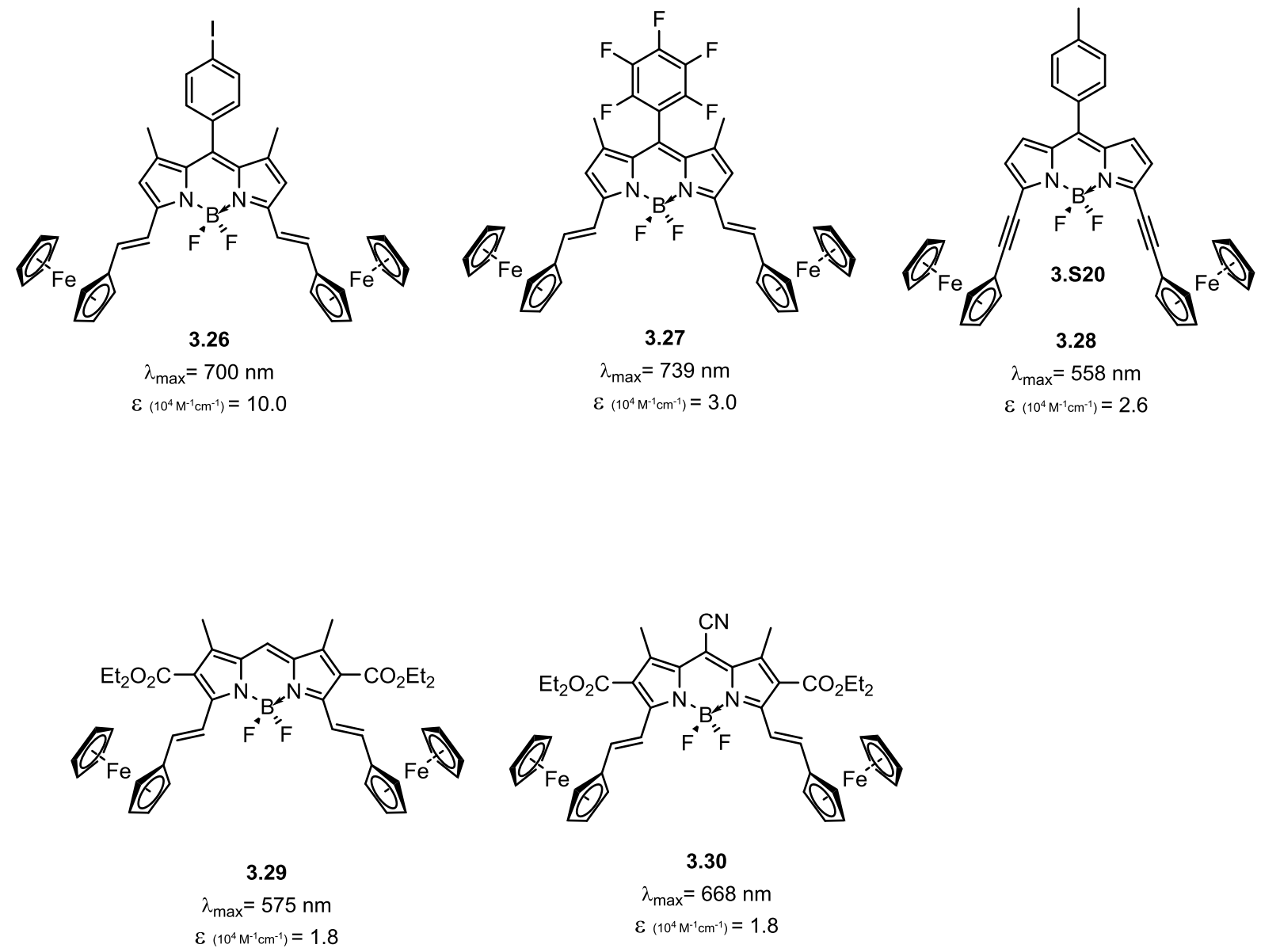

Figure 3.6: Novel diferrocene $\alpha$ substituted BODIPY dyads $33,10,12-13$

Yin et al. were the first to report a diferrocene BODIPY dyad 3.26 taking advantage of the two reactive $\alpha$ positions of BODIPY. ${ }^{40}$ The absorption profile revealed that the dominant transition was the MLCT at 700 $\mathrm{nm}$ due to the addition of a second ferrocene unit. When one ferrocene is oxidized it can undergo intervalence charge transfer between the metal centres of ferrocene, and 3.26 had a quenched fluorescence through PET like previously discussed ferrocene BODIPY dyads. The meso hexafluorophenyl derivative 3.27 has similar physicochemical properties as 3.27, but the absorption profile is red shifted due to the stabilization of the LUMO. ${ }^{41}$ The ethynyl spacer derivative $\mathbf{3 . 2 8}$ also shows that adding two ferrocene dramatically increases the MLCT band with the molecule, ${ }^{33}$ however not to the same degree as the vinyl bridges such as $\mathbf{3 . 2 6} \mathbf{\&} \mathbf{3 . 2 7}$. Compound 3.29, the diferrocene version of $\mathbf{3 . 2 3}^{\mathbf{3 8}}$, has an absorption profile that is red shifted compared to 3.23 , However unlike $3.26-28$, the dominant transition for 3.29 is $\pi-\pi^{*}$ of BODIPY, however the charge transfer is almost as intense. The reason for the observed switch is the HOMO electron density of $\mathbf{3 . 2 9}$ is more delocalized on to BODIPY compared to $\mathbf{3 . 2 7 - 2 9}$, in turn increasing 
the overlap of the $\pi$ and $\pi^{*}$ BODIPY molecular orbitals favouring the transition. The electron transfer rate from 3.30 was slower than the mono-ferrocene derivative 3.23 (176 ps and 136 ps respectively) meaning it has a longer lifetime for the excited state. Molecule $\mathbf{3 . 3 0}$ adds a cyano group to the meso position, making the BODIPY 3.30 a stronger acceptor which redshifts the absorption profile (668 $\mathrm{nm}$ and $964 \mathrm{~nm}$ ) due to the stabilization of the LUMO energy level. There was no electron transfer experiments done, but

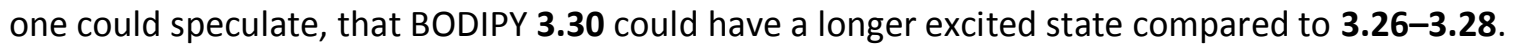

\subsection{4 $\beta$ substituted ferrocene- BODIPY adducts}

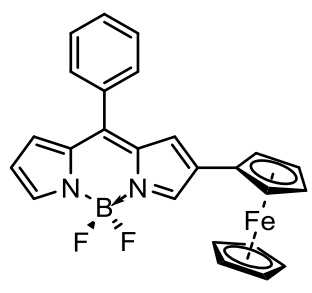

3.31

$\lambda_{\max }=513 \mathrm{~nm}$

$\varepsilon\left(10^{4} \mathrm{M}^{-1} \mathrm{~cm}^{-1}\right)=3.2$

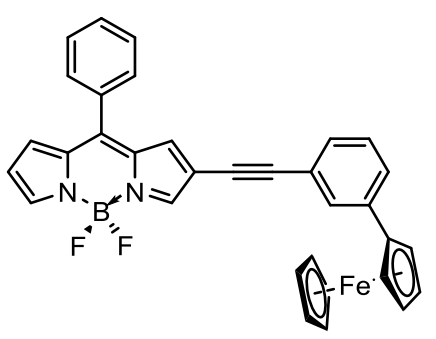

3.34

$\lambda_{\max }=544 \mathrm{~nm}$

$\varepsilon\left(10^{4} \mathrm{M}^{-1} \mathrm{~cm}^{-1}\right)=3.5$

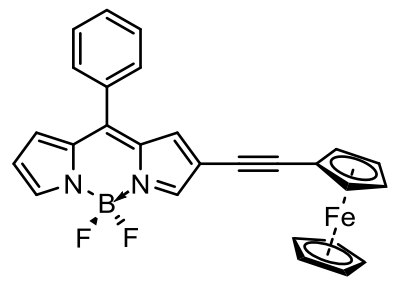

3.32

$\lambda_{\max }=523 \mathrm{~nm}$

$\varepsilon\left(10^{4} \mathrm{M}^{-1} \mathrm{~cm}^{-1}\right)=3.2$

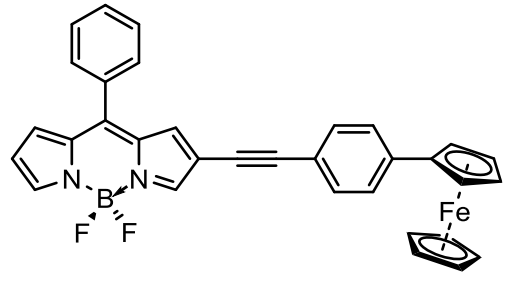

3.33

$\lambda_{\max }=543 \mathrm{~nm}$

$\varepsilon\left(10^{4} \mathrm{M}^{-1} \mathrm{~cm}^{-1}\right)=3.5$

Figure 3.7: $\beta$-substituted ferrocene- BODIPY adducts ${ }^{42}$

The only example of ferrocene added to the $\beta$ positions was reported by Dholake et al. with the synthesis of 3.31-3.34 (Figure 3.7). ${ }^{42}$ According to the absorption profiles of 3.31-3.34, only molecule $\mathbf{3 . 3 1}$ had a defined charge band ( $680 \mathrm{~nm}$ ), 3.32 the charge band is a shoulder on the dominant BODIPY $\pi-\pi^{*}$, and 3.33-3.34, experience the broadening of the $\pi-\pi^{*}$ transition (incorporation of MLCT bands). Unfortunately electrochemical studies were not done. Since little physicochemical studies were reported 
on $\beta$-substituted ferrocene BODIPY dyads, many questions remain unanswered with respect to the importance of substitution at the $\beta$-position.

\subsection{Thesis Objective}

This project explores the replacement of TPA as an electron donor with ferrocene towards BODIPY dyads for use in light harvesting dyes. The original target dyes for this project are presented in Figure 3.1. Target dyes 3.35a-d are readily solution soluble benchmark compounds prepared in order to study the $D-\pi-A$ motif with ferrocene substituted to the $\beta$ positions and target dyes $\mathbf{3 . 3 6 a - d}$ are the ferrocene BODIPY dyes for DSSC applications. The use of different electron rich substituents such as 2,5,6 trimethoxy phenyl group (b) and 4 ethoxy phenyl (d) and the electron deficient/neutral rings such as 4-nitro phenyl (c) or phenyl (a) are to probe the ability to change the frontier molecular orbital levels (particularly the LUMO level) of the dyes to match the energy level with $\mathrm{TiO}_{2}$ to allow for better electron injection.
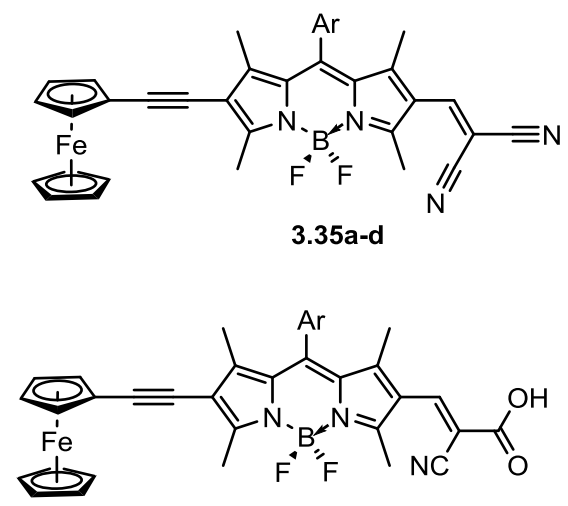

3.36a-d

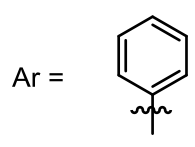

a

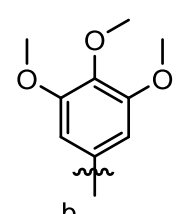

b

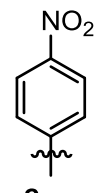

c

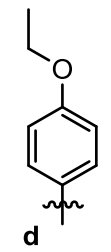

Figure 3.8: Family of target ferrocene BODIPY dyes 


\subsection{Synthesis}

\subsubsection{Ferrocene Donor}

Ferrocene was chosen as the electron donor for our BODIPY electron donor. Ferrocene is well studied compound within the literature, known for its redox stability and it was hoped that the incorporation of ferrocene BODIPY dyes should stabilize the photo-oxidized dye for DSSC and OPV applications. In previous attempts to incorporate ferrocene directly to pentamethyl BODIPY at the 2, 6 positions failed likely owing to steric hindrance (Scheme 3.2). An ethynyl spacer was then used to avoid the steric problems, as well as to extend conjugation (Scheme 3.4).
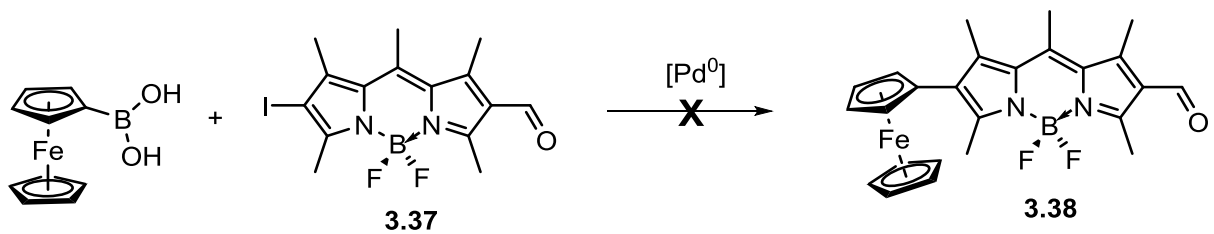

Scheme 3.3: Synthesis of ferrocene donor BODIPY dyad

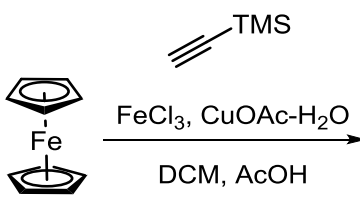

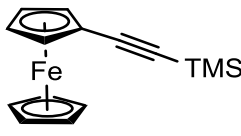

3. 39

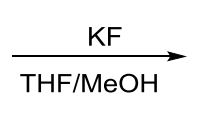

3. 40

Scheme 3.4: Synthesis of ethynyl ferrocene donor

Starting with ferrocene, the ferrocene was alkynylated using trimethylsilylacetylene using oxidative direct cross-coupling $^{43}$ to produce $\mathbf{3 . 3 9}$ in moderate yields. The protected ethynyl ferrocene $\mathbf{3 . 3 9}$ was then deprotected using potassium fluoride to get the desired ferrocene building block $\mathbf{3 . 4 0}$. 


\subsubsection{Synthesis BODIPY $\pi$ spacer}

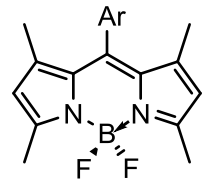

$3.41 \mathrm{a}=26 \%$

$3.41 b=48 \%$

$3.41 \mathrm{c}=15 \%$

$3.41 d=30 \%$ $\underset{\text { Dry DCM }}{\stackrel{\mathrm{DMCl}_{3}}{\stackrel{\mathrm{DMF}^{2}}{\longrightarrow}}}$<smiles></smiles>

$3.42 a=92 \%$

$3.42 b=80 \%$

$3.42 \mathrm{c}=74 \%$

3.42d $=71 \%$<smiles></smiles>

$3.43 a=73 \%$ $3.43 b=78 \%$

$3.43 \mathrm{c}=66 \%$

$3.43 d=42 \%$

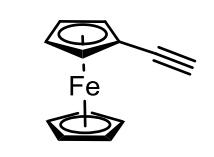

$\mathrm{Pd}\left(\mathrm{PPh}_{3}\right)_{2} \mathrm{Cl}_{2}$ $\mathrm{Cul} \longrightarrow$

$\mathrm{THF}_{\mathrm{NEt}} \mathrm{N}_{3}$

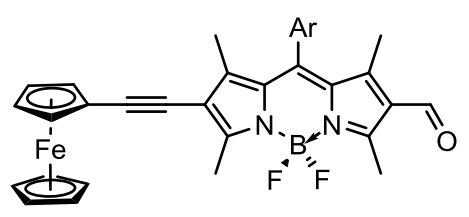

$3.44 a=30 \%$

$3.44 b=84 \%$

$3.44 \mathrm{c}=63 \%$

$3.44 d=43 \%$
$\mathrm{Ar}=$<smiles>CC(C)(C)c1ccccc1</smiles>

a<smiles>COc1cc(C(F)(F)F)cc(OC)c1OC</smiles>

b<smiles>CC(C)(C)c1ccc(OCCOc2ccc([N+](=O)[O-])cc2)cc1</smiles>

Scheme 3.5: Synthesis of aryl meso BODIPY $\pi$ spacer

In order to synthesize the BODIPY $\pi$ spacer, BODIPY 3.41a-d were synthesised using aryl aldehydes to introduce different electron rich/poor aryl rings in the meso position in moderate yields. BODIPYs 3.41a-d are formylated using the Vilsmeier-Haack reaction to yield the BODIPYs 3.42a-d. BODIPY 3.42ad underwent iodination reactions using NIS to afford the desired 3.43a-d. The ferrocene donor was conjugated via Sonogashira cross-couplings of 3.43a-d to produce compounds 3.44a-d with varying success. 


\subsubsection{Installation of the acceptor units}

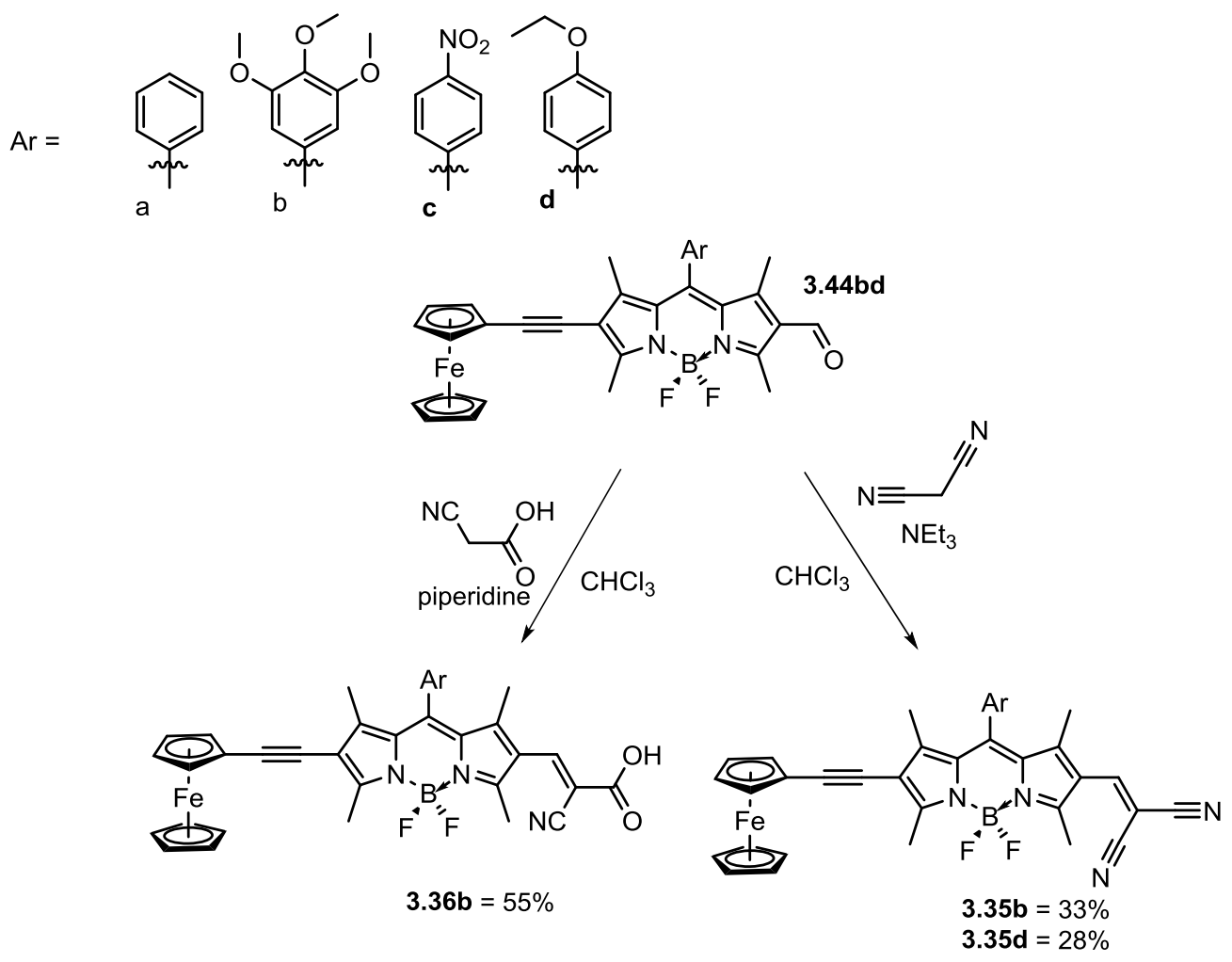

Scheme 3.6: Synthesis of aryl meso ferrocene BODIPY D- $\pi-A$ dyes

To make the bench mark targets 3.35a-d, Knoevenagel condensation was performed with malonitrile to make 3.35b and 3.35d. The nitro and phenyl compounds were difficult to isolate and seem to have issue with stability. Another problem was the base sensitive methyl groups at the 3,5 position which can also undergo a condensation reaction to make oligomers (Appendix Figure A.1). For target dyes 3.36a-d, due to the limited amount of 3.44a-d, only target dye 3.36b was carried forward because it had the highest LUMO level (a desirable trait for DSSC applications). The Knoevenagel condensation was performed with cyanoacetic acid to yield the DSSC 3.36b with a 55\% yield and a small amount of 3.44b as impurity (10\%) which could not be removed. An attempt was made to synthesis targets dyes 3.36a and 3.36c, however these molecules seemed to fall apart when trying to isolate them and decomposed rather quickly (a warning of what is yet to come, vide infra). 


\subsection{Results and Discussion}

The physicochemical properties for BODIPY derivatives 3.41a-d to 3.44a-d, 3.35bd, and 3.36b (in DCM solutions) have been explored and contrasted in Table 3.1. The physicochemical properties were examined through absorption spectroscopy, electrochemistry, density functional (DFT) theoretical calculations and spectroelectrochemical studies.

\subsubsection{UV-Vis Spectroscopy of 3.1b-3.36b}

A)

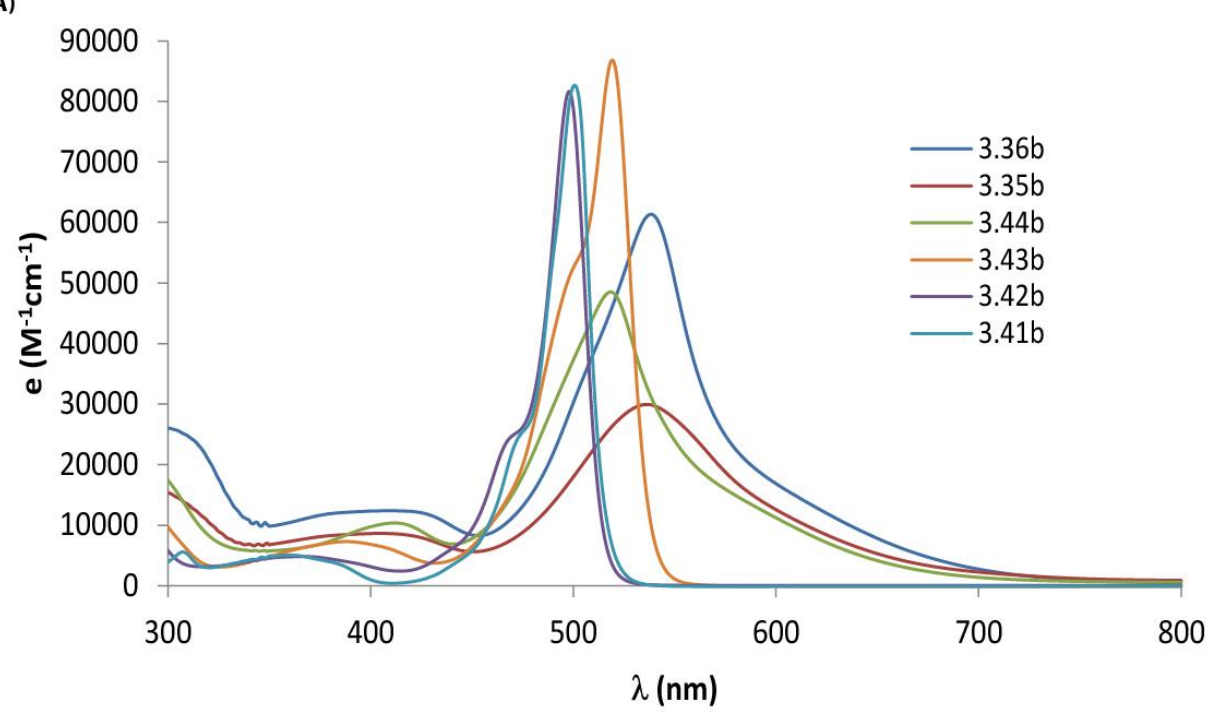

B)
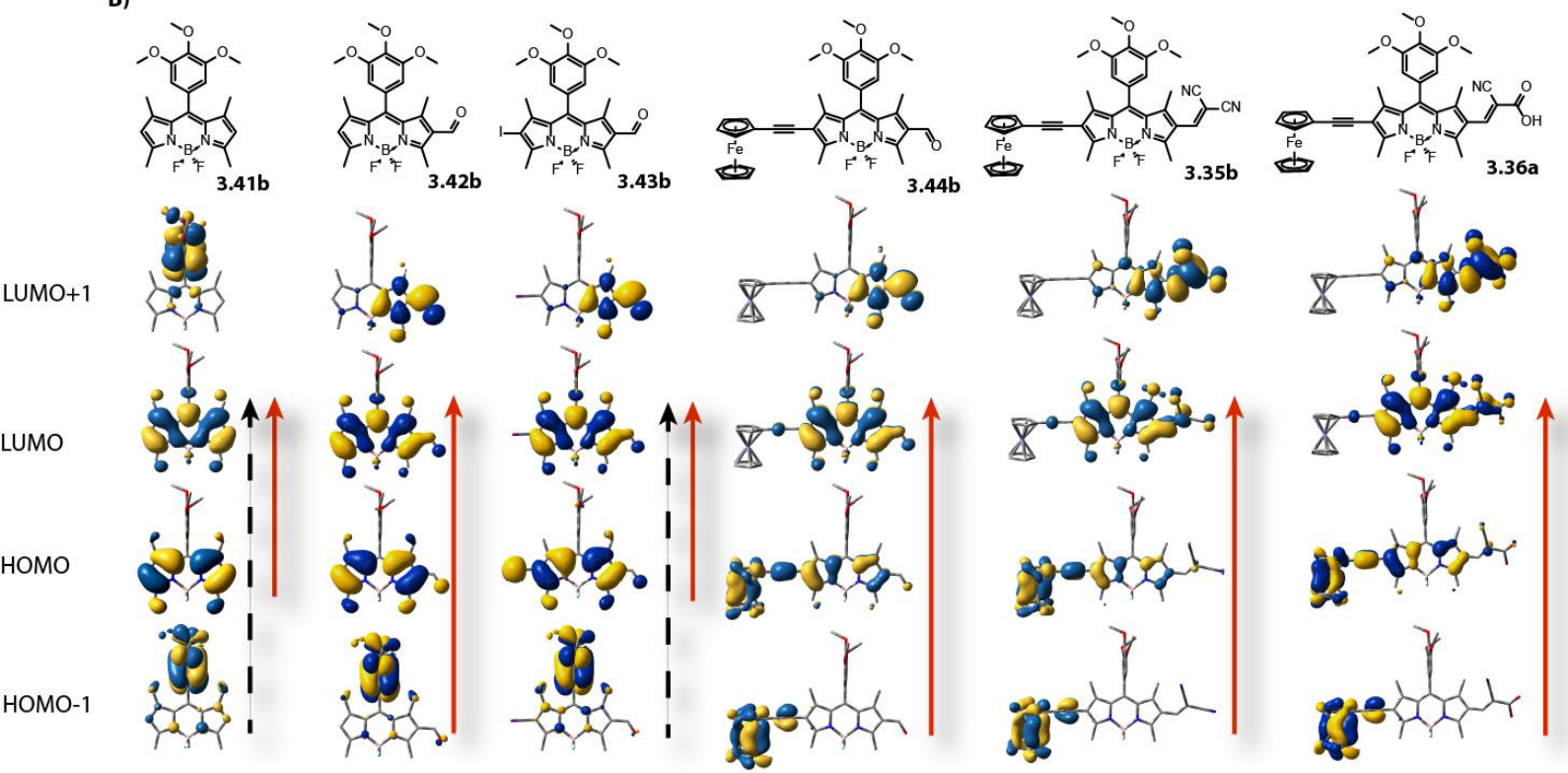

Figure 3.9: a) UV/Vis spectra of BODIPY 3.41b and target dyes 3.35-6b. Data collected in DCM b) DFT 
In order to have efficient power conversion in DSSCs and OPVs, panchromatic dyes are needed for absorbing light well into the Vis-NIR. The UV-Vis (in DCM) and TD-DFT for the trimethoxy phenyl derivatives 3.41b-3.44b and 3.35b-3.36b are collated in Figure 3.9. BODIPY 3.41b has a distinctive BODIPY absorption profile with a sharp absorption maximum at $502 \mathrm{~nm}$ from a dominant HOMO to LUMO $\left(\pi-\pi^{*}\right)$ transition with a shoulder representing the HOMO-1 to LUMO transition. Formyl BODIPY $\mathbf{3 . 4 2} \mathbf{b}$ absorption profile has a slight hypsochromic shift $\lambda_{\max }$ at $498 \mathrm{~nm}$; however the dominant transition for this $\lambda_{\max }$ is HOMO-1 to LUMO. The iodinated 3.43b $\lambda_{\max }$ has a bathochromic shift of $20 \mathrm{~nm}$ at $519 \mathrm{~nm}$ from the HOMO to LUMO transition. The installation of ethynyl ferrocene to make 3.44b makes the dominant transition from HOMO-1 to LUMO at $\lambda_{\max }$ at $519 \mathrm{~nm}$. However comparing the ferrocene derivative $\mathbf{3 . 4 4 b}$ to formyl BODIPY 3.42b, the bathochromic shift indicates that there is a modest decrease in the HOMO-LUMO gap. The ferrocene malonitrile derivative 3.35b and ferrocene cyanoacetic acid derivative 3.36b $\lambda_{\max }$ both undergoes a bathochromic shift of $\sim 17 \mathrm{~nm}$ to $536 \mathrm{~nm}$ (HOMO-1 to LUMO). All three ferrocene BODIPY derivatives have panchromatic absorption profiles which are desirable for light harvesting applications. Also all three ferrocene BODIPY derivatives have broad absorption tails from $550-750 \mathrm{~nm}$ that are attributed to the weak HOMO-LUMO transition and intramolecular charge transfer (MLCT) from ferrocene to BODIPY.

\subsubsection{UV-Vis spectroscopy of dyes 3.44a-d}

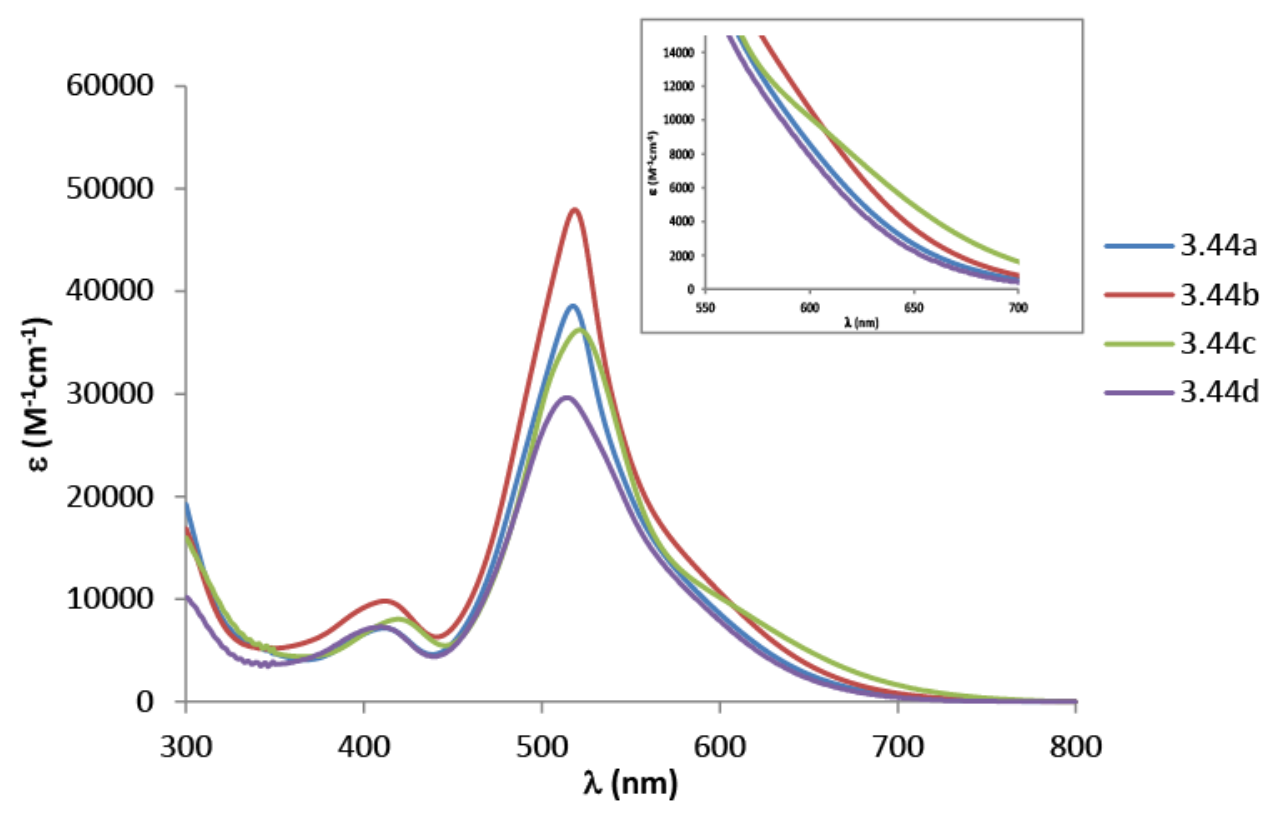

Figure 3.10: UV-Vis spectra of BODIPYs 3.44a-d. Data collected in DCM 
To explore the effect of the meso position on the physicochemical properties of the ferrocene push-pull BODIPY derivatives, UV/Vis data was collected on the 3.44a-d (figure). In general there are insignificant differences in $\lambda_{\max }$ of range $517-521 \mathrm{~nm}$. The largest bathochromic shift of $\lambda_{\max }$ was the nitro phenyl derivative 3.44c $(521 \mathrm{~nm})$ due to electron withdrawing moiety stabilizing the LUMO energy level. The largest hypsochromic shift of the $\lambda_{\max }$ was observed for the ethoxyphenyl derivative $\mathbf{3 . 4 4 d}$ due to the electron rich donating ring destabilizing the HOMO energy level while stabilizing the LUMO energy. All four derivatives have panchromatic absorption profiles and weak broad absorption from 550-750 nm attributed to the weak HOMO-LUMO transition and photo induced intramolecular charge transfer (MLCT) from ferrocene to BODIPY. However, 3.44c seems to favour the intramolecular charge transfer (Figure 3.10) where a weak transition band is more pronounced at $\sim 605 \mathrm{~nm}$, likely owing to the HOMO-LUMO transition.

\subsubsection{Preliminary testing of device 3.36b}

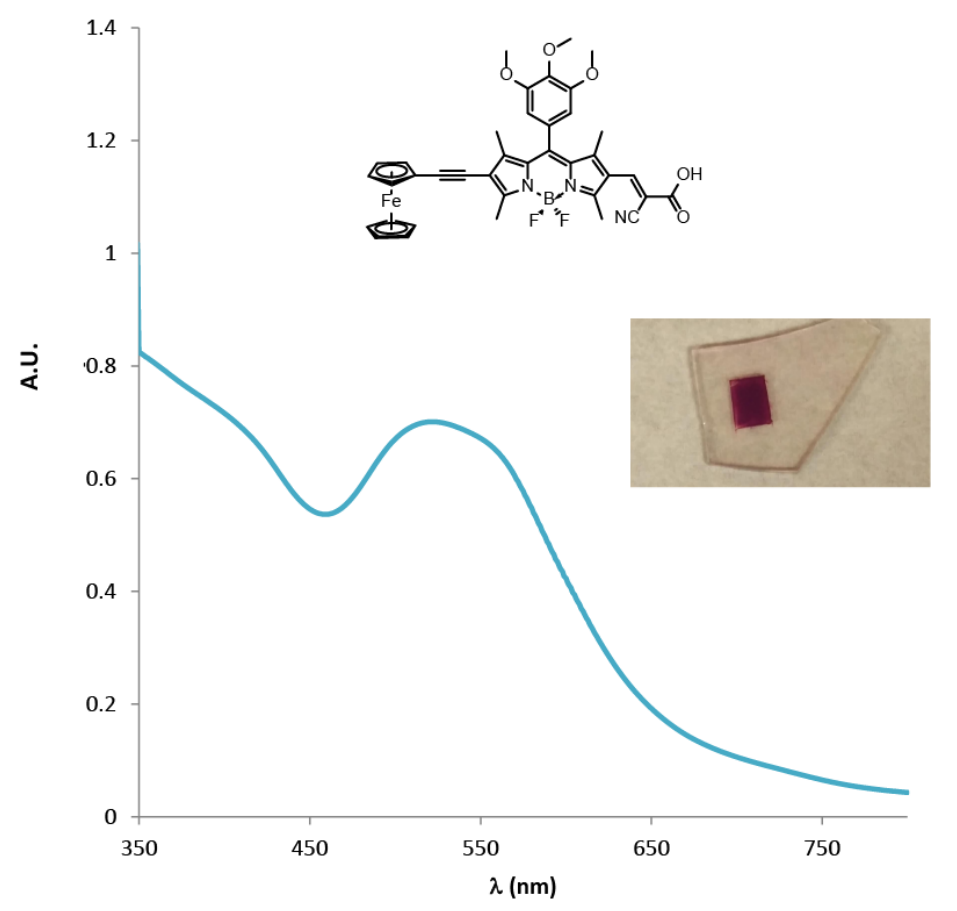

Figure 3.11: UV-Vis spectra of BODIPYs 3.36 adsorbed on the $\mathrm{TiO}_{2}$ film.

3.36b was adsorbed onto a $\mathrm{TiO}_{2}$-ITO cathode to assess the absorption profile for DSSC device purposes. The absorption was quite broad and panchromatic reaching $800 \mathrm{~nm}$ absorption (consistent with what was observed with other species). This UV-Vis showed promise for efficient performs of DSSC device based on 
3.36b. In collaboration with the Berlinguette group at University of British Columbia, 3.36b was examined for preliminary device performance. The device performed extremely poorly with power conversion efficiency of $0 \%$. There was a miserably low small open circuit voltage, but no short circuit current. This is mostly due to a low laying LUMO which is below the conduction band of $\mathrm{TiO}_{2}$, so there is no driving force to inject electrons into the cathode (however, dye stability may have also been a problem, vide infra).

Table 3. 1: Device Data for 3.36b

\begin{tabular}{lllll}
\hline & $\mathrm{J}_{\text {sc }}(\mathrm{A})$ & $\mathrm{V}_{\text {oc }}(\mathrm{mV})$ & $\mathrm{FF}$ & $\mathrm{P}_{\text {ce }}(\%)$ \\
\hline 3.6b & 0 & 0.253 & 0 & 0
\end{tabular}

\subsubsection{Electrochemistry of Ferrocene D- $\pi$-A BODIPY dyads}

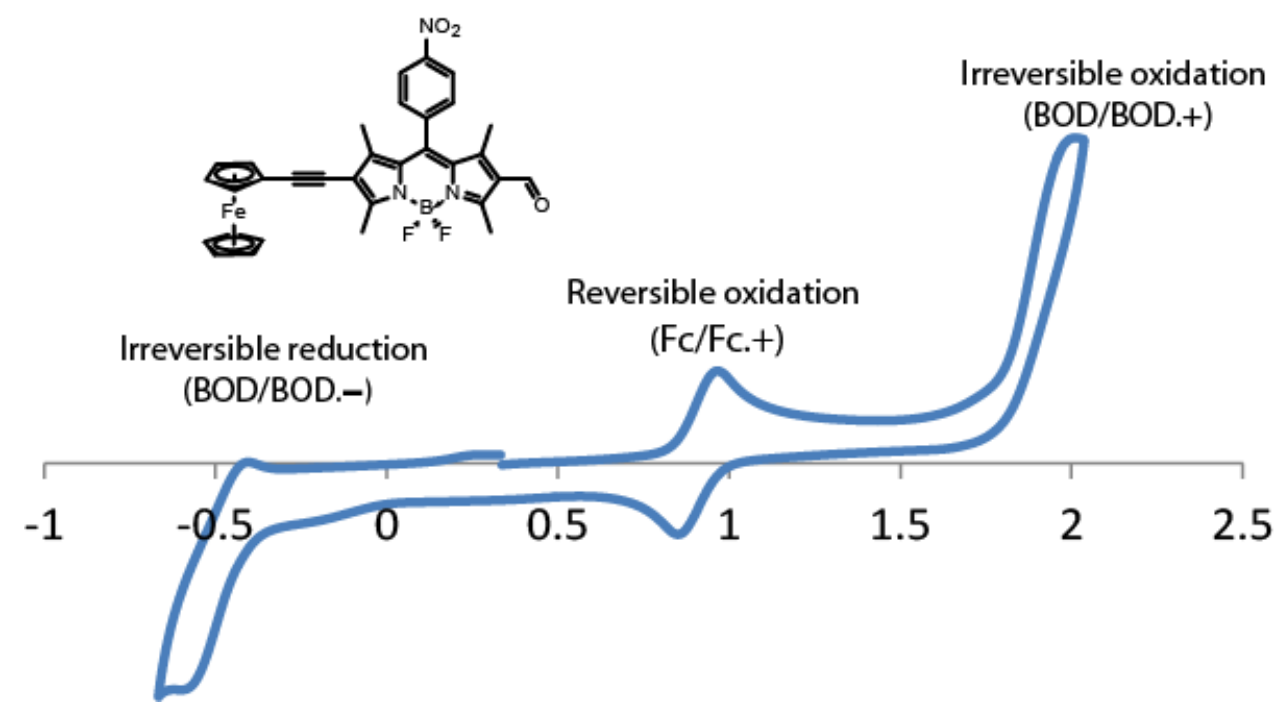

Figure 3.12: Example cyclic voltammogram of dye 3.44c. Data collected in $\mathrm{DCM}$ using $0.1 \mathrm{M}\left[\mathrm{nBu}_{4} \mathrm{~N}\right]\left[\mathrm{PF}_{6}\right]$ at $100 \mathrm{mV} / \mathrm{s}$ and reference to a $\left[\mathrm{Fc} / \mathrm{Fc}^{+}\right]$internal standard $(+0.765 \mathrm{~V}$ vs. NHE in DCM)

Electrochemical studies were performed on dyes 3.41a-d to 3.36b to determine the energy levels of Ferrocene D- $\pi$-A dyes and the effects ferrocene has on ground state dyad redox properties. Figure 3.6 refers to characteristic cyclic voltammogram of the ferrocene $D-\pi-A$ dyes $3.44 a-d$ to $3.36 b$. The cyclic voltammetry revealed (Table 3.1 ) that ferrocene $D-\pi$-A dyads and dyes experience a reversible oxidation of ferrocene (0.89-0.93 V), followed by an irreversible oxidation of BODIPY (1.81-1.96 V) and irreversible reduction of BODIPY (-0.50- $-0.61 \mathrm{~V})$. The ferrocene electron donor has a slightly lower oxidation potential 
compared to typical TPA donor ( $1 \mathrm{~V}$ ) which is desirable for DSSC applications (enhanced electron transfer rates would be expected). The effect of the electron withdrawing groups (aldehyde, malonitrile, and cyanoacetic acid) on the ferrocene oxidation potential does not seem to be significant. However the electron withdrawing groups do have effects on the second BODIPY oxidation potential. The trend observed is that the stronger the electron withdrawing group (aldehyde<malonitrile=cyanoacetic acid), the lower the oxidation potential. When looking at the reduction potential, the electron withdrawing acceptors decrease the reduction potential due to the stabilization of LUMO energy (this property is a undesirable for DSSC applications). TPA BODIPY dye has a higher LUMO energy level by approximately $0.30 \mathrm{~V}$, and its LUMO was below the conduction band of $\mathrm{TiO}_{2}$ which hinder the performance of the DSSC device. The low laying LUMO also seems to cause problems with decomposition, which will be discussed further. 
Table 3.2: Physicochemical properties for dyes 3.41a-d, 3.42a-d, 3.44a-d, 3.35b, d, 3.36b

\begin{tabular}{|c|c|c|c|c|c|}
\hline Compound & $\begin{array}{l}\lambda_{\max } \mathrm{nm} \\
\left(\varepsilon \times 10^{-4} \mathrm{M}^{-1} \mathrm{~cm}^{-1}\right)\end{array}$ & $E(V)_{(B O D / B O D ~-.) a}$ & $E(V)_{(\mathrm{Fc} / \mathrm{Fc}+)^{\mathrm{a}}}$ & $E(V)_{(B O D / B O D+.)^{a}}$ & $\begin{array}{l}\text { HOMO-LUMO } \\
\text { Gap (V) }\end{array}$ \\
\hline $3.41 \mathrm{a}$ & $501(8.2)$ & -0.96 & - & 1.52 & 2.48 \\
\hline $3.41 b$ & $502(8.1)$ & - & - & 1.58 & - \\
\hline $3.41 \mathrm{c}$ & $502(4.9)$ & -0.73 & - & 1.62 & 2.35 \\
\hline $3.41 \mathrm{~d}$ & $499(3.6)$ & -0.98 & - & 1.54 & 2.52 \\
\hline $3.42 \mathrm{a}$ & $497(5.0)$ & -0.73 & - & 1.87 & 2.60 \\
\hline $3.42 b$ & $498(8.2)$ & -0.74 & - & 1.75 & 2.49 \\
\hline $3.42 \mathrm{c}$ & $502(5.3)$ & -0.57 & - & 1.84 & 2.44 \\
\hline $3.42 \mathrm{~d}$ & $497(6.0)$ & -0.74 & - & 1.85 & 2.59 \\
\hline $3.44 a$ & $517(3.9)$ & -0.56 & 0.89 & 1.95 & 1.45 \\
\hline $3.44 b$ & $519(4.8)$ & -0.61 & 0.91 & 1.93 & 1.52 \\
\hline $3.44 c$ & $521(3.6)$ & -0.50 & 0.91 & 1.96 & 1.41 \\
\hline $3.44 d$ & $514(3.0)$ & -0.60 & 0.90 & 1.95 & 1.50 \\
\hline $3.35 b$ & $536(3.0)$ & -0.52 & 0.91 & 1.90 & 1.43 \\
\hline $3.35 d$ & $535(0.7)$ & -0.51 & 0.93 & 1.91 & 1.44 \\
\hline $3.36 \mathrm{~b}$ & $537(6.1)$ & -0.51 & 0.91 & 1.81 & 1.42 \\
\hline
\end{tabular}

${ }^{\mathrm{a}}$ Data collected using $0.1 \mathrm{M} \mathrm{NBu}_{4} \mathrm{PF}_{6} \mathrm{DCM}$ solutions at $100 \mathrm{mVs}^{-1}$ and referenced to a $[\mathrm{Fc}] /[\mathrm{Fc}]+$ internal standard followed by conversion to $\mathrm{NHE} ;[\mathrm{Fc}] /[\mathrm{Fc}+]=+765 \mathrm{mV}$ vs. NHE in DCM; 


\subsubsection{Solvent polarity absorption-dependence of 3.44c}

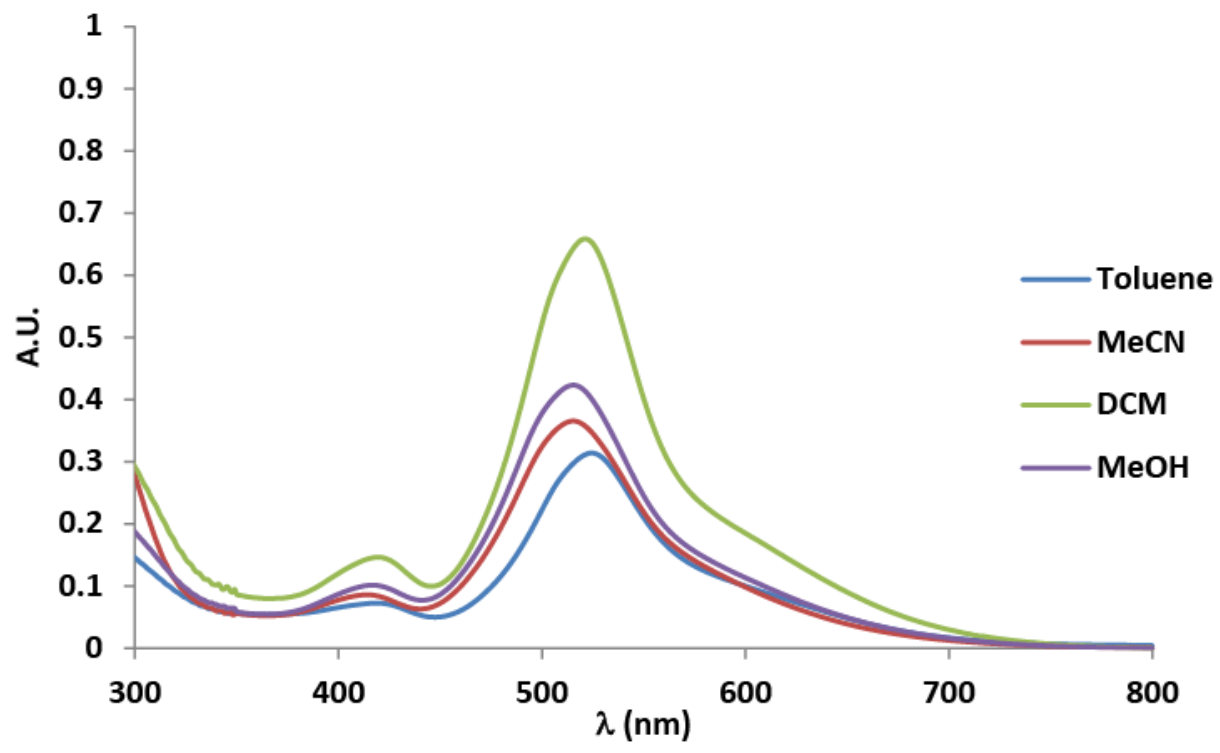

Figure 3.13: UV-Vis spectra of BODIPYs 3.44c. Data collected in DCM, MeCN, toluene, $\mathrm{MeOH}$.

Similar to previously reported work, the solvent used seems to have a noticeable effect on the absorption behaviour. In ferrocene BODIPY dyad 3.44c, more polar solvents like methanol and acetonitrile, there is a hypsochromic shift of $6 \mathrm{~nm}$ to $515 \mathrm{~nm}$ (Figure 3.12). This hypsochromic shift can be attributed to the destabilization of LUMO in the excited state. When toluene is used, there could be a intermolecular $\pi$ stacking that stabilizes the ferrocene BODIPY dyads, which redshifts the absorption to $524 \mathrm{~nm}$. 


\subsubsection{Spectroelectrochemical studies of Ferrocene $D-\pi-A$ dyes}

A)
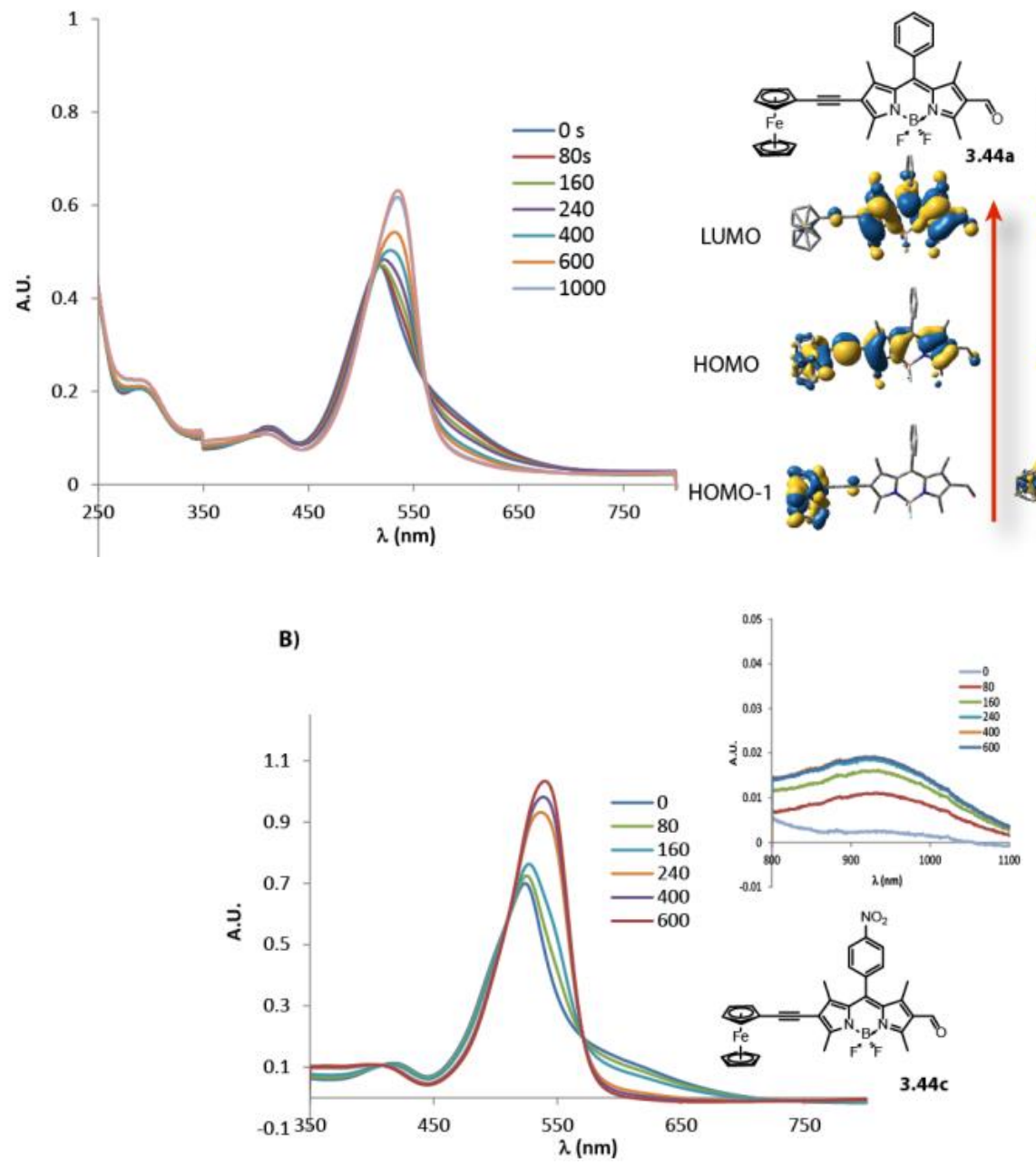

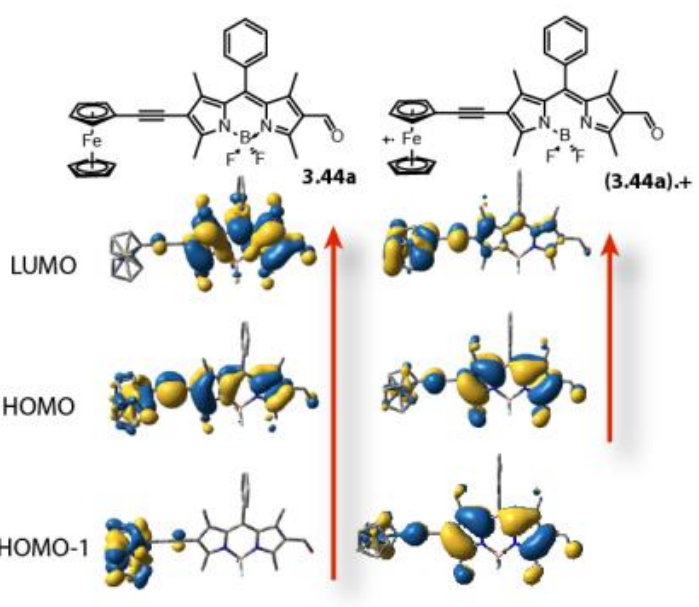

D)
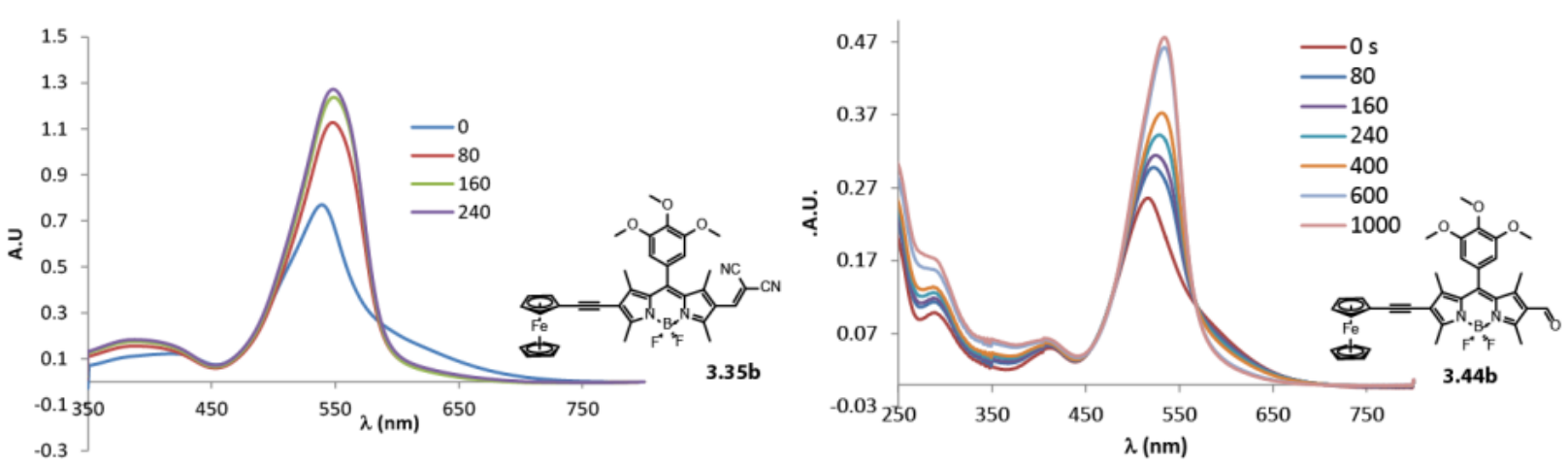

Figure 3.14: Spectrochemical data for dyes 3.44a-c and 3.35b. a) Transformation of UV-vis spectra of 3.44a during the first oxidation process and DFT calculation comparing the 3.44a and (3.44a).+ b) Transformation of UV-vis spectra of 3.44c during the first oxidation process and inset of the production of ferrocenium. c) Transformation of 
UV-vis spectra of 3.44b during the first oxidation process d) Transformation of UV-vis spectra of 3.35b during the first oxidation process

Table 3.3: Spectroelectrochemical data for dyes 3.44a-c and 3.35b

\begin{tabular}{|c|c|c|}
\hline Compound & $\lambda_{\max } \mathrm{nm}$ & $\lambda_{\max } \mathrm{nm}$ (oxidation) \\
\hline $3.4 a$ & 517 & 534 \\
\hline $3.4 \mathrm{~b}$ & 519 & 536 \\
\hline $3.4 \mathrm{c}$ & 521 & 546 \\
\hline $3.5 b$ & 536 & 549 \\
\hline
\end{tabular}

Data collected using $0.1 \mathrm{M} \mathrm{NBu}_{4} \mathrm{PF}_{6}$ DCM solutions at $100 \mathrm{mVs}-1$ and oxidized at Fc/Fc.+ of BODIPY derivatives

In order to assess the importance on the redox-activity in the ferrocene $D-\pi-A$ dyes $3.44 a-d$ to $3.36 b$ and the change in absorption of oxidized species, spectroelectrochemical studies were performed on 3.44a, 3.44b, 3.44c, and 3.36b. During the ferrocene oxidation (Figure 3.13), the MLCT band decreases intensity while $\pi \rightarrow \pi^{*}$ transition in the visible region undergoes a red shift in all cases with increasing intensity. TD-DFT revealed that when ferrocene is oxidized, the electron density liess on mostly the BODIPY and the main transition is the HOMO-LUMO transition $\left(\pi-\pi^{*}\right)$. In addition for 3.44c, the formation of a very weak NIR band ranging from 800-1000 $\mathrm{nm}$ due to the formation of ferrocenium-BODIPY species was observed. This evidence confirms that the oxidation of ferrocene $D-\pi-A$ occurs on ferrocene moiety.

\subsubsection{Decomposition of ferrocene BODIPYs dyads and dyes}

Another problem was that $\mathbf{3 . 3 6}$ b readily decomposed in ambient conditions which is surprising due to ferrocene redox robustness which should stabilize the BODIPY dye. Instead the molecule was found to readily photobleach (Figure 3.15 ), which may be due to a radical decomposition process. In addition, this photobleaching was observed even with the aldehyde compound as well. 

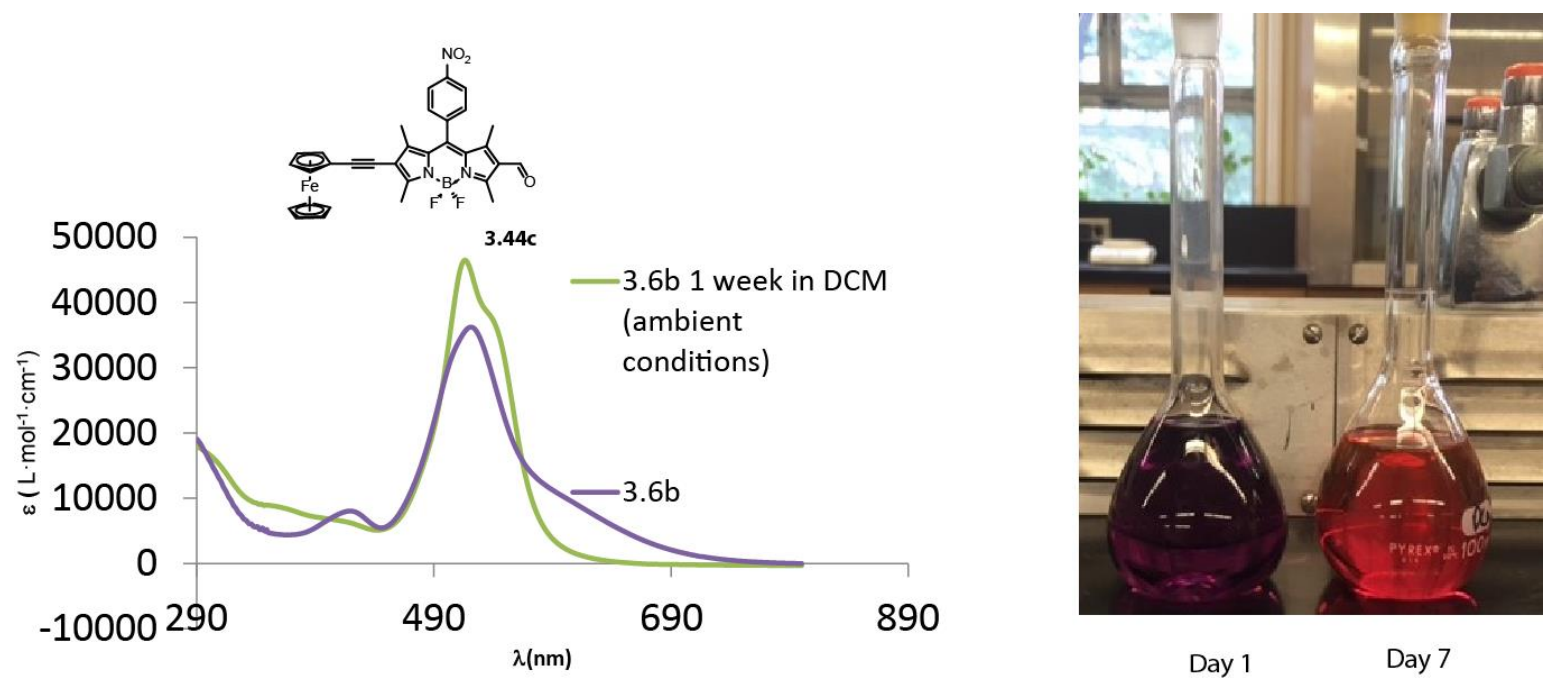

Figure 3.15: Decomposition of BODIPYs 3.44c in DCM.

\subsection{Conclusion and Future Work}

The ferrocene BODIPY D- $\pi$-A dyes proposed in this project have been synthesised with successes and have shown some interesting physicochemical properties. Incorporating ferrocene as electron donor and varying electron withdrawing groups (aldehyde, malonitrile and cyanoacetic acid) to BODIPY, has resulted in panchromatic intensely absorbing dyes which also exhibit unique charge transfer behaviour that will be explored in further detail in Jennifer Huynh's thesis. Unravelling the questionable stability of these dyes and dyads as seen in Figure 3.15 will also be the focus of Jennifer's thesis.

For light harvesting DSSC applications, 3.36b, did not perform as desired due to its low laying LUMO and recombination issues. In order to combat the low laying LUMO, additional electronic rich rings can be introduced at 3, 5 positions which should raise the LUMO. The addition of meso phenyl ring with different electron donor/withdrawing groups did not significantly change the LUMO level, but this proposed modification should.
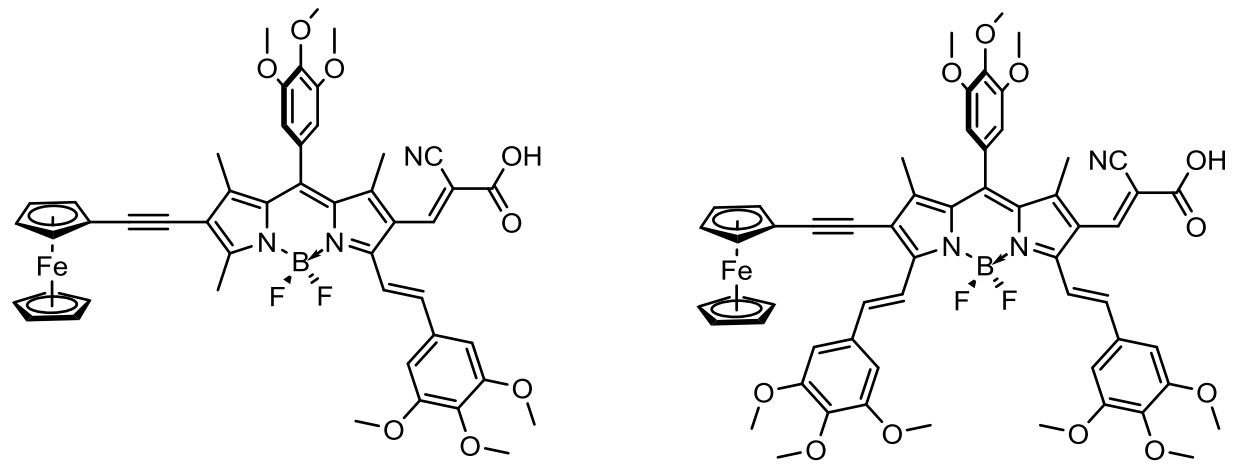

Figure 3.16: Proposed ferrocene BODIPY for raising LUMO energy level. 
This novel BODIPY ferrocene dyad family has challenged the idea of ferrocene incorporation into organic photosensitizer for stability issues, but perhaps the derivatives in Figure 3.16 would be able to overcome this hurdle. Further studies into the decomposition pathways and what causes it will be needed to make these ferrocene-BODIPY dyes a reality. 


\subsection{Experimental}

\section{General Considerations}

All reagents were purchased from Aldrich except Pd(PPh3)4 (Pressure Chemical Co., Pittsburg, PA). Purification by column chromatography was carried out using silica (Silicycle: ultrapure flash silica). Analytical thin-layer chromatography was performed on aluminum-backed sheets precoated with silica 60 F254 adsorbent ( $0.25 \mathrm{~mm}$ thick; Silicycle) and visualized under UV light. IR data was collected in $\mathrm{KBr}$ using a Perkin Elmer Spectrum 1 Fourier Transform Infrared spectrophotometer. Melting points were determined using a Perkin Elmer Diamond Differential Scanning Calorimeter. Routine $1 \mathrm{H}, 13 \mathrm{C}\{1 \mathrm{H}\}, 11 \mathrm{~B}\{1$ $\mathrm{H}$ \} and 19F NMR spectra were recorded at 400,100, 128 and $376 \mathrm{MHz}$, respectively, on a Bruker AV 400 instrument at ambient temperature. Chemical shifts $(\delta)$ are reported in parts per million $(\mathrm{ppm})$ from low to high field and referenced to a residual nondeuterated solvent $(\mathrm{CHCl} 3)$ for $1 \mathrm{H}$ and $13 \mathrm{C}$ nuclei and

$\mathrm{BF} 3 \bullet \mathrm{OEt2}$ (11B nucleus; $\delta=0 \mathrm{ppm}$ ) C6F6 (19F nucleus; $\delta=0 \mathrm{ppm}$ ). Standard abbreviations indicating multiplicity are used as follows: $\mathrm{s}=$ singlet; $\mathrm{d}=$ doublet; $\mathrm{m}=$ multiplet; $\mathrm{br}=$ broad. 4-methoxy- $\mathrm{N}-(4-$ methoxyphenyl)-N-(4-(4,4,5,5-tetramethyl-1,3,2-dioxaborolan-2-yl)phenyl)aniline (A) 1 , 4- hexyloxy- $\mathrm{N}$ (4-hexyloxyphenyl)-N-(4-(4,4,5,5-tetramethyl-1,3,2-dioxaborolan-2- yl)phenyl)aniline (B) 2 , 4,4,5,5tetramethyl-2-(thiophen-2-yl)-1,3,2-dioxaborolane (C) 3 and compound 14 were prepared as previously reported.

\section{Theoretical Calculations}

All DFT and TD-DFT calculation was done use Gaussian 09M version C software. All structure were optimized at ground state default spin using the B3LYP exchange correlation function. These calclations were done in the gas phase (no solvent). For TD-DFT calculations were done at TD-SCF using the B3LYP exchange correlation function. All calculation were optimized using $3.21 \mathrm{G}$ basis set $(6.31 \mathrm{G}$ basis set when mentioned).

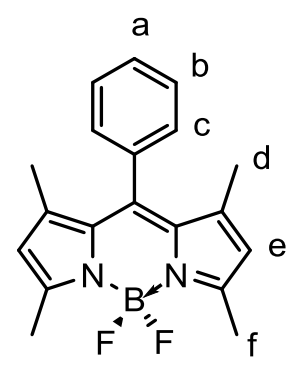

3.41a: To a solution of pyrrole $(3.07 \mathrm{~g}, 32.3 \mathrm{mmol}$,) in dry dichloromethane $(150 \mathrm{~mL}$ ) benzoyl chloride (0.75 mL, $6.45 \mathrm{mmol}$,) was refluxed for two hours. Once the reaction was complete, $\mathrm{N}, \mathrm{N}$ dispropylethylamine $(7 \mathrm{~mL})$ and $\mathrm{BF}_{3} \cdot \mathrm{OEt}_{2}(7 \mathrm{~mL})$ were added and stirred overnight. The mixture was concentrated in vacuo, re-dissolved in EtOAc and the combined organic layers were washed with $\mathrm{HCl} 1 \mathrm{M}$ $(50 \mathrm{~mL})$, sat. $\mathrm{NaHCO}_{3}(100 \mathrm{~mL})$ and water $(100 \mathrm{~mL})$. The combined organic layers dried $\left(\mathrm{MgSO}_{4}\right)$, filtered and volatiles were removed in vacuo. The crude product was then subjected to column chromatography, using DCM as an eluent to afford the desired product as an orange powder $(0.350 \mathrm{~g}, 16 \%) .{ }^{1} \mathrm{H}$ NMR (400 
$\left.\mathrm{MHz}, \mathrm{CDCl}_{3}\right) \delta 7.48\left(\mathrm{~m}, 3 \mathrm{H}, \mathrm{H}_{\mathrm{a}}, \mathrm{H}_{\mathrm{b}}\right), 7.28\left(\mathrm{dd},{ }^{3} \mathrm{~J}=4.5 \mathrm{~Hz},{ }^{4} \mathrm{~J}=3.1 \mathrm{~Hz}, 2 \mathrm{H}, \mathrm{H}_{\mathrm{c}}\right), 5.98\left(\mathrm{~s}, 2 \mathrm{H}, \mathrm{H}_{\mathrm{e}}\right), 2.56\left(\mathrm{~s}, 6 \mathrm{H}, \mathrm{H}_{\mathrm{f}}\right.$ ), $1.37\left(\mathrm{~s}, 6 \mathrm{H}, \mathrm{H}_{\mathrm{d}}\right)$. The remainder of the characterization was also consistent with previously reported literature. ${ }^{44}$

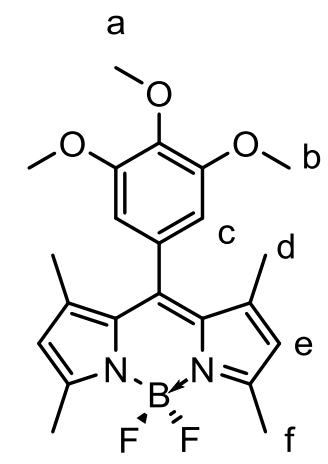

3.41b: To a solution of pyrrole (1.36 g, $20.4 \mathrm{mmol}$ ) in dry dichloromethane $(150 \mathrm{~mL})$ the appropriate benzaldehyde $(1.00 \mathrm{~g}, 5.10 \mathrm{mmol}$ ) was added followed by a catalytic amount of trifluoroacetic acid (TFA). After stirring overnight, resulting in a dark red solution, 2,3-dichoro-5,6-dicyanobenzoquinone (1.75 g, $14.1 \mathrm{mmol})$ was added and the mixture was stirred for $12 \mathrm{~h}$. After, $\mathrm{N}, \mathrm{N}$-dispropylethylamine $(7 \mathrm{~mL})$ and $\mathrm{BF}_{3} \bullet \mathrm{OEt}_{2}(7 \mathrm{~mL})$ were added. After the stirring overnight, the mixture was concentrated in vacuo, redissolved in EtOAc and the combined organic layers were dried $\left(\mathrm{MgSO}_{4}\right)$, filtered and volatiles were removed in vacuo. The crude product was then subjected to column chromatography, using hexanes:EtOAc 3:2 as an eluent to afford the desired product as an orange powder $(0.632 \mathrm{~g}, 29 \% \%) .{ }^{1} \mathrm{H}$ $\operatorname{NMR}\left(400 \mathrm{MHz}, \mathrm{CDCl}_{3}\right) \delta 6.52\left(\mathrm{~s}, 2 \mathrm{H}, \mathrm{H}_{\mathrm{c}}\right), 6.00\left(\mathrm{~s}, 2 \mathrm{H}, \mathrm{H}_{\mathrm{e}}\right), 3.91\left(\mathrm{~s}, 3 \mathrm{H}, \mathrm{H}_{\mathrm{a}}\right), 3.82\left(\mathrm{~s}, 6 \mathrm{H}, \mathrm{H}_{\mathrm{b}}\right), 2.55\left(\mathrm{~s}, 6 \mathrm{H}, \mathrm{H}_{\mathrm{f}}\right)$, $1.53\left(\mathrm{~s}, 6 \mathrm{H}, \mathrm{H}_{\mathrm{d}}\right)$. The remainder of the characterization was also consistent with previously reported literature. $^{45}$

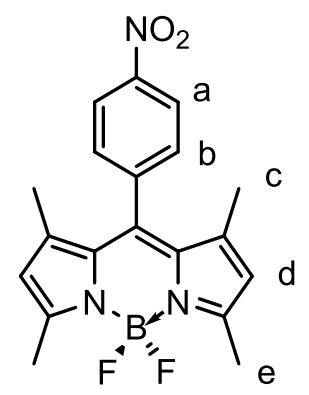

3.41c: To a solution of pyrrole $(0.460 \mathrm{~g}, 4.83 \mathrm{mmol}$,) in dry dichloromethane $(150 \mathrm{~mL})$ the appropriate benzaldehyde $(0.43 \mathrm{~g}, 2.42 \mathrm{mmol}$ ) was added followed by a catalytic amount of trifluoroacetic acid (TFA). After stirring overnight, resulting in a dark red solution, 2,3-dichoro-5,6-dicyanobenzoquinone $(0.659 \mathrm{~g}$, $2 . .90 \mathrm{mmol}$ ) was added and the mixture was stirred for $12 \mathrm{~h}$. After, $\mathrm{N}, \mathrm{N}$-dispropylethylamine $(3 \mathrm{~mL})$ and $\mathrm{BF}_{3} \bullet \mathrm{OEt}_{2}(3.5 \mathrm{~mL})$ were added. After the stirring overnight, the mixture was concentrated in vacuo, redissolved in EtOAc and the combined organic layers were washed with $\mathrm{HCl} 1 \mathrm{M}(50 \mathrm{~mL})$, sat. $\mathrm{NaHCO}_{3}(100$ $\mathrm{mL})$ and water $(100 \mathrm{~mL})$. The combined organic layers were dried $\left(\mathrm{MgSO}_{4}\right)$, filtered and volatiles were 
removed in vacuo. The crude product was then subjected to column chromatography, using DCM as an eluent to afford the desired product as an orange powder $(0.547 \mathrm{~g}, 22 \% \%) .{ }^{1} \mathrm{H} \mathrm{NMR}\left(400 \mathrm{MHz}, \mathrm{CDCl}_{3}\right) \delta$ $8.41\left(\mathrm{~d},{ }^{3} \mathrm{~J}=8.2 \mathrm{~Hz}, 2 \mathrm{H}, \mathrm{H}_{\mathrm{a}}\right), 7.57\left(\mathrm{~d},{ }^{3} \mathrm{~J}=8.3 \mathrm{~Hz}, 2 \mathrm{H}, \mathrm{H}_{\mathrm{b}}\right), 6.04\left(\mathrm{~s}, 2 \mathrm{H}, \mathrm{H}_{\mathrm{d}}\right), 2.59\left(\mathrm{~s}, 6 \mathrm{H}, \mathrm{H}_{\mathrm{e}}\right), 1.39\left(\mathrm{~s}, 6 \mathrm{H}, \mathrm{H}_{\mathrm{d}}\right)$. The remainder of the characterization was also consistent with previously reported literature. ${ }^{44}$

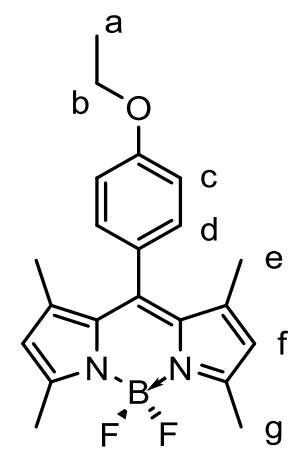

3.41d: To a solution of 2,4-dimethyl-1H-pyrrole $(0.790 \mathrm{~g}, 8.32 \mathrm{mmol})$ in dry DCM (120 mL), 4ethoxybenzaldehyde $(0.500 \mathrm{~g}, 3.33 \mathrm{mmol}$,) was added followed by a catalytic amount of TFA. After overnight stirring of the resulting reddish solution, DDQ (1.13 g, $1.5 \mathrm{eq})$ was added and the mixture was stirred for 3 hours. After TLC analysis revealed the completion of oxidation, DIPEA ( $4.0 \mathrm{~mL}$ ) and BF3OEt2 $(4.7 \mathrm{~mL})$ were added. After stirring for 6 hours, the mixture was concentrated in vacuo, dissolved in EtOAc and washed with water. The water layer was extracted with EtOAc, and the combined organic layers were dried over MgSO4, filtered, and concentrated in vacuo. Purification by column chromatography yielded the product as a red powder (367 mg, 30\%). ${ }^{1} \mathrm{H} \mathrm{NMR}\left(400 \mathrm{MHz}, \mathrm{CDCl}_{3}\right) \delta 7.15\left(\mathrm{~d},{ }^{3} \mathrm{~J}=8.6 \mathrm{~Hz}, 2 \mathrm{H}, \mathrm{H}_{\mathrm{c}}\right), 7.00$ $\left(\mathrm{d},{ }^{3} \mathrm{~J}=8.6 \mathrm{~Hz}, 2 \mathrm{H}, \mathrm{H}_{\mathrm{d}}\right), 5.97\left(\mathrm{~s}, 2 \mathrm{H}, \mathrm{H}_{\mathrm{f}}\right), 4.09\left(\mathrm{q},{ }^{3} \mathrm{~J}=7.0 \mathrm{~Hz}, 2 \mathrm{H}, \mathrm{H}_{\mathrm{b}}\right), 2.55\left(\mathrm{~s}, 6 \mathrm{H}, \mathrm{H}_{\mathrm{g}}\right), 1.47\left(\mathrm{t},{ }^{3} \mathrm{~J}=7.0 \mathrm{~Hz}, 3 \mathrm{H}\right.$, $\left.\mathrm{H}_{\mathrm{a}}\right), 1.43\left(\mathrm{~s}, 6 \mathrm{H}, \mathrm{H}_{\mathrm{e}}\right) .{ }^{13} \mathrm{C}\left\{{ }^{1} \mathrm{H}\right\} \mathrm{NMR}\left(101 \mathrm{MHz}, \mathrm{CDCl}_{3}\right) \delta 159.52,155.23,143.18,141.96,131.87,129.11$, $128.23,126.90,121.07,115.04,63.56,14.78,14.56 .{ }^{11} \mathrm{~B} \mathrm{NMR}\left(128 \mathrm{MHz}, \mathrm{CDCl}_{3}\right) \delta 0.79$ (t). ${ }^{19} \mathrm{~F} \mathrm{NMR}(376$ $\mathrm{MHz}, \mathrm{CDCl}_{3}$ ) $\delta-146.19--146.46$ (q).<smiles>O=Cc1c(Cl)c2n(c1I)[B-](F)(F)n1c(I)cc(I)c1C2=c1c[c+]cc1</smiles>

3.42a: A solution of $10 \mathrm{~mL}$ of dry DMF in $30 \mathrm{~mL}$ of dry DCM was cooled in an ice bath and $12 \mathrm{~mL}$ of $\mathrm{POCl}_{3}$ was added dropwise. The mixture was warmed to room temperature and stirred $30 \mathrm{~min}$. 3.41a $(0.300 \mathrm{mg}$, $0.925 \mathrm{mmol}$ ) was added in one portion and the mixture was stirred $3 \mathrm{~h}$. The mixture was neutralized in 30 $\mathrm{mL}$ of saturated aqueous $\mathrm{NaHCO}_{3}$ cooled to $0{ }^{\circ} \mathrm{C}$ and the mixture was stirred $30 \mathrm{~min}$ at room temperature. The organic layer was separated and the aqueous layer was extracted with DCM $(3 \times 25 \mathrm{~mL})$. The combined 
organic layers were dried over $\mathrm{MgSO}_{4}$, filtered and volatiles were removed in vacuo. The crude product was then subjected to column chromatography, using DCM as an eluent to afford the desired product as an orange powder $(0.301 \mathrm{~g}, 92 \% \%)^{1} \mathrm{H} N M R\left(400 \mathrm{MHz}, \mathrm{CDCl}_{3}\right) \delta 10.01\left(\mathrm{~s}, 1 \mathrm{H}, \mathrm{He}_{\mathrm{e}}\right), 7.53\left(\mathrm{~m}, 3 \mathrm{H}, \mathrm{H}_{\mathrm{a}}, \mathrm{H}_{\mathrm{b}}\right), 7.28$ $\left(\mathrm{d},{ }^{3} \mathrm{~J}=12.5 \mathrm{~Hz}, 2 \mathrm{H}, \mathrm{H}_{\mathrm{c}}\right), 6.15\left(\mathrm{~s}, 1 \mathrm{H}, \mathrm{H}_{\mathrm{h}}\right), 2.83\left(\mathrm{~s}, 3 \mathrm{H}, \mathrm{H}_{\mathrm{f}}\right), 2.62\left(\mathrm{~s}, 3 \mathrm{H}, \mathrm{H}_{\mathrm{d}}\right), 1.66\left(\mathrm{~s}, 3 \mathrm{H}, \mathrm{H}_{\mathrm{g}}\right), 1.43\left(\mathrm{~s}, 3 \mathrm{H}, \mathrm{H}_{\mathrm{i}}\right)$. The remainder of the characterization was also consistent with previously reported literature. ${ }^{46}$<smiles></smiles>

3.42b: A solution of $10 \mathrm{~mL}$ of dry DMF in $30 \mathrm{~mL}$ of dry DCM was cooled in an ice bath and $12 \mathrm{~mL}$ of $\mathrm{POCl}_{3}$ was added dropwise. The mixture was warmed to room temperature and stirred $30 \mathrm{~min}$. 3.41b $(0.300 \mathrm{mg}$, $0.724 \mathrm{mmol}$ ) was added in one portion and the mixture was stirred $3 \mathrm{~h}$. The mixture was neutralized in 30 $\mathrm{mL}$ of saturated aqueous $\mathrm{NaHCO}_{3}$ cooled to $0{ }^{\circ} \mathrm{C}$ and the mixture was stirred $30 \mathrm{~min}$ at room temperature. The organic layer was separated and the aqueous layer was extracted with DCM $(3 \times 25 \mathrm{~mL})$. The combined organic layers were dried over $\mathrm{MgSO}_{4}$, filtered and volatiles were removed in vacuo. The crude product was then subjected to column chromatography, using DCM as an eluent to afford desired product as an orange powder $(0.258 \mathrm{~g}, 81 \%){ }^{1} \mathrm{H}$ NMR $\left(400 \mathrm{MHz}, \mathrm{CDCl}_{3}\right) \delta 10.04\left(\mathrm{~s}, 1 \mathrm{H}, \mathrm{H}_{\mathrm{e}}\right), 6.54\left(\mathrm{~s}, 2 \mathrm{H}, \mathrm{H}_{\mathrm{c}}\right), 6.19(\mathrm{~s}, 1 \mathrm{H}$, $\left.\mathrm{H}_{\mathrm{h}}\right), 3.94\left(\mathrm{~s}, 3 \mathrm{H}, \mathrm{H}_{\mathrm{a}}\right), 3.86\left(\mathrm{~s}, 6 \mathrm{H}, \mathrm{H}_{\mathrm{b}}\right), 2.83\left(\mathrm{~s}, 3 \mathrm{H}, \mathrm{H}_{\mathrm{f}}\right), 2.63\left(\mathrm{~s}, 3 \mathrm{H}, \mathrm{H}_{\mathrm{d}}\right), 1.83\left(\mathrm{~s}, 3 \mathrm{H}, \mathrm{H}_{\mathrm{g}}\right), 1.61\left(\mathrm{~s}, 3 \mathrm{H}, \mathrm{H}_{\mathrm{i}}\right) .{ }^{13} \mathrm{C}\left\{{ }^{1} \mathrm{H}\right\}$ $\operatorname{NMR}\left(101 \mathrm{MHz}, \mathrm{CDCl}_{3}\right) \delta 185.85,161.76,156.59,154.51,147.18,143.21,142.78,139.19,134.03,129.72$, $129.13,126.35,123.99,104.99,61.38,56.46,53.41,15.07,14.71,12.97,11.54 .{ }^{11} \mathrm{~B} \mathrm{NMR}\left(128 \mathrm{MHz}, \mathrm{CDCl}_{3}\right)$ $\delta$ 0.93-0.43 (t). $\left.{ }^{19} \mathrm{~F} \mathrm{NMR} \mathrm{(376} \mathrm{MHz,} \mathrm{CDCl}_{3}\right) \delta-144.42--144.90$ (q).<smiles></smiles> 
3.42c : A solution of $10 \mathrm{~mL}$ of dry DMF in $30 \mathrm{~mL}$ of dry DCM was cooled in an ice bath and $12 \mathrm{~mL}$ of $\mathrm{POCl}_{3}$ was added dropwise. The mixture was warmed to room temperature and stirred $30 \mathrm{~min} . \mathbf{3 . 4 1 \mathrm { c }}(0.300 \mathrm{~g}$, $0.813 \mathrm{mmol}$ ) was added in one portion and the mixture was stirred $3 \mathrm{~h}$. The mixture was neutralized in 30 $\mathrm{mL}$ of saturated aqueous $\mathrm{NaHCO}_{3}$ cooled to $0{ }^{\circ} \mathrm{C}$ and the mixture was stirred $30 \mathrm{~min}$ at room temperature. The organic layer was separated and the aqueous layer was extracted with DCM $(3 \times 25 \mathrm{~mL})$. The combined organic layers were dried over $\mathrm{MgSO}_{4}$, filtered and volatiles were removed in vacuo. The crude product was then subjected to column chromatography, using DCM as an eluent to afford desired product as an orange powder (0.238 g, 74\%). ${ }^{1} \mathrm{H}$ NMR $\left(400 \mathrm{MHz}, \mathrm{CDCl}_{3}\right) \delta 10.04\left(\mathrm{~s}, 1 \mathrm{H}, \mathrm{H}_{\mathrm{d}}\right), 8.46\left(\mathrm{~d},{ }^{3} \mathrm{~J}=8.8 \mathrm{~Hz}, 2 \mathrm{H}, \mathrm{H}_{\mathrm{a}}\right)$, $7.58\left(\mathrm{~d},{ }^{3} \mathrm{~J}=8.8 \mathrm{~Hz}, 2 \mathrm{H}, \mathrm{H}_{\mathrm{b}}\right), 6.22\left(\mathrm{~s}, 1 \mathrm{H}, \mathrm{H}_{\mathrm{g}}\right), 2.86\left(\mathrm{~s}, 3 \mathrm{H}, \mathrm{H}_{\mathrm{e}}\right), 2.66\left(\mathrm{~s}, 3 \mathrm{H}, \mathrm{H}_{\mathrm{c}}\right), 1.68\left(\mathrm{~s}, 3 \mathrm{H}, \mathrm{H}_{\mathrm{f}}\right), 1.44(\mathrm{~s}, 3 \mathrm{H}$, $H_{h}$ ). The remainder of the characterization was also consistent with previously reported literature. ${ }^{46}$

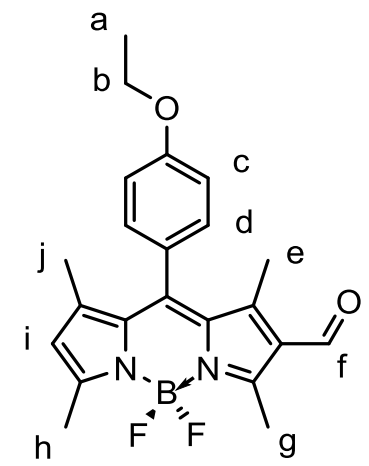

3.42d: A solution of $10 \mathrm{~mL}$ of dry DMF in $30 \mathrm{~mL}$ of dry DCM was cooled in an ice bath and $12 \mathrm{~mL}$ of $\mathrm{POCl}_{3}$ was added dropwise. The mixture was warmed to room temperature and stirred $30 \mathrm{~min}$. $3.41 \mathrm{~d}(0.200 \mathrm{mg}$, $0.543 \mathrm{mmol}$ ) was added in one portion and the mixture was stirred $3 \mathrm{~h}$. The mixture was neutralized in 30 $\mathrm{mL}$ of saturated aqueous $\mathrm{NaHCO}_{3}$ cooled to $0^{\circ} \mathrm{C}$ and the mixture was stirred $30 \mathrm{~min}$ at room temperature. The organic layer was separated and the aqueous layer was extracted with DCM $(3 \times 25 \mathrm{~mL})$. The combined organic layers were dried over $\mathrm{MgSO}_{4}$, filtered and volatiles were removed in vacuo. The crude product was then subjected to column chromatography, using DCM as an eluent to afford desired product as an orange powder (0.153 g, 71\%). ${ }^{1} \mathrm{H}$ NMR $\left(400 \mathrm{MHz}, \mathrm{CDCl}_{3}\right) \delta 9.99\left(\mathrm{~s}, 1 \mathrm{H}, \mathrm{H}_{\mathrm{f}}\right), 7.13\left(\mathrm{~d},{ }^{3} \mathrm{~J}=8.6 \mathrm{~Hz}, 2 \mathrm{H}, \mathrm{H}_{\mathrm{c}}\right)$, $7.01\left(\mathrm{~d},{ }^{3} \mathrm{~J}=8.6 \mathrm{~Hz}, 2 \mathrm{H}, \mathrm{H}_{\mathrm{d}}\right), 6.13\left(\mathrm{~s}, 1 \mathrm{H}, \mathrm{H}_{\mathrm{i}}\right), 4.16-3.99\left(\mathrm{q},{ }^{3} \mathrm{~J}=6.9 \mathrm{~Hz}, 3 \mathrm{H}, \mathrm{H}_{\mathrm{b}}\right), 2.79\left(\mathrm{~s}, 3 \mathrm{H}, \mathrm{H}_{\mathrm{g}}\right), 2.58(\mathrm{~s}, 3 \mathrm{H}$, $\left.\mathrm{H}_{\mathrm{e}}\right), 1.70\left(\mathrm{~s}, 3 \mathrm{H}, \mathrm{H}_{\mathrm{h}}\right), 1.47\left(\mathrm{~s}, 3 \mathrm{H}, \mathrm{H}_{\mathrm{j}}\right), 1.44\left(\mathrm{t},{ }^{3} \mathrm{~J}=7.0 \mathrm{~Hz}, 3 \mathrm{H}, \mathrm{H}_{\mathrm{a}}\right) .{ }^{13} \mathrm{C}\left\{{ }^{1} \mathrm{H}\right\} \mathrm{NMR}\left(101 \mathrm{MHz}, \mathrm{CDCl}_{3}\right) \delta 185.88$, $161.35,159.97,156.27,147.35,143.88,142.86,134.51,130.21,128.99,126.27,125.91,123.88,115.38$, 63.66, 15.04, 14.71, 12.97, 11.73. ${ }^{11} \mathrm{~B} N M R\left(128 \mathrm{MHz}, \mathrm{CDCl}_{3}\right) \delta 0.70(\mathrm{t}) . \mathrm{HRMS}: \mathrm{m} / \mathrm{z} 396.1821(\mathrm{M}+)$, calculated for $\mathrm{C}_{23} \mathrm{H}_{23} \mathrm{BF}_{2} \mathrm{IN}_{2} \mathrm{O}_{2}: \mathrm{m} / \mathrm{z} 396.1821$ 


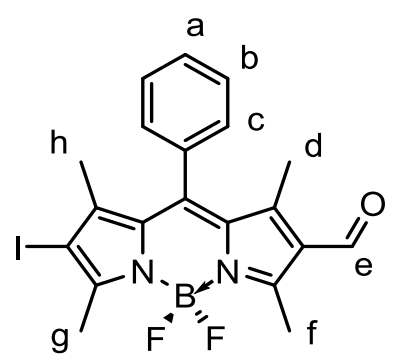

3.43a: BODIPY 3.42a ( $0.200 \mathrm{~g}, 0568 \mathrm{mmol})$ was dissolved in a minimum amount of DCM and NIS ( $0.141 \mathrm{~g}$, $0.625 \mathrm{mmol}$ ) was added in one portion. The mixture was stirred $12 \mathrm{~h}$ with the flask covered with Al foil. Volatiles were removed in vacuo and the crude material was purified via column chromatography using DCM as the eluent, affording the desired compound as an orange solid $(0.194 \mathrm{~g}, 73 \%) .{ }^{1} \mathrm{H} \mathrm{NMR}(400 \mathrm{MHz}$, $\left.\mathrm{CDCl}_{3}\right) \delta 10.01\left(\mathrm{~s}, 1 \mathrm{H}, \mathrm{H}_{\mathrm{e}}\right), 7.58-7.50\left(\mathrm{~m}, 3 \mathrm{H}, \mathrm{H}_{\mathrm{a}}, \mathrm{H}_{\mathrm{b}}\right), 7.27\left(\mathrm{dd},{ }^{3} \mathrm{~J}=6.4,{ }^{4} \mathrm{~J}=2.8 \mathrm{~Hz}, 2 \mathrm{H}, \mathrm{H}_{\mathrm{c}}\right), 2.83\left(\mathrm{~s}, 3 \mathrm{H}, \mathrm{H}_{\mathrm{f}}\right)$, $2.70\left(\mathrm{~s}, 3 \mathrm{H}, \mathrm{H}_{\mathrm{d}}\right), 1.65\left(\mathrm{~s}, 3 \mathrm{H}, \mathrm{H}_{\mathrm{g}}\right), 1.43\left(\mathrm{~s}, 3 \mathrm{H}, \mathrm{H}_{\mathrm{h}}\right)$. The remainder of the characterization was also consistent with previously reported literature. ${ }^{23}$

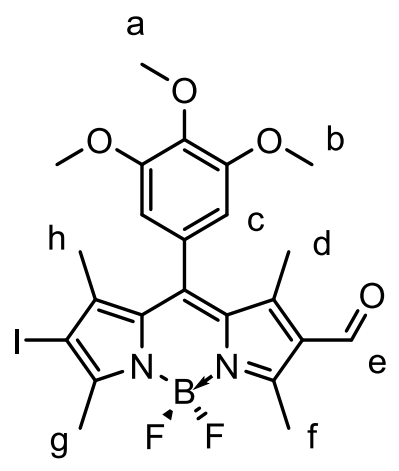

3.43b: BODIPY 3.42b (0.200 g, $0.452 \mathrm{mmol})$ was dissolved in a minimum amount of DCM and NIS (0.122 $\mathrm{g}, 0.543 \mathrm{mmol}$ ) was added in one portion. The mixture was stirred $12 \mathrm{~h}$ with the flask covered with Al foil. Volatiles were removed in vacuo and the crude material was purified via column chromatography using hexanes:DCM 1:4 as the eluent, affording the desired compound as an orange solid $(0.203 \mathrm{~g}, 78 \%) .{ }^{1} \mathrm{H}$ $\operatorname{NMR}\left(400 \mathrm{MHz}, \mathrm{CDCl}_{3}\right) \delta 10.02\left(\mathrm{~s}, 1 \mathrm{H}, \mathrm{H}_{\mathrm{e}}\right), 6.51\left(\mathrm{~s}, 2 \mathrm{H}, \mathrm{H}_{\mathrm{c}}\right), 3.93\left(\mathrm{~s}, 3 \mathrm{H}, \mathrm{H}_{\mathrm{a}}\right), 3.84\left(\mathrm{~s}, 6 \mathrm{H}, \mathrm{H}_{\mathrm{b}}\right), 2.82\left(\mathrm{~s}, 3 \mathrm{H}, \mathrm{H}_{\mathrm{f}}\right)$, $2.61\left(\mathrm{~s}, 3 \mathrm{H}, \mathrm{H}_{\mathrm{d}}\right), 1.81\left(\mathrm{~s}, 3 \mathrm{H}, \mathrm{H}_{\mathrm{g}}\right), 1.59\left(\mathrm{~s}, 3 \mathrm{H}, \mathrm{H}_{\mathrm{h}}\right) .{ }^{13} \mathrm{C}\left\{{ }^{1} \mathrm{H}\right\}$ NMR $(101 \mathrm{MHz}): \delta 185,171.1,160.8,158.0,154.7$, 147.8, 145.1, 143.4, 139.4, 133.4, 129.9, 129.0, 127.0, 104.8, 61.4 60.4, 56.5, 21.0, 17.3, 16.38, 14.2 13.3, 11.8. ${ }^{11} \mathrm{~B} \mathrm{NMR}\left(\mathrm{CDCl}_{3}, 128 \mathrm{MHz}\right): \delta 0.60$ (t). ${ }^{19} \mathrm{~F} \mathrm{NMR}\left(\mathrm{CDCl}_{3}, 376 \mathrm{MHz}\right): \delta-144.5$ (q). HRMS: $\mathrm{m} / \mathrm{z} 568.0832$ $(\mathrm{M}+)$, calculated for $\mathrm{C}_{23} \mathrm{H}_{24} \mathrm{BF}_{2} \mathrm{IN}_{2} \mathrm{O}_{4}: \mathrm{m} / \mathrm{z} 568.0842$ 
<smiles>Cc1c(C=O)c(C)n2c1c(-c1ccc([N+](=O)[O-])cc1)c1n2[P](F)(F)n2c(I)c(I)c(Cl)c2-1</smiles>

3.43c: BODIPY 3.42c $(0.200 \mathrm{~g}, 0503 \mathrm{mmol})$ was dissolved in a minimum amount of DCM and NIS ( $0.136 \mathrm{~g}$, $0.604 \mathrm{mmol}$ ) was added in one portion. The mixture was stirred $12 \mathrm{~h}$ with the flask covered with Al foil. Volatiles were removed in vacuo and the crude material was purified via column chromatography using $\mathrm{DCM}$ as the eluent, affording the desired compound as an orange solid $(0.171 \mathrm{~g}, 65 \%) .{ }^{1} \mathrm{H} \mathrm{NMR}(400 \mathrm{MHz}$, $\left.\mathrm{CDCl}_{3}\right) \delta 10.04\left(\mathrm{~s}, 1 \mathrm{H}, \mathrm{H}_{\mathrm{d}}\right), 8.47\left(\mathrm{~d},{ }^{3} \mathrm{~J}=8.2 \mathrm{~Hz}, 2 \mathrm{H}, \mathrm{H}_{\mathrm{a}}\right), 7.58\left(\mathrm{~d},{ }^{3} \mathrm{~J}=8.3 \mathrm{~Hz}, 2 \mathrm{H}, \mathrm{H}_{\mathrm{b}}\right), 2.85\left(\mathrm{~s}, 3 \mathrm{H}, \mathrm{H}_{\mathrm{e}}\right), 2.74(\mathrm{~s}$, $\left.3 \mathrm{H}, \mathrm{H}_{\mathrm{c}}\right), 1.67\left(\mathrm{~s}, 3 \mathrm{H}, \mathrm{H}_{\mathrm{f}}\right), 1.45\left(\mathrm{~s}, 3 \mathrm{H}, \mathrm{H}_{\mathrm{g}}\right) .{ }^{13} \mathrm{C}\left\{{ }^{1} \mathrm{H}\right\} \mathrm{NMR}\left(101 \mathrm{MHz}, \mathrm{CDCl}_{3}\right) \delta 185.47(\mathrm{~s}), 162.12(\mathrm{~s}), 158.81(\mathrm{~s})$, 148.80 (s), 147.10 (s), 144.52 (s), 140.87 (s), 140.21, 132.80, 129.56, 129.29, 127.26, 124.81, 77.33, 77.01, 76.70, 17.71, 16.55, 13.29, 12.20. ${ }^{11} \mathrm{~B} N M R\left(128 \mathrm{MHz}, \mathrm{CDCl}_{3}\right) \delta 0.82-0.34(\mathrm{t}, J=31.4 \mathrm{~Hz}) .{ }^{19} \mathrm{~F} \mathrm{NMR}(376$ $\left.\mathrm{MHz}, \mathrm{CDCl}_{3}\right) \delta$-144.15- -144.36 (q). HRMS: $\mathrm{m} / \mathrm{z} 523.0386(\mathrm{M}+)$, calculated for $\mathrm{C}_{35} \mathrm{H}_{33} \mathrm{BF}_{2} \mathrm{FeN}_{2} \mathrm{O}_{4}: \mathrm{m} / \mathrm{z}$ 523.0376

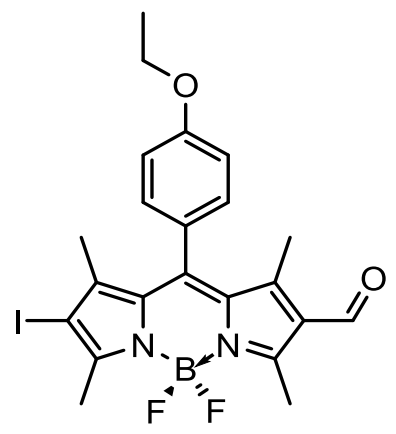

3.43d: Refer to Jennifer Huynh thesis (2017) for full characterization

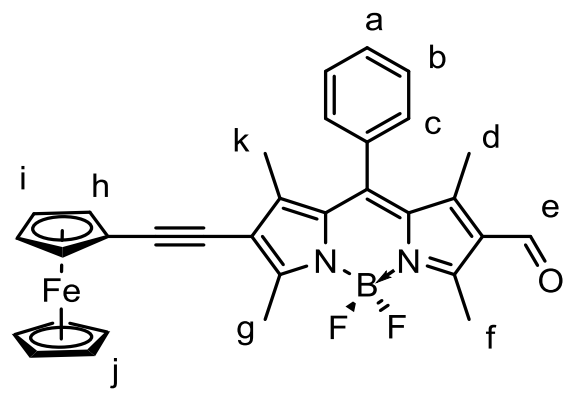


3.44a: In a $100 \mathrm{~mL}$ schlenk flask under nitrogen, BODIPY 3.43a (0.100 g, $0.209 \mathrm{mmol})$ and ethynyl ferrocene $(0.066 \mathrm{~g}, 0.314 \mathrm{mmol}$ ) was were solubilized in $25 \mathrm{~mL}$ of THF:DiPEA 3:2 and the solution was degassed for 30 minutes. $\mathrm{Pd}(\mathrm{PPh} 3)_{2} \mathrm{Cl}_{2}(0.022 \mathrm{~g}, 0.031 \mathrm{mmol})$, and $\mathrm{Cul}(0.005 \mathrm{~g}, 0.021 \mathrm{mmol})$ added and the mixture was stirred $35{ }^{\circ} \mathrm{C}$ for $12 \mathrm{~h}$. After the reaction was complete, the solvents were removed in vacuo and the crude product was purified via column chromatography using hexane:EtOAc 8:2 as the eluent, affording the desired product as (0.044 g, 38\%). ${ }^{1} \mathrm{H}$ NMR (400 MHz, CDCl $) \delta 10.02\left(\mathrm{~s}, 1 \mathrm{H}, \mathrm{He}_{\mathrm{e}}\right), 7.60$ $-7.51\left(\mathrm{~m}, 3 \mathrm{H}, \mathrm{H}_{\mathrm{a}}, \mathrm{H}_{\mathrm{b}}\right), 7.34-7.28\left(\mathrm{~m}, 2 \mathrm{H}, \mathrm{H}_{\mathrm{c}}\right), 4.45\left(\mathrm{~s}, 2 \mathrm{H}, \mathrm{H}_{\mathrm{h}}\right), 4.25\left(\mathrm{~s}, 2 \mathrm{H}, \mathrm{H}_{\mathrm{i}}\right), 4.19\left(\mathrm{~m}, 5 \mathrm{H}, \mathrm{H}_{\mathrm{j}}\right), 2.84(\mathrm{~s}, 3 \mathrm{H}$, $\left.\mathrm{H}_{\mathrm{f}}\right), 2.74\left(\mathrm{~s}, 3 \mathrm{H}, \mathrm{H}_{\mathrm{d}}\right), 1.68\left(\mathrm{~s}, 3 \mathrm{H}, \mathrm{H}_{\mathrm{g}}\right), 1.52\left(\mathrm{~m}, 3 \mathrm{H}, \mathrm{H}_{\mathrm{k}}\right) .{ }^{13} \mathrm{C}\left\{{ }^{1} \mathrm{H}\right\} \mathrm{NMR}\left(101 \mathrm{MHz}, \mathrm{CDCl}_{3}\right) \delta$ 185.78, 162.43, 157.41, 146.11, 143.88, 134.12, 129.62, 127.72, 97.00, 77.33, 77.02, 76.70, 71.52, 70.05, 69.09, 64.69, 13.83, 11.70. ${ }^{11} \mathrm{~B} \mathrm{NMR}\left(128 \mathrm{MHz}, \mathrm{CDCl}_{3}\right) \delta 0.66(\mathrm{t}) .{ }^{19} \mathrm{~F} \mathrm{NMR}\left(376 \mathrm{MHz}, \mathrm{CDCl}_{3}\right) \delta$-144.71- 144.97 (q). HRMS: $\mathrm{m} / \mathrm{z} 560.1545(\mathrm{M}+)$, calculated for $\mathrm{C}_{32} \mathrm{H}_{27} \mathrm{BF}_{2} \mathrm{FeN}_{2} \mathrm{O}: \mathrm{m} / \mathrm{z} 560.1534$

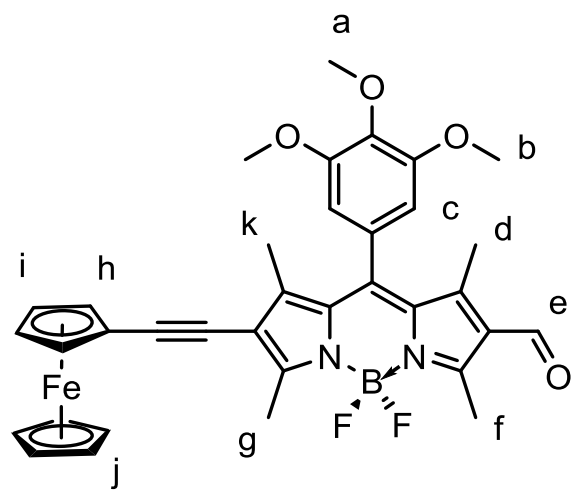

3.4b: In a $100 \mathrm{~mL}$ schlenk flask under nitrogen, BODIPY 3.43b (0.100 g, $0.191 \mathrm{mmol})$ and ethynyl ferrocene $(0.048 \mathrm{~g}, 0.229 \mathrm{mmol})$ was were solubilized in $25 \mathrm{~mL}$ of THF:DiPEA 3:2 and the solution was degassed for 30 minutes. $\mathrm{Pd}(\mathrm{PPh} 3)_{2} \mathrm{Cl}_{2}(0.020 \mathrm{~g}, 0.029 \mathrm{mmol})$, and $\mathrm{Cul}(0.004 \mathrm{~g}, 0.019 \mathrm{mmol})$ added and the mixture was stirred $35{ }^{\circ} \mathrm{C}$ for $12 \mathrm{~h}$. After the reaction was complete, the solvents were removed in vacuo and the crude product was purified via column chromatography using hexane:EtOAc 9:1 as the eluent, affording the desired product as (0.105 g, 84\%). ${ }^{1} \mathrm{H}$ NMR $\left(400 \mathrm{MHz}, \mathrm{CDCl}_{3}\right) \delta 10.07\left(\mathrm{~s}, 1 \mathrm{H}, \mathrm{H}_{\mathrm{e}}\right), 6.55(\mathrm{~s}, 2 \mathrm{H}, \mathrm{H} \mathrm{c}), 4.48(\mathrm{~s}$, $\left.2 \mathrm{H}, \mathrm{H}_{\mathrm{h}}\right), 4.28\left(\mathrm{~s}, 2 \mathrm{H}, \mathrm{H}_{\mathrm{i}}\right), 4.23\left(\mathrm{~s}, 5 \mathrm{H}, \mathrm{H}_{\mathrm{j}}\right), 3.97\left(\mathrm{~s}, 3 \mathrm{H}, \mathrm{H}_{\mathrm{a}}\right), 3.88\left(\mathrm{~s}, 6 \mathrm{H}, \mathrm{H}_{\mathrm{b}}\right), 2.86\left(\mathrm{~s}, 3 \mathrm{H}, \mathrm{H}_{\mathrm{f}}\right), 2.76\left(\mathrm{~s}, 3 \mathrm{H}, \mathrm{H}_{\mathrm{d}}\right)$, $1.86\left(\mathrm{~s}, 3 \mathrm{H}, \mathrm{H}_{\mathrm{g}}\right), 1.72\left(\mathrm{~s}, 3 \mathrm{H}, \mathrm{H}_{\mathrm{k}}\right) .{ }^{13} \mathrm{C}\left\{{ }^{1} \mathrm{H}\right\} \mathrm{NMR}\left(101 \mathrm{MHz}, \mathrm{CDCl}_{3}\right) \delta 185.80,162.57,157.46,154.56,145.94$, 143.75, 143.43, 139.26, 133.02, 130.31, 129.04, 126.61, 104.90, 97.20, 71.53, 70.07, 69.14, 64.61, 61.43, $56.47,14.09,13.81,13.16,11.72 .{ }^{11} \mathrm{~B}$ NMR $\left(128 \mathrm{MHz}, \mathrm{CDCl}_{3}\right) \delta 0.64(\mathrm{t}) .{ }^{19} \mathrm{~F} \mathrm{NMR}\left(376 \mathrm{MHz}, \mathrm{CDCl}_{3}\right) \delta-144.75$ - -145.15 (q). HRMS: $\mathrm{m} / \mathrm{z} 650.1852(\mathrm{M}+)$, calculated for $\mathrm{C}_{35} \mathrm{H}_{33} \mathrm{BF}_{2} \mathrm{FeN}_{2} \mathrm{O}_{4}: \mathrm{m} / \mathrm{z} 650.1851$ 


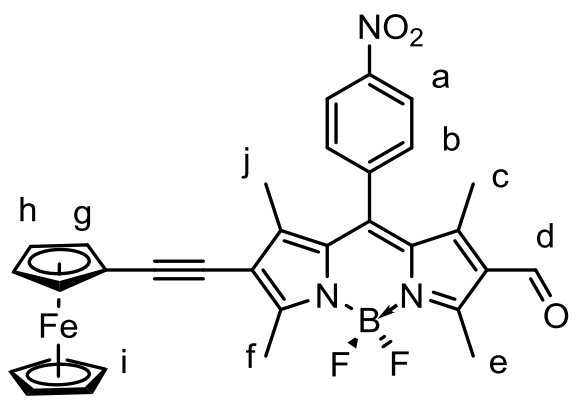

3.4c: In a $100 \mathrm{~mL}$ schlenk flask under nitrogen, BODIPY 3.43c (0.100 g, $0.191 \mathrm{mmol})$ and ethynyl ferrocene $(0.048 \mathrm{~g}, 0.229 \mathrm{mmol})$ was were solubilized in $25 \mathrm{~mL}$ of THF:DiPEA 3:2 and the solution was degassed for 30 minutes. $\mathrm{Pd}\left(\mathrm{PPh}_{3}\right)_{2} \mathrm{Cl}_{2}(0.020 \mathrm{~g}, 0.029 \mathrm{mmol})$, and $\mathrm{Cul}(0.004 \mathrm{~g}, 0.019 \mathrm{mmol})$ added and the mixture was stirred $35{ }^{\circ} \mathrm{C}$ for $12 \mathrm{~h}$. After the reaction was complete, the solvents were removed in vacuo and the crude product was purified via column chromatography using hexane:EtOAc 8:2 as the eluent, affording the desired product as (0.072 mg, 63\%). ${ }^{1} \mathrm{H}$ NMR $\left(400 \mathrm{MHz}, \mathrm{CDCl}_{3}\right) \delta 10.05\left(\mathrm{~s}, 1 \mathrm{H}, \mathrm{H}_{\mathrm{d}}\right), 8.48\left(\mathrm{~d},{ }^{3} \mathrm{~J}=8.5 \mathrm{~Hz}, 2 \mathrm{H}\right.$, $\left.\mathrm{H}_{\mathrm{a}}\right), 7.59\left(\mathrm{~d},{ }^{3} \mathrm{~J}=8.3 \mathrm{~Hz}, 2 \mathrm{H}, \mathrm{H}_{\mathrm{b}}\right), 4.49\left(\mathrm{~d},{ }^{3} \mathrm{~J}=1.7 \mathrm{~Hz}, 2 \mathrm{H}, \mathrm{H}_{\mathrm{g}}\right), 4.29\left(\mathrm{~d}, 3^{3} \mathrm{~J}=1.7 \mathrm{~Hz}, 2 \mathrm{H}, \mathrm{H}_{\mathrm{h}}\right), 4.22\left(\mathrm{~s}, 5 \mathrm{H}, \mathrm{H}_{\mathrm{i}}\right)$, $2.87\left(\mathrm{~s}, 3 \mathrm{H}, \mathrm{H}_{\mathrm{e}}\right), 2.78\left(\mathrm{~s}, 3 \mathrm{H}, \mathrm{H}_{\mathrm{c}}\right), 1.70\left(\mathrm{~s}, 3 \mathrm{H}, \mathrm{H}_{\mathrm{f}}\right), 1.53\left(\mathrm{~s}, 3 \mathrm{H}, \mathrm{H}_{\mathrm{j}}\right) .{ }^{13} \mathrm{C}\left\{{ }^{1} \mathrm{H}\right\} \mathrm{NMR}\left(101 \mathrm{MHz}, \mathrm{CDCl}_{3}\right) \delta$ 185.57, $163.83,158.20,148.76,144.97,143.09,140.89,140.26,129.47,126.85,124.72,98.09,71.58,70.09$, 69.26, 29.69, 14.17, 12.13. ${ }^{11} \mathrm{~B}$ NMR (128 MHz, CDCl $) \delta 0.60$ (t). ${ }^{19} \mathrm{~F} \mathrm{NMR}\left(376 \mathrm{MHz}_{3} \mathrm{CDCl}_{3}\right) \delta-144.60$ - 144.84 (q)

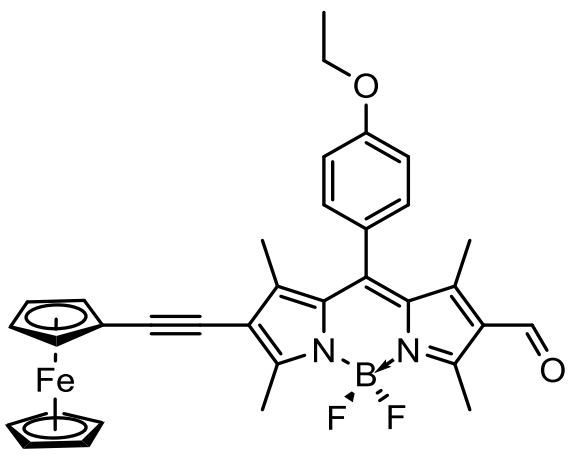

3.4d: Refer to Jennifer Huynh thesis (2017) for full characterization 


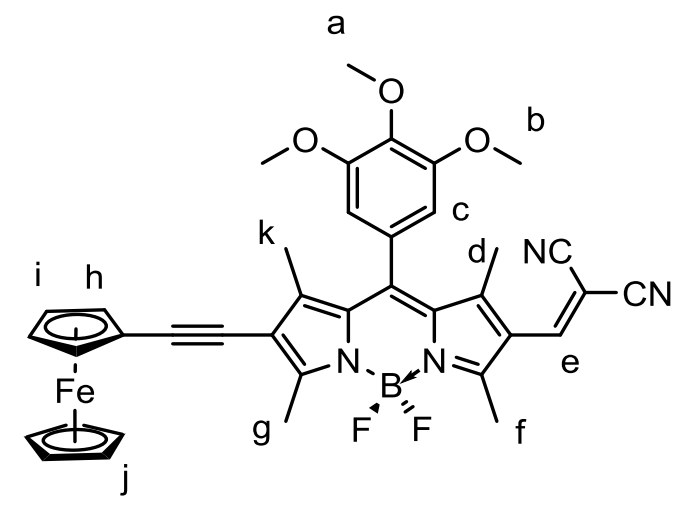

3.5b: $3.44 b$ ( $0.050 \mathrm{~g}, 0.077 \mathrm{mmol}$ ) was dissolved in minimal amount of $\mathrm{CHCl}_{3} 1: 1$ and malononitrile $(0.011$ $\mathrm{mg}, 0.173 \mathrm{mmol}$ ) was added, followed by $\mathrm{NEt}_{3}$ ( 2 drops). The mixture was refluxed $12 \mathrm{~h}$ and after being cooled at room temperature, organic layer was washed with $\mathrm{HCl} 1 \mathrm{~N}(3 \times 25 \mathrm{~mL})$. The organic layer was dried over $\mathrm{MgSO}_{4}$, filtered and volatiles were removed in vacuo. The crude product was purified via column chromatography using DCM as the eluent affording a dark purple solid ( $0.018 \mathrm{~g}, 33 \%) .{ }^{1} \mathrm{H}$ NMR $\left(400 \mathrm{MHz}, \mathrm{CDCl}_{3}\right) \delta 7.73\left(\mathrm{~s}, 1 \mathrm{H}, \mathrm{H}_{\mathrm{e}}\right), 6.55\left(\mathrm{~s}, 2 \mathrm{H}, \mathrm{H}_{\mathrm{c}}\right), 4.49\left(\mathrm{~s}, 2 \mathrm{H}, \mathrm{H}_{\mathrm{h}}\right), 4.29\left(\mathrm{~s}, 2 \mathrm{H}, \mathrm{H}_{\mathrm{h}}\right), 4.23\left(\mathrm{~s}, 5 \mathrm{H}, \mathrm{H}_{\mathrm{j}}\right), 3.97$ $\left(\mathrm{s}, 3 \mathrm{H}, \mathrm{H}_{\mathrm{a}}\right), 3.88\left(\mathrm{~s}, 6 \mathrm{H}, \mathrm{H}_{\mathrm{b}}\right), 2.77\left(\mathrm{~s}, 3 \mathrm{H}, \mathrm{H}_{\mathrm{f}}\right), 2.68\left(\mathrm{~s}, 3 \mathrm{H}, \mathrm{H}_{\mathrm{d}}\right), 1.73\left(\mathrm{~s}, 3 \mathrm{H}, \mathrm{H}_{\mathrm{g}}\right), 1.65\left(\mathrm{~s}, 3 \mathrm{H}, \mathrm{H}_{\mathrm{k}}\right) .{ }^{13} \mathrm{C}\left\{{ }^{1} \mathrm{H}\right\} \mathrm{NMR}$ $\left(101 \mathrm{MHz}, \mathrm{CDCl}_{3}\right) \delta 163.97,154.66,153.19,142.73,139.89,139.37,128.67,114.22,113.14,104.81,97.91$, $82.91,71.58,70.10,69.25,61.47,56.50,14.23,13.99 .{ }^{11} \mathrm{~B} \mathrm{NMR}\left(128 \mathrm{MHz}, \mathrm{CDCl}_{3}\right) \delta 0.55(\mathrm{t}) .{ }^{19} \mathrm{~F} \mathrm{NMR}(376$ $\left.\mathrm{MHz}, \mathrm{CDCl}_{3}\right) \delta-145.44--145.69$ (q). HRMS: $\mathrm{m} / \mathrm{z} 698.1979(\mathrm{M}+)$, calculated for $\mathrm{C}_{38} \mathrm{H}_{33} \mathrm{BF}_{2} \mathrm{FeN}_{4} \mathrm{O}_{3}: \mathrm{m} / \mathrm{z}$ 698.1963

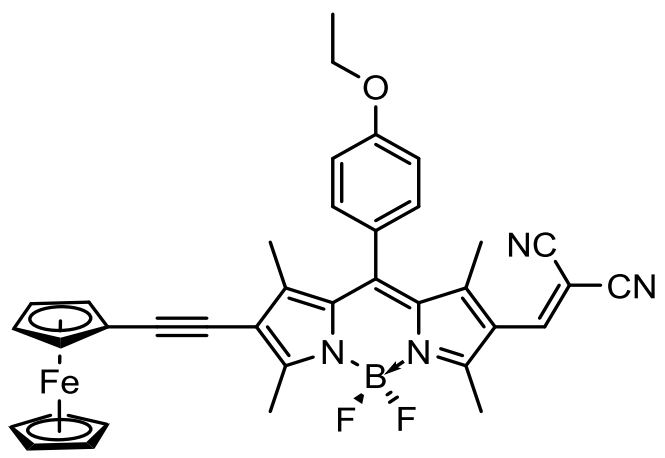

3.5d: Refer to Jennifer Huynh thesis (2017) for full characterization 
a

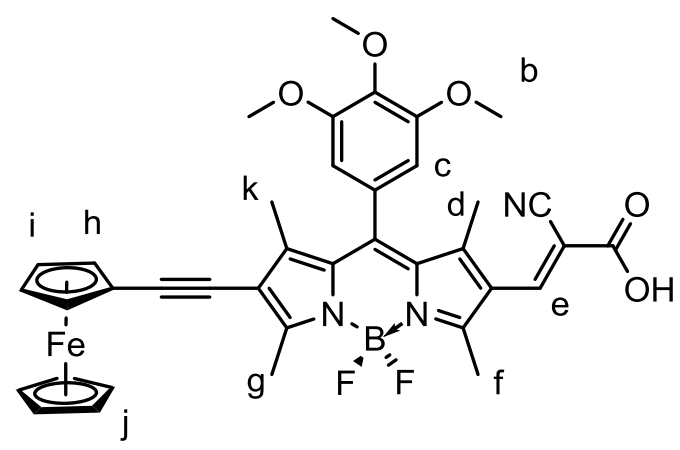

3.6b: 3.44b ( $0.060 \mathrm{~g}, 0.092 \mathrm{mmol}$ ) was dissolved in minimal amount of $\mathrm{CHCl}_{3} 1: 1$ and cyanoacetic acid ( $0.016 \mathrm{mg}, 0.185 \mathrm{mmol}$ ) was added, followed by piperidine $(2 \mu \mathrm{L}, 0.02 \mathrm{mmol})$. The mixture was refluxed $12 \mathrm{~h}$ and after being cooled at room temperature, organic layer was washed with $\mathrm{HCl} 1 \mathrm{~N}(3 \times 25 \mathrm{~mL})$. The organic layer was dried over $\mathrm{MgSO}_{4}$, filtered and volatiles were removed in vacuo. The crude product was recrystallized from hexanes:EtOAc 10:1, affording a dark purple solid( $0.037 \mathrm{~g}, 55 \%) .{ }^{1} \mathrm{H} \mathrm{NMR}(400 \mathrm{MHz}$, $\left.\mathrm{CDCl}_{3}\right) \delta 8.25\left(\mathrm{~s}, 1 \mathrm{H}, \mathrm{H}_{\mathrm{e}}\right), 6.56\left(\mathrm{~s}, 2 \mathrm{H}, \mathrm{H}_{\mathrm{c}}\right), 4.62\left(\mathrm{~m}, 2 \mathrm{H}, \mathrm{H}_{\mathrm{h}}\right), 4.48\left(\mathrm{~s}, 2 \mathrm{H}, \mathrm{H}_{\mathrm{i}}\right), 4.28\left(\mathrm{~s}, 5 \mathrm{H}, \mathrm{H}_{\mathrm{j}}\right), 3.97\left(\mathrm{~s}, 3 \mathrm{H}, \mathrm{H}_{\mathrm{a}}\right)$, $3.88\left(\mathrm{~s}, 6 \mathrm{H}, \mathrm{H}_{\mathrm{b}}\right), 2.75\left(\mathrm{~s}, 3 \mathrm{H}, \mathrm{H}_{\mathrm{f}}\right), 2.69\left(\mathrm{~s}, 3 \mathrm{H}, \mathrm{H}_{\mathrm{d}}\right), 1.72\left(\mathrm{~s}, 3 \mathrm{H}, \mathrm{H}_{\mathrm{g}}\right), 1.67\left(\mathrm{~s}, 3 \mathrm{H}, \mathrm{H}_{\mathrm{k}}\right) .{ }^{11} \mathrm{~B} \mathrm{NMR}\left(128 \mathrm{MHz}, \mathrm{CDCl}_{3}\right)$ $\delta 0.63(\mathrm{t}) . \delta-145.51--145.76(\mathrm{q})$. 


\section{Chapter 4: Towards BODIPY-Anthracene Cyclophanes: Tuneable HOMO-LUMO gap}

\subsection{Introduction}

Cyclophanes are ubiquitous in literature due to the defined cavity size and are efficient in encapsulating and stabilising guest molecules, with unique donor/acceptor motifs through intramolecular/intermolecular interactions. This review highlights the use of various interesting pyrrolic cofacial dimers and cyclophane architectures

\subsection{1 $\pi-\pi$ Interaction}

$\pi$-stacking occurs as a result of intermolecular or intramolecular forces between unique aromatic systems. These interactive forces come from $\pi$ orbital mixing between aromatic ring systems and electrostatic polarization of $\pi$ electron density. ${ }^{47}$ Hunter and Sanders first described this interaction and have since established criteria for explaining aromatic-aromatic interactions. ${ }^{47-48}$ Hunter and Sanders argue that $\pi$ electron density in most aromatic systems produces a quadrupole moment ${ }^{1,49}$ resulting in electrostatic interactions between the various aromatic ring systems; as a result electron rich areas and partially positive area's build in predictable ways along the periphery of the conjugated system. For benzene and other electron rich aromatics, the quadrupole moment consists of partial positive charges that can result both above and below the plane of the aromatic ring. For electron deficient aromatics, such as naphthalene tetracarboxylic acid diimide, the addition of electron withdrawing groups pulls electronic density from the aromatic core to produce an electrostatic redistribution seen in Figure 4.1. ${ }^{47}$ 


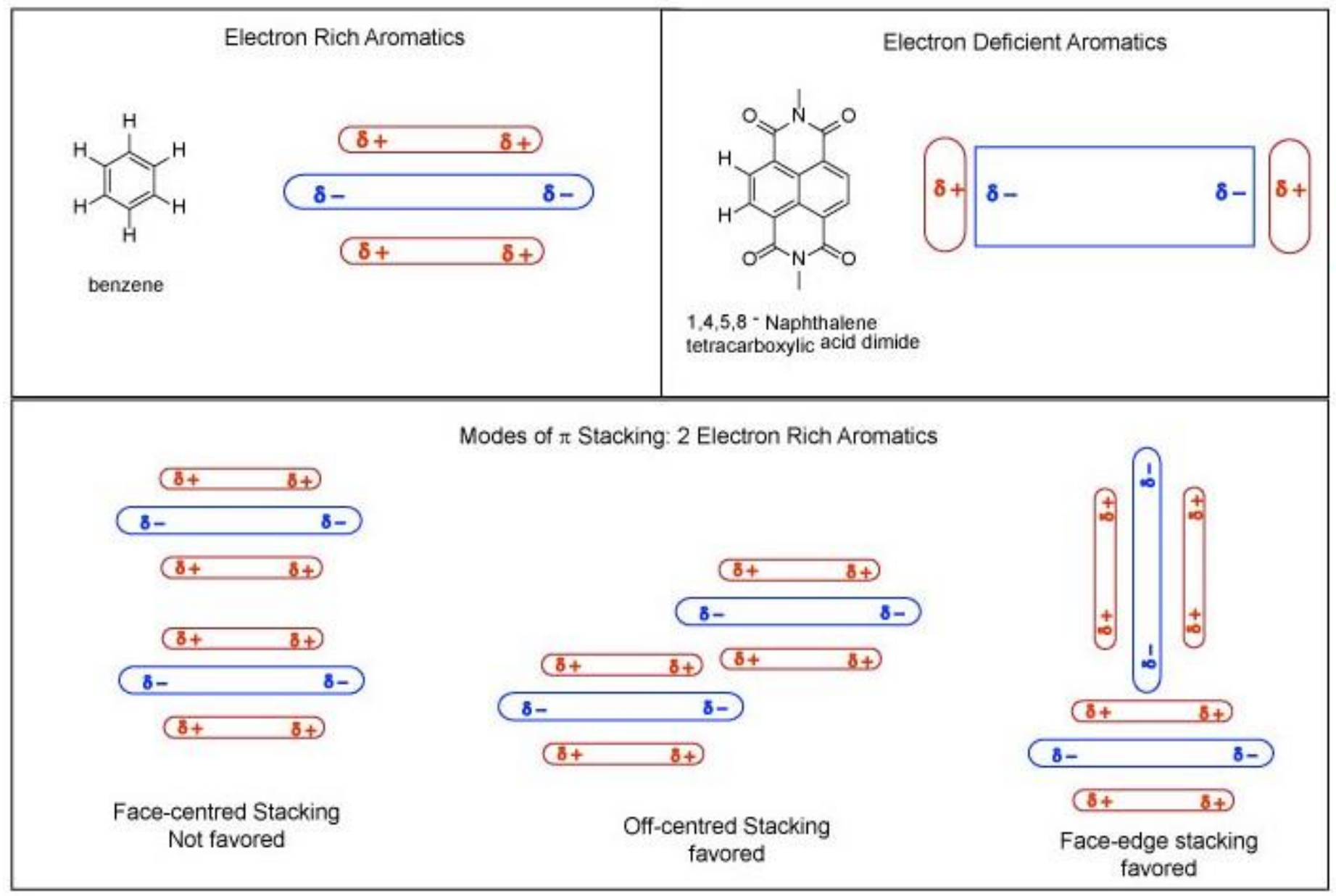

Figure 4.1: Electrostatic view of aromatic-aromatic interaction. ${ }^{47}$

The modes of $\pi$ stacking between aromatics are as follows; face-centred parallel stacking, face to edge stacking and off-centered parallel stacking (Figure 4.1). ${ }^{47}$ For $\pi$-stacking between electron rich aromatics, both off-centered and face-edge stacking is favoured, however, face-face stacking does not occur due to the repulsion of the quadrupole moment of the two aromatics. ${ }^{50} \pi$-stacking between electron rich aromatics and electron deficient aromatics allows for face centred stacking (Figure 4.2). ${ }^{1,4}$ This $\pi$-stacking leads to a "donor-acceptor" motif due the polarization of the $\pi$ orbital mixing. The electron rich aromatic acts donor and electron deficient acts the acceptor in this case. This acceptor-donor relationship can often be observed in solution and the solid state and has been exploited extensively in supramolecular chemistry. 


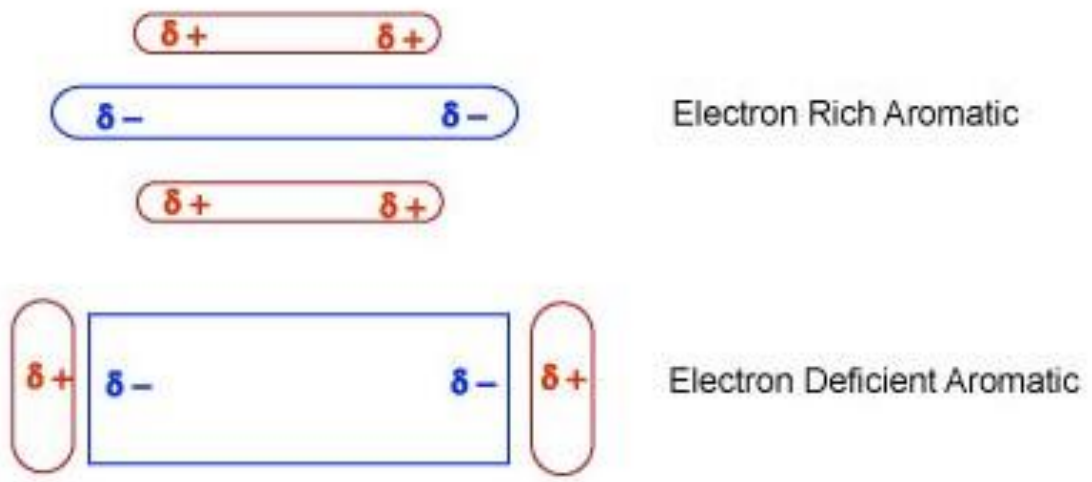

Face centred stacking favoured

Figure 4. 2: Electrostatic view of electron rich/electron deficient aromatic-aromatic interaction

To understand intramolecular $\pi$-stacking within a molecule and the effects it has on the physiochemical properties, a review of supramolecular $\pi$-stacking will be discussed further.

\subsubsection{Cofacial dimers}

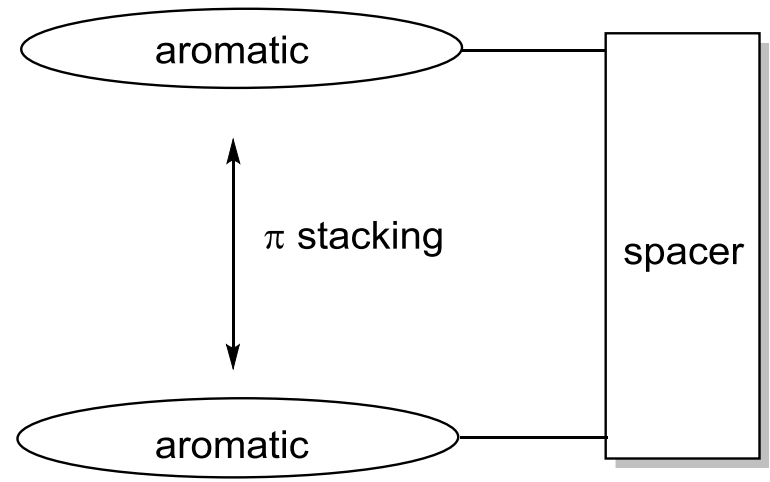

face-centred stacking allowed

Figure 4. 3: Cofacial dimers motif 
An example of face-centred intramolecular $\pi$-stacking system is the cofacial dimer (Figure 4.3$).{ }^{51-52}$ Cofacial dimers consist of rigid spacer allowing the aromatic planes to stack in a face-centred manner, near the Van der Waals radius, as such, the distance between the aromatic dimer must be close enough for $\pi$ orbital stacking to occur. Steric moieties are sometimes added to both the spacer or the aromatic dimer to force face centred stacking. Cofacial dimers are of great interest because of their ubiquitous presence in Nature and their importance in photosynthetic processes. ${ }^{53}$ Cofacial dimers are part of antenna complexes within bacteriochlorophyll involved in electron transfer processes as "special pairs" .54 Cofacial dimers within these antenna complexes are usually porphyrin based and have been extensively studied in the literature. ${ }^{53}$

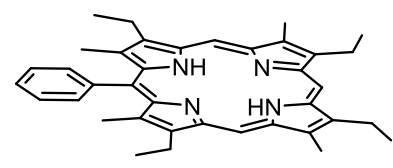

$\mathrm{Zn}\left(\mathrm{Et}_{4} \mathrm{Me}_{4} \mathrm{PhP}\right)$

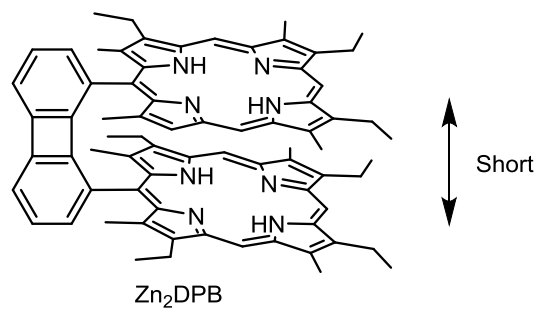

Figure 4.4: Porphyrin cofacial dimers motifs and the different spacers and the reference porphyrin monomer..$^{53}$

Takai et al. studied electron transfer properties within porphyrin cofacial dimers ( $\left.Z \mathrm{n}_{2} \mathrm{DPB}\right)$ facilitated by $\pi-\pi$ interactions (Figure 4.4). ${ }^{53}$ It was observed that, delocalization of $\pi$-electrons between discrete $\pi$ systems in aromatic dimers allows electron transfer from one ring to the other to occur. Strong $\pi-\pi$ interactions; especially in aromatic radical cation dimers were observed once electron transfer had occurred. ${ }^{55}$

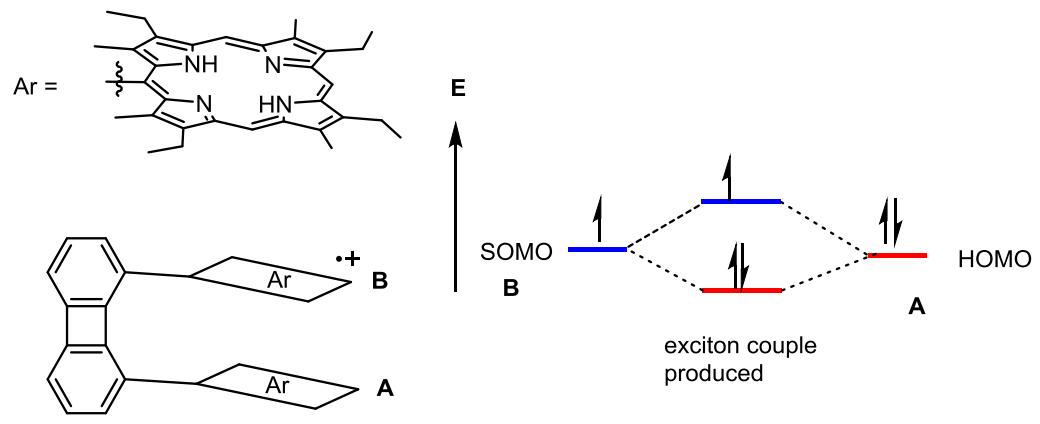

Figure 4.5: Porphyrin cofacial dimers oxidation cause the formation of an exciton 
In order to study $\pi$-dimer radical cations (Figure 4.5 ), the oxidation of $\mathrm{Zn}_{2} \mathrm{DPB}$ was done using $\left[\mathrm{Ru}(\mathrm{bpy})_{3}\right]^{3+}$ in $\mathrm{MeCN}$ to form the $\pi$-dimer radical cation. ${ }^{53} \mathrm{Vis} / \mathrm{NIR}$ spectroscopy and cyclic voltammetry, revealed that the radical cation was delocalized through both rings via $\pi$ orbital mixing to form a new energy level from the SOMO of the oxidized ring and the HOMO of the neutral ring; similar to exciton coupling. ${ }^{7,56}$ This exciton coupling can be confirmed by appearance of an intervalence charge-transfer transition band (IVCT) in the electronic spectra around $2950 \mathrm{~nm}$ due to the small energy gap. Cyclic voltammetry also showed that an electron from this dimer system is easier to remove compared to the monomeric parent due to the stabilization effects from $\pi$-stacking.

Benniston et al. presented the synthesis of the first cofacial BODIPY dimers (Figure 4.6). ${ }^{57}$ The cofacial dimers were designed to study BODIPY exciton coupling in cofacial motifs and how these interactions will affect/enhance their optical \& electrochemical properties.
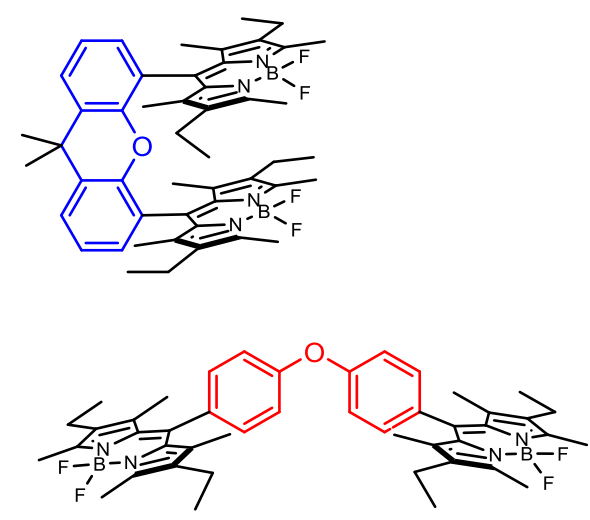

Figure 4.6: BODIPY Cofacial Dimers

In BODIPY cofacial dimer, a 9,9-dimethylxanthene spacer was used to induce a cofacial conformation. ${ }^{11}$ 1,4-phenoxybenzene spacer serves as a benchmark where the BODIPYs are too far apart to interact. Electrochemical studies revealed that 1,4-phenoxybenzene BODIPY dimer behave like isolated BODIPYs, with one reversible oxidation and one reversible reduction. However for 9,9-dimethylxanthene BODIPY dimer, splitting of both the oxidation and reduction waves was observed (two reversible oxidation and reduction waves). The $\pi$ system overlap in this system allows for electron delocalization between both BODIPYs, promoting oxidation due to the stabilization of the $\pi$ dimer radical cation as in the porphyrin system of Takai et al. ${ }^{7}$ However, the opposite trend is observed for reductions, where the strong $\pi$ interactions make it difficult to add an electron into the $\pi$ system due to electron repulsion. Spectroelectrochemical studies suggested that significant exciton coupling was present as evidenced by the intervalence charge-transfer transition in the absorption profile. 


\subsubsection{Cyclophanes}

Sandwich molecules or cyclophanes are a class of compounds that consist of a strained ring system Figure 4.7. ${ }^{58-61} \mathrm{~A}$ simple cyclophane consists of two benzene rings that are bound by at least two bridges in para positions or meta positions. These two regioisomers are called paracyclophane (a) and metacyclophane (b). The minimum number of atoms in the bridge of these structures is two, any less would be too strained and not possible to synthesize. $\pi$-stacking in cyclophanes with novel structures has attracted attention in the applications of host-guest chemistry, molecular motors, and nanostructure devices. ${ }^{58-61}$

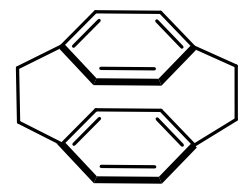

a

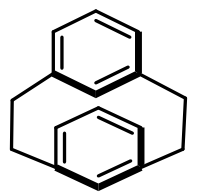

b

Figure 4.7: paracyclophane and metacyclophane

The cyclophane HOMO-LUMO gap is heavily affected by the presence of the intramolecular $\pi-\pi$ interactions. ${ }^{1}$ According to Hunter and Sanders studies, the overlap of the $\pi$ orbitals of both ring systems should result in $\pi$ orbital mixing between both aromatic molecules within a cyclophane. ${ }^{1}$ This $\pi$ orbital mixing should create new charge transfer absorption bands indicative of the electron density transfer from the HOMO state (the electron rich aromatic) to the LUMO state (electron deficient aromatic) via $\pi$ orbital mixing. This transition should be lower in energy than the charge redistribution within the aromatic ring (Figure 4.8) resulting in aromatic cyclophanes having a smaller (and tunable!) HOMO-LUMO gap compared to the HOMO-LUMO gaps of the individual aromatics. Evidence of these phenomena are seen in multi-aromatic systems (one electron rich and one electron deficient) that absorb light in the UV region. When confined together through $\pi$ stacking, the absorption red shifts into the visible region. This absorption in the visible region corresponds to the charge transfer caused from strong intramolecular $\pi$ $\pi$ interactions. 


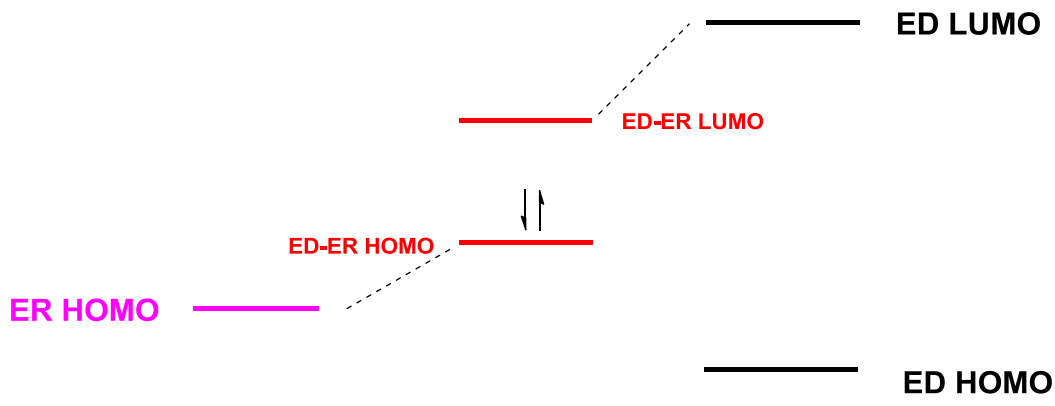

Figure 4.8: $\mathrm{HOMO}-\mathrm{LUMO}$ gap of cyclophane: $E D=$ electron deficient aromatic, $E R$ =electron rich aromatic

Organic macrocycles containing cyclophanes have been explored in various host-guest chemistry motifs. ${ }^{62}$ Host-guest chemistry involves the complexation of small molecules within the cavities of macrocycles through non-covalent interactions. Host-guest chemistry has been incorporated in applications such as small molecule detection, ${ }^{63}$ supramolecular catalysis and energy transfer. ${ }^{64}$

\subsubsection{Cyclophanes: Host-guest chemistry}

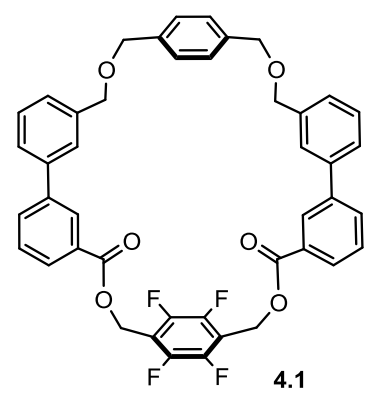

Guests

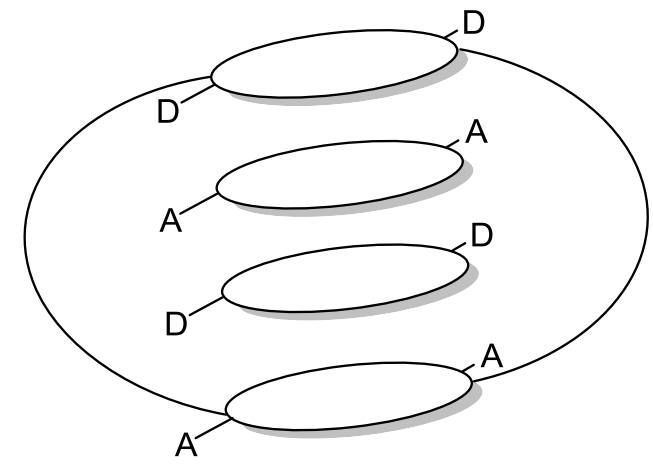

Host-guest interaction

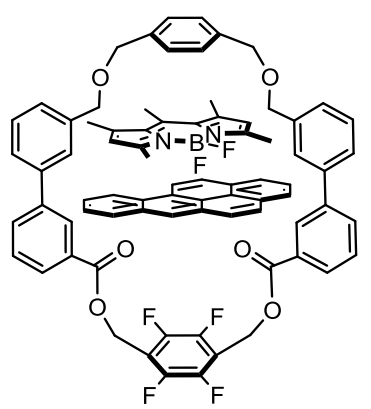

4.1- $G_{1} G_{2}$<smiles></smiles>

$\mathrm{G}_{1}$

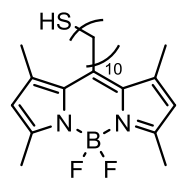

$\mathrm{G}_{2}$

Figure 4.9: Macrocycles and guest molecules

Radaram et al. prepared organic cyclophane to test non-covalent energy transfer between the host \& guest molecules through intermolecular $\pi$ stacking of hosts and guests (Figure 4.9). ${ }^{62} \mathbf{4 . 1}$ was used to test 
energy transfer between an electron rich donor $\left(G_{1}\right)$ and a BODIPY fluorophore energy acceptor $\left(G_{2}\right)$. 4.1 possess electronic dissymmetry from the electron deficient fluorinated portion and electron rich components that facilitate $\pi$ stacking interactions between the macrocycle and the guests. The authors discovered that $\mathbf{4 . 1}$ effectively bound polyaromatic $\mathbf{G}_{\mathbf{1}}$ due to the electronic deficient acceptor. Upon addition of the second guest, the 1 host: 2 guest complex formed $4.1-\mathbf{G}_{1} \mathbf{G}_{2}$ (Figure 4.9). ${ }^{62}$ In order to test non-covalent energy transfer between guests, $\mathrm{G}_{2}$ was used as a fluorophore acceptor and $\mathrm{G}_{1}$ was the energy donor; the macrocycle was innocent in energy transfer, but structurally $\mathbf{4 . 1}$ facilitated the energy transfer from the energy donor $G_{1}$ to the $G_{2}$ accepter fluorophore. The energy transfer was confirmed by fluorescence studies through the observed increase in the intensity in the emission from BODIPY $\left(G_{2}\right)$ and the decreased intensity in emission of $\mathrm{G}_{1}$.

\subsubsection{Cyclophanes: Helical modes}

In biological systems, helicity is an important structural feature in DNA and proteins. ${ }^{65}$ In supramolecular chemistry, incorporating helicity has also aided in the development of catalysts for asymmetric synthesis. ${ }^{66}$ Maeda et. al used cyclic bis-bidipyrrin ligands to make Zn-dipyrrin dimer double helices. ${ }^{67}$ The use of two Zn metal centres stabilizes the double helix structure (Figure 4.10). Strapped Zn double helices (4.2a-d) were made using olefin metathesis in order to make a rigid structure promoting exciton transition.

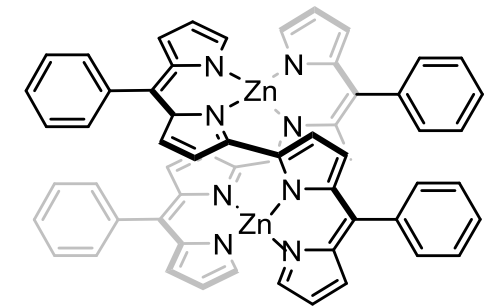

strap-free $\mathrm{Zn}$ double helix

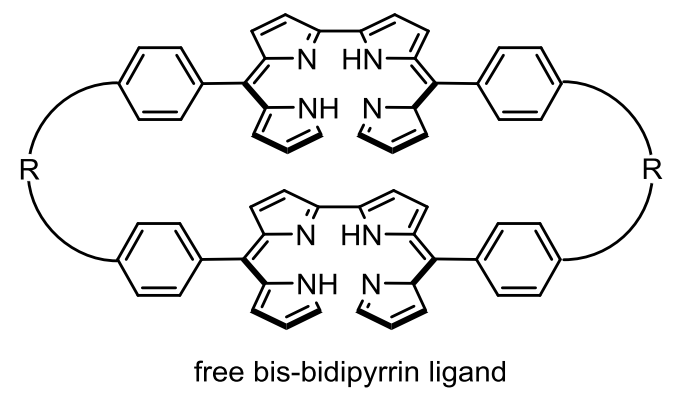

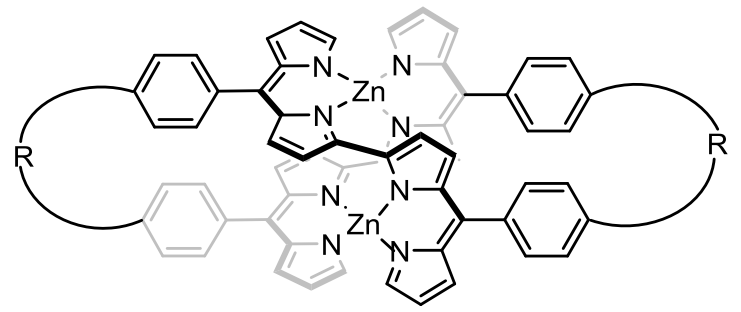

straped Zn double helix

4.2

a $\mathrm{R}=\mathrm{O}\left(\mathrm{CH}_{2}\right) \mathrm{CH}=\mathrm{CH}\left(\mathrm{CH}_{2}\right) \mathrm{O}$ b R $=\mathrm{O}\left(\mathrm{CH}_{2}\right)_{2} \mathrm{CH}=\mathrm{CH}\left(\mathrm{CH}_{2}\right)_{2} \mathrm{O}$ c R $=\mathrm{O}\left(\mathrm{CH}_{2}\right)_{3} \mathrm{CH}=\mathrm{CH}\left(\mathrm{CH}_{2}\right)_{3} \mathrm{O}$ d R $=\mathrm{O}\left(\mathrm{CH}_{2}\right)_{4} \mathrm{CH}=\mathrm{CH}\left(\mathrm{CH}_{2}\right)_{4} \mathrm{O}$

Figure 4.10: Zinc double helices

Computation studies (B3LYP/6-31G) revealed that there are two modes of these $\mathrm{Zn}$ double helices. Short or long modes (Figure 4.11) are possible and this is ultimately affects the distance between the $\mathrm{Zn}$ atoms 
of the dimer. ${ }^{18}$ In the strap-free $\mathrm{Zn}$ double helix, the short mode is preferred; however, for $\mathbf{4 . 2 a - d ~ t h e ~}$ mode of the dimer depends on the length of the bridges. For $\mathbf{4 . 2 a}$, the long mode is more stable compared to $4.2 \mathrm{~d}$ which is stable in the short mode.

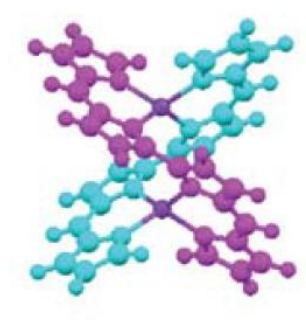

$S$

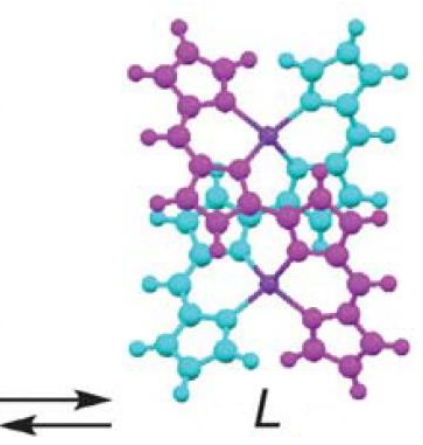

Figure 4.11: Short $(S)$ and long $(L)$ modes of the $\mathrm{Zn}$-dipyrrin double helices

Owing to $\pi$ stacking within the double helix structure, the electronic states of $\mathrm{Zn}$ double helices were determined by Kasha's molecular exciton model. ${ }^{18}$ The $\pi$ stacking creates four electronic transitions between electronic transitions dipoles (Figure 4.12) that interact with each other.

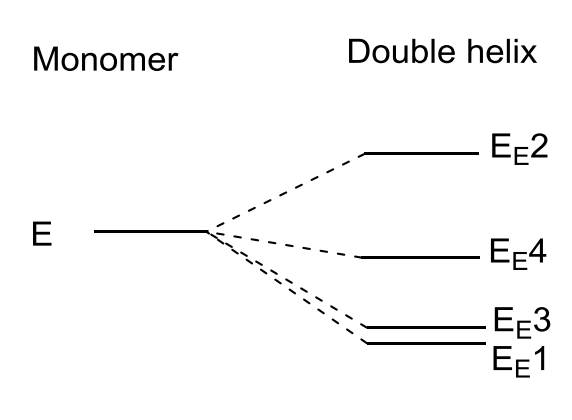

$E_{E} 1$

$E_{E} 2$
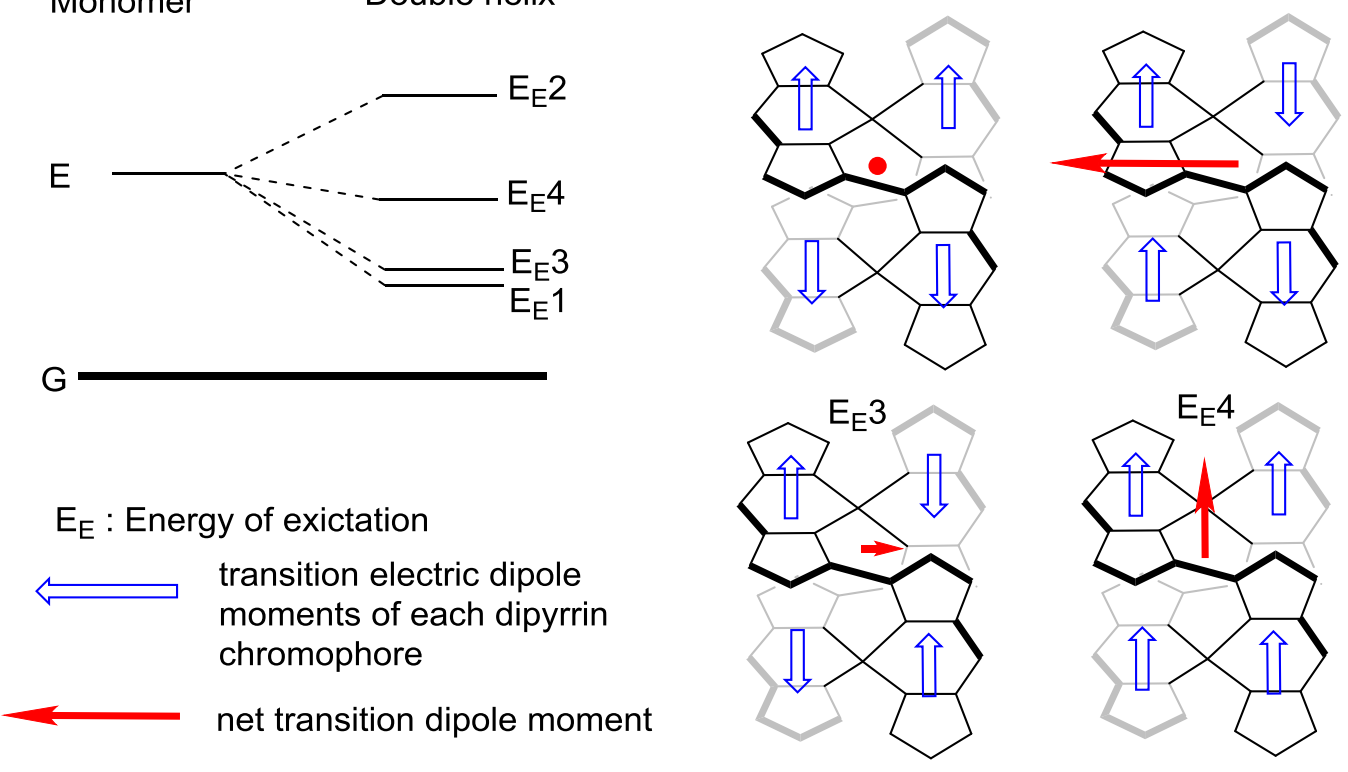

Figure 4.12: Electronic structure of Zn-dipyrrin double helices 
The transition from $\mathrm{G}$ to $\mathrm{E}_{\mathrm{E}}{ }^{1}$ is optically forbidden due to the net cancelation of dipole moment of the complex. Calculated spectra reproduced experimental absorption spectra confirming calculated electronic transitions. ${ }^{18}$

\subsubsection{Cyclophanes: Subporphyrin cages cyclophanes}

Inokuma et al. synthesized novel capped subporphyrin cages (Scheme 4.1) that can trap guest molecules and exhibit intramolecular $\pi-\pi$ stacking. ${ }^{68}$ These cage subporphyrins were synthesized using imine chemistry for attaching the caps (Scheme 4.1) followed by reduction to produce the amine bridges. Capped subporphyrins with varying bridge lengths were utilized (4.3a-e), as well as also electron withdrawing esters (4.3c, 4.3e) were used to test electronic effects.

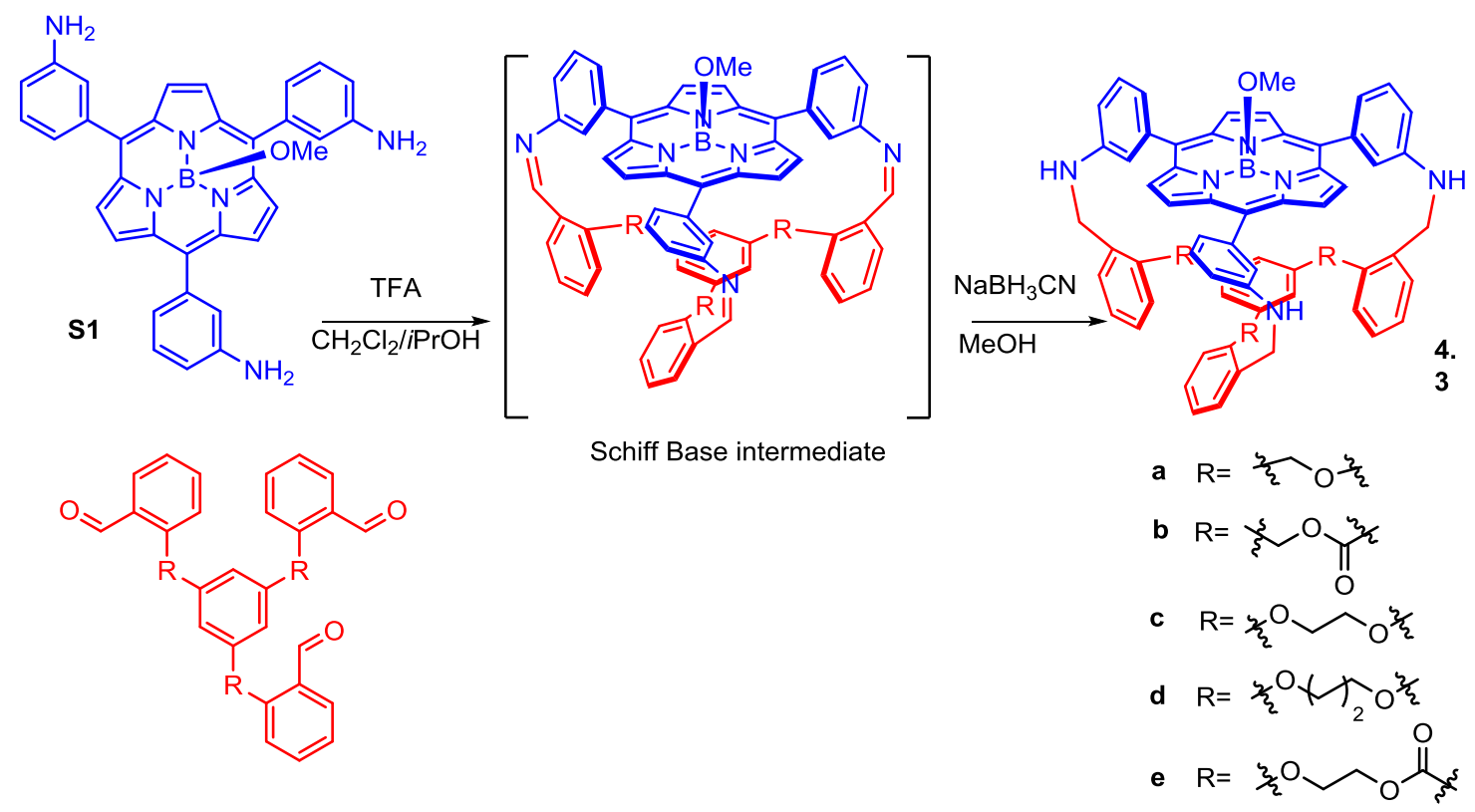

Scheme 4.1: Capped subporphyrins

The crystal structures of $\mathbf{4 . 3 a}$ and $\mathbf{4 . 3 b}$ revealed there are two conformations of capped subporphyrins, $\mathrm{P}$ and $M$ spiral forms (form of axial chirality $P(+)$ and $M(-)$ ). ${ }^{68}$ The crystal structures of $\mathbf{4 . 3 a}$ and $\mathbf{4 . 3 b}$ also revealed that there was a strong possibility of $\pi-\pi$ stacking with the molecule due to the interplanar distances (3.56 $\AA$ and $3.13 \AA$ respectively) are within the van der Waals radius. ${ }^{68}$ The crystal structures of 4.3d and 4.3e showed that the cavities within these molecules were too large to $\pi-\pi$ stacking; however, compound $4.3 \mathrm{~d}$ and $4.3 \mathrm{e}$ were able to trap guest molecules $\left(\mathrm{CHCl}_{3}\right.$, mesitylene) within the cavities of the capped subporphyrins. 


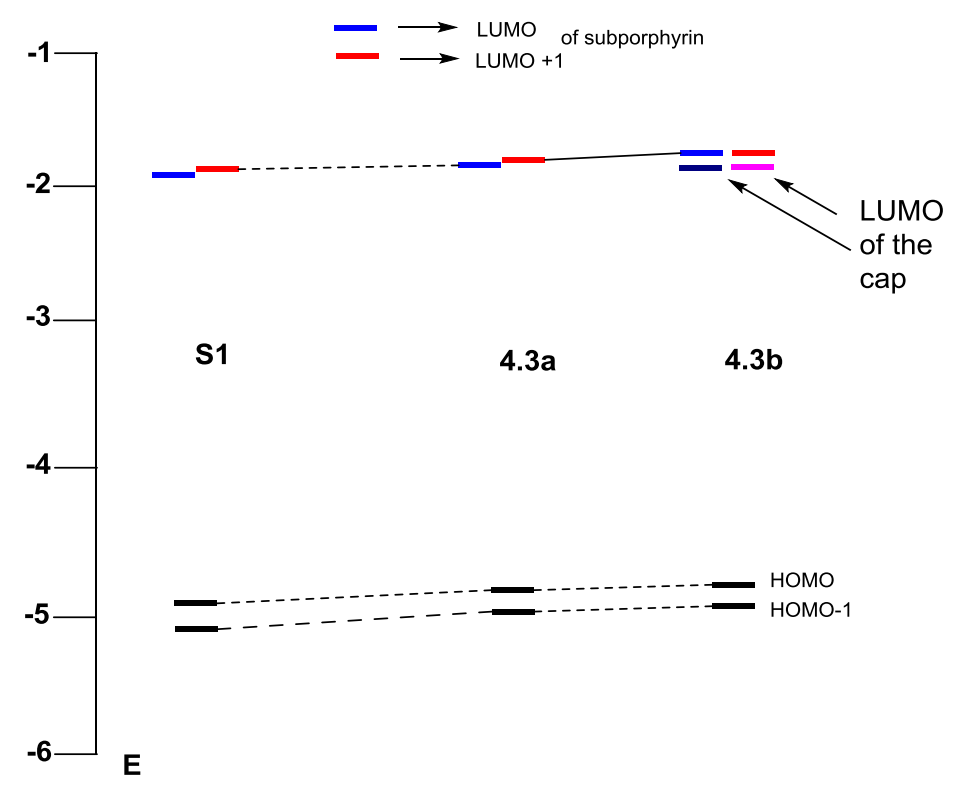

Figure 4.13: Molecular orbitals of capped subporphyrins ${ }^{68}$

Density function theory (DFT) calculations were performed on the capped subporphyrins S1, 4.3a, 4.3b. S1 and 4.3a both had similar frontier molecular orbital distributions (4 MOs: HOMO, HOMO-1, LUMO, LUMO+1) (Figure 4.13) where electron density resided only within the subporphyrins. ${ }^{68}$ The similarity of 4.3b and S1 MOs indicate there is no significant effect on optical properties from $\pi-\pi$ stacking within 4.3a. However, 4.3b has electron density on the aryl cap due to the presence of the electron withdrawing group, enhancing $\pi-\pi$ stacking. The absorption spectra (Table 4.1) ${ }^{69}$ of the capped subporphyrins (4.3a-e) indicated insignificant changes to absorption properties compared to S1 owing to poor $\pi-\pi$ stacking, however, 4.3b had the largest red shift. Fluorescence studies (Table 4.1) showed that for subporphyrins 4.3a-, there was a blue shift in emission compared to $\mathbf{S 1 .}$ 
Table 4.1: Optical properties of capped subporphyrins ${ }^{69}$

\begin{tabular}{lllll}
\hline & $\begin{array}{l}\text { Absorption } \\
\text { Soret (nm) }\end{array}$ & $\begin{array}{l}\mathbf{Q} \\
\text { band } \\
(\mathbf{n m})\end{array}$ & $\begin{array}{l}\text { Fluorescence } \\
\lambda_{\max }(\mathbf{n m})\end{array}$ & $\boldsymbol{\Phi}_{\mathrm{f}} \%$ \\
\hline S1 & 377 & 489 & 525 & 12.2 \\
$\mathbf{1}$ & 377 & 487 & 517 & 13.2 \\
$\mathbf{2}$ & 387 & 497 & 515 & 16.6 \\
$\mathbf{3}$ & 377 & 487 & 515 & 13.3 \\
$\mathbf{4}$ & 382 & 493 & 517 & 15.6 \\
$\mathbf{5}$ & 380 & 492 & 534 & 16.7 \\
\hline
\end{tabular}

These capped subporphyrins are the first example of cyclophane subporphyrins with the potential to have significant intramolecular $\pi-\pi$ stacking. However, there were insignificant changes to the electronic properties in these capped motifs most likely due to the bowel shape of subporphyrins.

\subsection{Thesis Objective}

\subsubsection{BODIPY-anthracene cyclophane}

The aim of this project is to study how confined intramolecular $\pi$ stacking affects the HOMO-LUMO gap of BODIPY in $\pi$ dimer cyclophane architectures, and if it is possible to tune the HOMO-LUMO gap within the $\pi$ dimer cyclophane using the confined intramolecular $\pi$ stacking. The two planar ring systems that will make up this dimer are BODIPY and anthracene (Figure 4.14). The requirement for this cyclophane is that it must have $\pi-\pi$ stacking in order to test the intramolecular effects on this HOMO-LUMO gap. BODIPY was chosen as the molecule for the $\pi-\pi$ dimer cyclophane due it desirable light harvesting properties. Anthracene was selected as the second molecule due its rigid three fused conjugated ring system, where this rigidity should negate energy loss through vibration when excited. Another advantage of using anthracene is the $\pi$ system physically overlaps with the $\pi$ system of BODIPY. In addition, anthracene can be readily functionalized allowing for many structure-property relationships to be explored. This controlled functionalization of both BODIPY and anthracene can allow for the individual tuning of both the HOMO and LUMO energy levels. The proposed cyclophane architectures are presented below. 

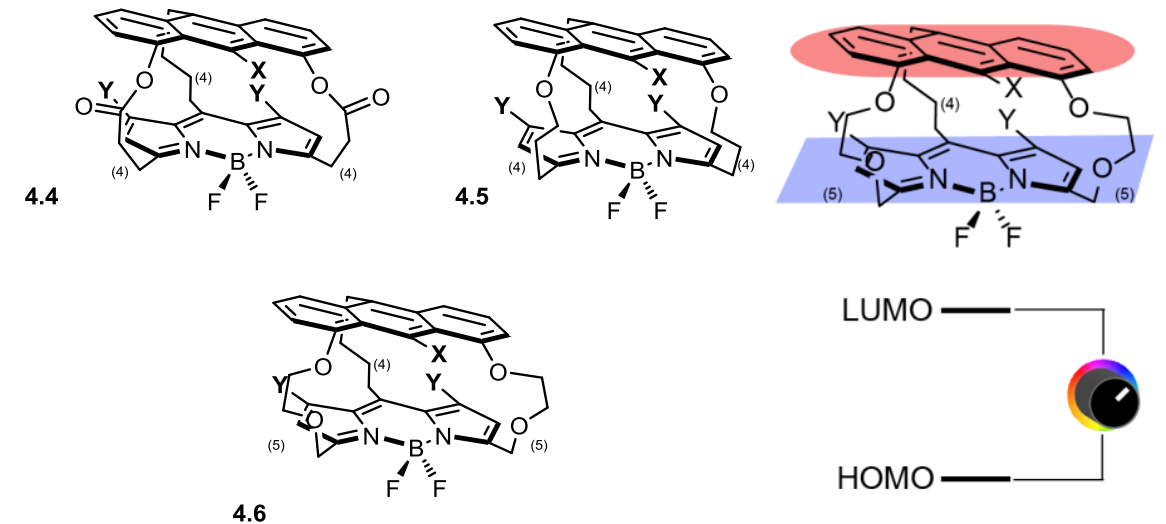

Figure 4.14: Proposed BODIPY-anthracene cyclophane architecture

\subsubsection{Tethers of BODIPY-anthracene cyclophane}

Effective $\pi-\pi$ stacking between BODIPY and anthracene in this cyclophane architecture will require precise tethers. In order to make sure that both aromatic molecules are locked into place (avoiding vibrational relaxation that would be detrimental to our energy study), three bridges are desirable. If only two tethers are used, there would be excessive flexibility in the molecule which will hinder $\pi-\pi$ stacking. Three tethers will dramatically decrease the flexibility of the molecule which in turn increases the probability of an intramolecular $\pi-\pi$ stacking occurring between the BODIPY and anthracene. The three tethers will be bound to the meso, and 3, 5 positions of BODIPY (Figure 4.14) as the reactivity of these positions are well established in the literature. ${ }^{2}$ The corresponding anthracene tether positions will be 1,8 , and 10 positions. Another issue that needs to be considered for the bridges is the number of atoms in each bridge. If the bridges are too long, then the distance between both the aromatic compounds will be larger than the $\pi$ $\pi$ stacking radius. However, if the bridges are too short, then the steric bulk of both aromatics molecules will prohibit the ring closure of the cyclophane. Thorough DFT calculations were performed, and in order to make the cyclophane, there needs to be 4-5 atoms in each bridge in order to have the intramolecular $\pi-\pi$ stacking near to the van der Waals radius.

\subsection{Theoretical Calculations}

According to DFT studies performed on the BODIPY cyclophane architecture $\mathbf{4 . 5}$ proposed in this project (Figure 4.14), there is evidence of confined intramolecular $\pi-\pi$ stacking. In the HOMO state, the electron density mostly lays in the $\pi$ system of the anthracene and in the LUMO state, the electron density lies on 
the BODIPY $\pi$ system. The tether atoms are all $\mathrm{sp}^{3}$ hybridized meaning that there is no orbital overlap through the bridges. This localized HOMO and LUMO should permit the ability to tune the HOMO and LUMO independently and find the sweet spot for intramolecular charge transfer. In the TD-DFT, the UVVis spectrum of the proposed cyclophane 4.5 shows three main transitions. A strong transition at $260 \mathrm{~nm}$ corresponding to the $\pi-\pi^{*}$ transition occurring in the anthracene, $460 \mathrm{~nm}$ corresponding to the $\pi-\pi^{*}$ transition occurring in BODIPY and the HOMO-LUMO transition that occurs at $750 \mathrm{~nm}$, however the extinction coefficient is extremely low $(\varepsilon<0.1)$. Also the DFT calculation is performed in the gas phase, so solvent effects can considerably affect the extinction coefficient.

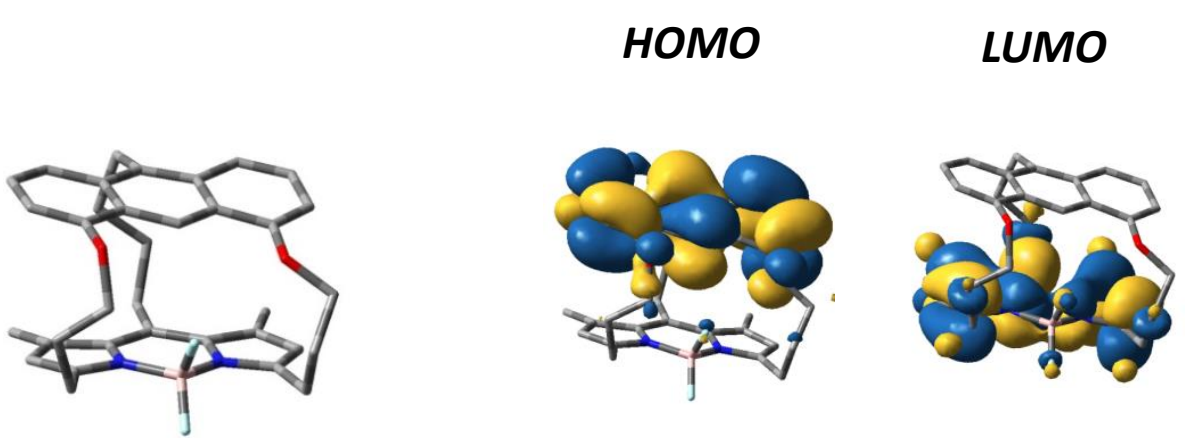

Figure 4.15: DFT: B3LYP 3-21G of BODIPY-anthracene and predicted molecular orbitals of HOMO-LUMO states

In collaboration with Dr. Eduardo Schott (Pontifical Catholic University, Chile), further DFT studies were performed to see how electronic donating/withdrawing groups on the anthracene and BODIPY compounds could alter where the electron density sits within the molecule in the ground/excited state. 4.6 was used as a model for this DFT study (Appendix). Table 4.2 is summary of the HOMO/LUMO level of all the different combination of functional groups as well as HOMO-LUMO energy gap

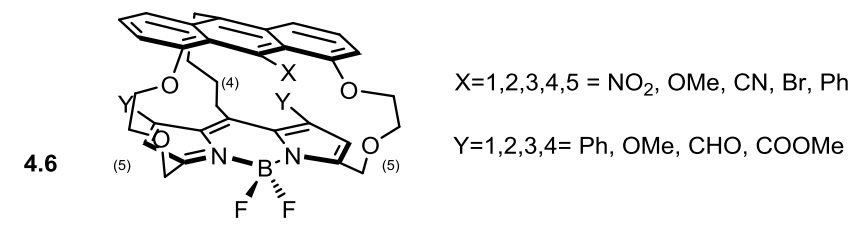

Figure 4.16: The Modification of 4.6 for DFT Study

Table 4.2: Summary of Calculated Energy states of 4.6

\begin{tabular}{lllll} 
Compound & F.G. & $E_{\text {Hомо }}(\mathrm{eV})$ & $\mathrm{E}_{\mathrm{LUMO}}(\mathrm{eV})$ & $\begin{array}{l}\mathrm{GAP}_{\mathrm{H}-\mathrm{L}} \\
(\mathrm{eV})\end{array}$ \\
\hline $\mathrm{X} 1 \_\mathrm{Y1}$ & $\mathrm{X}=\mathrm{NO}_{2}$ & -6.68 & -1.90 & 4.78
\end{tabular}




\begin{tabular}{|c|c|c|c|c|}
\hline & $\mathrm{Y}=\mathrm{Ph}$ & & & \\
\hline X1_Y2 & $\begin{array}{l}\mathrm{X}=\mathrm{NO}_{2} \\
\mathrm{Y}=\mathrm{OMe}\end{array}$ & -6.64 & -1.39 & 5.25 \\
\hline X1_Y3 & $\begin{array}{l}\mathrm{X}=\mathrm{NO}_{2} \\
\mathrm{Y}=\mathrm{CHO}\end{array}$ & -6.87 & -2.90 & 3.97 \\
\hline X1_Y4 & $\begin{array}{l}\mathrm{X}=\mathrm{NO}_{2} \\
\mathrm{Y}=\mathrm{COOMe}\end{array}$ & -6.47 & -2.08 & 4.39 \\
\hline X2_Y1 & $\begin{array}{l}X=O M e \\
Y=P h\end{array}$ & -6.20 & -1.81 & 4.40 \\
\hline X2_Y2 & $\begin{array}{l}X=O M e \\
Y=O M e\end{array}$ & -6.17 & -1.30 & 4.88 \\
\hline X2_Y3 & $\begin{array}{l}\mathrm{X}=\mathrm{OMe} \\
\mathrm{Y}=\mathrm{CHO}\end{array}$ & -6.42 & -2.83 & 3.58 \\
\hline X2_Y4 & $\begin{array}{l}X=O M e \\
Y=\text { COOMe }\end{array}$ & -6.27 & -2.34 & 3.93 \\
\hline X3_Y1 & $\begin{array}{l}\mathrm{X}=\mathrm{CN} \\
\mathrm{Y}=\mathrm{Ph}\end{array}$ & -6.55 & -1.95 & 4.59 \\
\hline X3_Y2 & $\begin{array}{l}\mathrm{X}=\mathrm{CN} \\
\mathrm{Y}=\mathrm{OMe}\end{array}$ & -6.59 & -1.46 & 5.13 \\
\hline X3_Y3 & $\begin{array}{l}\mathrm{X}=\mathrm{CN} \\
\mathrm{Y}=\mathrm{CHO}\end{array}$ & -6.83 & -2.97 & 3.86 \\
\hline X3_Y4 & $\begin{array}{l}X=C N \\
Y=\text { COOMe }\end{array}$ & -6.69 & -2.49 & 4.20 \\
\hline X4_Y1 & $\begin{array}{l}X=B r \\
Y=P h\end{array}$ & -6.31 & -1.87 & 4.44 \\
\hline X4_Y2 & $\begin{array}{l}\mathrm{X}=\mathrm{Br} \\
\mathrm{Y}=\mathrm{OMe}\end{array}$ & -6.27 & -1.38 & 4.89 \\
\hline X4_Y3 & $\begin{array}{l}\mathrm{X}=\mathrm{Br} \\
\mathrm{Y}=\mathrm{CHO}\end{array}$ & -6.52 & -2.91 & 3.61 \\
\hline X4_Y4 & $\begin{array}{l}X=\mathrm{Br} \\
\mathrm{Y}=\mathrm{COOMe}\end{array}$ & -6.36 & -2.42 & 3.94 \\
\hline X5_Y1 & $\begin{array}{l}X=P h \\
Y=P h\end{array}$ & -6.20 & -1.88 & 4.32 \\
\hline X5_Y2 & $\begin{array}{l}\mathrm{X}=\mathrm{Ph} \\
\mathrm{Y}=\mathrm{OMe}\end{array}$ & -6.17 & -1.37 & 4.80 \\
\hline X5_Y3 & $\begin{array}{l}\mathrm{X}=\mathrm{Ph} \\
\mathrm{Y}=\mathrm{CHO}\end{array}$ & -6.41 & -2.89 & 3.52 \\
\hline X5_Y4 & $\begin{array}{l}X=P h \\
Y=\text { coOMe }\end{array}$ & -6.25 & -2.41 & 3.84 \\
\hline
\end{tabular}

*DFT calculation were done as B3LYP, 6-311G*, in the gas phase, DFT images in appendix

According to the Table 4.2, regardless of varying $X$ and $Y$, the HOMO resides on the more electronic rich anthracene while the LUMO resides on BODIPY. Overall, while keeping $X$ the same and independently varying $\mathrm{Y}$, the HOMO energy level is not significantly affected. However, the LUMO energy level can be changed based on introducing electron donating groups (OMe, $\mathrm{Ph})$ and electron withdrawing (CHO, 
COOMe) in a predictable, controlled manner. According to Table 4.2, the lowest laying LUMO to the highest LUMO energy level in all cases of $\mathrm{X}$ is as follows; $\mathrm{CHO}<\mathrm{COOMe}<\mathrm{Ph}<\mathrm{OMe}$. Also the differences between the energy levels of the $4 Y$ variations in all cases of $X$ are almost constant. The effects that $Y$ variations have on the HOMO levels also is quite constant, revealing that there could be electronic communication between the two aromatic systems (BODIPY, anthracene) through space.

\subsection{Synthesis and Results}

4.4.1 Synthetic route for BODIPY-anthracene cyclophanes

The proposed retrosynthetic route are presented for 4.4, 4.5, 4.6 are in Scheme 4.2 

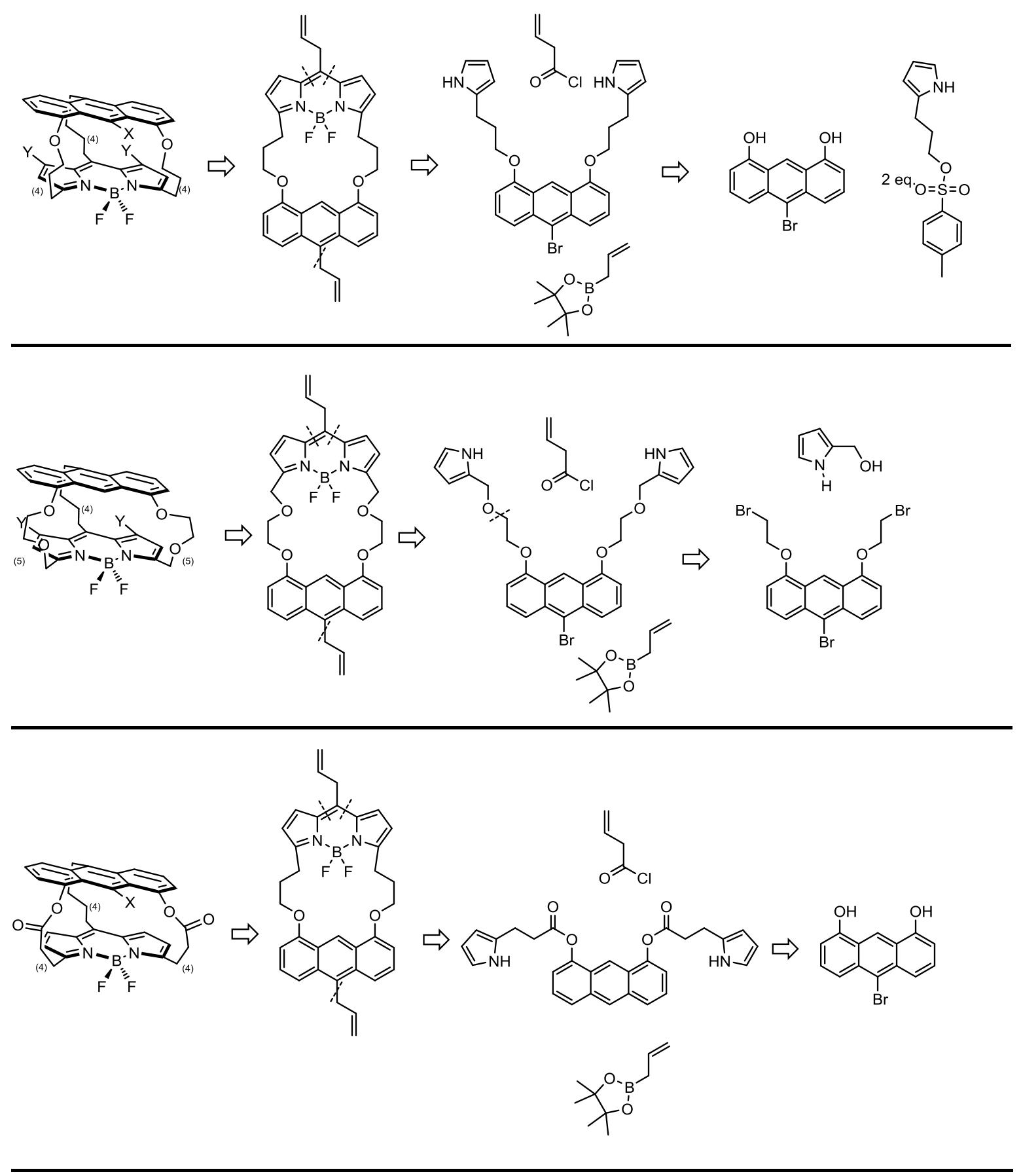

Scheme 4.2: retrosynthetic approach to synthesis cyclophanes 4.4, 4.5, 4.6

For each BODIPY anthracene cyclophane proposed, the desired ring closing reaction will be performed using olefin metathesis and the Grubbs $1^{\text {st }}$ generation catalyst. In order to get to that step, allylic tether units are needed for ring closing reaction. This allylic BODIPY-anthracene macrocycle can be made using Suzuki coupling to attach the allylic tether to anthracene and BODIPY formation occurs using 3-butenoyl 
chloride. The pyrrole and anthracene units for the synthesis of the BODIPY-anthracene cyclophanes are discussed further.

\subsubsection{Synthesis of Anthracene Building Blocks}

Starting with commercially available 1,8-dihydroxyanthraquinone, the hydroxyl groups were protected using acetyl chloride in the presence of $\mathrm{NEt}_{3}$ leading to 1,8 diacteoxyanthraquinone, 4.7. 4.7 was then reduced using $\mathrm{Zn}$ in acetic acid, and reprotected with acetyl chloride in the presence of $\mathrm{NEt}_{3}$ to create the anthracene derivative $4.8 .^{70}$ This reaction proceeds rather sluggishly with a yield of $61 \%$ and, the purification was difficult due to the presence of 4.9 as a by-product (Scheme 4.3 ) due to over-reduction. 4.9 has similar polarity as $\mathbf{4 . 8}$ so separating these two products using chromatography was not possible. Recrystallization did improve the purity of 4.8 and the use of ethanol with a slow recrystallization allowed for the ratio of (4.8:4.9) 95:5. This ratio was deemed clean enough to carry to the next reaction.

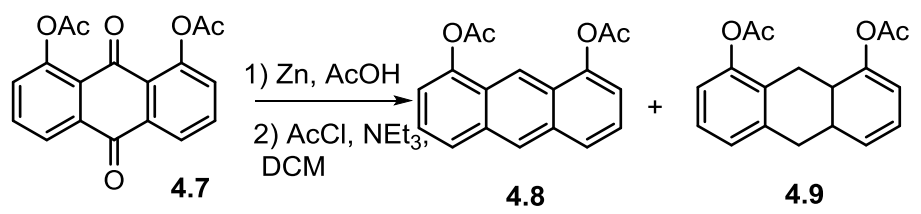

Scheme 4.3: Synthesis of 1,8 diacetoxyanthracene through the reduction of anthraquinone using $\mathrm{Zn}$

In order to eliminate the messy work up involved with producing molecule $\mathbf{4 . 8}$ and cut reaction time, the reaction in Scheme 4.4 was used. ${ }^{71}$ The advantage of this reaction was that less acetic acid and overall solvent was need. This reaction also eliminates of the reprotection step of the hydroxyl groups with $\mathrm{NEt}_{3}$ and $\mathrm{AcCl}$ due to the reduction of the ketones. Instead, the presence of acetic anhydride and sodium acetate reprotects the hydroxyl groups in situ.
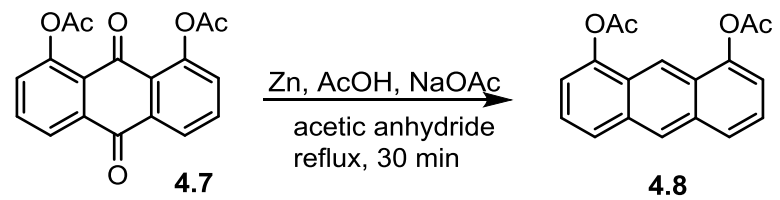

Scheme 4.4: Improved synthesis of 1,8 diacetoxyanthracene through the reduction of anthraquinone using $\mathrm{Zn}$ Originally the synthesis of $\mathbf{4 . 1 0}$ was done using $\mathrm{Br}_{2}$ in acetic acid at high dilution. ${ }^{71}$ However, no precipitate was observed after multiple attempts and the reaction mixture was extracted with DCM in order to recover the starting material. After column chromatography, a dark green residue was acquired which by ${ }^{1} \mathrm{H}$ NMR was determined to be a mixture of 4.10 and the isomer 4.11 (Scheme 4.5). According to ${ }^{1} \mathrm{H}$ NMR the major product was $\mathbf{4 . 1 1}$ while $\mathbf{4 . 1 0}$ was the minor product. After multiple purification attempts, the 
desired 4.10 was attained pure, however the yield for this reaction was only $20 \%$. This method of bromination was abandoned and instead NBS was used at $50^{\circ} \mathrm{C}$ in a moderately high boiling halogenated solvent (DCE) using $I_{2}$ in catalytic amounts in order to retard radical formation. This led to selective formation of 4.10 between $65-80 \%$ which was higher than previously reported literature $(45 \%){ }^{72}$ The saponification of the acetoxy protecting groups of $\mathbf{4 . 1 0}$ proceed efficiently producing quantitative yields of molecule 4.12.<smiles>CC(=O)Oc1cccc2cc3cccc(OC(C)=O)c3cc12</smiles>

4.8<smiles>CC(=O)Oc1cccc2cc3cccc(OC(C)=O)c3cc12</smiles>

4.8

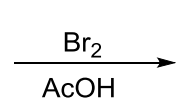

$\mathrm{AcOH}$

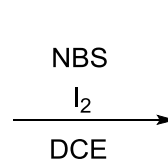

DCE

$\mathrm{Br} 4.10$<smiles></smiles><smiles>CC(=O)Oc1cccc2c(Br)c3cccc(OC(C)=O)c3cc12</smiles><smiles></smiles><smiles>Oc1cccc2c(Br)c3c(O)cccc3cc12</smiles>

Scheme 4.5: Synthesis of 10-bromo-1,8 diacetoxyanthracene

To install the allylic tether unit for the ring closing metathesis, a Suzuki coupling was used on compound 4.10 as a test (Scheme 4.6). Due the sensitivity of protecting acetyl groups, basic aqueous conditions were not feasible, so a fluoride source was used to activate the boronic ester. The first attempt used TBAF as the fluoride source which resulted in saponification of one the protecting groups. Attempts using KF yielded only a trace amount of the desired product; however, saponification still occurred in the molecule. This saponification problem is most likely due to water contamination.

Starting from both 4.13 and 4.17, a Wittig olefination was performed using (carbethoxymethylene) triphenyl phosphorance resulting in the pyrrole vinyl esters 4.19 and 4.20. After the alkene was reduced using $\mathrm{Pd} / \mathrm{C}$ with hydrogen gas bubbled in $\mathrm{MeOH}$ to produce 4.21 and 4.22. The saponification of the pyrrole esters 4.21 and 4.22 using $\mathrm{NaOH}$ produced the carboxylic acids 4.25 and 4.26 in moderate yields. 
4.21 was further reacted with $\mathrm{LiAlH}_{4}$ in $\mathrm{THF}$ to reduce ester to make 4.23. The alcohol of $\mathbf{4 . 2 3}$ was reacted with $\mathrm{TsCl}$ to make a good leaving group for tethering pyrrole to 4.12 .

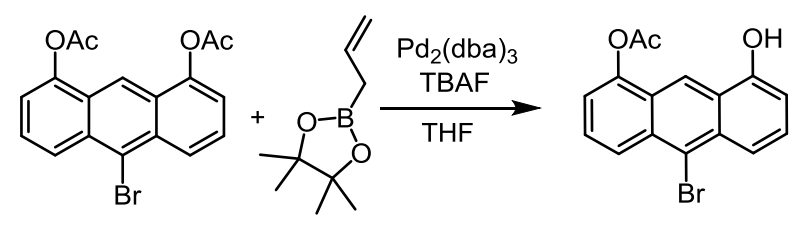

4.10

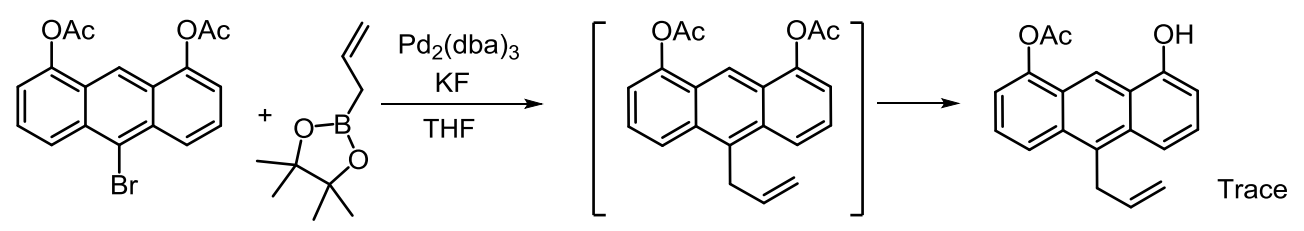

4.10

Scheme 4.6: Synthesis of 10-bromo-1,8 diacetoxyanthracene

\subsubsection{Synthesis of Pyrrole Building Blocks}

The commercially available pyrrole-2-carboxyaldehyde 4.13 was chosen as the pyrrole starting material (Scheme 4.7). First, the pyrrole-2-carboxyaldehyde was brominated using NBS in THF at $0^{\circ} \mathrm{C}$ for 15 minutes to produce 4.14 in quantitative yields. ${ }^{73}$ In order to convert the bromide leaving group to a methyl group, tert-butyllithium was required. However, the carbonyl group is electrophilic and it was necessary to protect this functional group. The method of the protection chosen was to form the pyrrole dimer 4.15 using aqueous dimethyl amine. ${ }^{73}$ Refer to appendix A.4 for the proposed mechanism. The reaction yields were moderate (50-65\%), but it was easily purified through recrystallization in EtOH/water.

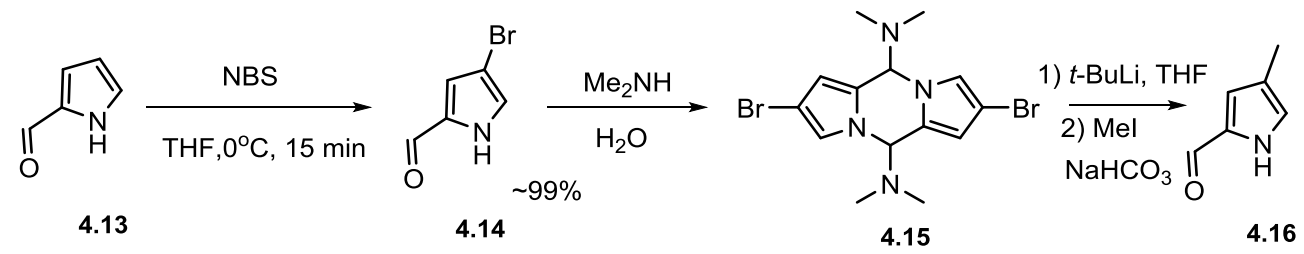

Scheme 4.7: Original synthetic towards the pyrrole building blocks

Once the pyrrole dimer 4.15 was synthesised, the next step was a metal-halogen exchange and quench with methyl iodide (Scheme 4.8) to form the dimethyl dimer. The dimer was then broken using sodium bicarbonate to produce methyl pyrrole-2-carboxyaldehyde 4.16. The methyl pyrrole carboxyaldehyde was 
obtained as a mixture with the original pyrrole carboxyaldehyde 4.13. The best ratio obtained for this reaction was 77:23 for the methyl pyrrole carboxyaldehyde: pyrrole carboxyaldehyde mixture.

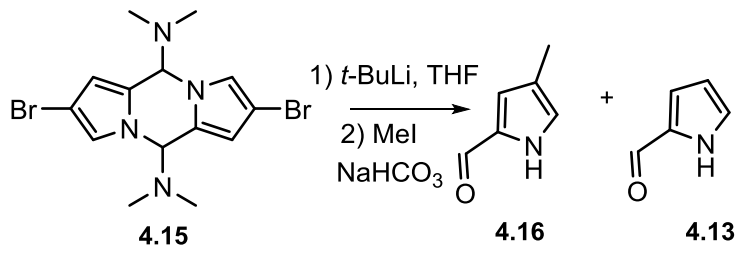

Scheme 4.8: Synthesis towards the methyl pyrrole carboxyaldehyde

In order to move on with the project, instead of protecting the pyrrole with the methyl group, the amine of 4.13 was protected with $\mathrm{TsCl}$ to form the tosylated 4.17 (Scheme 4.9). An advantage of using the tosyl group to protect the nitrogen is the crystalline property of this motif which makes purification easier. 4.17 is then reduced using $\mathrm{NaBH}_{4}$ to produce $\mathbf{4 . 1 8}$ quantitative yields.

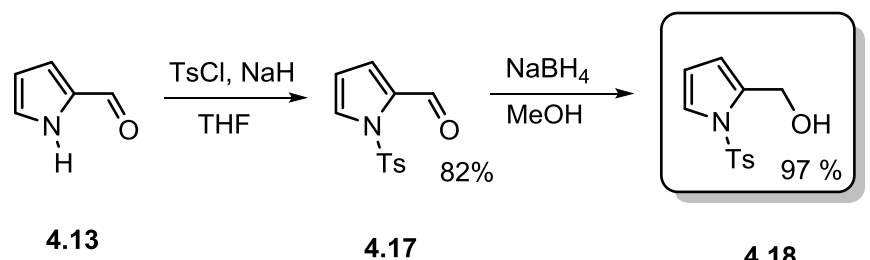

Scheme 4.9: Synthesis of protected pyrrole propanol 4.18

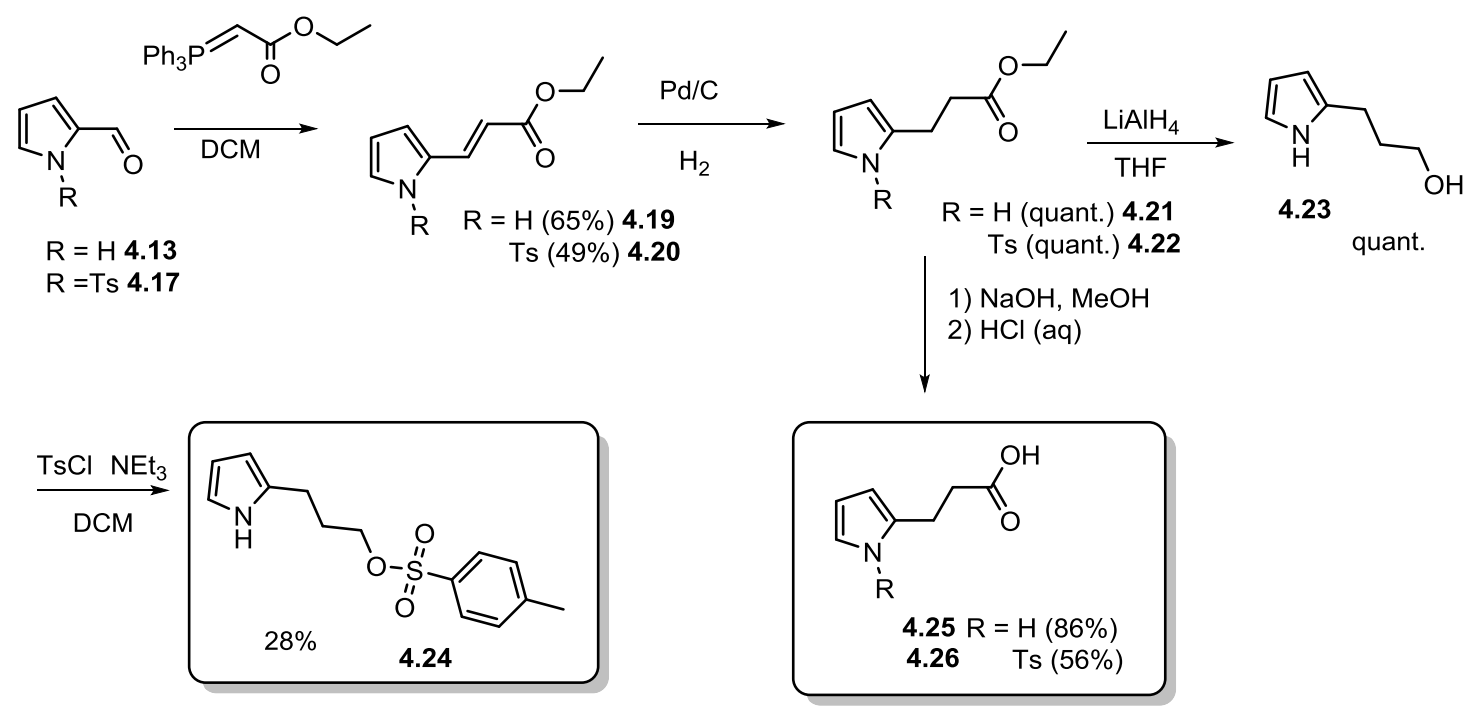

Scheme 4.10: Synthesis of pyrrole building blocks 


\subsubsection{Tethering of Pyrrole and Anthracene for BODIPY-Anthracene Cyclophane 4.5}

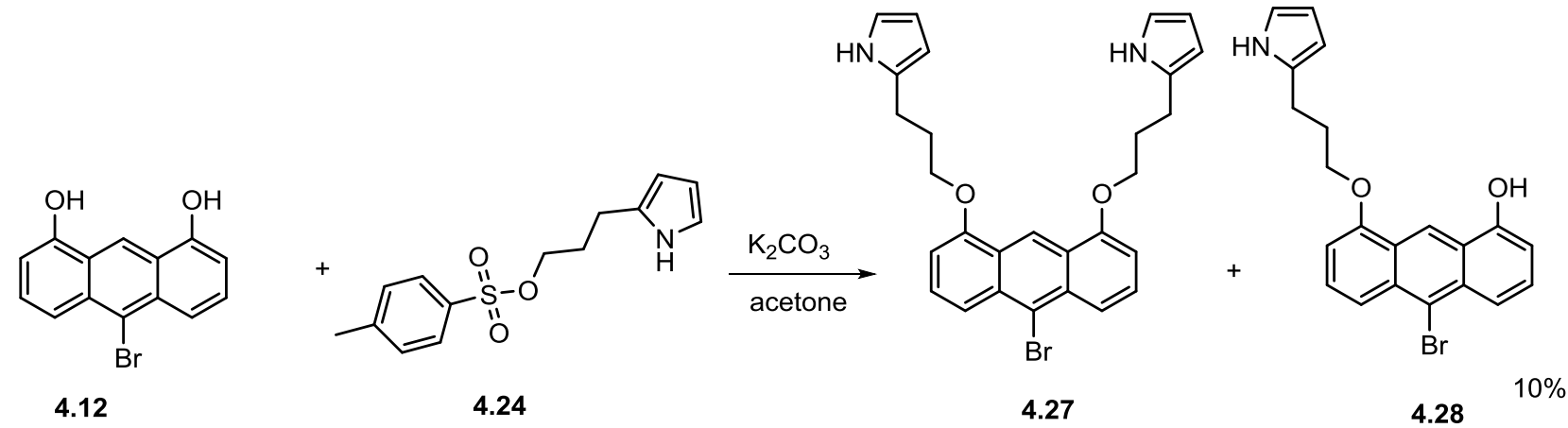

Scheme 4.11: Synthesis of anthracene-pyrrole building block for 4.5

In an attempt to prepare cyclophane 4.5, 4.12 was chosen as the anthracene containing moiety and 4.24 was the source of the pyrrole units (Scheme 4.11). A Williamson ether synthesis was attempted, however, only a minuscule amount of the desired 4.27 was obtained along with the mono substituted product in a $10 \%$ yield. A possible reason for the low yields is most likely due to the cyclic intramolecular reaction between the depronated anime from the pyrrole and the electrophile carbon with the tosyl leaving group. This pathway showed some promise as a way to tether the pyrrolic units to anthracene; however the yield was too low to be viable. For these reasons, the synthesis of $\mathbf{4 . 5}$ was abandoned, and a greater focus was given to the preparation of cyclophanes 4.4 \& 4.6 as more realistic targets for synthesis of a BODIPYAnthracene cyclophane. 


\subsubsection{Tethering of Pyrrole and Anthracene for BODIPY-Anthracene Cyclophane 4.6}
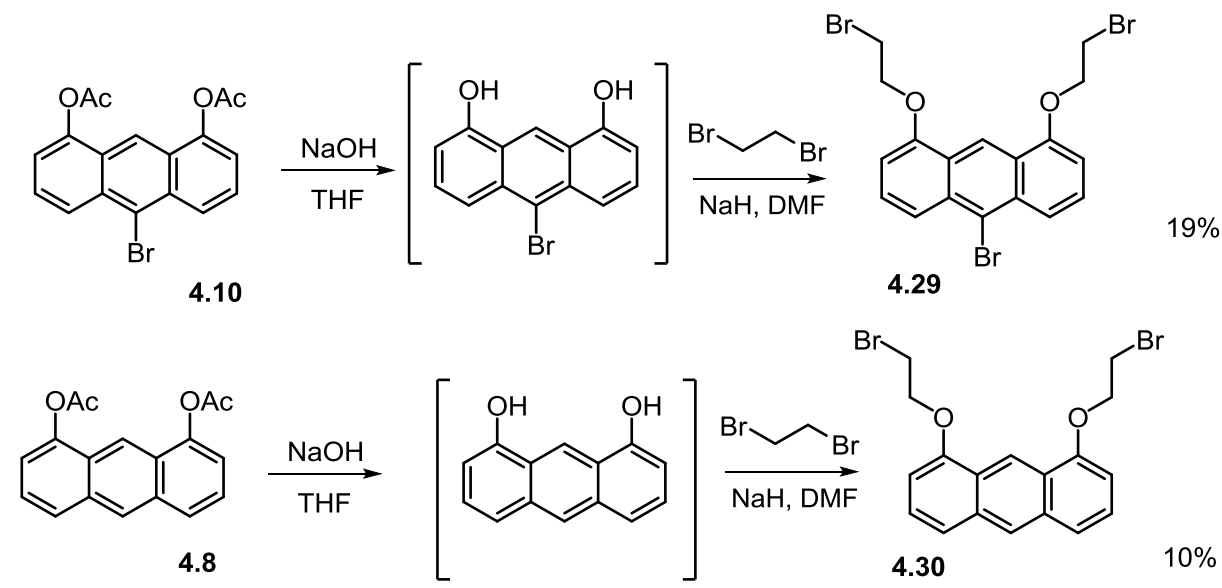<smiles>O=C1c2cccc(O)c2C(=O)c2c(O)cccc21</smiles>

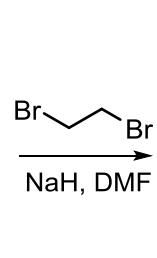<smiles>O=C1c2cccc(OCCBr)c2C(=O)c2c(OCCBr)cccc21</smiles>

$5 \%$

Scheme 4.12: Williamson ether synthesis of anthracene/anthraquinone

For cyclophane 4.6, 4.10 were used as the test substrate for a Williamson ether synthesis with dibromoethane (Scheme 4.12) to make 4.29. The reaction was successful in producing 4.29 , however the product was produced in poor yield. The same Williamson ether synthesis was attempted using the 1,8dihydroxyanthraquinone and starting anthracene with success yielding 4.31 and $\mathbf{4 . 3 0}$ respectively. These ether reactions also could be done on large scale. 


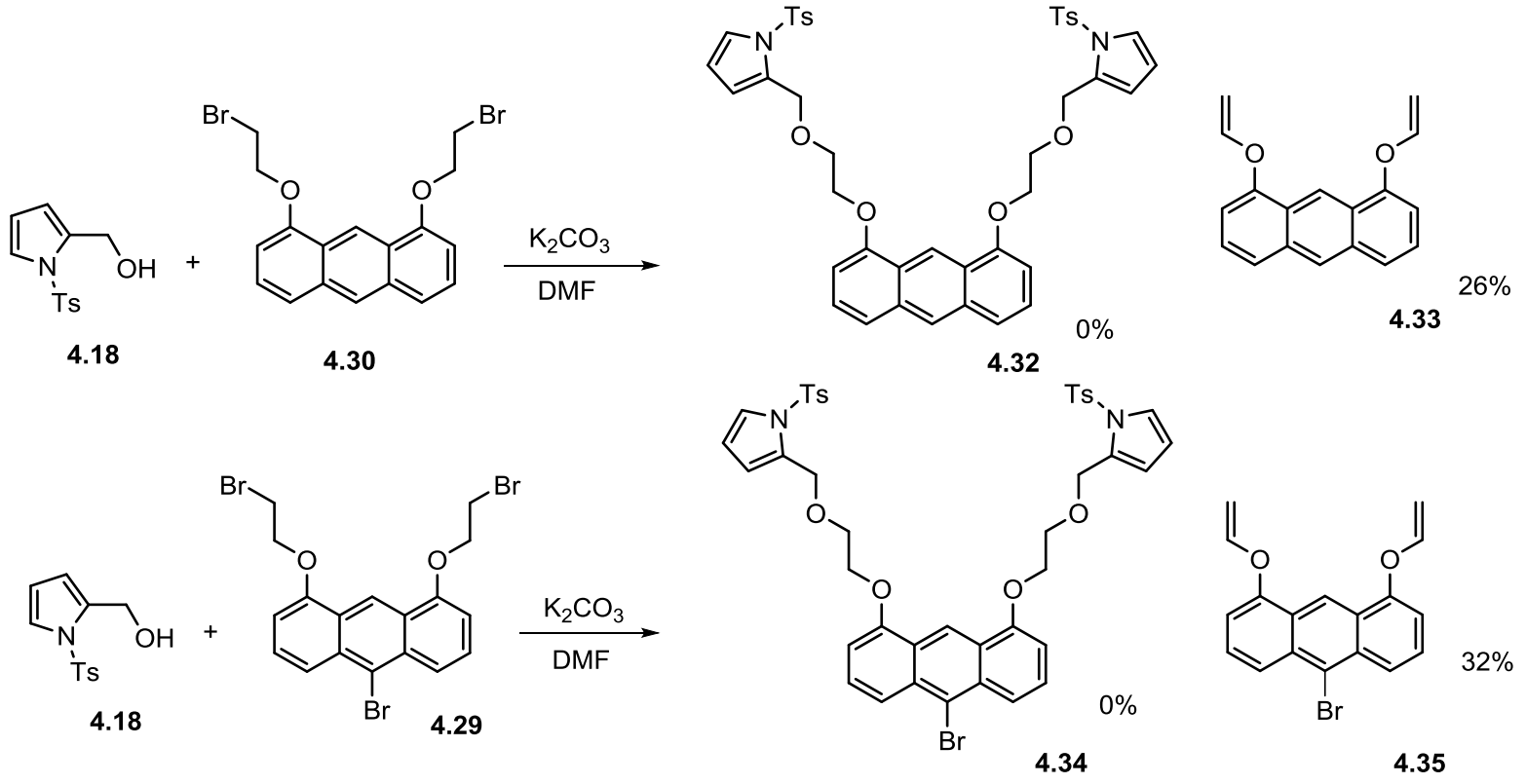

Scheme 4.13: Synthesis of anthracene-pyrrole building block for $\mathbf{4 . 6}$

Continuing towards cyclophane 4.6, 4.29 and 4.30 were used as the anthracene moieties and 4.18 contained the pyrrole units (Scheme 4.13). Williamson ether synthesis was employed in order to tether the pyrrole units 4.18 to anthracene 4.30. The reaction did not yield the desired product 4.32 , but did produce a significant byproduct that could not initially be interpreted by ${ }^{1} \mathrm{H}-\mathrm{NMR}$. The same reaction was repeated with 4.29; however, the desired product was not isolated. Instead, a significant amount of similar byproduct ( ${ }^{1} \mathrm{H}$ NMR) was collected. A crystal of unknown molecule from the reaction with 4.30 was grown and crystallography revealed the formation of $\mathbf{4 . 3 3}$ (Figure 4.17 ). The vinyl ether $\mathbf{4 . 3 3}$ produced was from elimination reaction instead of the desired substitution product. Due to time constraints, no further work was done to make cyclophane 4.6.

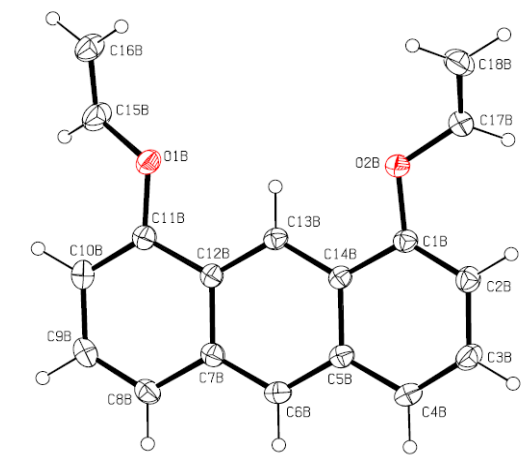

Figure 4.17: Crystal Structure of 4.33 


\subsubsection{Tethering of Pyrrole and Anthracene for BODIPY-Anthracene Cyclophane 4.4}

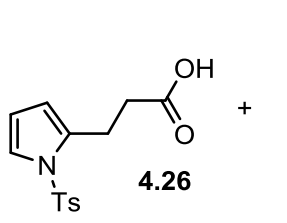<smiles>Oc1cccc2cc3cccc(O)c3cc12</smiles>

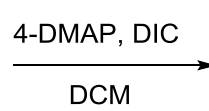<smiles>CCc1cccn1C</smiles><smiles>CC(=O)Oc1ccccc1</smiles><smiles>C=c1cc2cccc(OC(=O)CCc3cccn3[Te])c2cc1=CO</smiles>

Scheme 4. 14: Synthesis of anthracene-pyrrole building block for 4.4

In an attempt to prepare cyclophane 4.4, 1,8-dihydroxyanthracene was used as the anthracene containing starting material and $\mathbf{4 . 2 6}$ contained the pyrrole units, where esterification would tether the pyrrole units to anthracene. This esterification using 4-DMAP as the organic base, and DIC in DCM was successful, producing the desired product 4.36 in a moderate yield. Unfortunately, 4.36 was not light/air stable and was quick to decompose in solution. An attempt to deprotect the pyrrole using TBAF resulted in decomposition due to the rather sensitive ester link of the pyrrole and anthracene. This stability issues hindered the synthesis of cyclophane 4.4 , and this target was also abandoned.

\subsection{Conclusion and Future Work}
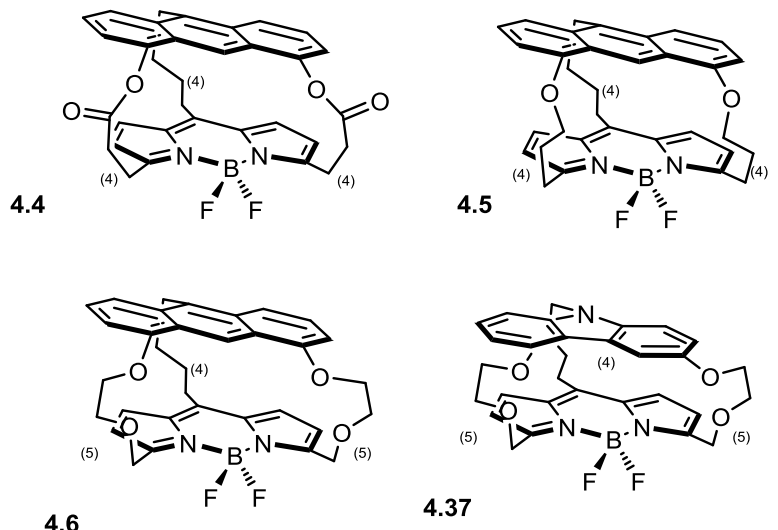

Figure 4.18: BODIPY-anthracene cyclophane architectures

For all three BODIPY-anthracene cyclophanes $(4.4,4.5,4.6)$, the theoretical calculations show that the cyclophanes proposed have intramolecular $\pi$ stacking and the in-depth DFT study of 4.6 illustrated the HOMO and LUMO energy levels can be tuned in a controlled manner. Synthesis towards these BODIPYanthracene cyclophanes did lead to the synthesis of interesting anthracene/pyrrole building blocks and one successful tethered anthracene-pyrrole compound 4.36. However, the following insurmountable synthetic challenges prevented the synthesis of these novel cyclophanes. 
- The sensitivity of anthracene to light/air (oxidation) and its tendency to oligomerize in ambient conditions was challenging. In the future, a light/air stable aromatic should be considered instead of anthracene for these cyclophane motifs.

- The protection of the amine in the pyrrolic unit was another challenge. While tosyl protecting group did allow for easier purification, the deprotection reaction was harsh and lead to the decomposition of 4.36. A better protection strategy should be employed to ensure that the cyclophane product can be made.

A possible proposed method of achieving a more stable anthracene BODIPY is using amide linkages instead of ester linkages (4.4). Another proposed derivative is using carboazole (4.37) instead of anthracene to avoid the oligomorization issue. Once the cyclophanes are synthesized, the physicochemical properties should be examined using optical spectroscopy (UV-Vis, fluorescence) and cyclic voltammetry to study the effects of the confined intramolecular $\pi$ stacking and the tunability of the HOMO-LUMO levels. 


\subsection{Experimental}

\section{General Considerations}

Unless stated otherwise, all reactions were performed under $\mathrm{N}_{2}$, using dry solvents and standard Schlenk techniques. All reactants and solvents were purchased from Sigma Aldrich except $\mathrm{PdCl}_{2}$ (Pressure Chemical Co.) and used without further purification. All nuclear magnetic resonance (NMR) spectroscopy analyses were performed on a Bruker $400 \mathrm{MHZ}$ spectrometer. ${ }^{1} \mathrm{H}$ NMR spectra were calibrated according to the residual solvent peak $\left(\mathrm{CHCl}_{3}, \delta=7.26 \mathrm{ppm}\right)$. Abbreviations used to indicate multiplicity are as followed: $\mathrm{s}=$ singlet, $\mathrm{d}=$ doublet, $\mathrm{t}=$ triplet, $\mathrm{m}=$ multiplet. Density function theory calculations for molecular modelling were done using Gaussian 03. Thin layer chromatography was performed on a aluminum backed sheets precoated with silica F254 ( $0.25 \mathrm{~nm}$ thick; Silicycle, Quebec, Canada) adsorbent and visualized under UV light. Purification via column chromatography was carried out using silica (Silicycle: P60 ultrapure flash silica). Common acronyms in this experimental section include dichloromethane (DCM), dichloroethane (DCE), tetrahyrofuran (THF), acetic acid (AcOH), acetyl chloride $(\mathrm{AcCl})$, ethyl acetate $(\mathrm{EtOAC})$, Ether $\left(\mathrm{Et}_{2} \mathrm{O}\right)$

\section{Theoretical Calculations}

All DFT and TD-DFT calculation was done use Gaussian 09M version C software. All structure were optimized at ground state default spin using the B3LYP exchange correlation function. These calclations were done in the gas phase (no solvent). For TD-DFT calculations were done at TD-SCF using the B3LYP exchange correlation function. All calculation were optimized using $3.21 \mathrm{G}$ basis set $(6.31 \mathrm{G}$ basis set when mentioned).<smiles>CC(=O)Oc1cccc2c1C(=O)c1cccc(OC(C)=O)c1C2=O</smiles>

Synthesis of 1,8-diacetoxyanthracene (4.7): 1,8 dihydroxyanthraquinone $(4.00 \mathrm{~g}, 16.65 \mathrm{mmol})$ was dissolved in DCM (minimal amount) and triethylamine $(10.9 \mathrm{~mL}, 99.9 \mathrm{mmol})$ was added slowly for 10 minutes while being stirred. Acetyl chloride $(4.90 \mathrm{~g}, 99.9 \mathrm{mmol})$ was added dropwise at $0^{\circ} \mathrm{C}$ over 10 minutes and the reaction mixture was stirred overnight. Product was recrystallized in hot ethanol to obtain a yellow solid $(5.05 \mathrm{~g}, 93 \%){ }^{1} \mathrm{H}$ NMR $\left(400 \mathrm{MHz}, \mathrm{CDCl}_{3}\right): \delta=8.23\left(\mathrm{~d}, 2 \mathrm{H}, \mathrm{H}_{\mathrm{c}}\right), 7.76\left(\mathrm{t}, 2 \mathrm{H}, \mathrm{H}_{\mathrm{b}}\right) 7.40$ (d, $\left.2 \mathrm{H}, \mathrm{H}_{\mathrm{a}}\right), 2.45\left(\mathrm{~s}, 12 \mathrm{H}, \mathrm{H}_{\mathrm{Ac}}\right)$. The remainder of the characterization was also consistent with previously reported literature. ${ }^{70}$<smiles>CC(=O)Oc1cccc2cc(Cl)ccc12</smiles> 
Synthesis of 1,8-diacetoxyanthracene (4.8): 1,8-Diacetoxyanthraquinone (5.00 g, $15.42 \mathrm{mmol}$ ) was dissolved in $\mathrm{AcOH}(300 \mathrm{~mL})$ and heated to $140^{\circ} \mathrm{C}$ with stirring and a reflux condenser under nitrogen until everything dissolved. The reaction vessel was removed from the oil bath and 3 equivalents of $\mathrm{Zn}(3.03 \mathrm{~g}$, $46.25 \mathrm{mmol}$ ) was added slowly portion-wise. The reaction vessel was put back into the oil bath and left to stir for 3 hours at $135^{\circ} \mathrm{C}$. After 3 hours, 1 equivalent of $\mathrm{Zn}(1.00 \mathrm{~g}, 15.42 \mathrm{mmol})$ was added to the mixture and was heated at $135^{\circ} \mathrm{C}$ for 3 hours. After 3 hours, another equivalent of $\mathrm{Zn}(1.00 \mathrm{~g}, 15.42 \mathrm{mmol})$ was added to the mixture and was heated at $135^{\circ} \mathrm{C}$ for $15 \mathrm{~h}$. The reaction mixture was passed through a short silica plug to remove zinc and zinc salts. The $\mathrm{AcOH}$ was removed in vacuo. Then the crude product was dissolved in a minimum amount of DCM and 6 equiv of $\mathrm{NEt}_{3}(10.13 \mathrm{ml}, 92.52 \mathrm{mmol})$ was added dropwise. The reaction was left to stir for 20 minutes, at $0{ }^{\circ} \mathrm{C}$, then $\mathrm{AcCl}(4.53 \mathrm{ml}, 92.52 \mathrm{mmol})$ was added dropwise and the mixture was stirred overnight. The solvent was removed in vacuo and purified through a silica column with DCM as eluent. The crude product was then recrystallized in ethanol to obtain an off white solid (2.25 g, 50\%). ${ }^{1} \mathrm{H} \mathrm{NMR}\left(400 \mathrm{MHz}, \mathrm{CD}_{3} \mathrm{Cl}\right): \delta=8.50\left(\mathrm{~s}, 1 \mathrm{H}, \mathrm{H}_{\mathrm{a}}\right), 8.46\left(\mathrm{~s}, 1 \mathrm{H}, \mathrm{H}_{\mathrm{e}}\right), 7.90\left(\mathrm{~d}, 2 \mathrm{H}, \mathrm{H}_{\mathrm{b}}\right), 7.47(\mathrm{t}$, $\left.2 \mathrm{H}, \mathrm{H}_{\mathrm{c}}\right) 7.29\left(\mathrm{~d}, 2 \mathrm{H}, \mathrm{H}_{\mathrm{d}}\right) 2.53\left(\mathrm{~s}, 12 \mathrm{H}, \mathrm{H}_{\mathrm{Ac}}\right)$. The remainder of the characterization was also consistent with previously reported literature. ${ }^{70}$<smiles>CC(=O)Oc1cccc2c(Br)c3cccc(OC(C)=O)c3cc12</smiles>

Synthesis of 10-bromo-1,8-diacetoxyanthracene (4.10): 1,8 diacetoxyanthracene (0.400 g, $1.36 \mathrm{mmol})$ and NBS $(0.306 \mathrm{~g}, 1.36 \mathrm{mmol})$ were dissolved in DCE $(12 \mathrm{~mL})$ and a catalytic amount of iodine (one drop of solution containing $0.05 \mathrm{~g}$ of iodine in DCM $(2.5 \mathrm{~mL})$. The mixture was heated at $50{ }^{\circ} \mathrm{C}$ for $3 \mathrm{~h}$. The solvent was removed in vacuo and the crude product was then purified using column chromatography with DCM as the eluent. Light green solid was obtained $(0.396 \mathrm{~g}, 80 \%){ }^{1} \mathrm{H} \mathrm{NMR}\left(400 \mathrm{MHz}, \mathrm{CDCl}_{3}\right): \delta=8.57$ $\left(\mathrm{s}, 1 \mathrm{H}, \mathrm{H}_{\mathrm{a}}\right), 8.45\left(\mathrm{~d}, 2 \mathrm{H}, \mathrm{H}_{\mathrm{b}}\right), 7.63\left(\mathrm{t}, 2 \mathrm{H}, \mathrm{H}_{\mathrm{c}}\right), 7.37\left(\mathrm{~d}, 2 \mathrm{H}, \mathrm{H}_{\mathrm{d}}\right), 2.55\left(\mathrm{~s}, 12 \mathrm{H}, \mathrm{H}_{\mathrm{Ac}}\right)$. The reminder of the characterization was also consistent with previously reported literature. ${ }^{70}$<smiles>Oc1cccc2c(Br)c3c(O)cccc3cc12</smiles>

Synthesis of 10-bromo-1,8-dihydroxyanthracene(4.12): A solution of 10-bromo-1,8-diacetoxyanthracene $(0.633 \mathrm{~g}, 1.70 \mathrm{mmol})$ in THF $(40 \mathrm{~mL})$ was sparged for 15 minutes with $\mathrm{N}_{2}$. To this mixture, $1 \mathrm{M} \mathrm{NaOH}(15$ $\mathrm{mL}$ ) was added dropwise and stirred under nitrogen. The solution turned deep yellow and then bright red and then back to yellow. Upon completion, by TLC, the reaction was quenched with $1.0 \mathrm{M} \mathrm{HCl}(25 \mathrm{~mL})$. The mixture was then washed with $0.1 \mathrm{M} \mathrm{HCl}(30 \mathrm{~mL} \times 2)$ and extracted with ether $(50 \mathrm{~mL})$. The mixture was then dried using $\mathrm{MgSO}_{4}$ and filtered. The crude product was passed through a short silica plug using EtOAc as eluent. A dark green solid was obtained $(0.481 \mathrm{~g}, 98 \%)$. ${ }^{1} \mathrm{H}$ NMR $\left(400 \mathrm{MHz}, \mathrm{CD}_{3} \mathrm{OD}\right): \delta=9.32(\mathrm{~s}$, $\left.1 \mathrm{H}, \mathrm{H}_{\mathrm{a}}\right), 7.92\left(\mathrm{~d}, 2 \mathrm{H}, \mathrm{H}_{\mathrm{b}}\right), 7.42\left(\mathrm{t}, 2 \mathrm{H}, \mathrm{H}_{\mathrm{c}}\right), 6.81\left(\mathrm{~d}, 2 \mathrm{H}, \mathrm{H}_{\mathrm{d}}\right)$. The reminder of the characterization was also consistent with previously reported literature. ${ }^{70}$ 


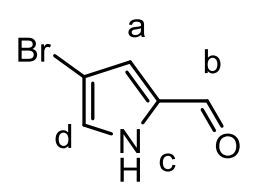

Synthesis of 4-bromo-1H-pyrrole-2-carbaldehyde (4.14): A stirred solution of pyrrole-2-carbozaldehyde $(1.00 \mathrm{~g}, 10.51 \mathrm{mmol})$ in $10.5 \mathrm{ml}$ of THF was cooled to $0^{\circ} \mathrm{C}$ under $\mathrm{N}_{2}$. NBS $(1.87 \mathrm{~g}, 10.51 \mathrm{mmol})$ was added all at once. The reaction mixture was stirred for $15 \mathrm{~min}$ at $0^{\circ} \mathrm{C}$ under $\mathrm{N}_{2}$. Solvent was removed in vacuo and the resulting solid was washed with water $(50 \mathrm{~mL})$ and filtered. The solid was then recrystallized in ethanol ( $3 \mathrm{~mL}$ ) and water $(30 \mathrm{~mL})$. The crystals were filtered through vacuum filtration and off white solid was obtained $(1.65 \mathrm{~g}, 90 \%){ }^{1} \mathrm{H} \mathrm{NMR}\left(400 \mathrm{MHz}, \mathrm{CD}_{3} \mathrm{Cl}\right): \delta=9.49\left(\mathrm{~s}, 1 \mathrm{H}, \mathrm{H}_{\mathrm{b}}\right), 7.13\left(\mathrm{~s}, 1 \mathrm{H}, \mathrm{H}_{\mathrm{a}}\right), 6.98\left(\mathrm{~s}, 1 \mathrm{H}, \mathrm{H}_{\mathrm{d}}\right)$. The remainder of the characterization was also consistent with previously reported literature. ${ }^{73}$<smiles>CN(C)C1c2cc(Br)cn2C(N(C)C)c2cc(Br)cn21</smiles>

Synthesis of 3-bromo-6-(dimethyamino)-1-azafulvene (4.15): A solution of 4-bromo-1H-pyrrole-2carbaldehyde $(0.200 \mathrm{~g}, 1.15 \mathrm{mmol})$ in aqueous dimethylamine was stirred at room temperature for $3.5 \mathrm{~h}$ and then diluted with an equal volume of water. The solid was collected by filtration, washed with ethyl acetate and dried in vacuo. The crude product was recrystallized from ether-ethyl acetate (9:1) to give a brown solid $(0.365 \mathrm{~g}, 78 \%){ }^{1} \mathrm{H} \mathrm{NMR}\left(400 \mathrm{MHz}, \mathrm{CDCl}_{3}\right): \delta=6.96\left(\mathrm{~s}, 2 \mathrm{H}, \mathrm{H}_{\mathrm{c}}\right), 6.22\left(\mathrm{~s}, 2 \mathrm{H}, \mathrm{H}_{\mathrm{d}}\right), 5.77\left(\mathrm{~s}, 2 \mathrm{H}, \mathrm{H}_{\mathrm{b}}\right)$, $2.26\left(\mathrm{~s}, 12 \mathrm{H}, \mathrm{H}_{\mathrm{a}}\right)$. The remainder of the characterization was also consistent with previously reported literature. ${ }^{73}$

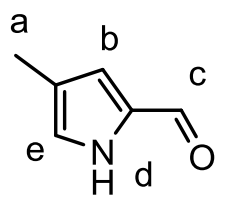

Synthesis of 4-methyl-1H-pyrrole-2-carbaldehyde (4.16): A solution of 1.7 M $t$-butyllithium in pentane (5.85 $\mathrm{mL}, 9.95 \mathrm{mmol})$ was added dropwise to a stirred solution of 3-bromo-6-(dimethyamino)-1azafulvene $(1.00 \mathrm{~g}, 2.49 \mathrm{mmol})$ in anhydrous THF $\left(40 \mathrm{~mL}, \mathrm{~N}_{2}\right.$ atmosphere $)$ at $-78^{\circ} \mathrm{C}$. The yellow coloured solution was stirred for $0.5 \mathrm{~h}$ at $-78^{\circ} \mathrm{C}$ and then methyl iodide $(0.619 \mathrm{~mL}, 9.95 \mathrm{mmol})$ was added. The reaction temperature was left to rise to $-50^{\circ} \mathrm{C}$ during $1 \mathrm{~h}$ and then stirred at room temperature for $0.5 \mathrm{~h}$. Water $(40 \mathrm{~mL})$ and saturated aqueous sodium bicarbonate $(40 \mathrm{~mL})$ were added and the mixture was heated at reflux for $15 \mathrm{~h}$. The mixture was poured into water $(150 \mathrm{~mL})$, extracted with $\mathrm{DCM}(50 \mathrm{~mL})$ and the mixture was then dried using $\mathrm{MgSO}_{4}$. The crude product was purified using a silica column (6:1 Hexane/EtOAc). A mixture of 4-methyl-1H-pyrrole-2-carbaldehyde/pyrrole-2-carbaldehyde (80:20) with an overall yield of $31 \%(0.163 \mathrm{~g})$ for the desired product . ${ }^{1} \mathrm{H} \mathrm{NMR}\left(400 \mathrm{MHz}, \mathrm{CDCl}_{3}\right): \delta=10.37$ (broad s, $\left.1 \mathrm{H}, \mathrm{H}_{\mathrm{d}}\right), 9.42\left(\mathrm{~s}, 1 \mathrm{H}, \mathrm{H}_{\mathrm{c}}\right), 6.97\left(\mathrm{~s}, 1 \mathrm{H}, \mathrm{H}_{\mathrm{b}}\right), 6.82\left(\mathrm{~s}, 1 \mathrm{H}, \mathrm{H}_{\mathrm{e}}\right), 2.15\left(\mathrm{~s}, 3 \mathrm{H}, \mathrm{H}_{\mathrm{a}}\right)$. The remainder of the characterization was also consistent with previously reported literature. ${ }^{73}$ 


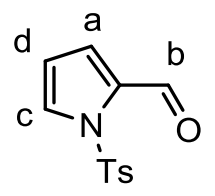

Synthesis of 1-tosyl-1H-pyrrole-2-carbaldehyde (4.17): A solution of 1H-pyrrole-2-carbaldehyde 4.13 (5.0 $\mathrm{g}, 52.6 \mathrm{mmol})$ in THF $(80 \mathrm{~mL})$ was prepared, $\mathrm{NaH}(2.31 \mathrm{~g}, 57.8 \mathrm{mmol})$ was added slowly at $0{ }^{\circ} \mathrm{C}$. After the mixture had been stirred at this temperature for $30 \mathrm{~min}$, tosyl chloride $(4.208 \mathrm{~g}, 55.2 \mathrm{mmol})$ in $20 \mathrm{~mL}$ of THF was added dropwise. The reaction mixture was stirred at $0{ }^{\circ} \mathrm{C}$ for $2 \mathrm{~h}$ and then diluted with ethyl acetate $(200 \mathrm{~mL})$. The organic phase was washed with water $(100 \mathrm{~mL})$ and brine $(50 \mathrm{~mL})$, dried over $\mathrm{Na}_{2} \mathrm{SO}_{4}$, and evaporated in vacuo. The residue was purified by silica gel column chromatography eluting with 8:2 hexanes/ethyl acetate ether to give $4.17(11 \mathrm{~g}, 82 \%)$ as a white solid: ${ }^{1} \mathrm{H} \mathrm{NMR}\left(400 \mathrm{MHz}, \mathrm{CDCl}_{3}\right)$ $\delta 2.42\left(\mathrm{~s}, 3 \mathrm{H}, \mathrm{H}_{\mathrm{Ts}}\right), 6.40\left(\mathrm{t},{ }^{3} \mathrm{~J}=3.6 \mathrm{~Hz}, 1 \mathrm{H}, \mathrm{H}_{\mathrm{c}}\right), 7.16\left(\mathrm{~m}, 1 \mathrm{H}, \mathrm{H}_{\mathrm{c}}\right), 7.32\left(\mathrm{~d},{ }^{3} \mathrm{~J}=8.0 \mathrm{~Hz}, 2 \mathrm{H}, \mathrm{H}_{\mathrm{Ts}}\right), 7.62(\mathrm{~m}, 1 \mathrm{H}$, $\left.\mathrm{H}_{\mathrm{a}}\right), 7.82\left(\mathrm{~d}, \mathrm{~J}=8.0 \mathrm{~Hz}, 2 \mathrm{H}, \mathrm{H}_{\mathrm{Ts}}\right), 9.97\left(\mathrm{~s}, 1 \mathrm{H}, \mathrm{H}_{\mathrm{b}}\right)$. The remainder of the characterization was also consistent with previously reported literature ${ }^{74}$

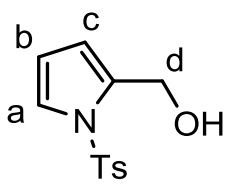

Synthesis of (1-tosyl-1H-pyrrol-2-yl)methanol (4.18): A solution of $4.17(1 \mathrm{~g}, 4.01 \mathrm{mmol})$ in $20 \mathrm{ml}$ methanol cooled to $0^{\circ} \mathrm{C}$ and $0.201 \mathrm{mg}(6.00 \mathrm{mmol}) \mathrm{NaBH}_{4}$ was added. The mixture was stirred overnight at room temperature, then water was added $(50 \mathrm{ml})$ and the solution was extracted with dichloromethane, dried over $\mathrm{MgSO}_{4}$, filtered and evaporated in vacuo. The residue was recrystallized in hexanes afforded 4.18 as a beige solid $(0.956 \mathrm{~g}, 94 \%):{ }^{1} \mathrm{H} \mathrm{NMR}\left(400 \mathrm{MHz}, \mathrm{CDCl}_{3}\right) \delta 7.73\left(\mathrm{~d},{ }^{3} \mathrm{~J}=8.3 \mathrm{~Hz}, 2 \mathrm{H}\right.$, $\left.\mathrm{H}_{\mathrm{Ts}}\right), 7.33\left(\mathrm{~d},{ }^{3} \mathrm{~J}=8.5 \mathrm{~Hz}, 2 \mathrm{H}, \mathrm{H}_{\mathrm{Ts}}\right), 7.29\left(\mathrm{dd},{ }^{3} \mathrm{~J}=3.4,{ }^{4} \mathrm{~J}=1.6 \mathrm{~Hz}, 1 \mathrm{H}, \mathrm{H}_{\mathrm{a}}\right), 6.30-6.23\left(\mathrm{~m}, 2 \mathrm{H}, \mathrm{H}_{\mathrm{b}}, \mathrm{H}_{\mathrm{c}}\right), 4.62(\mathrm{~s}$, $\left.2 \mathrm{H}, \mathrm{H}_{\mathrm{d}}\right), 2.71$ (broad s, $\left.1 \mathrm{H}, \mathrm{H}_{\mathrm{OH}}\right), 2.43\left(\mathrm{~s}, 3 \mathrm{H}, \mathrm{H}_{\mathrm{Ts}}\right)$. The remainder of the characterization was also consistent with previously reported literature. The remainder of the characterization was also consistent with previously reported literature. ${ }^{75}$

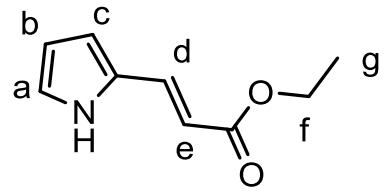

Synthesis of ethyl (E)-3-(1H-pyrrol-2-yl)acrylate (4.19): A solution of pyrrole-2-carbaldehyde 4.13 (0.5 g, $8.02 \mathrm{mmol}$ ) in $60 \mathrm{ml}$ was prepared, the Wittig reagent (carbethoxymethylene) triphenyl phosphorance $(3.75 \mathrm{~g}, 10.52 \mathrm{mmol})$ was added at r.t. After stirring for 1 day, the solution was poured into a mixture of diethyl ether and $\mathrm{H}_{2} \mathrm{O}(200 \mathrm{~mL})$. The organic was separated and washed with water $(2 \times 50 \mathrm{~mL})$ and brine $(20 \mathrm{~mL})$, dried over $\mathrm{MgSO}$, and concentrated in vacuo. The residue was purified via column chromatography eluted with DCM to yield 4.19 as a pink solid $(0.530 \mathrm{~g}, 40 \%) .{ }^{1} \mathrm{H}$ NMR $\left(400 \mathrm{MHz}, \mathrm{CDCl}_{3}\right) \delta$ $8.73\left(\mathrm{~s}, 1 \mathrm{H}, \mathrm{H}_{\mathrm{NH}}\right), 7.58\left(\mathrm{~d},{ }^{3} \mathrm{~J}=15.9 \mathrm{~Hz}, 1 \mathrm{H}, \mathrm{H}_{\mathrm{e}}\right), 6.95\left(\mathrm{~d},{ }^{3} \mathrm{~J}=1.2 \mathrm{~Hz}, 1 \mathrm{H}, \mathrm{H}_{\mathrm{c}}\right), 6.58\left(\mathrm{~s}, 1 \mathrm{H}, \mathrm{H}_{\mathrm{a}}\right), 6.30\left(\mathrm{dd},{ }^{3} \mathrm{~J}=6.0,{ }^{4} \mathrm{~J}=2.6\right.$ $\left.\mathrm{Hz}, 1 \mathrm{H}, \mathrm{H}_{\mathrm{b}}\right), 6.03\left(\mathrm{~d},{ }^{3} \mathrm{~J}=15.9 \mathrm{~Hz}, 1 \mathrm{H}, \mathrm{Hd}_{\mathrm{d}}\right), 4.26\left(\mathrm{q},{ }^{3} \mathrm{~J}=7.1 \mathrm{~Hz}, 2 \mathrm{H}, \mathrm{H}_{\mathrm{f}}\right), 1.34\left(\mathrm{t},{ }^{3} \mathrm{~J}=7.1 \mathrm{~Hz}, 3 \mathrm{H}, \mathrm{H}_{\mathrm{g}}\right)$. The remainder of the characterization was also consistent with previously reported literature. ${ }^{76}$ 


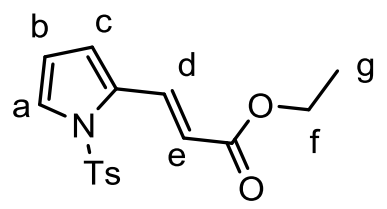

Synthesis of ethyl (E)-3-(1-tosyl-1H-pyrrol-2-yl)acrylate (4.20): A solution of $4.18(2 \mathrm{~g}, 8.02 \mathrm{mmol})$ in 60 $\mathrm{ml}$ was prepared, the Wittig reagent (carbethoxymethylene) triphenyl phosphorance $(3.50 \mathrm{~g}, 9.63 \mathrm{mmol})$ was added at r.t. After stirring for 1 day, the solution was poured into a mixture of diethyl ether and $\mathrm{H}_{2} \mathrm{O}$ $(200 \mathrm{~mL})$. The organic was separated and washed with water $(2 \times 50 \mathrm{~mL})$ and brine $(20 \mathrm{~mL})$, dried over $\mathrm{MgSO}_{4}$, and concentrated in vacuo. The residue was purified via column chromatography eluted with DCM to yield 4.20 as a beige solid (1.26 g, 50\%): ${ }^{1} \mathrm{H} \mathrm{NMR}\left(400 \mathrm{MHz}, \mathrm{CDCl}_{3}\right) \delta 8.10\left(\mathrm{~d},{ }^{3} \mathrm{~J}=15.8 \mathrm{~Hz}, 1 \mathrm{H}, \mathrm{He}_{\mathrm{e}}\right), 7.74\left(\mathrm{~d},{ }^{3} \mathrm{~J}=\right.$ $\left.8.0 \mathrm{~Hz}, 2 \mathrm{H}, \mathrm{H}_{\mathrm{Ts}}\right), 7.48\left(\mathrm{~s}, 1 \mathrm{H}, \mathrm{H}_{\mathrm{c}}\right), 7.31\left(\mathrm{~d},{ }^{3} \mathrm{~J}=7.9 \mathrm{~Hz}, 2 \mathrm{H}, \mathrm{H}_{\mathrm{Ts}}\right), 6.70\left(\mathrm{~s}, 1 \mathrm{H}, \mathrm{H}_{\mathrm{a}}\right), 6.32\left(\mathrm{~s}, 1 \mathrm{H}, \mathrm{H}_{\mathrm{b}}\right), 6.15(\mathrm{~d}, 3 \mathrm{~J}=15.8 \mathrm{~Hz}$, $\left.1 \mathrm{H}, \mathrm{H}_{\mathrm{d}}\right), 4.26\left(\mathrm{q},{ }^{3} \mathrm{~J}=7.1 \mathrm{~Hz}, 2 \mathrm{H}, \mathrm{H}_{\mathrm{f}}\right), 2.41\left(\mathrm{~s}, 3 \mathrm{H}, \mathrm{H}_{\mathrm{s}}\right), 1.35\left(\mathrm{t},{ }^{3} \mathrm{~J}=7.0 \mathrm{~Hz}, 3 \mathrm{H}, \mathrm{H}_{\mathrm{g}}\right)$. The remainder of the characterization was also consistent with previously reported literature ${ }^{77}$

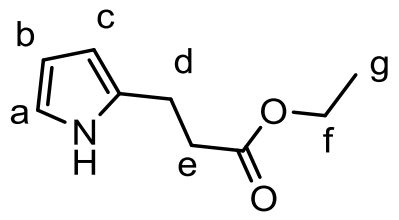

Synthesis of ethyl 3-(1H-pyrrol-2-yl)propanoate (4.21): A solution of 4.19 (1.40 g, $8.475 \mathrm{mmol})$ in $\mathrm{MeOH}$ $(60 \mathrm{~mL})$ was placed under at nitrogen. $\mathrm{Pd} / \mathrm{C}(0.150 \mathrm{~g}, 10 \%)$ was added, followed by replacement of the nitrogen with a hydrogen atmosphere. The reaction was then stirred at r.t. until completion as indicated by ${ }^{1} \mathrm{H}$ NMR analysis (16 h). The crude mixture was filtered over a bed of Celite, and the Celite plug was eluted further with an aliquot of $\mathrm{MeOH}(50 \mathrm{~mL})$. The combined $\mathrm{MeOH}$ washes were then concentrated in vacuo to afford 4.21 (1.40 g, 98\%) as a pale yellow oil. $\left.{ }^{1} \mathrm{H} \mathrm{NMR} \mathrm{(400} \mathrm{MHz,} \mathrm{CDCl} 3\right) \delta 8.59\left(\mathrm{~s}, 1 \mathrm{H}, \mathrm{H}_{\mathrm{NH}}\right), 6.69$ $\left(\mathrm{dd},{ }^{3} \mathrm{~J}=4.2,{ }^{4} \mathrm{~J}=2.6 \mathrm{~Hz}, 1 \mathrm{H}, \mathrm{H}_{\mathrm{a}}\right), 6.15-6.08\left(\mathrm{~m}, 1 \mathrm{H}, \mathrm{H}_{\mathrm{b}}\right), 5.94\left(\mathrm{~m}, 1 \mathrm{H}, \mathrm{H}_{\mathrm{c}}\right), 4.18\left(\mathrm{q},{ }^{3} \mathrm{~J}=7.1 \mathrm{~Hz}, 2 \mathrm{H}, \mathrm{H}_{\mathrm{f}}\right), 2.94\left(\mathrm{t},{ }^{3} \mathrm{~J}=6.8\right.$ $\left.\mathrm{Hz}, 2 \mathrm{H}, \mathrm{He}_{\mathrm{e}}\right), 2.65\left(\mathrm{t},{ }^{3} \mathrm{~J}=6.8 \mathrm{~Hz}, 2 \mathrm{H}, \mathrm{Hd}\right), 1.29\left(\mathrm{t},{ }^{3} \mathrm{~J}=7.1 \mathrm{~Hz}, 3 \mathrm{H}, \mathrm{H}_{\mathrm{g}}\right)$. The remainder of the characterization was also consistent with previously reported literature. ${ }^{76}$

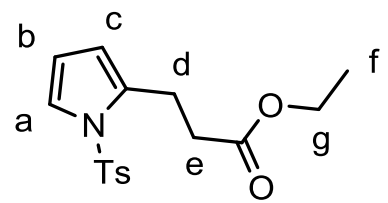

Synthesis of ethyl 3-(1-tosyl-1H-pyrrol-2-yl)propanoate (4.22): A solution of 4.20(0.400 g, $1.25 \mathrm{mmol})$ in $\mathrm{MeOH}(60 \mathrm{~mL})$ was placed under at nitrogen. $\mathrm{Pd} / \mathrm{C}(0.013 \mathrm{~g}, 10 \%)$ was added, followed by replacement of the nitrogen with a hydrogen atmosphere. The reaction was then stirred at r.t. until completion as indicated by ${ }^{1} \mathrm{H}$ NMR analysis (16 h). The crude mixture was filtered over a bed of Celite, and the Celite plug was eluted further with an aliquot of $\mathrm{MeOH}(50 \mathrm{~mL})$. The combined $\mathrm{MeOH}$ washes were then concentrated in vacuo to afford $4.22(0.390 \mathrm{~g}, \sim 98 \%)$ as a white solid. ${ }^{1} \mathrm{H} \mathrm{NMR}\left(400 \mathrm{MHz}, \mathrm{CDCl}_{3}\right) \delta 7.68\left(\mathrm{~d},{ }^{3} \mathrm{~J}\right.$ $\left.=8.4 \mathrm{~Hz}, 2 \mathrm{H}, \mathrm{H}_{\mathrm{Ts}}\right), 7.31\left(\mathrm{~m}, 3 \mathrm{H}, \mathrm{H}_{\mathrm{a}}, \mathrm{H}_{\mathrm{Ts}}\right), 6.21\left(\mathrm{t},{ }^{3} \mathrm{~J}=3.3 \mathrm{~Hz}, 1 \mathrm{H}, \mathrm{H}_{\mathrm{b}}\right), 6.02\left(\mathrm{~s}, 1 \mathrm{H}, \mathrm{H}_{\mathrm{c}}\right), 4.14\left(\mathrm{q},{ }^{3} \mathrm{~J}=7.1 \mathrm{~Hz}, 2 \mathrm{H}, \mathrm{H}_{\mathrm{f}}\right), 3.03$ $\left(\mathrm{t},{ }^{3} \mathrm{~J}=7.5 \mathrm{~Hz}, 2 \mathrm{H}, \mathrm{He}_{\mathrm{e}}\right), 2.65-2.59\left(\mathrm{~m}, 2 \mathrm{H}, \mathrm{H}_{\mathrm{d}}\right), 2.43\left(\mathrm{~s}, 3 \mathrm{H}, \mathrm{H}_{\mathrm{Ts}}\right), 1.25\left(\mathrm{t},{ }^{3} \mathrm{~J}=7.1 \mathrm{~Hz}, 3 \mathrm{H}, \mathrm{H}_{\mathrm{f}}\right)$. The remainder of the characterization was also consistent with previously reported literature ${ }^{77}$ 


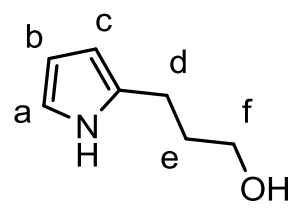

Synthesis of 3-(1H-pyrrol-2-yl)propan-1-ol (4.23): A solution of 4.21 (1.1 g, $6.57 \mathrm{mmol})$ in THF in $130 \mathrm{~mL}$ was prepared. This solution was cooled to $0{ }^{\circ} \mathrm{C}$ and then treated by the slow addition of $1.0 \mathrm{M} \mathrm{LiAlH}_{4} \mathrm{THF}$ solution $(14 \mathrm{~mL})$. The resulting the suspension was allowed to warm up to r.t. and stirred until completion as indicated by TLC analysis $(24 \mathrm{~h})$. The reaction mixture was then quenched with the dropwise addition of $1 \mathrm{M}$ aqueous $\mathrm{NaOH}$ until $\mathrm{pH}$ was neutral. The organic layer was decanted off and the remaining aluminium salts were washed with diethyl ether $(3 \times 50 \mathrm{~mL})$. The combined organic phases were dried over $\mathrm{MgSO}_{4}$, filter and concentrated in vacuo to yield 4.23 (0.810 g, quant.) as brown oil. ${ }^{1} \mathrm{H}$ NMR (400 $\left.\mathrm{MHz}, \mathrm{CDCl}_{3}\right): \delta 8.24$ (broad s, $\left.1 \mathrm{H}, \mathrm{H}_{\mathrm{NH}}\right), 6.69\left(\mathrm{td},{ }^{3} \mathrm{~J}=2.6,{ }^{4} \mathrm{~J}=1.6 \mathrm{~Hz}, 1 \mathrm{H}, \mathrm{H}_{\mathrm{a}}\right), 6.14\left(\mathrm{dd},{ }^{3} \mathrm{~J}=5.7,{ }^{4} \mathrm{~J}=2.8 \mathrm{~Hz}\right.$, $\left.3 \mathrm{H}, \mathrm{H}_{\mathrm{b}}\right), 5.95-5.93\left(\mathrm{~m}, 1 \mathrm{H}, \mathrm{H}_{\mathrm{c}}\right), 3.73\left(\mathrm{t},{ }^{3} \mathrm{~J}=6.3 \mathrm{~Hz}, 2 \mathrm{H}, \mathrm{H}_{\mathrm{f}}\right), 2.75\left(\mathrm{t},{ }^{3} \mathrm{~J}=6.6 \mathrm{~Hz}, 2 \mathrm{H}, \mathrm{H}_{\mathrm{d}}\right), 1.93-1.87\left(\mathrm{~m}, 2 \mathrm{H}, \mathrm{H}_{\mathrm{e}}\right)$. The remainder of the characterization was also consistent with previously reported literature ${ }^{76}$

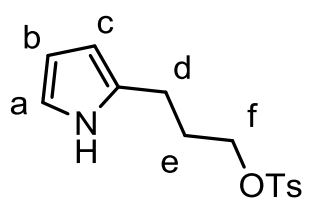

Synthesis of 3-(1H-pyrrol-2-yl)propyl 4-methylbenzenesulfonate (4.24): A solution of 4.23 (1.2 g, 9.58 mmol) in DCM $(60 \mathrm{~mL})$ was treated sequentially with $\mathrm{NEt}_{3}(2.67 \mathrm{~mL}, 19.17 \mathrm{mmol})$ and Trifluoromethanesulfonic anhydride $(1.85 \mathrm{ml}, 11.0 \mathrm{mmol})$ at $0{ }^{\circ} \mathrm{C}$. The resulting reaction mixture was stirred at $0{ }^{\circ} \mathrm{C}$ for $1 \mathrm{~h}$ before being allowed to warm up to r.t. at which point, TLC analysis indicated reaction completion. The reaction was then transferred to a separating funnel and was washed with $1 \mathrm{M} \mathrm{HCl}(20$ $\mathrm{mL})$, followed by aqueous saturated $\mathrm{NaHCO}_{3}(20 \mathrm{~mL})$ and brine $(20 \mathrm{~mL})$. The solution was dried over $\mathrm{Na}_{2} \mathrm{SO}_{4}$, filtered and concentrated in vacuo. The residue was purified via column chromatography using 9/1 hexanes/ethyl acetate to afford 4.24 ( $0.730 \mathrm{~g}, 28 \%$ ) as yellow oil. ${ }^{1} \mathrm{H} \mathrm{NMR}\left(400 \mathrm{MHz}, \mathrm{CDCl}_{3}\right) \delta 8.20(\mathrm{~s}, 1 \mathrm{H}$, $\left.\mathrm{H}_{\mathrm{NH}}\right), 7.84-7.79\left(\mathrm{~m}, 2 \mathrm{H}, \mathrm{H}_{\mathrm{Ts}}\right), 7.41-7.35\left(\mathrm{~m}, 2 \mathrm{H}, \mathrm{H}_{\mathrm{Ts}}\right), 6.65\left(\mathrm{dd},{ }^{3} \mathrm{~J}=2.6,{ }^{4} \mathrm{~J}=1.1 \mathrm{~Hz}, 1 \mathrm{H}, \mathrm{H}_{\mathrm{a}}\right), 6.10\left(\mathrm{~d},{ }^{3} \mathrm{~J}=3.0 \mathrm{~Hz}, 1 \mathrm{H}\right.$, $\left.\mathrm{H}_{\mathrm{c}}\right), 5.93-5.70\left(\mathrm{~m}, 1 \mathrm{H}, \mathrm{H}_{\mathrm{b}}\right), 4.08\left(\mathrm{t},{ }^{3} \mathrm{~J}=6.1 \mathrm{~Hz}, 2 \mathrm{H}, \mathrm{He}_{\mathrm{e}}\right), 2.68\left(\mathrm{t},{ }^{3} \mathrm{~J}=7.3 \mathrm{~Hz}, 2 \mathrm{H}, \mathrm{H}_{\mathrm{d}}\right), 2.48\left(\mathrm{~s}, 3 \mathrm{H}, \mathrm{H}_{\mathrm{s}}\right), 2.02-1.92(\mathrm{~m}$, $2 \mathrm{H}, \mathrm{H}_{\mathrm{e}}$ ). The remainder of the characterization was also consistent with previously reported literature ${ }^{76}$

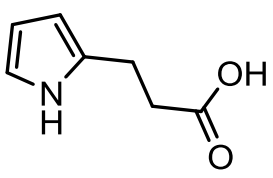

Synthesis of 3-(1H-pyrrol-2-yl)propanoic acid (4.25): A solution of $4.21(0.20 \mathrm{~g}, 1.19 \mathrm{mmol})$ in EtOH/ $\mathrm{H}_{2} \mathrm{O}$ $(8 / 4 \mathrm{~mL})$ was prepared, $\mathrm{NaOH}(0.100 \mathrm{~g}, 2.50 \mathrm{mmol})$ was added in portions at $0-5^{\circ} \mathrm{C}$. The resulting solution was stirred for $5 \mathrm{hr}$ at room temperature, monitored by TLC, and then concentrated in vacuo. After the $\mathrm{pH}$ value of the solution was adjusted to $3-4$ with $\mathrm{HCl}(10 \%)$, it was extracted with $3 \times 100 \mathrm{~mL}$ of ethyl acetate. The combined organic layers were dried over anhydrous magnesium sulfate and concentrated in vacuo, affording $4.25(0.138 \mathrm{~g}, 86 \%)$ as brown solid. No further characterization was done, the product was used as is. 


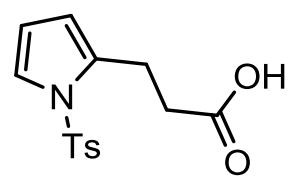

Synthesis of 3-(1-tosyl-1H-pyrrol-2-yl)propanoic acid (4.26): A solution of $4.22(0.350 \mathrm{~g}, 1.09 \mathrm{mmol})$ in $\mathrm{EtOH} / \mathrm{H}_{2} \mathrm{O}(8 / 4 \mathrm{~mL})$ was prepared, $\mathrm{NaOH}(0.080 \mathrm{~g}, 1.96 \mathrm{mmol})$ was added in portions at $0-5^{\circ} \mathrm{C}$. The resulting solution was stirred for $3 \mathrm{hr}$ at room temperature, monitored by TLC, and then concentrated in vacuo. After the $\mathrm{pH}$ value of the solution was adjusted to $3-4$ with $\mathrm{HCl}(10 \%)$, it was extracted with $3 \times 100$ $\mathrm{mL}$ of ethyl acetate. The combined organic layers were dried over anhydrous magnesium sulfate and concentrated in vacuo, affording $4.26(0.185 \mathrm{~g}, 56 \%)$ as brown solid. No further characterization was done, the product was used as is.<smiles>BrCCOc1cccc2c(Br)c3cccc(OCCBr)c3cc12</smiles>

Synthesis of 10-bromo-1,8-bis(2-bromoethoxy)anthracene (4.29): A solution of 4.10 (0.220 g, 0.589 mmol) in THF $40 \mathrm{ml}$ sparaged for 15 minutes with $\mathrm{N}_{2}$. To this mixture, a $1 \mathrm{M}$ aqueous $\mathrm{NaOH}(2 \mathrm{~mL})$ was added and stirred under nitrogen. The solution turned deep yellow and then bright red and then back to yellow. The reaction was monitored by TLC. At completion, the reaction was quenched with $\mathrm{HCl}$ to neutral $\mathrm{pH}$. The mixture was then washed with $0.1 \mathrm{M} \mathrm{HCl}(50 \mathrm{~mL})$ to further quench the reaction and extracted with diethyl ether. The mixture was then filtered and passed through a short silica plug using EtOAc as eluent to purify the crude 4.12. A solution of residue 4.12 in DMF $(10 \mathrm{~mL}) / \mathrm{DBE}(10 \mathrm{~mL})$ was stirred at r.t. for 10 mins, $\mathrm{Cs}_{2} \mathrm{CO}_{3}$ was added at once and the reaction was heated at $100^{\circ} \mathrm{C}$ under nitrogen overnight. The product was extracted with EtOAc and then washed with water $(3 \times 50 \mathrm{~mL})$, followed by aqueous saturated $\mathrm{NaHCO}_{3}(20 \mathrm{~mL})$ and brine $(20 \mathrm{~mL})$. The combined organic layers were dried over anhydrous magnesium sulfate and concentrated in vacuo. The residue was purified via column chromatography using $7 / 3$ hexanes/ethyl acetate to afford $4.29(0.058 \mathrm{~g}, 19 \%)$ as green solid. ${ }^{1} \mathrm{H} \mathrm{NMR}\left(400 \mathrm{MHz}, \mathrm{CDCl}_{3}\right) \delta 9.47$ $(\mathrm{s}, 1 \mathrm{H}), 8.12\left(\mathrm{~d},{ }^{3} \mathrm{~J}=8.9 \mathrm{~Hz}, 2 \mathrm{H}\right), 7.55-7.48(\mathrm{~m}, 2 \mathrm{H}), 6.81\left(\mathrm{~d},{ }^{3} \mathrm{~J}=7.5 \mathrm{~Hz}, 2 \mathrm{H}\right), 4.58\left(\mathrm{t},{ }^{3} \mathrm{~J}=6.2 \mathrm{~Hz}, 4 \mathrm{H}\right), 3.89$ $\left(t,{ }^{3} \mathrm{~J}=6.2 \mathrm{~Hz}, 4 \mathrm{H}\right)$. No further characterization was done due to the exploratory nature of this molecule

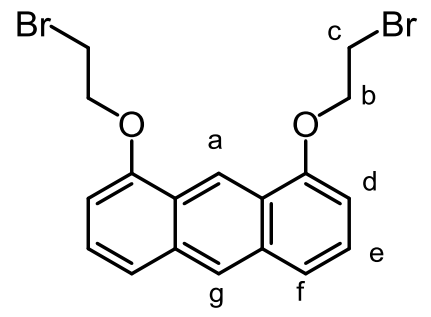


Synthesis of 1,8-bis(2-bromoethoxy)anthracene (4.30): A solution of $4.8(0.500 \mathrm{~g}, 1.69 \mathrm{mmol})$ in THF 40 $\mathrm{ml}$ sparaged for 15 minutes with $\mathrm{N}_{2}$. To this mixture, a $1 \mathrm{M}$ aqueous $\mathrm{NaOH}(10 \mathrm{~mL})$ was added and stirred under nitrogen. The solution turned deep yellow and then bright red and then back to yellow. The reaction was monitored by TLC. At completion, the reaction was quenched with aqueous $\mathrm{HCl}$ to neutral $\mathrm{pH}$. The mixture was then washed with $0.1 \mathrm{M} \mathrm{HCl}(50 \mathrm{~mL})$ to further quench the reaction and extracted with diethyl ether. The mixture was then filtered and passed through a short silica plug using EtOAc as eluent to purify the crude. A solution of residue in DMF $(10 \mathrm{~mL}) / \mathrm{DBE}(10 \mathrm{~mL})$ was stirred at r.t. for $10 \mathrm{mins}, \mathrm{Cs}_{2} \mathrm{CO}_{3}$ was added at once and the reaction was heated at $100^{\circ} \mathrm{C}$ under nitrogen overnight. The product was extracted with EtOAc and then washed with water $(3 \times 50 \mathrm{~mL})$, followed by aqueous saturated $\mathrm{NaHCO}_{3}(20 \mathrm{~mL})$ and brine $(20 \mathrm{~mL})$. The combined organic layers were dried over anhydrous magnesium sulfate and concentrated in vacuo. The residue was purified via column chromatography using DCM to afford 4.30 $(0.068 \mathrm{~g}, 10 \%)$ as green solid. ${ }^{1} \mathrm{H} \mathrm{NMR}\left(400 \mathrm{MHz}, \mathrm{CDCl}_{3}\right) \delta 9.36\left(\mathrm{~s}, 1 \mathrm{H}, \mathrm{H}_{\mathrm{a}}\right), 8.35\left(\mathrm{~s}, 1 \mathrm{H}, \mathrm{H}_{\mathrm{g}}\right), 7.63\left(\mathrm{~d},{ }^{3} \mathrm{~J}=8.5\right.$ $\left.\mathrm{Hz}, 2 \mathrm{H}, \mathrm{H}_{\mathrm{f}}\right), 7.47\left(\mathrm{t}, 2 \mathrm{H}, \mathrm{H}_{\mathrm{e}}\right), 6.77\left(\mathrm{~d},{ }^{3} \mathrm{~J}=7.4 \mathrm{~Hz}, 2 \mathrm{H}, \mathrm{H}_{\mathrm{d}}\right), 4.58\left(\mathrm{t},{ }^{3} \mathrm{~J}=6.3 \mathrm{~Hz}, 4 \mathrm{H}, \mathrm{H}_{\mathrm{b}}\right), 3.90\left(\mathrm{t},{ }^{3} \mathrm{~J}=6.3 \mathrm{~Hz}, 4 \mathrm{H}\right.$, $\mathrm{H}_{c}$ ). No further characterization was done due to the exploratory nature of this molecule

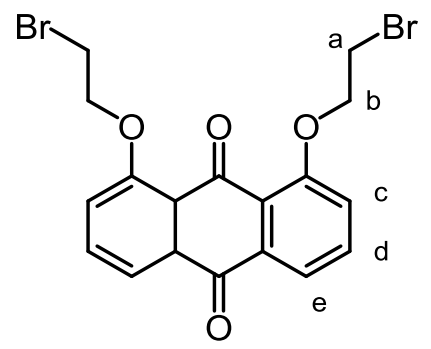

Synthesis of 1,8-bis(2-bromoethoxy)anthraquinone (4.31) A solution of 1,8-dihydoxyanthraquinone (2 g, $8.26 \mathrm{mmol})$ in DMF $(10 \mathrm{~mL}) / \mathrm{DBE}(10 \mathrm{~mL})$ was stirred at r.t. for $10 \mathrm{mins}, \mathrm{Cs}_{2} \mathrm{CO}_{3}$ was added at once and the reaction was heated at $100^{\circ} \mathrm{C}$ under nitrogen overnight. The product was extracted with EtOAc and then washed with water $(3 \times 50 \mathrm{~mL})$, followed by aqueous saturated $\mathrm{NaHCO}_{3}(20 \mathrm{~mL})$ and brine $(20 \mathrm{~mL})$. The combined organic layers were dried over anhydrous magnesium sulfate and concentrated in vacuo. The residue was purified via column chromatography using 6/4 hexanes/EtOAc to afford $4.32(0.216 \mathrm{~g}, 5 \%)$ as yellow solid. ${ }^{1} \mathrm{H} \mathrm{NMR}\left(400 \mathrm{MHz}, \mathrm{CDCl}_{3}\right) \delta 7.91\left(\mathrm{dd},{ }^{3} \mathrm{~J}=7.7,{ }^{4} \mathrm{~J}=0.9 \mathrm{~Hz}, 2 \mathrm{H}, \mathrm{H}_{\mathrm{e}}\right), 7.65\left(\mathrm{t},{ }^{3} \mathrm{~J}=8.0 \mathrm{~Hz}, 2 \mathrm{H}, \mathrm{H}_{\mathrm{d}}\right)$, $7.34\left(\mathrm{~d},{ }^{3} \mathrm{~J}=8.3 \mathrm{~Hz}, 1 \mathrm{H}, \mathrm{H}_{\mathrm{c}}\right), 4.47\left(\mathrm{t},{ }^{3} \mathrm{~J}=6.7 \mathrm{~Hz}, 2 \mathrm{H}, \mathrm{H}_{\mathrm{b}}\right), 3.79\left(\mathrm{t},{ }^{3} \mathrm{~J}=6.7 \mathrm{~Hz}, 2 \mathrm{H}, \mathrm{H}_{\mathrm{a}}\right) .{ }^{13} \mathrm{C}\left\{{ }^{1} \mathrm{H}\right\} \mathrm{NMR}(100 \mathrm{MHz}$, $\left.\mathrm{CDCl}_{3}\right) \delta 183.46,182.03,157.90,134.84,133.93,125.21,121.55,120.49$, 70.39, 28.79.

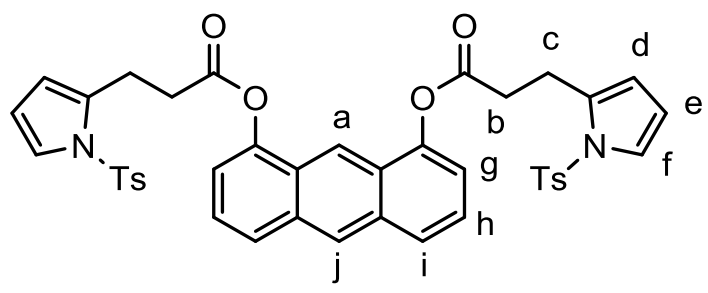

Synthesis of 8-((3-(1-tosyl-1H-pyrrol-2-yl)propanoyl)oxy)anthracen-1-yl-3-(1-tosyl-1H-pyrrol-2yl)propanoate (4.36): A solution of $4.26(0.188 \mathrm{~g}, 0.642 \mathrm{mmol})$ and 1,8 dihydroxyanthracene $(0.045 \mathrm{~g}$, $0.214 \mathrm{mmol})$ in DCM $(20 \mathrm{~mL})$ was prepared. 4-DMAP $(0.008 \mathrm{~g}, 0.064 \mathrm{mmol})$ and DIC $(0.12 \mathrm{~mL}, 0.771 \mathrm{mmol})$ was added to the mixture at r.t. The resulting solution was warmed to $40{ }^{\circ} \mathrm{C}$ and stirred for $2 \mathrm{~h}$. Then the reaction is cooled to r.t., the reaction was stirred for another $1 \mathrm{~h}$. The reaction was filtered through a short 
pad of silica gel and eluted with EtOAc. The filtrate was concentrated and purified by flash column chromatography using 6/4 hexanes/EtOAc as the eluted to afford $4.36(0.065 \mathrm{~g}, 41 \%)$ as green wax. ${ }^{1} \mathrm{H}$ $\operatorname{NMR}\left(400 \mathrm{MHz}, \mathrm{CDCl}_{3}\right) \delta 8.52\left(\mathrm{~s}, 1 \mathrm{H}, \mathrm{H}_{\mathrm{a}}\right), 8.48\left(\mathrm{~s}, 1 \mathrm{H}, \mathrm{H}_{\mathrm{j}}\right), 7.92\left(\mathrm{~d},{ }^{3} \mathrm{~J}=8.6 \mathrm{~Hz}, 2 \mathrm{H}, \mathrm{H}_{\mathrm{g}}\right), 7.69\left(\mathrm{~d},{ }^{3} \mathrm{~J}=8.2 \mathrm{~Hz}\right.$, $\left.4 \mathrm{H}, \mathrm{H}_{\mathrm{Ts}}\right), 7.47\left(\mathrm{t},{ }^{3} \mathrm{~J}=7.9 \mathrm{~Hz}, 2 \mathrm{H}, \mathrm{H}_{\mathrm{h}}\right), 7.35\left(\mathrm{~s}, 2 \mathrm{H}, \mathrm{H}_{\mathrm{f}}\right), 7.25-7.17\left(\mathrm{~m}, 4 \mathrm{H}, \mathrm{H}_{\mathrm{Ts}}\right), 6.26\left(\mathrm{t},{ }^{3} \mathrm{~J}=3.3 \mathrm{~Hz}, 2 \mathrm{H}_{\mathrm{i}}\right), 6.15$ $\left(\mathrm{s}, 2 \mathrm{H}, \mathrm{H}_{\mathrm{e}}\right), 3.25\left(\mathrm{t},{ }^{3} \mathrm{~J}=7.3 \mathrm{~Hz}, 4 \mathrm{H}, \mathrm{H}_{\mathrm{b}}\right), 3.14\left(\mathrm{t},{ }^{3} \mathrm{~J}=7.3 \mathrm{~Hz}, 4 \mathrm{H}, \mathrm{H}_{\mathrm{c}}\right)$. No further characterization was done due to instability of this molecule.

\section{Conclusions and Future work}

Overall, the objectives of this thesis were met with varying success. The development of $\mathrm{CHY} 399$ and $\mathrm{CHY}$ 307 laboratory courses have permitted undergraduate students from multiple fields of science (Table A1) to be exposed to a myriad of practical skills such as Schlenk line techniques, column chromatography, vacuum distillation, advance characterization techniques such as NMR, IR, UV-Vis spectroscopy, cyclic voltammetry, and theoretical calculations (DFT, TD-DFT). Learners also had the opportunity to learn how present scientific data (poster presentation at conferences \&amp; manuscript writing). To this end it has pointed students in a graduate school direction that otherwise would not have considered it. For the 
benefit of the research program, each cohort was able to produce at least $20 \mathrm{~g}$ of BODIPY which is equivalent to $\$ 200,000$ from commercial sources (Sigma Aldrich). In addition, students were able to elucidate a large number of structure property relationships illustrated in Chapter 2 that allowed researchers the opportunity to quickly glean a deeper insight into possible research avenues worth (or not worth) exploring. Furthermore, a manuscript based off the student's work has been submitted and is currently under review. The next step in this lab venture will be to find a new benchmark molecule for students to manipulate and study, such as a metallocene derivative.

Ferrocene derivatives are of particular interests to this thesis research, as we have reported a number of optical and electrochemical insights relating to ferrocene-BODIPY dyes discussed in Chapter 3. Even with the desired panchromatic absorption profile and desired reversible oxidative in this novel family of dyes, the ferrocene BODIPY dyads that were synthesized were surprisingly problematic in that the stability of these dyes and dyads (Figure 3.15) are not yet suitable for materials applications. Further insight regarding these ferrocene stability challenges will be discussed in Jennifer Huynh's thesis).

For the ambitious BODIPY-cyclophane project (Chapter 4) the synthesis leading to the target was more challenging than initially surmised. This was partially due to a number of overtly hopeful synthetic strategies, but also owing to the low oxidation potential of the anthracene building block employed. Instability of a number of key anthracene intermediates led to significant scale up and purification challenges. While the synthesis has not been completed, there is precedent for these types of supramolecular architectures, and with modest changes in the synthetic strategies, this author is still hopeful that such a target could be synthesized. These architectures are predicted to have unique energy transfer properties that exploit confined $\pi-\pi$ interactions, which would enable tuning of the absorption and emission spectra of BODIPY in a unique way. 


\section{Appendix}

Table A1: Student Demographics

Table S1. Student demographics completing Stage 1

(molecules 1-7) (over a 2 year period).

\# of Program of Study Year of Study

\begin{tabular}{|c|c|c|c|}
\hline \multirow{5}{*}{46} & 2 & biology & \multirow{5}{*}{$\begin{array}{l}1 \text { first year } \\
17 \text { second year } \\
14 \text { third year } \\
15 \text { fourth year }\end{array}$} \\
\hline & 4 & physics & \\
\hline & & & \\
\hline & 4 & $\begin{array}{l}\text { cnemical } \\
\text { engineering }\end{array}$ & \\
\hline & 36 & chemistry & \\
\hline
\end{tabular}




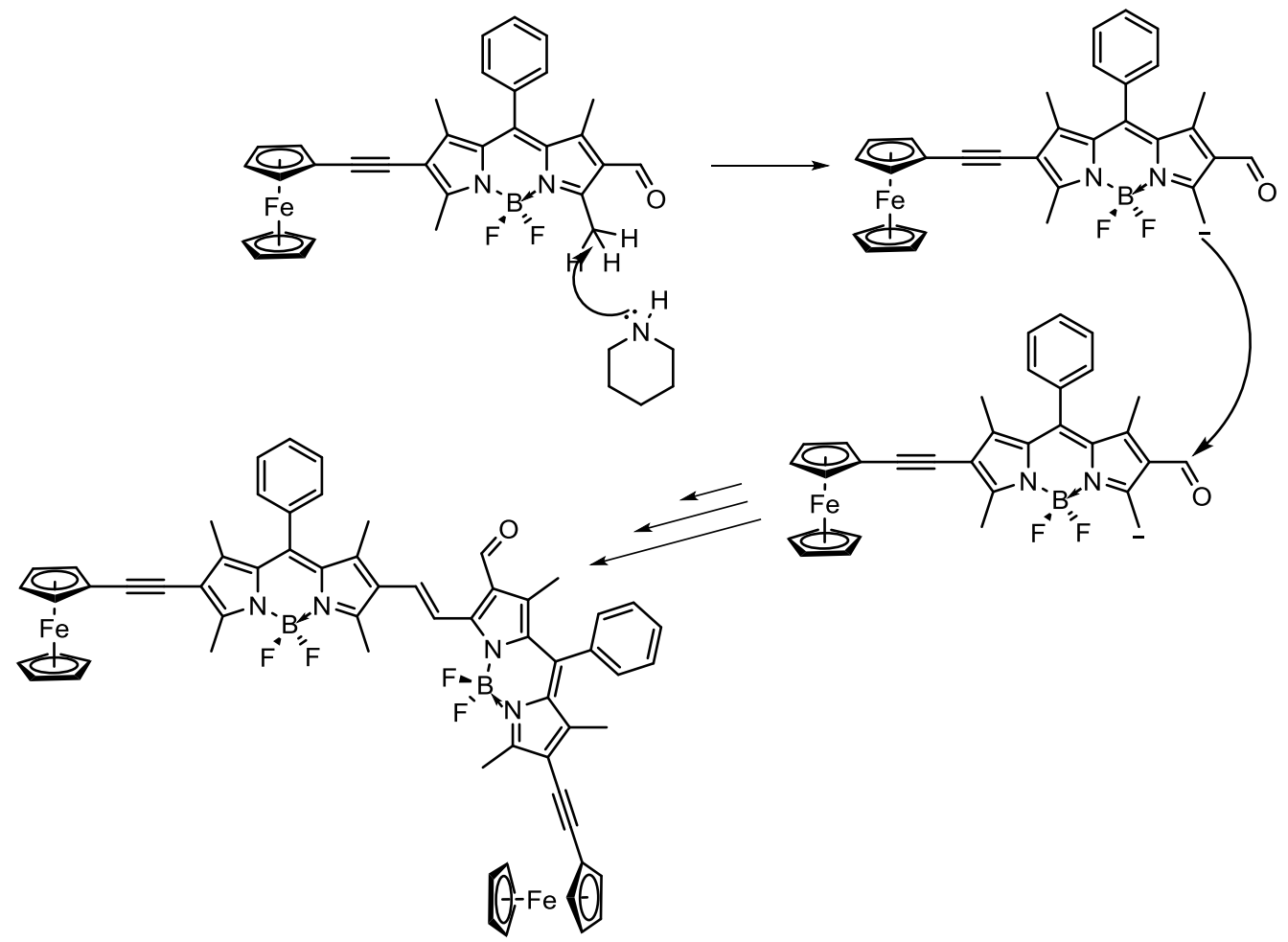

Figure A.1 : Undesired dimerization of BODIPY 3.44a-d

DFT Study of $\mathbf{4 . 6}$

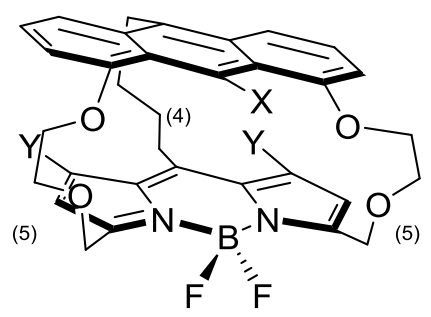

HOMO

LUMO

$X=\mathrm{NO}_{2}$

$\mathrm{Y}=\mathrm{Ph}$
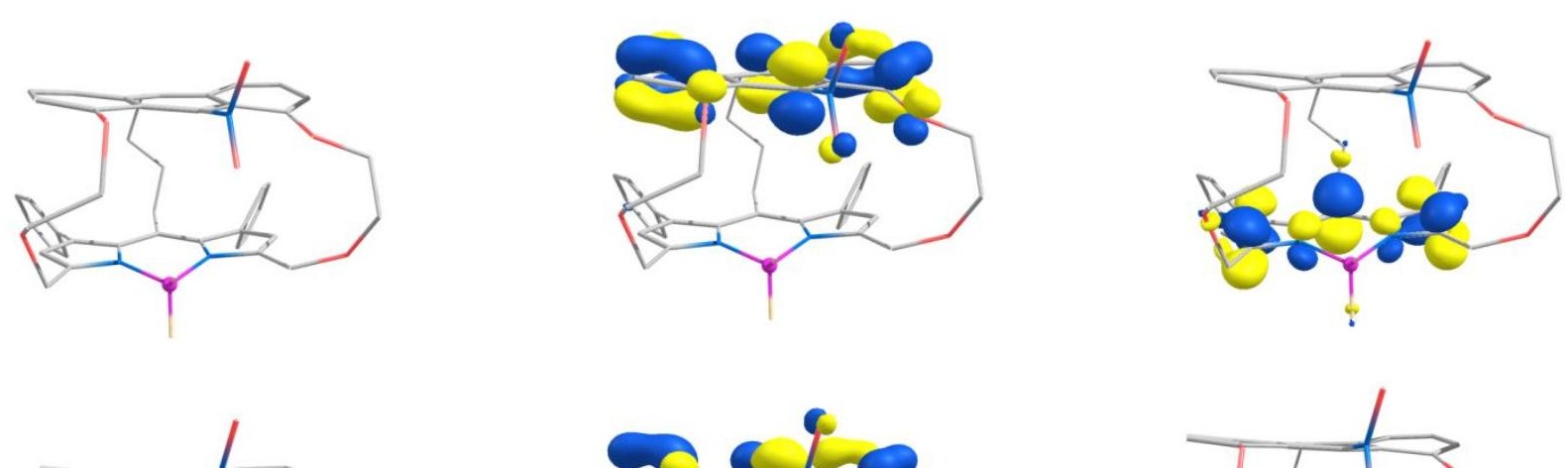


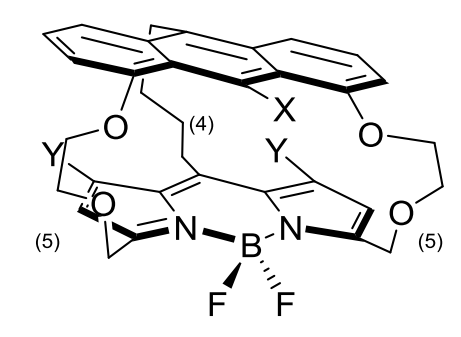

$X=$ OMe

$\mathrm{Y}=\mathrm{Ph}$

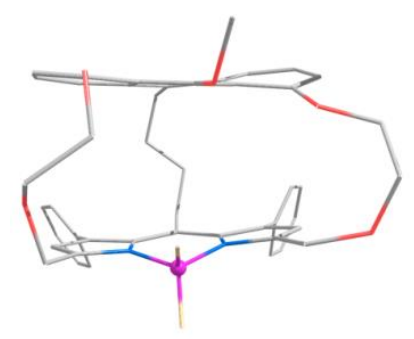

$X=$ OMe

$\mathrm{Y}=\mathrm{OMe}$

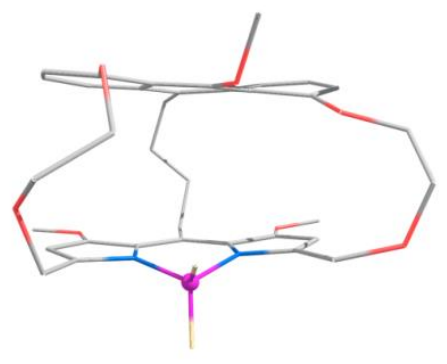

$X=\mathrm{OMe}$
$\mathrm{Y}=\mathrm{CHO}$

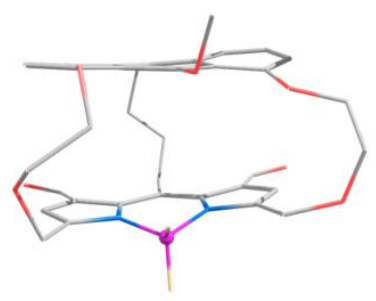

$\mathrm{X}=\mathrm{OMe}$

$\mathrm{Y}=\mathrm{COOMe}$
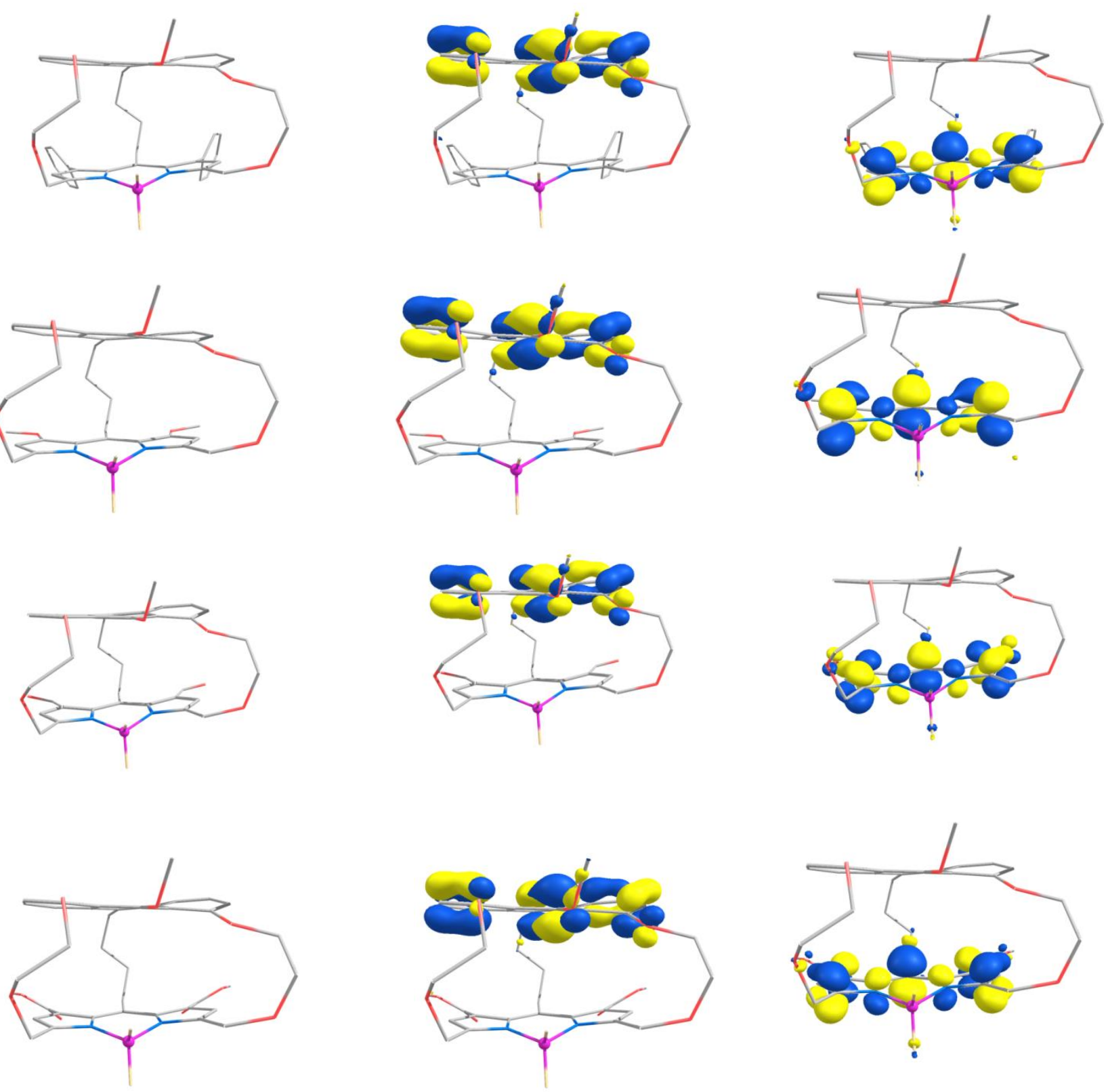

HOMO
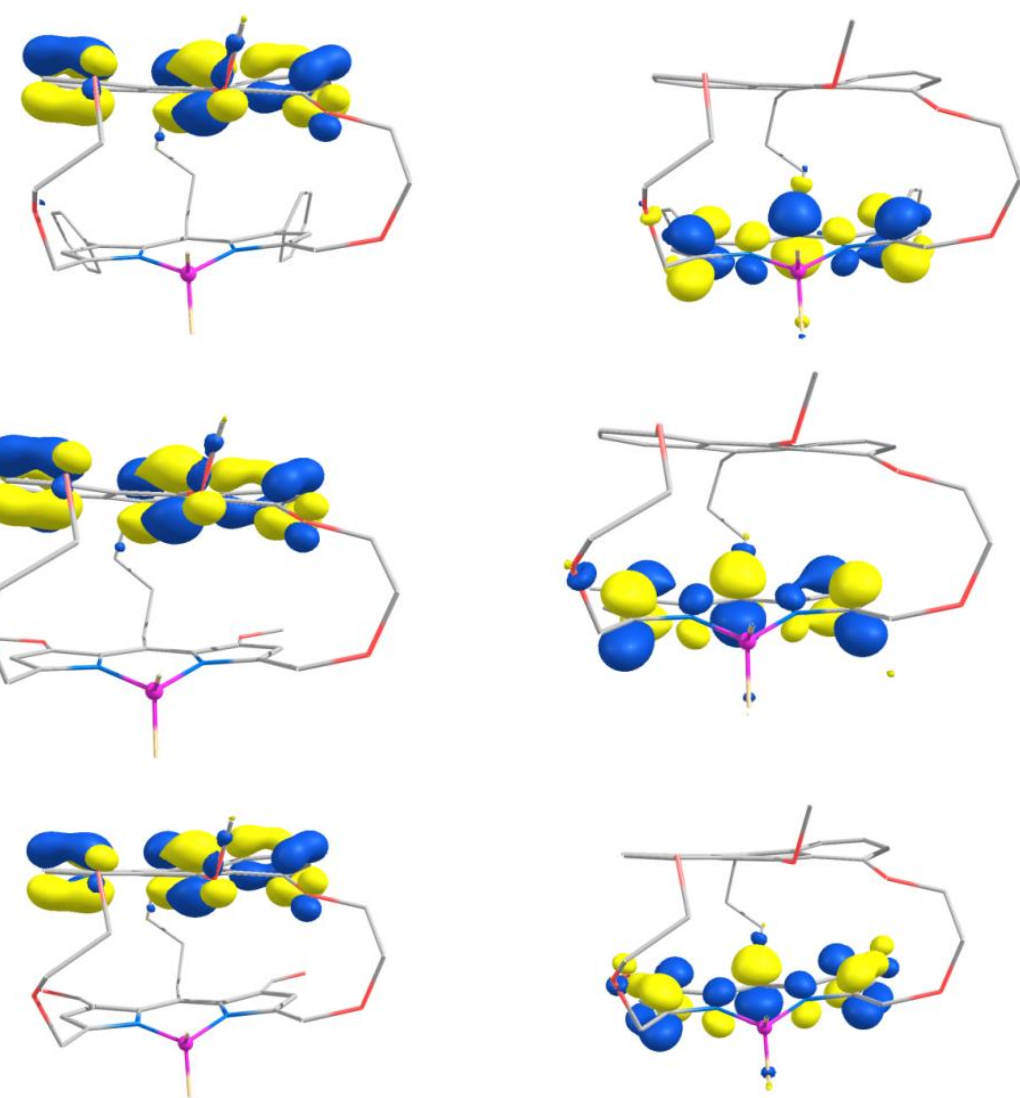

LUMO
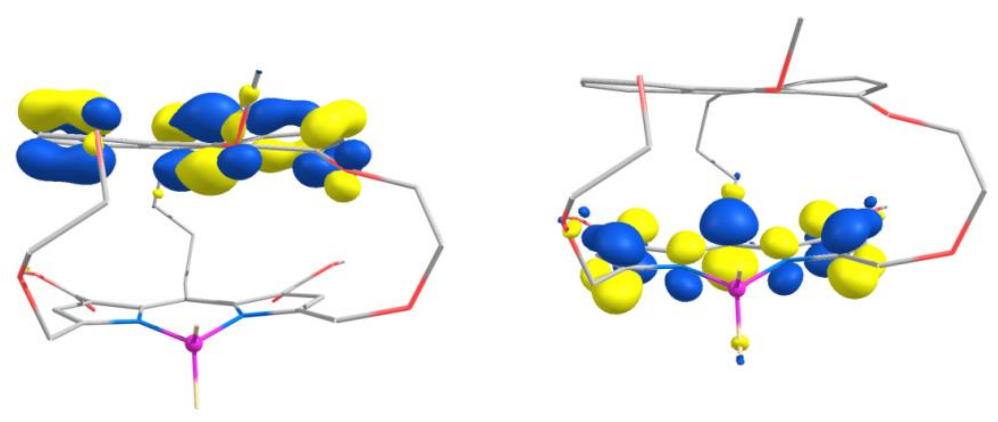


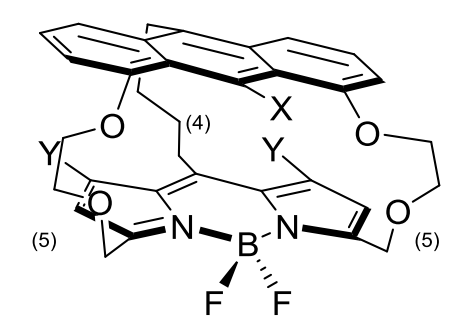

$X=$ OMe

$\mathrm{Y}=\mathrm{Ph}$

$\mathrm{X}=\mathrm{OMe}$

$\mathrm{Y}=\mathrm{OMe}$

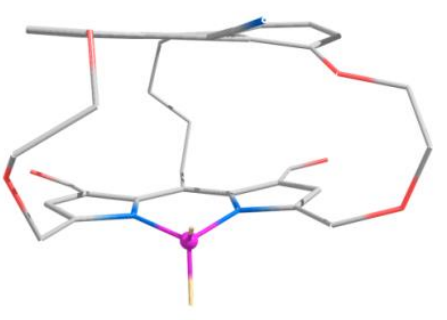

$X=$ OMe

$\mathrm{Y}=\mathrm{CHO}$

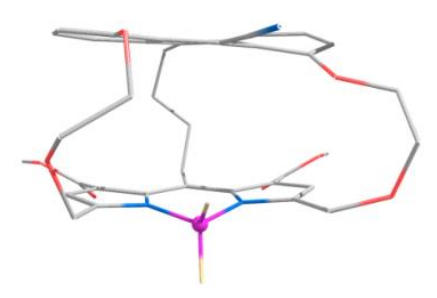

$X=\mathrm{OMe}$

$\mathrm{Y}=\mathrm{COOMe}$
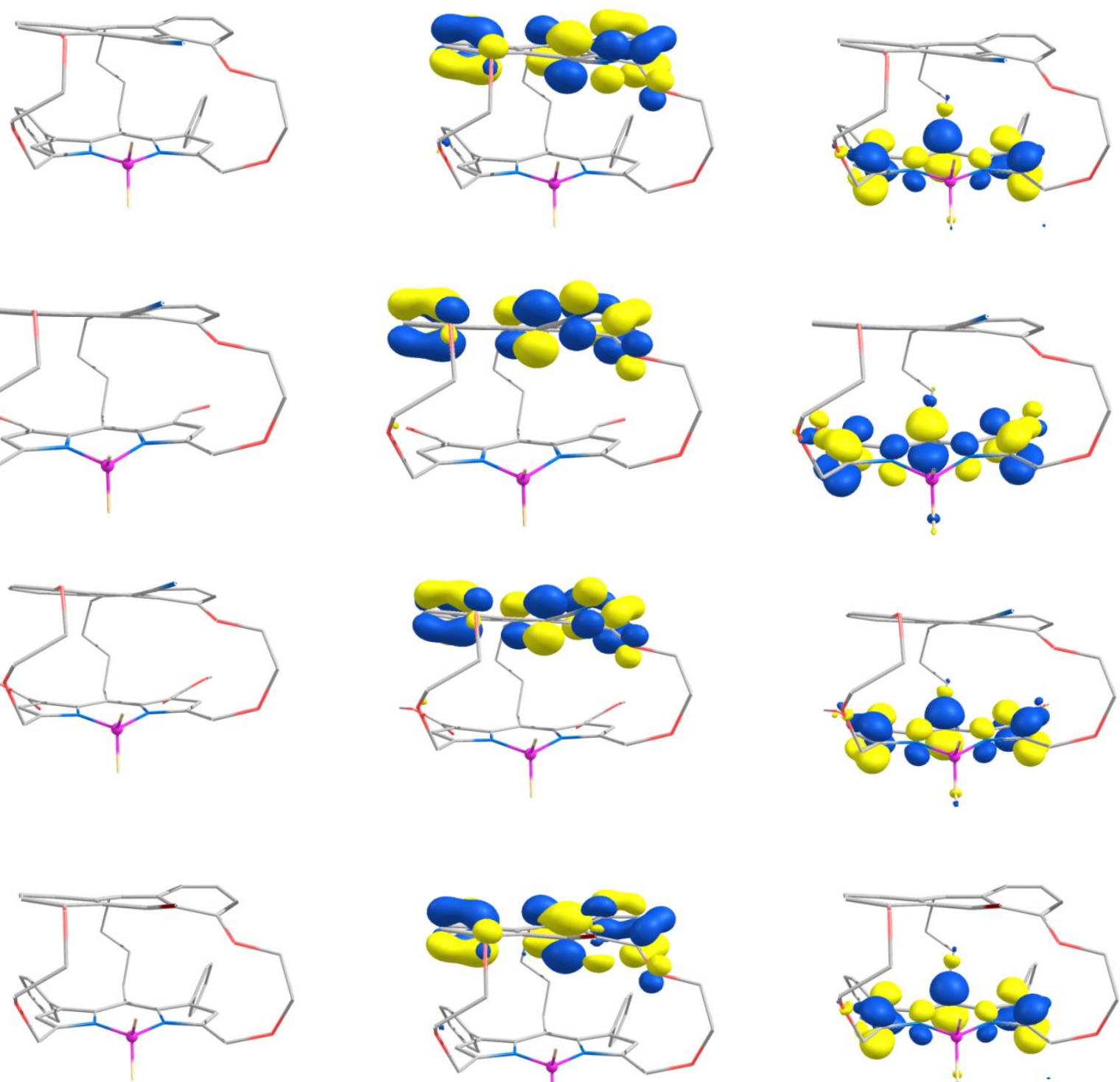

HOMO

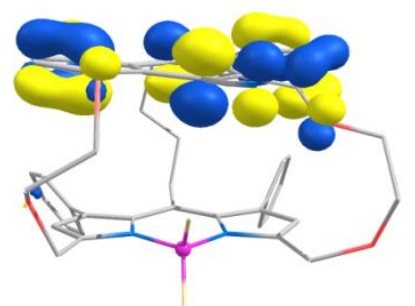

LUMO

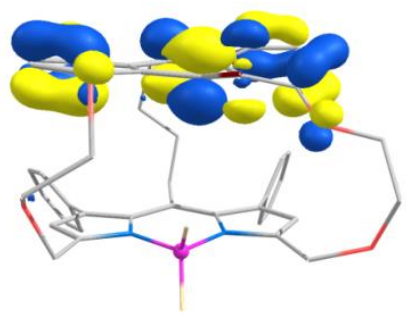

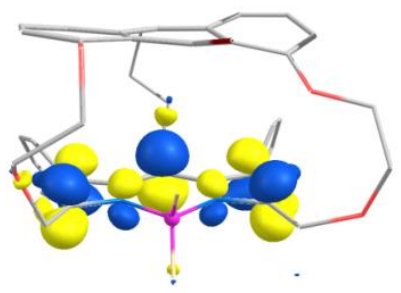




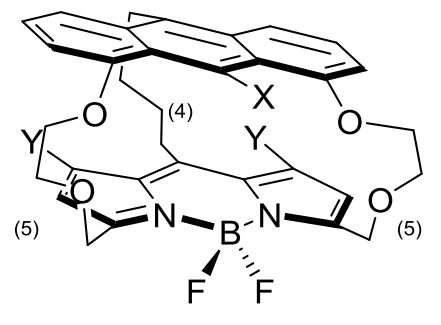

$\mathrm{X}=\mathrm{NO}_{2}$
$\mathrm{Y}=\mathrm{Ph}$
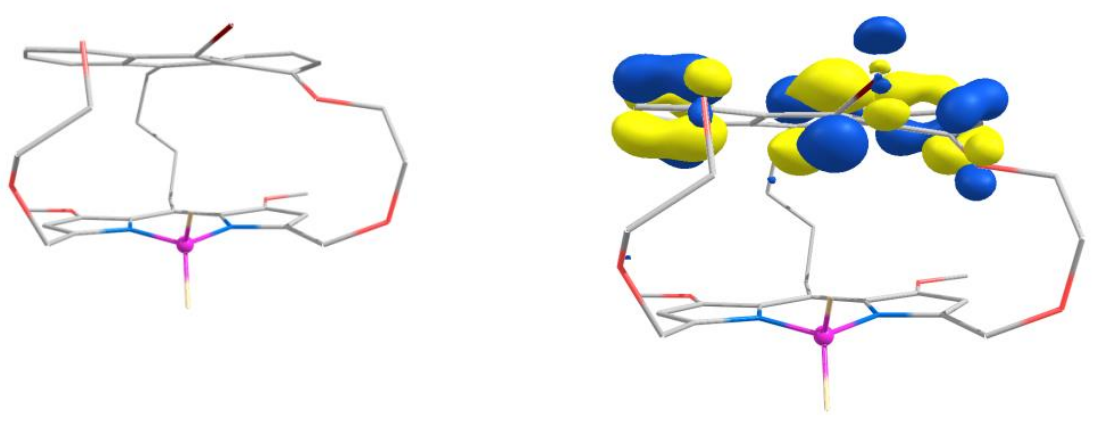

$\mathrm{X}=\mathrm{NO}_{2}$

$\mathrm{Y}=\mathrm{OMe}$
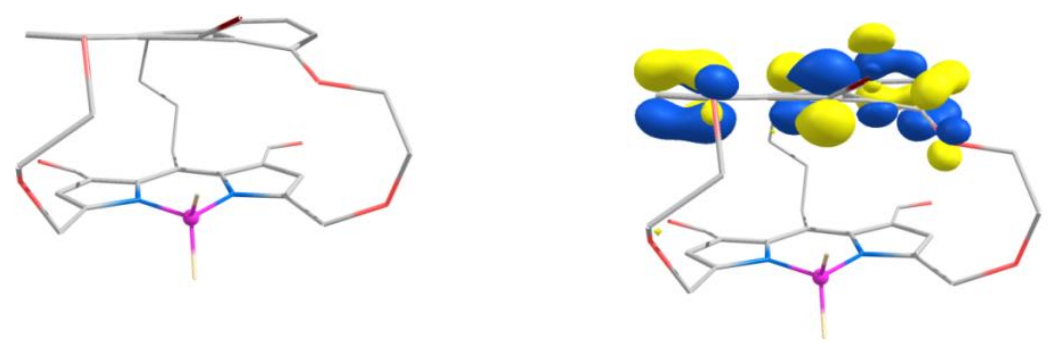

$\mathrm{X}=\mathrm{NO}_{2}$
$\mathrm{Y}=\mathrm{CHO}$

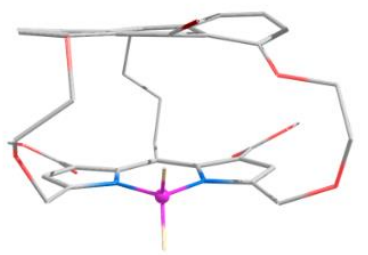

$\mathrm{X}=\mathrm{NO}_{2}$

$\mathrm{Y}=\mathrm{COOMe}$

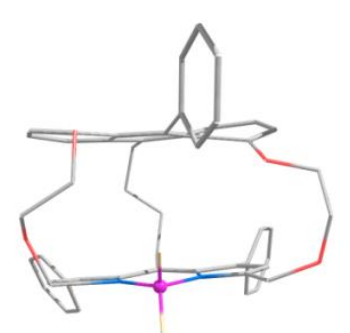

HOMO

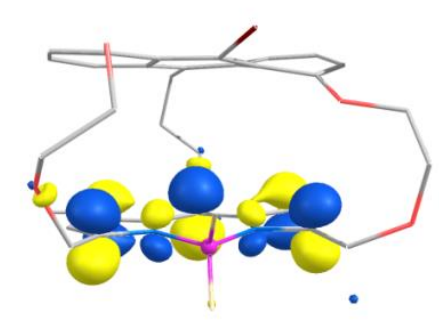

LUMO
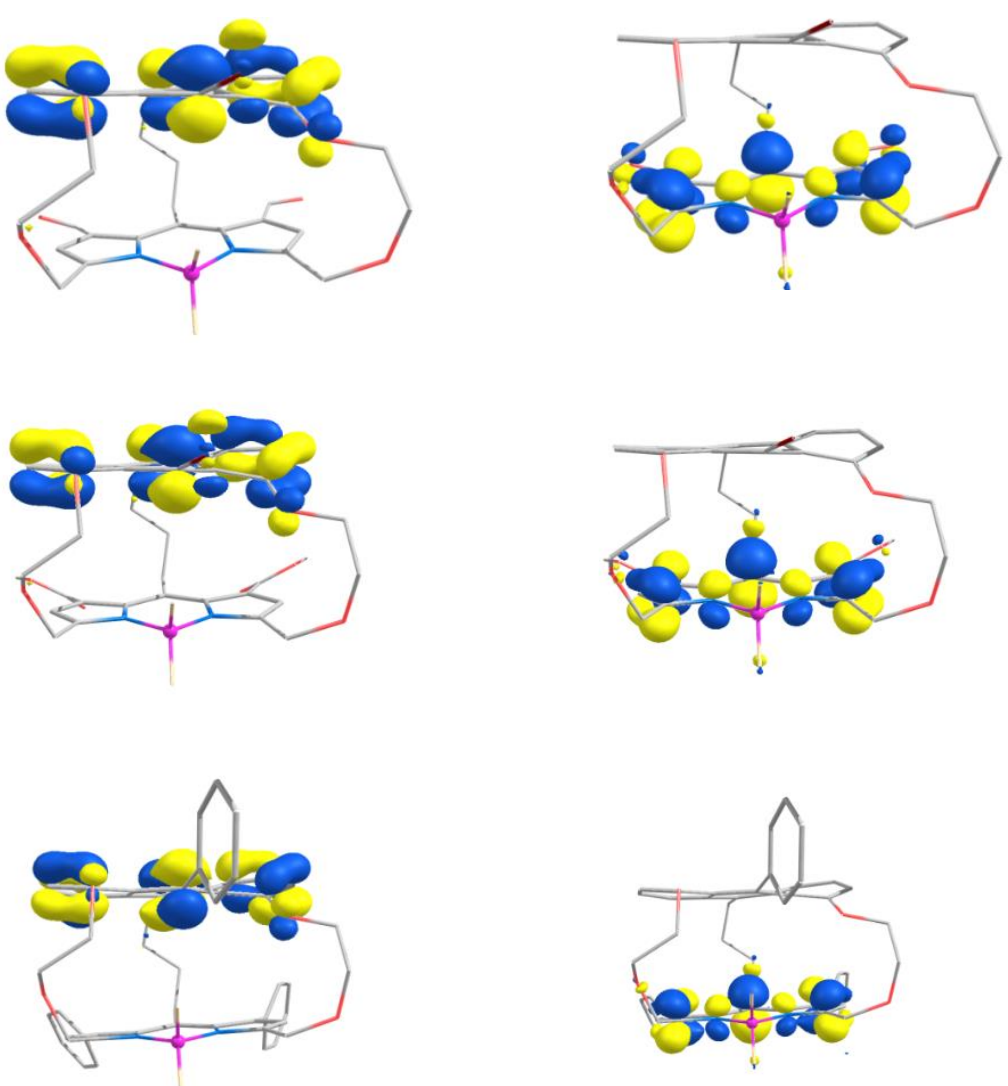


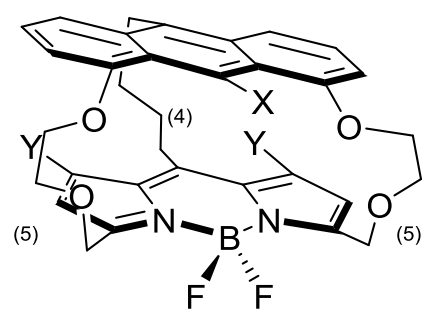

HOMO

LUMO

$X=\mathrm{NO}_{2}$

$\mathrm{Y}=\mathrm{Ph}$
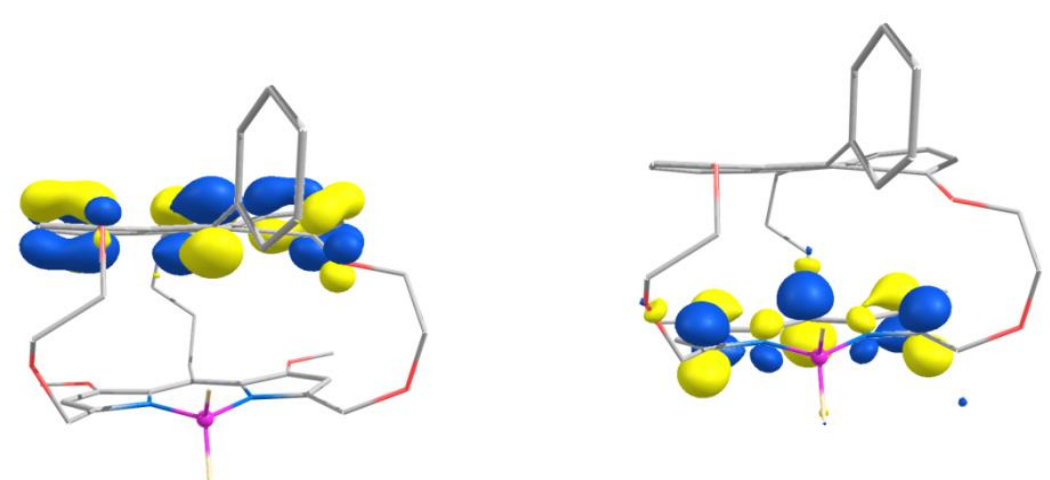

$X=\mathrm{NO}_{2}$

$Y=O M e$
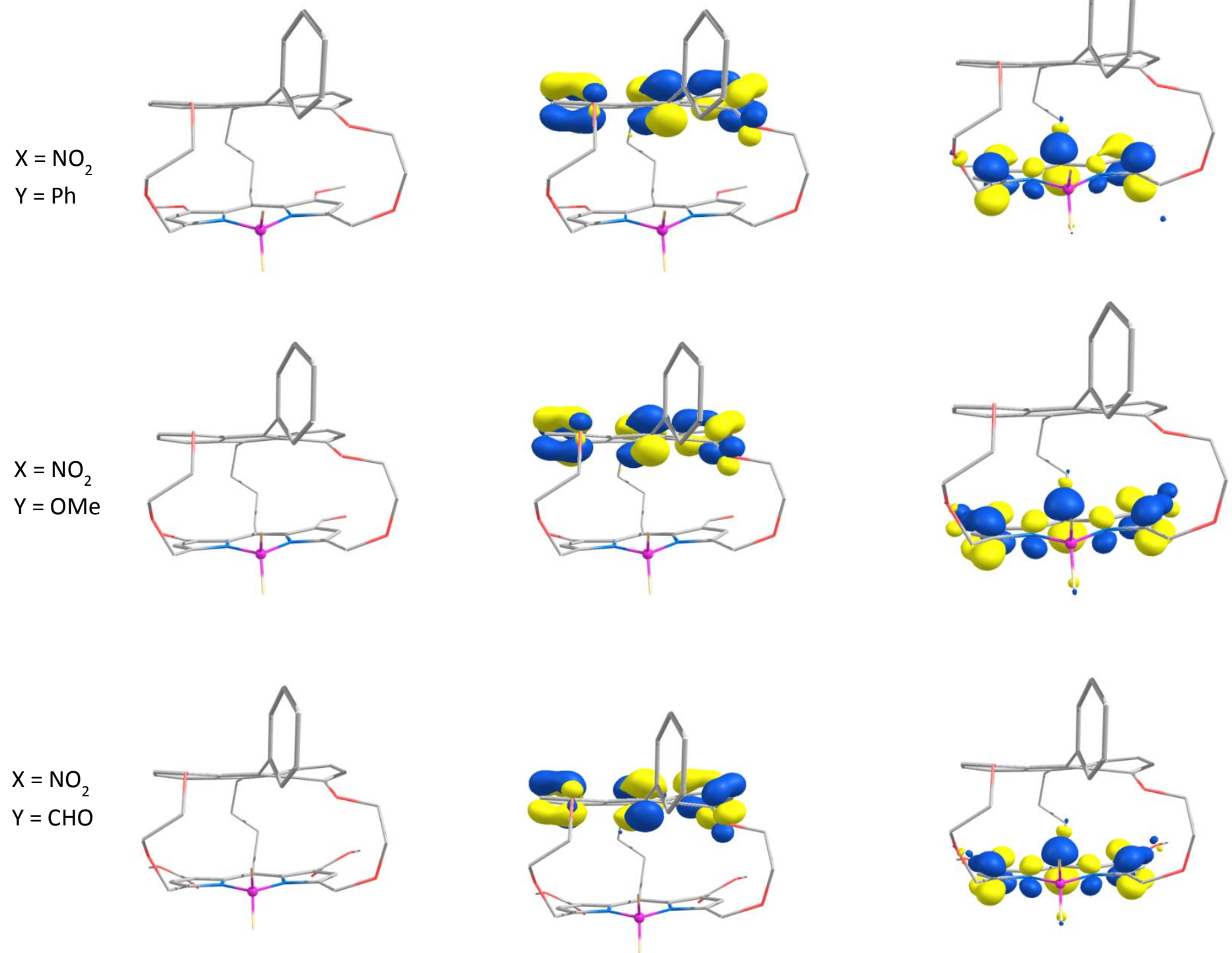


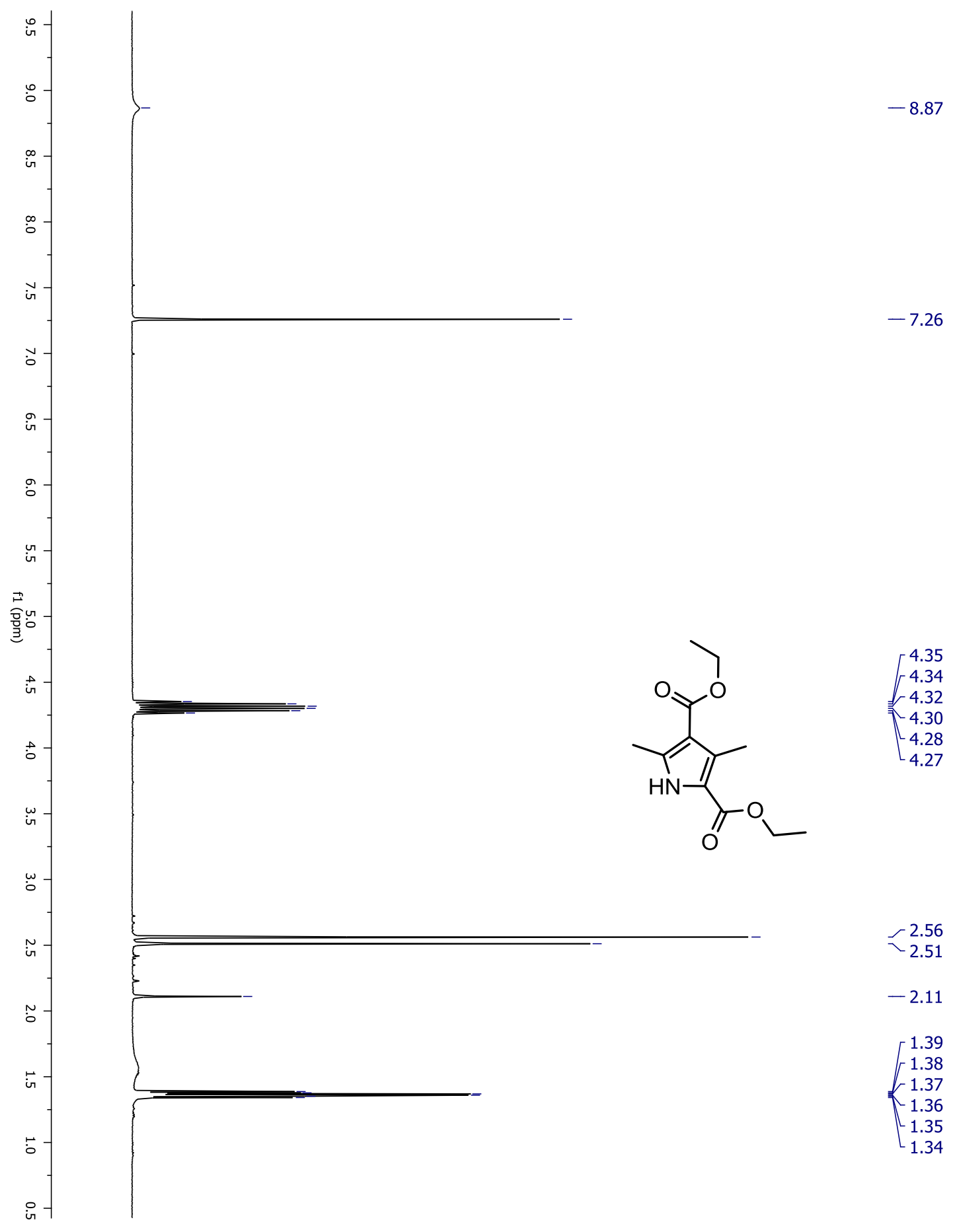

Figure A.2: ${ }^{1} \mathrm{H}$ NMR of $\mathbf{2 . 1}$ 


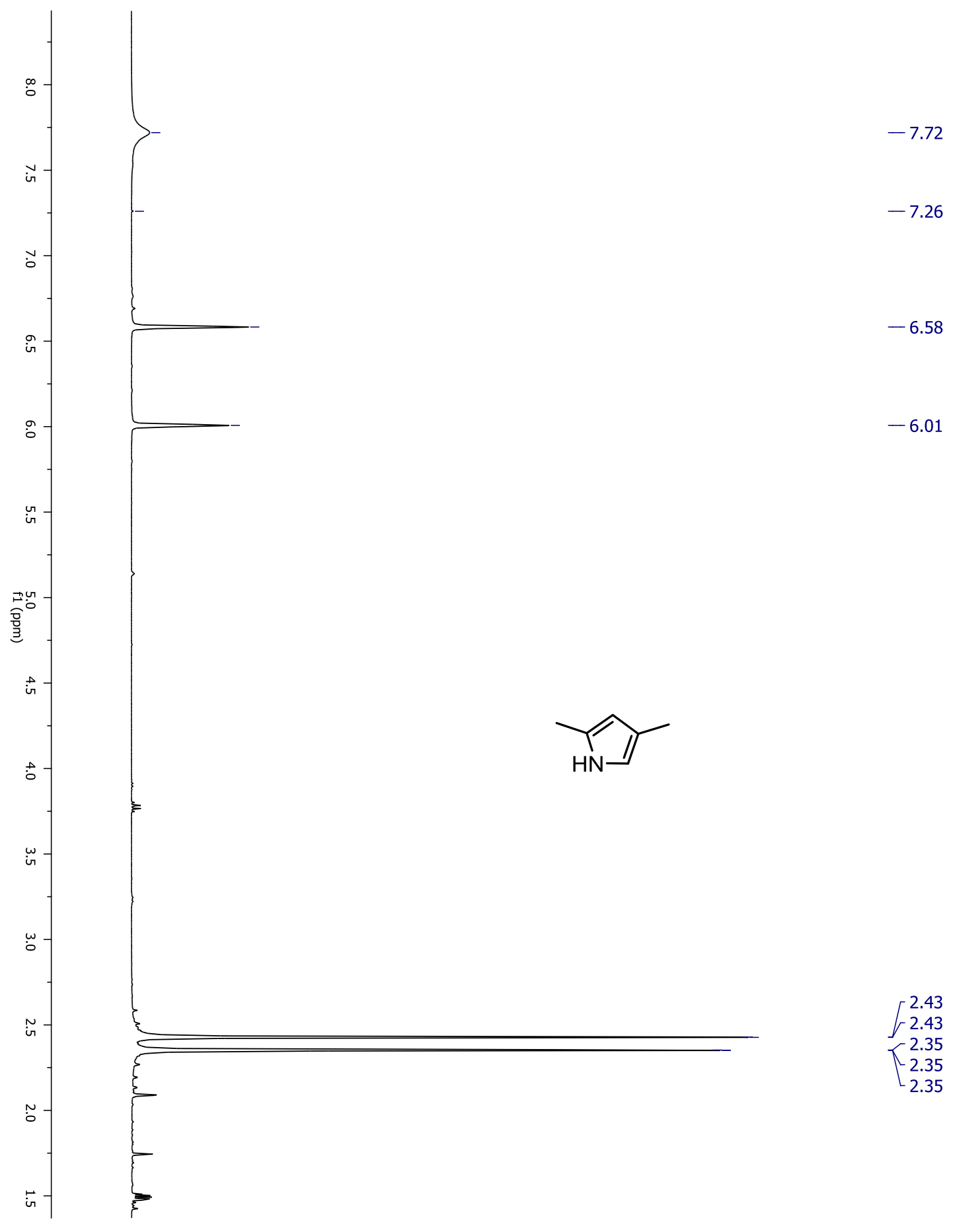

Figure A.3: ${ }^{1} \mathrm{H}$ NMR of $\mathbf{2 . 2}$ 

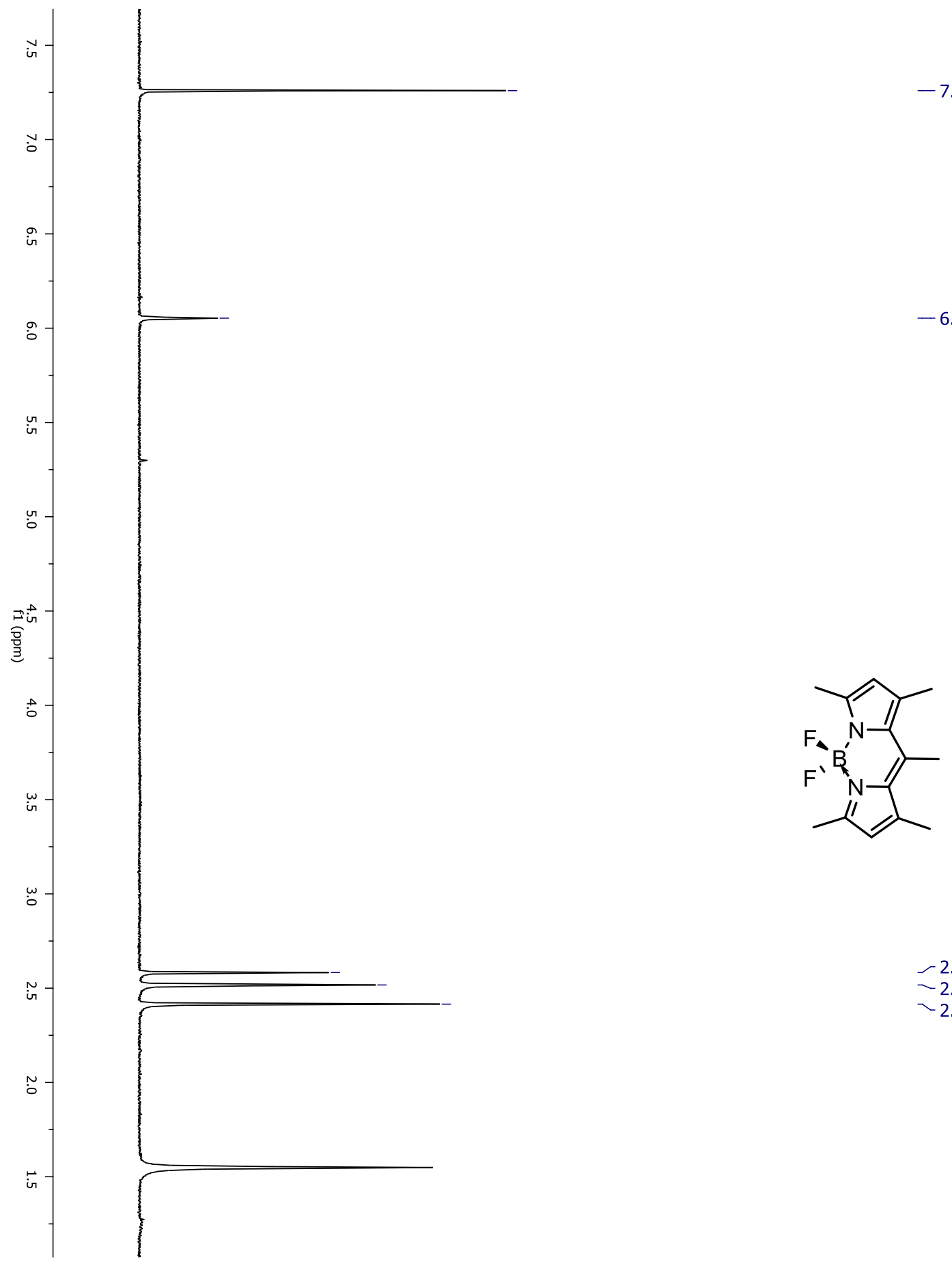

Figure A.4: ${ }^{1} \mathrm{H}$ NMR of $\mathbf{2 . 3}$ 


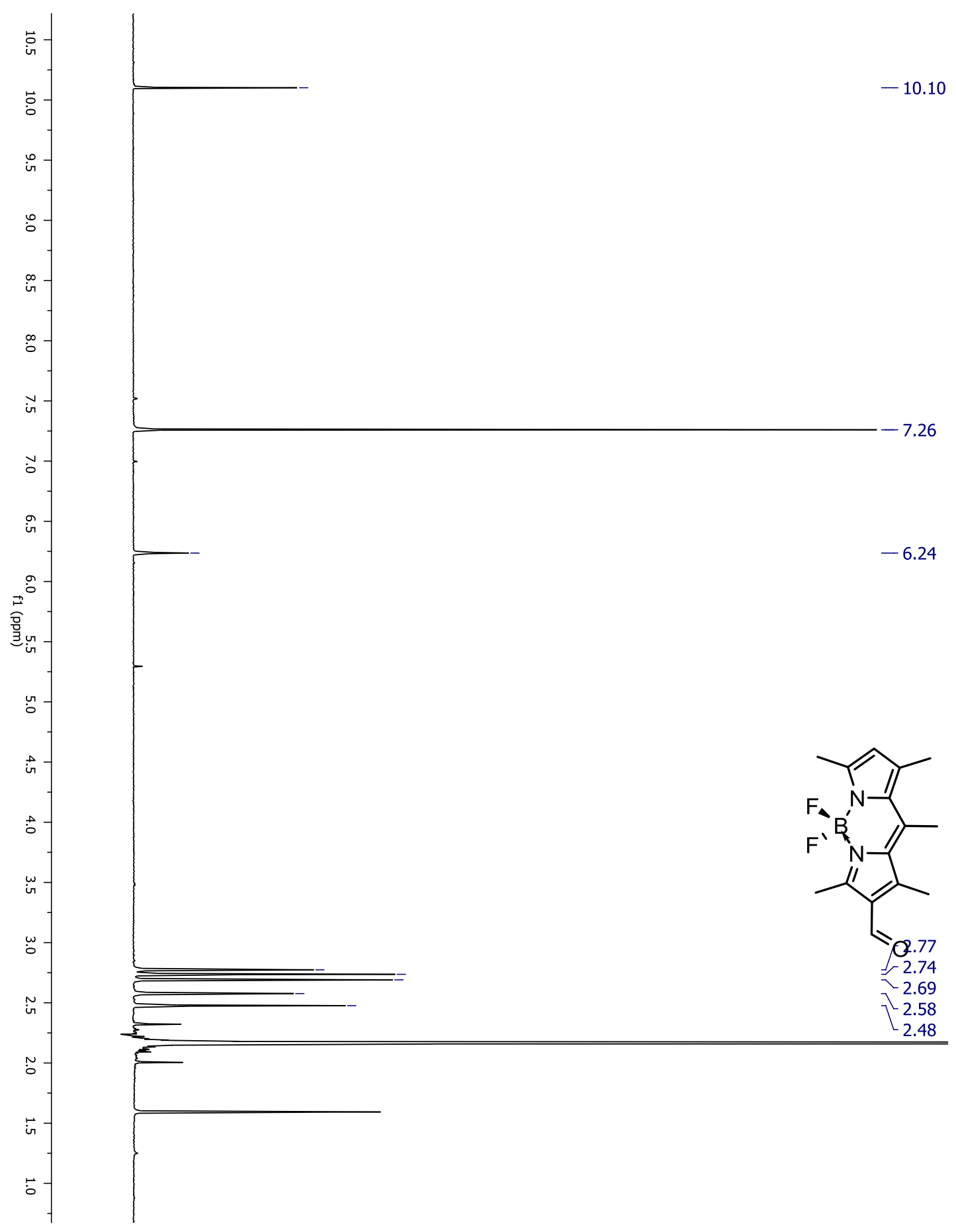

Figure A.5: ${ }^{1} \mathrm{H}$ NMR of $\mathbf{2 . 4}$ 


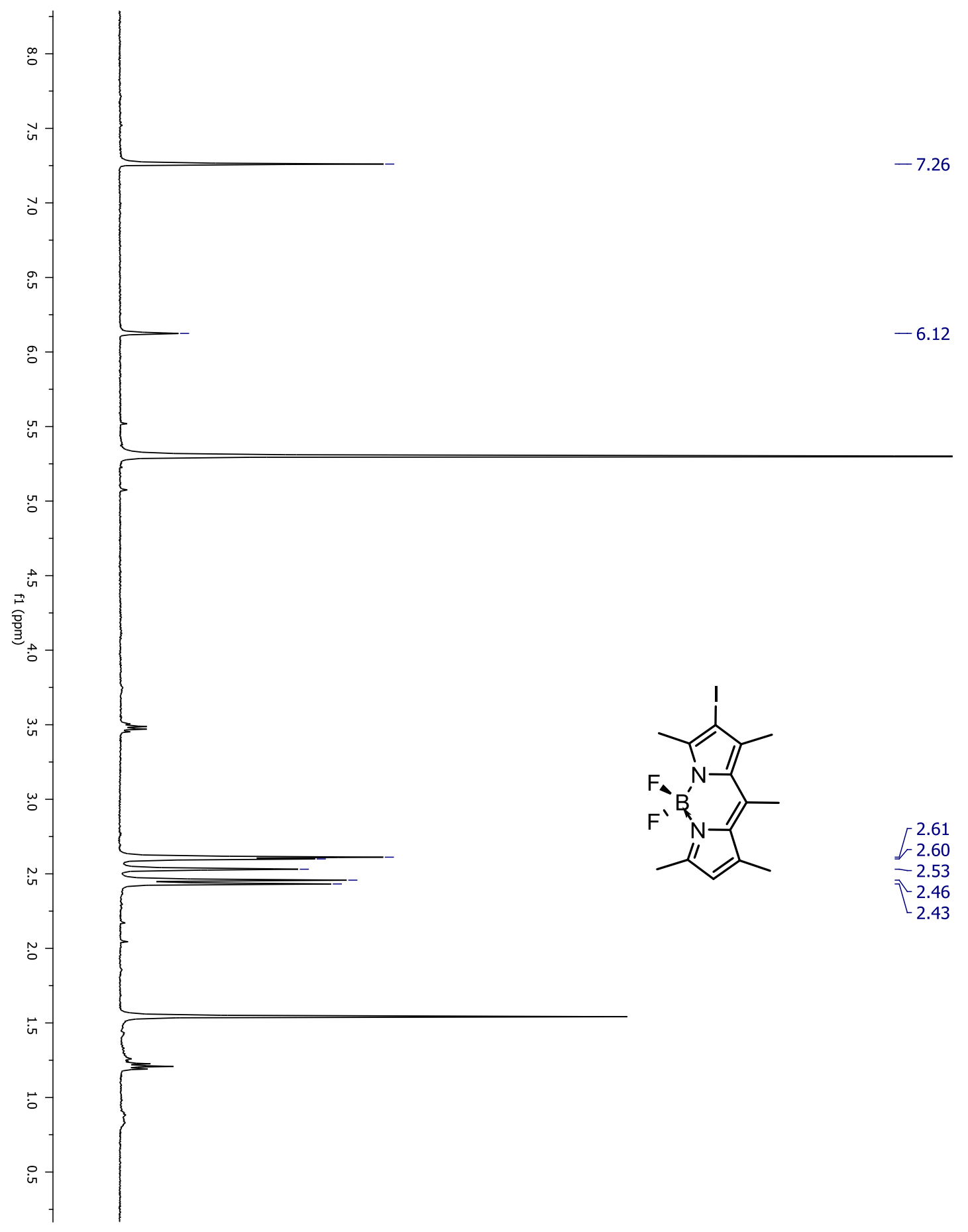

Figure A.6: ${ }^{1} \mathrm{H}$ NMR of $\mathbf{2 . 5}$ 


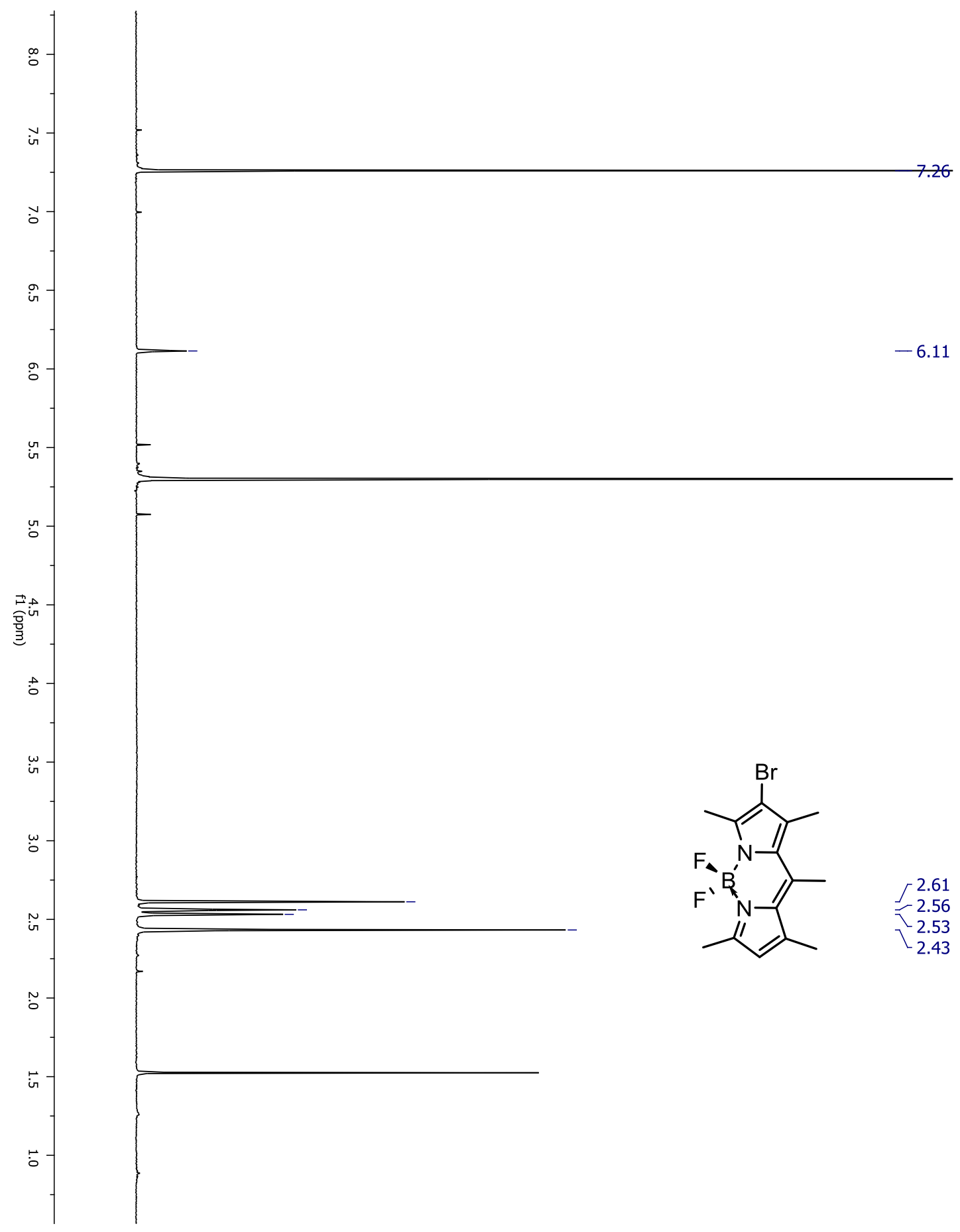

Figure A.7: ${ }^{1} \mathrm{H}$ NMR of $\mathbf{2 . 6}$ 


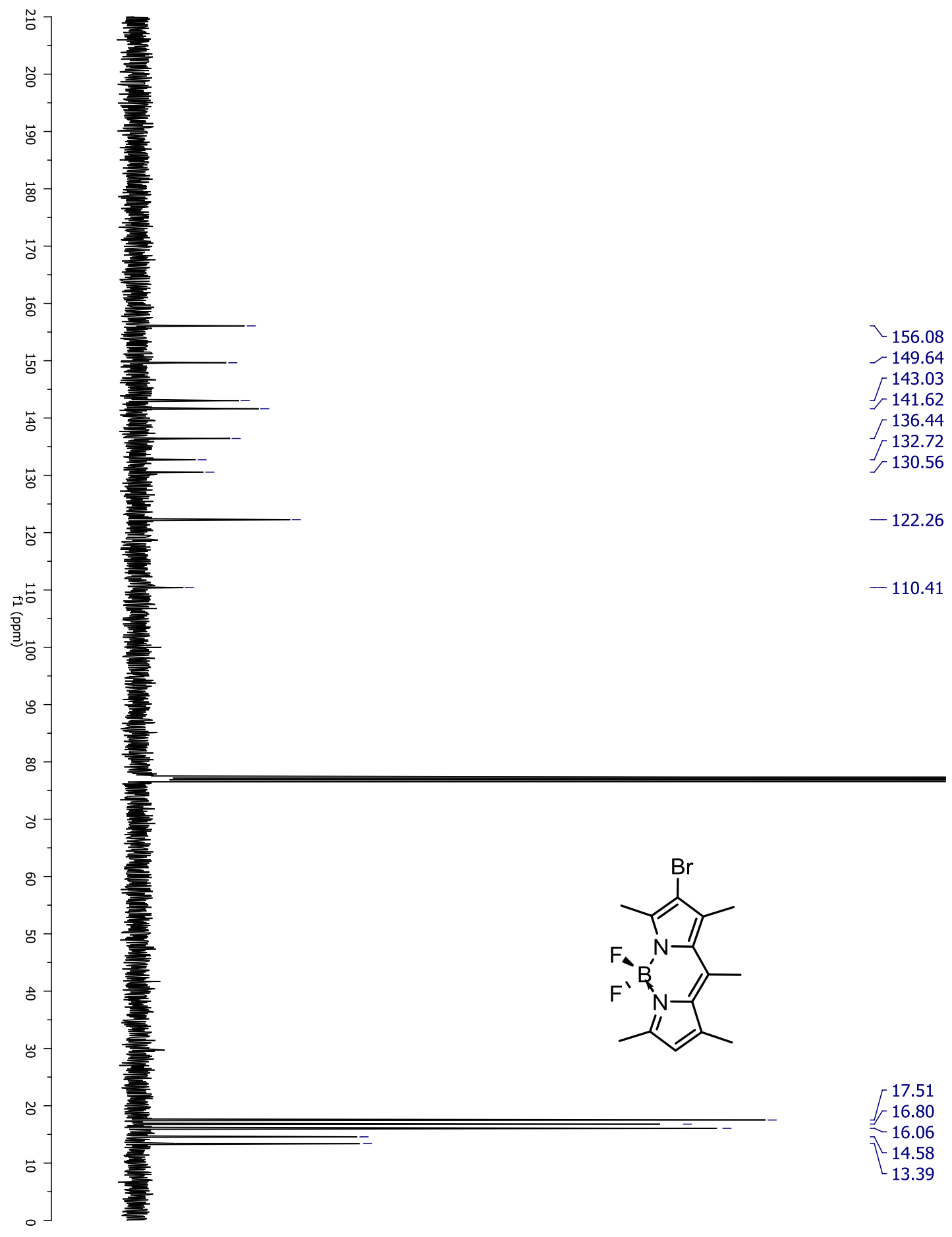

Figure A.8: ${ }^{13} \mathrm{C}$ NMR of 2.6 


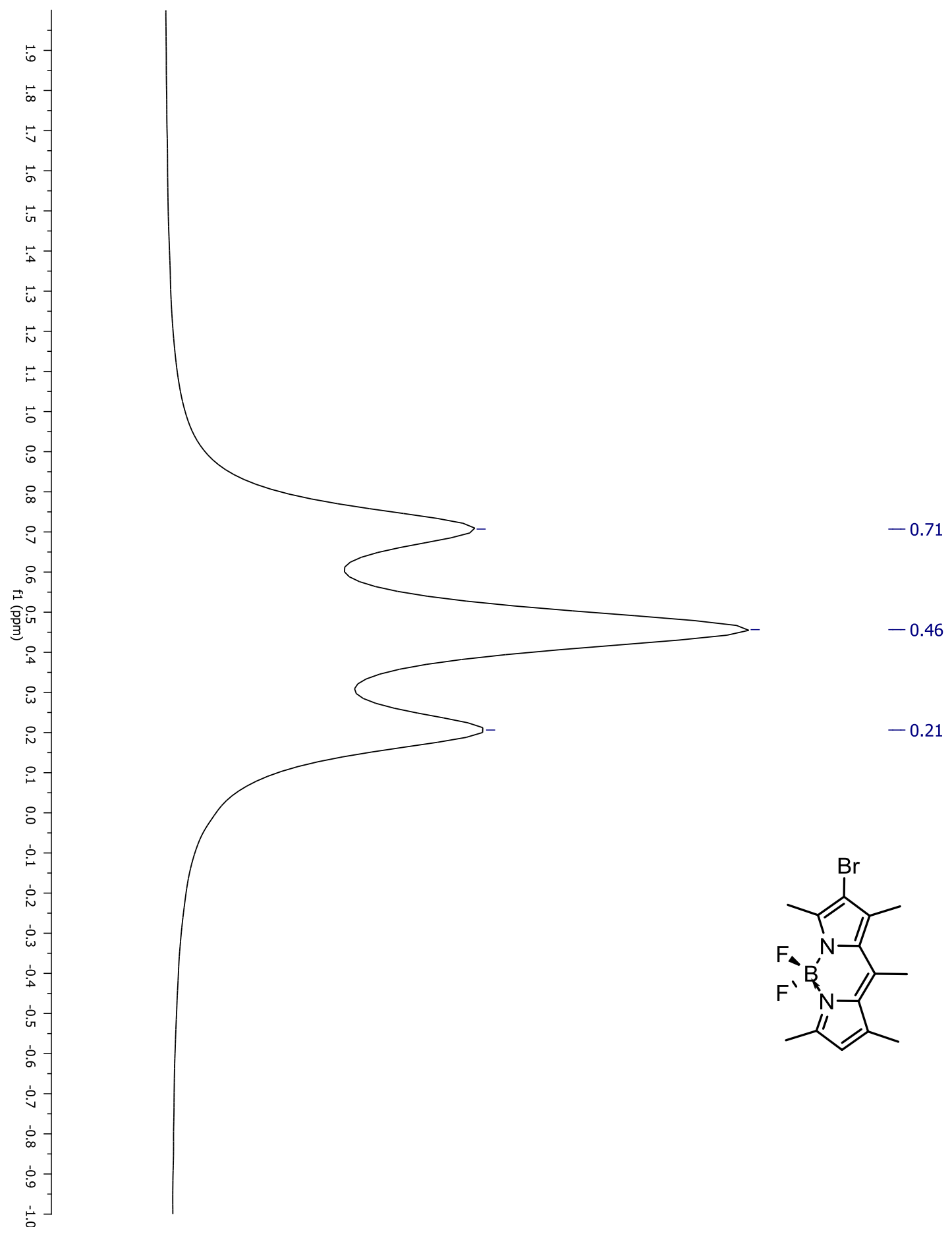

Figure A.9: ${ }^{11} B$ NMR of 2.6 

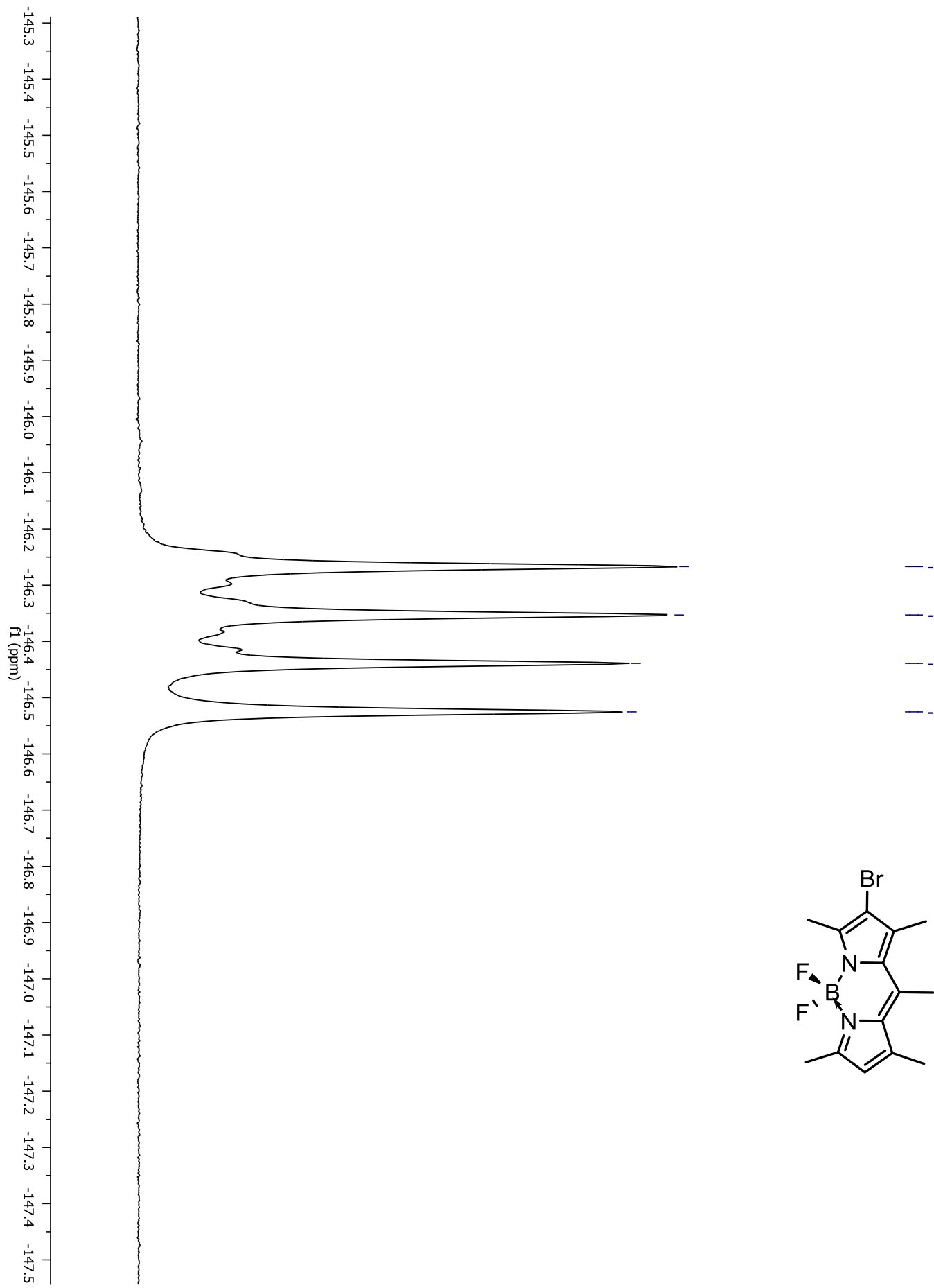

Figure A.10: ${ }^{19} \mathrm{~F}$ NMR of 2.6 


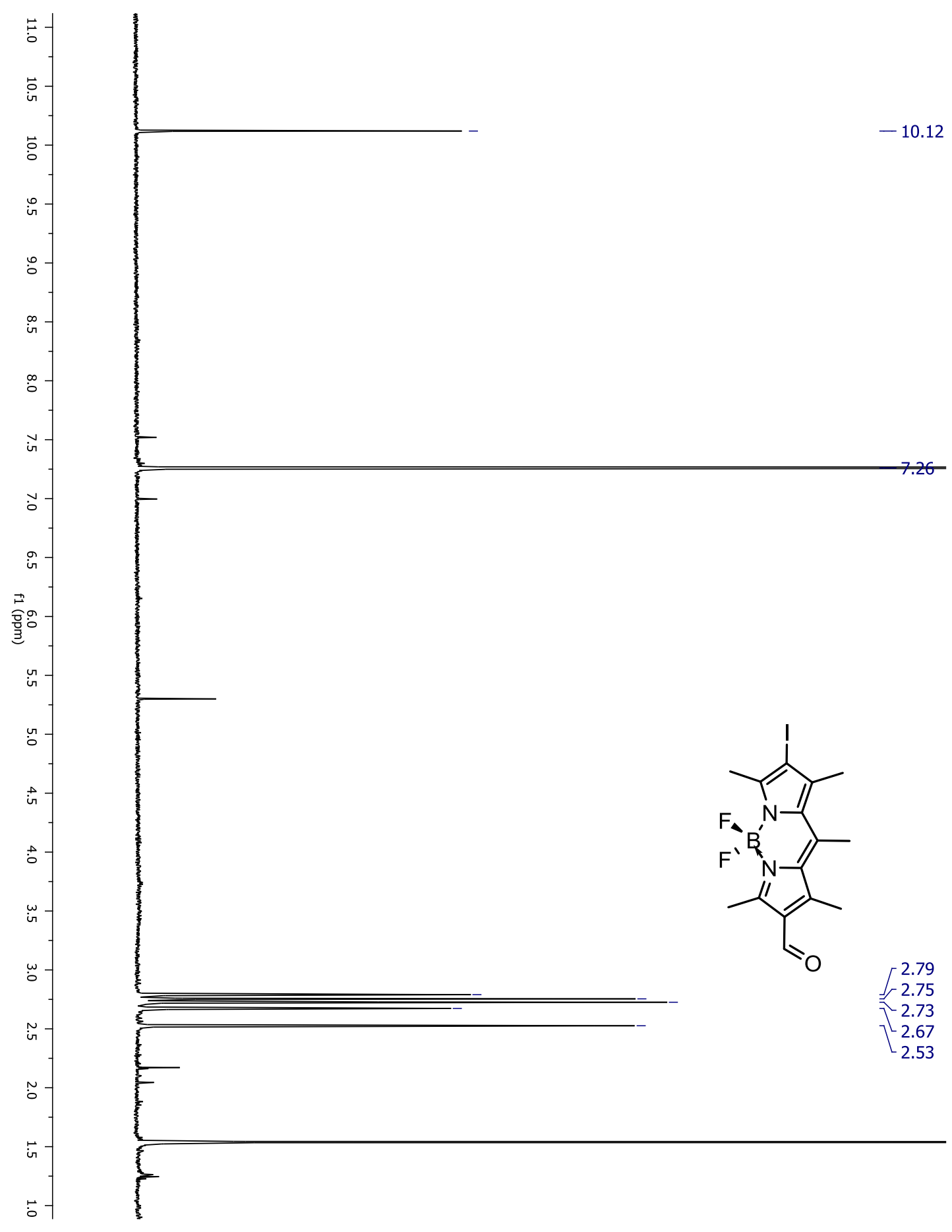

Figure A.11: ${ }^{1} \mathrm{H}$ NMR of 2.7 

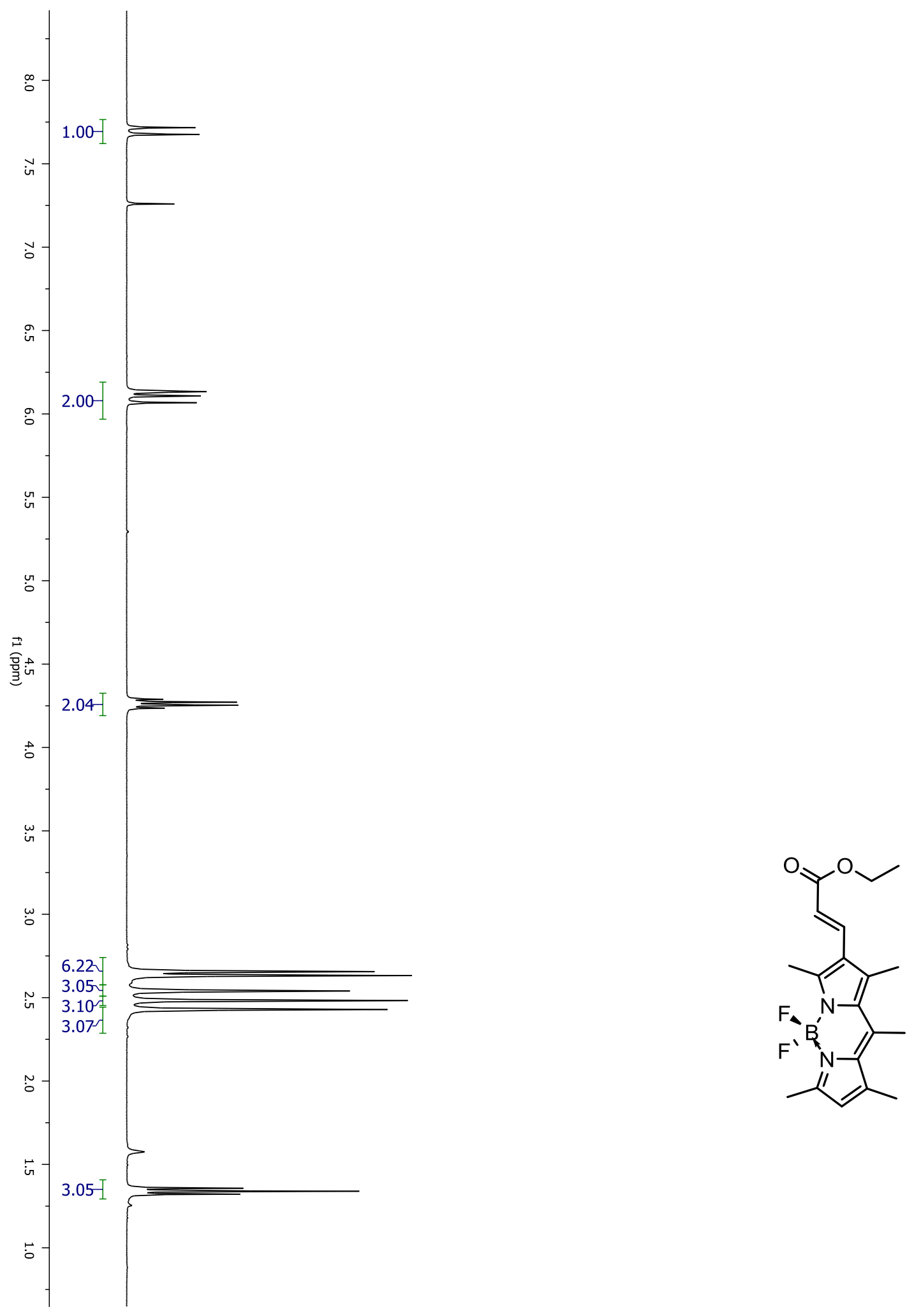

Figure A.12: ${ }^{1} \mathrm{H}$ NMR of $\mathbf{2 . 1 0}$ 


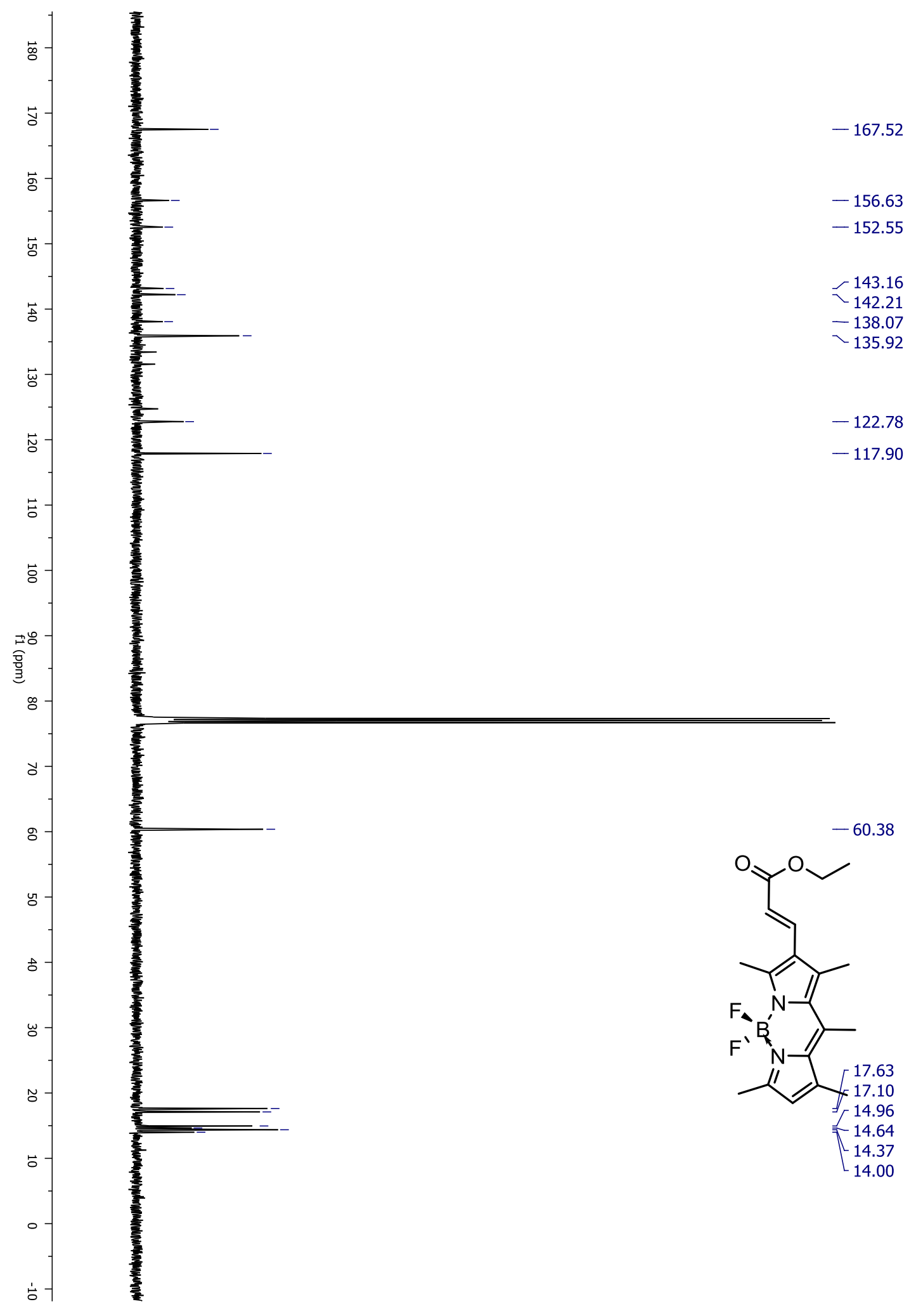

Figure A.13: ${ }^{13} \mathrm{C}$ NMR of $\mathbf{2 . 1 0}$ 

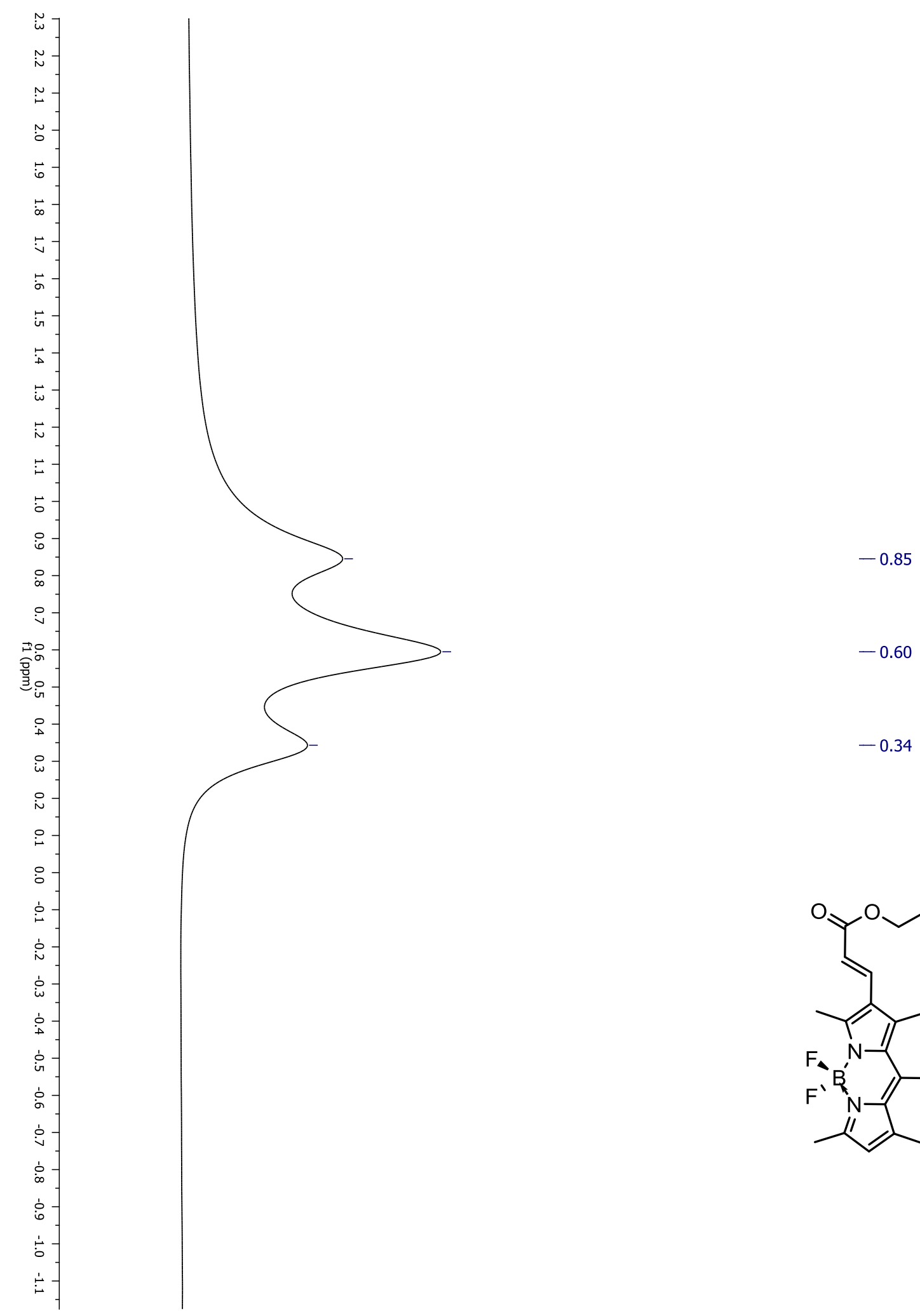

$$
-0.60
$$$$
-0.34
$$

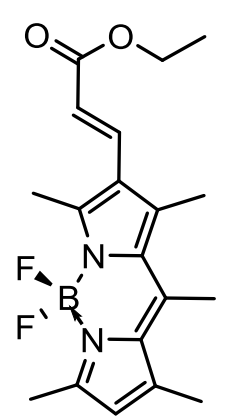

Figure A.14: ${ }^{11} B$ NMR of 2.10 


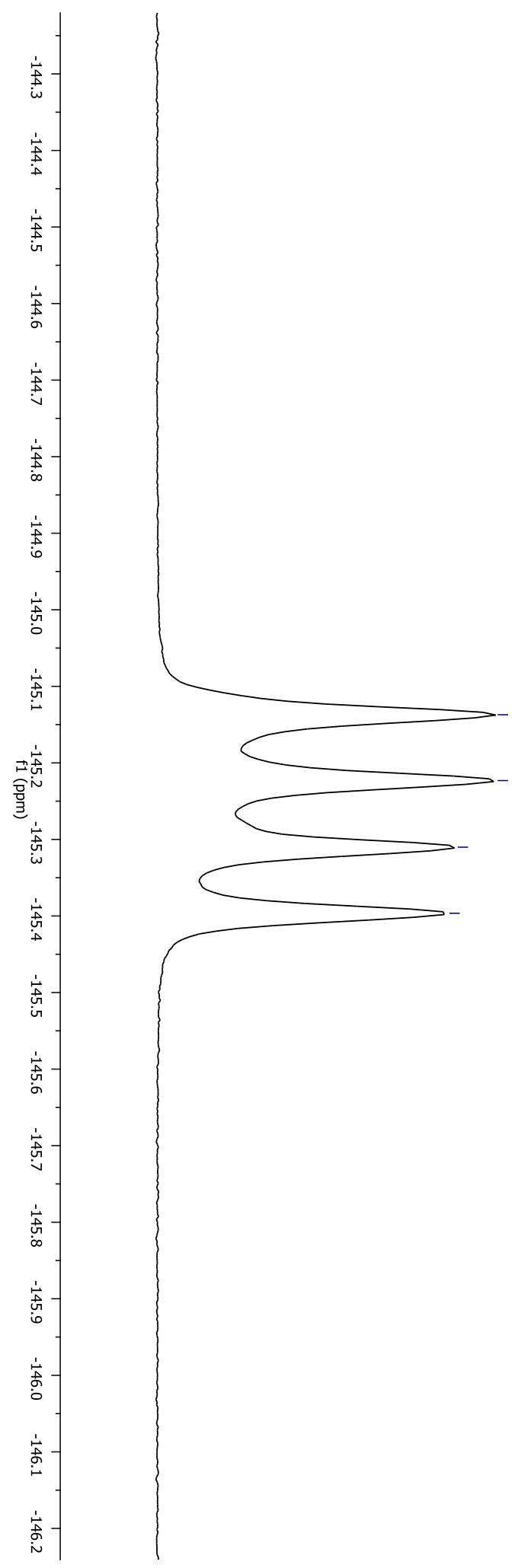

$--145.14$

$--145.22$

$--145.31$

$--145.40$

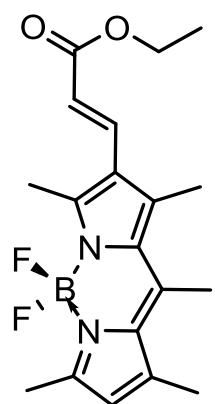

Figure A.15: ${ }^{19} \mathrm{~F}$ NMR of $\mathbf{2 . 1 0}$ 

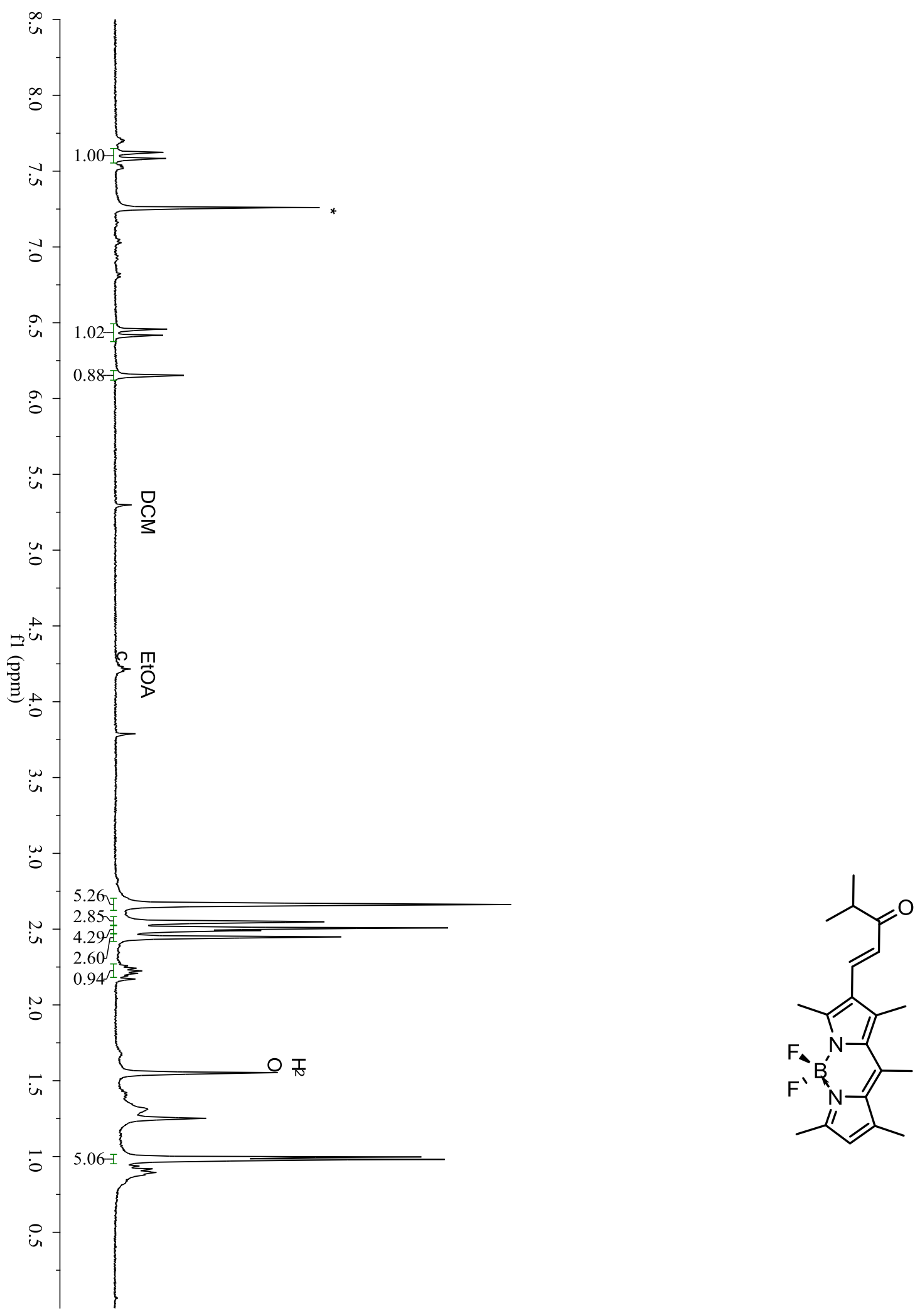

Figure A.16: ${ }^{1} \mathrm{H}$ NMR of $\mathbf{2 . 1 1}$ 


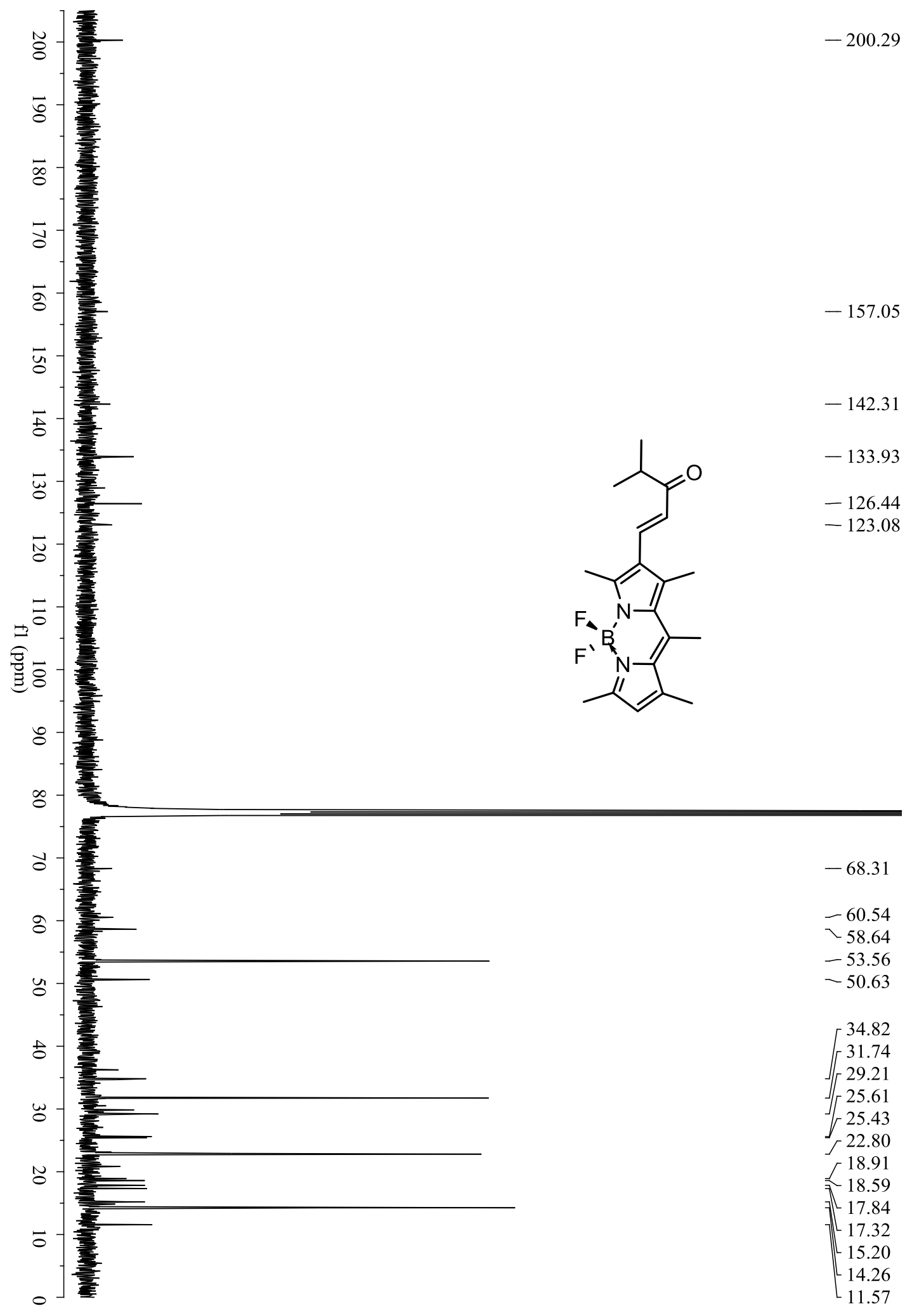

Figure A.17: ${ }^{13} \mathrm{C}$ NMR of $\mathbf{2 . 1 1}$ 

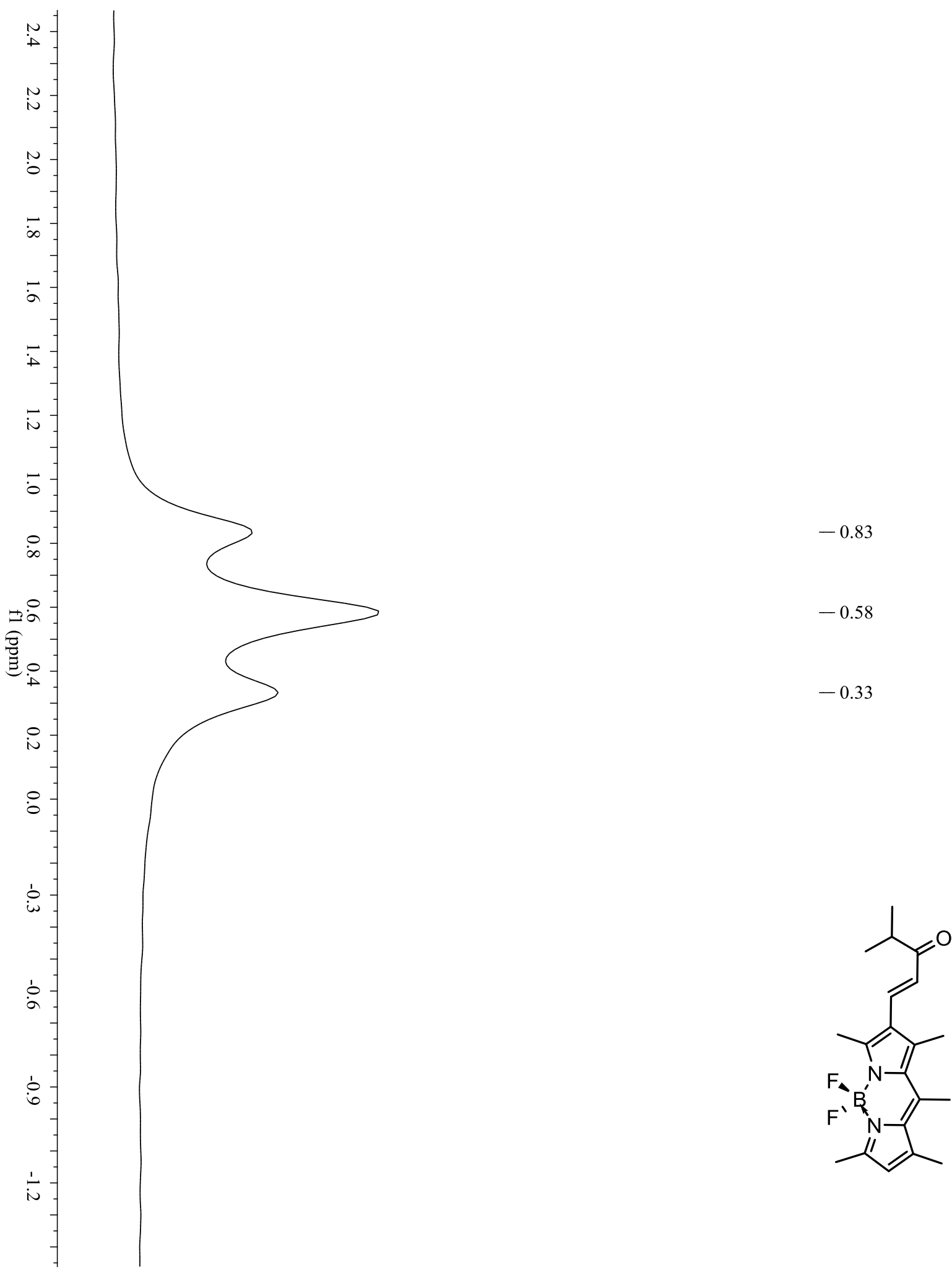

Figure A.18: ${ }^{11} B$ NMR of 2.11 


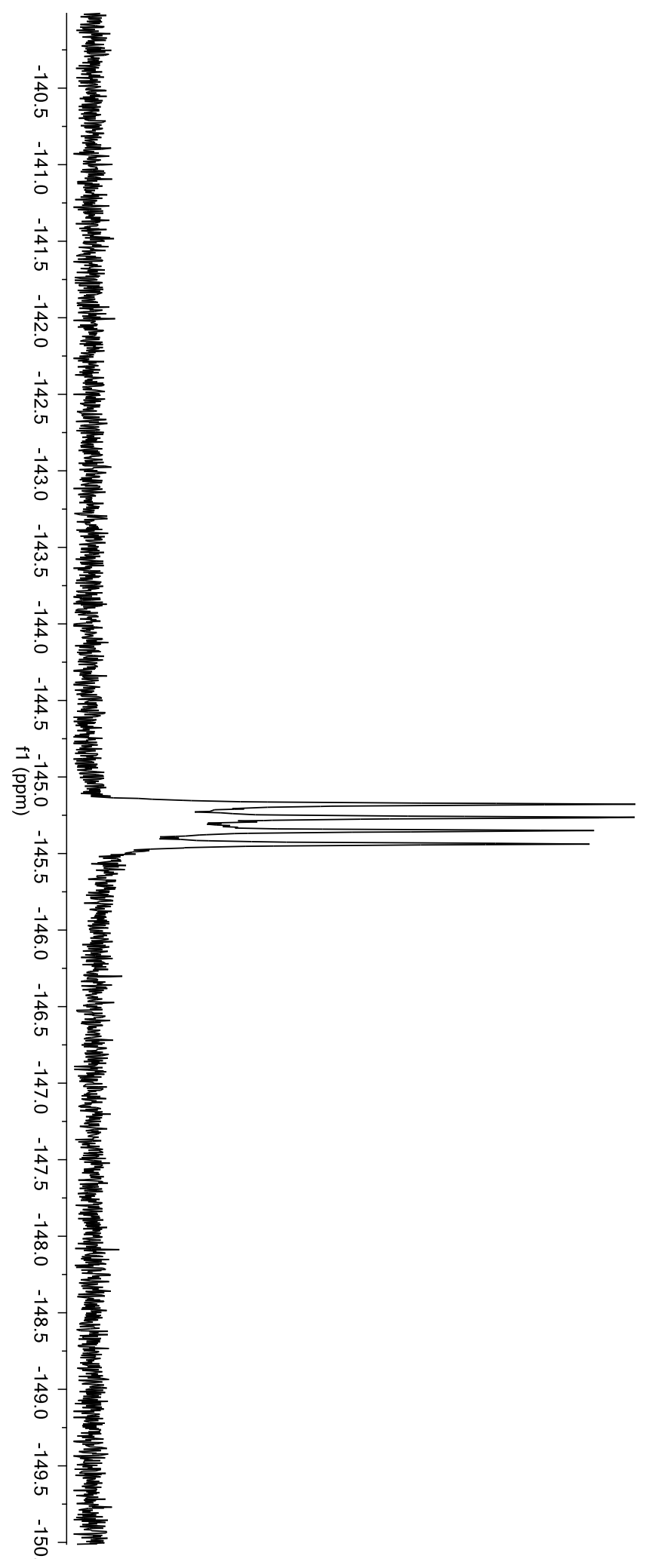

-145.18
$\simeq-145.26$

$-145.35$

$-145.44$

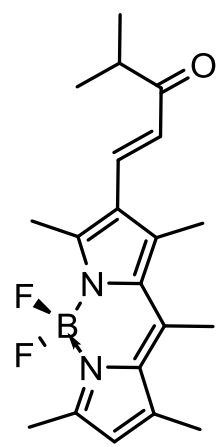

Figure A.19: ${ }^{19}$ FNMR of 2.11 


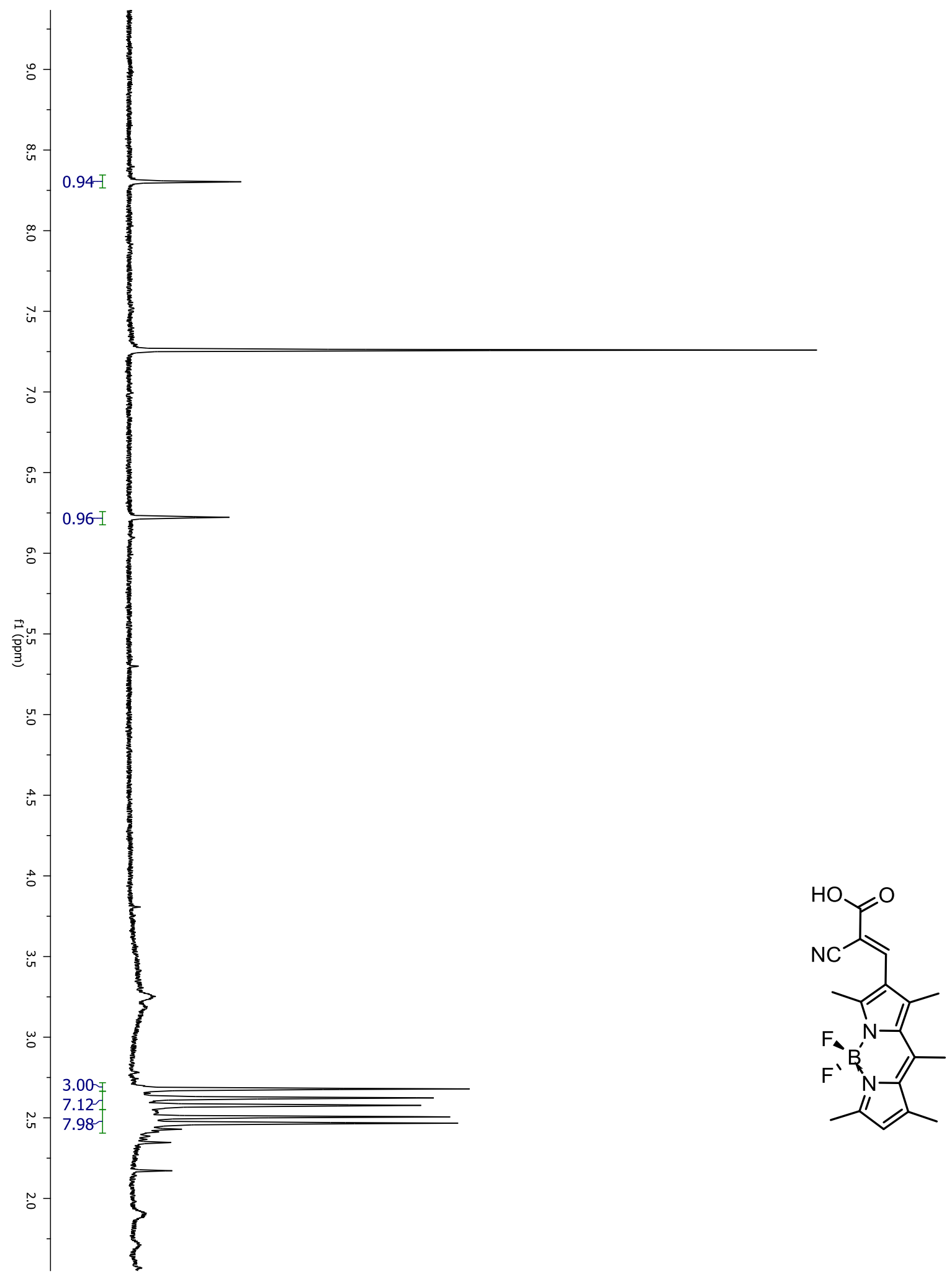

Figure A.20: ${ }^{1} \mathrm{H}$ NMR of $\mathbf{2 . 1 3}$ 

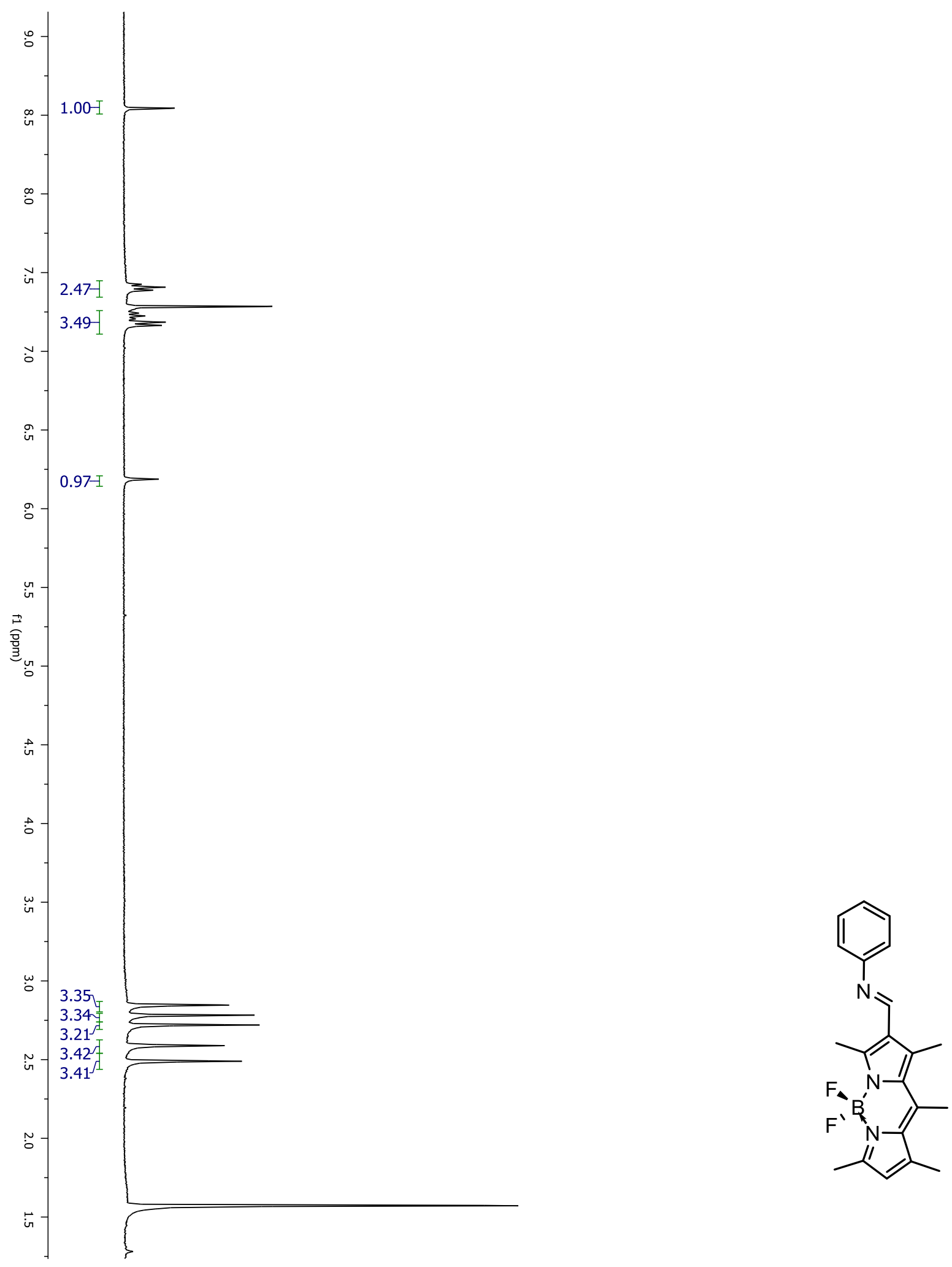

Figure A.21: ${ }^{1} \mathrm{H}$ NMR of $\mathbf{2 . 1 4}$ 


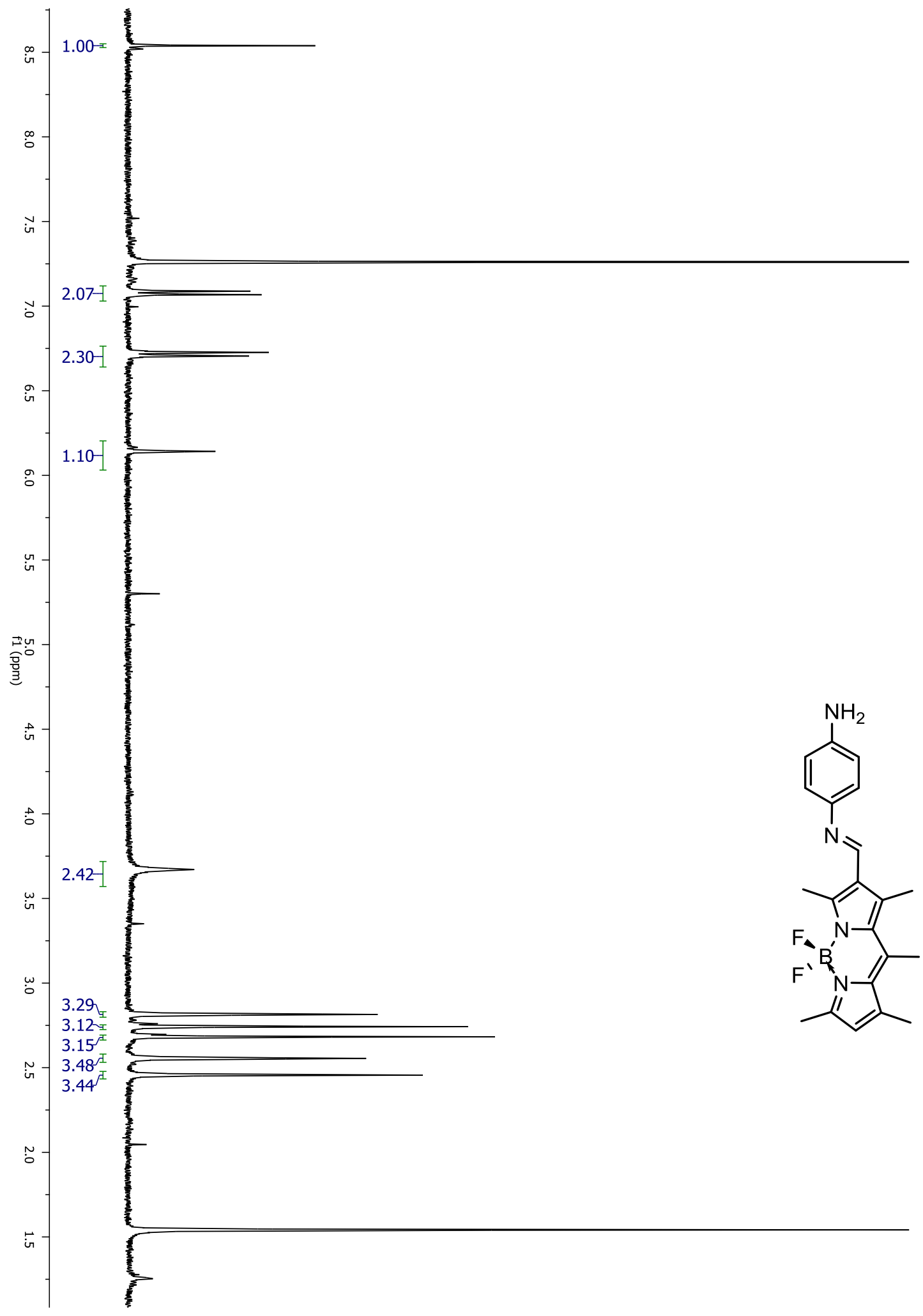

Figure A.23: ${ }^{1} \mathrm{H}$ NMR of $\mathbf{2 . 1 5}$ 


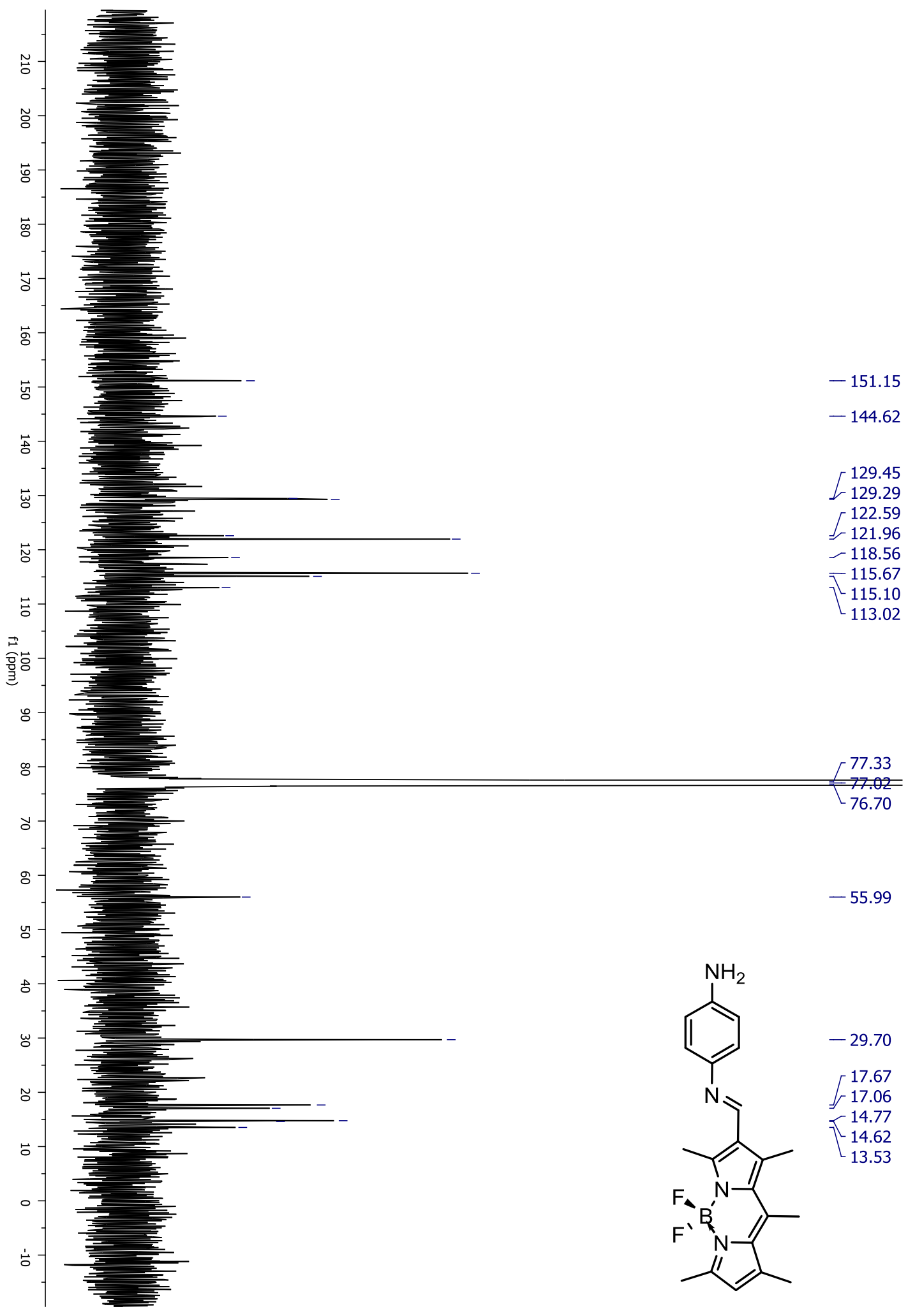

Figure A.24: ${ }^{13} \mathrm{C}$ NMR of 2.15 


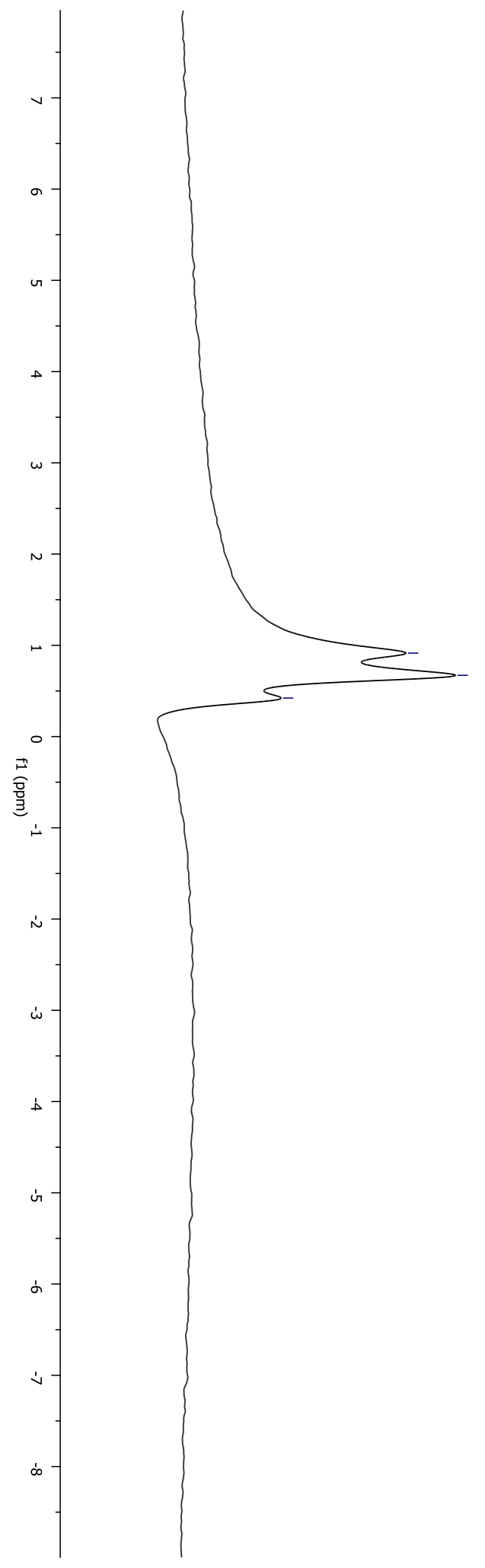

$$
\begin{array}{r}
-0.91 \\
-0.67 \\
-0.42
\end{array}
$$

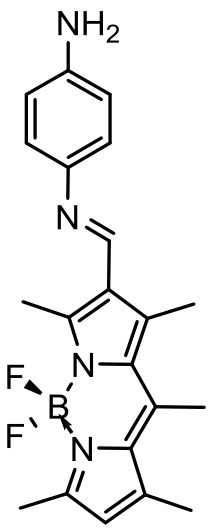

Figure A.25: ${ }^{11} B$ NMR of 2.15 


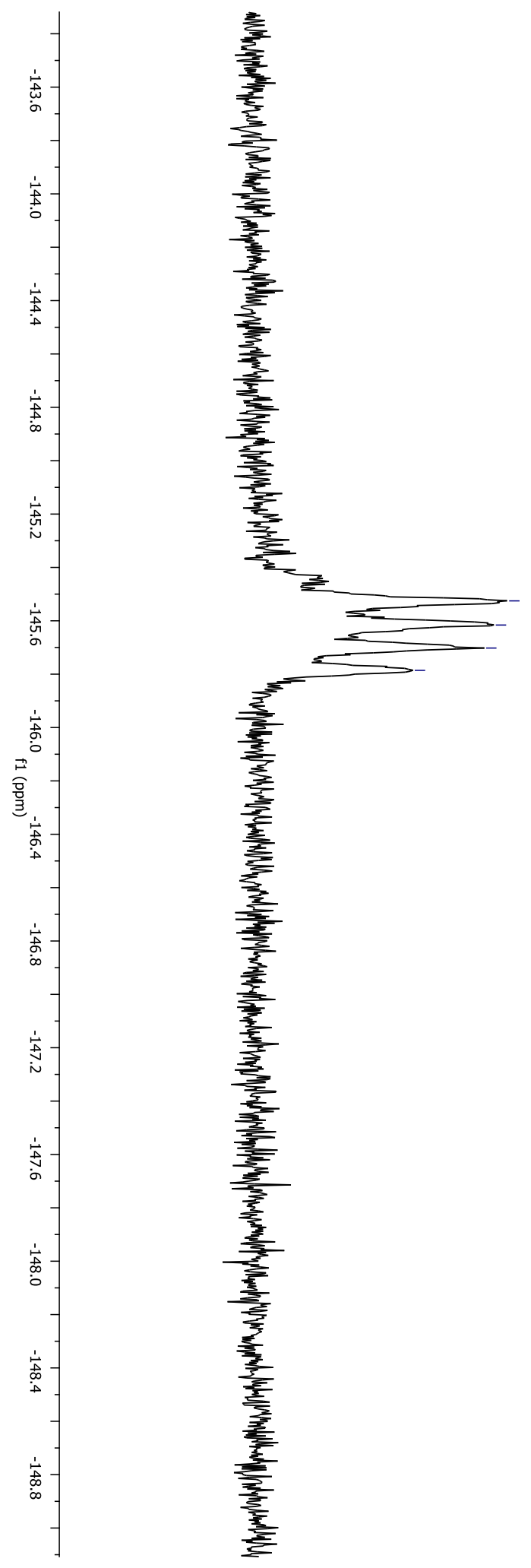

$--145.53$

$--145.62$

$--145.70$

$--145.79$

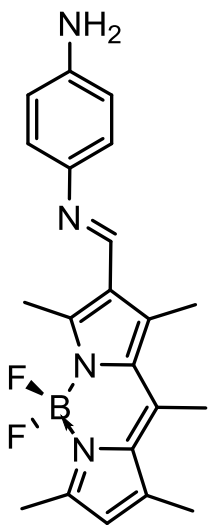

Figure A.26: ${ }^{19} \mathrm{~F}$ NMR of $\mathbf{2 . 1 5}$ 


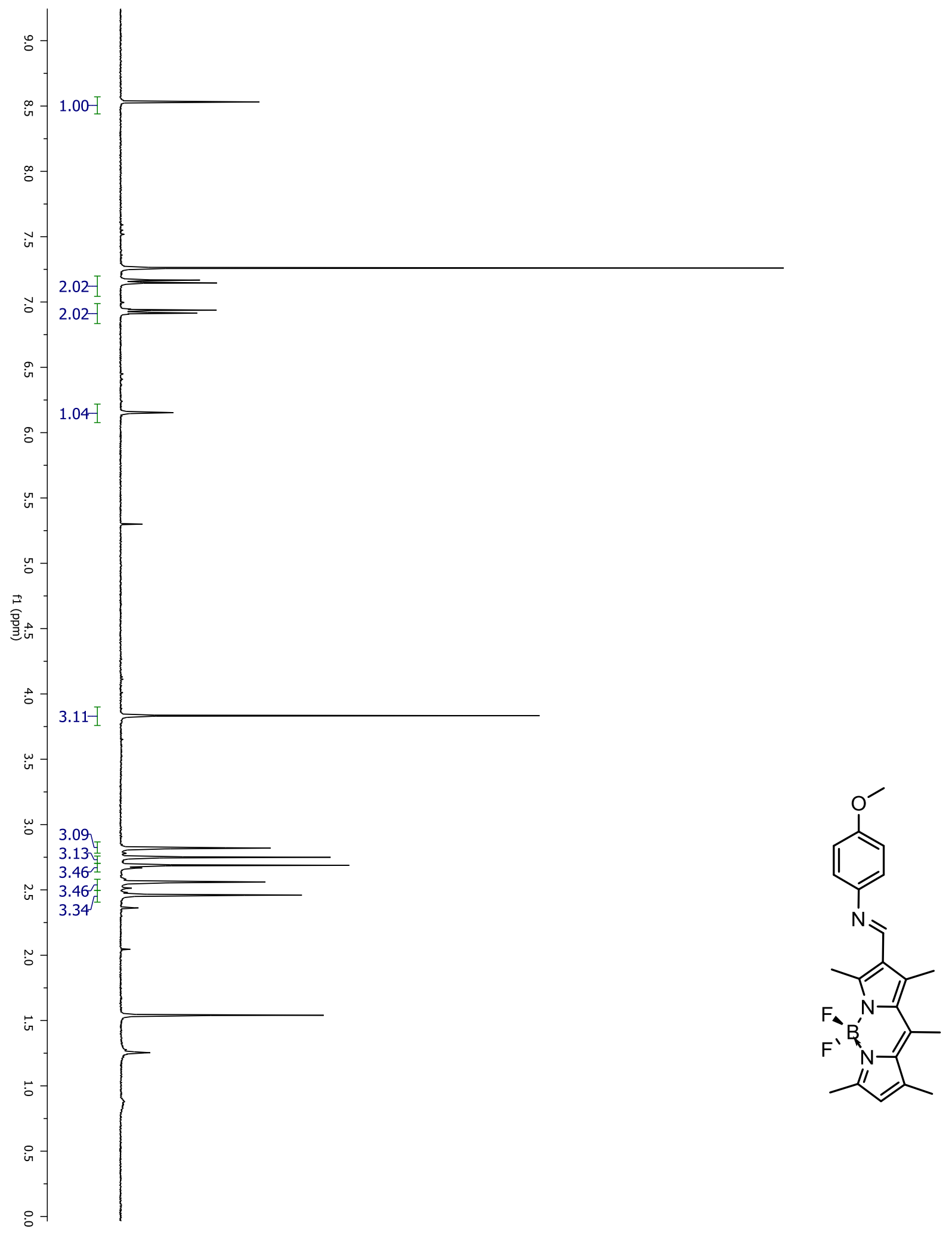

Figure A.27: ${ }^{1} \mathrm{H}$ NMR of $\mathbf{2 . 1 6}$ 


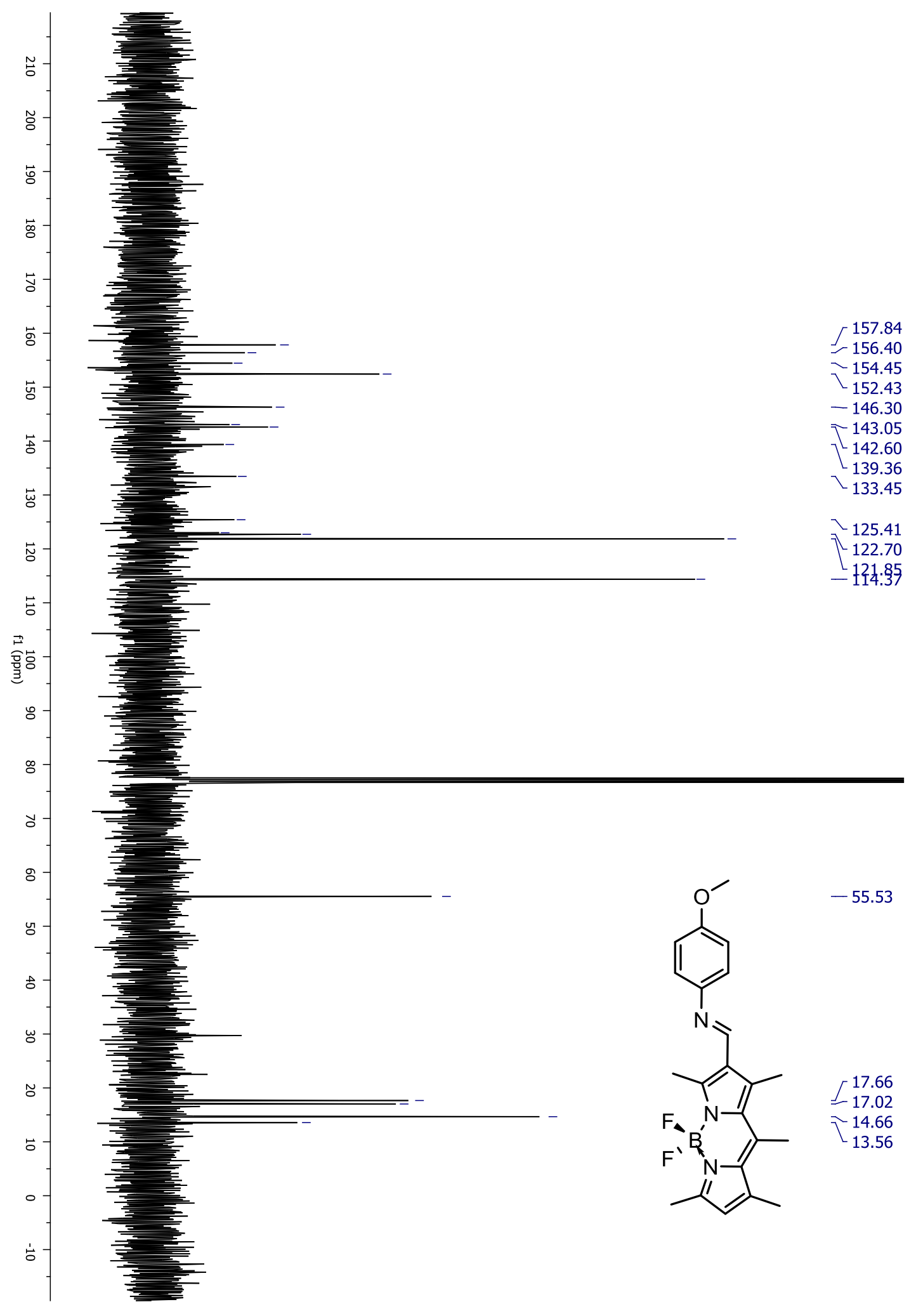

Figure A.28: ${ }^{13} \mathrm{C}$ NMR of 2.16 


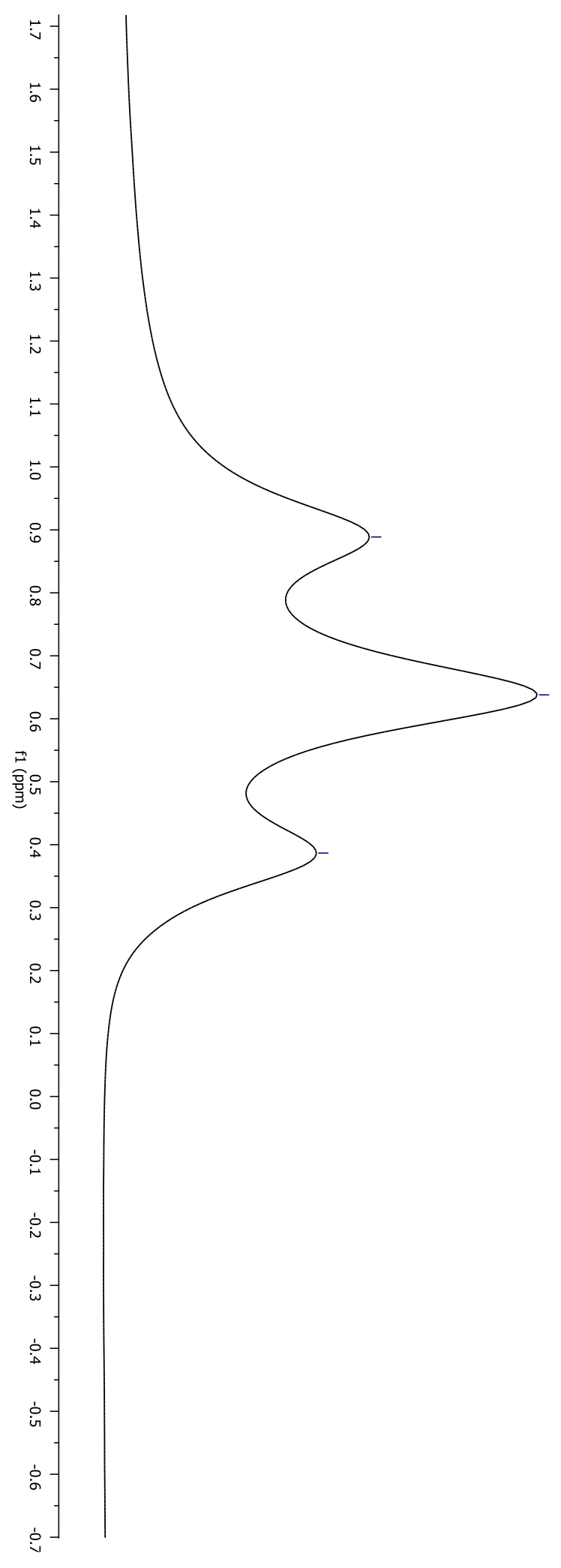

$-0.89$

$-0.64$

$-0.39$

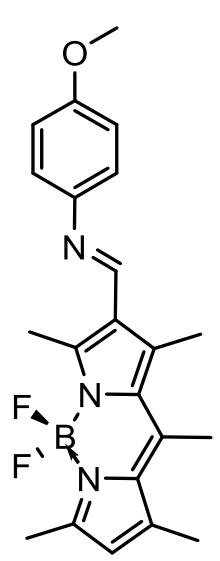

Figure A.29: ${ }^{11} B$ NMR of 2.16 


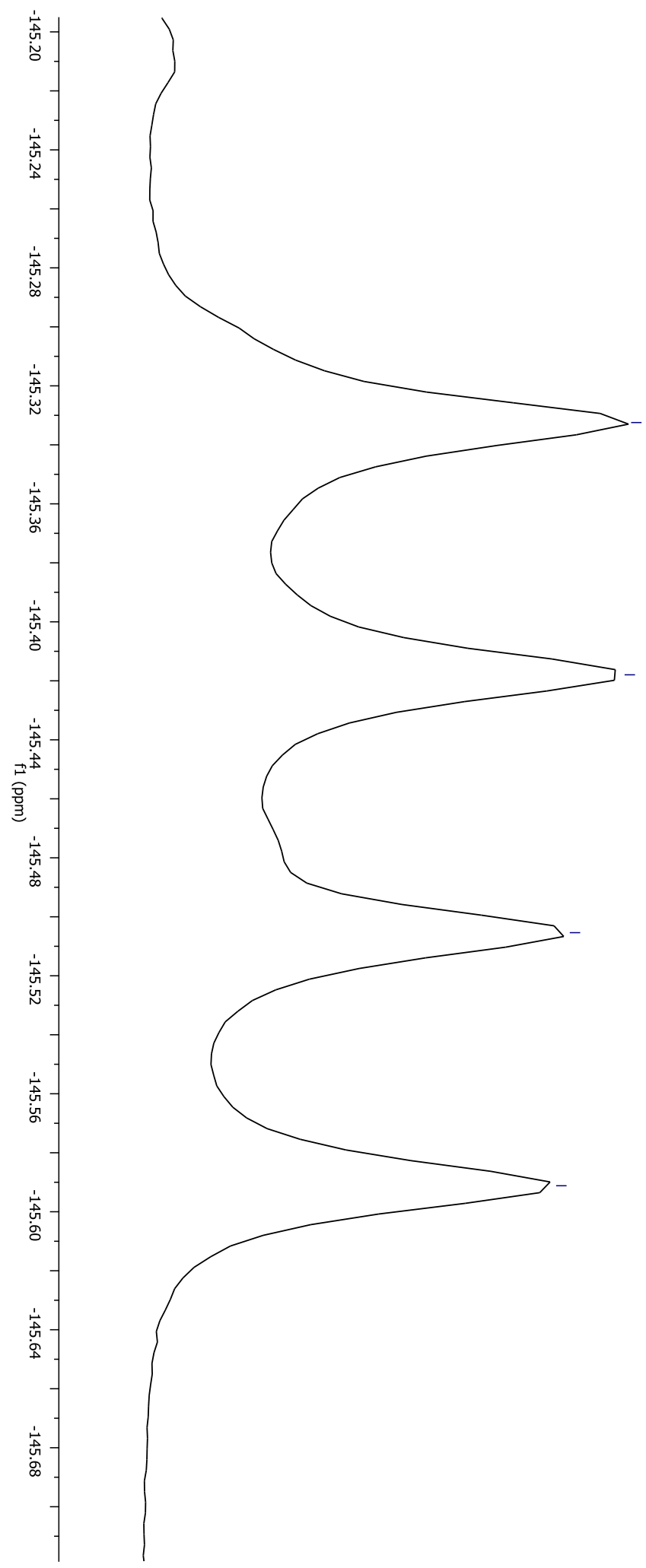

$--145.33$

$--145.42$

$--145.51$

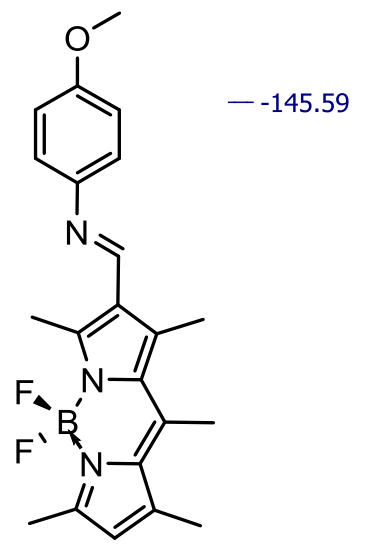

Figure A.30: ${ }^{19} \mathrm{~F}$ NMR of 2.16 


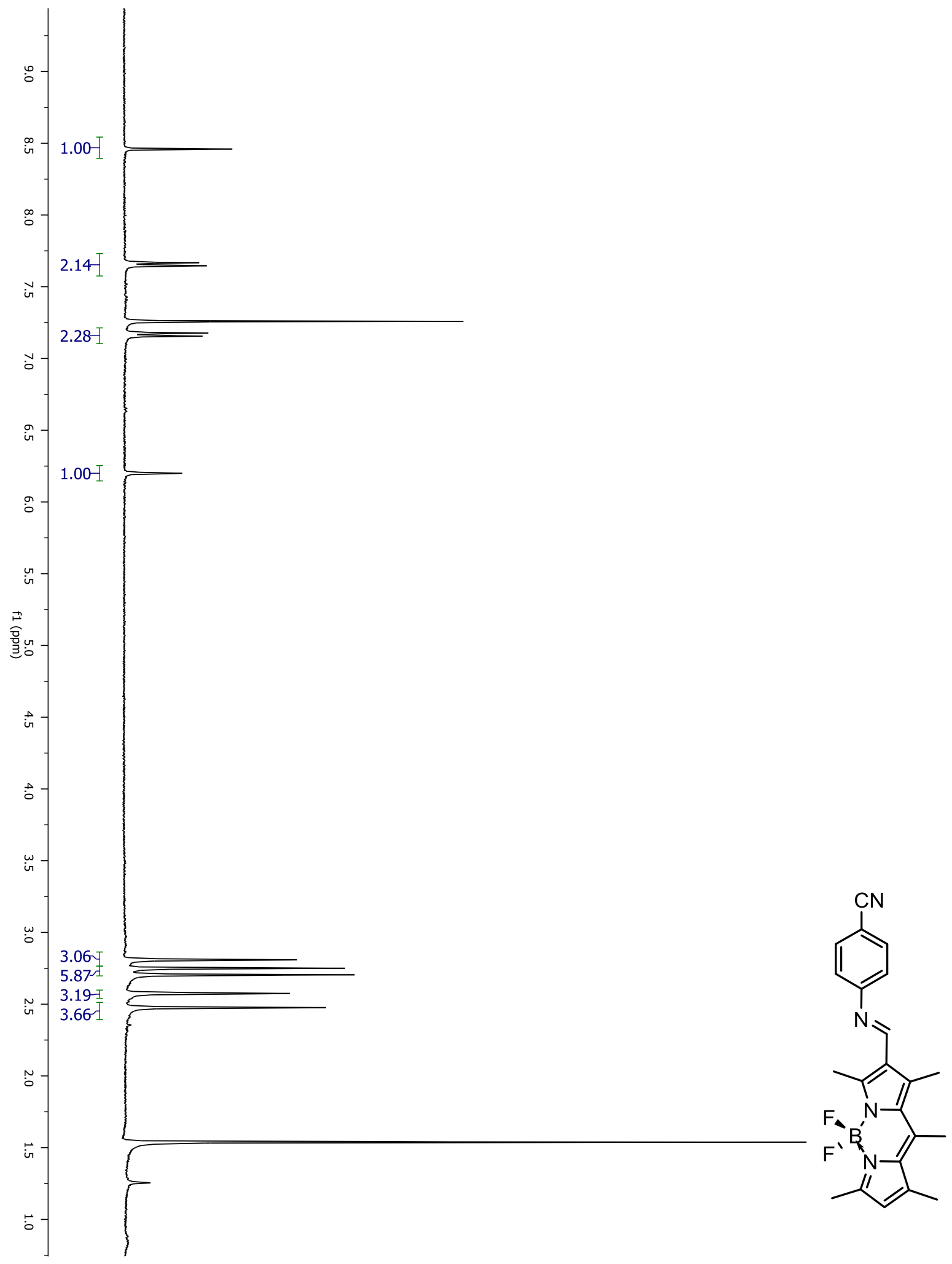

Figure A.31: ${ }^{1} \mathrm{H}$ NMR of $\mathbf{2 . 1 7}$ 


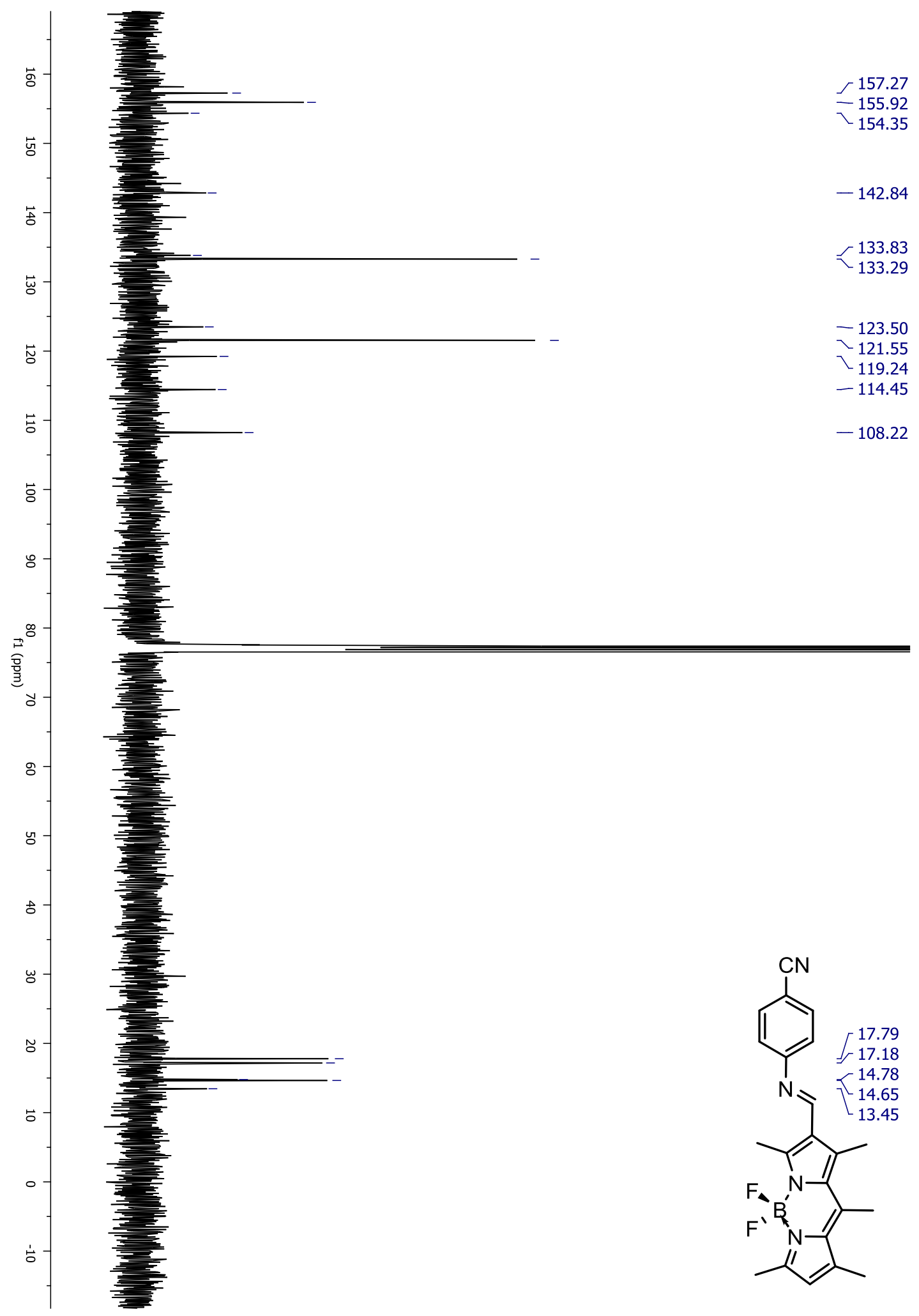

Figure A.32: ${ }^{13} \mathrm{C}$ NMR of $\mathbf{2 . 1 7}$ 

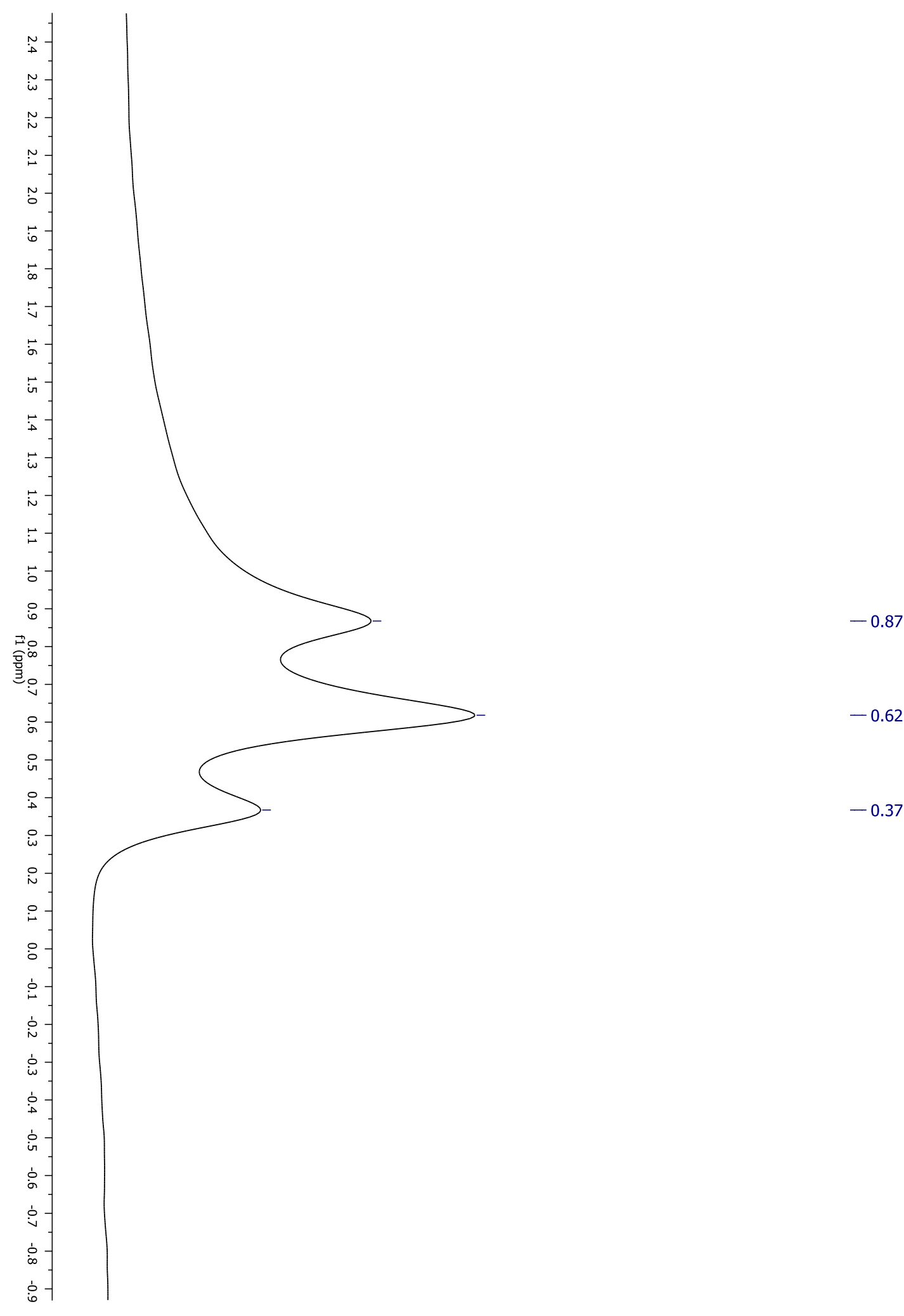

$-0.62$

$-0.37$

Figure A.33: ${ }^{11} B$ NMR of 2.17 

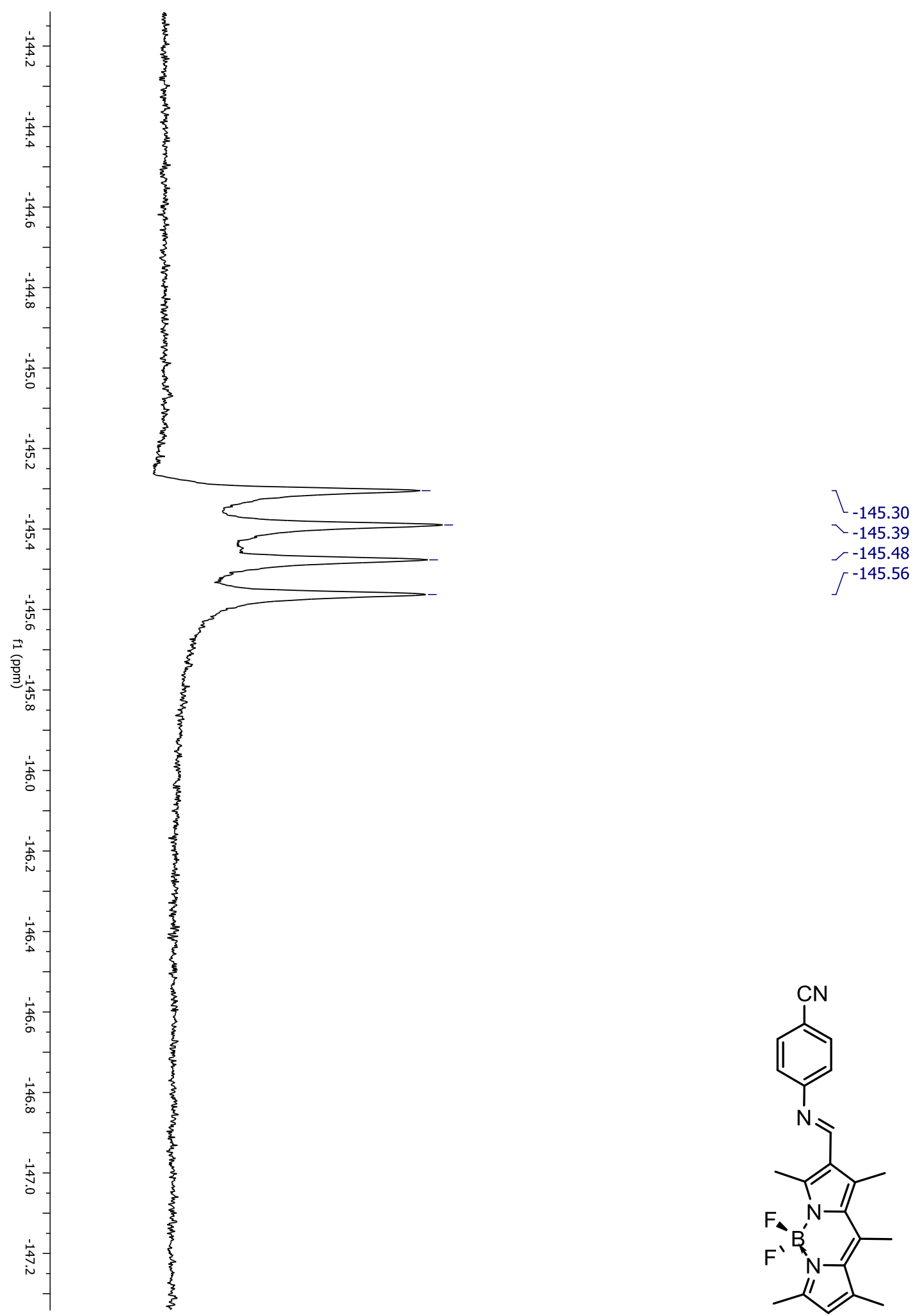

Figure A.33: ${ }^{19} \mathrm{~F}$ NMR of 2.17

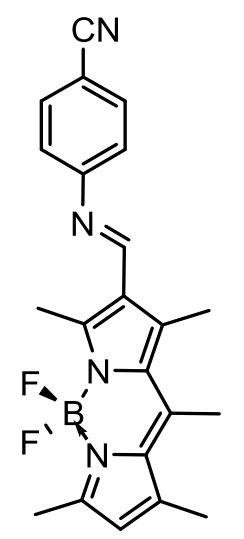



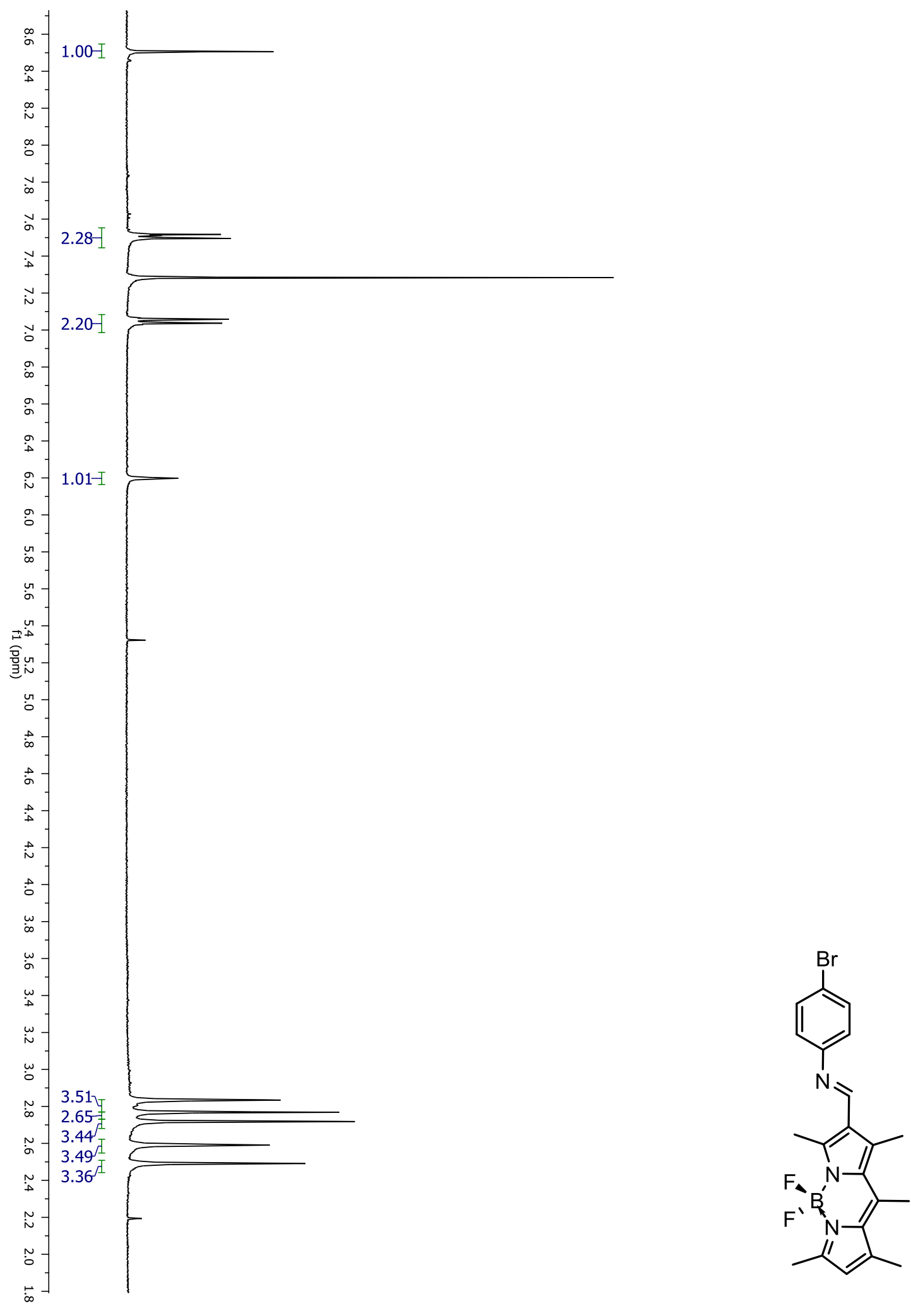

Figure A.34: ${ }^{1} \mathrm{H}$ NMR of $\mathbf{2 . 1 8}$ 


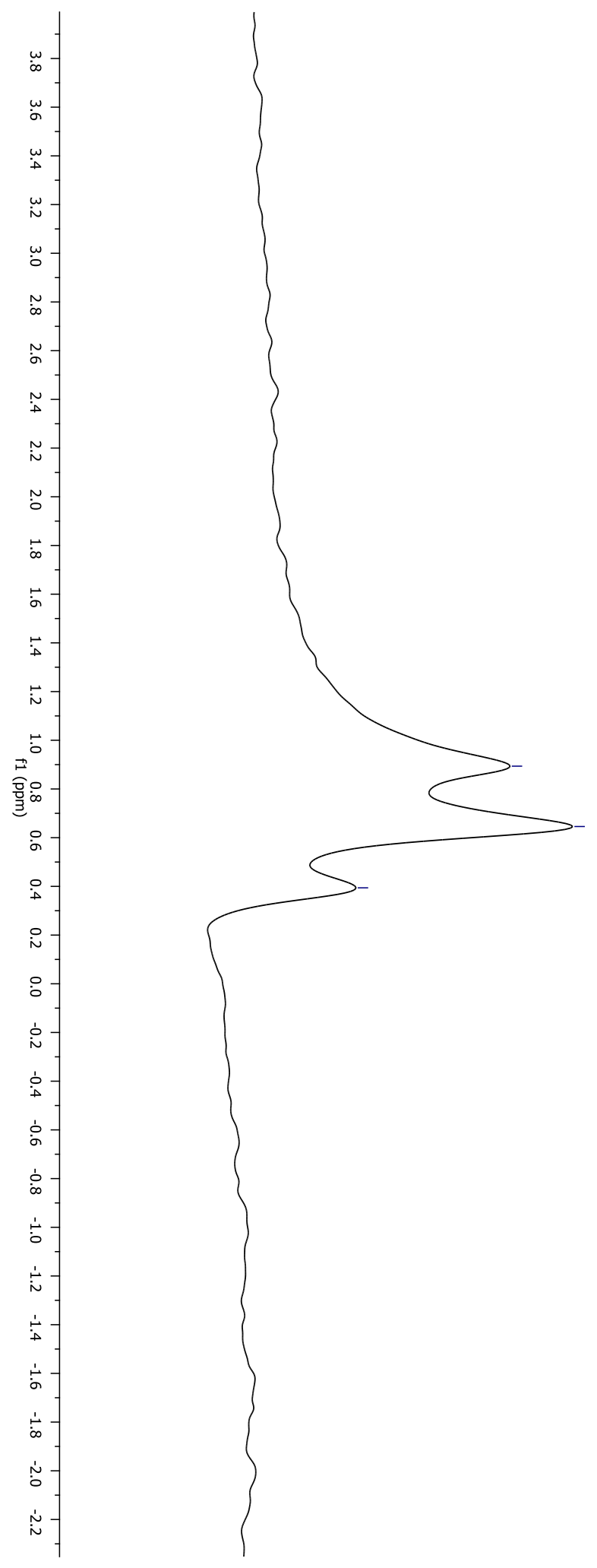

$-0.89$

$-0.65$

$-0.39$

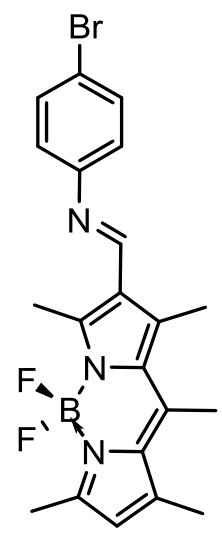

Figure A.35: ${ }^{11} B$ NMR of 2.18 

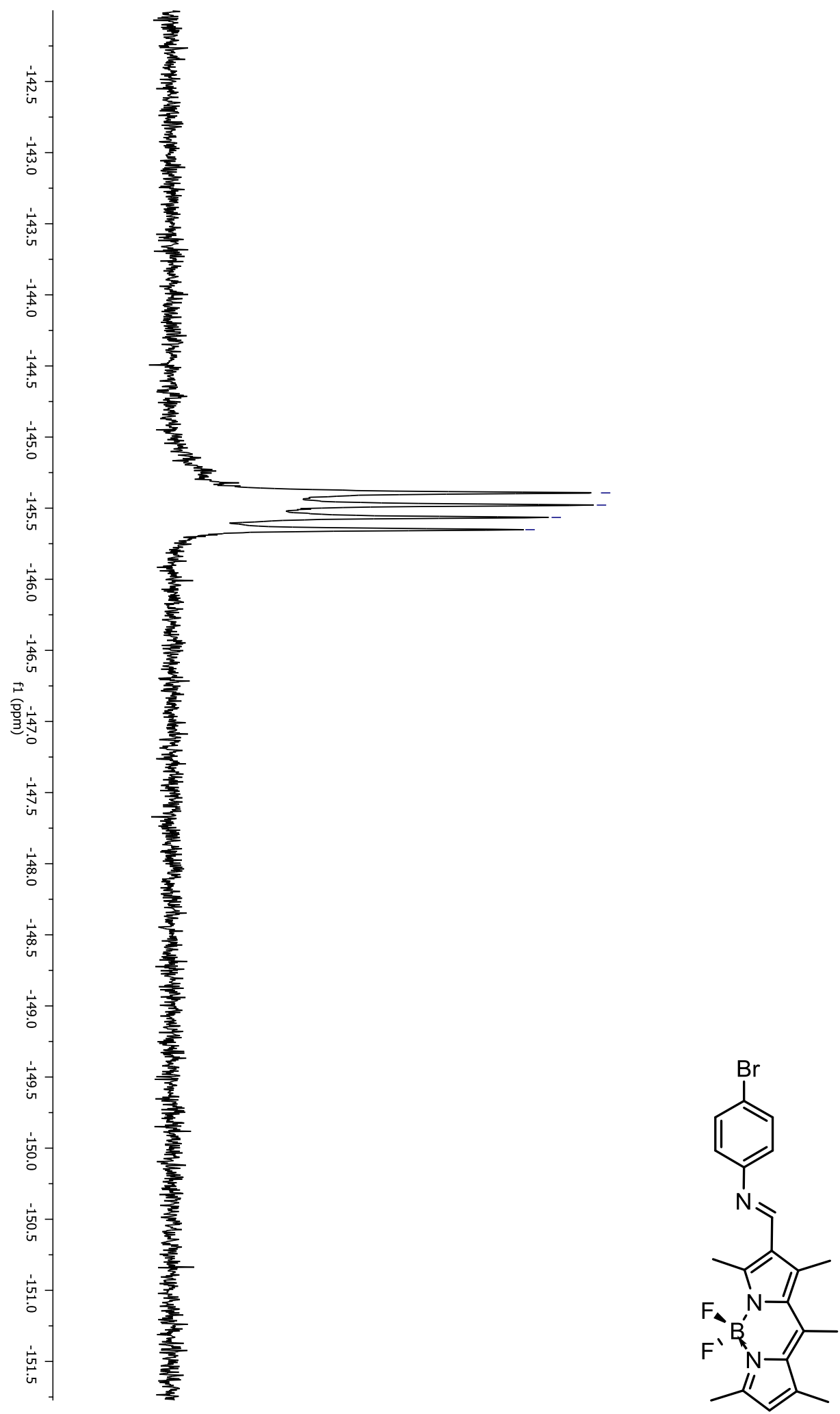

Figure A.36: ${ }^{19} \mathrm{~F}$ NMR of $\mathbf{2 . 1 8}$ 

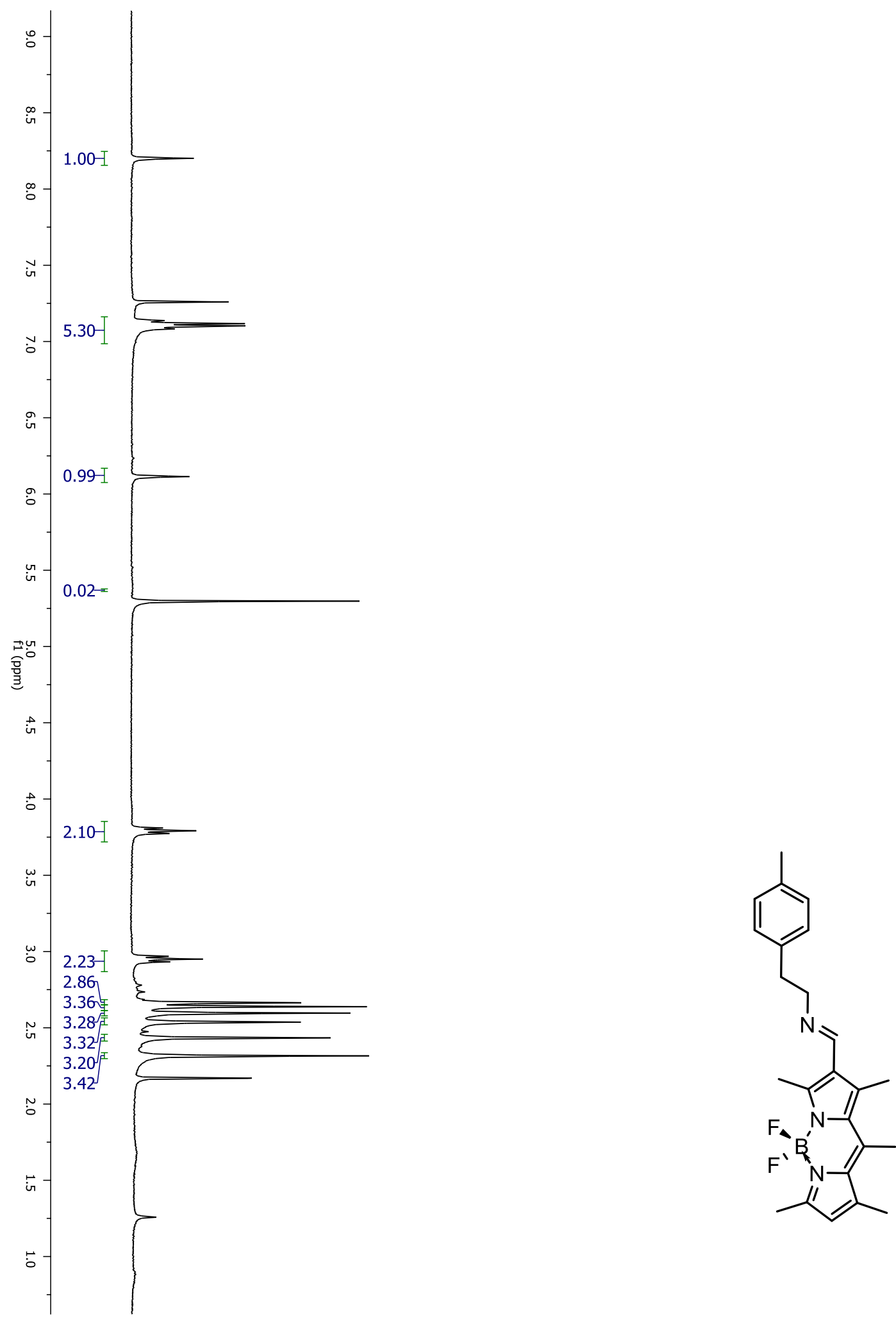

Figure A.37: ${ }^{1} \mathrm{H}$ NMR of $\mathbf{2 . 1 9}$ 


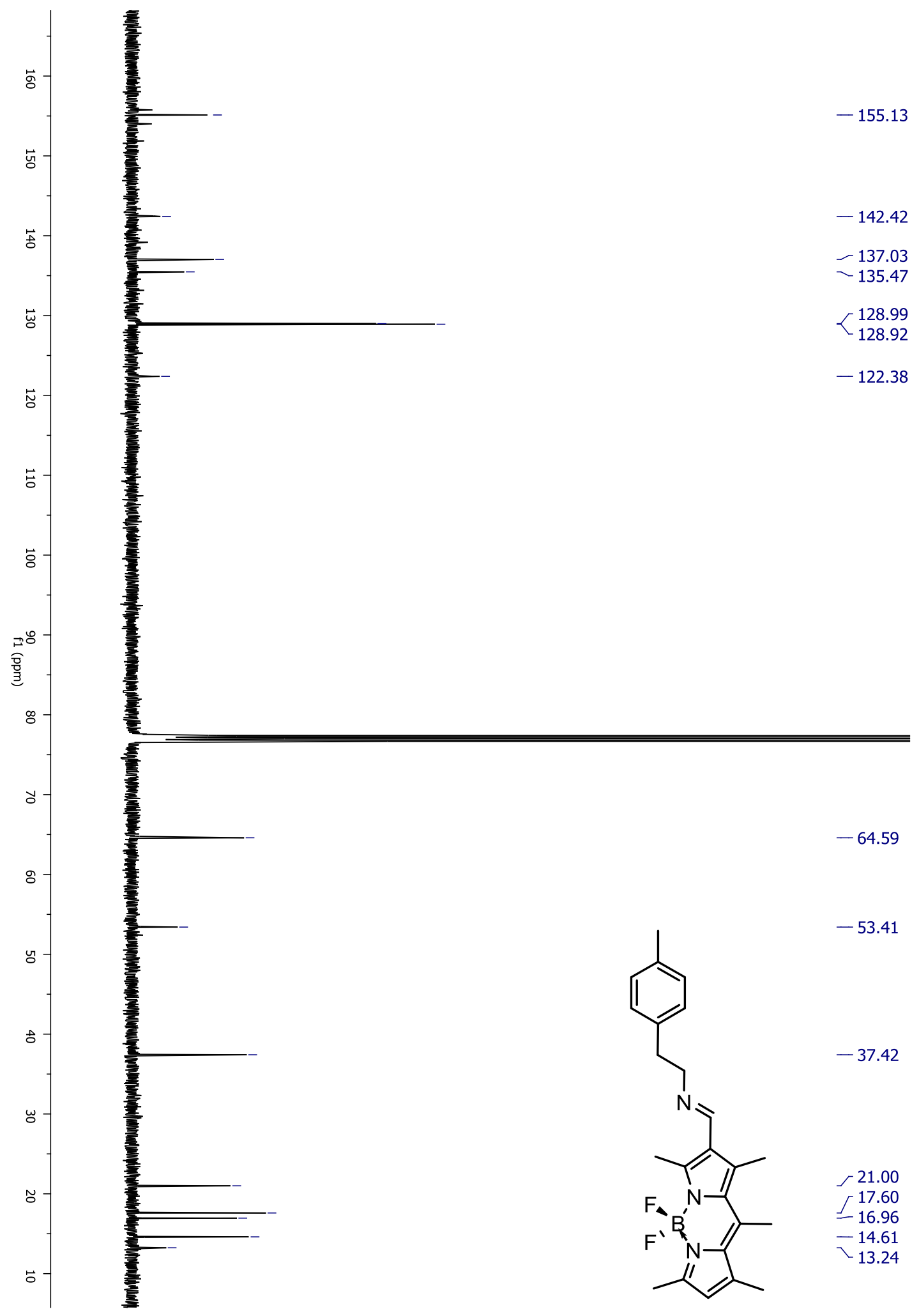

Figure A.38: ${ }^{13} \mathrm{C}$ NMR of 2.19 


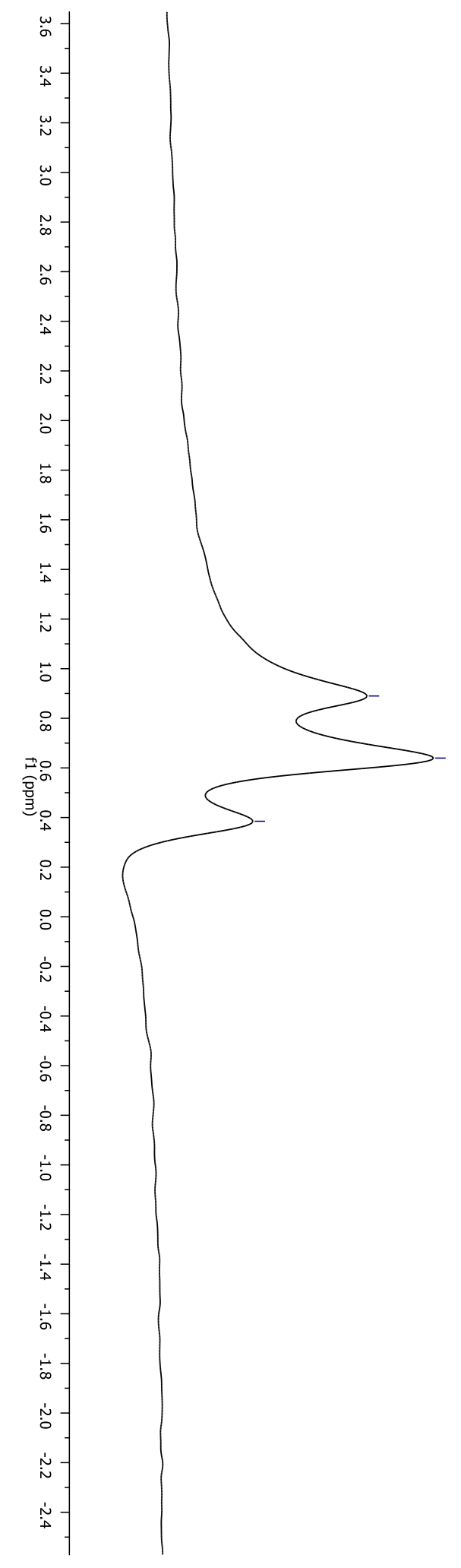

$$
\begin{aligned}
& -0.89 \\
& -0.64 \\
& -0.38
\end{aligned}
$$

Figure A.39: ${ }^{11} B$ NMR of 2.19 


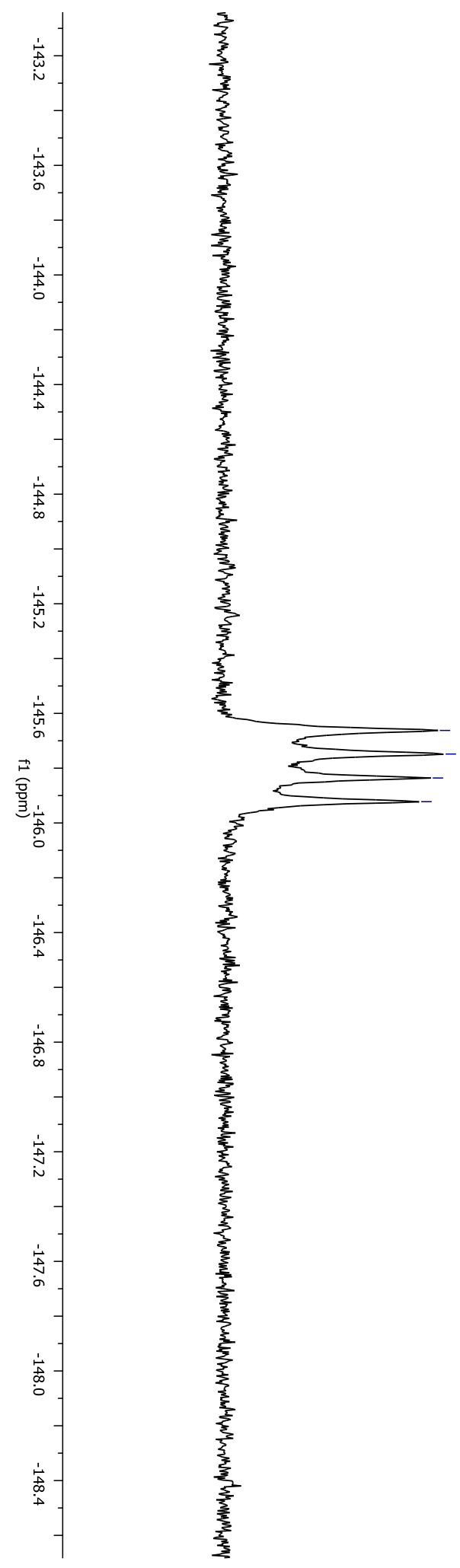

-145.66
--14575

$--145.84$

$--145.92$

Figure A.40: ${ }^{19} \mathrm{~F}$ NMR of $\mathbf{2 . 1 9}$

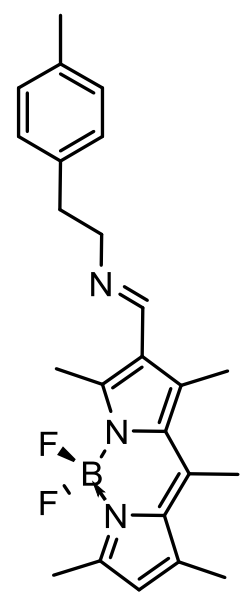




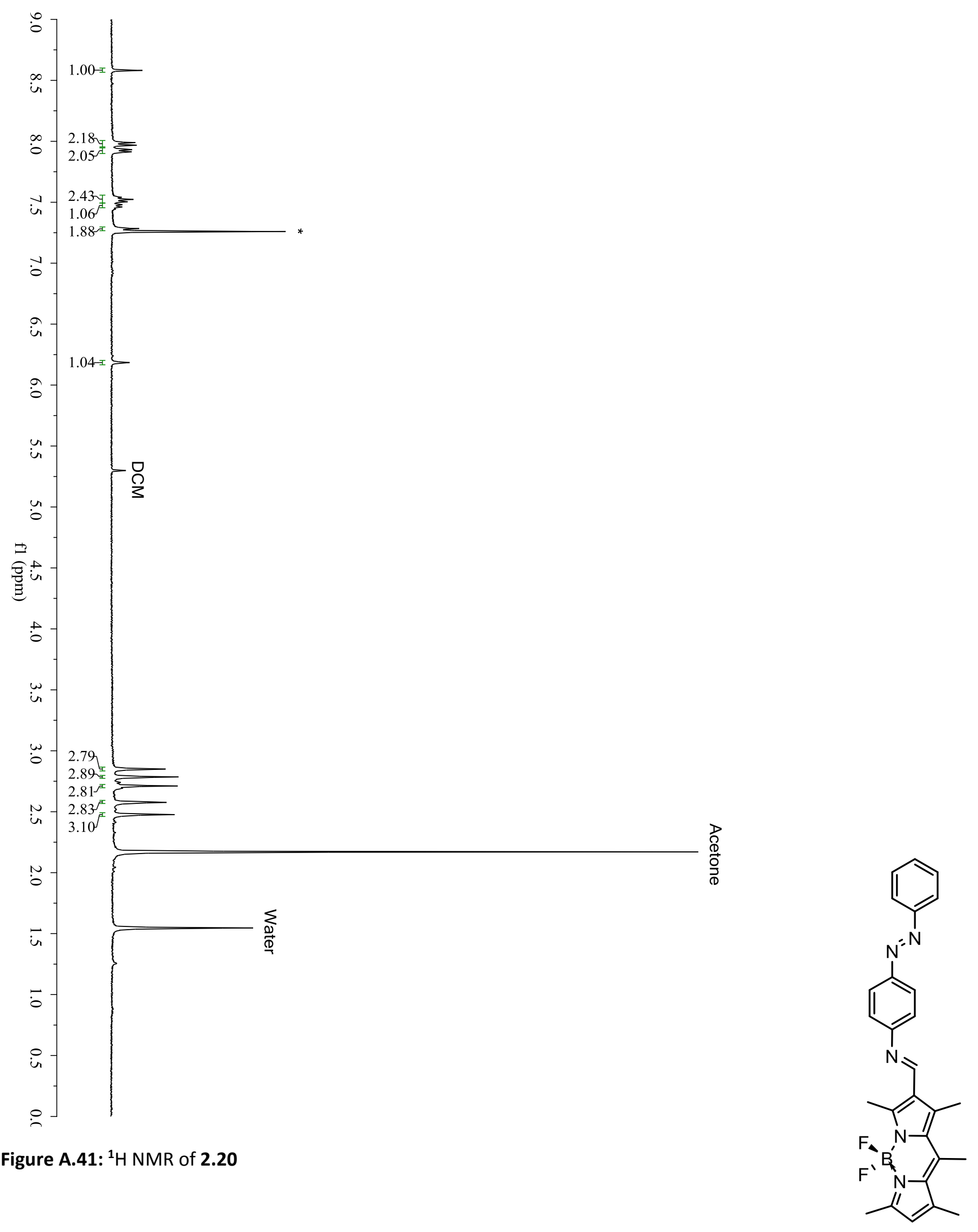




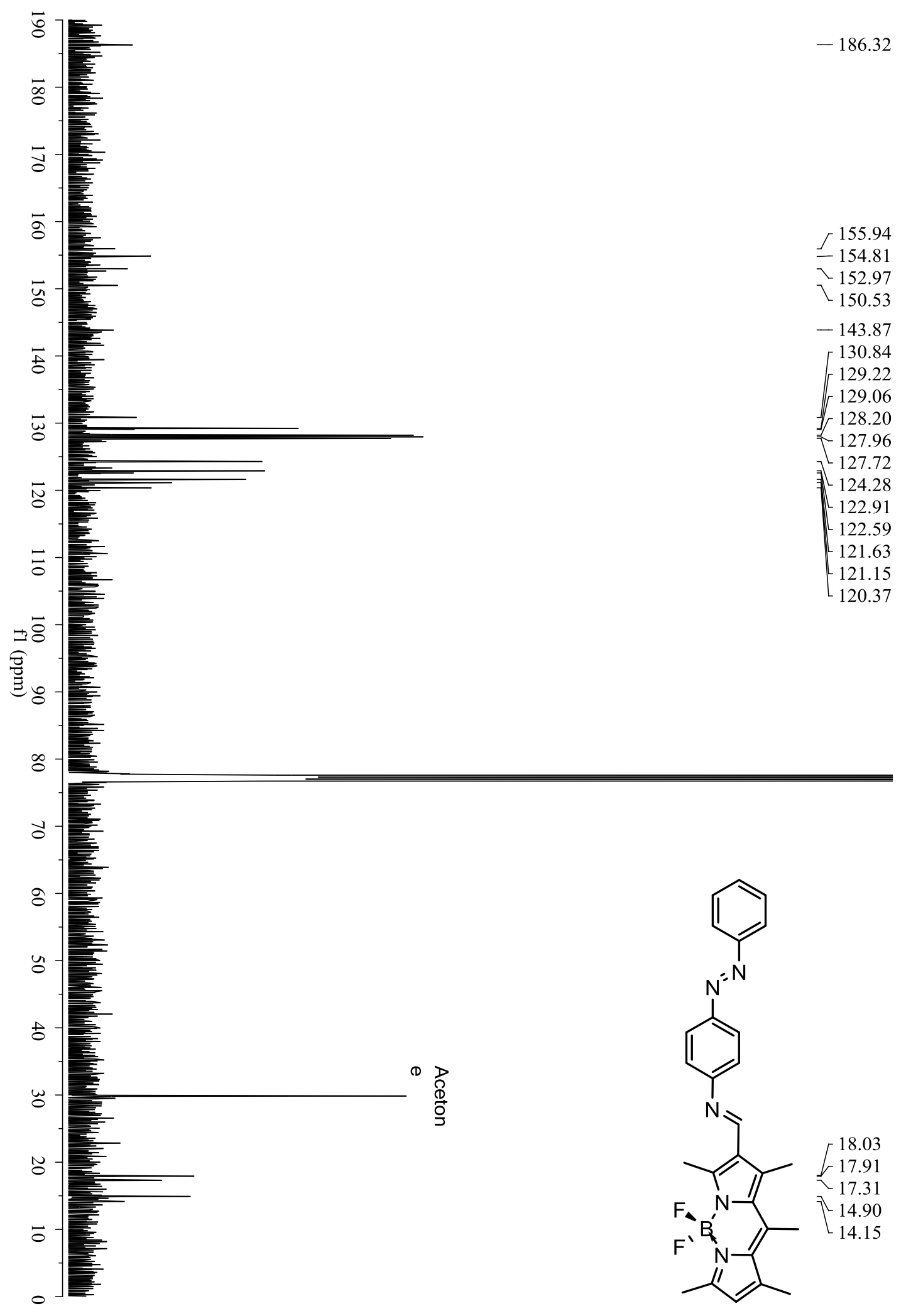

Figure A.42: ${ }^{13} \mathrm{C}$ NMR of $\mathbf{2 . 2 0}$ 


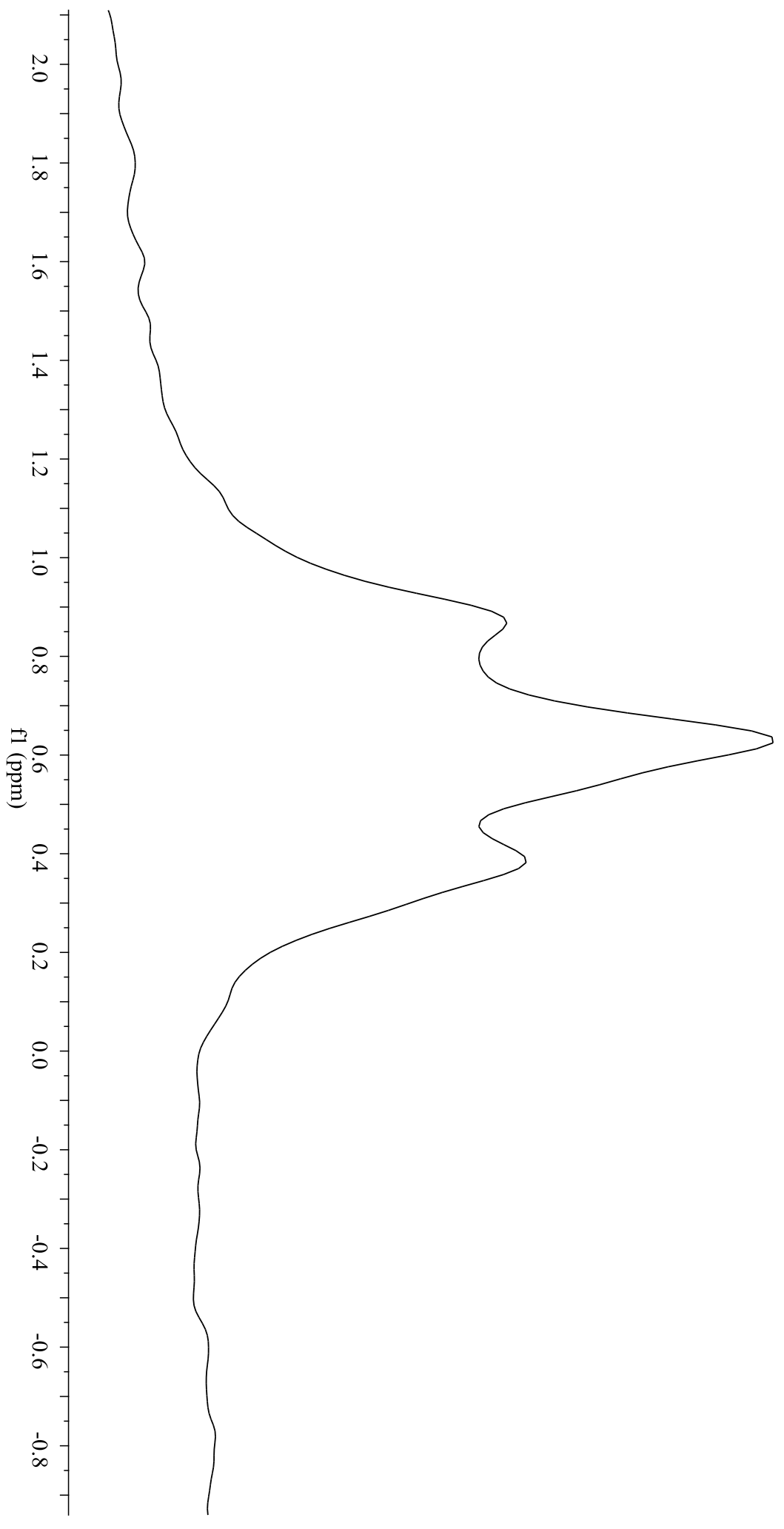

Figure A.43: ${ }^{11} B$ NMR of $\mathbf{2 . 2 0}$

$-0.87$

$-0.63$

$-0.39$

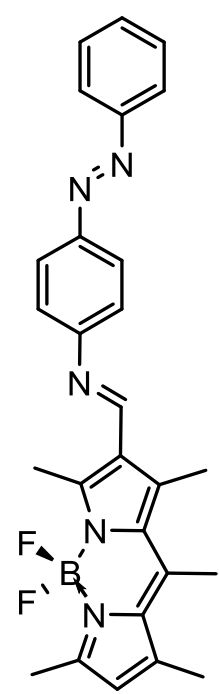




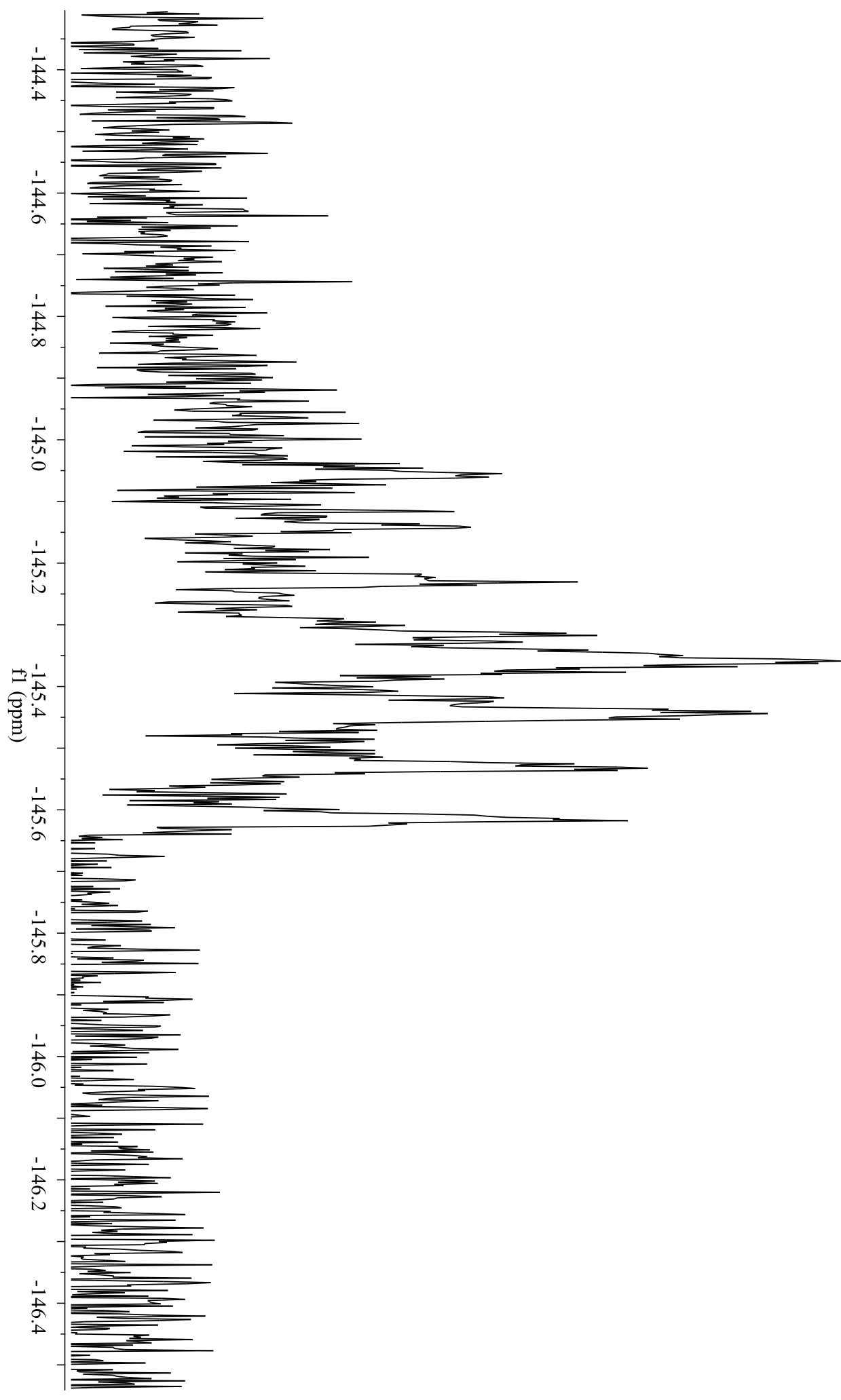

$--145.36$

$--145.44$

$--145.53$

$--145.62$

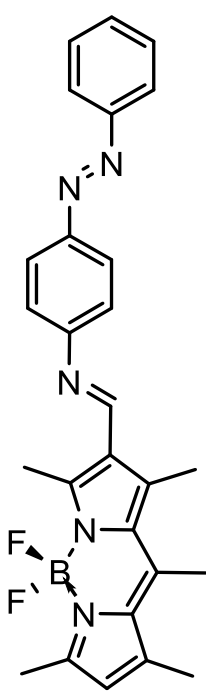

Figure A.44: ${ }^{19} \mathrm{~F}$ NMR of $\mathbf{2 . 2 0}$ 

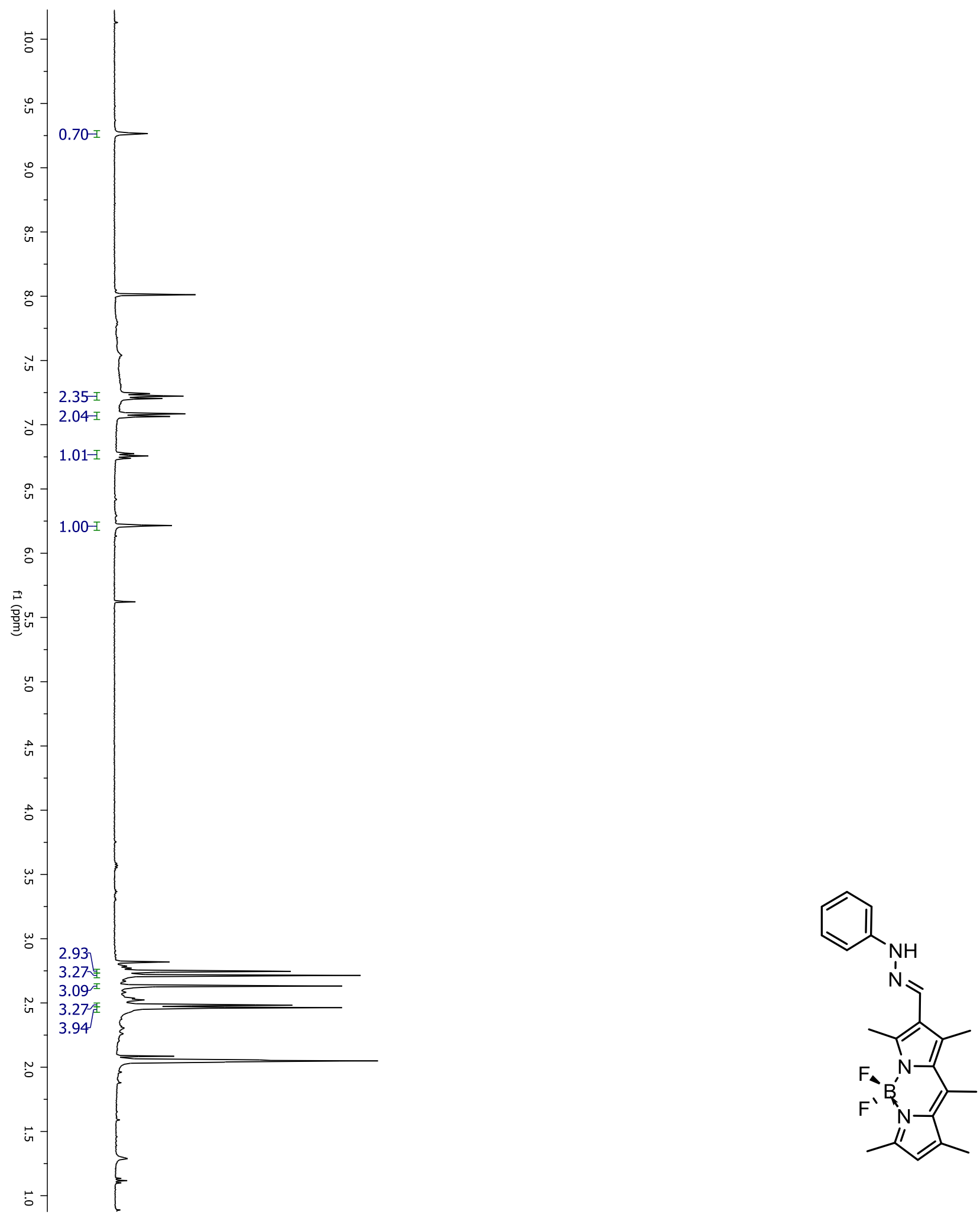

Figure A.45: ${ }^{1} \mathrm{H}$ NMR of $\mathbf{2 . 2 2}$ 


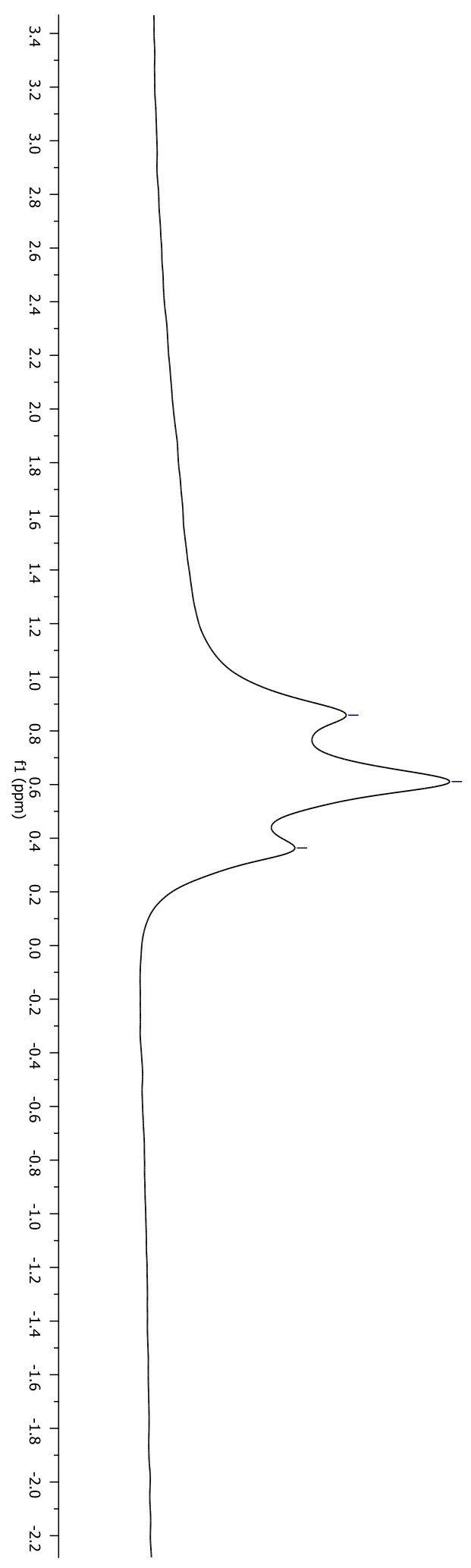

$$
\begin{aligned}
& -0.86 \\
& -0.61 \\
& -0.36
\end{aligned}
$$

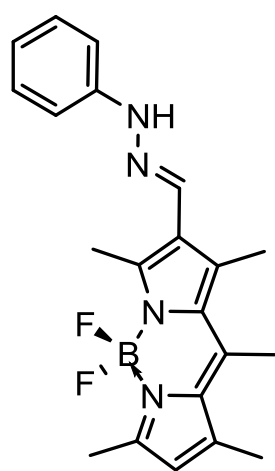

Figure A.46: ${ }^{11} B$ NMR of 2.22 

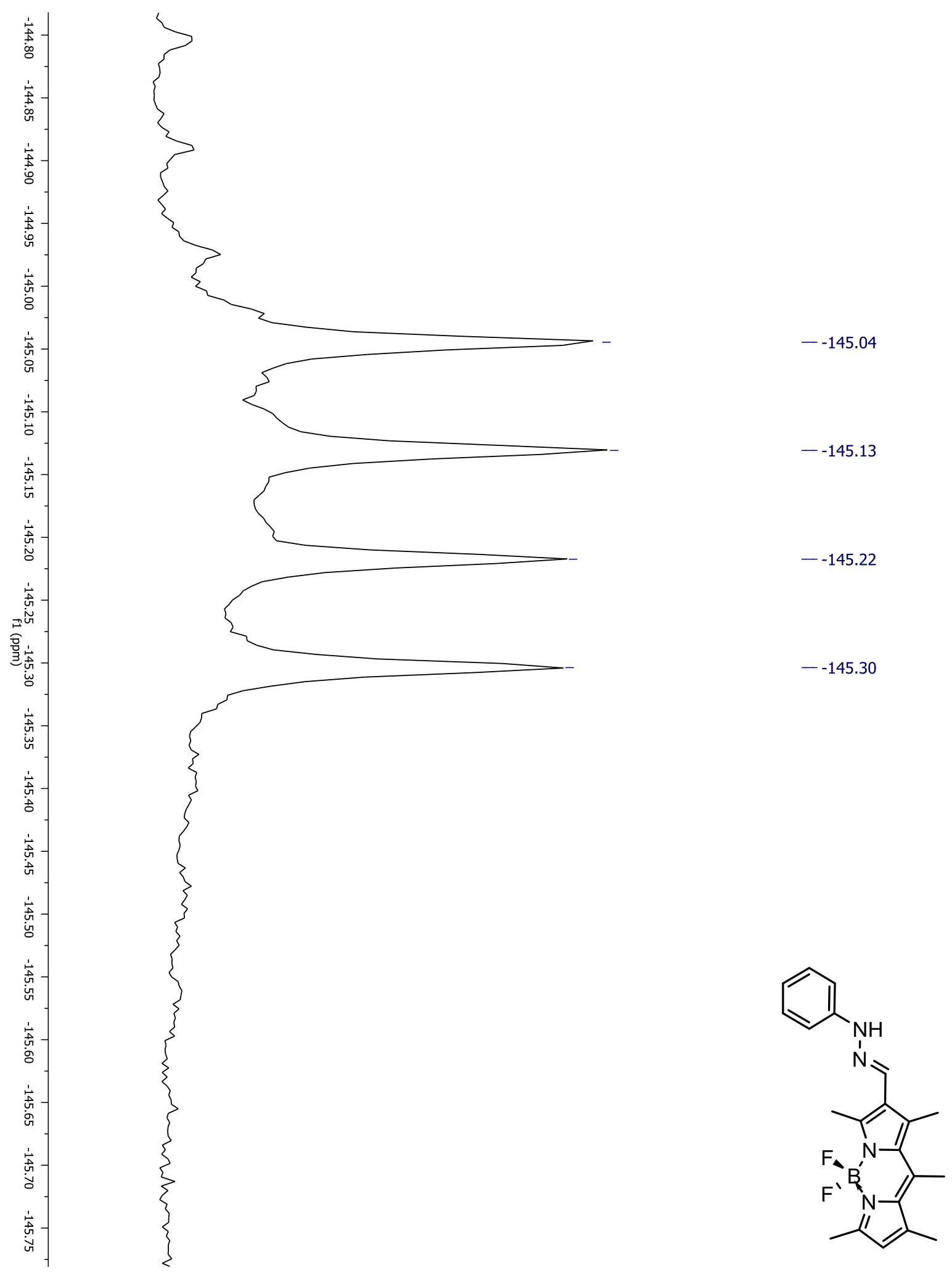

Figure A.47: ${ }^{19} \mathrm{~F}$ NMR of $\mathbf{2 . 2 2}$ 


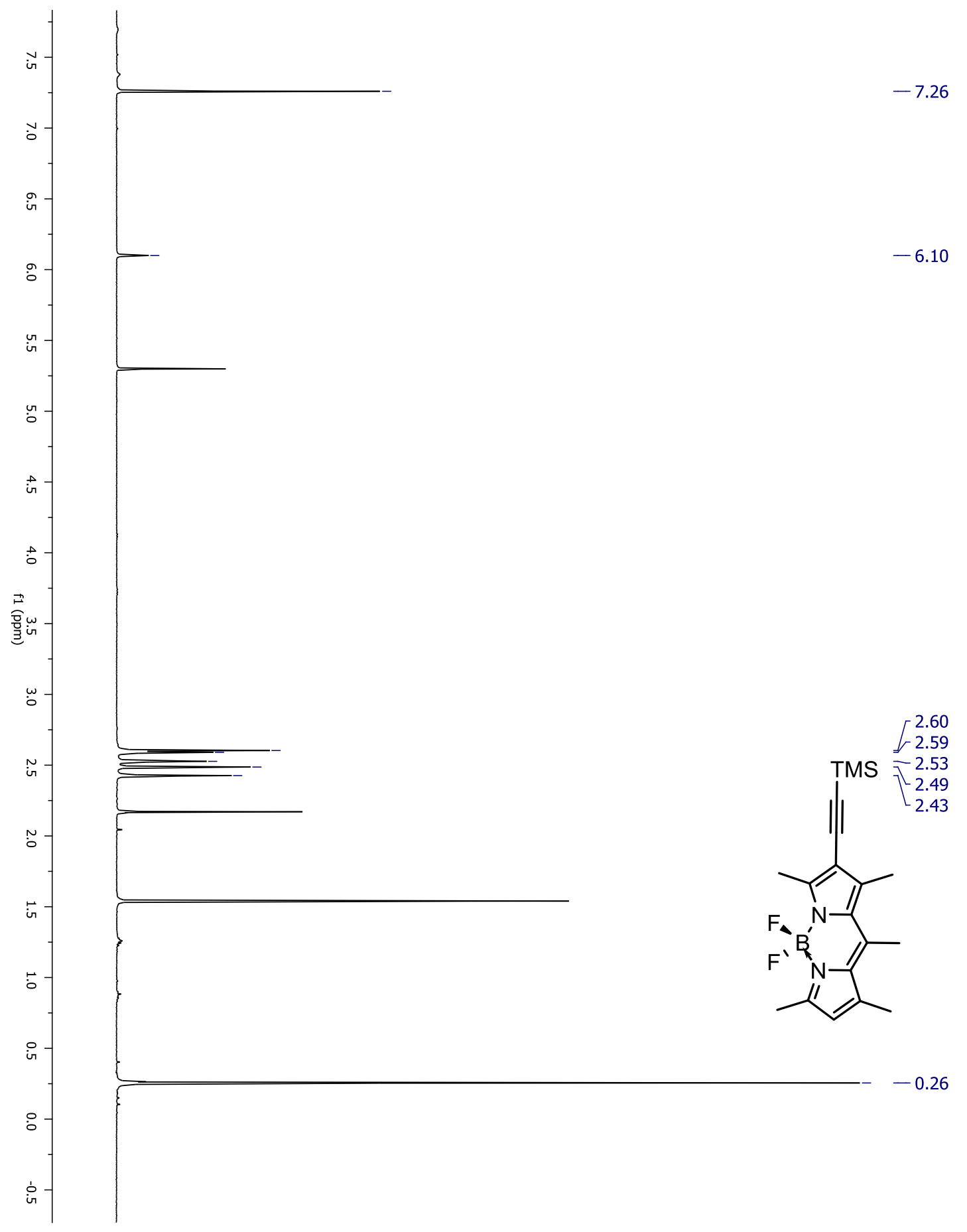

Figure A.48: ${ }^{1} \mathrm{H}$ NMR of $\mathbf{2 . 2 3}$ 


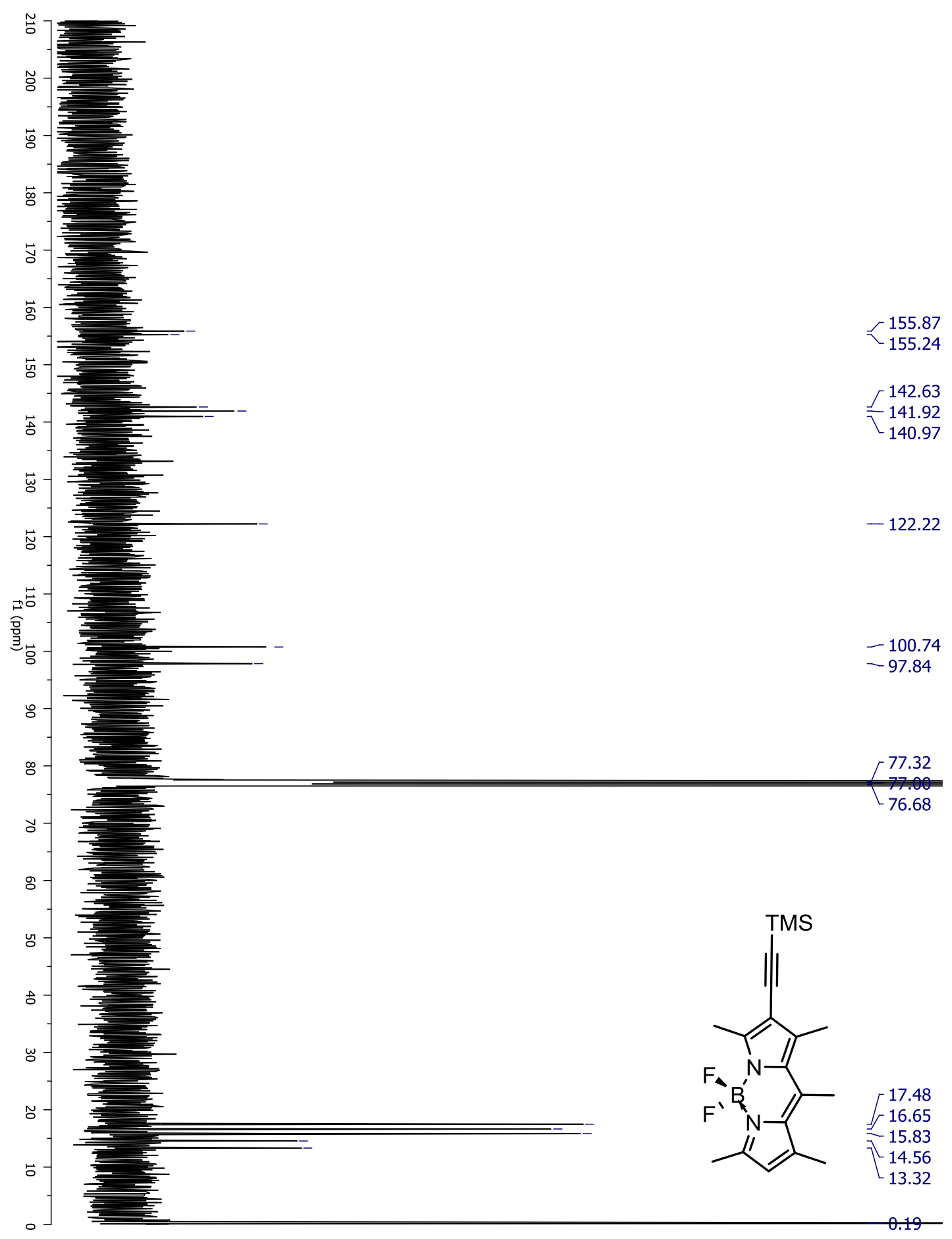

Figure A.49: ${ }^{13} \mathrm{C}$ NMR of $\mathbf{2 . 2 3}$ 


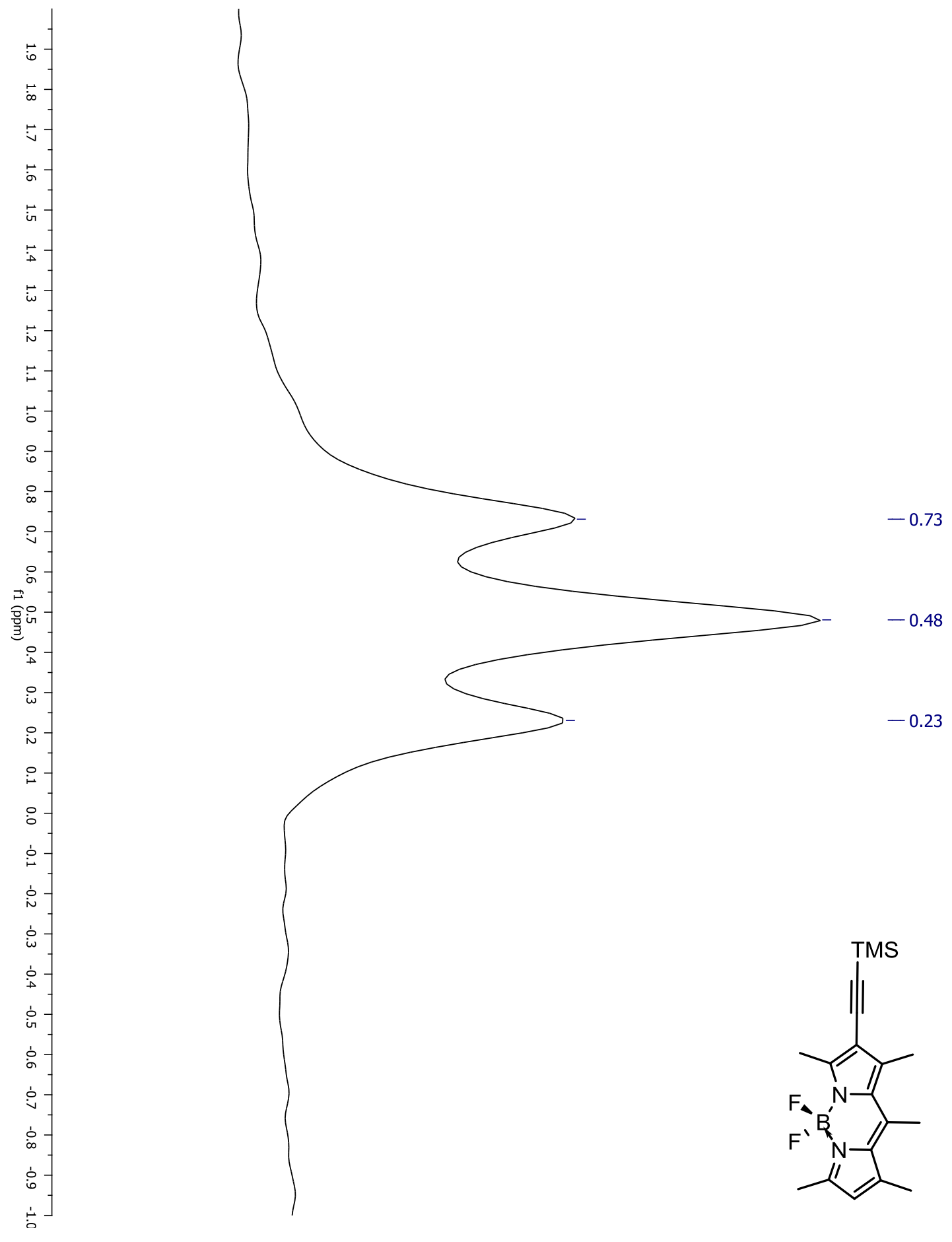

Figure A.50: ${ }^{11} B$ NMR of 2.23 


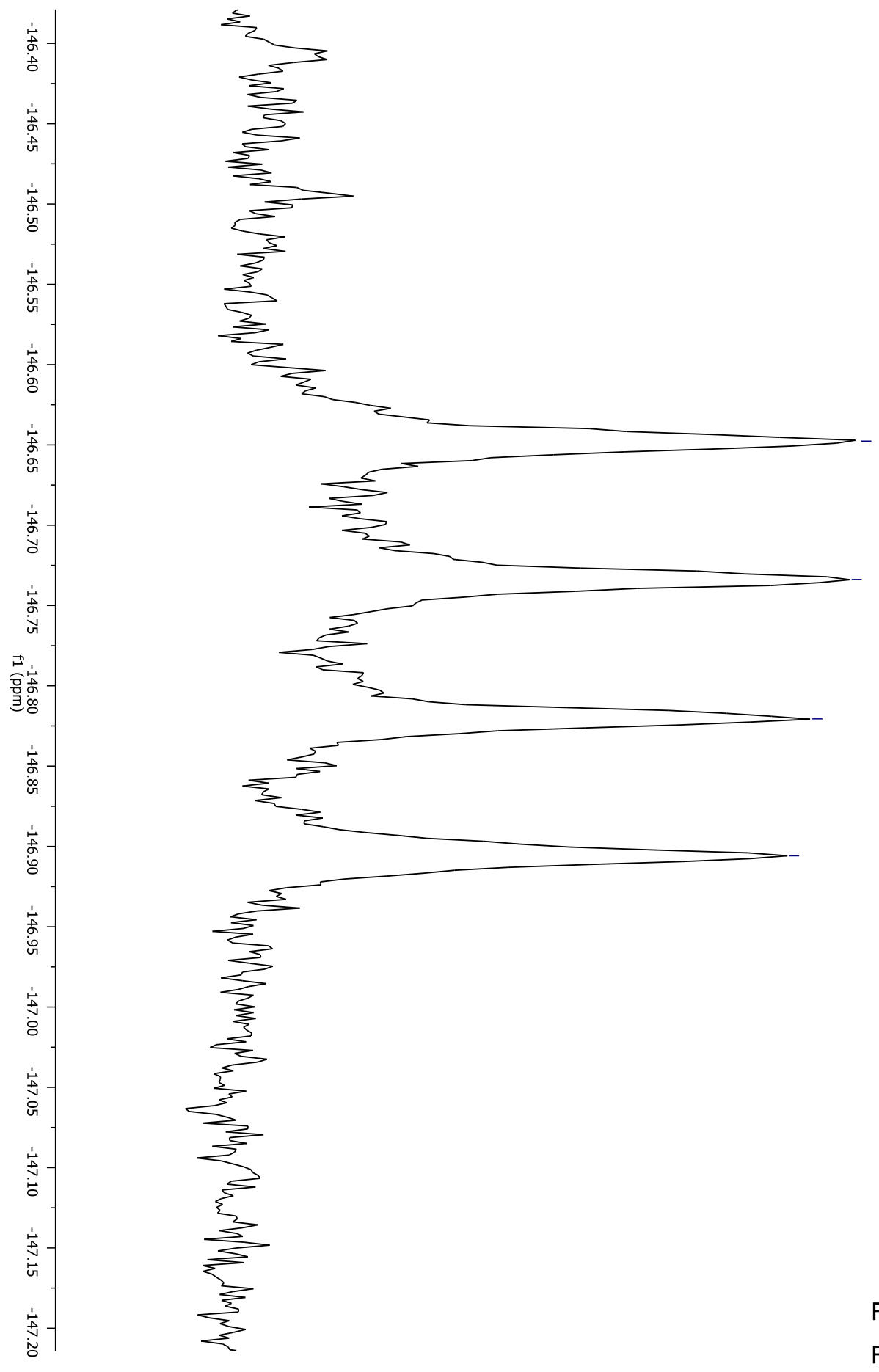

$--146.65$

$--146.73$

$--146.82$

$--146.91$

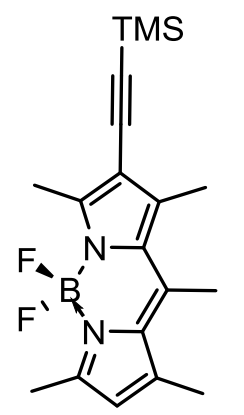

Figure A.51: ${ }^{19} \mathrm{~F}$ NMR of $\mathbf{2 . 2 3}$ 


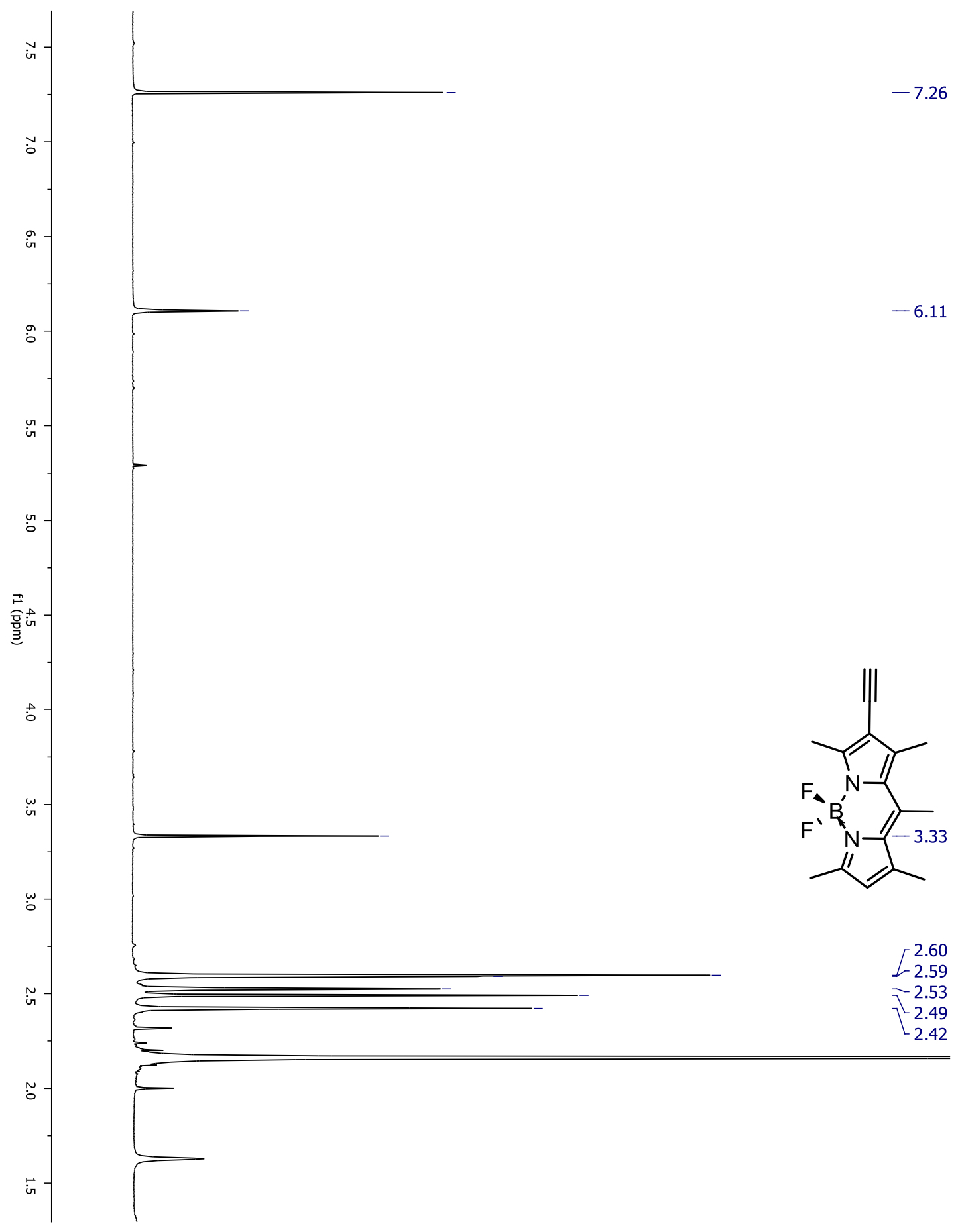

Figure A.52: ${ }^{1} \mathrm{H}$ NMR of $\mathbf{2 . 2 4}$ 


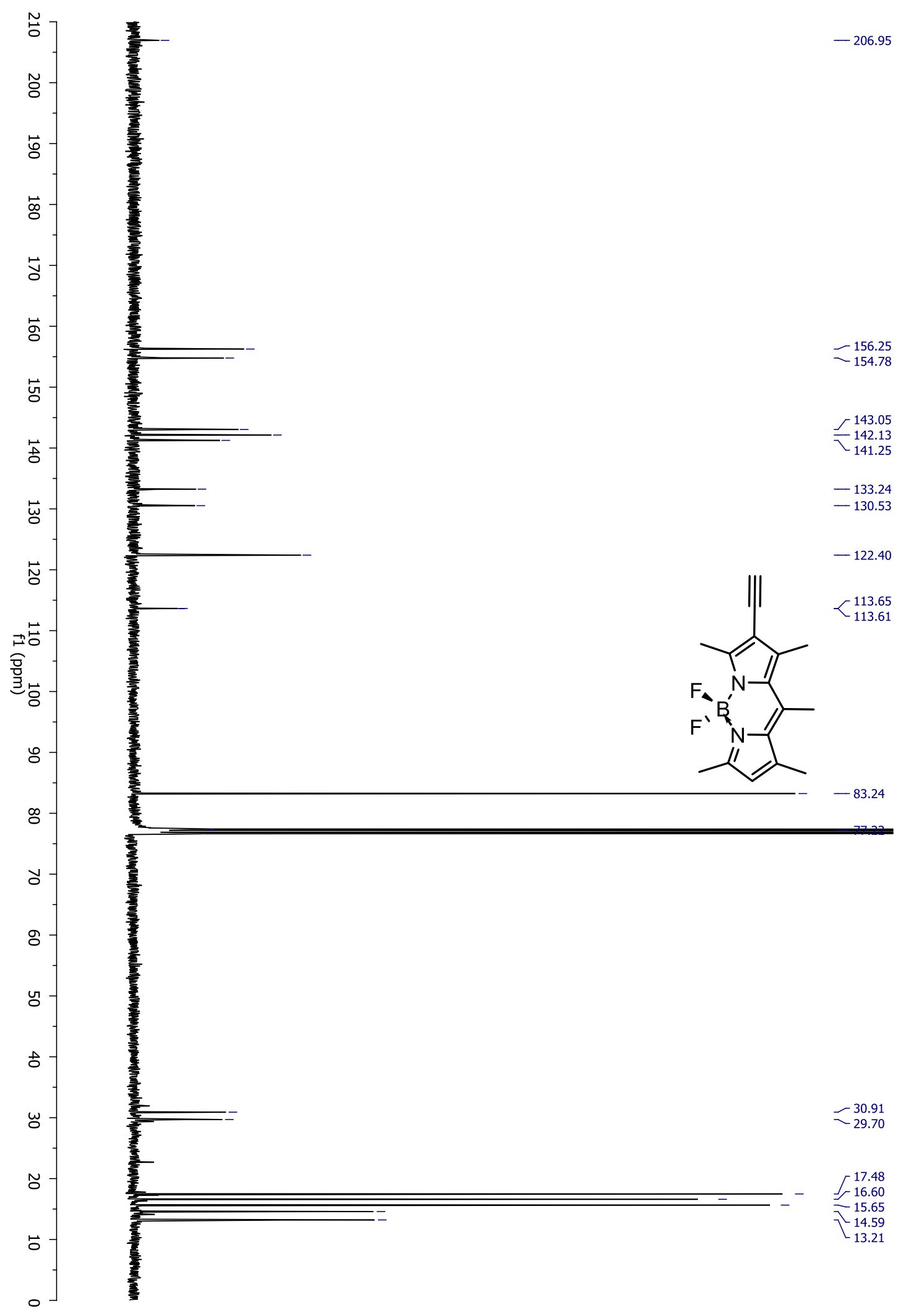

Figure A.53: ${ }^{13} \mathrm{C}$ NMR of $\mathbf{2 . 2 4}$ 


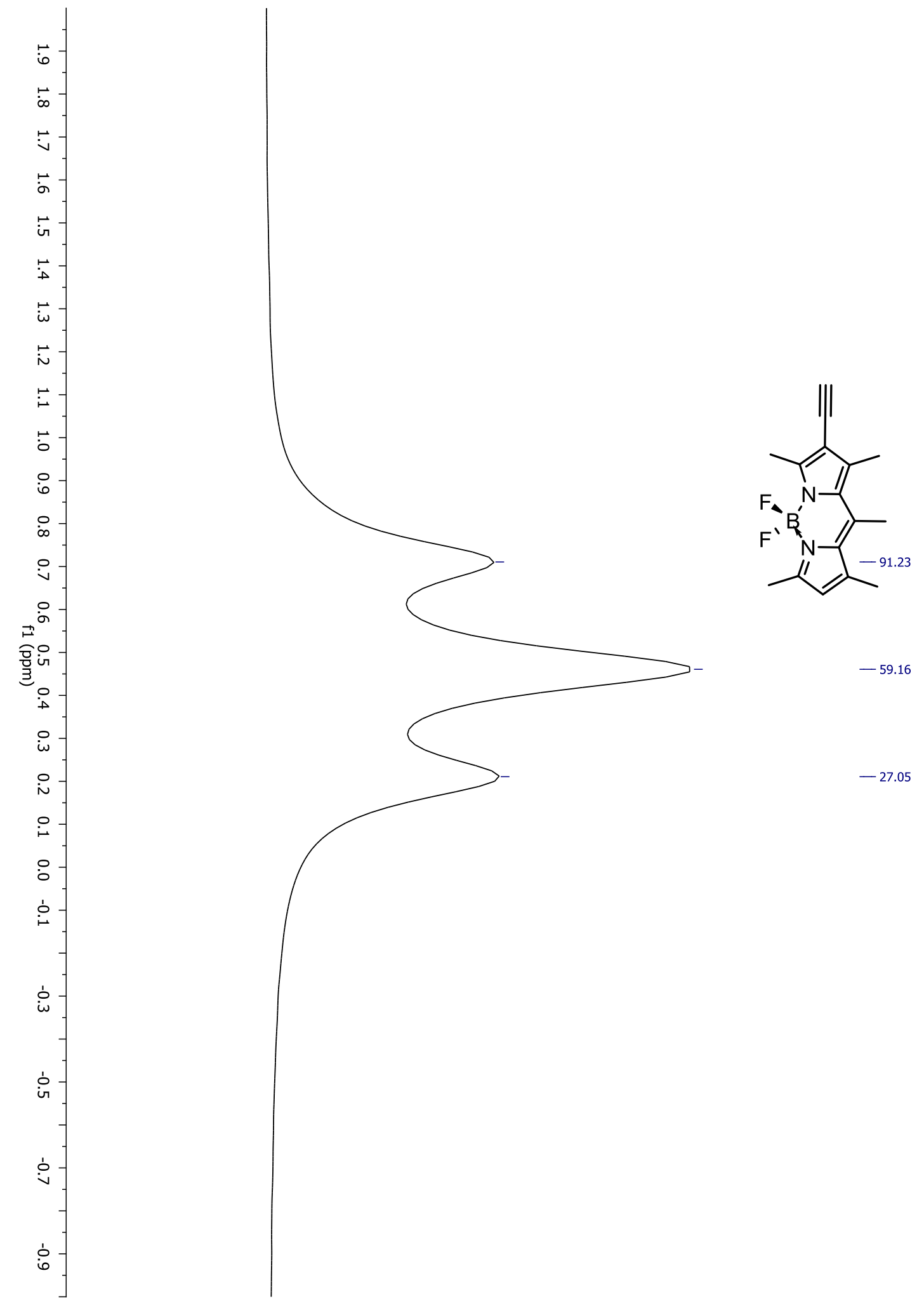

Figure A..54: ${ }^{11} B$ NMR of 2.24 


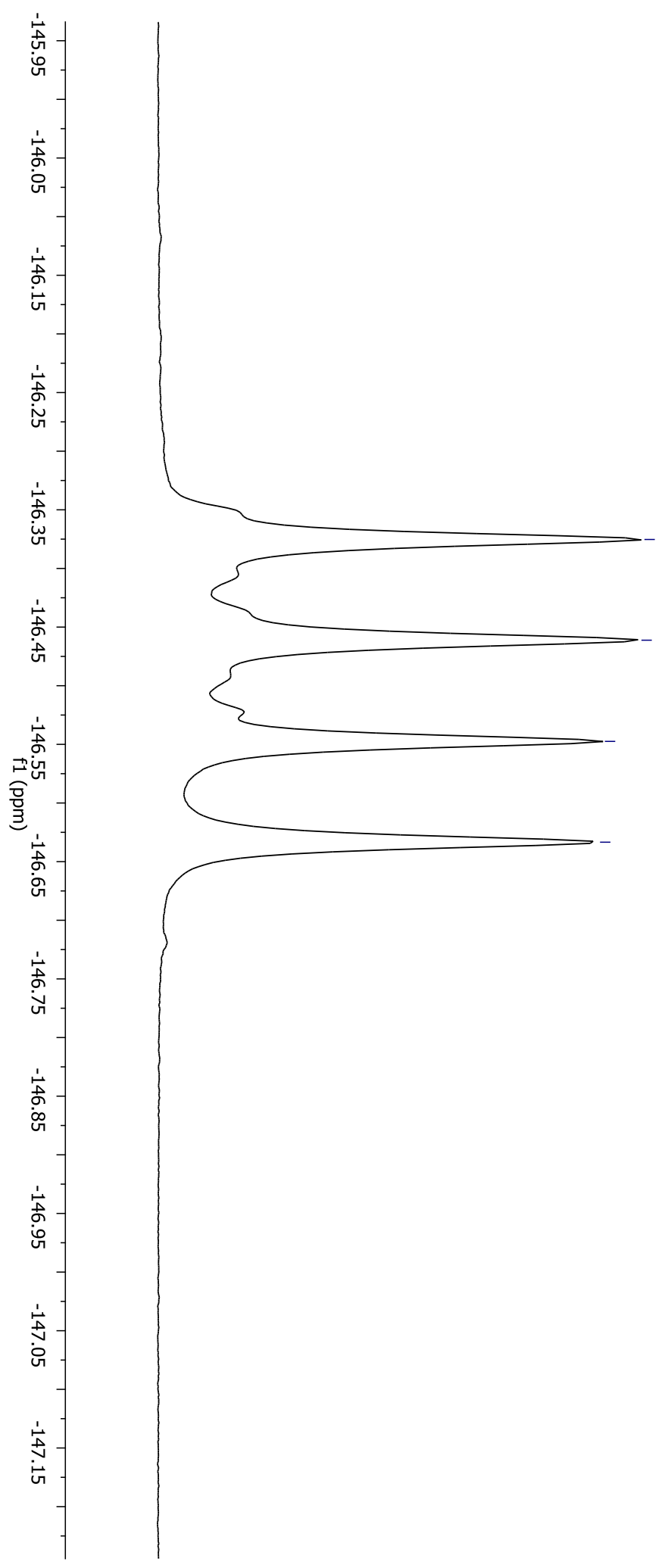

Figure A.54: ${ }^{19} \mathrm{~F}$ NMR of $\mathbf{2 . 2 4}$ 


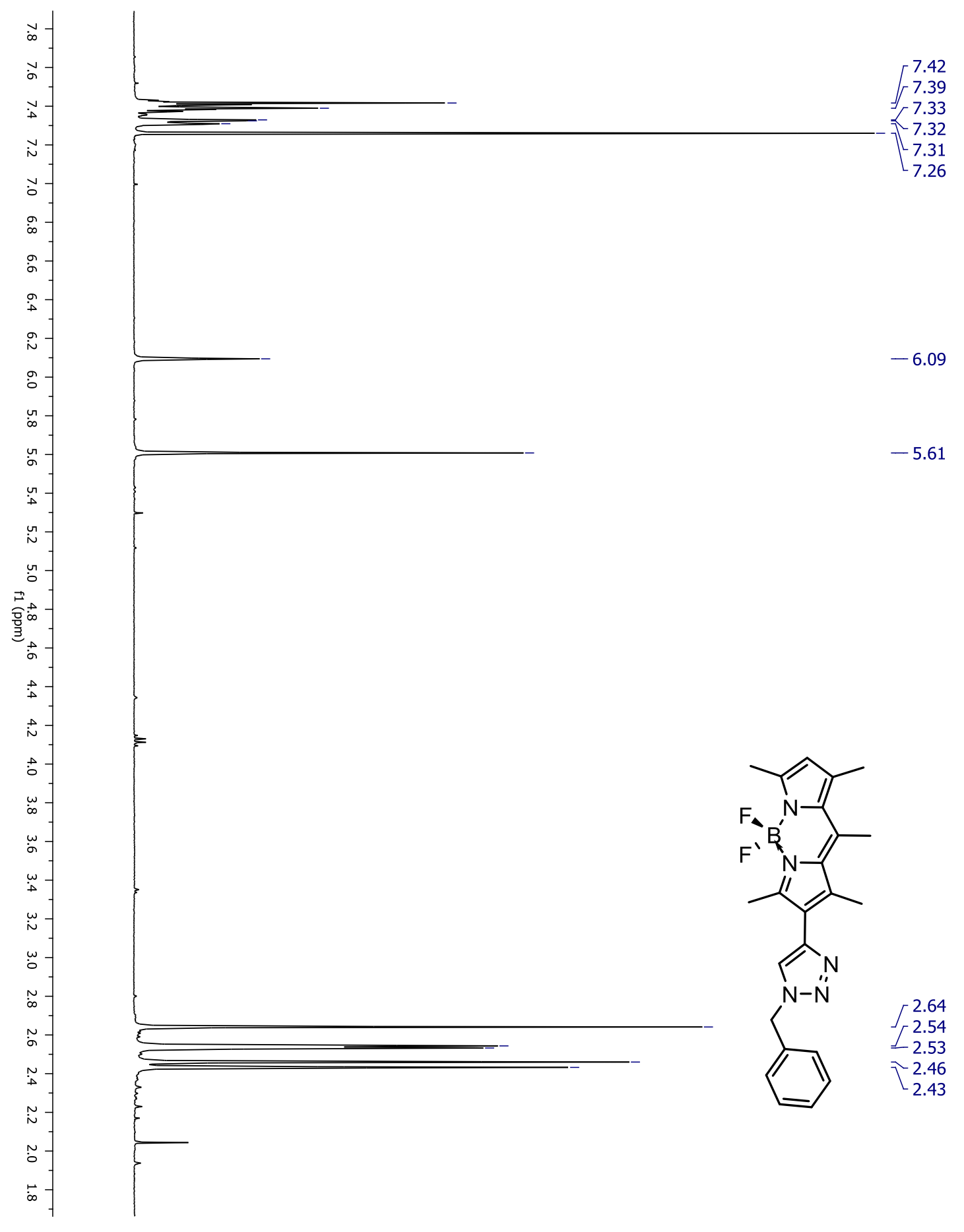

Figure A.55: ${ }^{1} \mathrm{H}$ NMR of 2.25a 


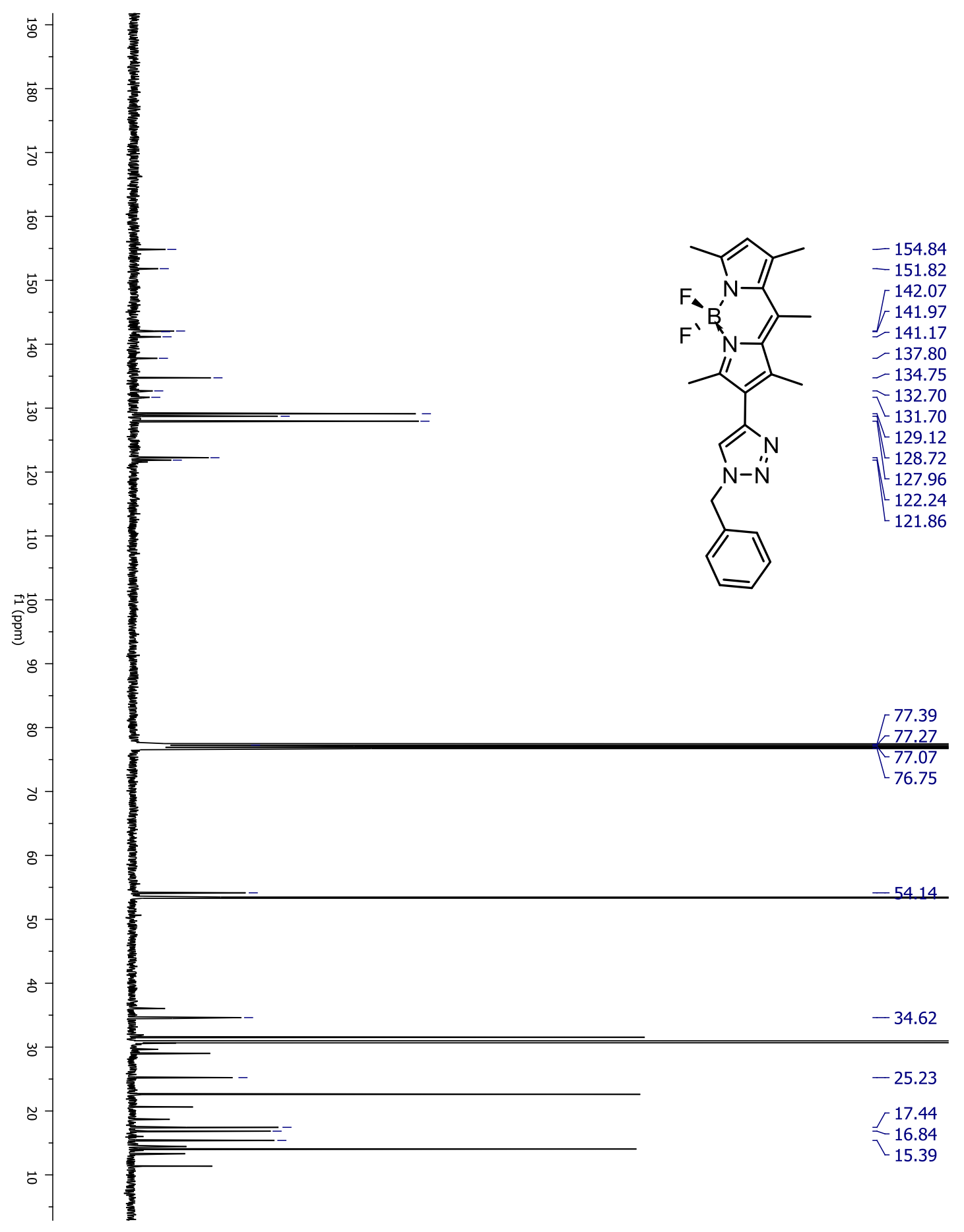

Figure A.56: ${ }^{13} \mathrm{C}$ NMR of 2.25a 


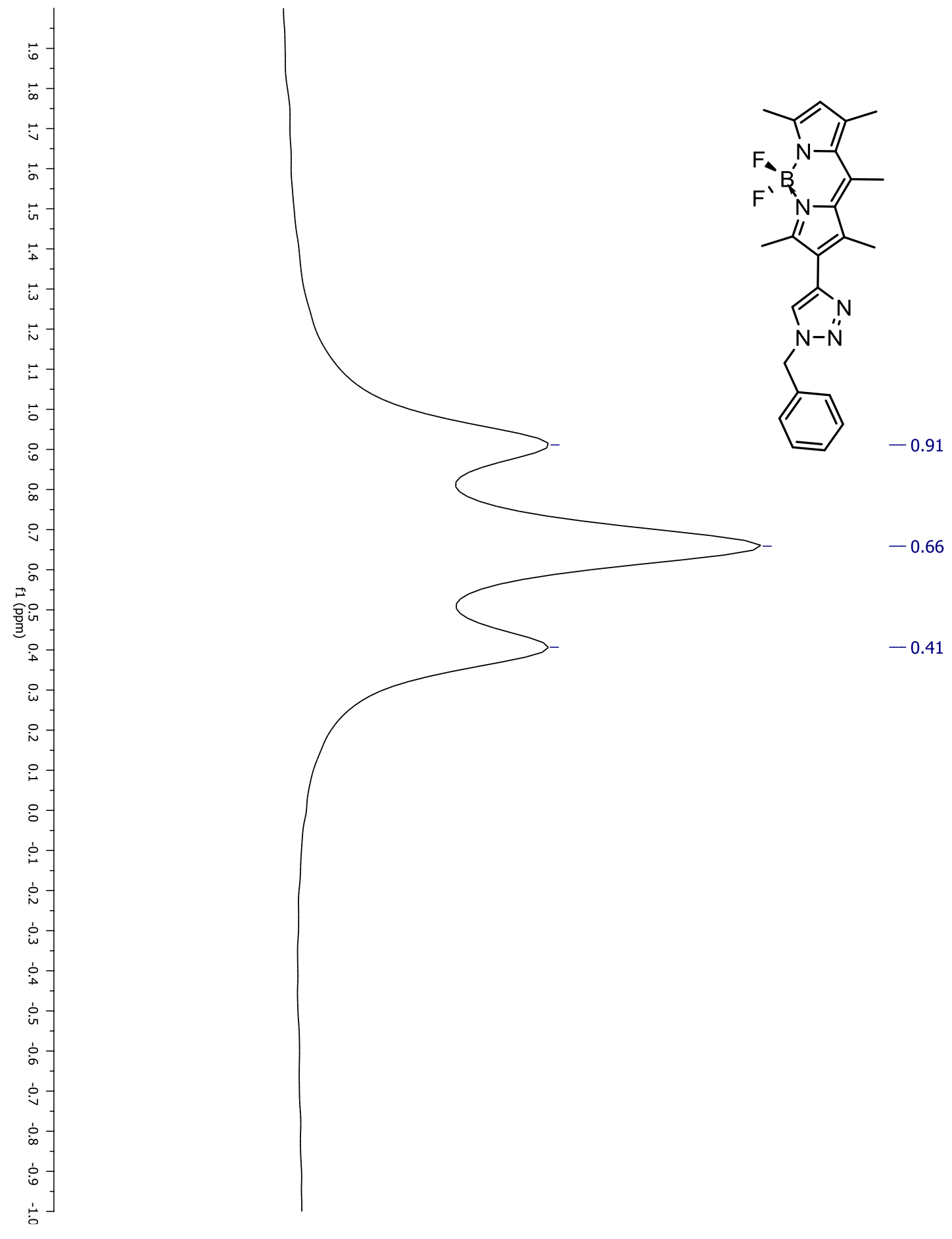

Figure A.57: ${ }^{11} \mathrm{~B}$ NMR of 2.25a 

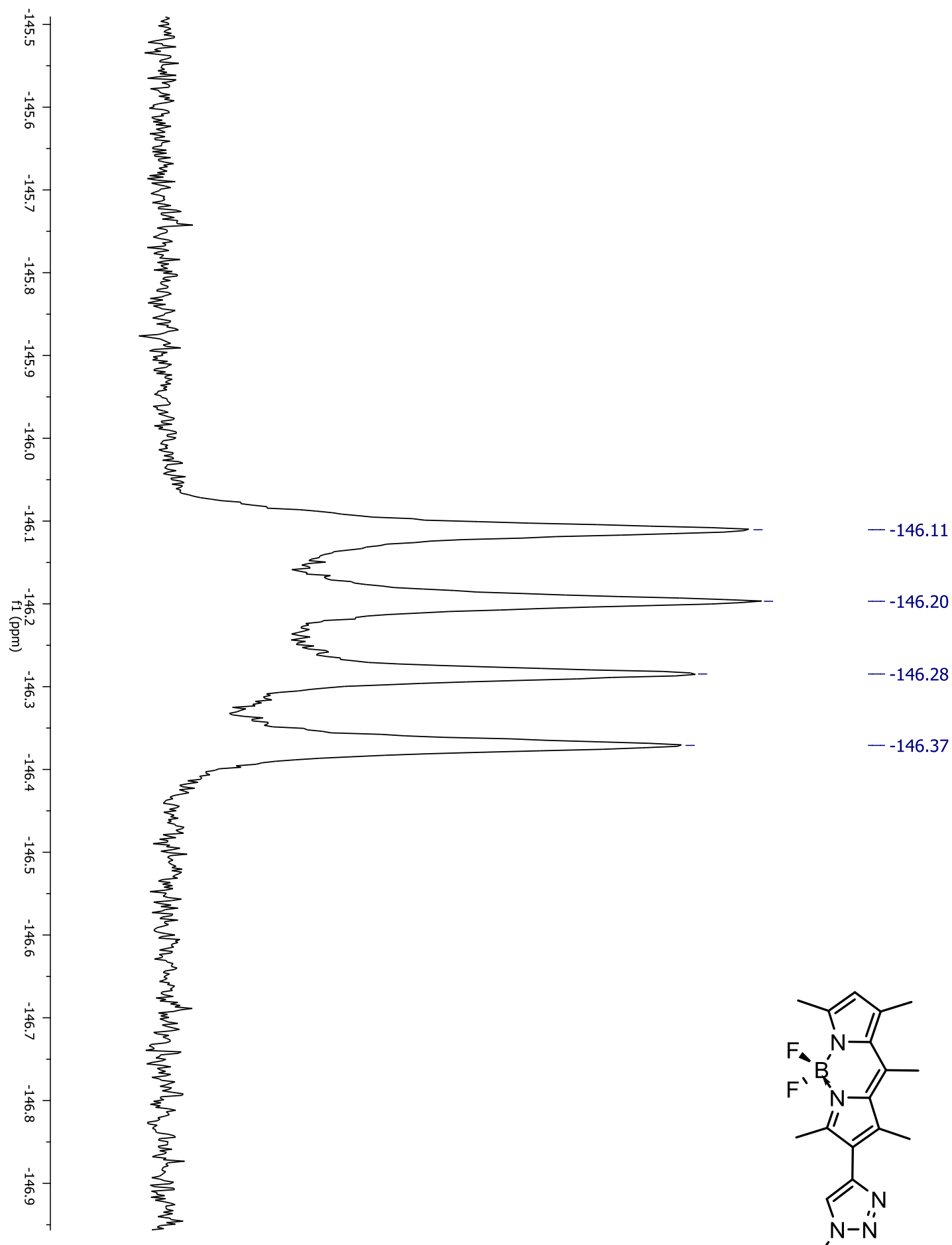

$--146.20$

$--146.28$

$--146.37$

Figure A.58: ${ }^{19} \mathrm{~F}$ NMR of $2.25 a$

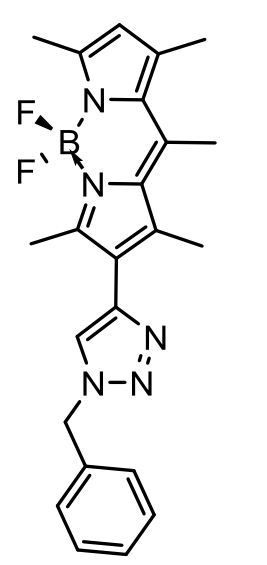




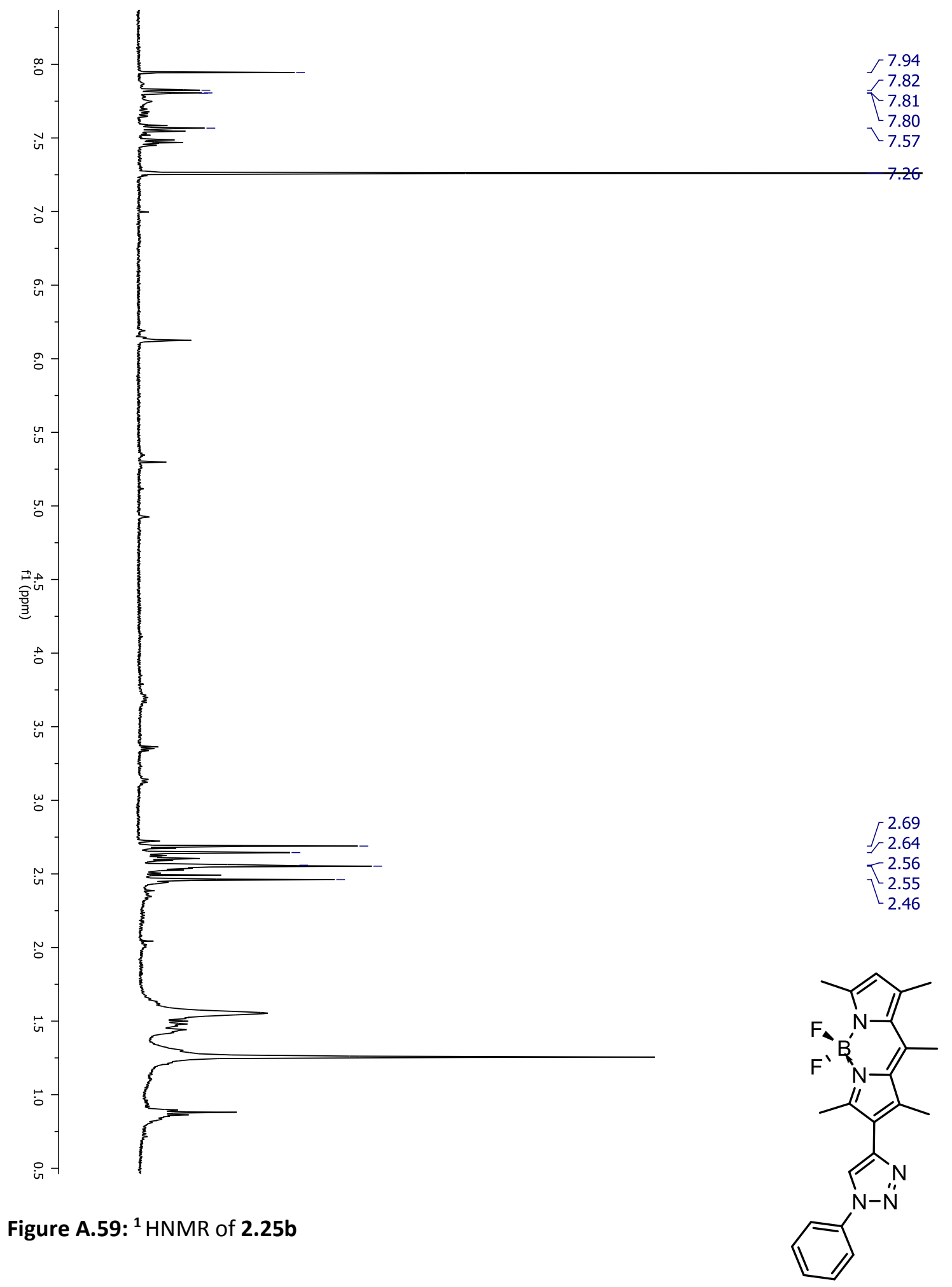




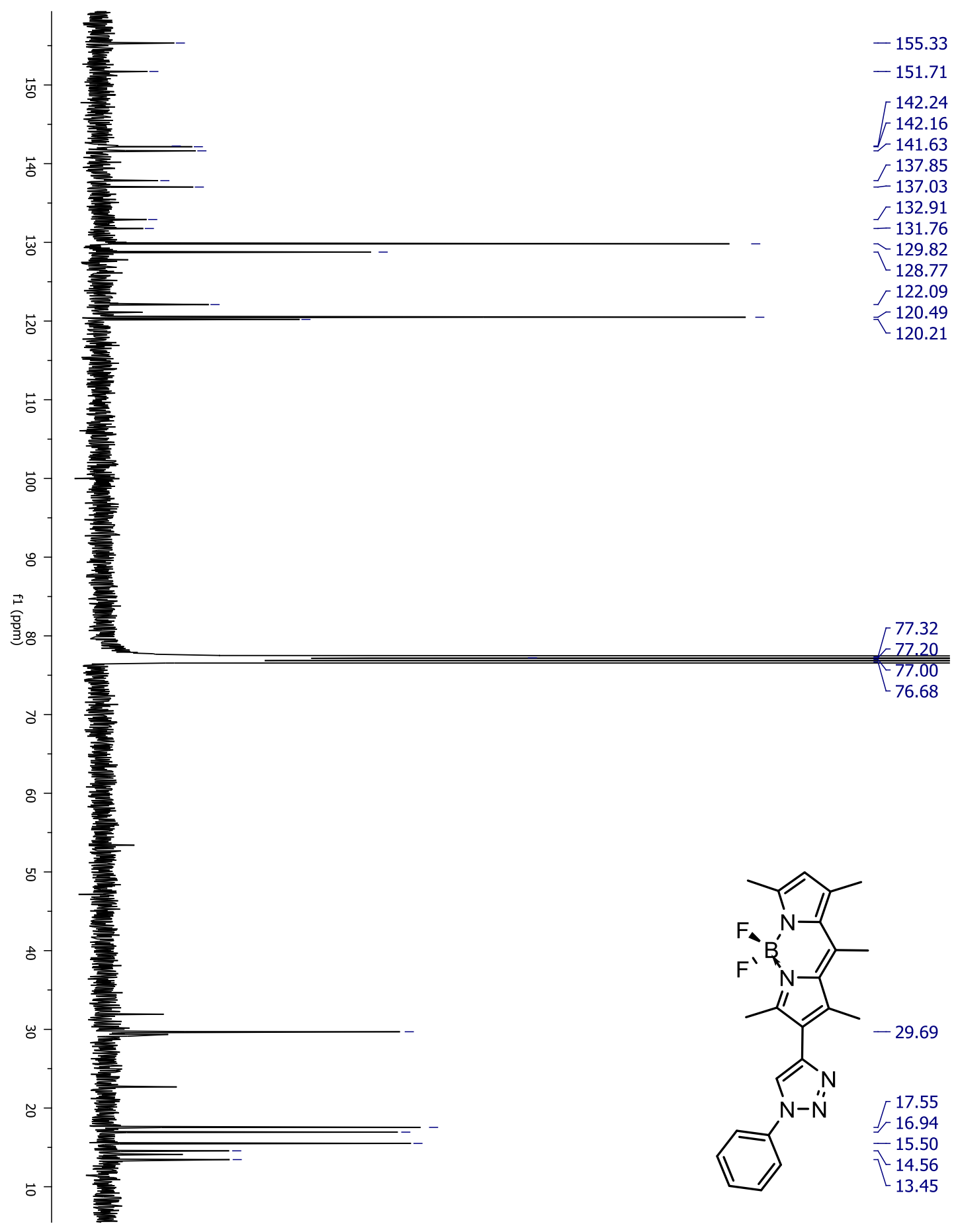

Figure A.60: ${ }^{13} \mathrm{C}$ NMR of $2.25 b$ 


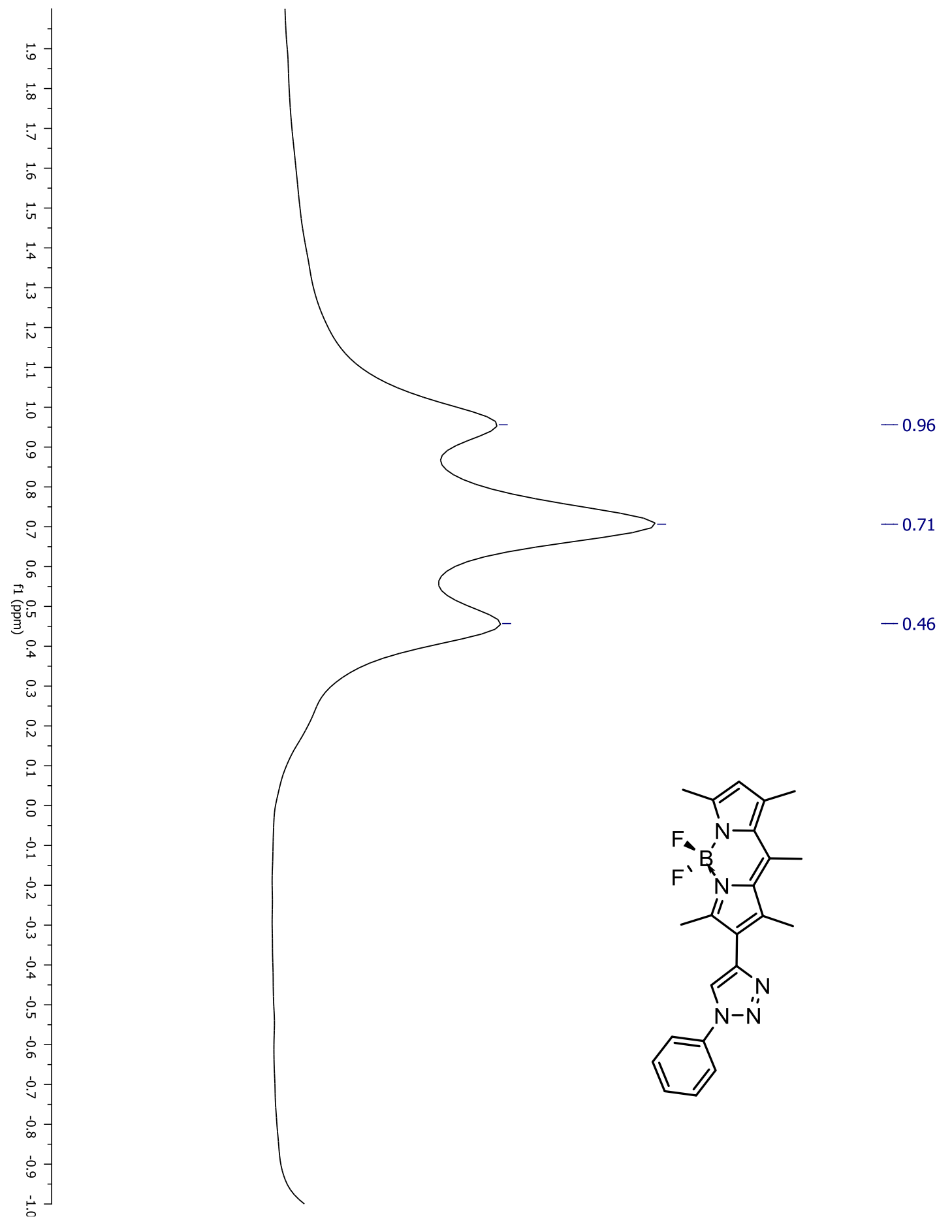

Figure A.61: ${ }^{11} B$ NMR of $2.25 b$ 


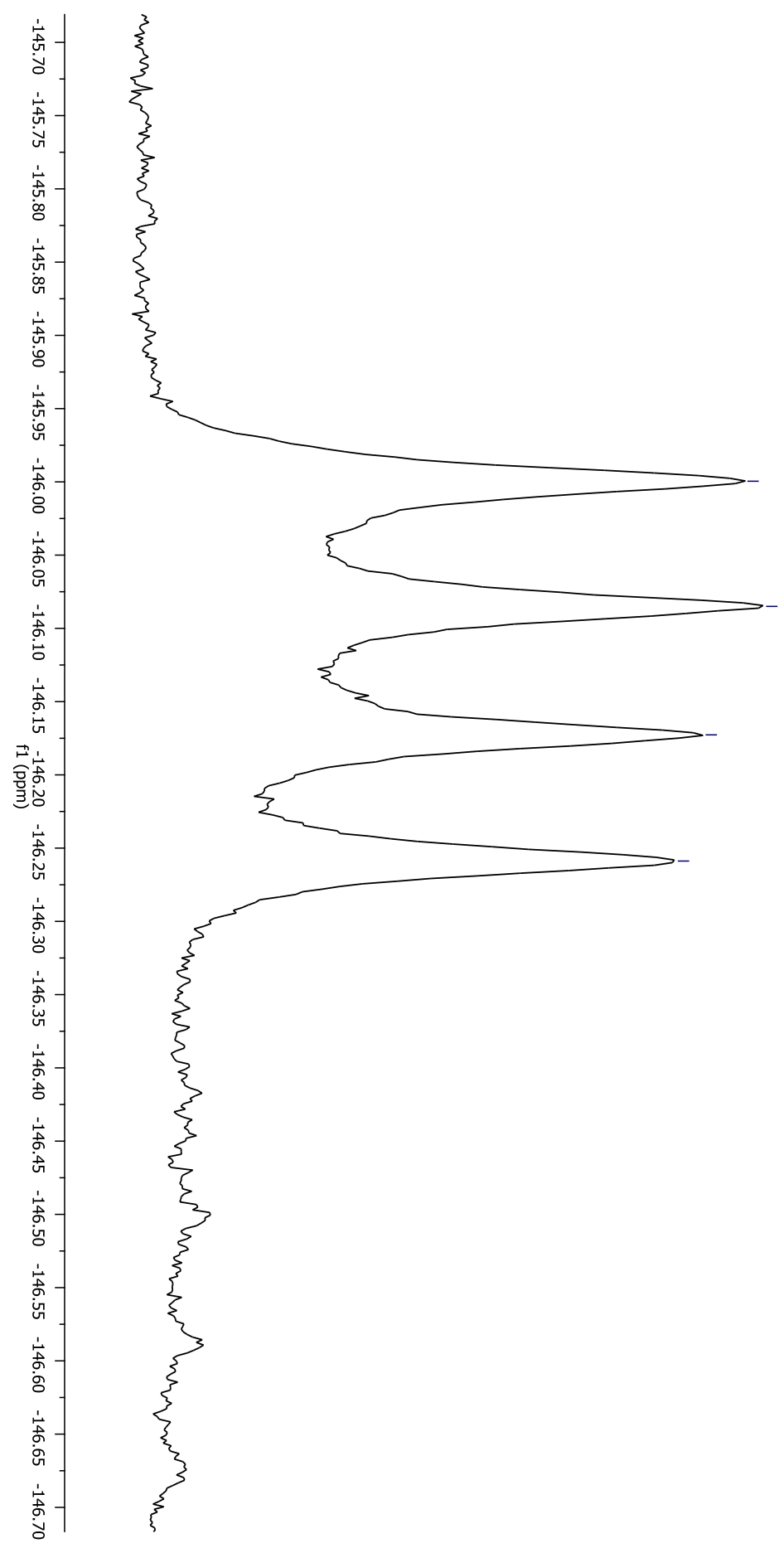

$--146.00$

$--146.08$

$--146.17$

$--146.26$

Figure A.62: ${ }^{19} \mathrm{~F}$ NMR of $\mathbf{2 . 2 5 b}$

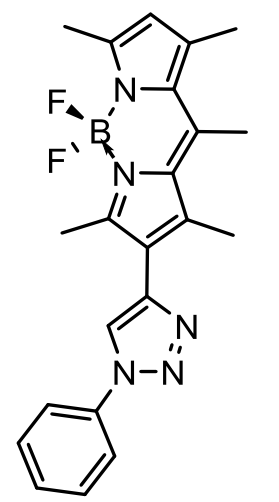




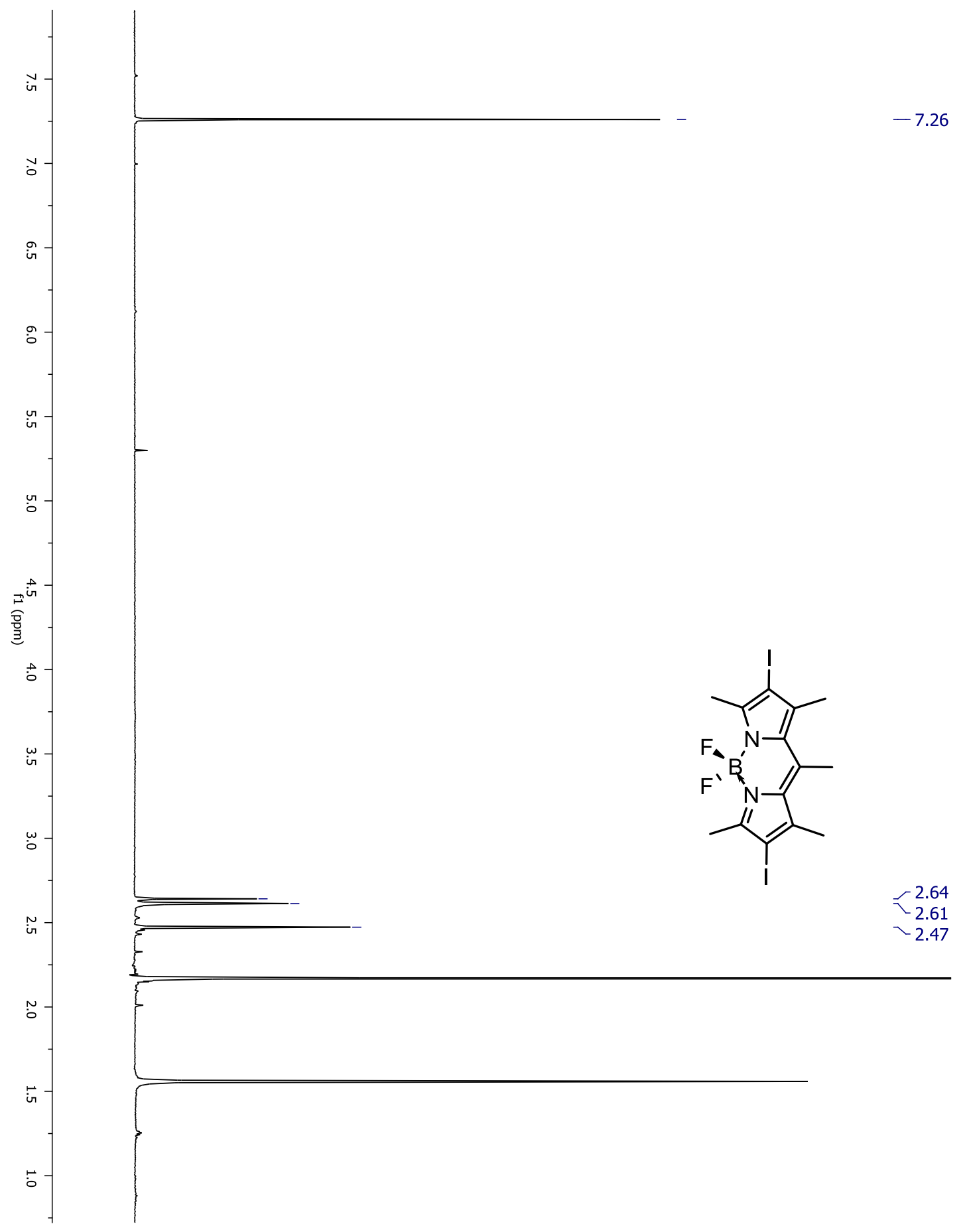

Figure A.63: ${ }^{1} \mathrm{H}$ NMR of $\mathbf{2 . 2 6}$ 


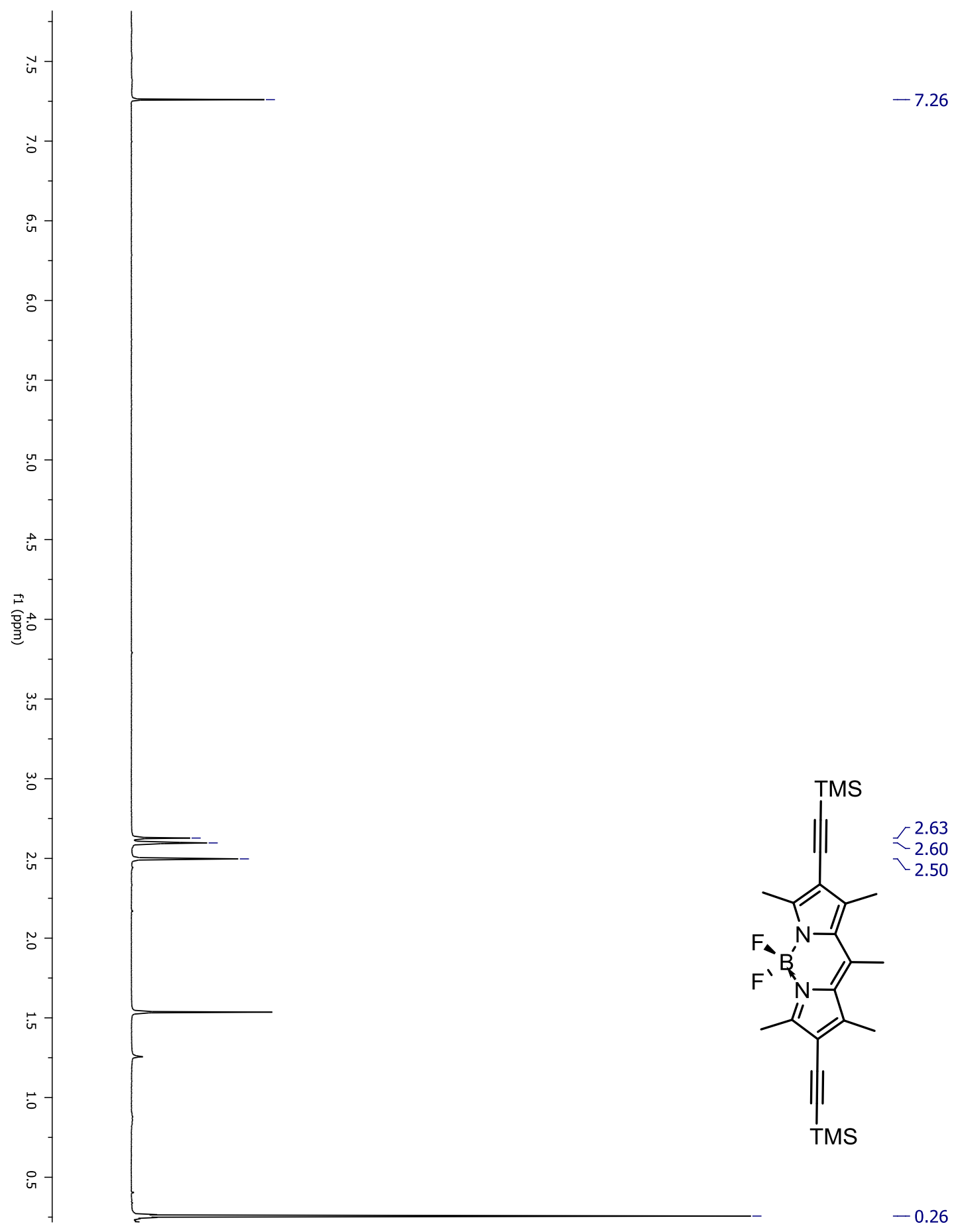

Figure A..64: ${ }^{1} \mathrm{H}$ NMR of $\mathbf{2 . 2 7}$ 


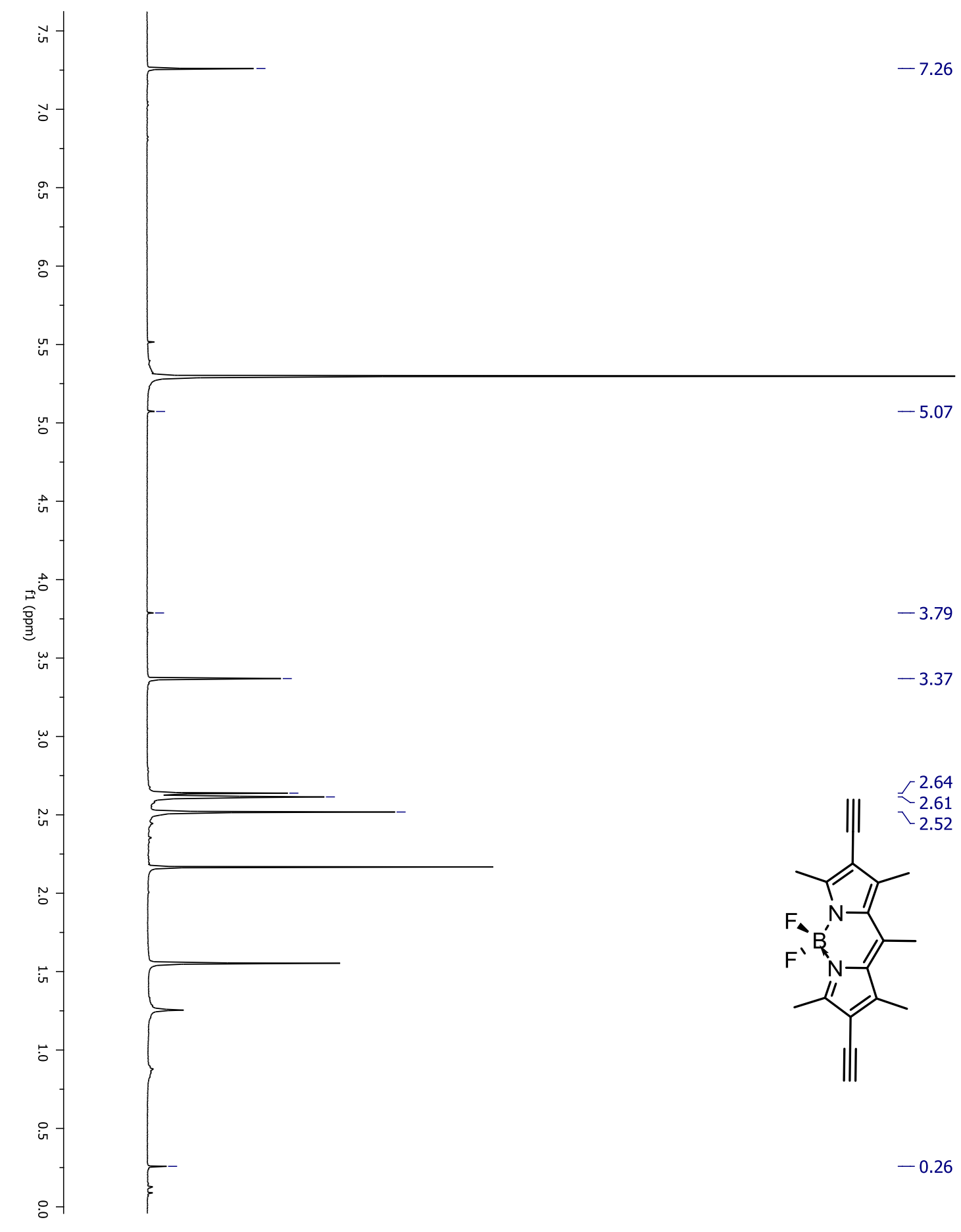

Figure A.65: ${ }^{1} \mathrm{H}$ NMR of $\mathbf{2 . 2 8}$ 


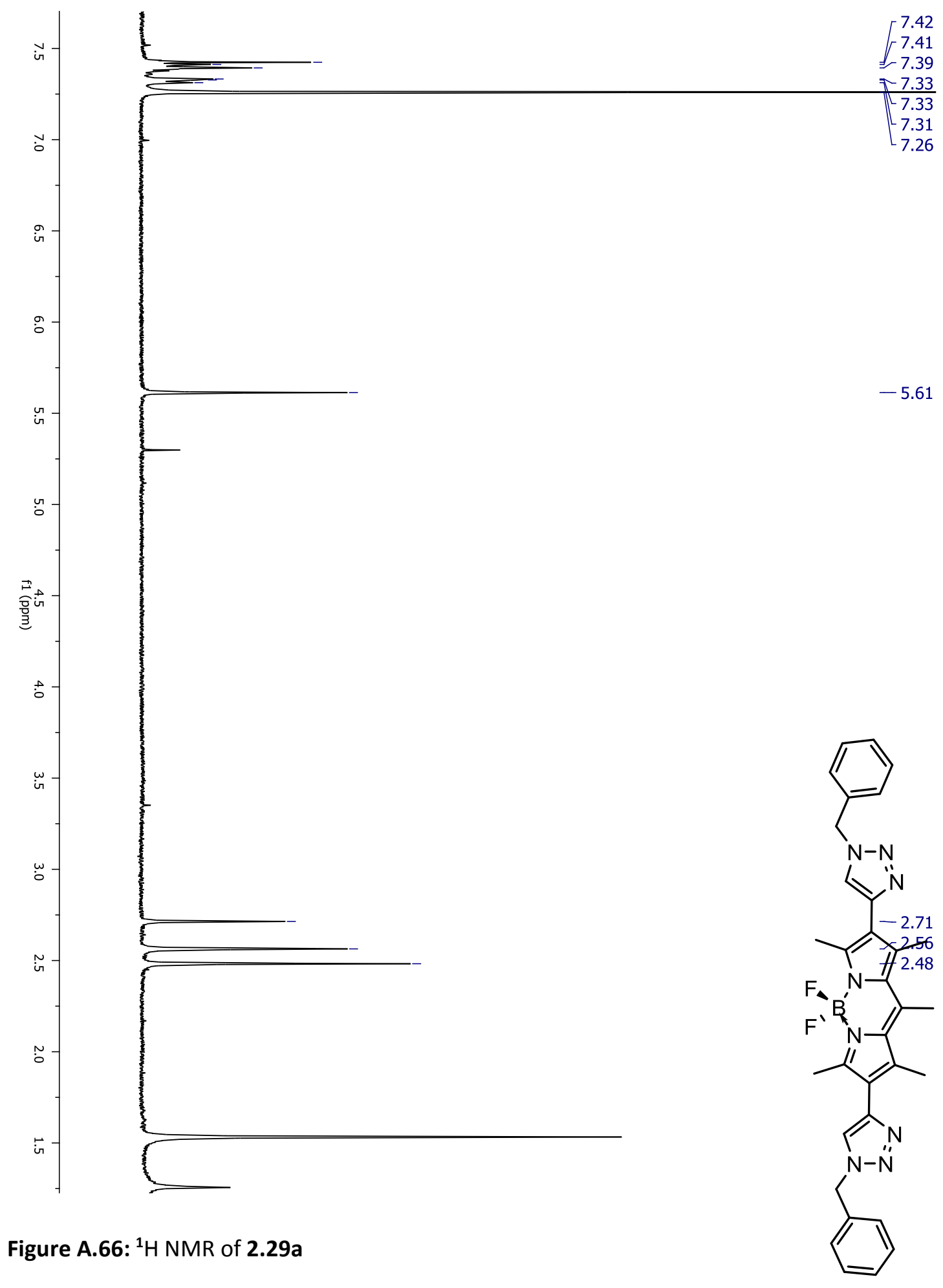




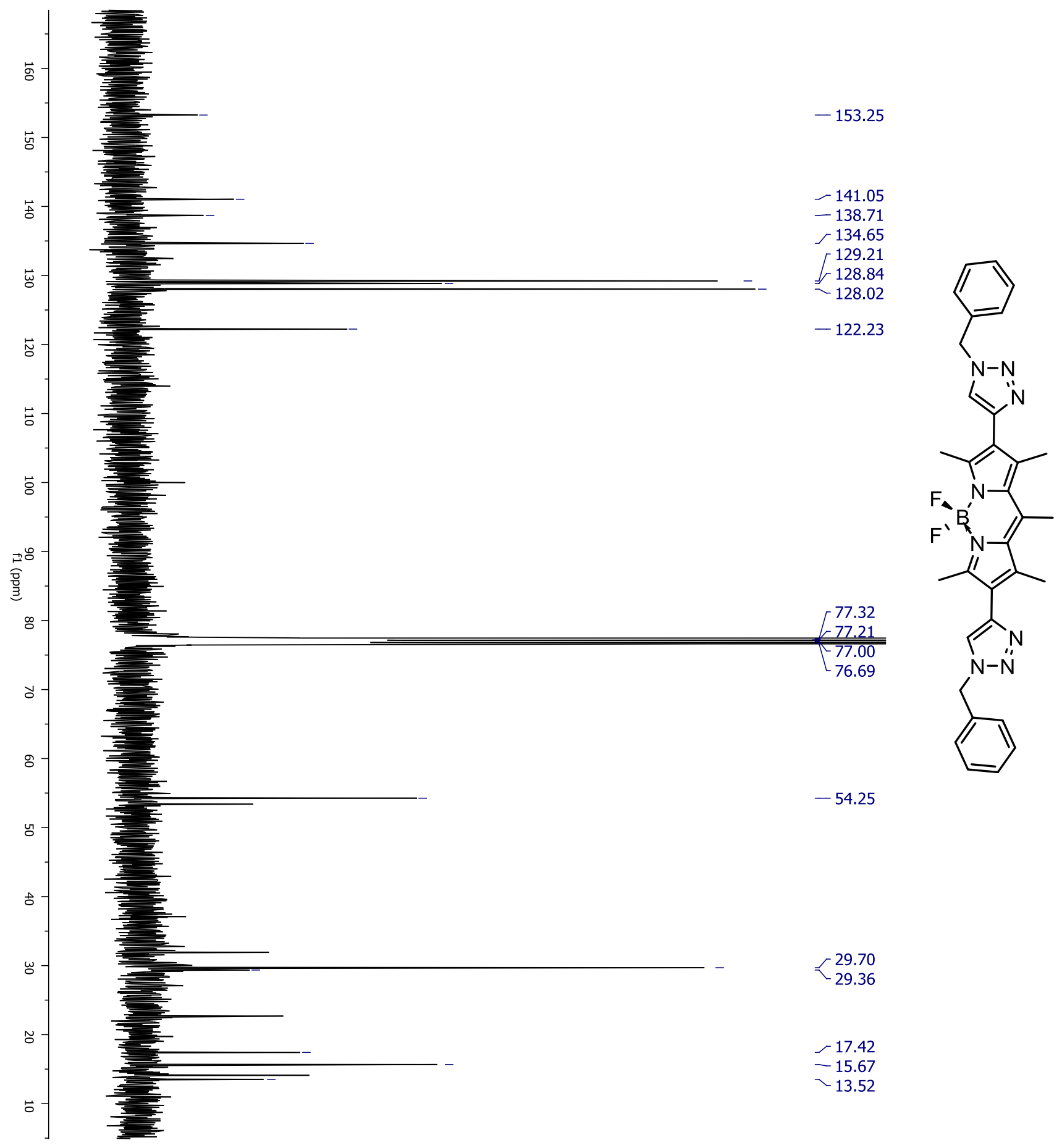

Figure A.67: ${ }^{13} \mathrm{C}$ NMR of 2.29a 

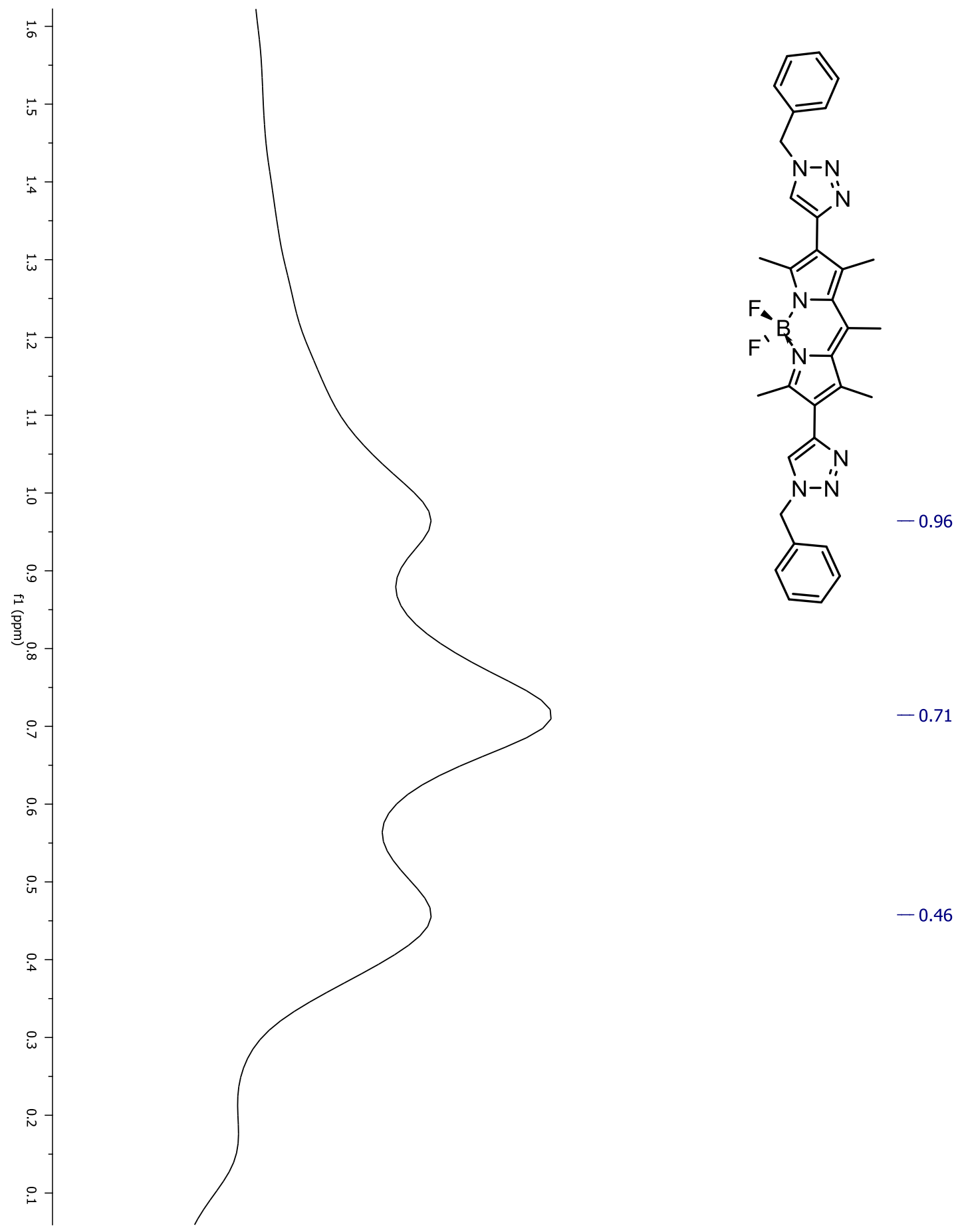

$-0.71$

$-0.46$

Figure A.68: ${ }^{11} B$ NMR of 2.29a 


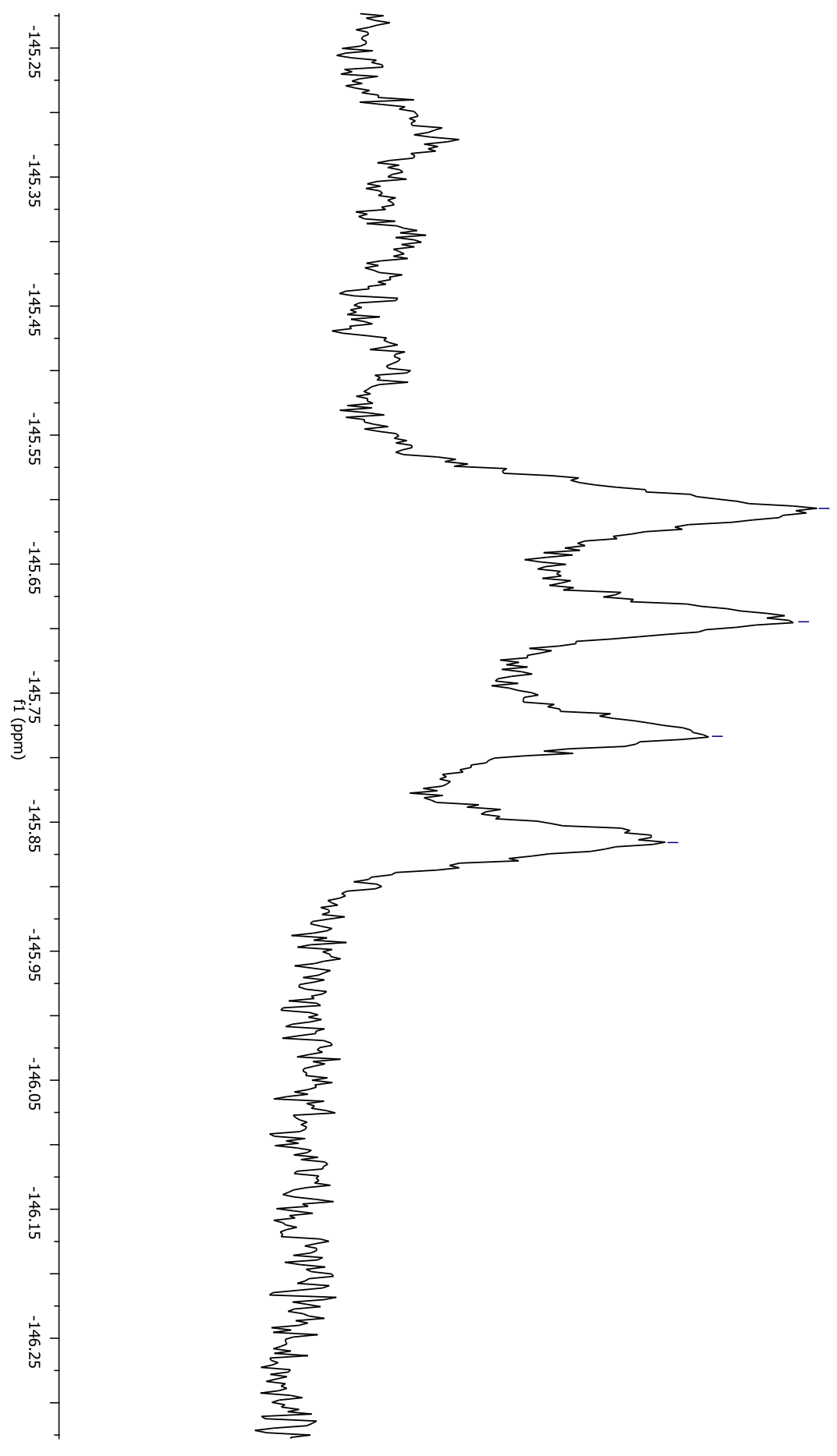

Figure A.69: ${ }^{19} \mathrm{~F}$ NMR of 2.29a

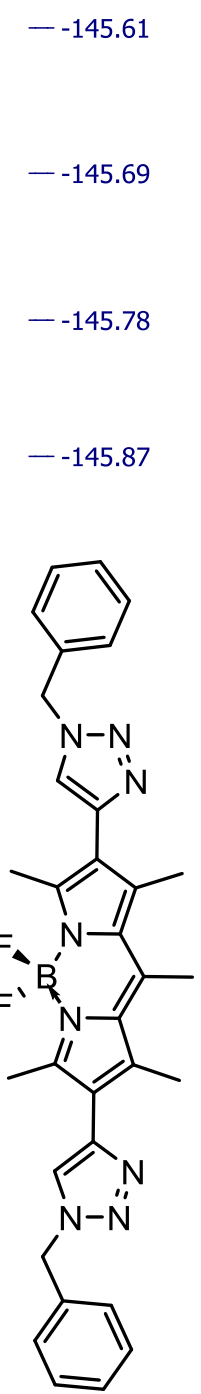




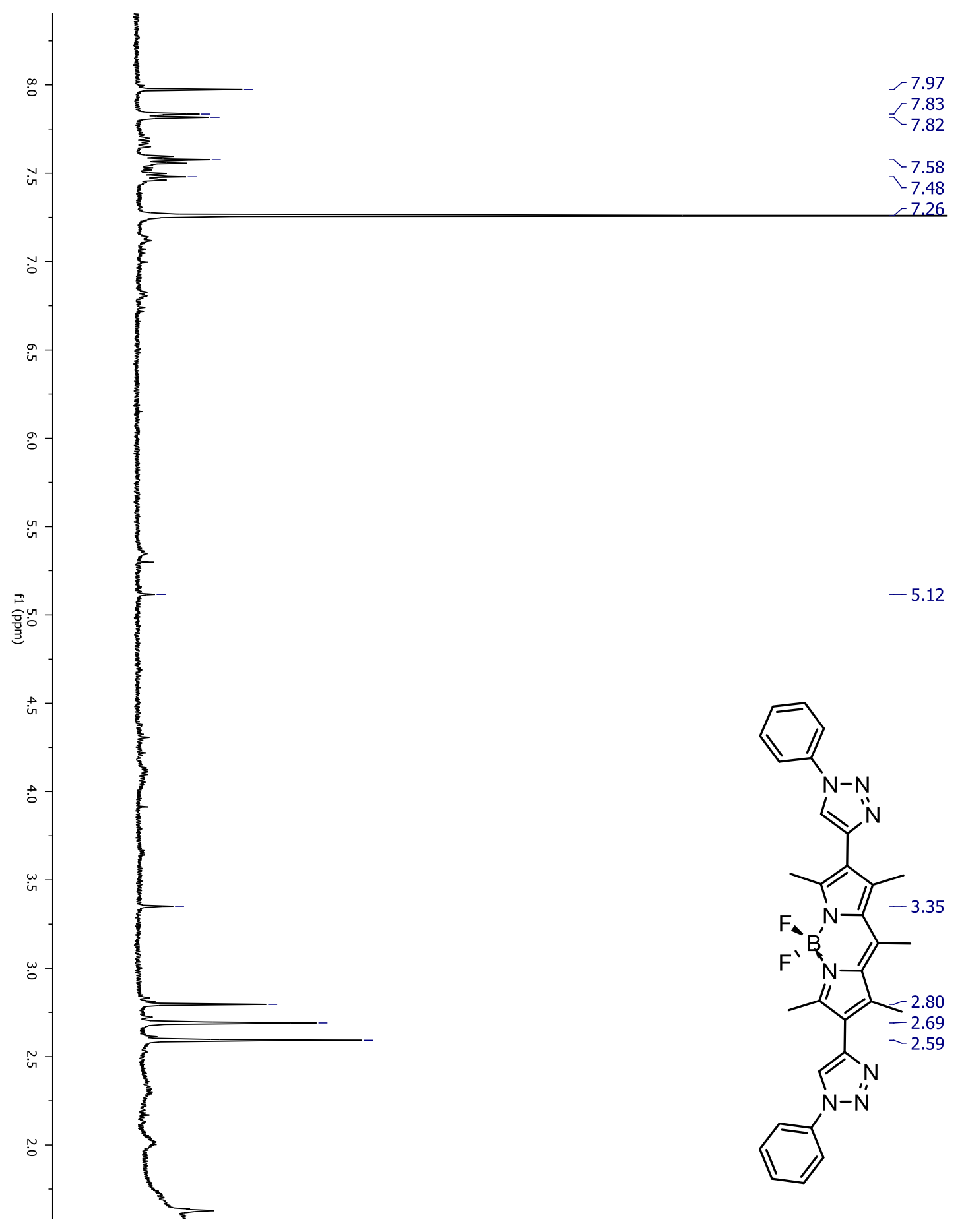

Figure A.70: ${ }^{13} \mathrm{C}$ NMR of $2.29 \mathrm{~b}$ 


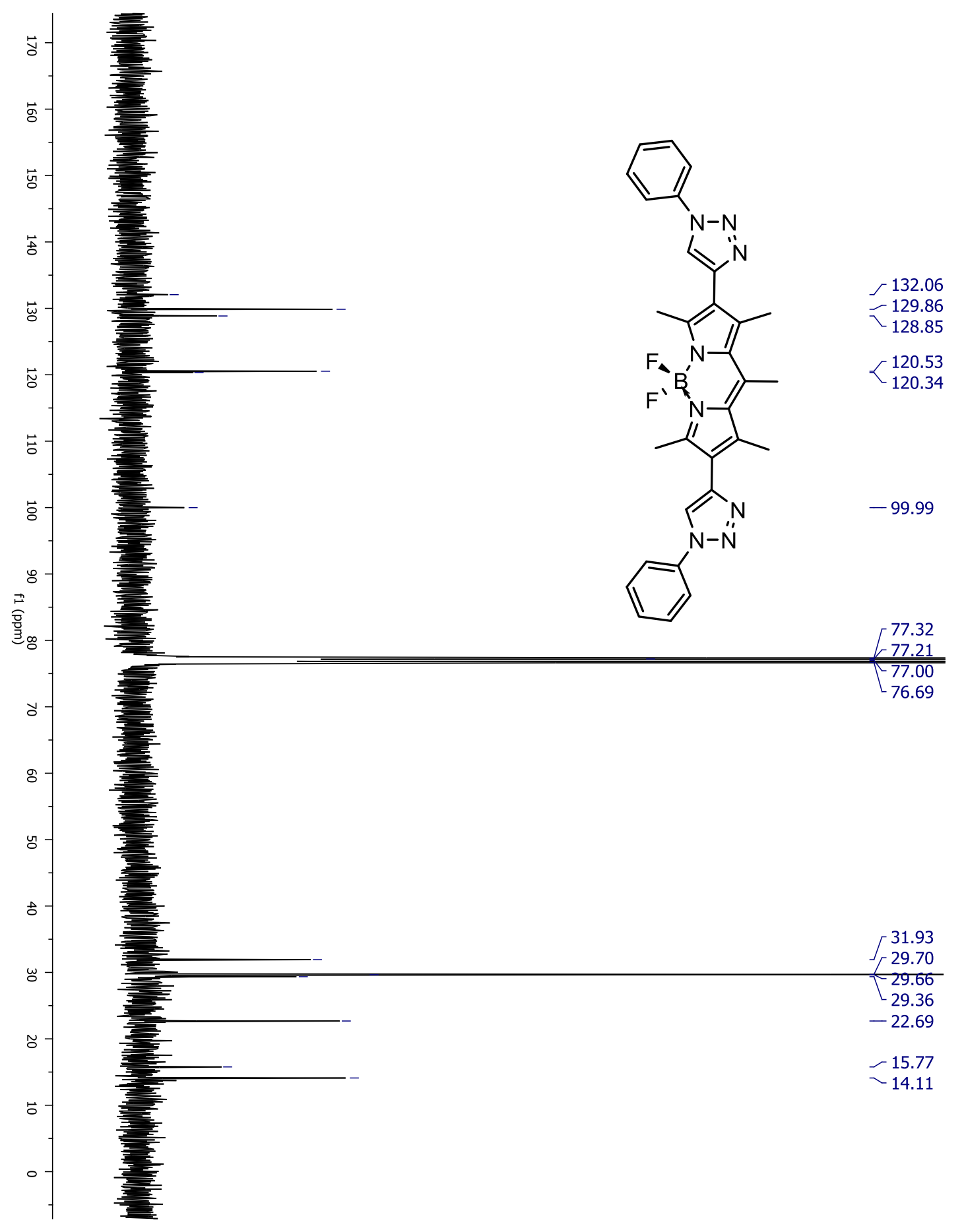

Figure A..71: ${ }^{13} \mathrm{C}$ NMR of $2.29 \mathrm{~b}$ 

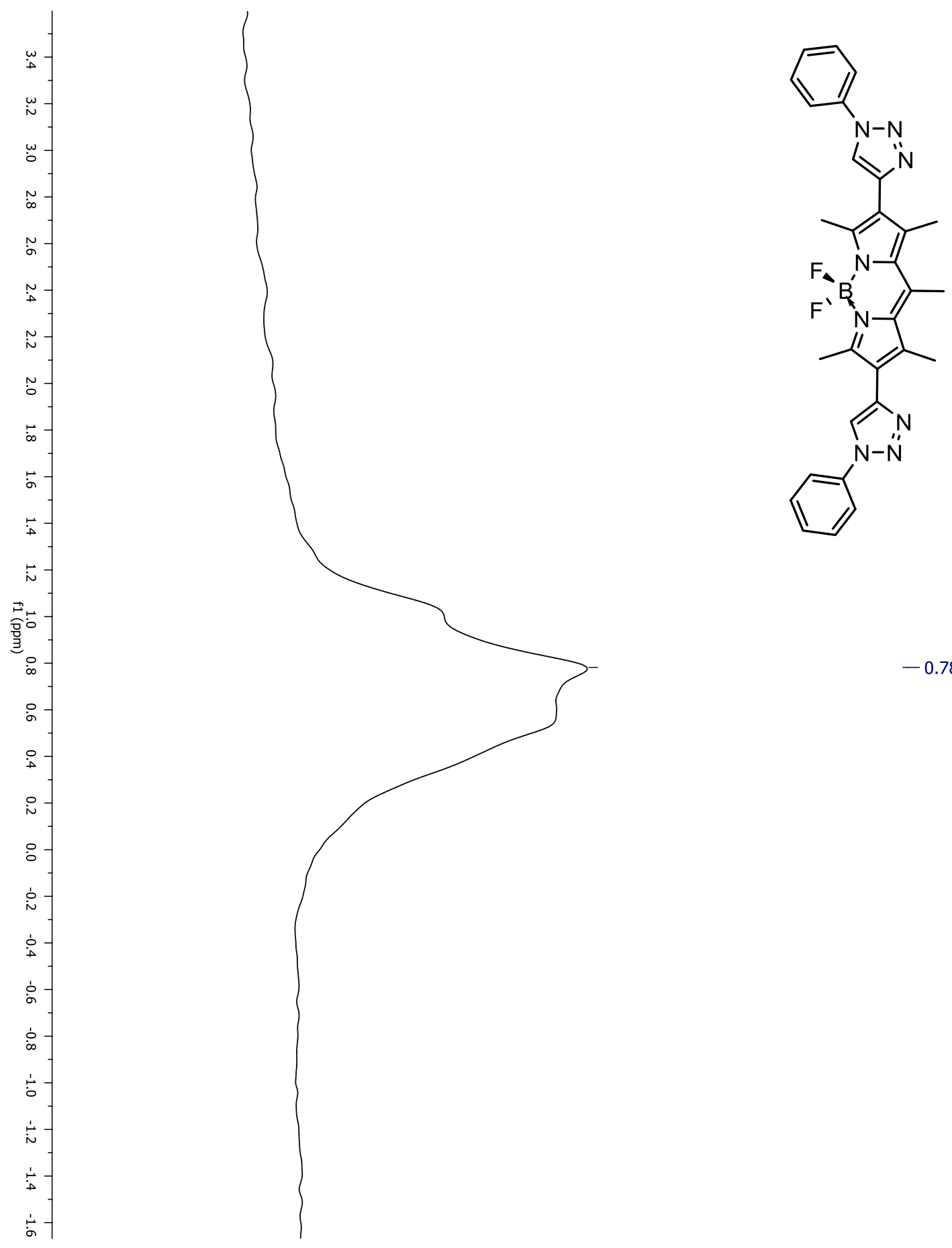

$-0.78$

Figure A.72: ${ }^{11}$ B NMR of $2.29 b$ 


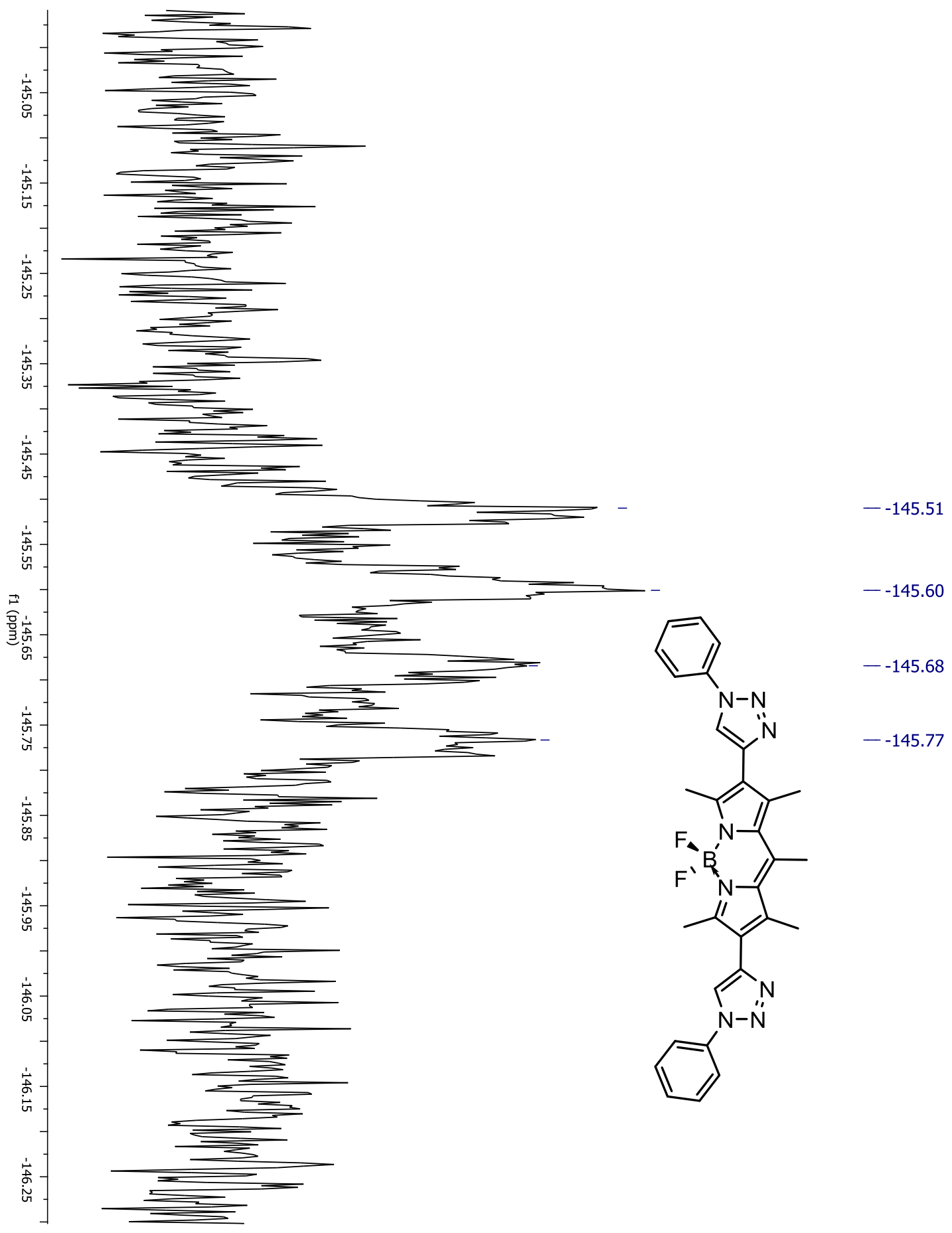

Figure A..73: ${ }^{19} \mathrm{~F}$ NMR of $2.29 \mathrm{~b}$ 


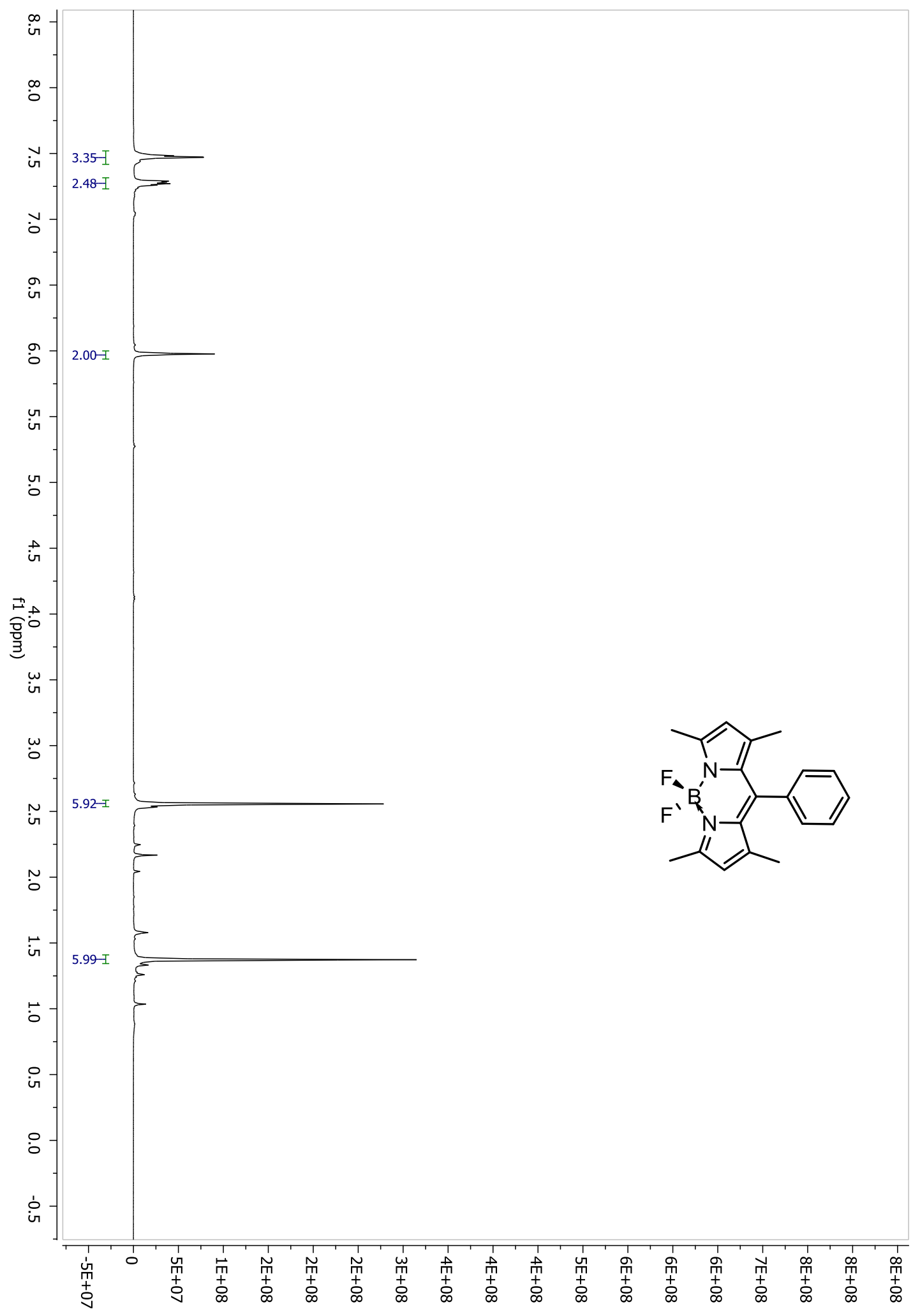

Figure A.74: ${ }^{1} \mathrm{H}$ NMR of $3.41 \mathrm{a}$ 


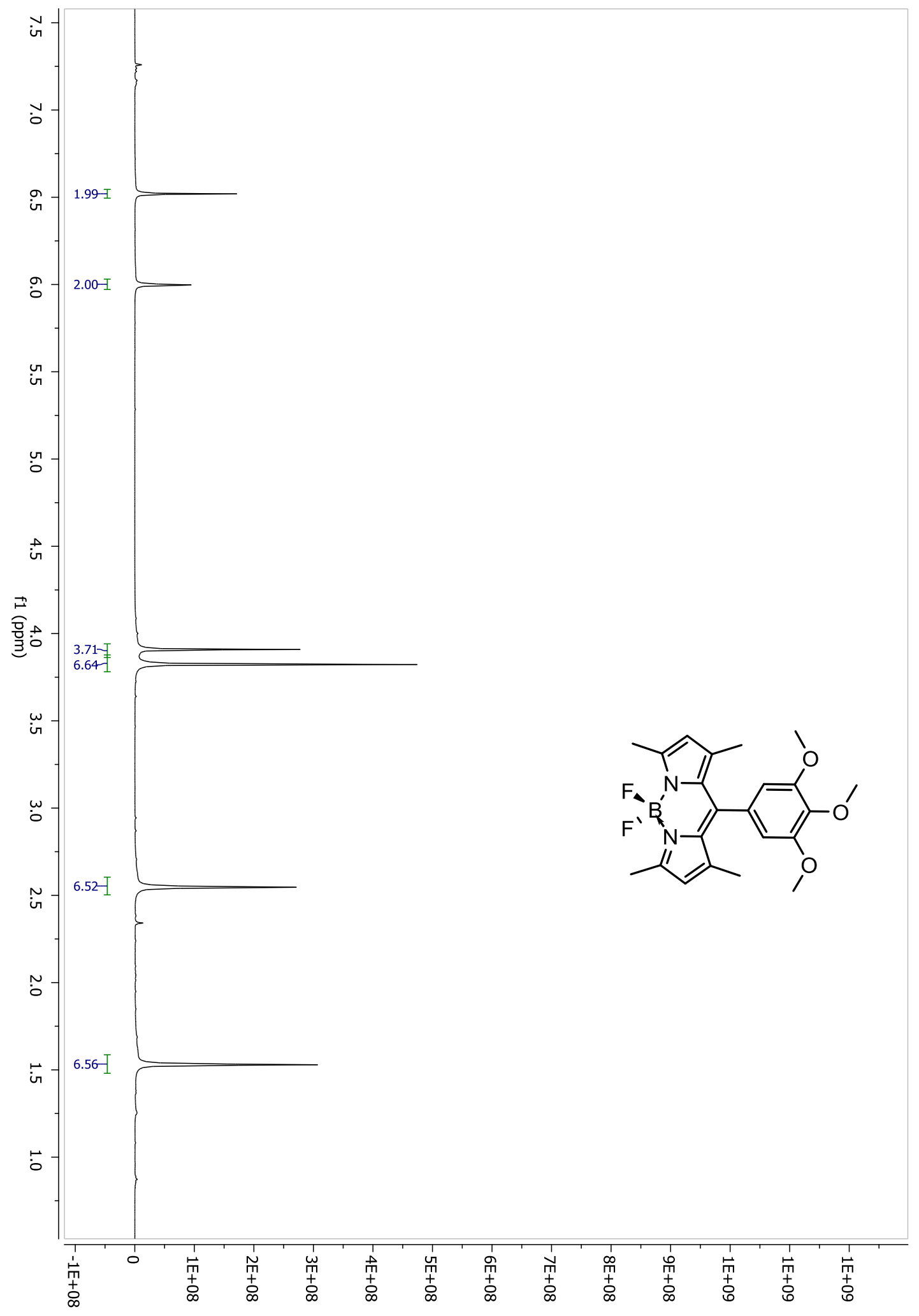

Figure A.75: ${ }^{1} \mathrm{H}$ NMR of 3.41b 


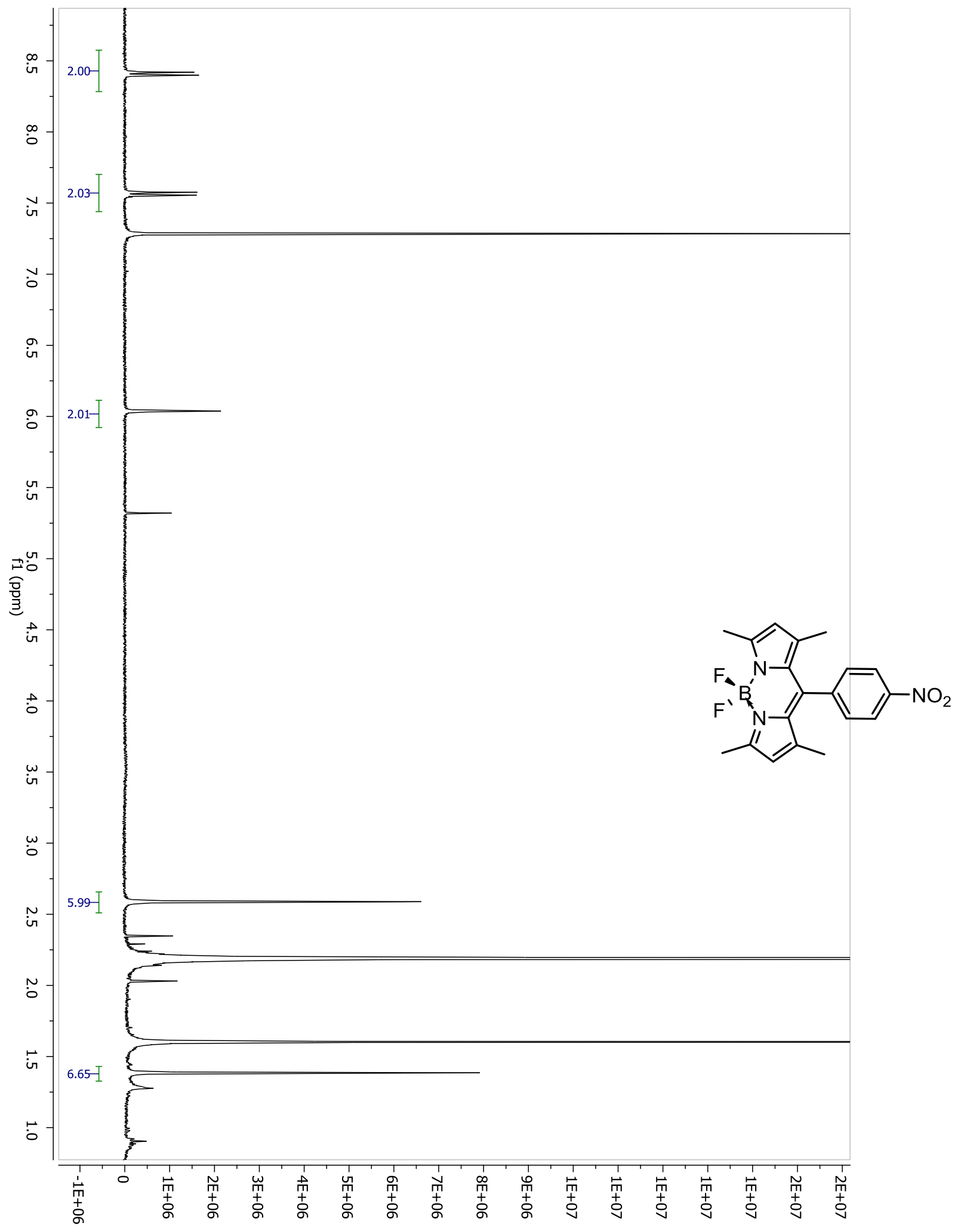

Figure A.76 ${ }^{1} \mathrm{H}$ NMR of $3.41 \mathrm{C}$ 


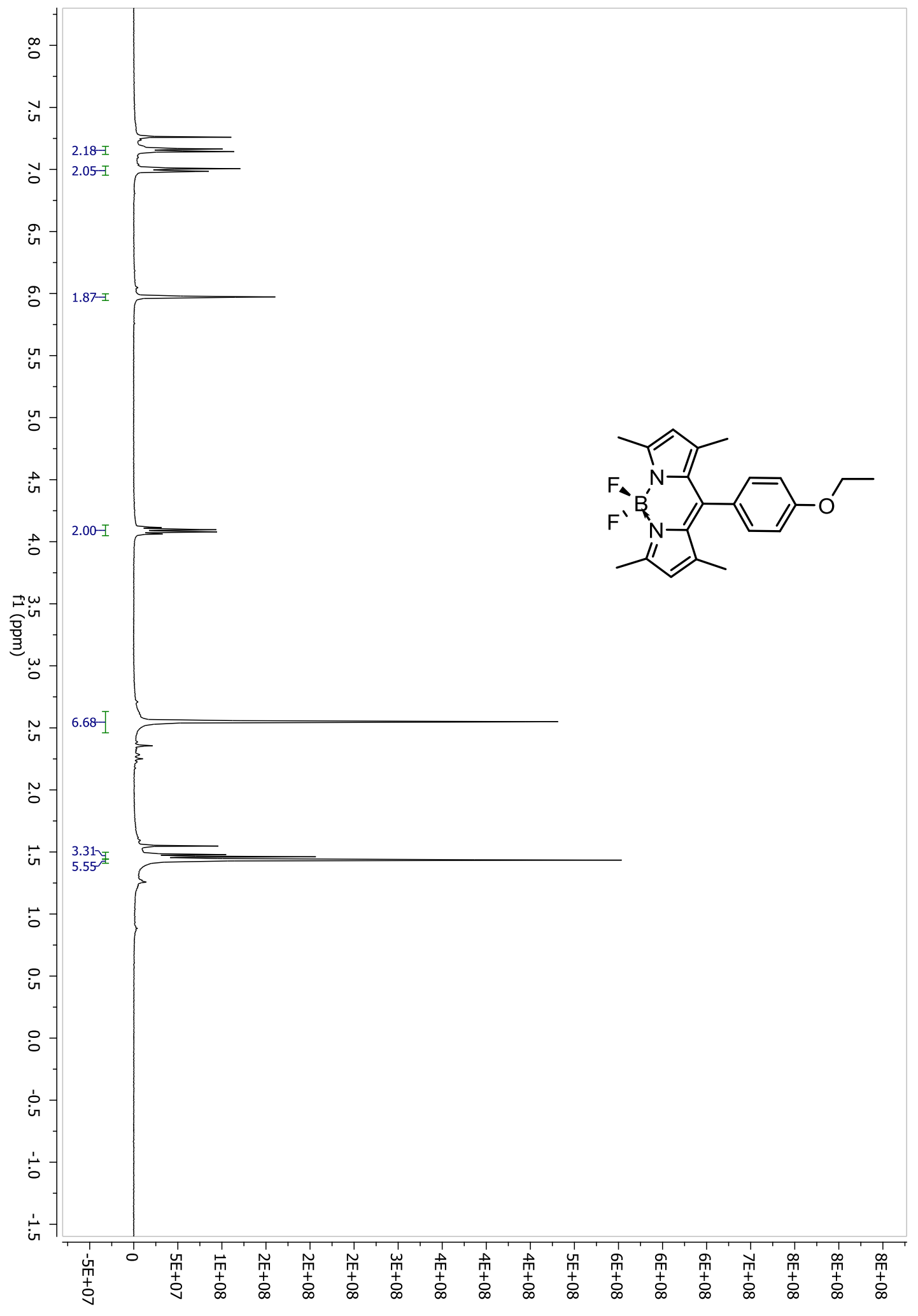

Figure A.74: ${ }^{1} \mathrm{H}$ NMR of $3.41 \mathrm{~d}$ 


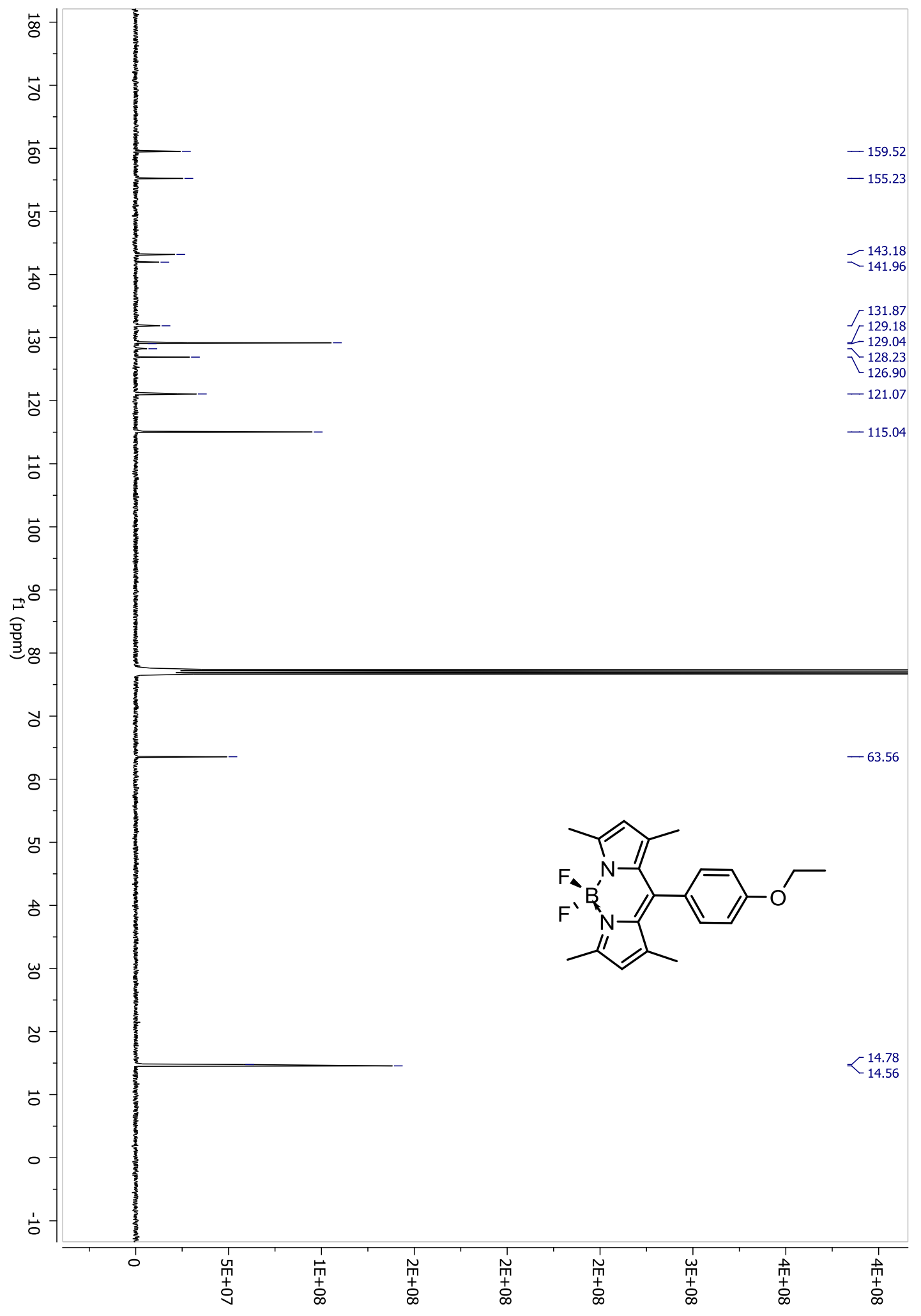

Figure A.75: ${ }^{13} \mathrm{C}$ NMR of 3.41d 


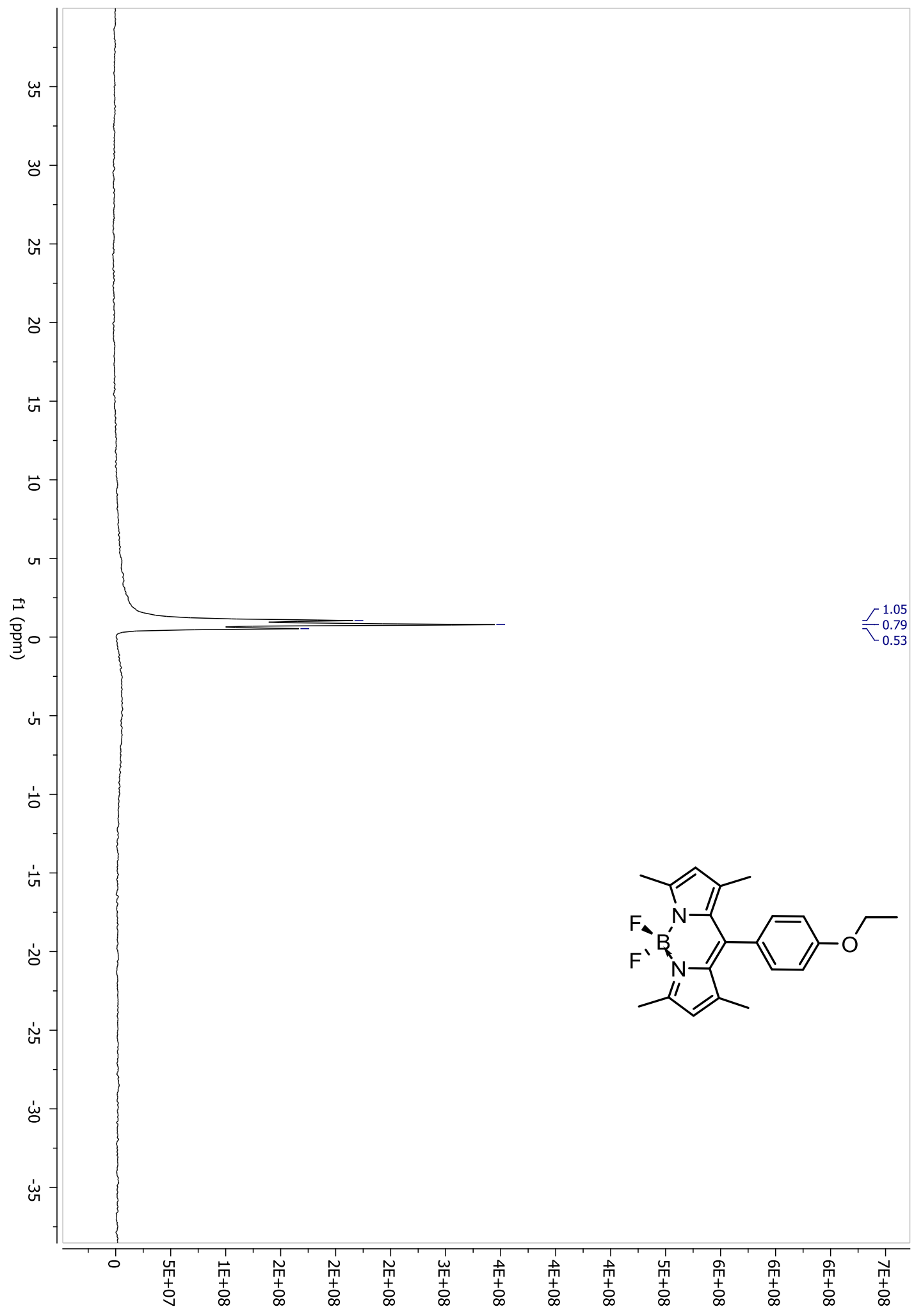

Figure A.76: ${ }^{11} B$ NMR of $3.41 d$ 


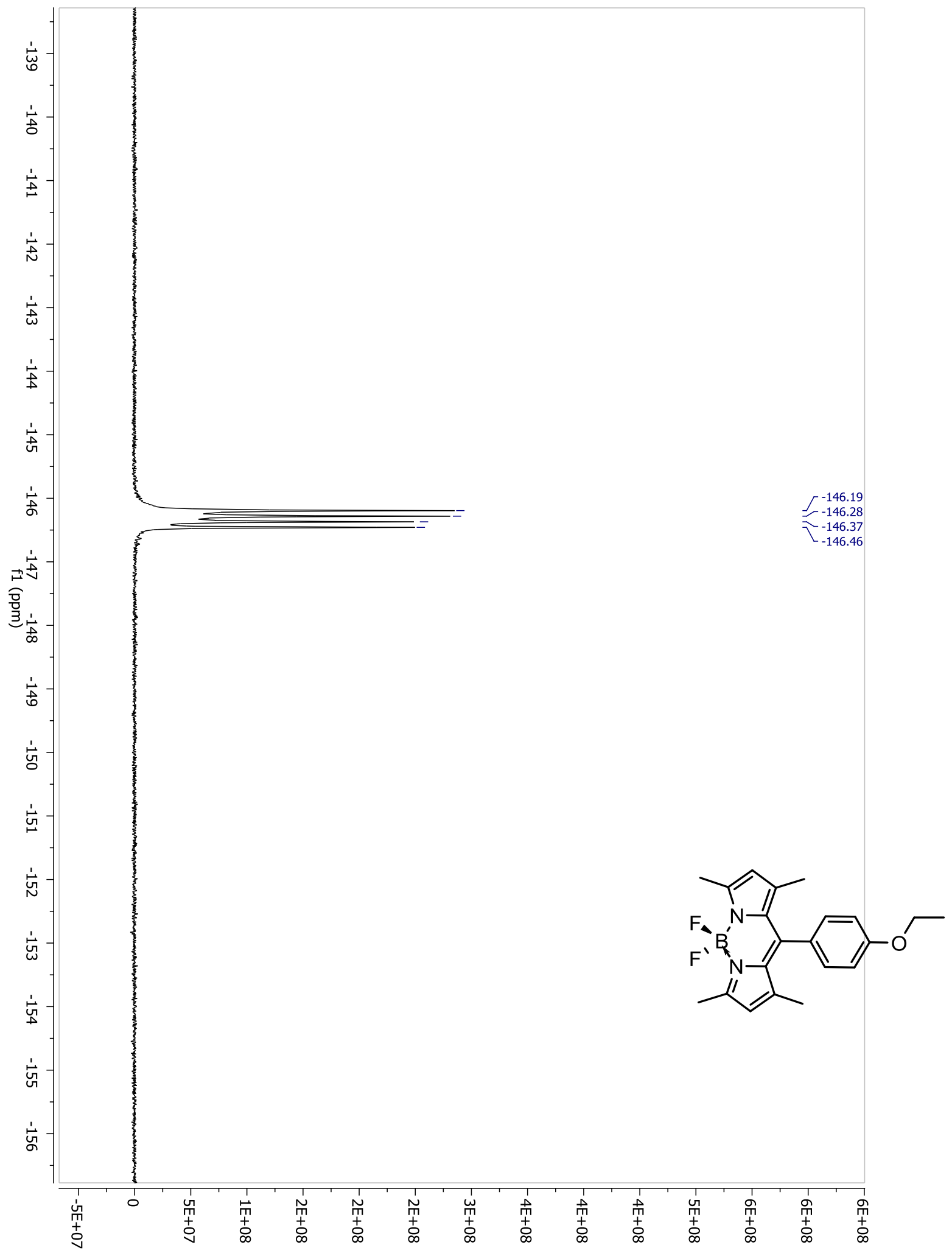

Figure A.76.: ${ }^{19} \mathrm{~F}$ NMR of 3.41d 


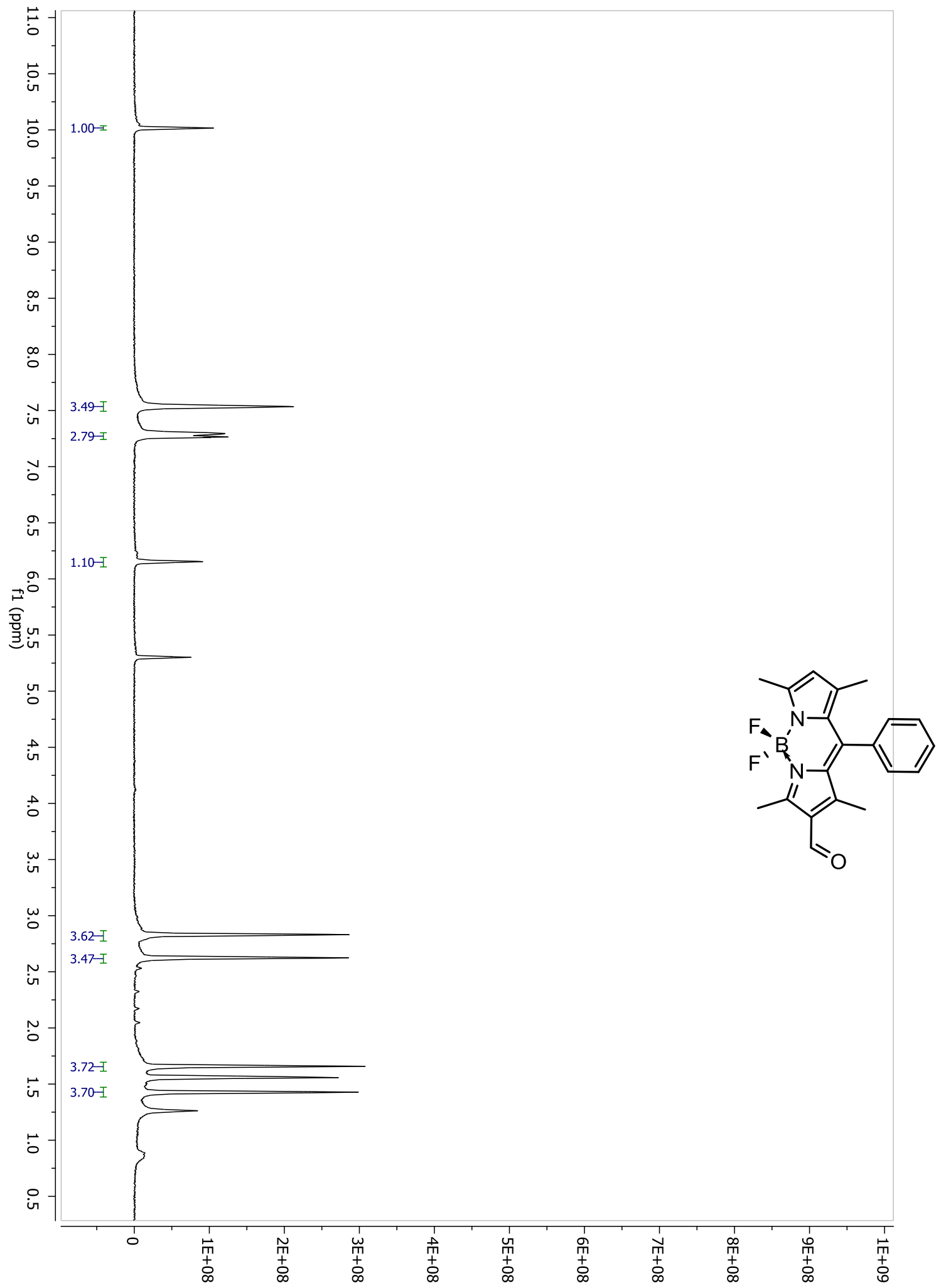

Figure A.77: ${ }^{1} \mathrm{H}$ NMR of $3.42 \mathrm{a}$ 


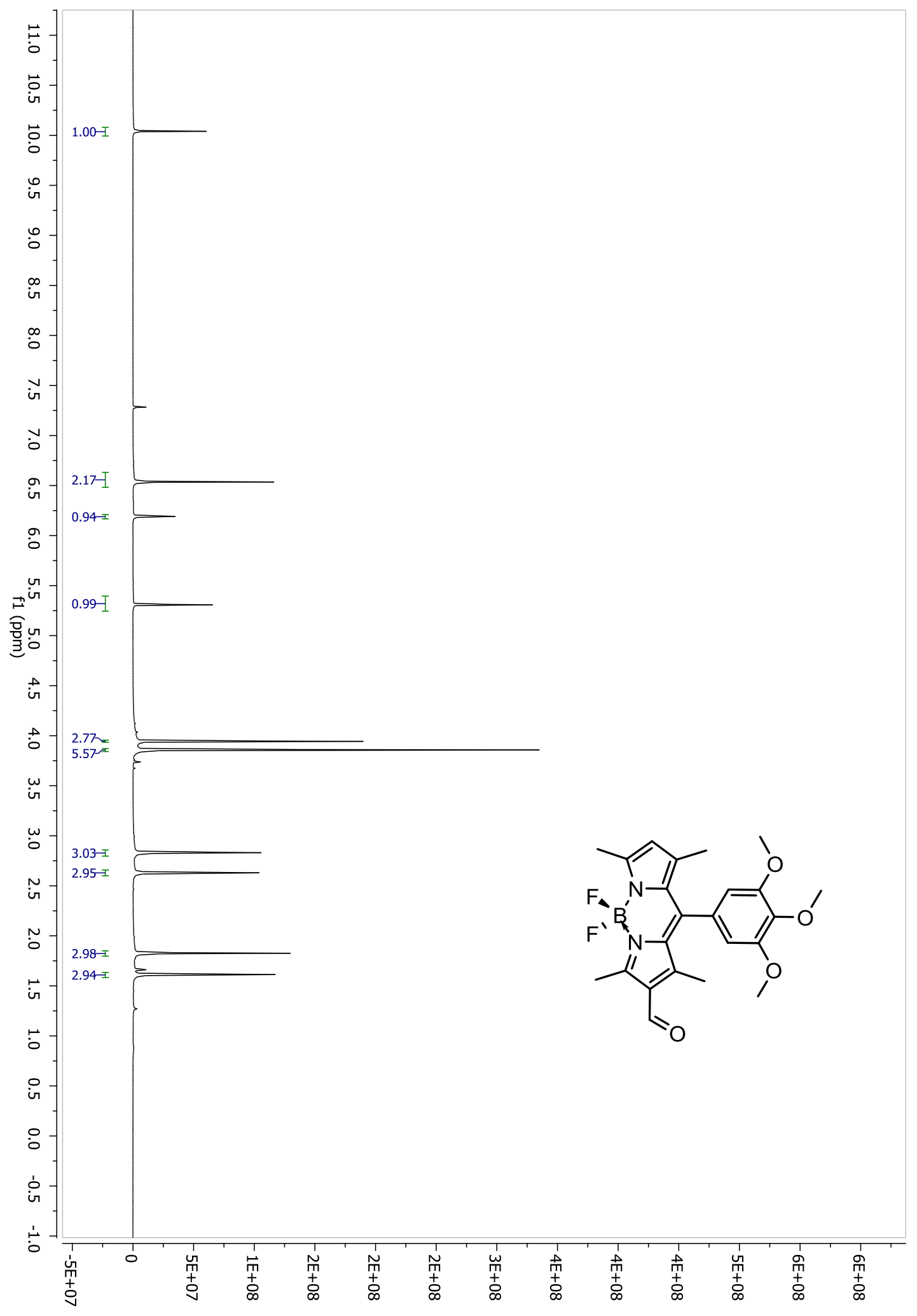

Figure A.78: ${ }^{1} \mathrm{H}$ NMR of $\mathbf{3 . 4 2 b}$ 


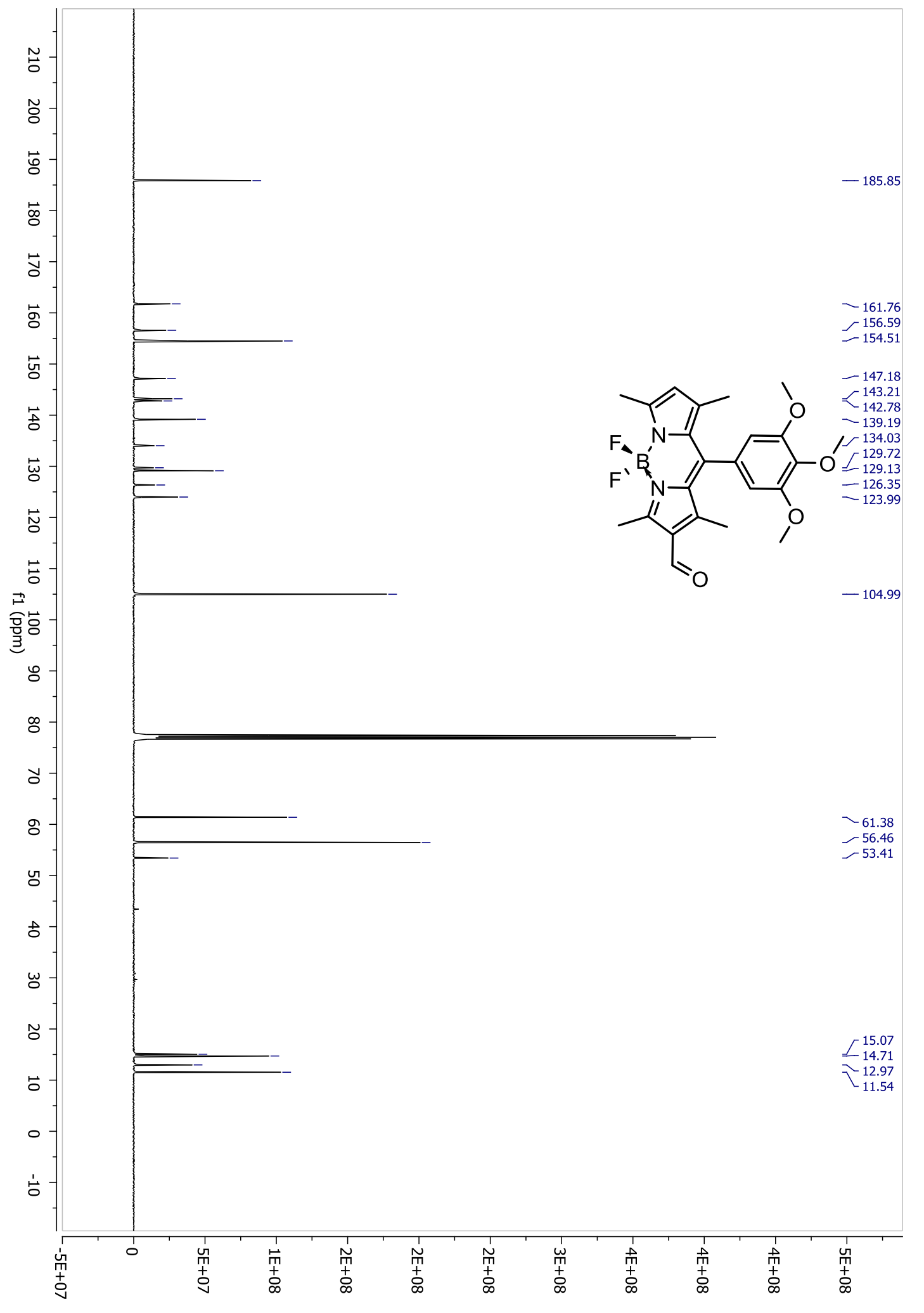

Figure A.79: ${ }^{13} \mathrm{C}$ NMR of $\mathbf{3 . 4 2 b}$ 


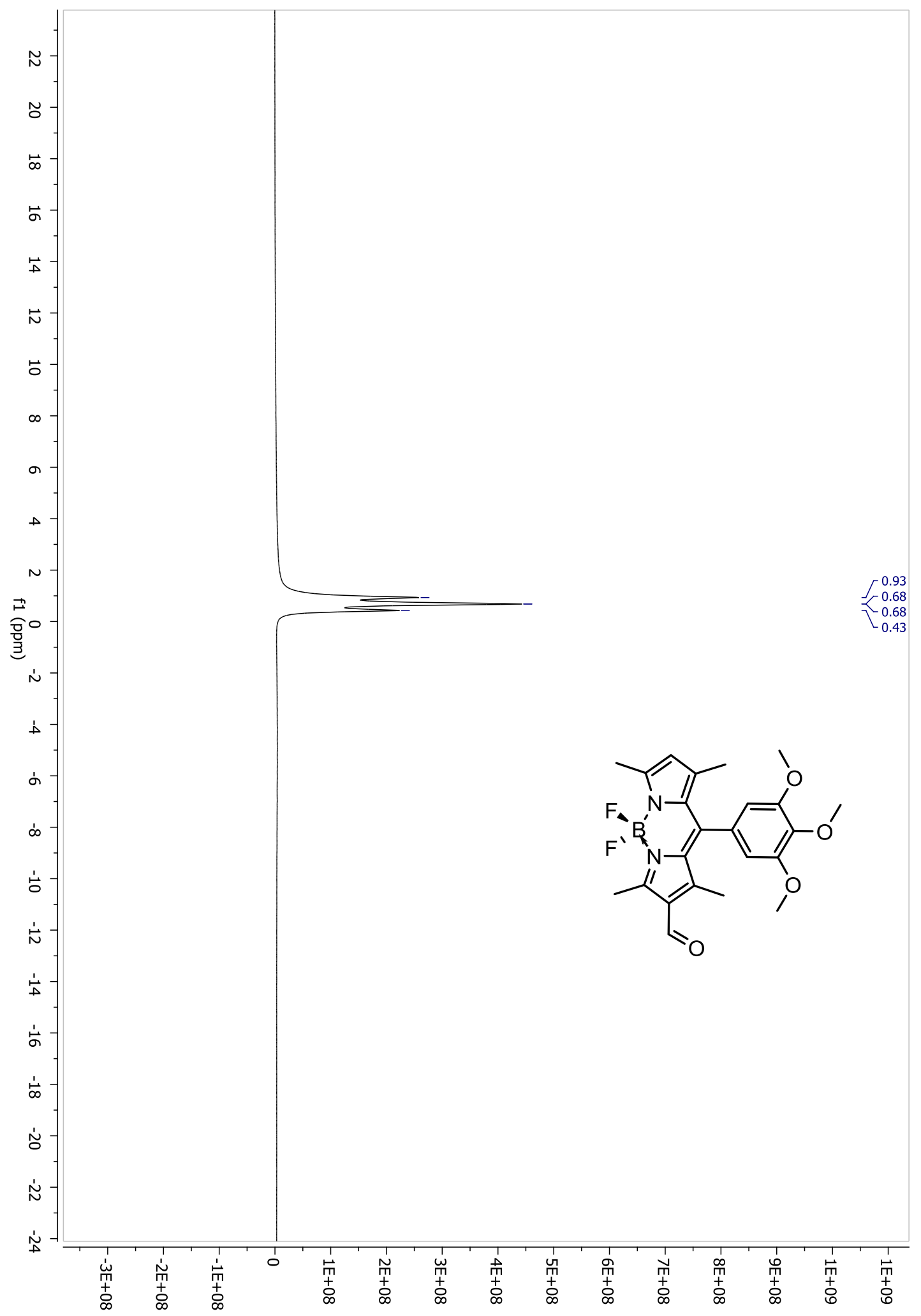

Figure A.80: ${ }^{11} B$ NMR of $\mathbf{3 . 4 2 b}$ 


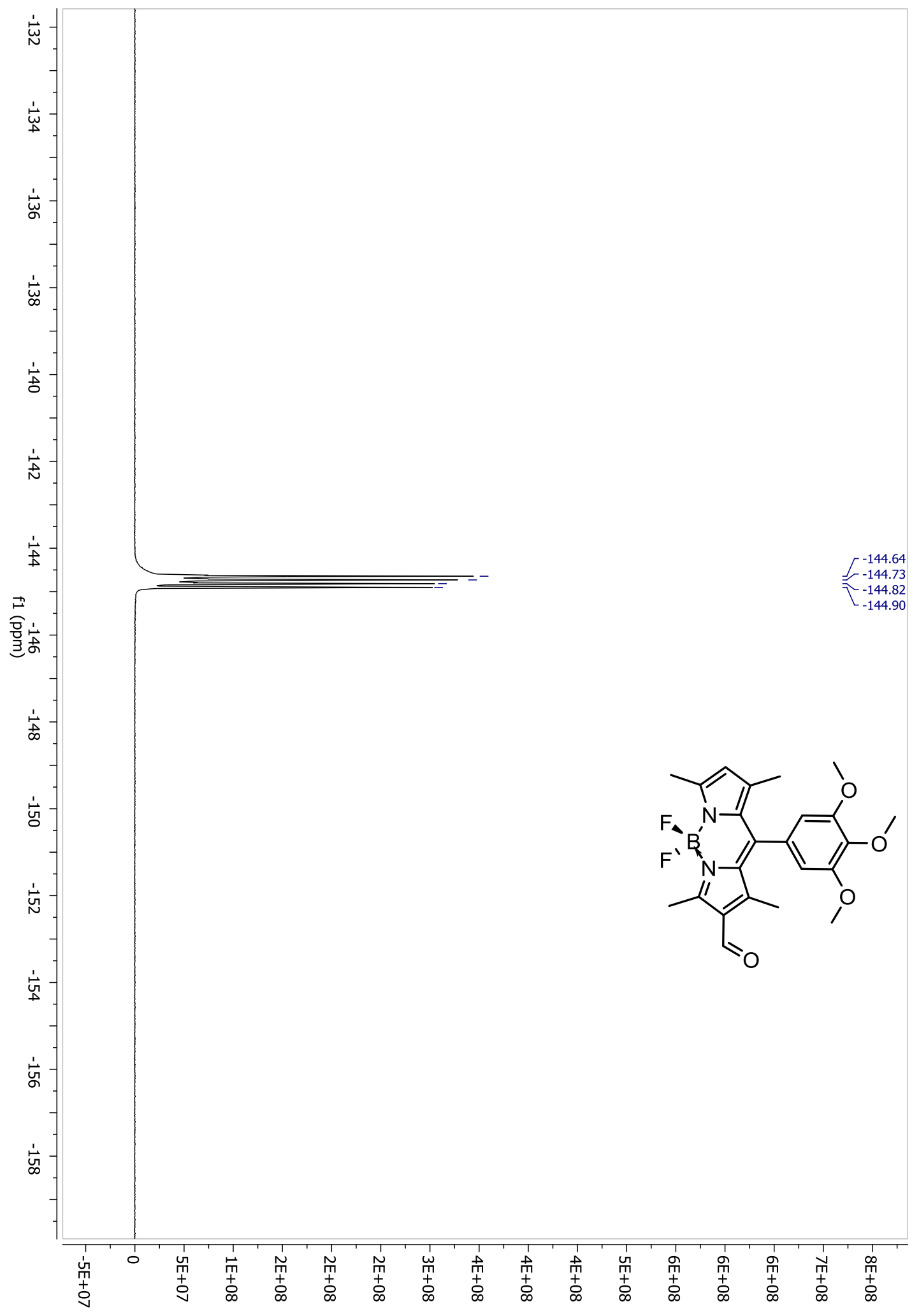

Figure A.81: ${ }^{19} \mathrm{~F} \mathrm{NMR}$ of $\mathbf{3 . 4 2 b}$ 


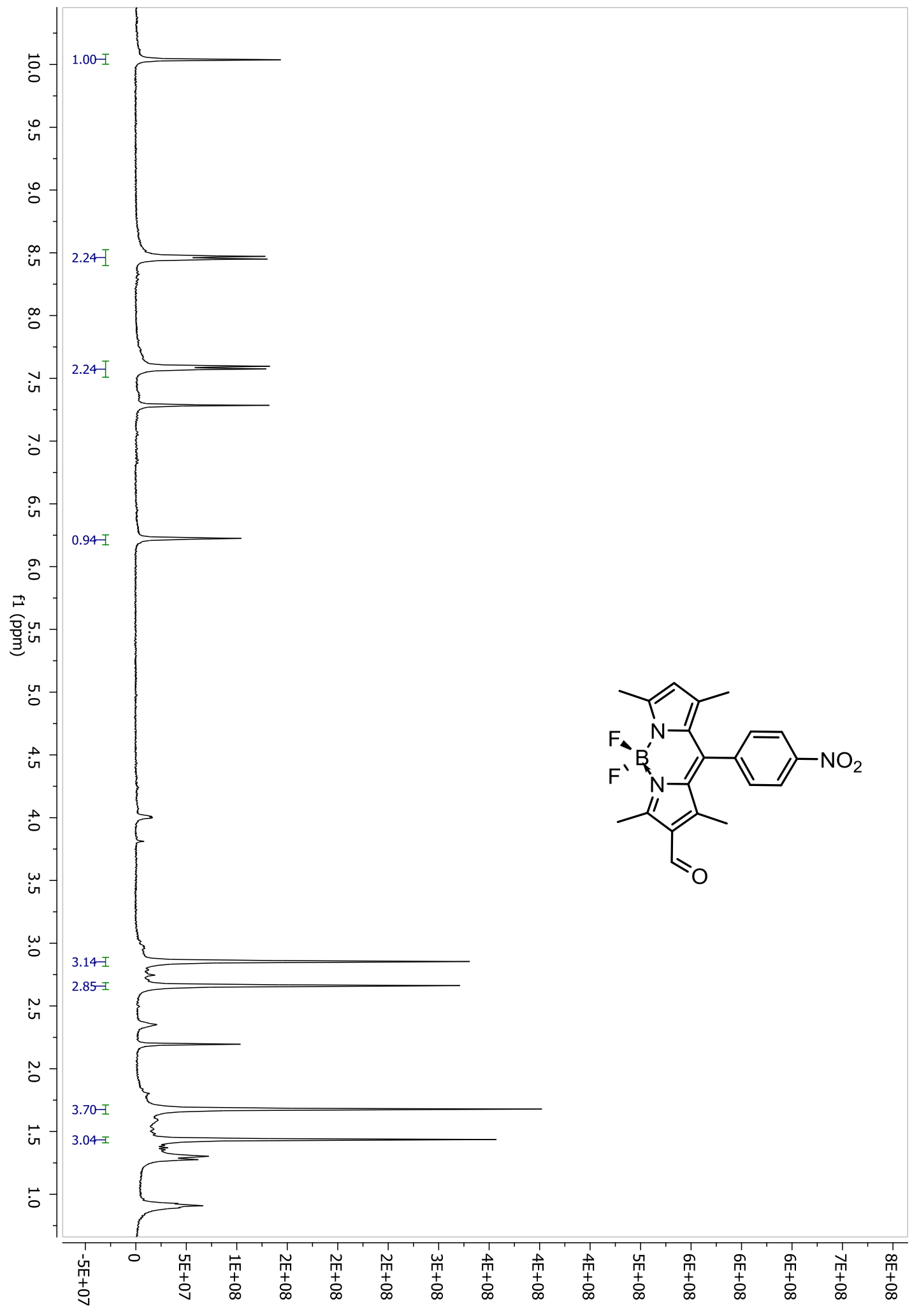

Figure A.82: ${ }^{1} \mathrm{H}$ NMR of $3.42 \mathrm{C}$ 


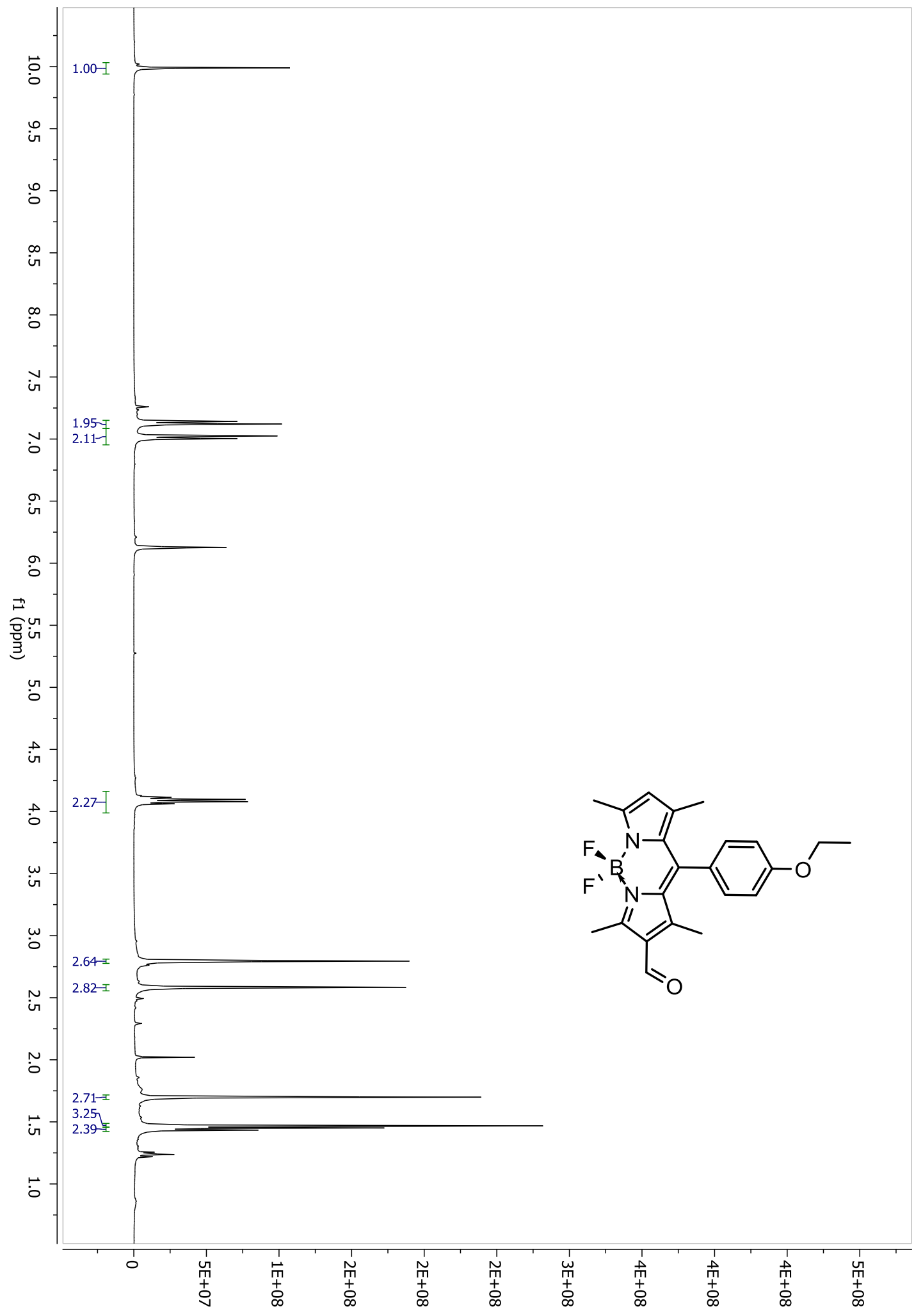

Figure A.83: ${ }^{1} \mathrm{H}$ NMR of $\mathbf{3 . 4 2 d}$ 


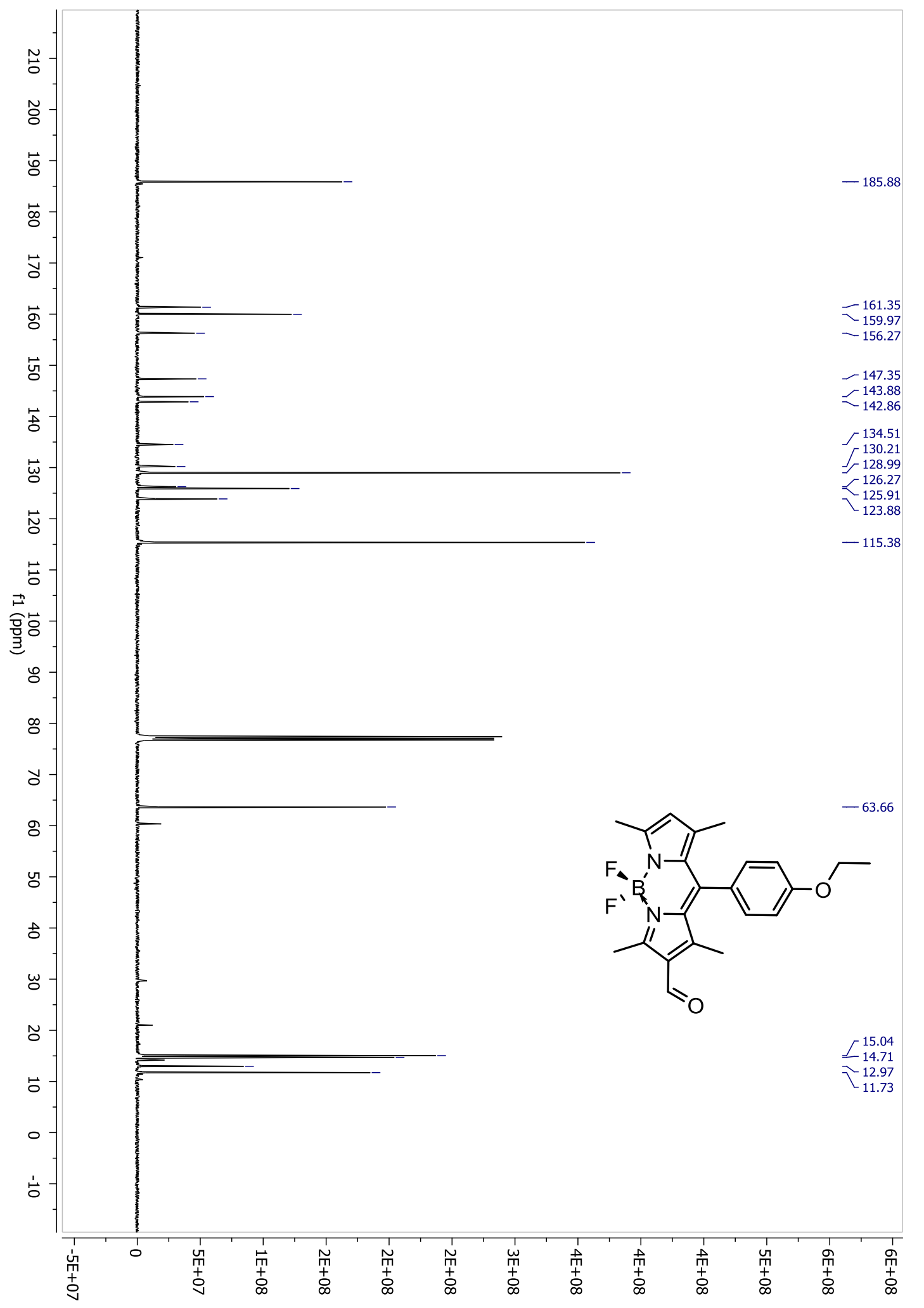

Figure A.84: ${ }^{13} \mathrm{C}$ NMR of $\mathbf{3 . 4 2 d}$ 


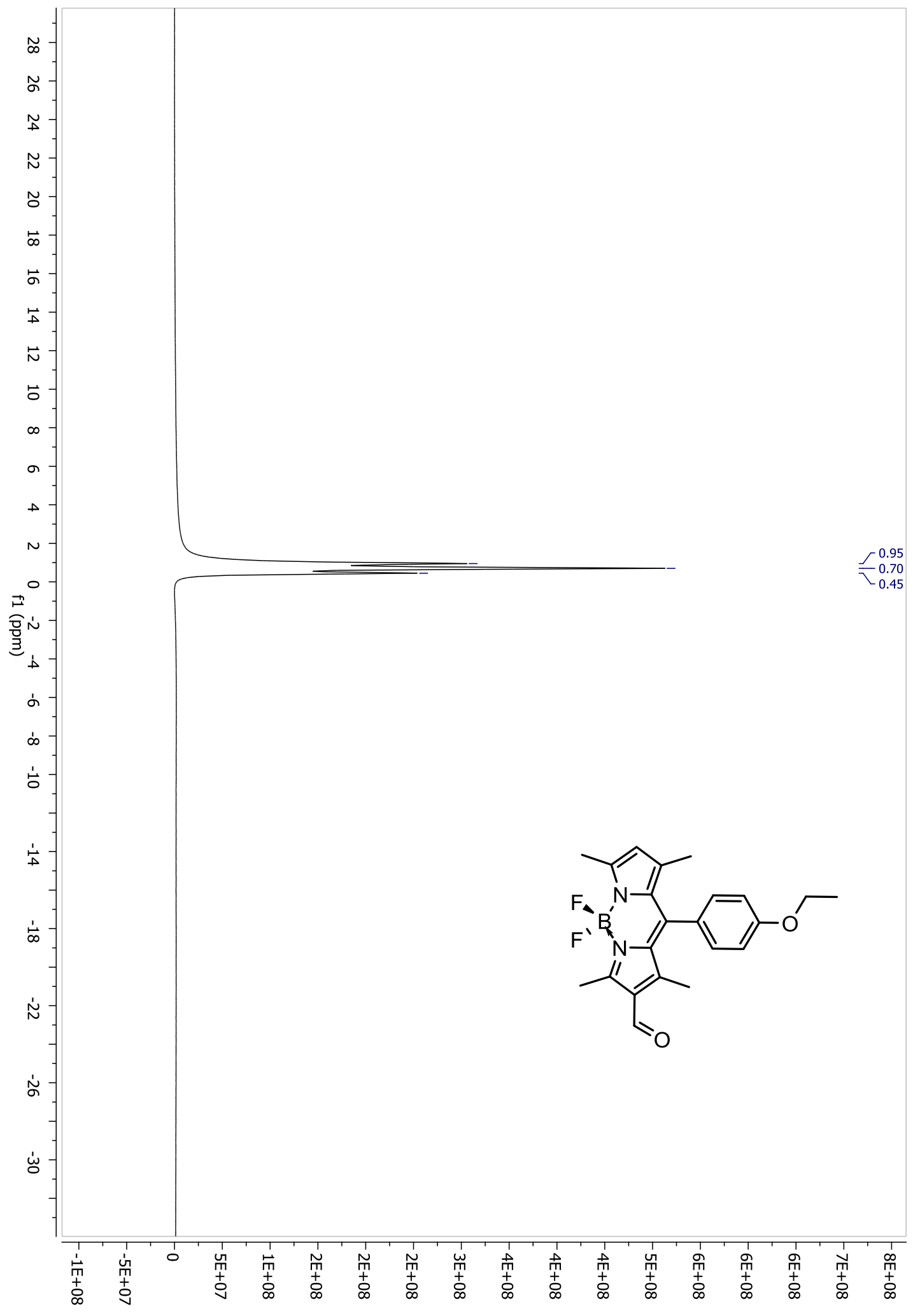

Figure A..85: ${ }^{11} B$ NMR of $\mathbf{3 . 4 2 d}$ 


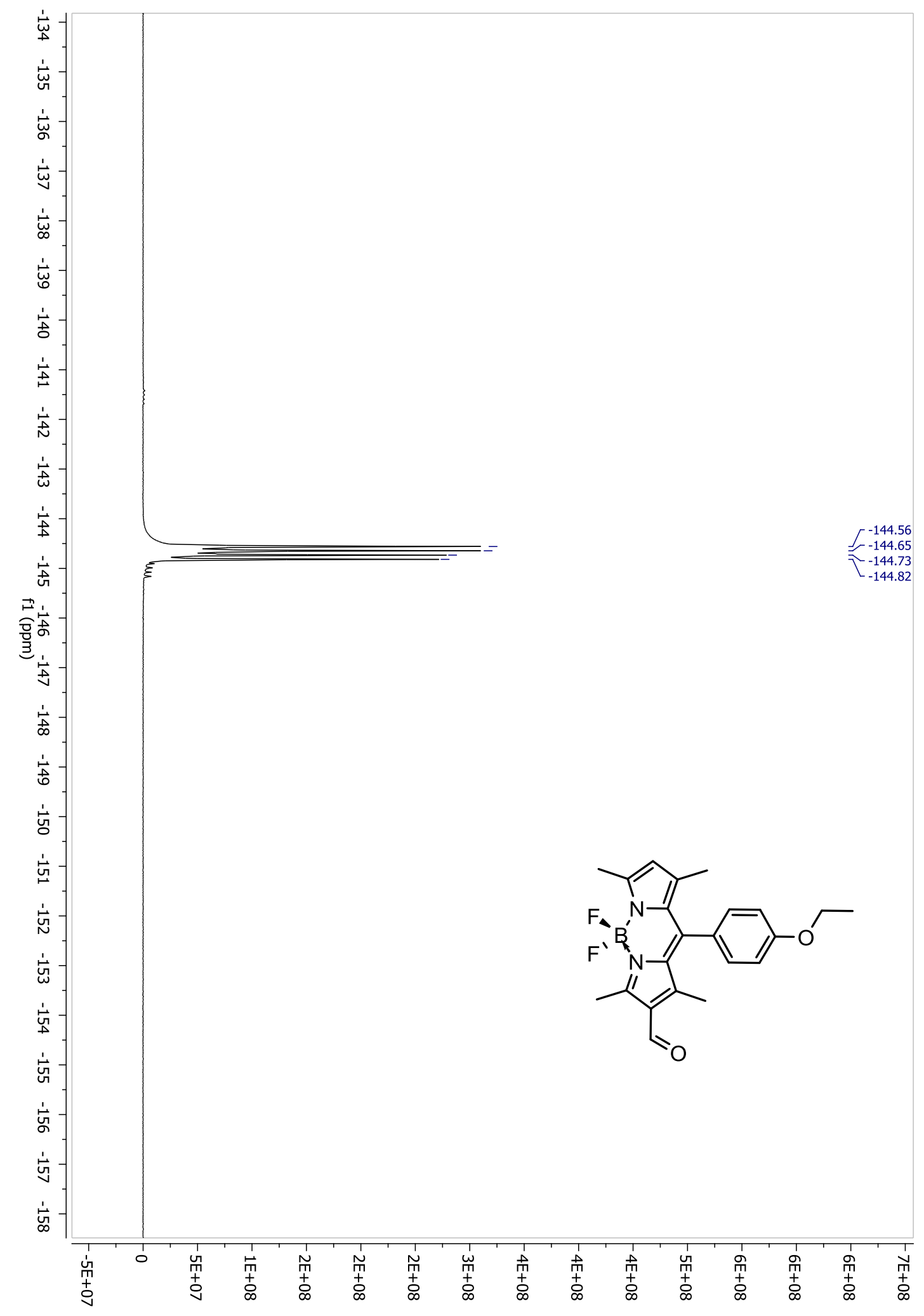

Figure A.86: ${ }^{19} \mathrm{~F}$ NMR of $\mathbf{3 . 4 2 d}$ 


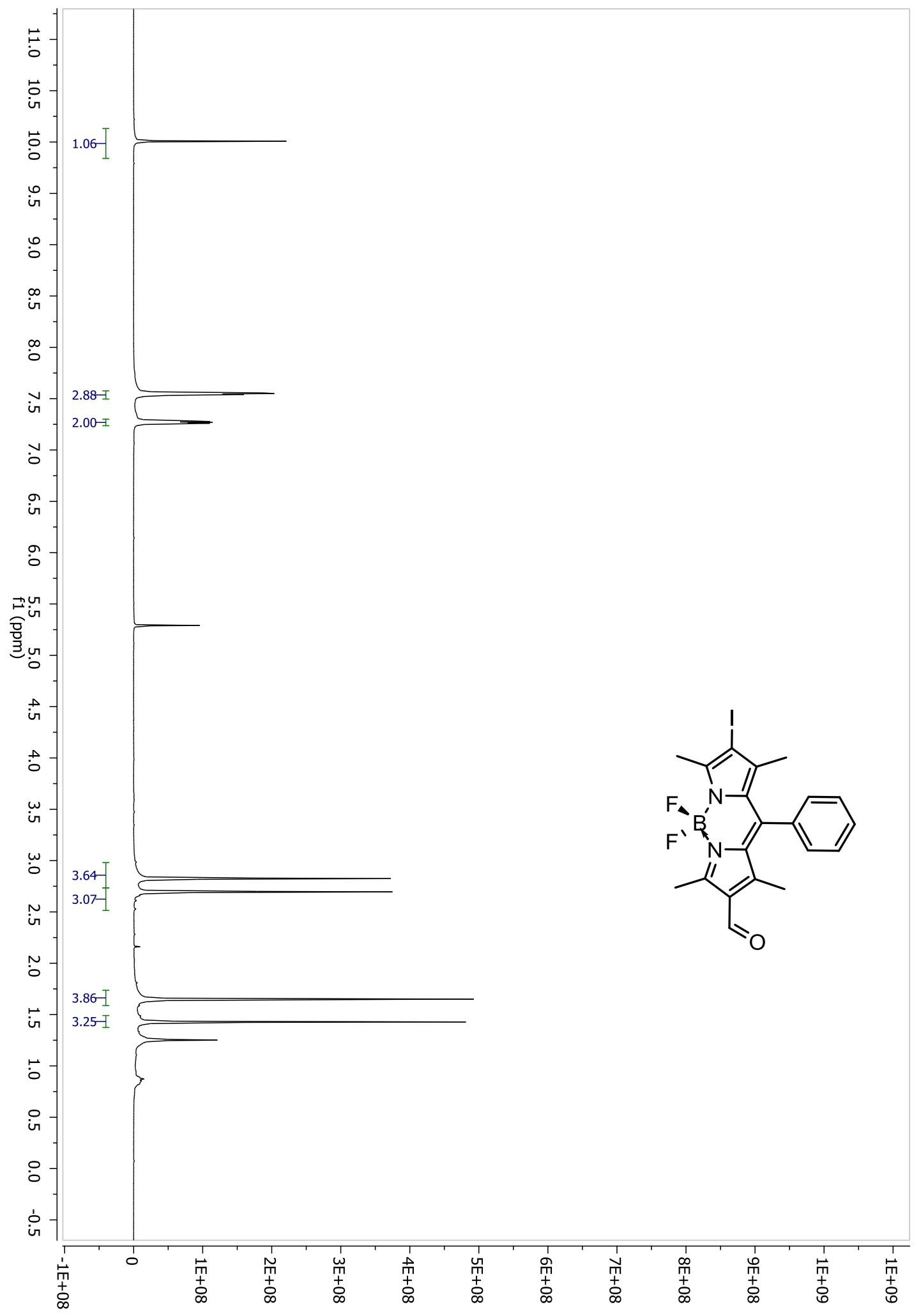

Figure A..87: ${ }^{1} \mathrm{H}$ NMR of $\mathbf{3 . 4 3 a}$ 


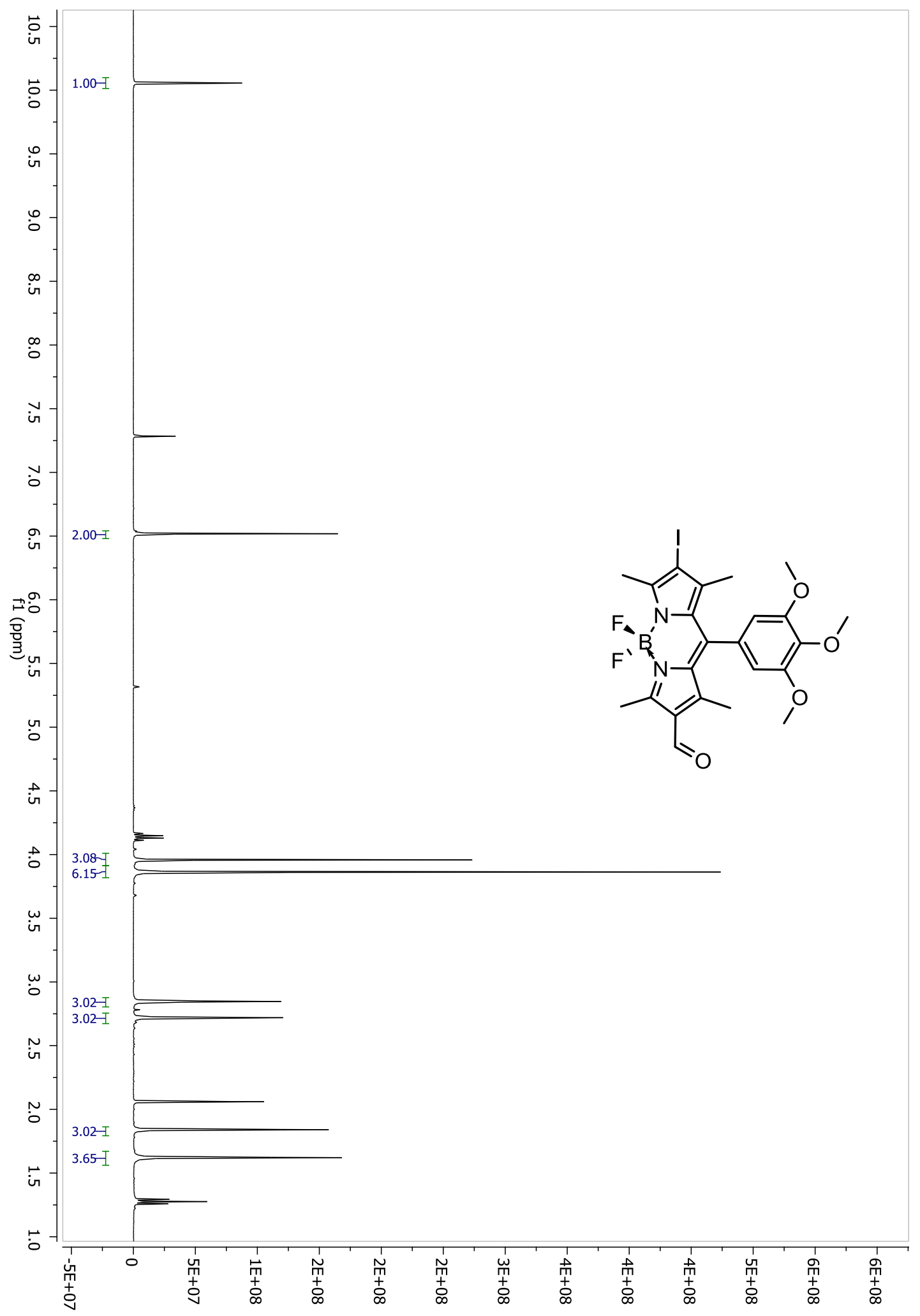

Figure A.88: ${ }^{1} \mathrm{H}$ NMR of $\mathbf{3 . 4 3 b}$ 


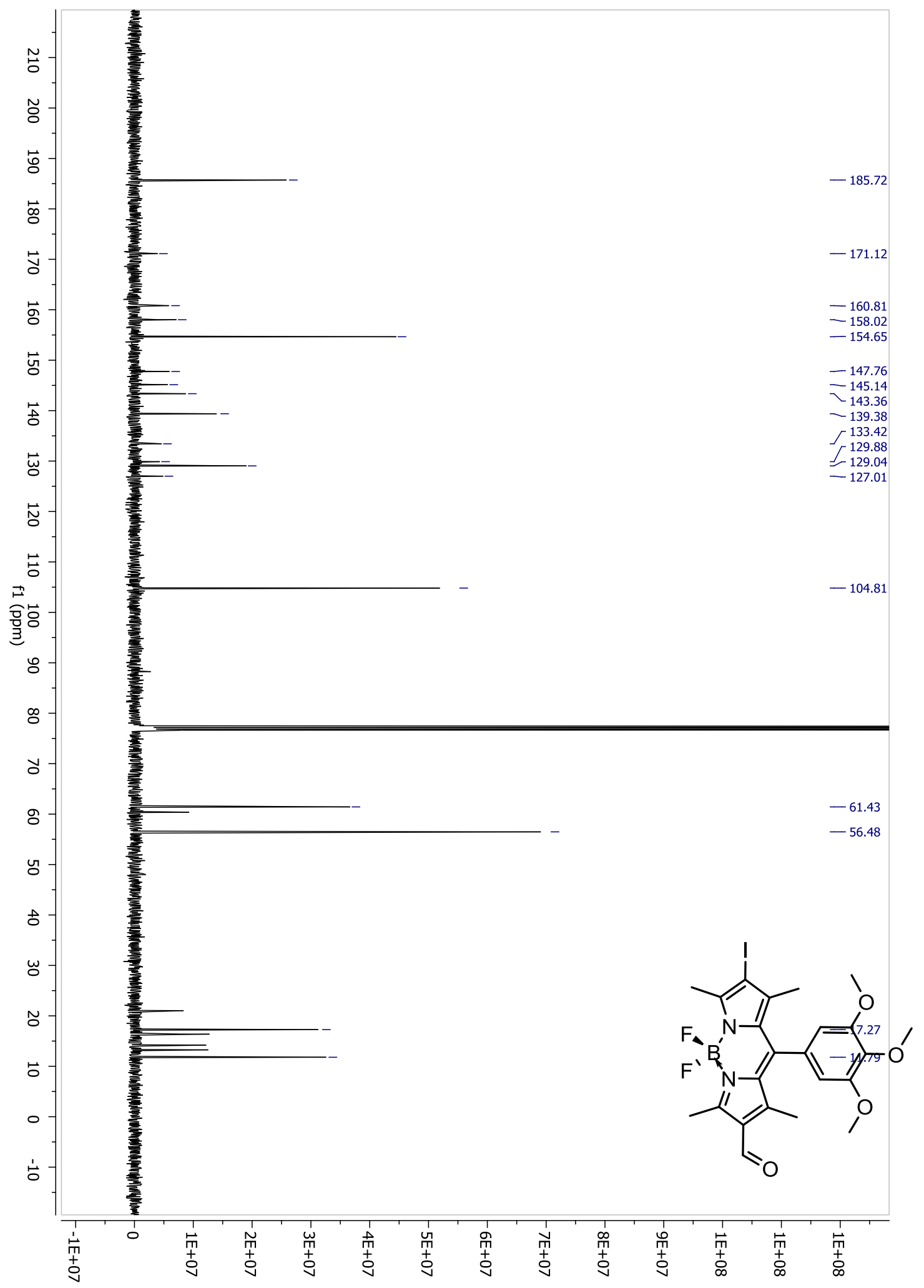

Figure A..89: ${ }^{13} \mathrm{C}$ NMR of $3.43 b$ 


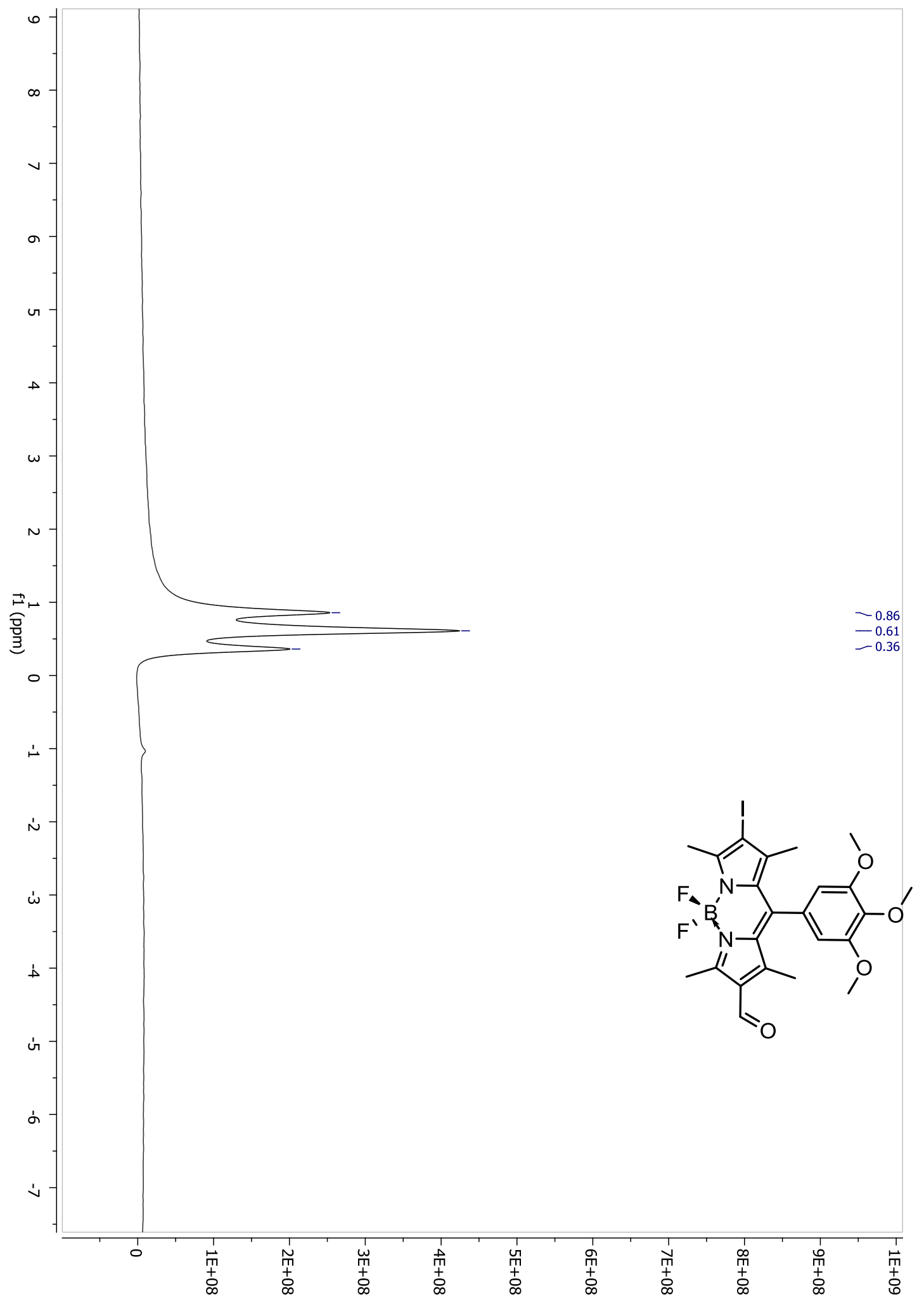

Figure A..90: ${ }^{11} \mathrm{~B}$ NMR of $3.43 \mathrm{~b}$ 


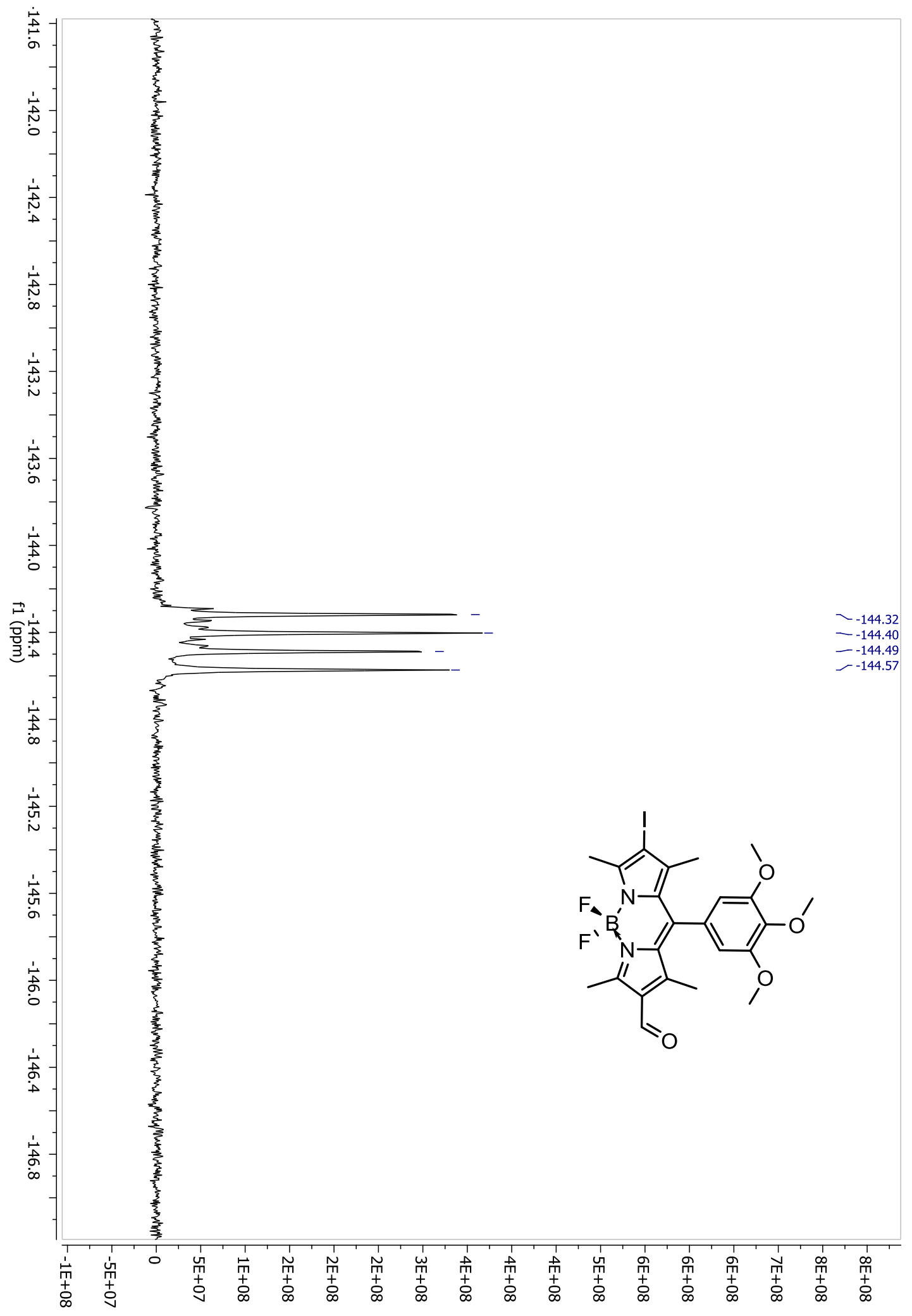

Figure A..91: ${ }^{19} \mathrm{~F}$ NMR of 3.43b 


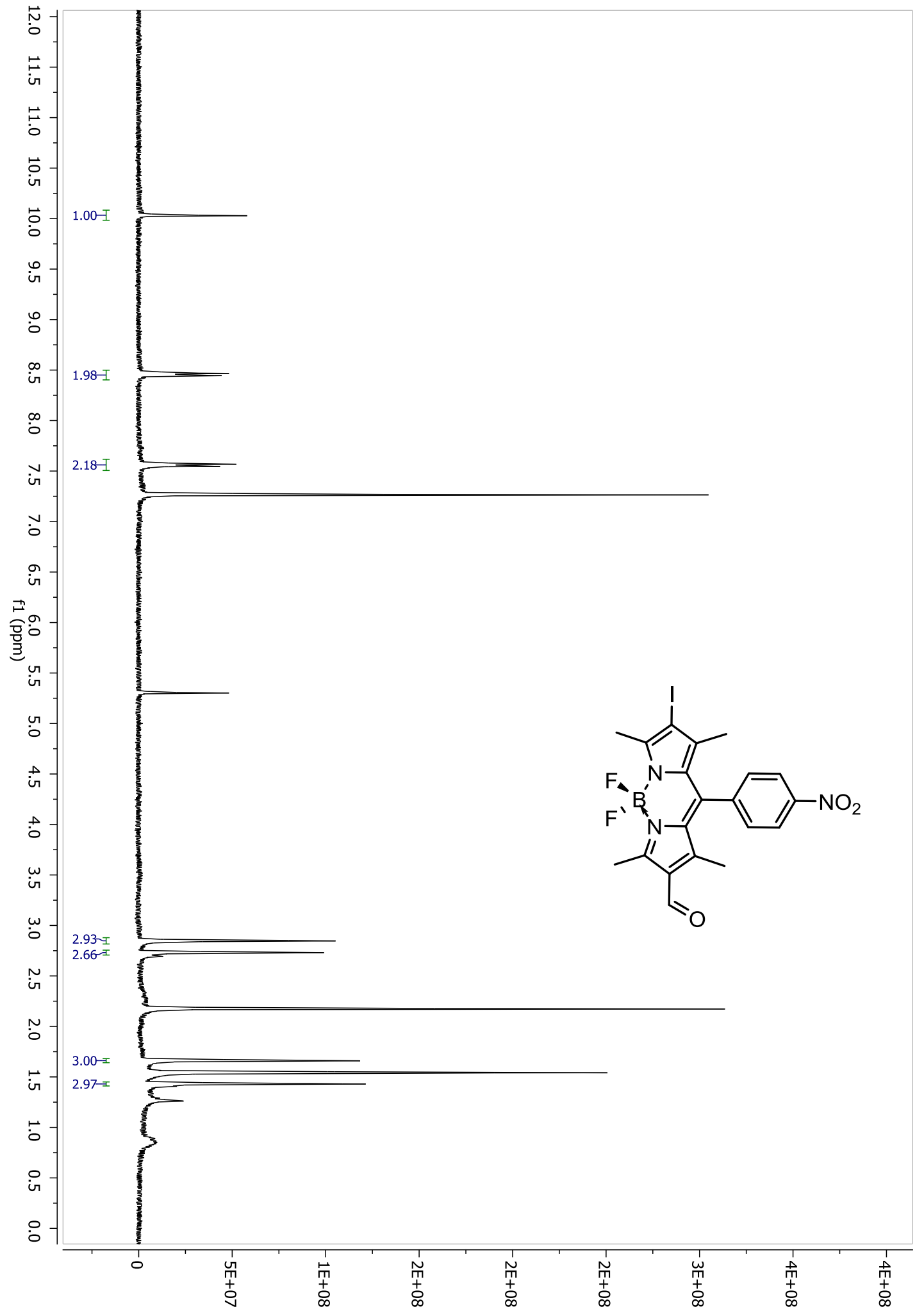

Figure A..92: ${ }^{1} \mathrm{H}$ NMR of $3.43 \mathrm{C}$ 


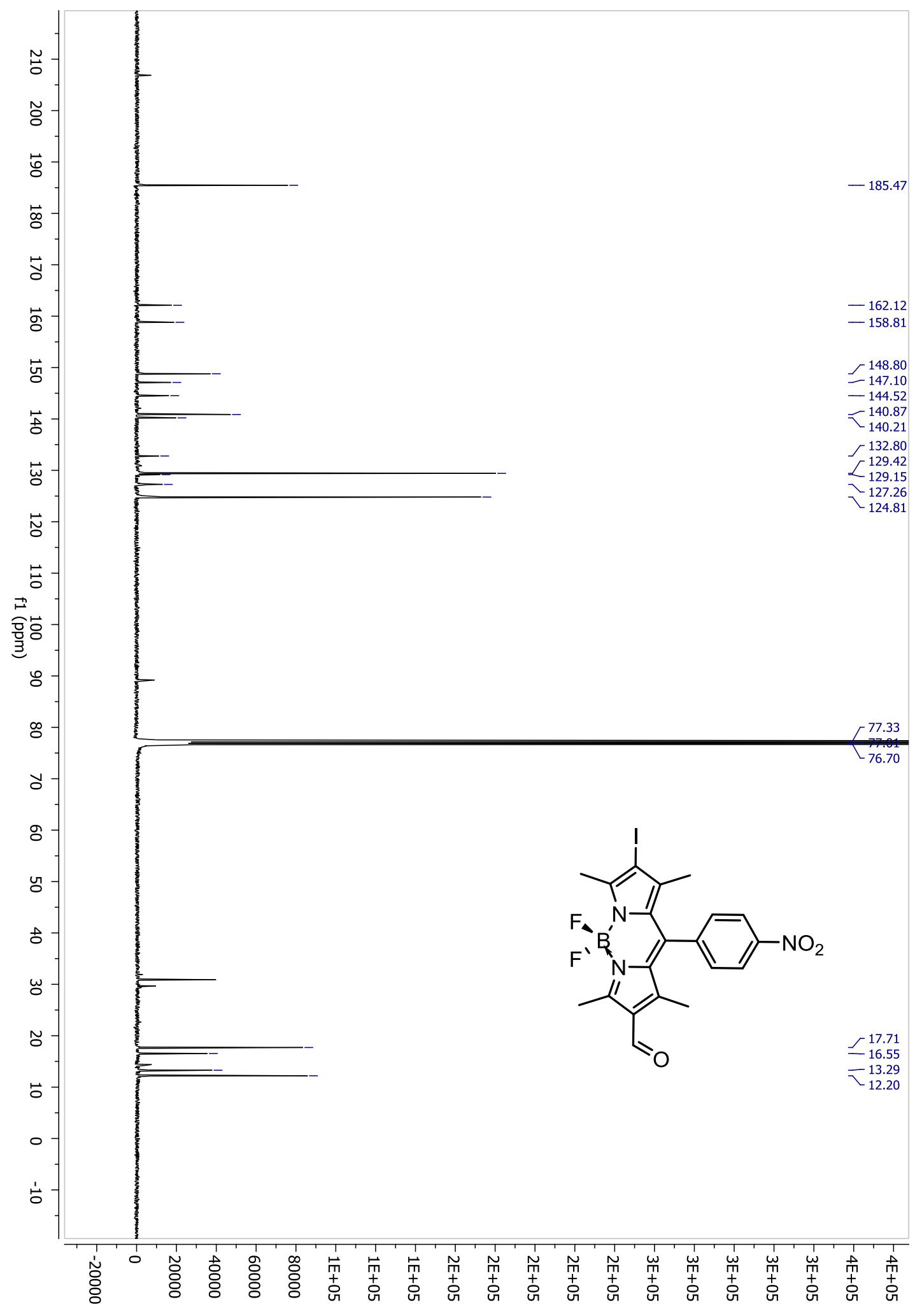

Figure A.93: ${ }^{13} \mathrm{C}$ NMR of $3.43 \mathrm{C}$ 


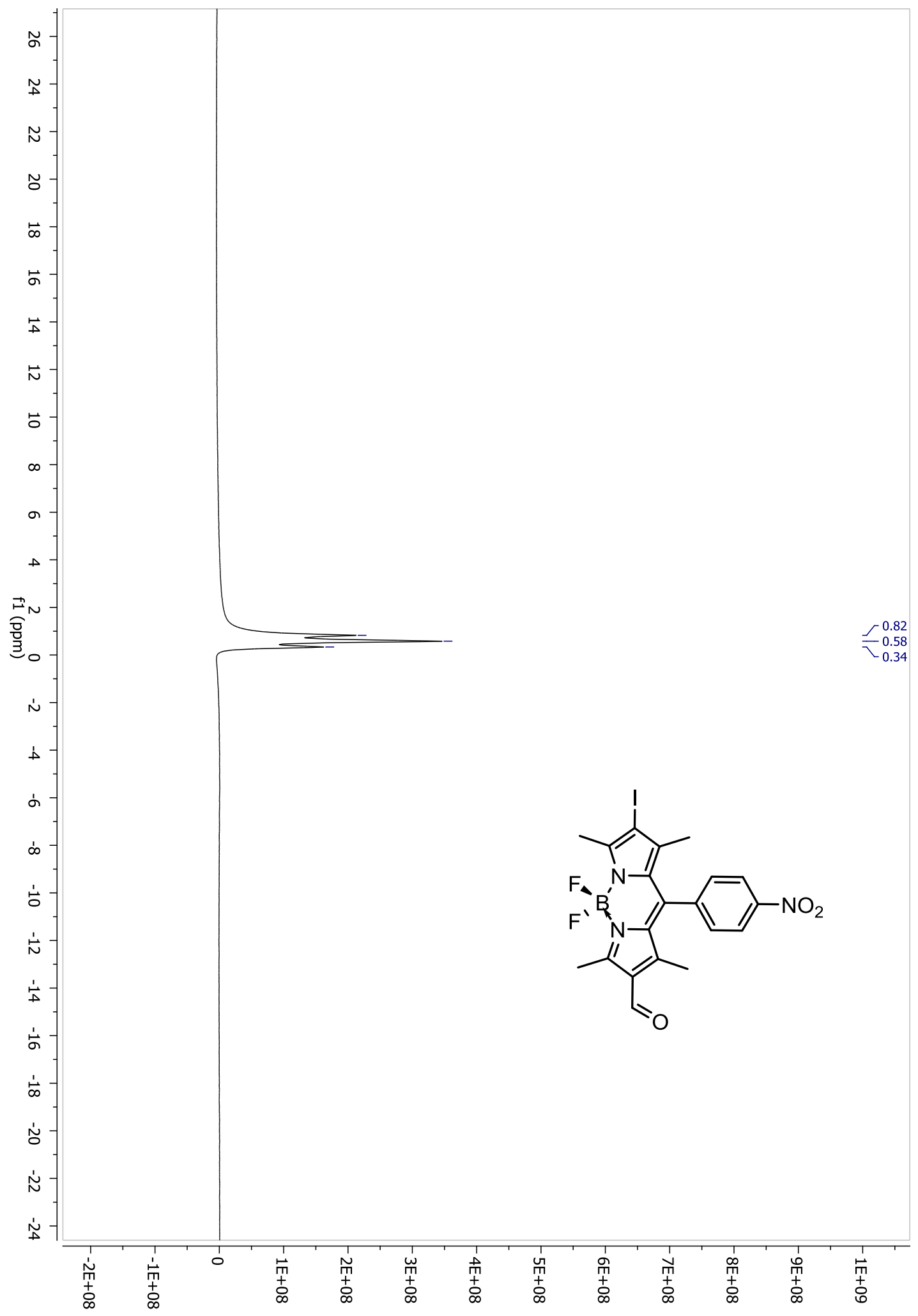

Figure A.94: ${ }^{11} \mathrm{~B}$ NMR of $3.43 \mathrm{C}$ 


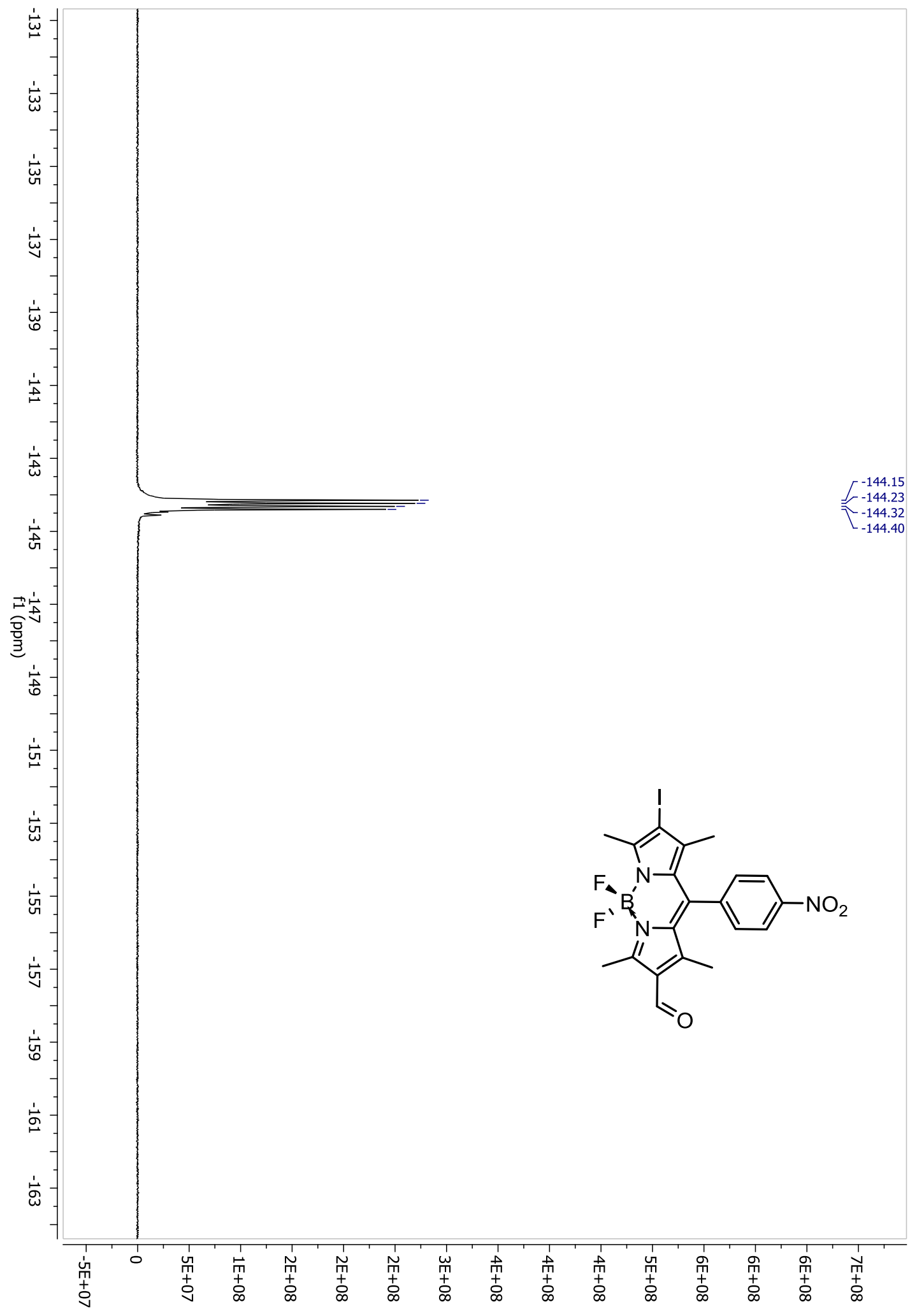

Figure A.95: ${ }^{19} \mathrm{~F}$ NMR of $3.43 \mathrm{C}$ 


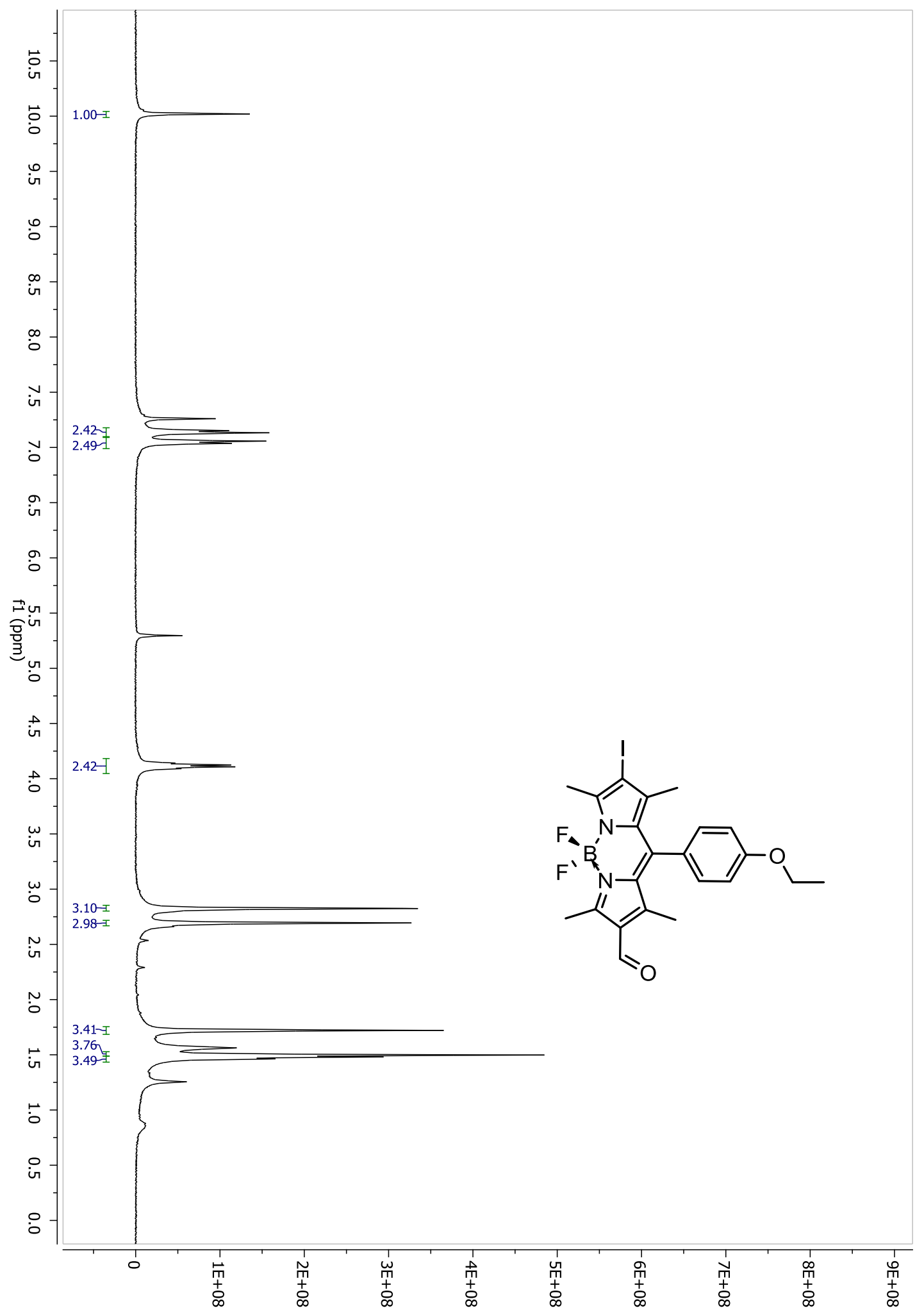

Figure A.96: ${ }^{1} \mathrm{H}$ NMR of $\mathbf{3 . 4 2 d}$ 


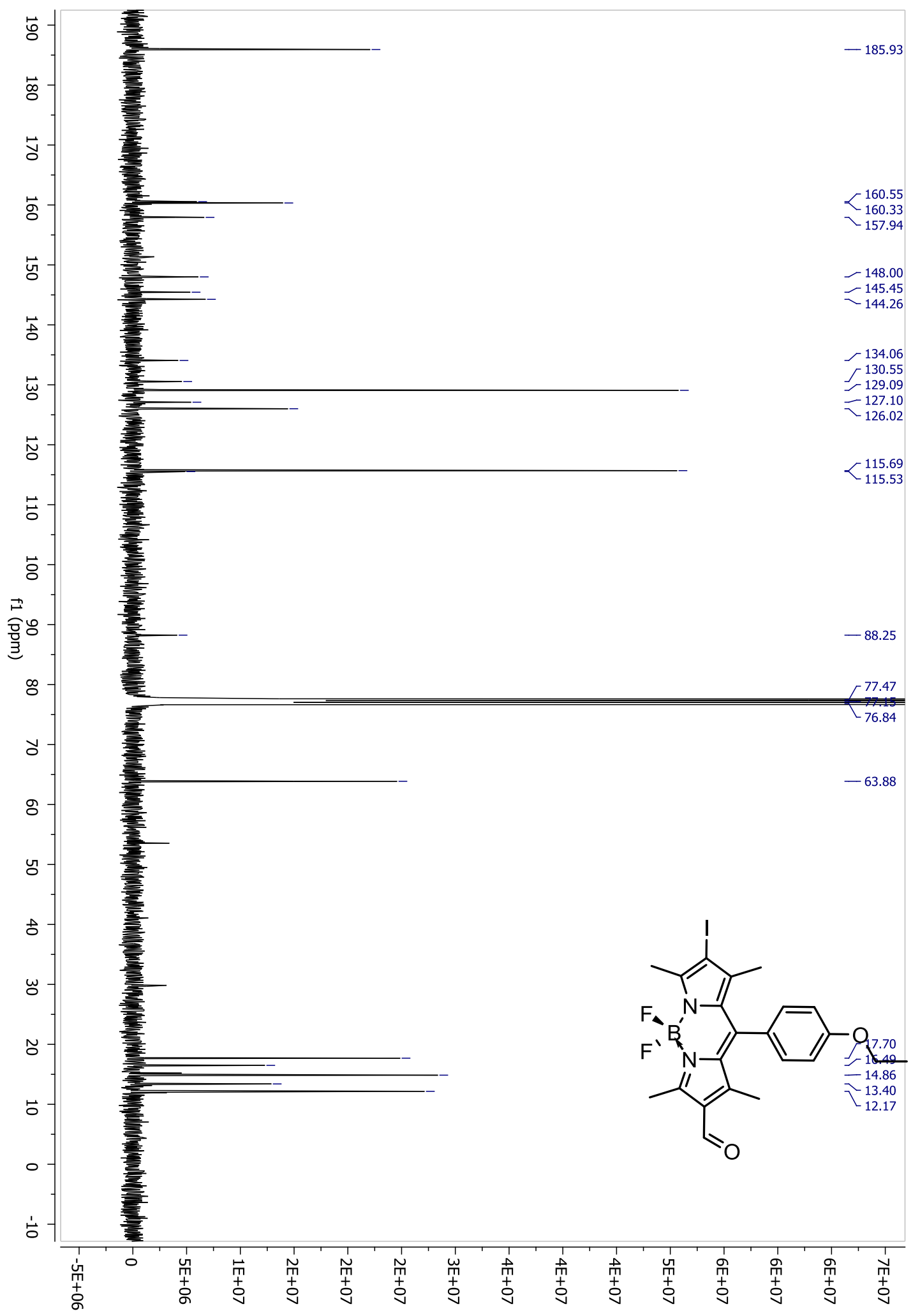

Figure A.97: ${ }^{13} \mathrm{C}$ NMR of $\mathbf{3 . 4 3 b}$ 


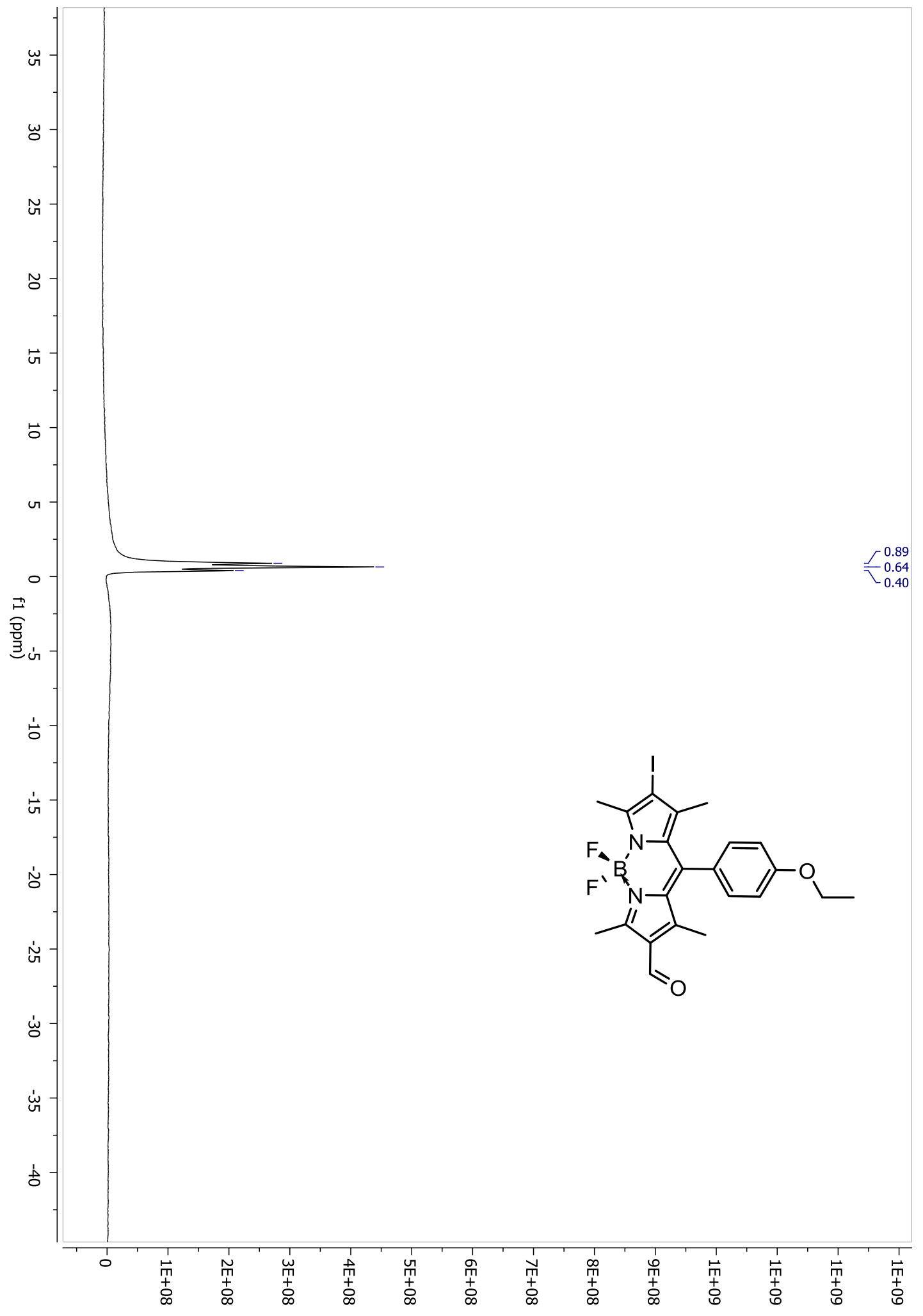

Figure A.98: ${ }^{11} B$ NMR of 3.43d 


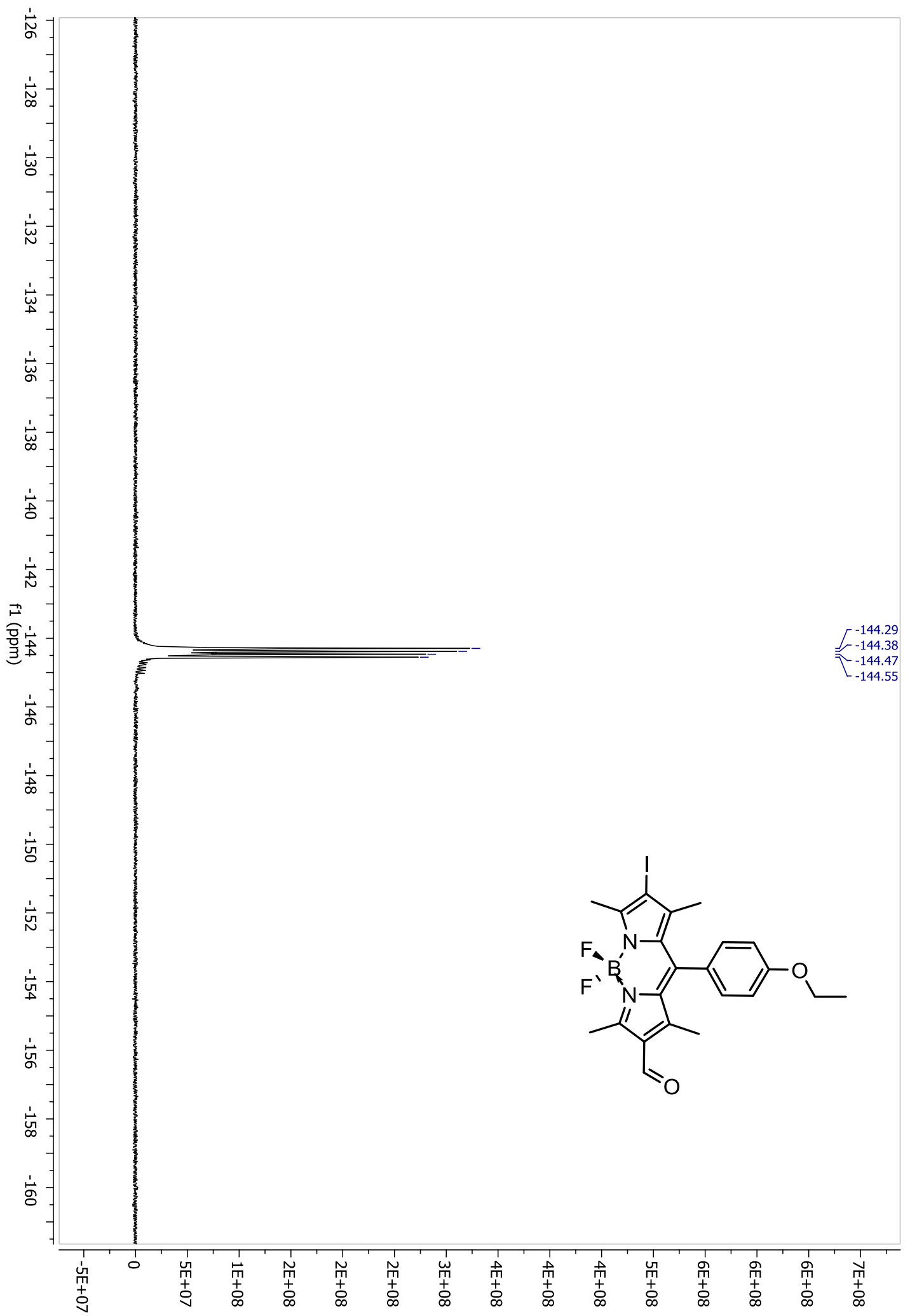

Figure A.99: ${ }^{19} \mathrm{~F}$ NMR of 3.43d 


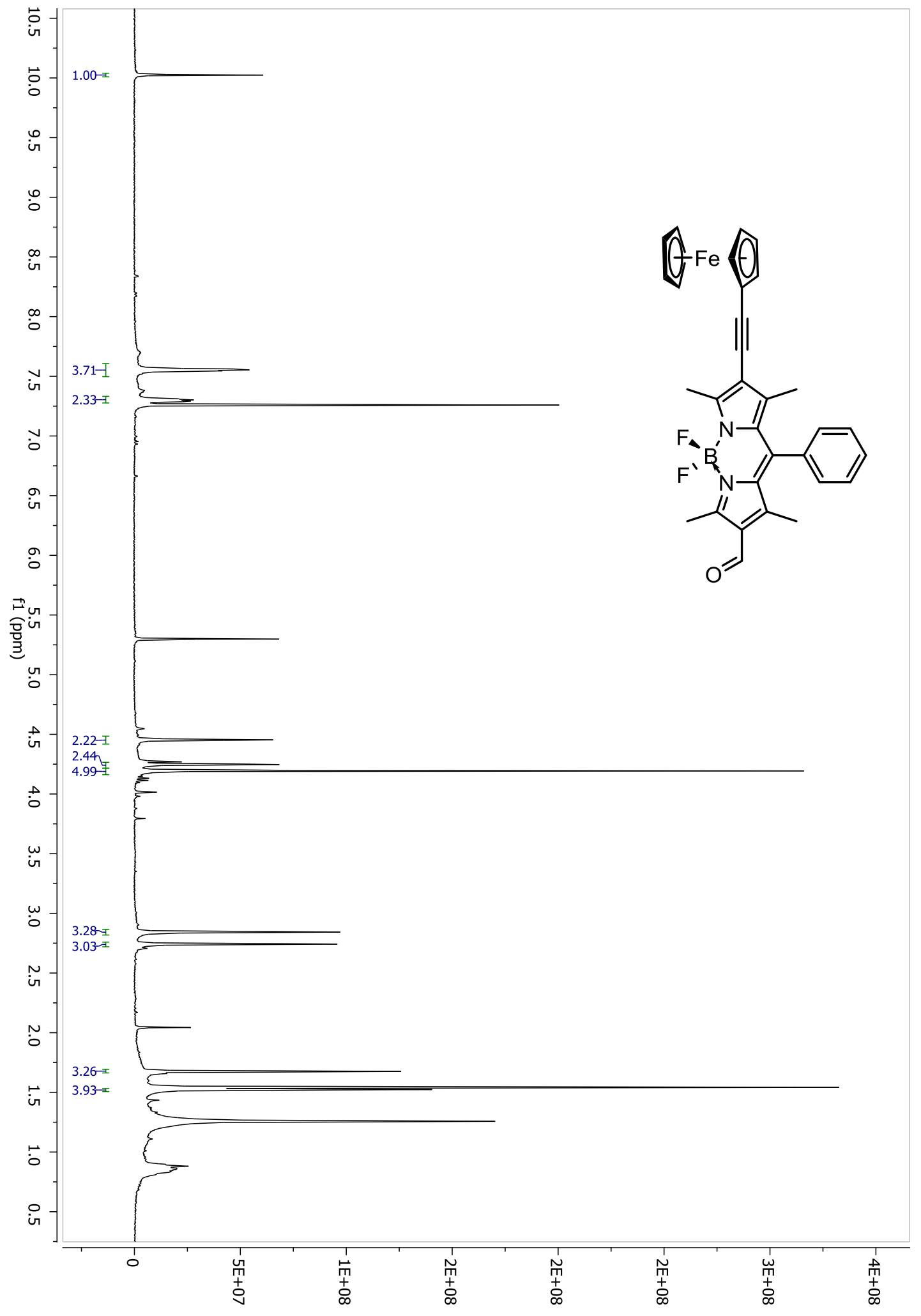

Figure A.100: ${ }^{1} \mathrm{H}$ NMR of $3.44 a$ 


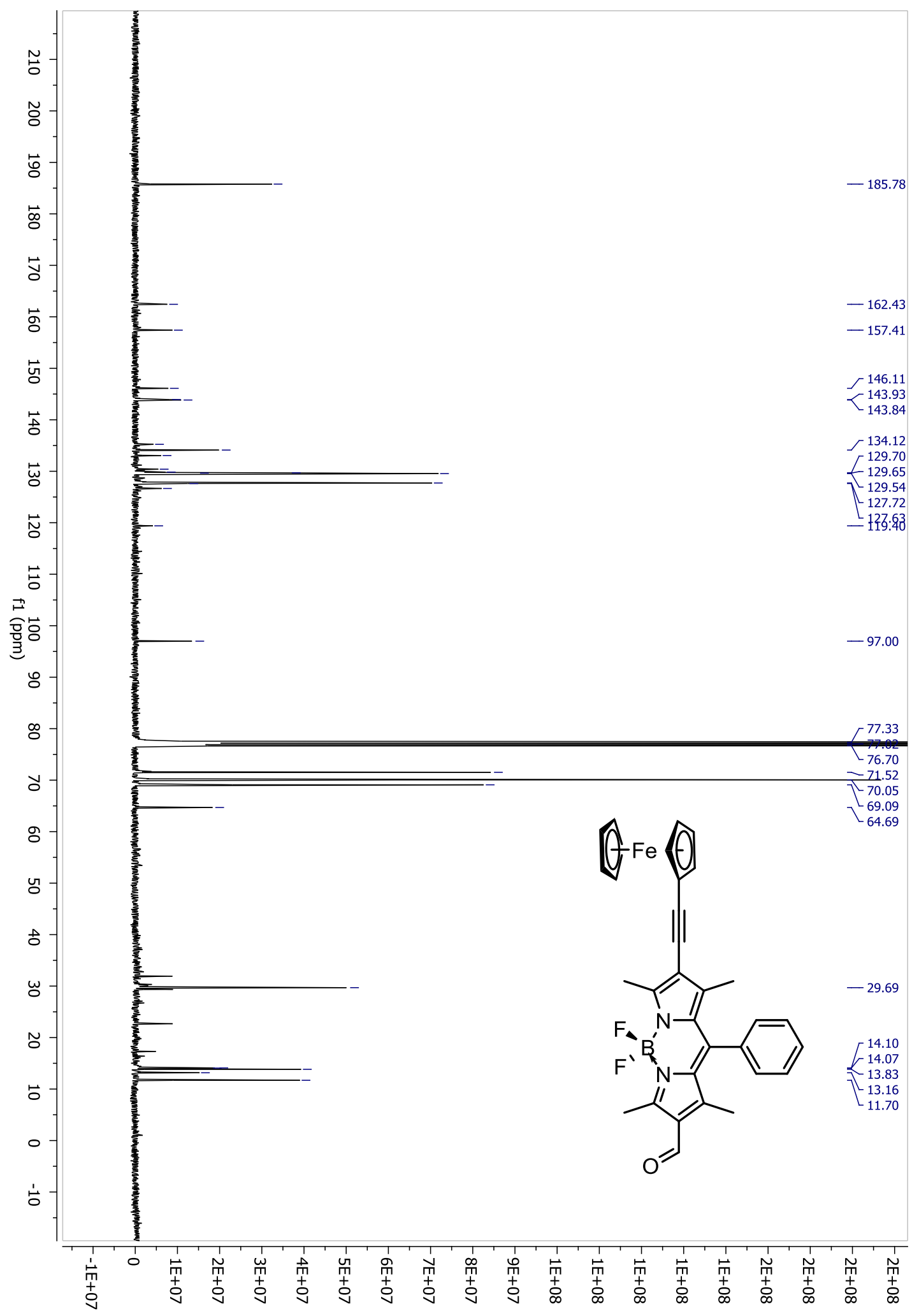

Figure A.101: ${ }^{13} \mathrm{C}$ NMR of $3.44 a$ 


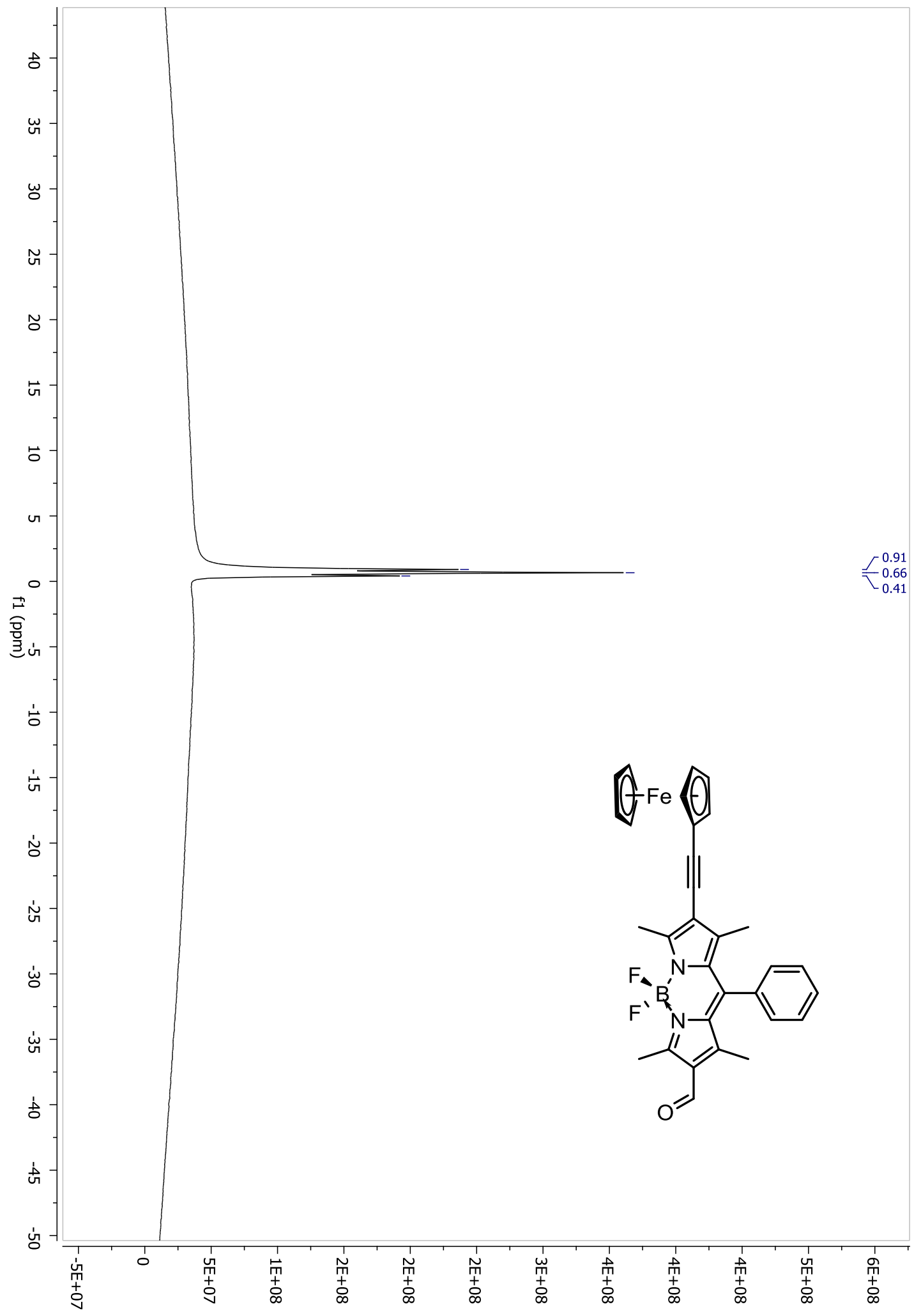

Figure A..102: ${ }^{11} B$ NMR of 3.44a 


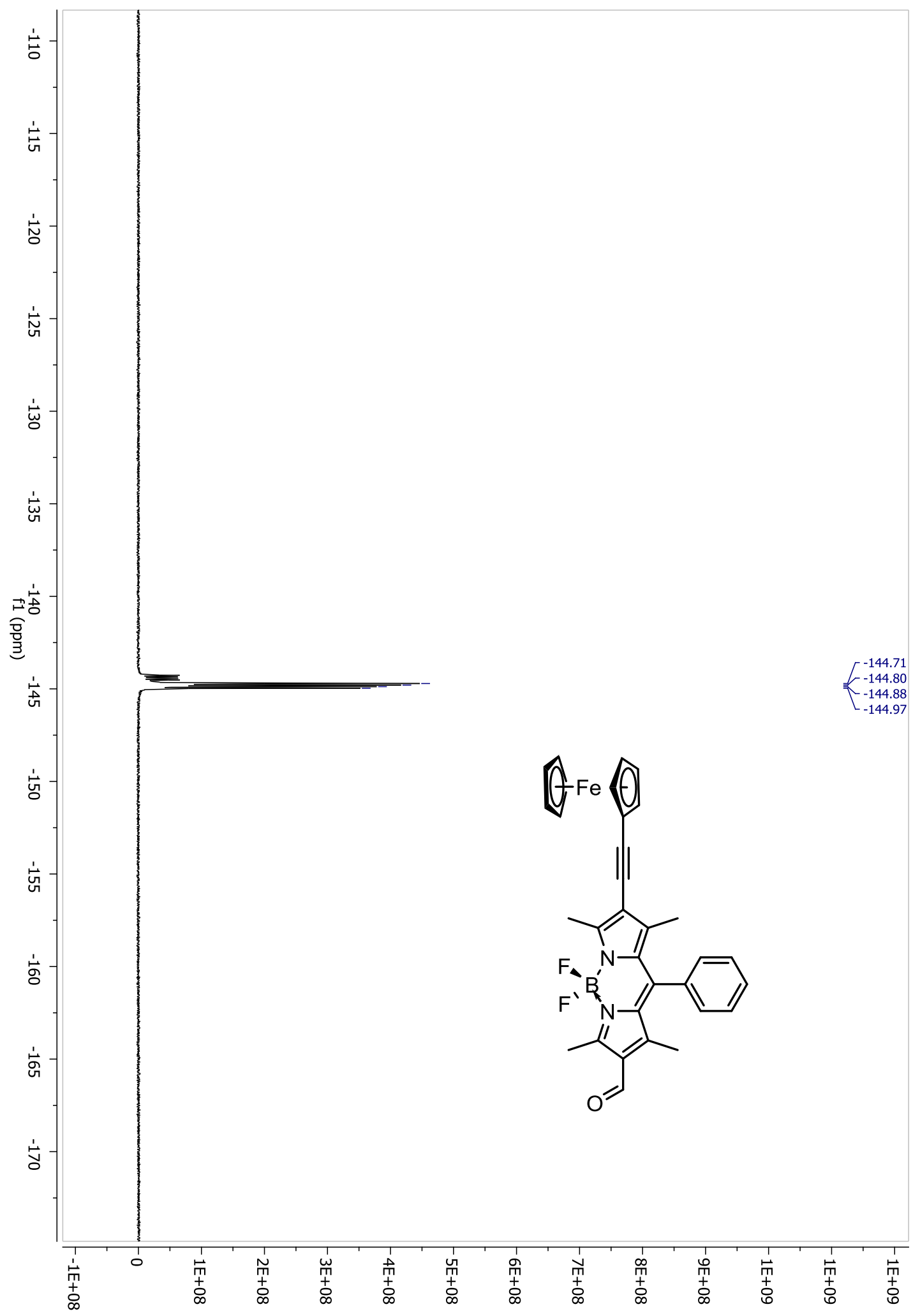

Figure A.103: ${ }^{19} \mathrm{~F}$ NMR of $3.44 a$ 


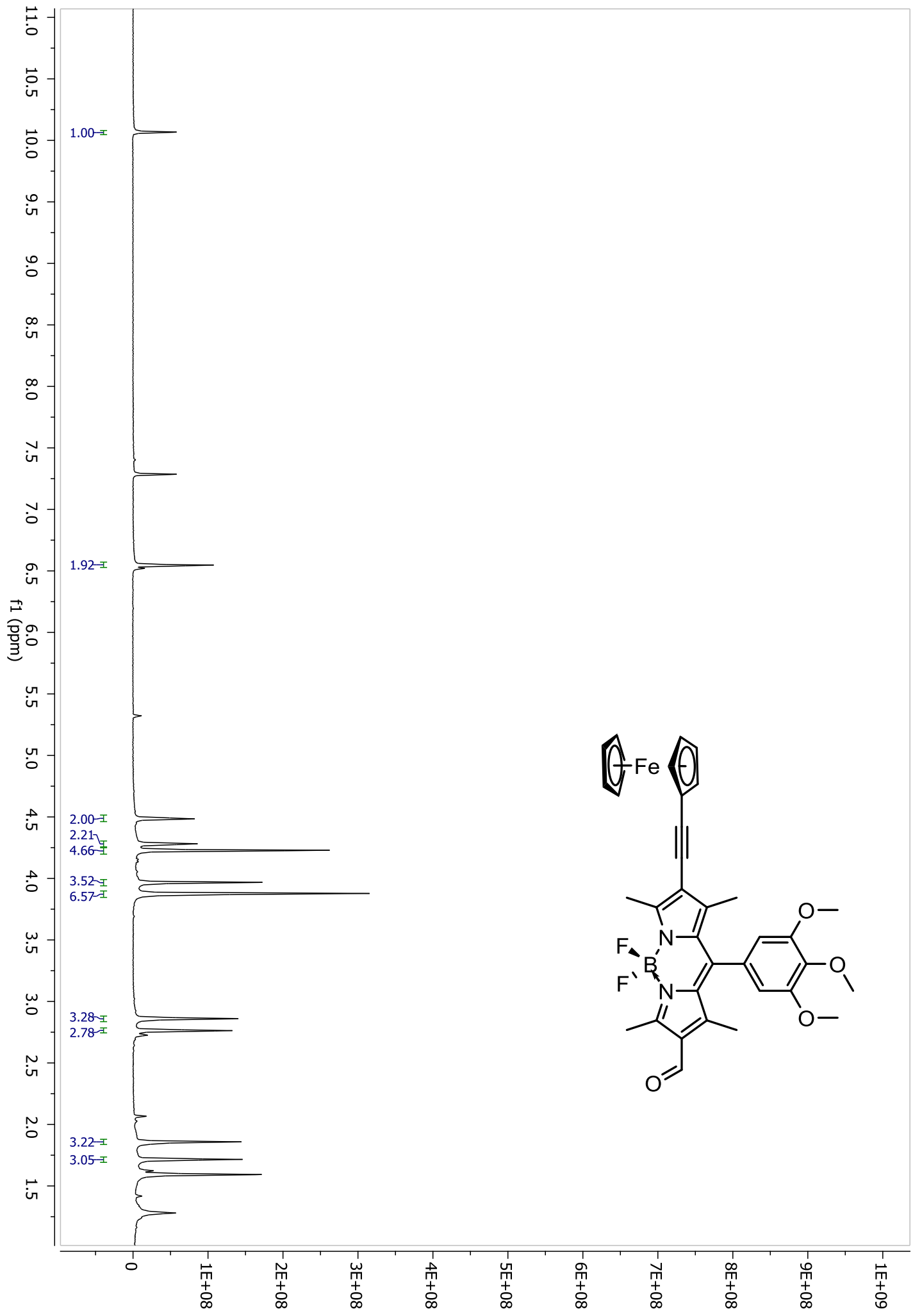

Figure A.104: ${ }^{1} \mathrm{H}$ NMR of $3.44 b$ 


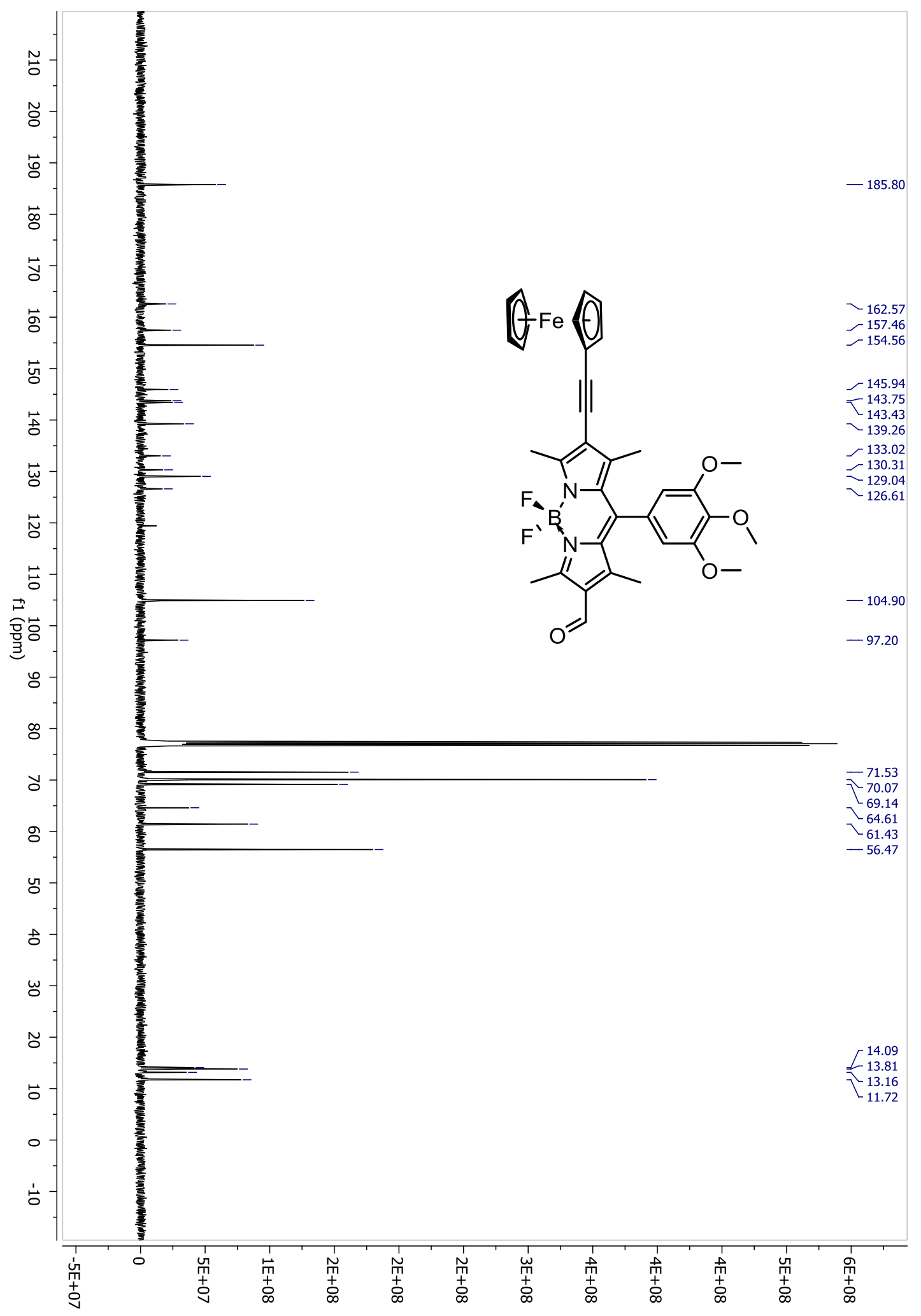

Figure A.105: ${ }^{13} \mathrm{C}$ NMR of 3.44b

235 


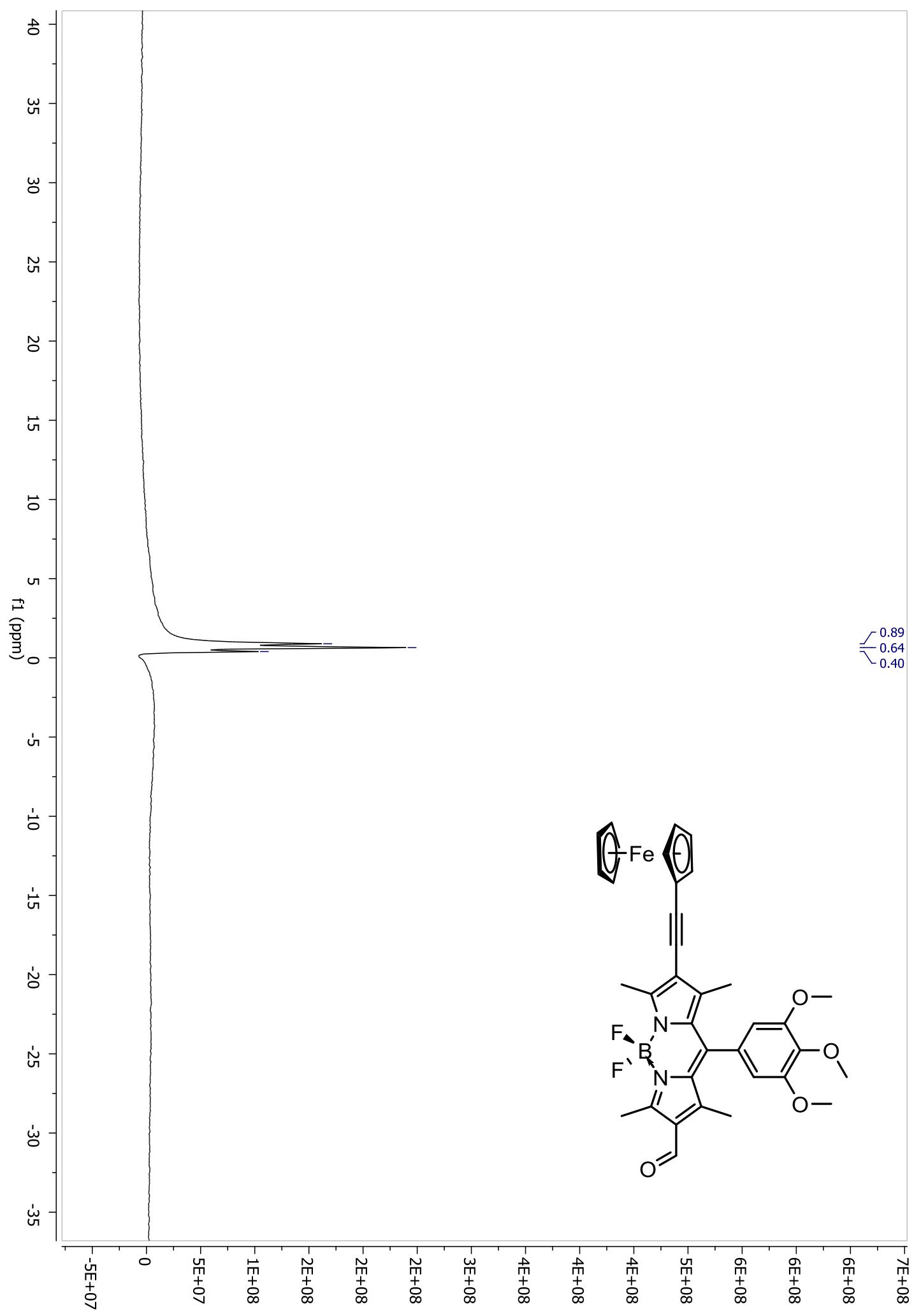

Figure A.106: ${ }^{11} B$ NMR of 3.44b 


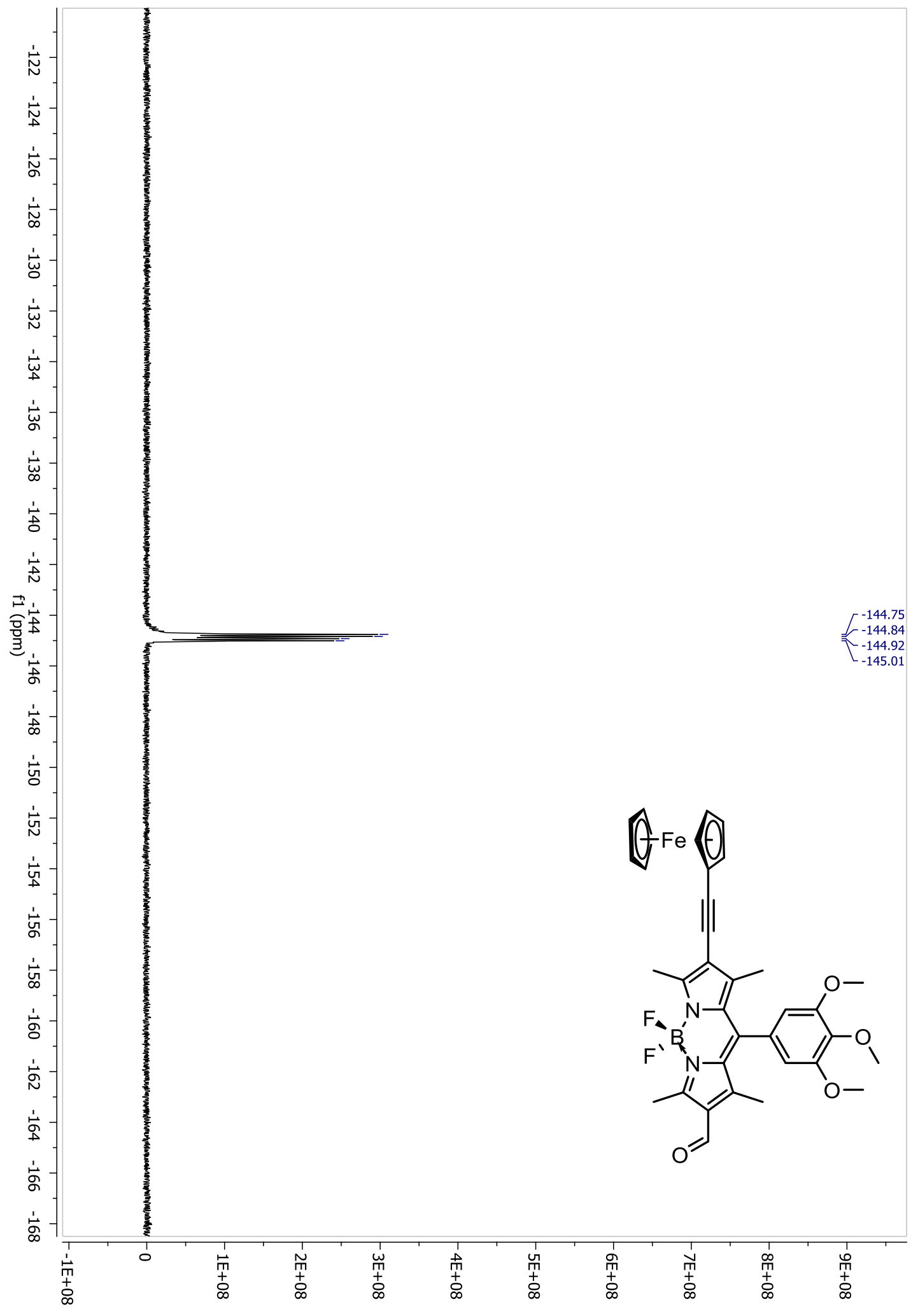

Figure A.107: ${ }^{19} \mathrm{~F}$ NMR of $3.44 b$ 


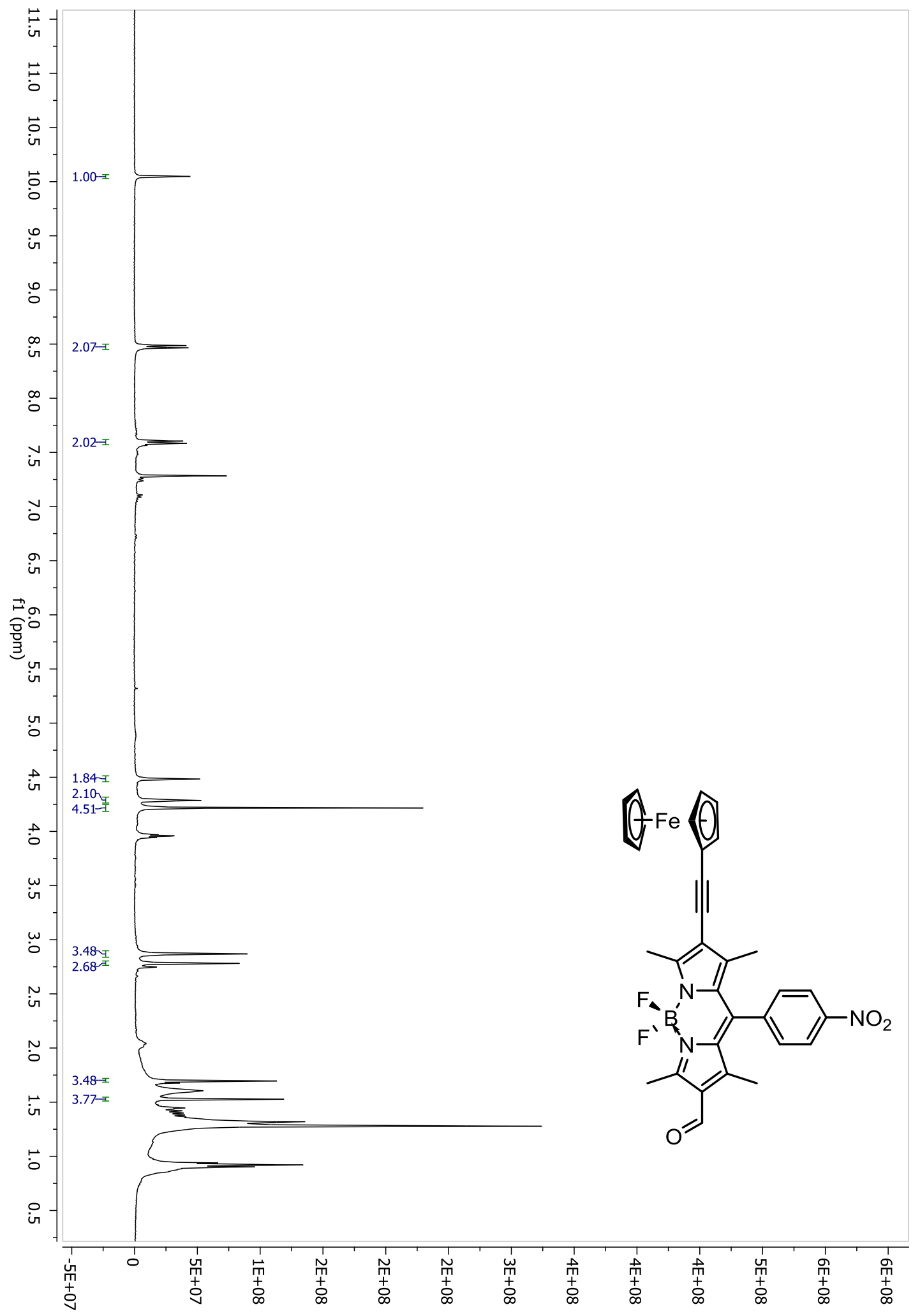

Figure A.108: ${ }^{11} \mathrm{H}$ NMR of $3.44 \mathrm{c}$ 


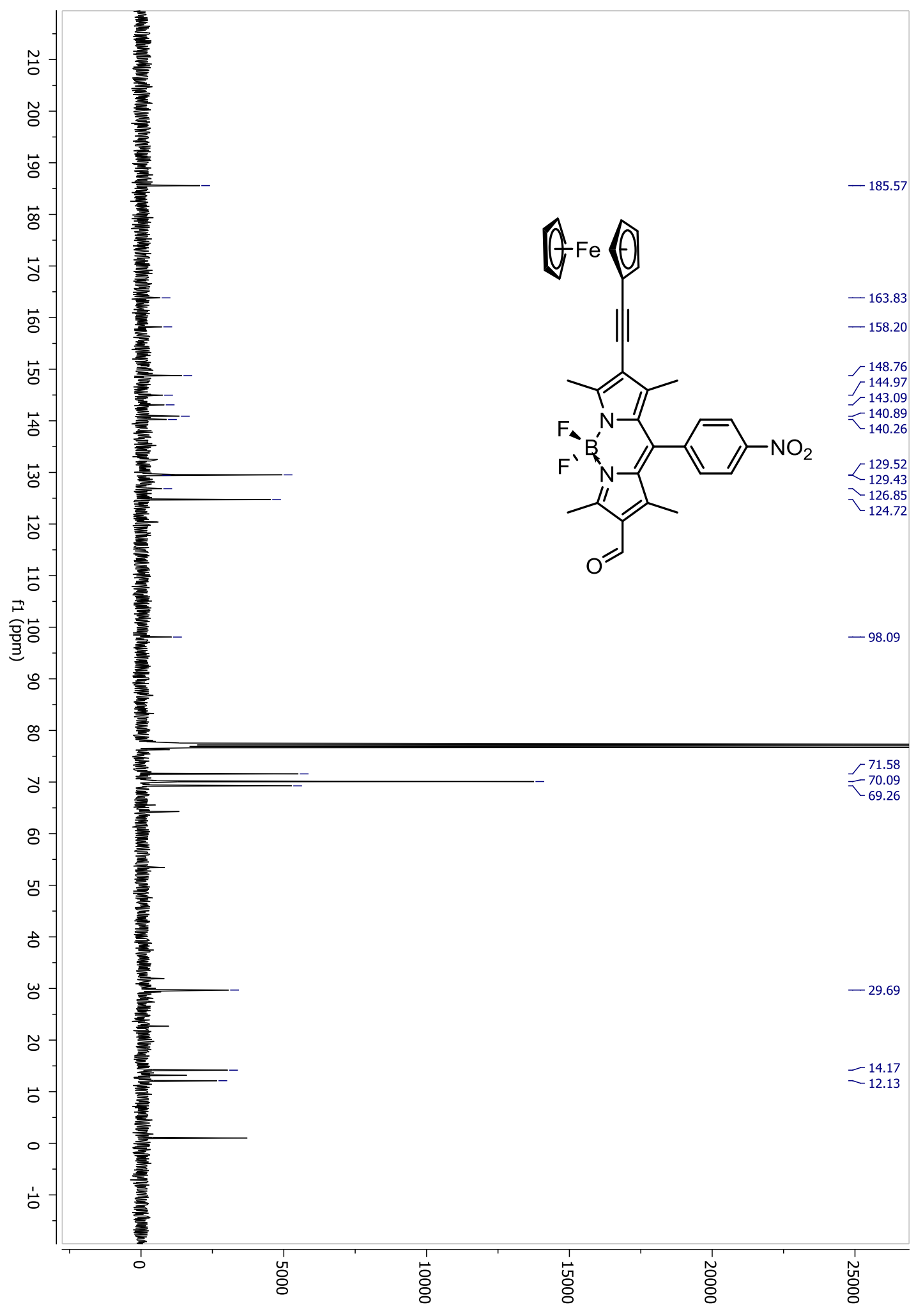

Figure A.109: ${ }^{13} \mathrm{C}$ NMR of $3.44 \mathrm{c}$ 


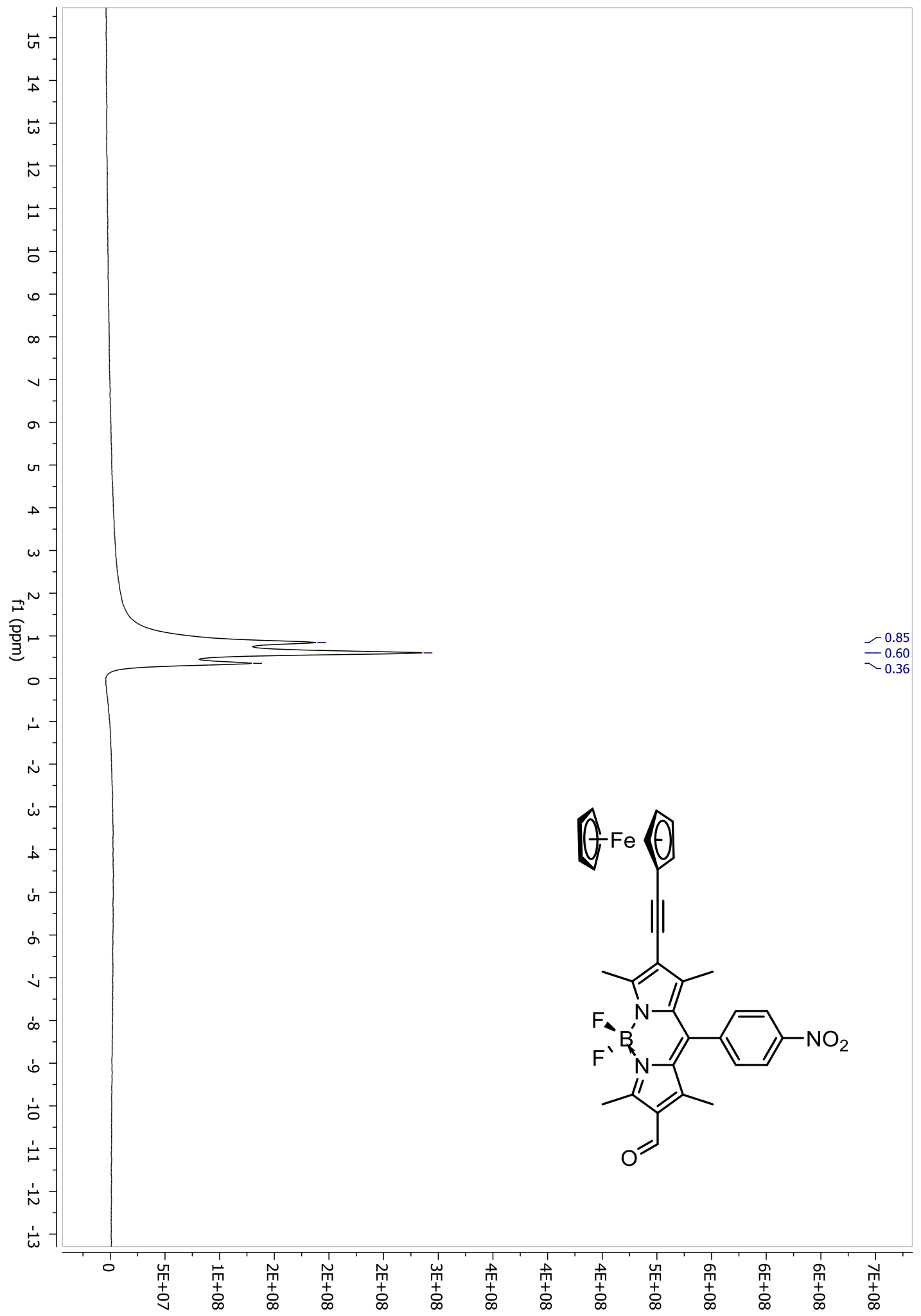

Figure A.110: ${ }^{13} \mathrm{~B}$ NMR of $3.44 \mathrm{c}$ 


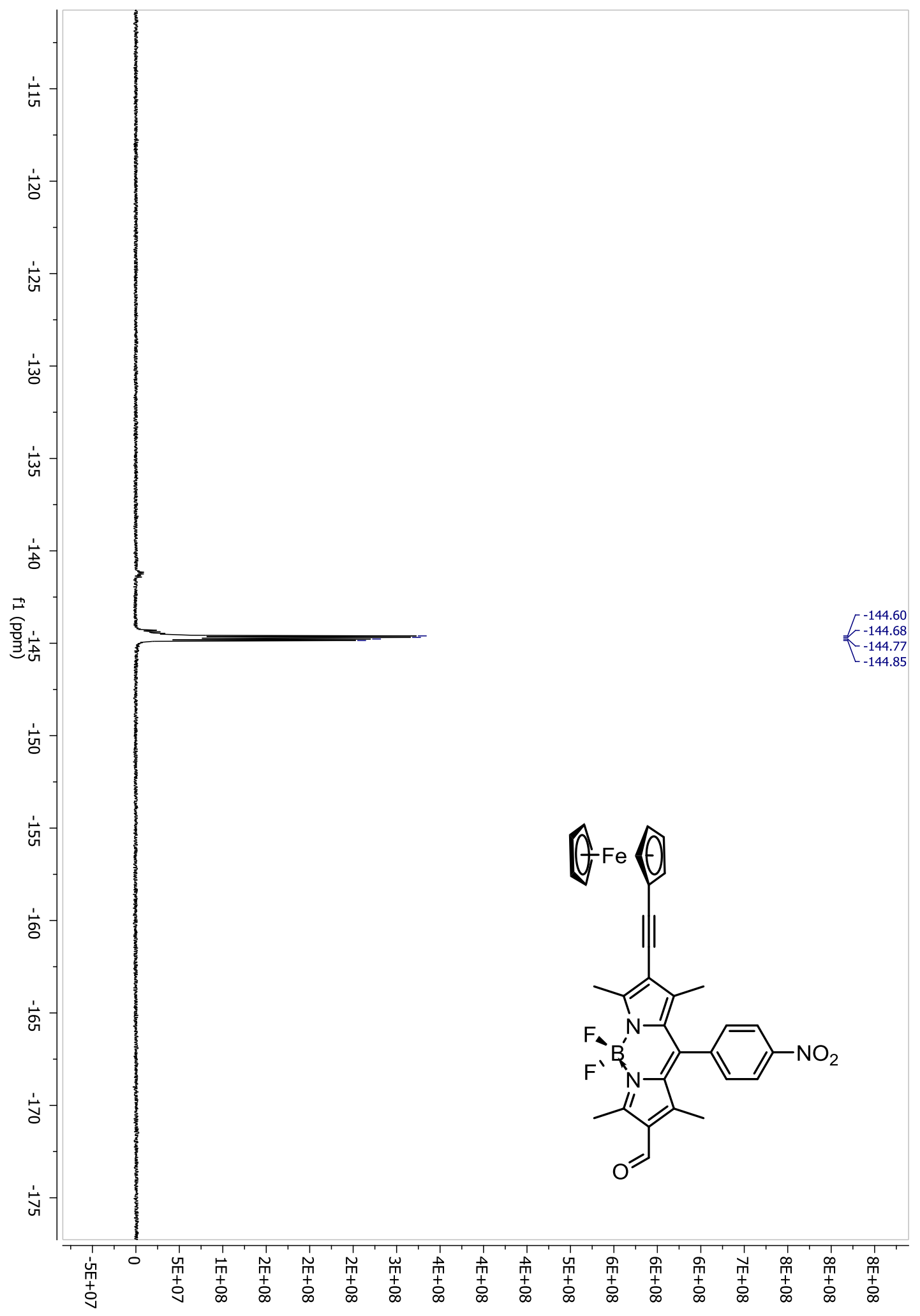

Figure A..111: ${ }^{19} \mathrm{~F}$ NMR of $3.44 \mathrm{c}$ 


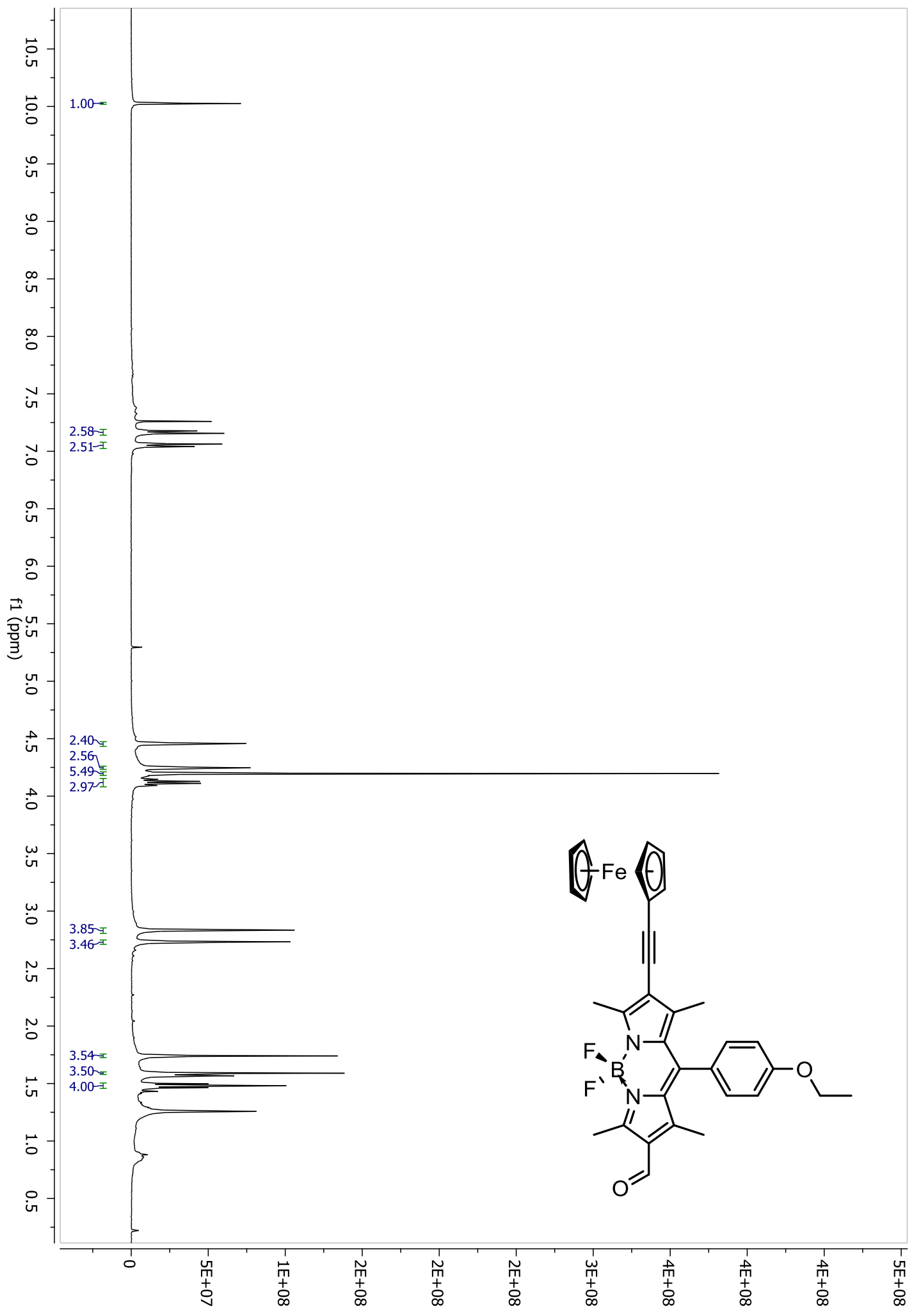

Figure A.112: ${ }^{1} \mathrm{H}$ NMR of $\mathbf{3 . 4 4 d}$ 


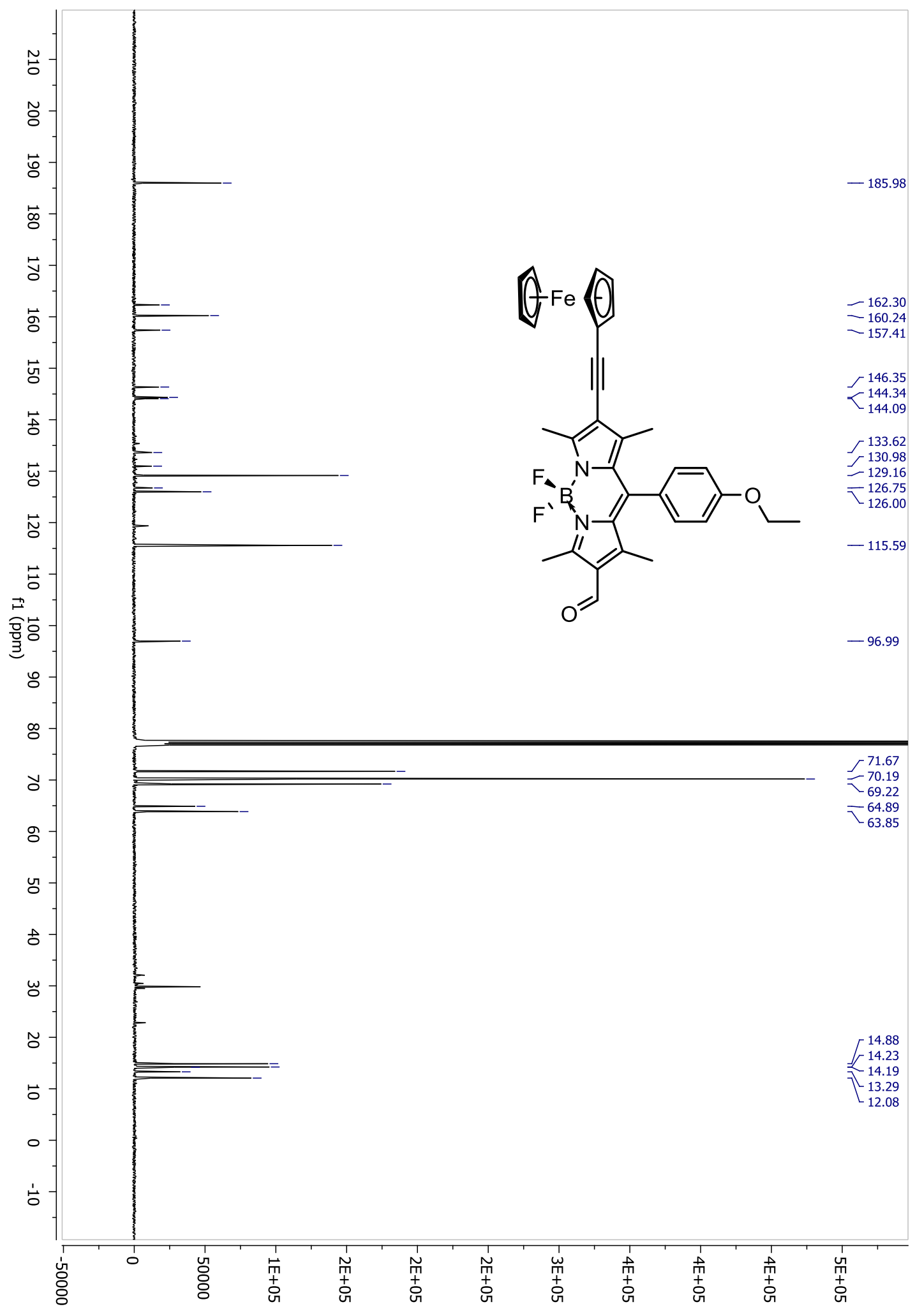

Figure A.113: ${ }^{13} \mathrm{C}$ NMR of $\mathbf{3 . 4 4 d}$ 


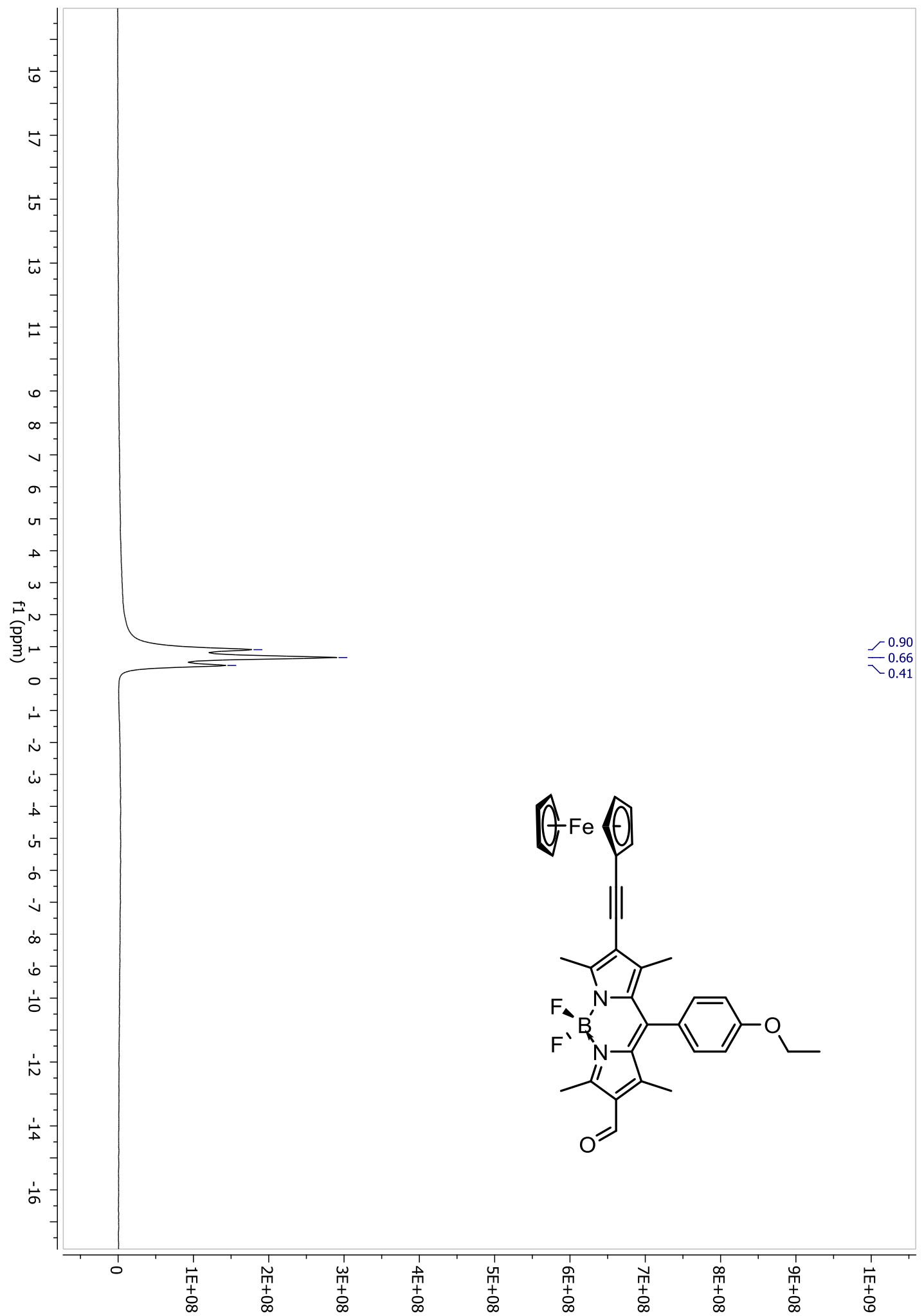

Figure A.114: ${ }^{11} B$ NMR of $3.44 d$ 


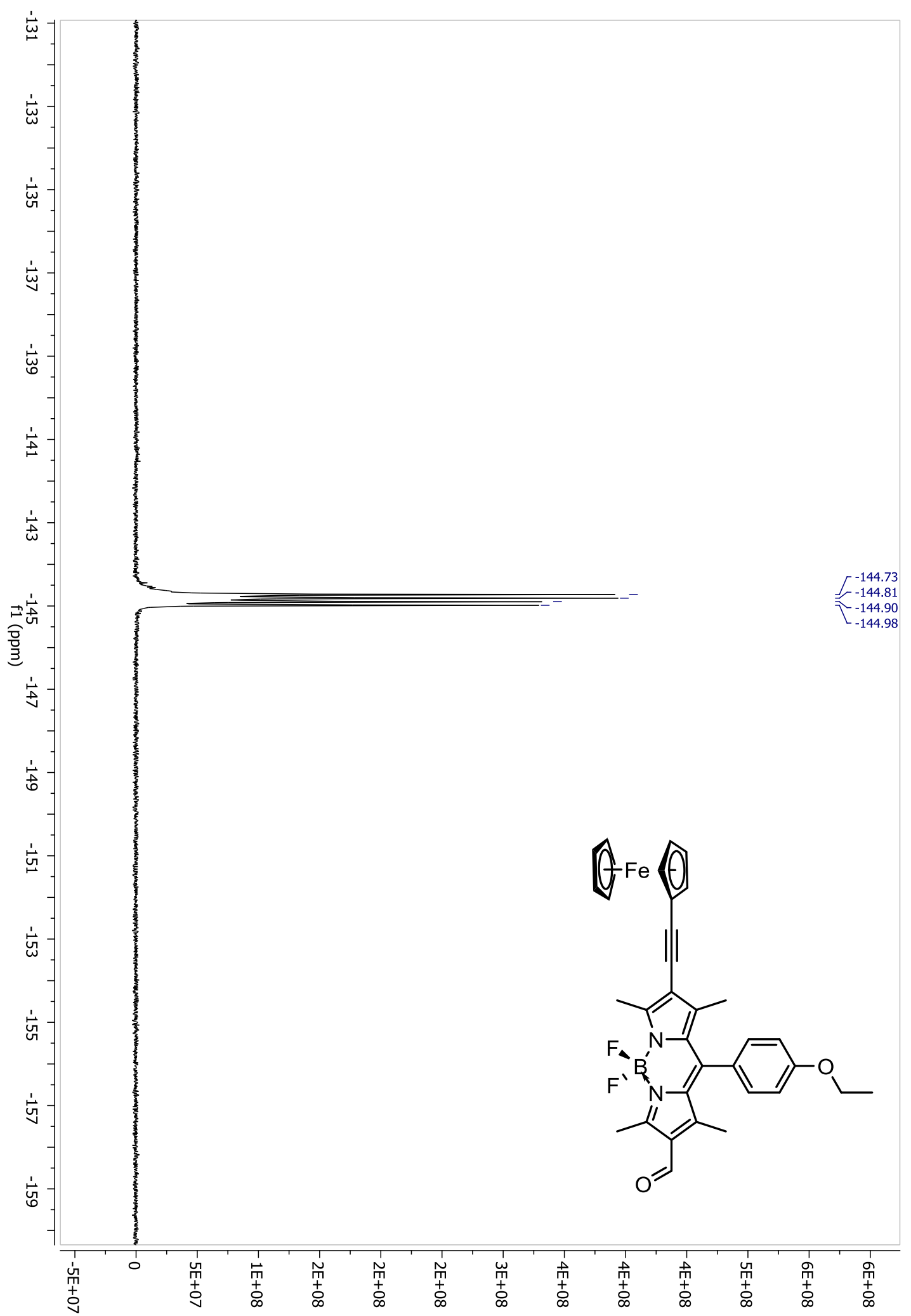

Figure A.115: ${ }^{19} \mathrm{~F}$ NMR of $\mathbf{3 . 4 4 d}$ 


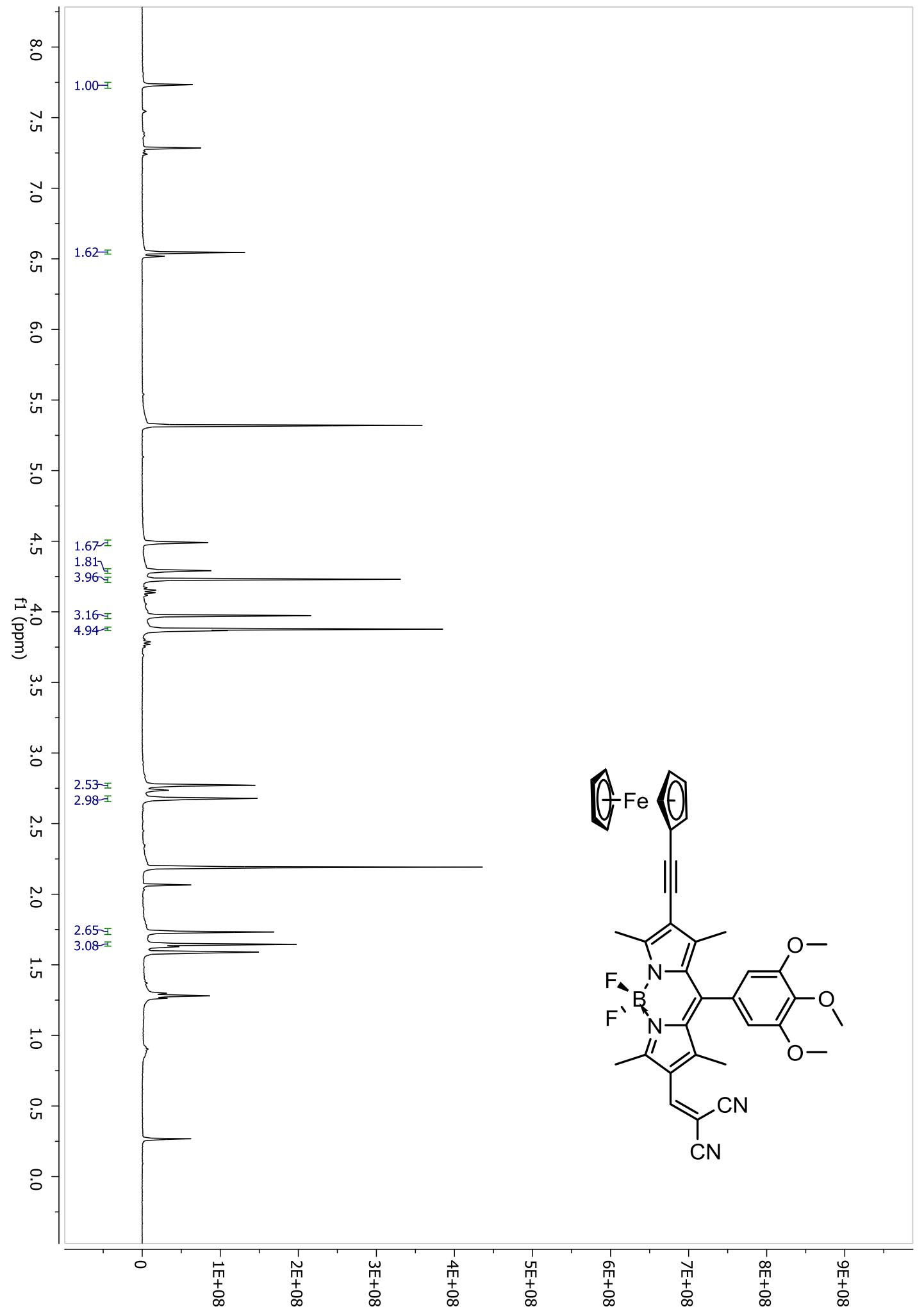

Figure A.116: ${ }^{1} \mathrm{H}$ NMR of $3.35 b$ 


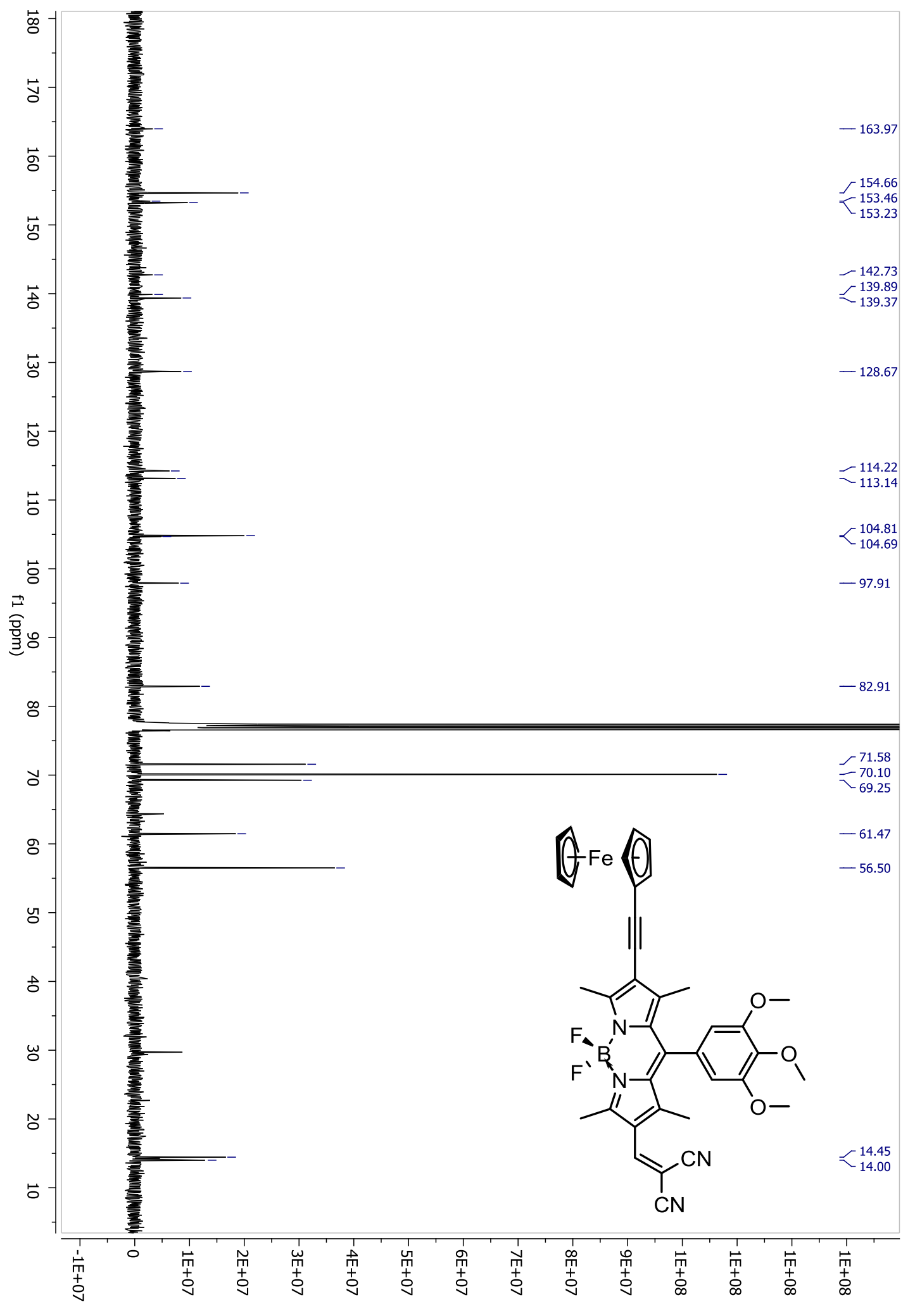

Figure A.117: ${ }^{13} \mathrm{C}$ NMR of $\mathbf{3 . 3 5}$ 


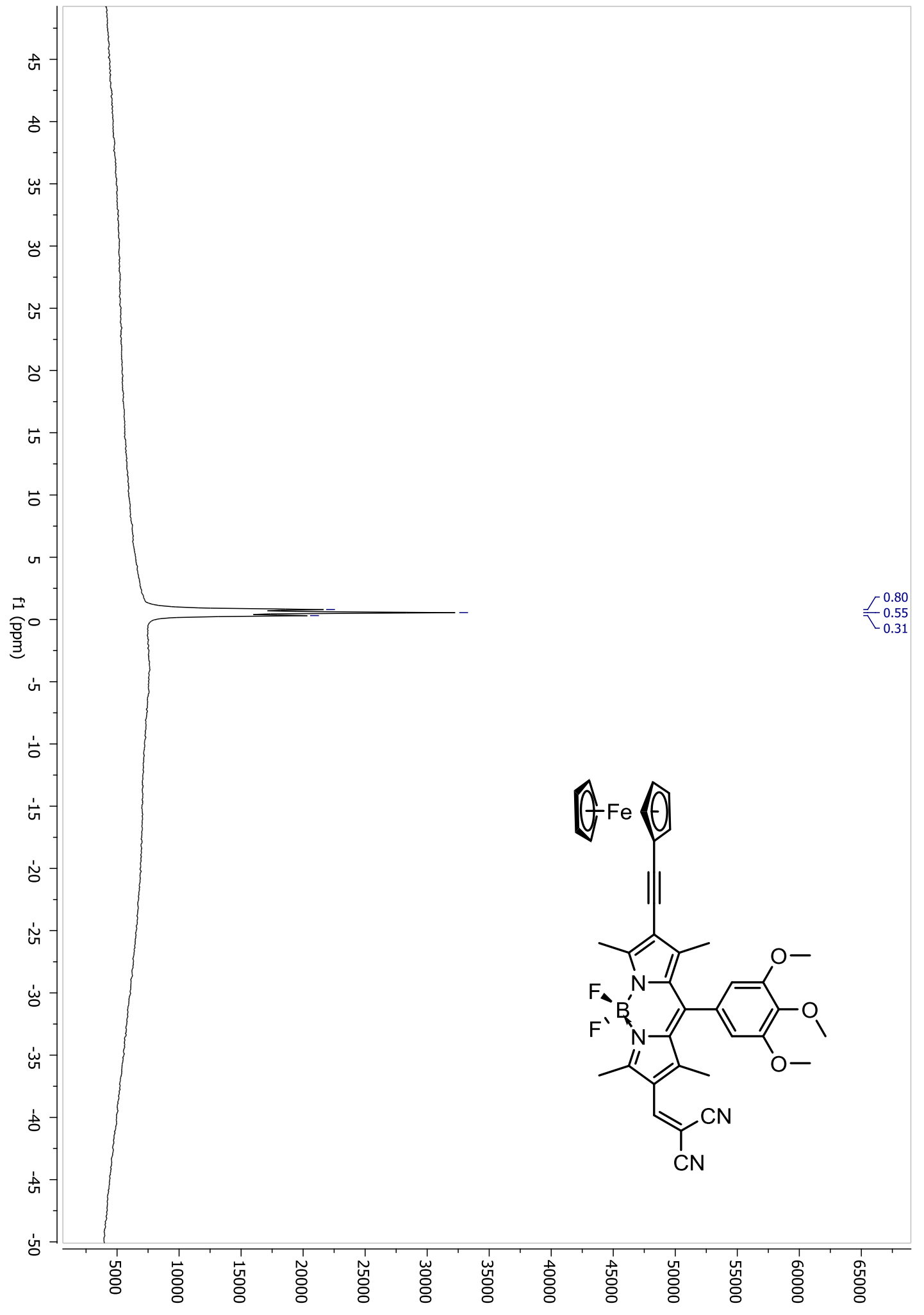

Figure A.118: ${ }^{11} B$ NMR of $3.35 b$ 


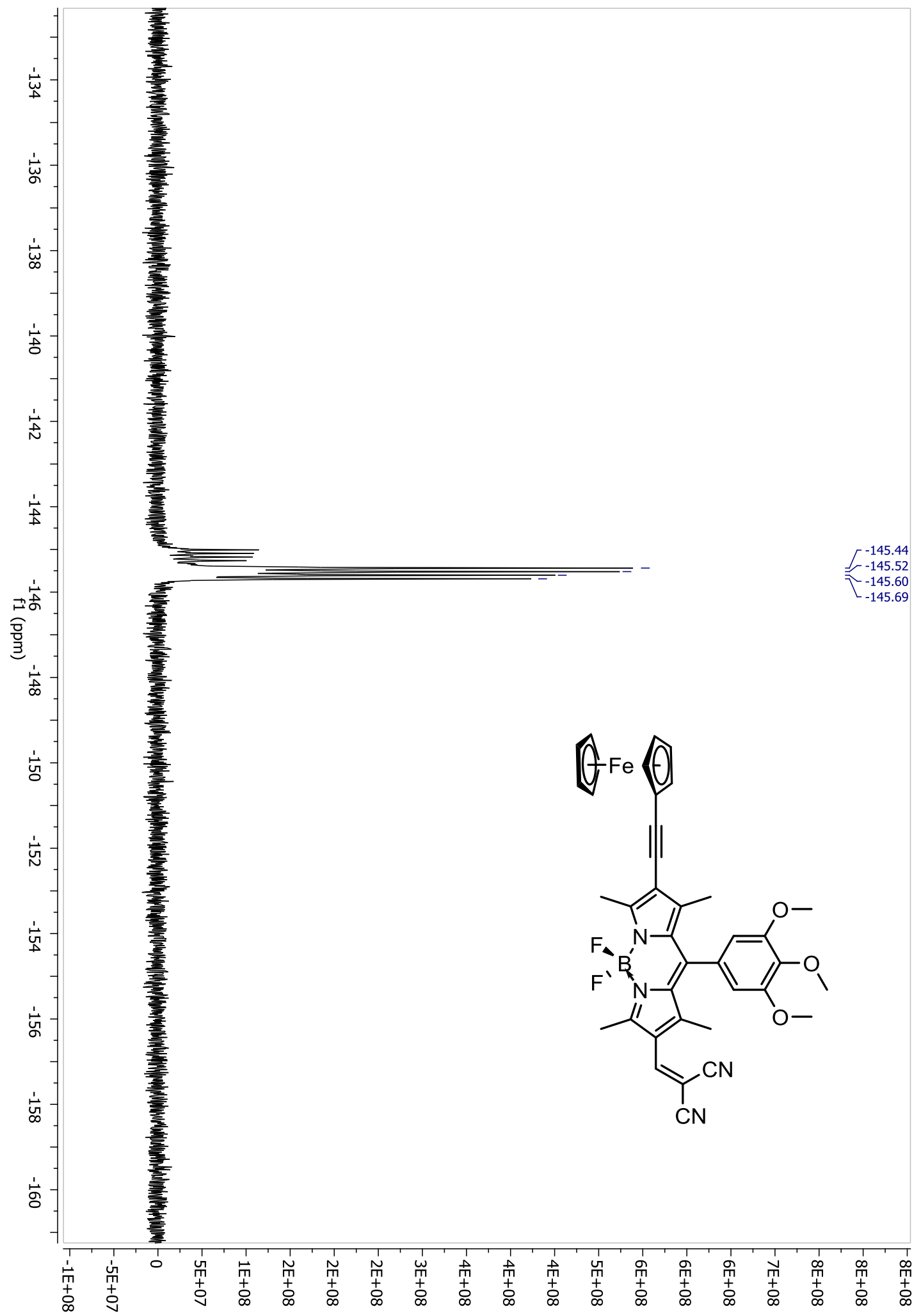

Figure A.119: ${ }^{19} \mathrm{~F}$ NMR of $3.35 b$ 


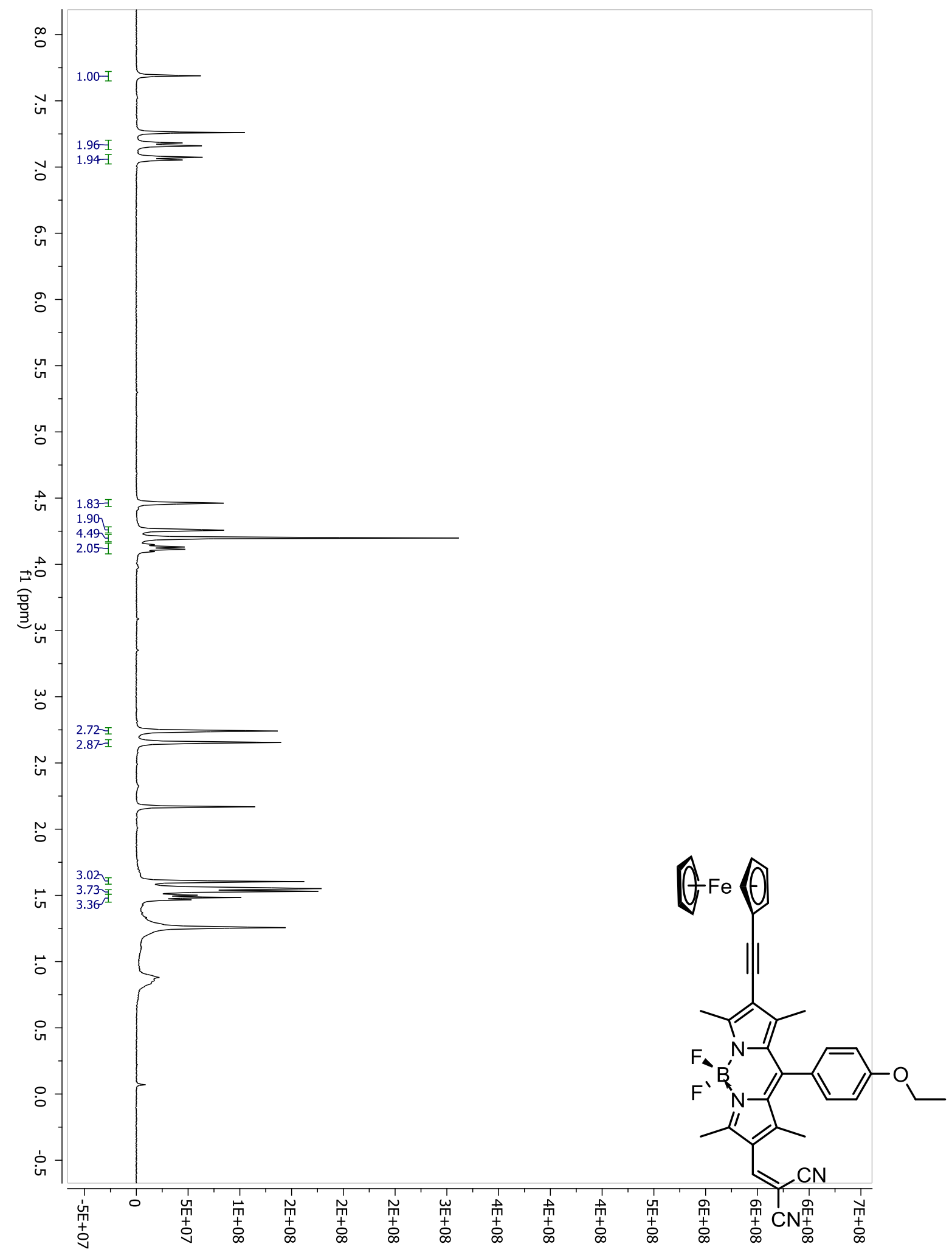

Figure A.120: ${ }^{1} \mathrm{H}$ NMR of $3.35 \mathrm{~d}$ 


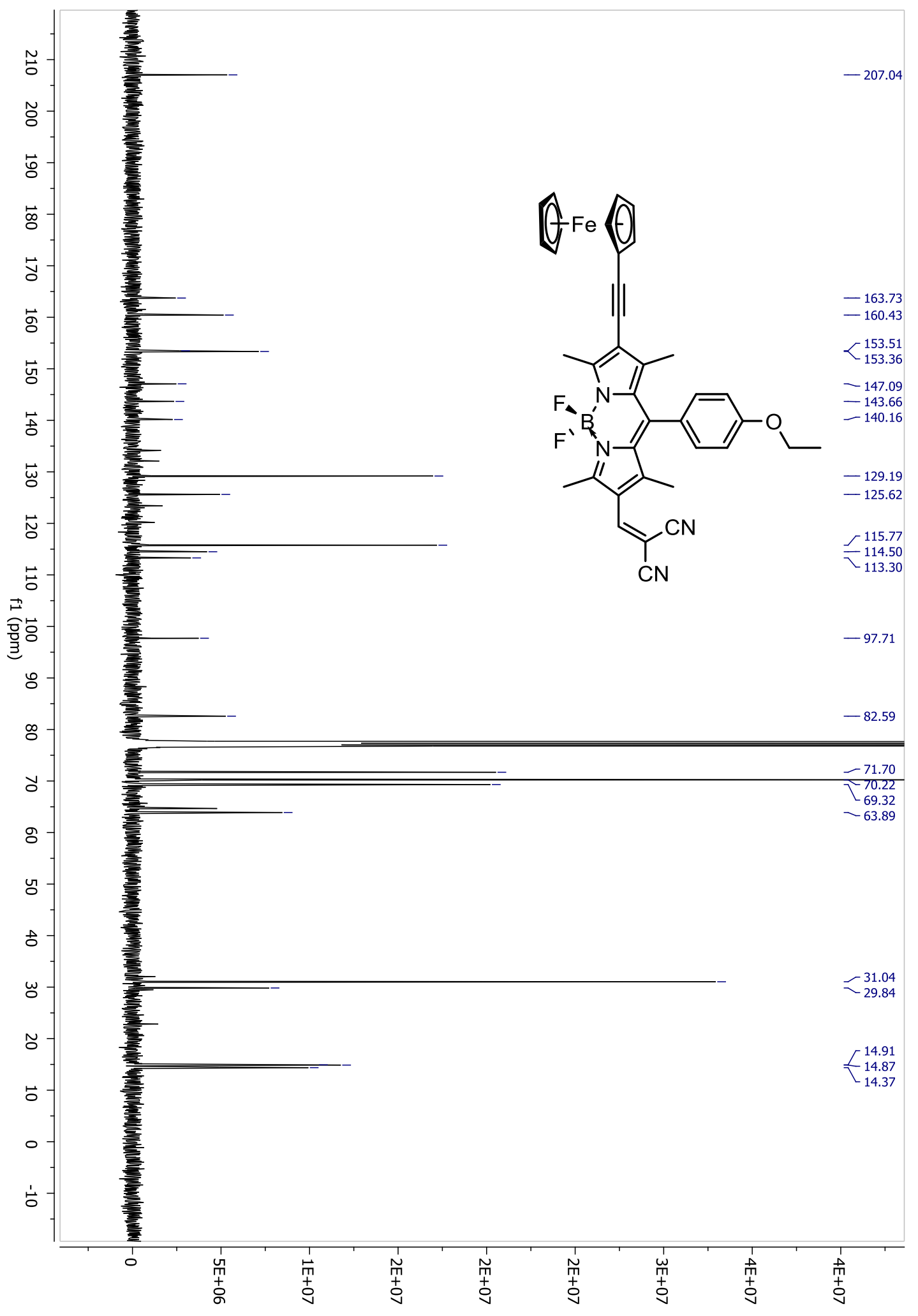

Figure A.121: ${ }^{13} \mathrm{C}$ NMR of $3.35 d$ 


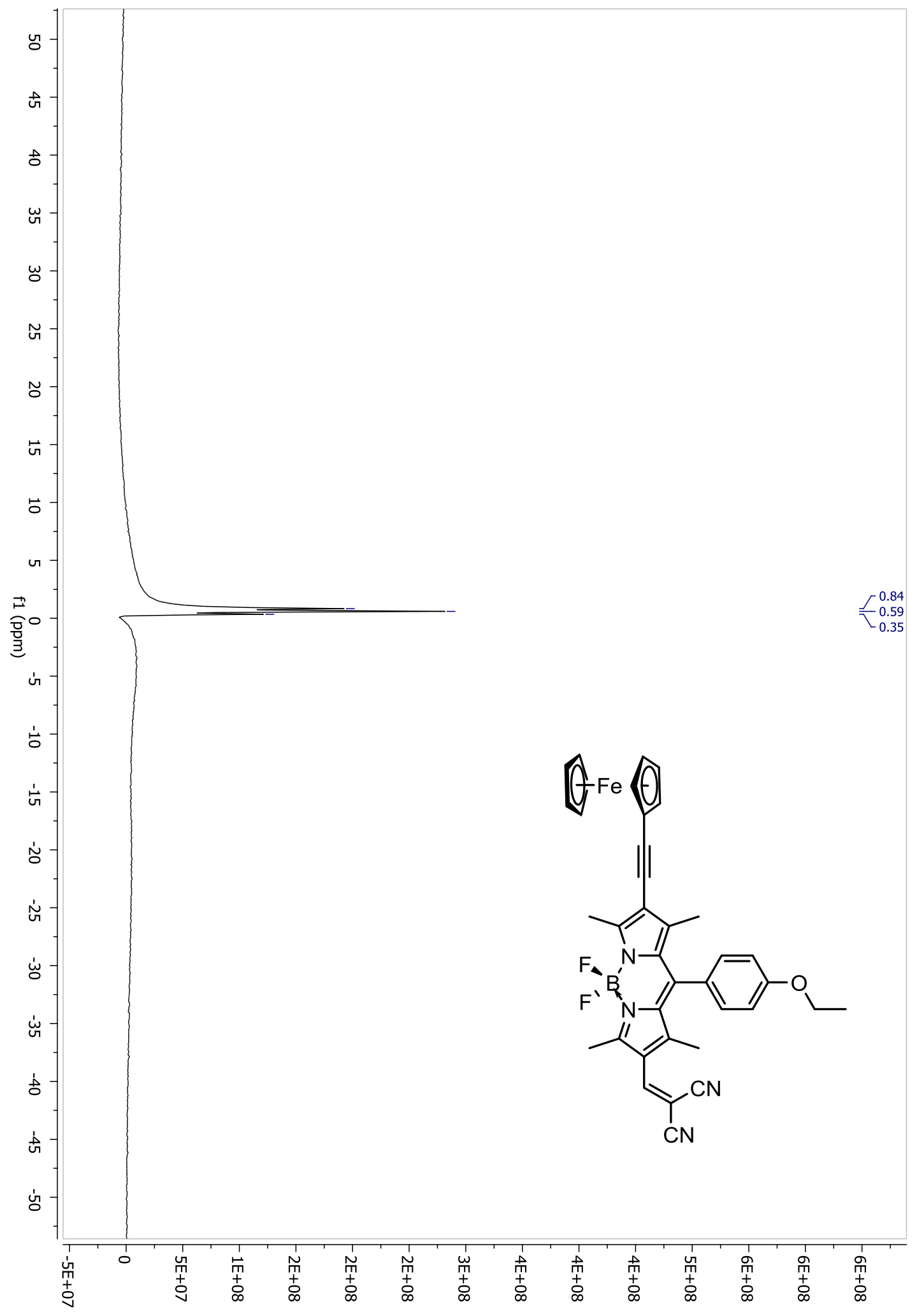

Figure A.122: ${ }^{11} B$ NMR of $3.35 d$ 


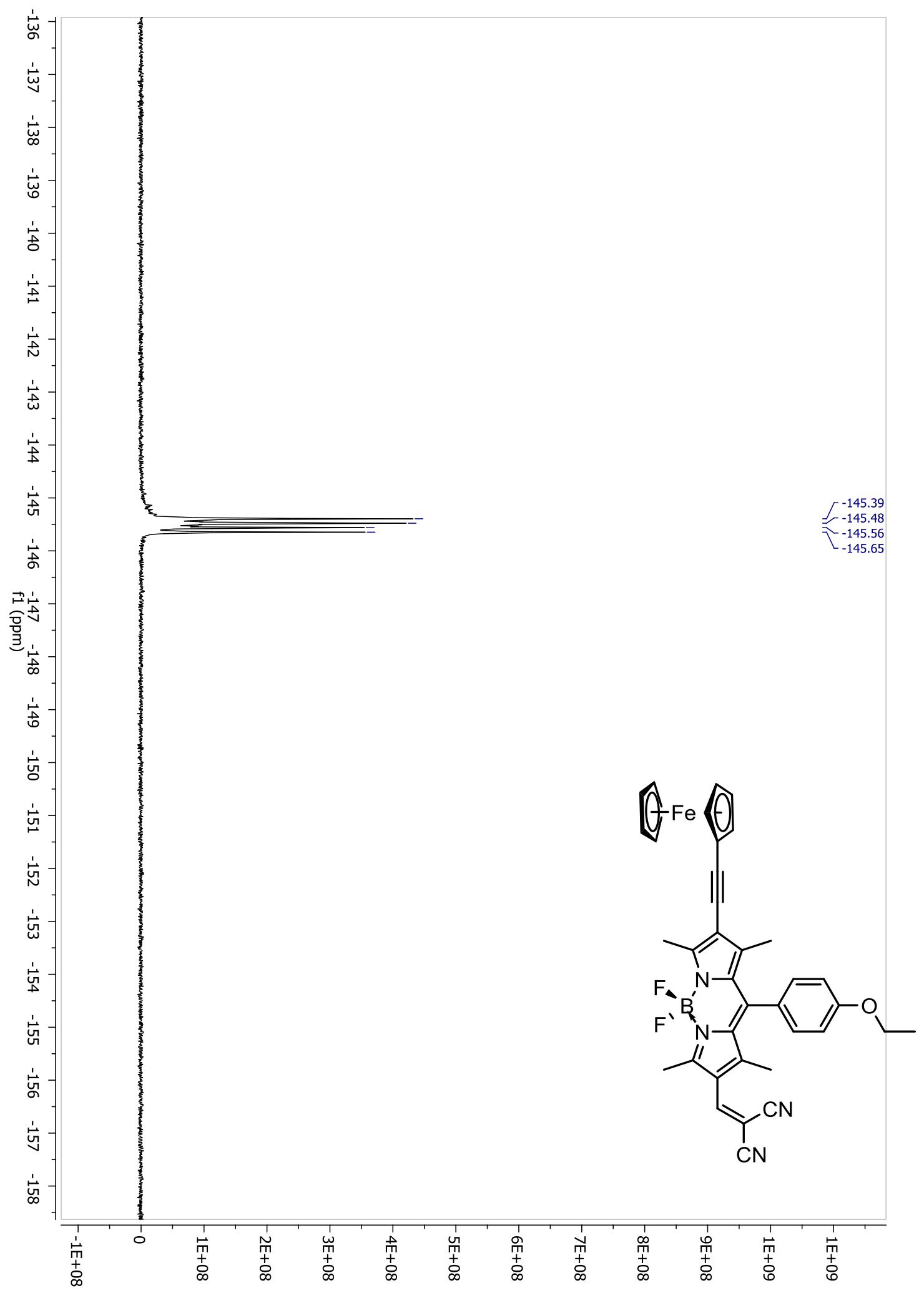

Figure A.123: ${ }^{19} \mathrm{~F}$ NMR of $3.35 \mathrm{~d}$ 


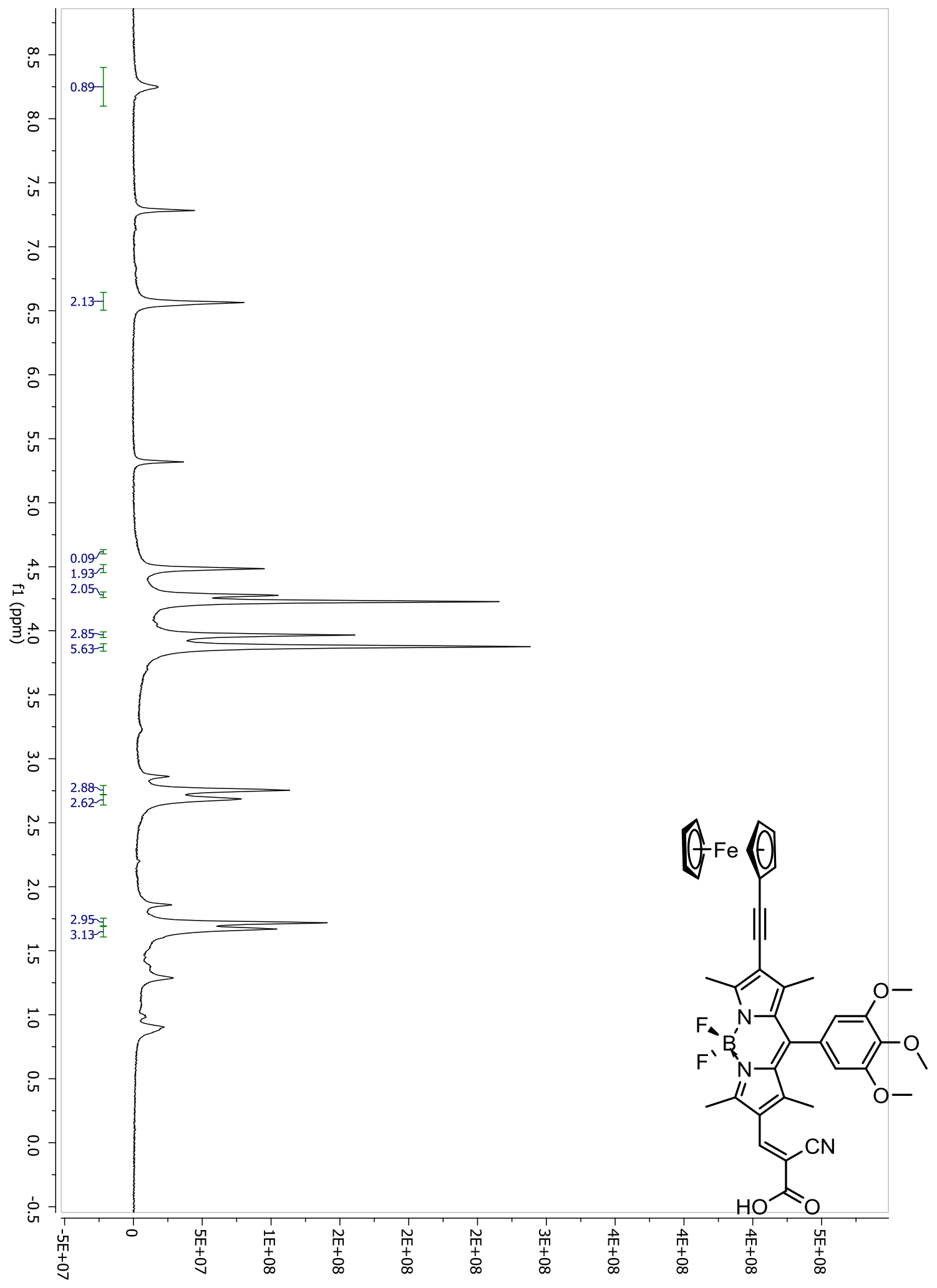

Figure A.124: ${ }^{1} \mathrm{H}$ NMR of $3.36 \mathrm{~b}$ 


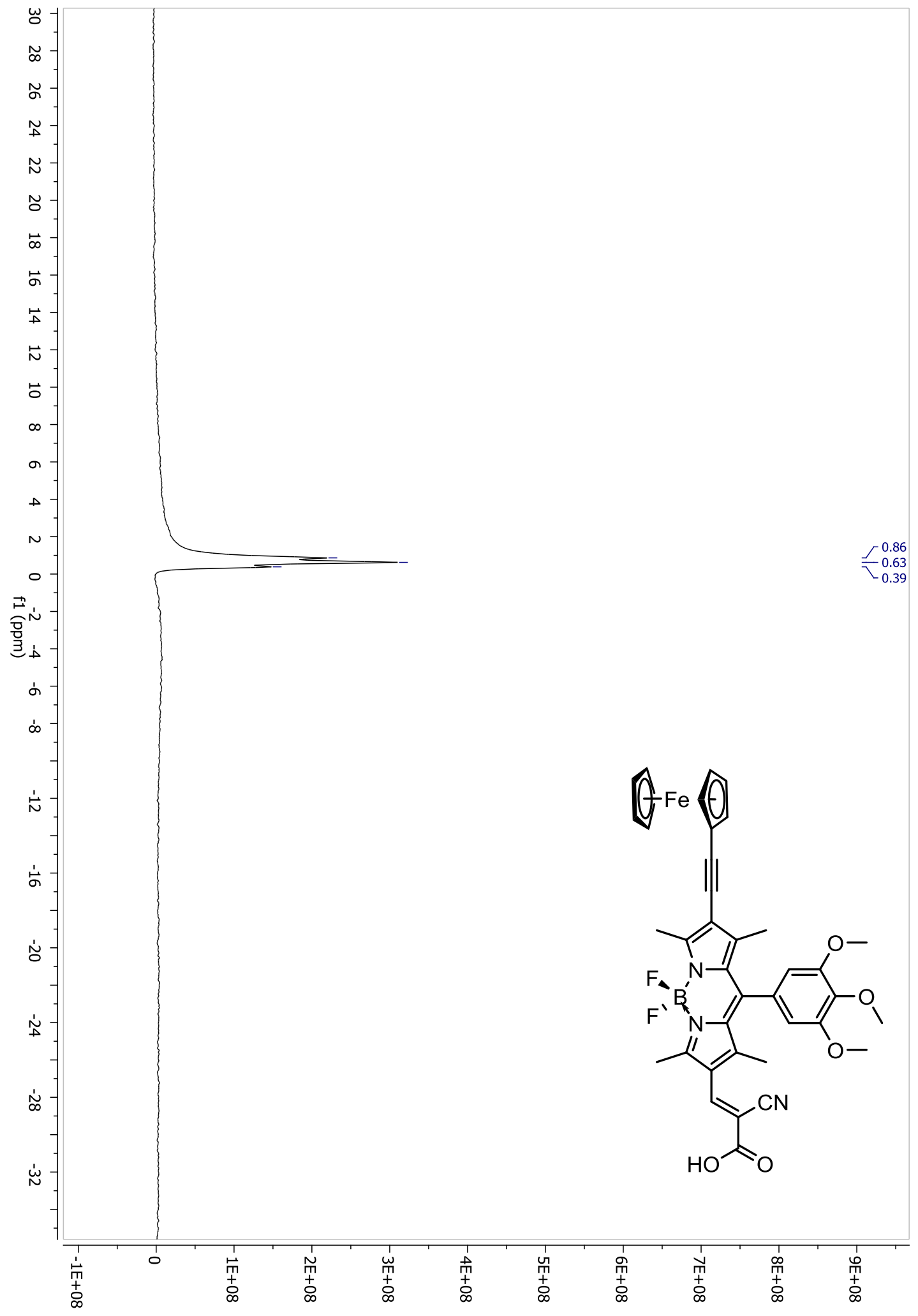

Figure A.125: ${ }^{11} B$ NMR of 3.36b 


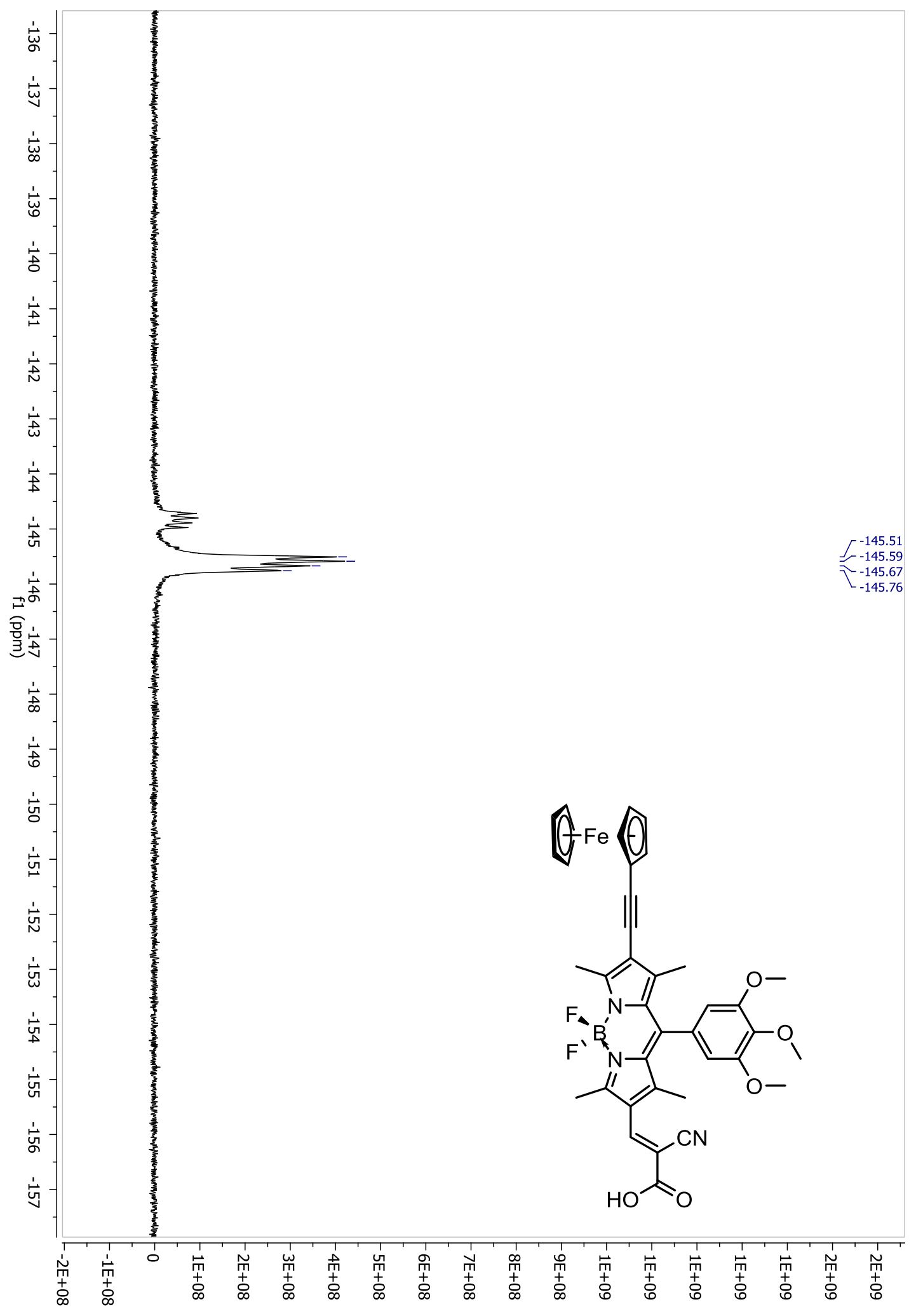

Figure A.126: ${ }^{19} \mathrm{~F}$ NMR of $3.36 \mathrm{~b}$ 


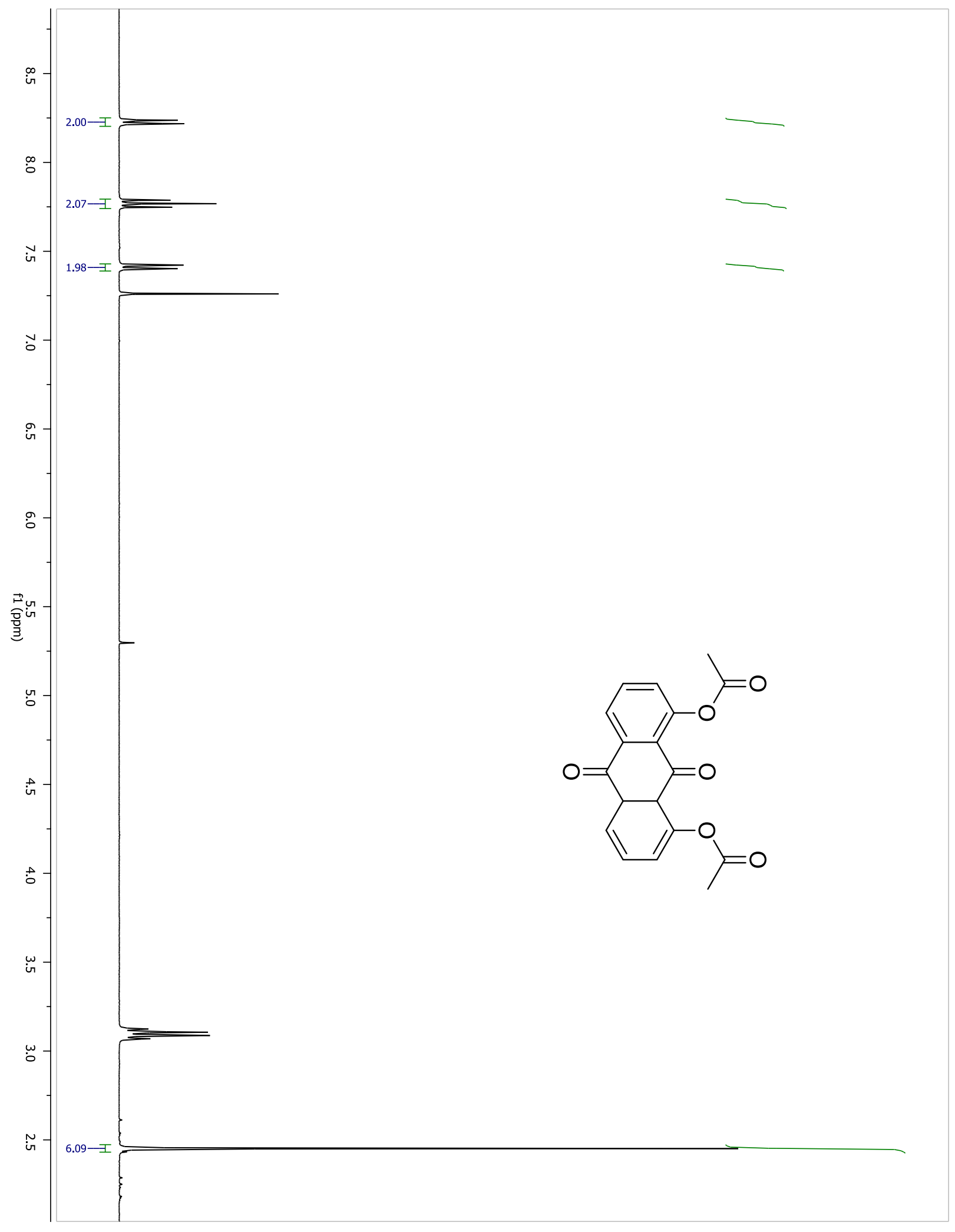

Figure A.127: ${ }^{1} \mathrm{H}$ NMR of 4.7 


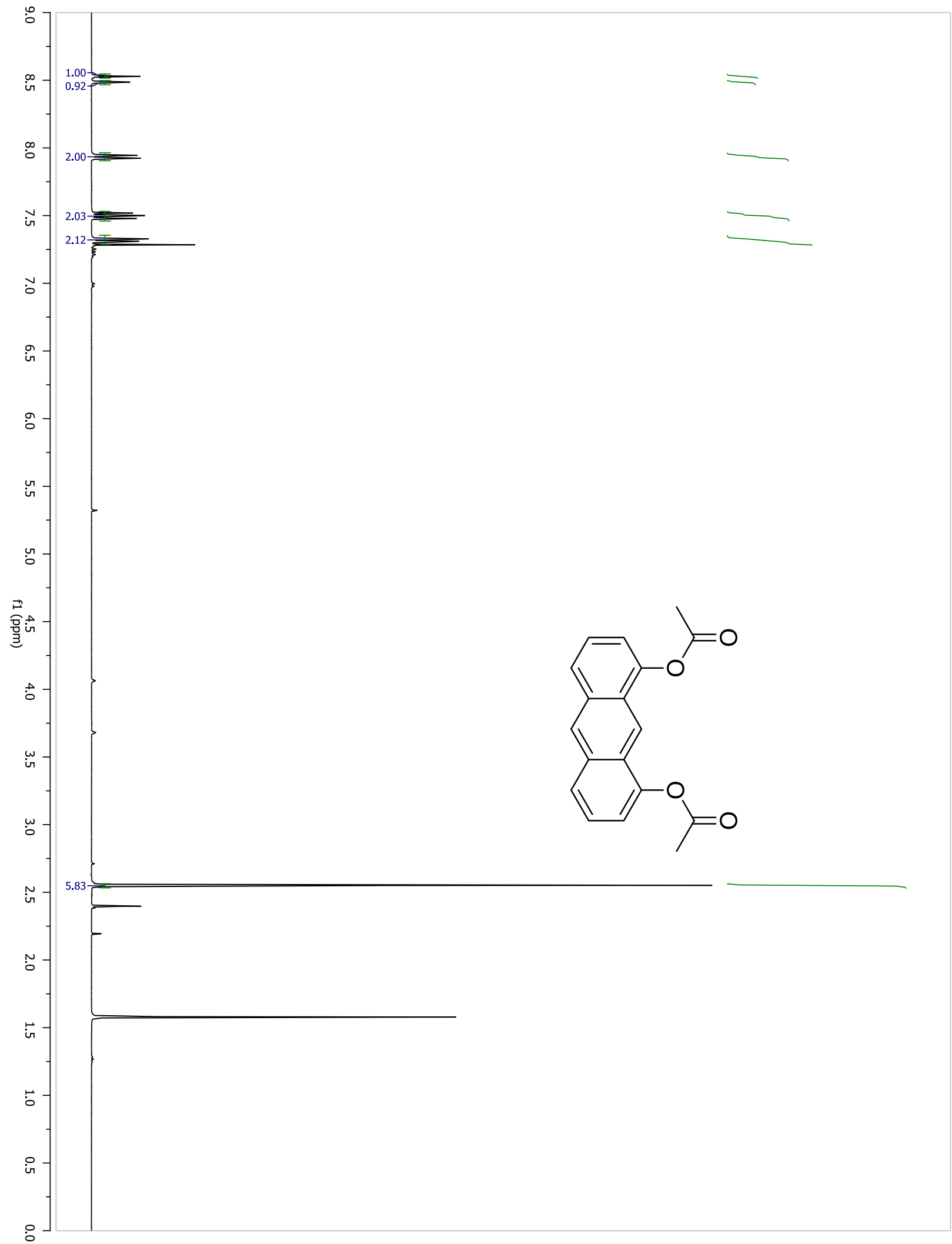

Figure A.128: ${ }^{1}$ H NMR of 4.8 


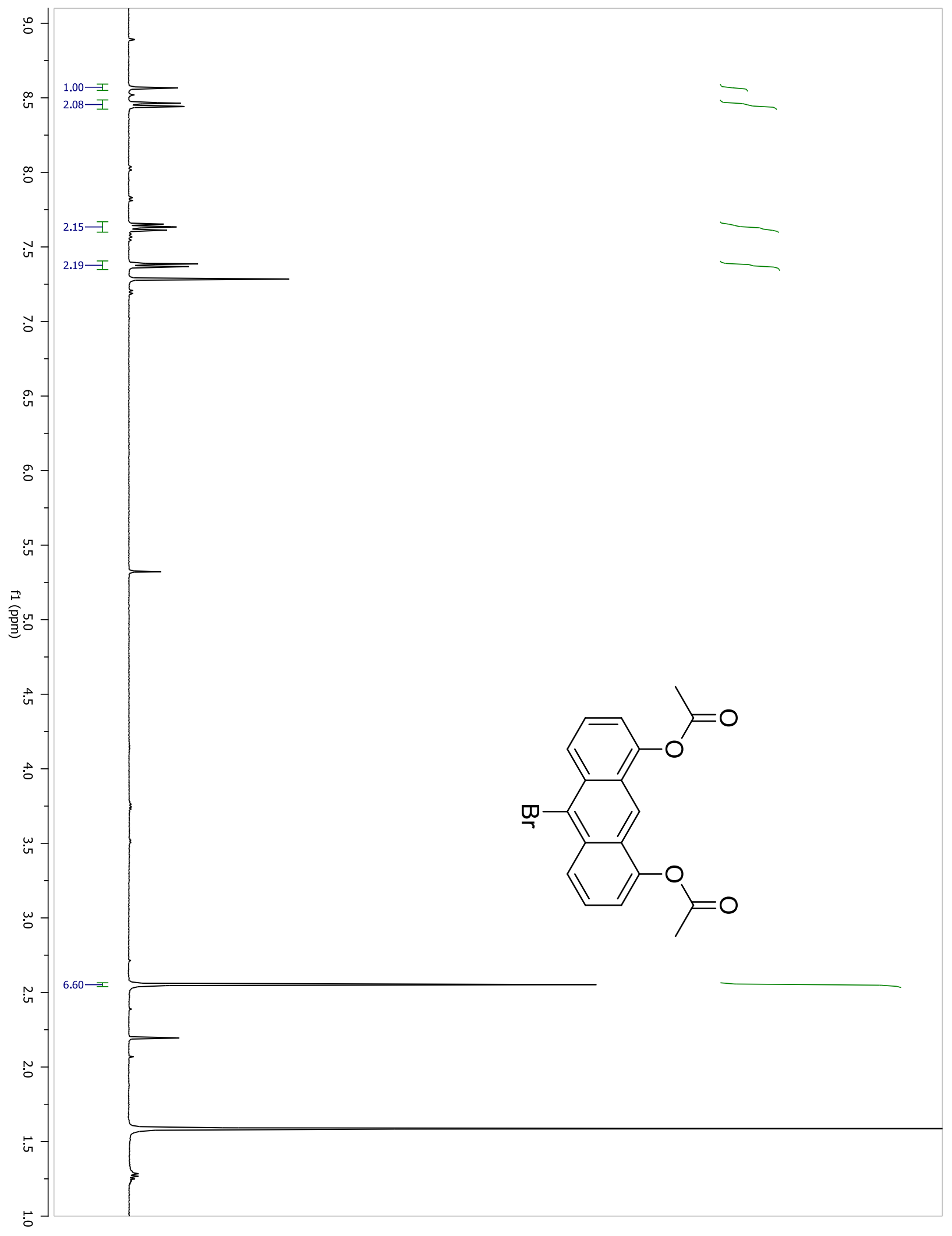

Figure A.129: ${ }^{1} \mathrm{H}$ NMR of 4.9 


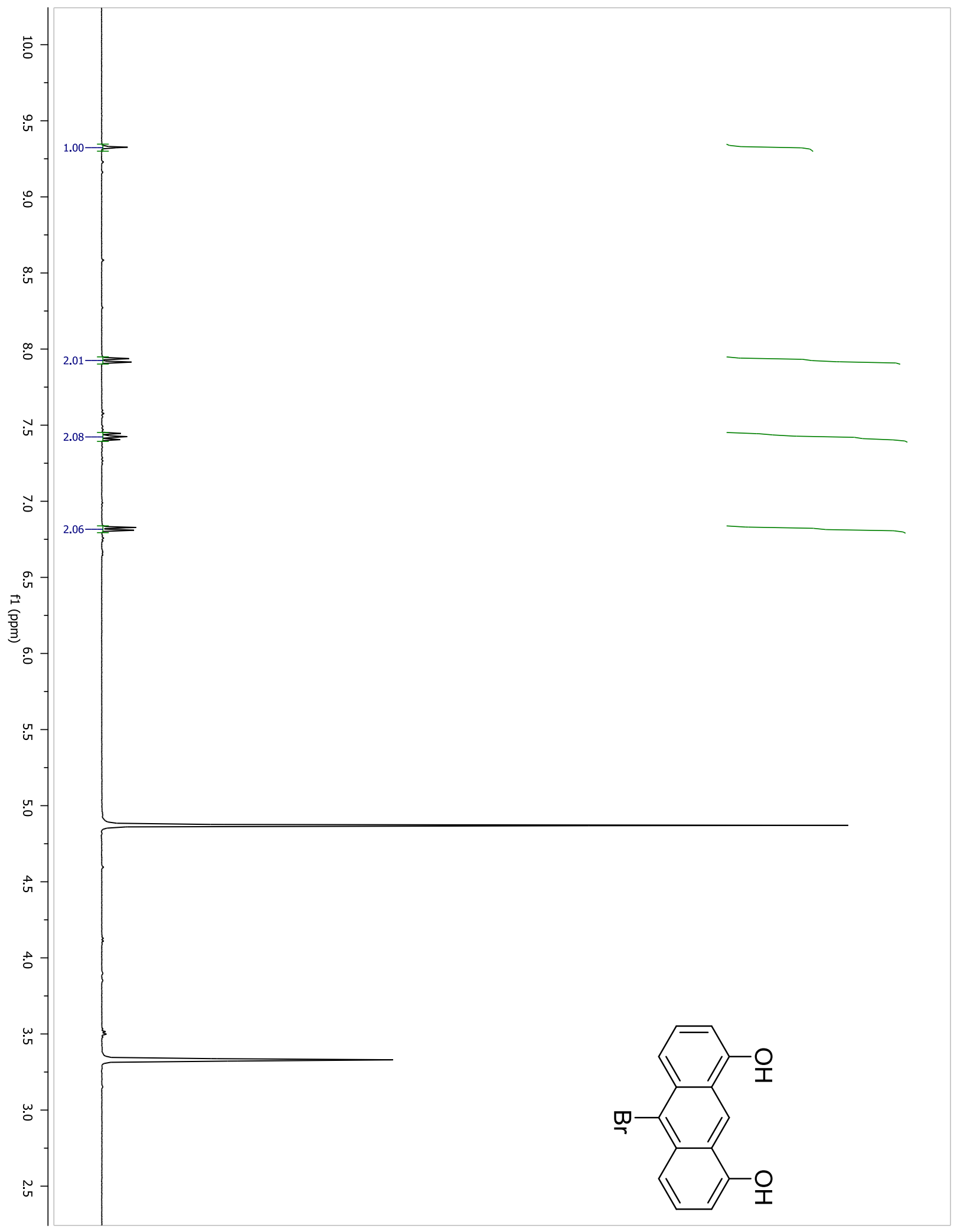

Figure A. 130: ${ }^{1} \mathrm{H}$ NMR of 4.10 


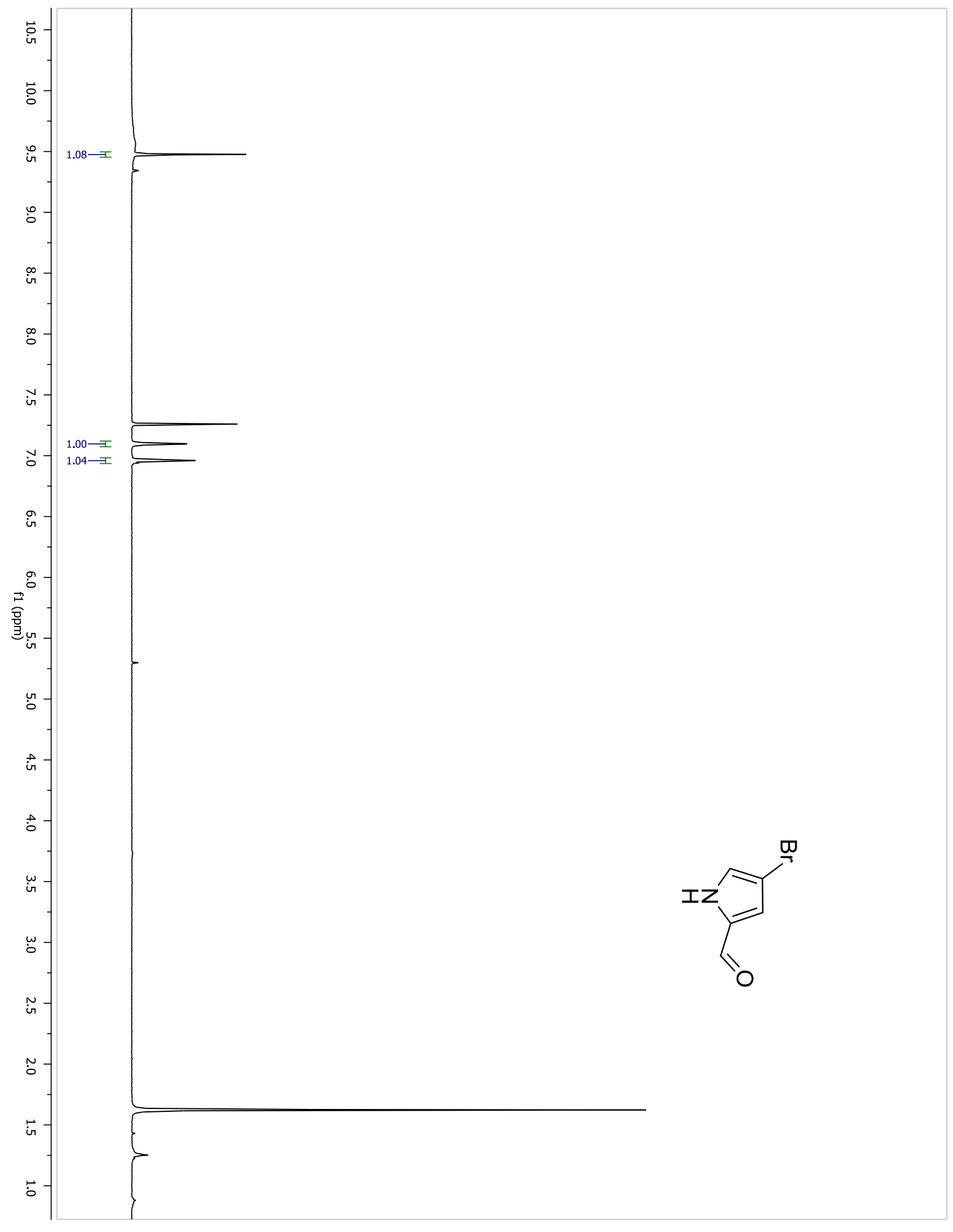

Figure A.131.: ${ }^{1} \mathrm{H}$ NMR of 4.14 


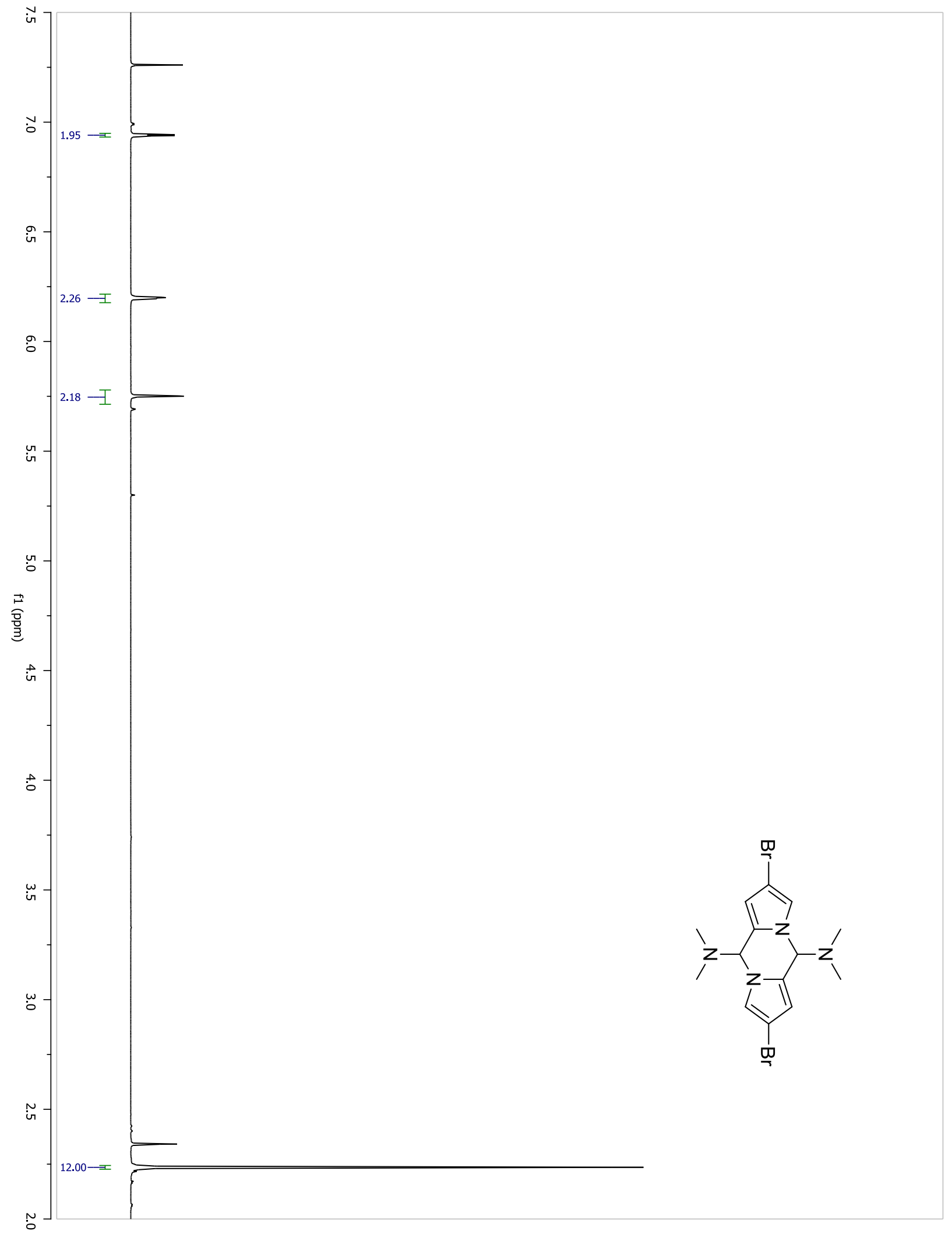

Figure A.132: ${ }^{1} \mathrm{H}$ NMR of 4.15 


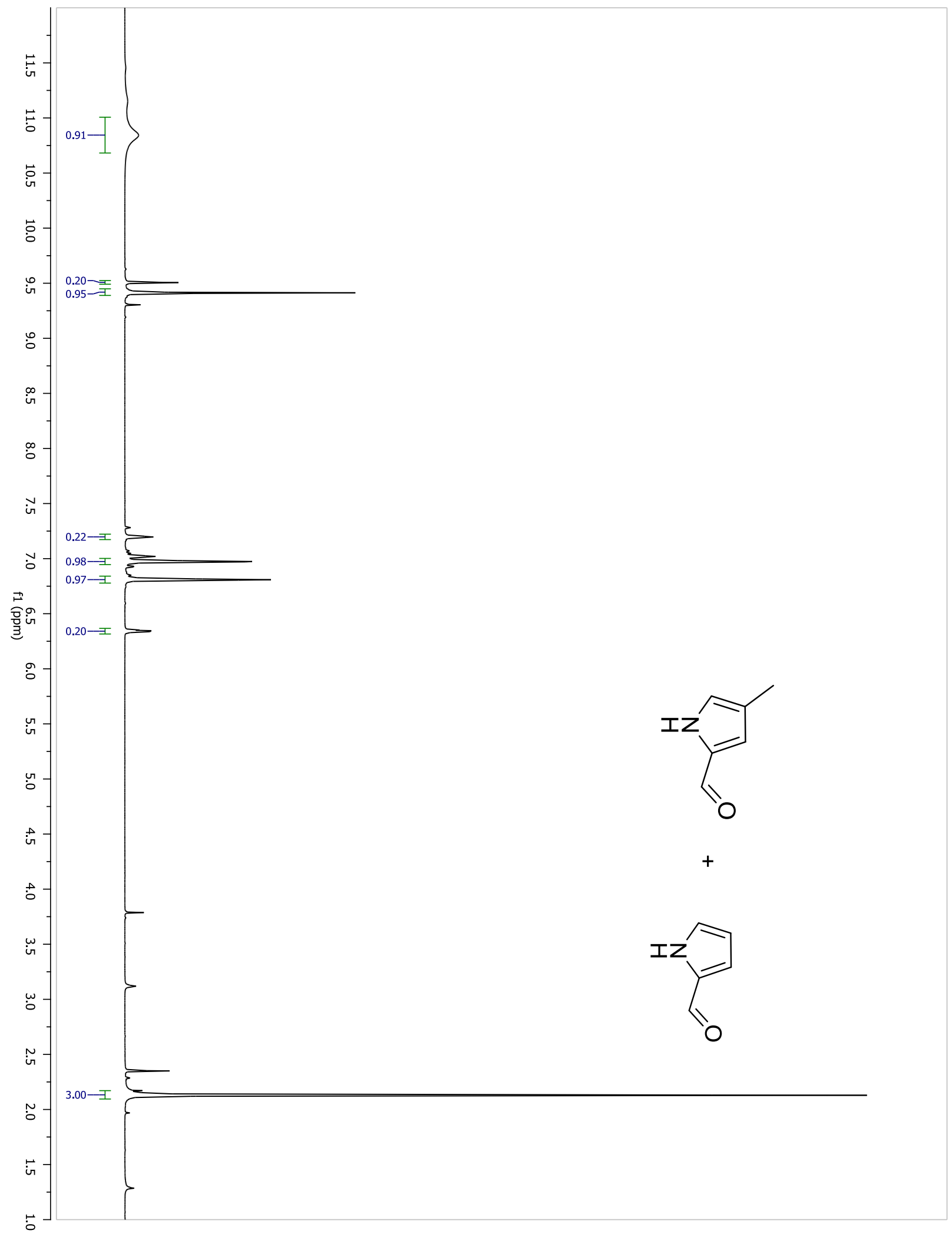

Figure A.133: ${ }^{1} \mathrm{H}$ NMR of 4.16 


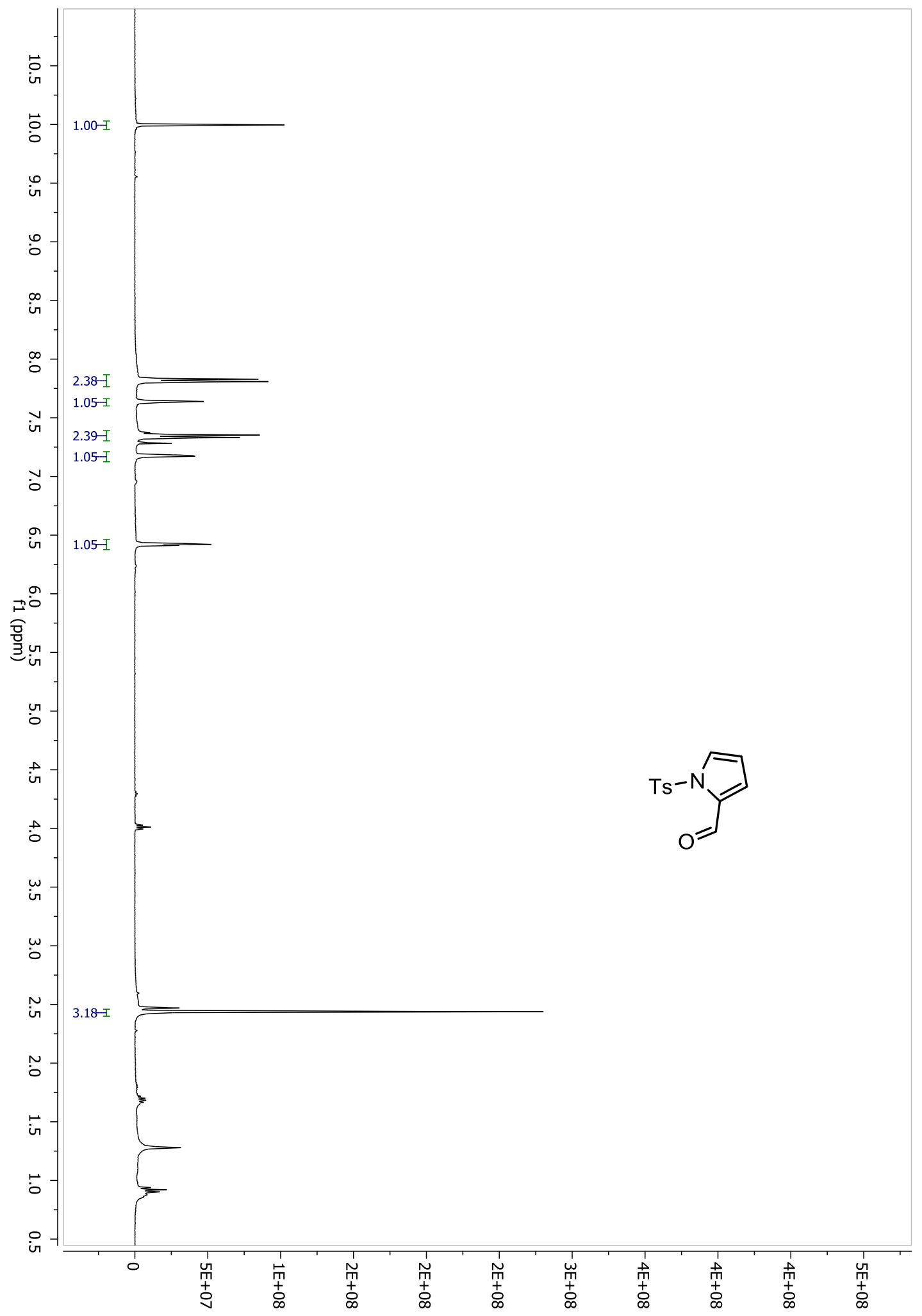

Figure A.134: ${ }^{13} \mathrm{C}$ NMR of 4.17 


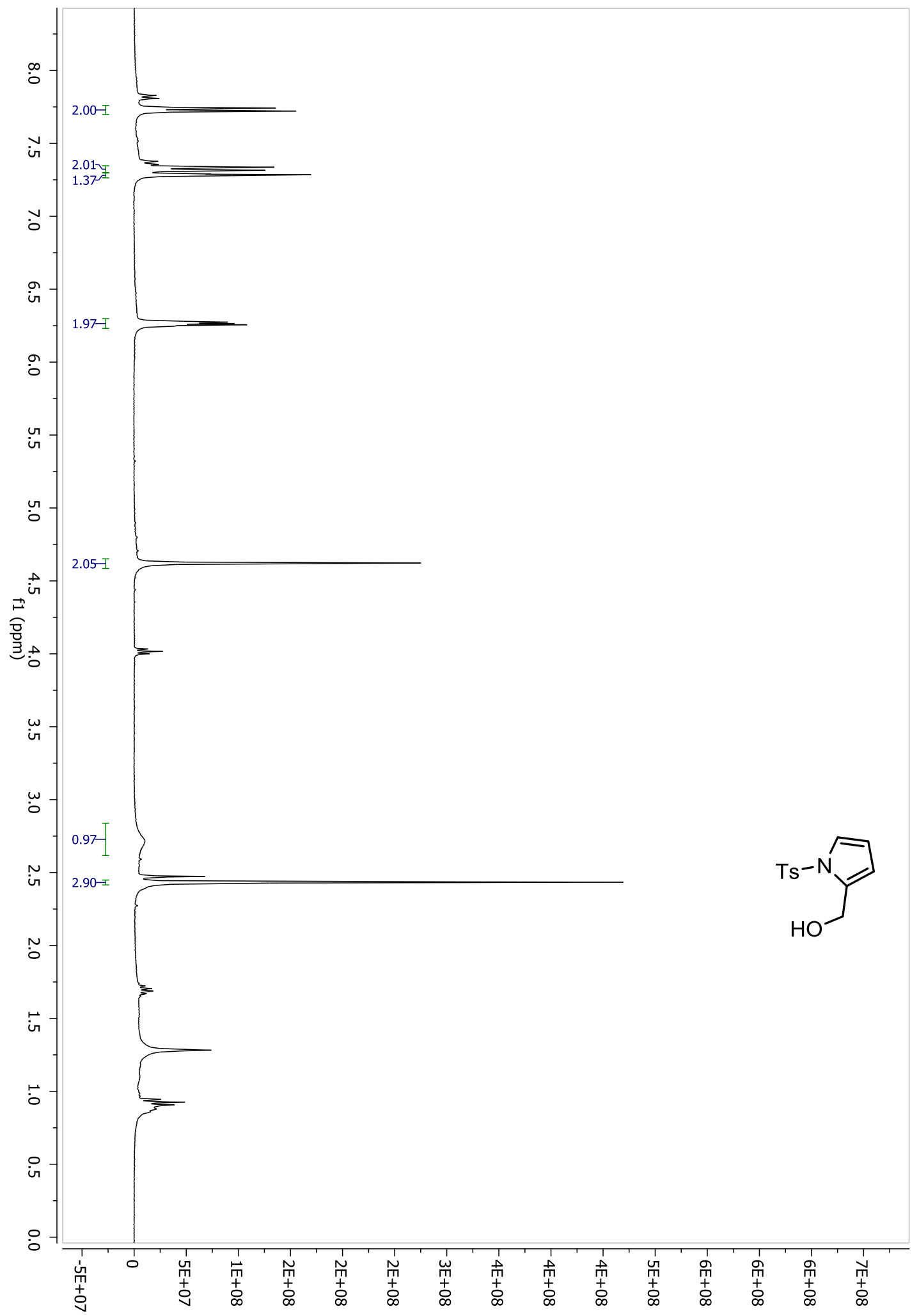

Figure A.135: ${ }^{1} \mathrm{H}$ NMR of 4.18 


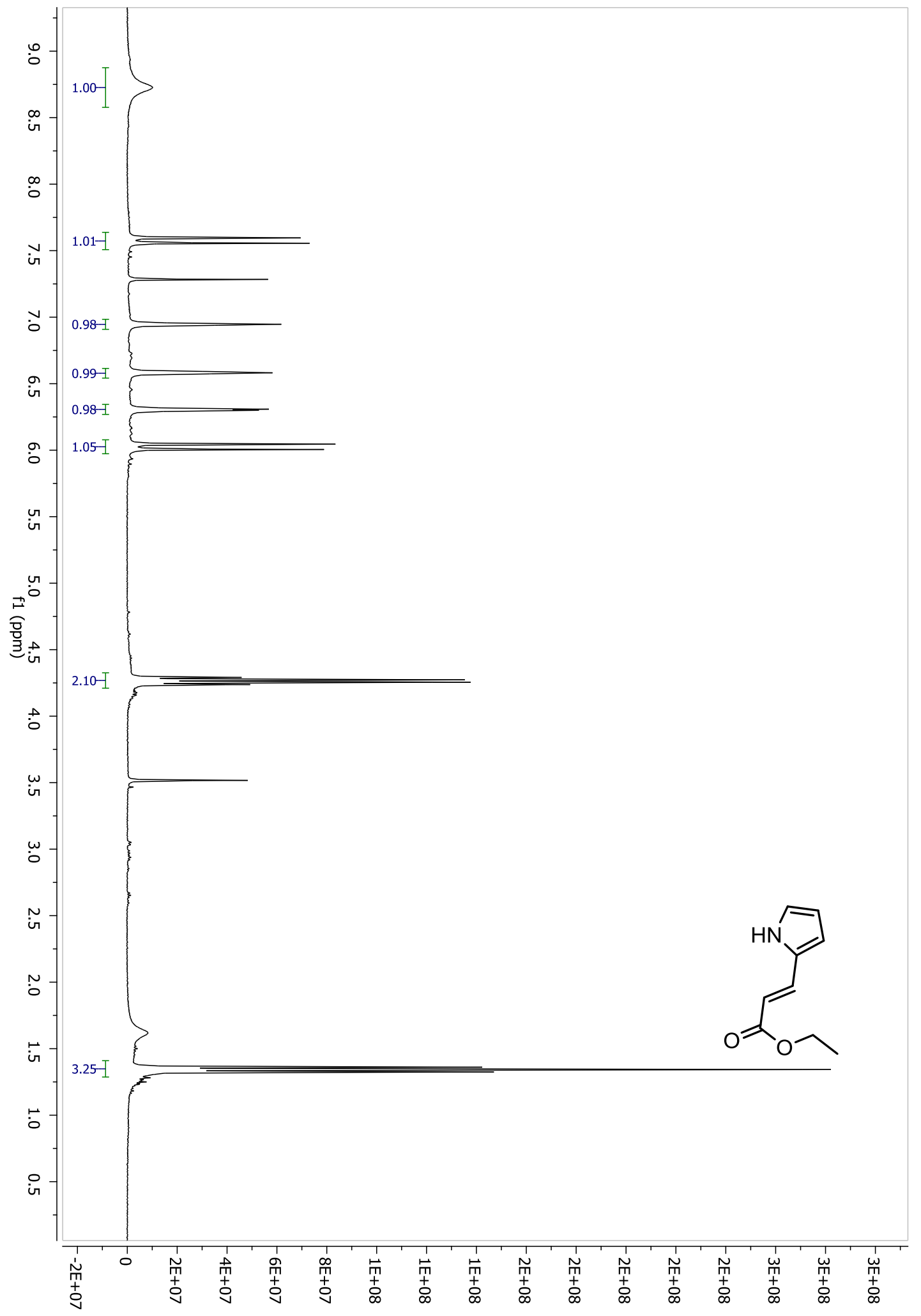

Figure A.136: ${ }^{1} \mathrm{H}$ NMR of 4.19 


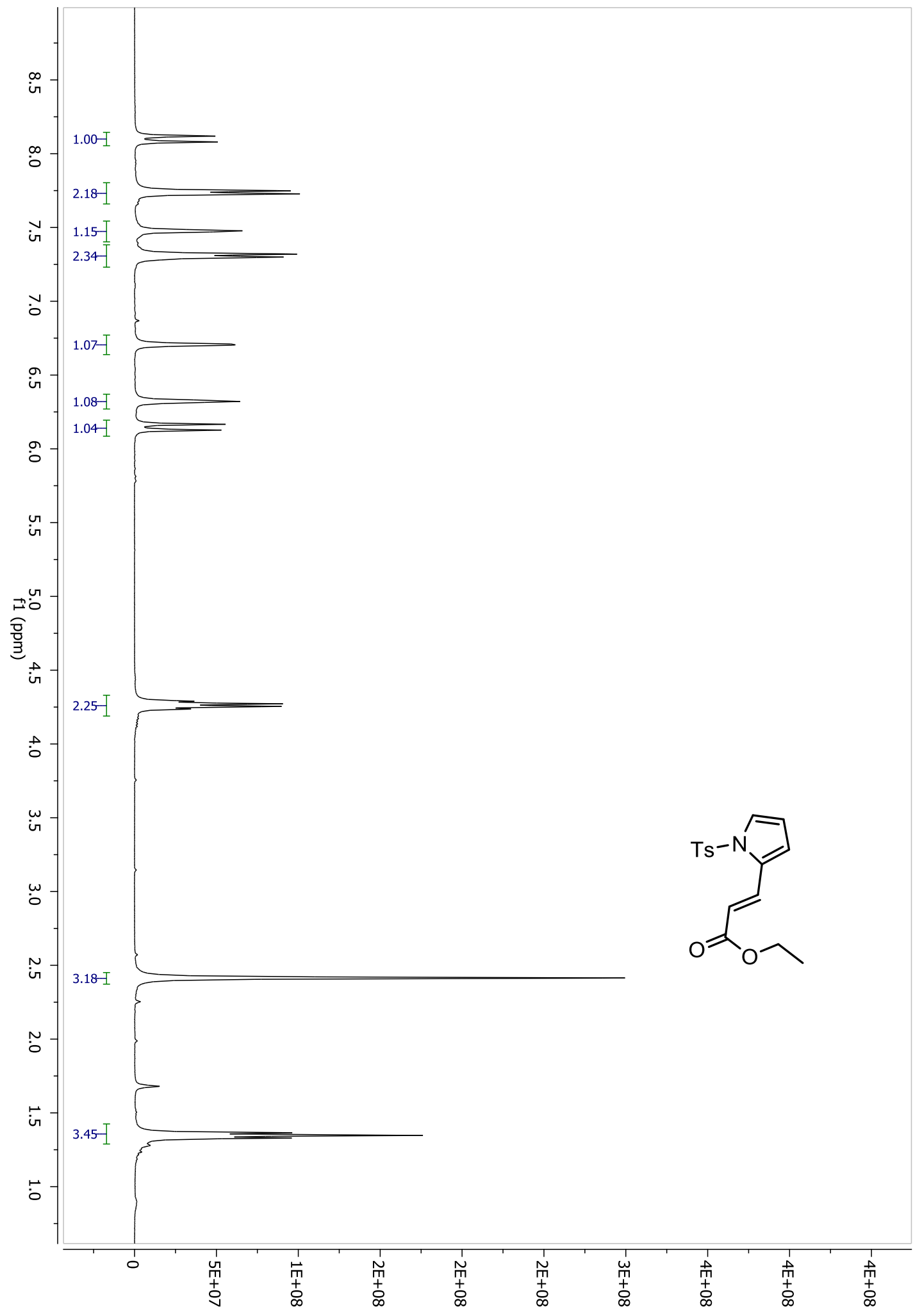

Figure A.137: ${ }^{1} \mathrm{H}$ NMR of 4.20 


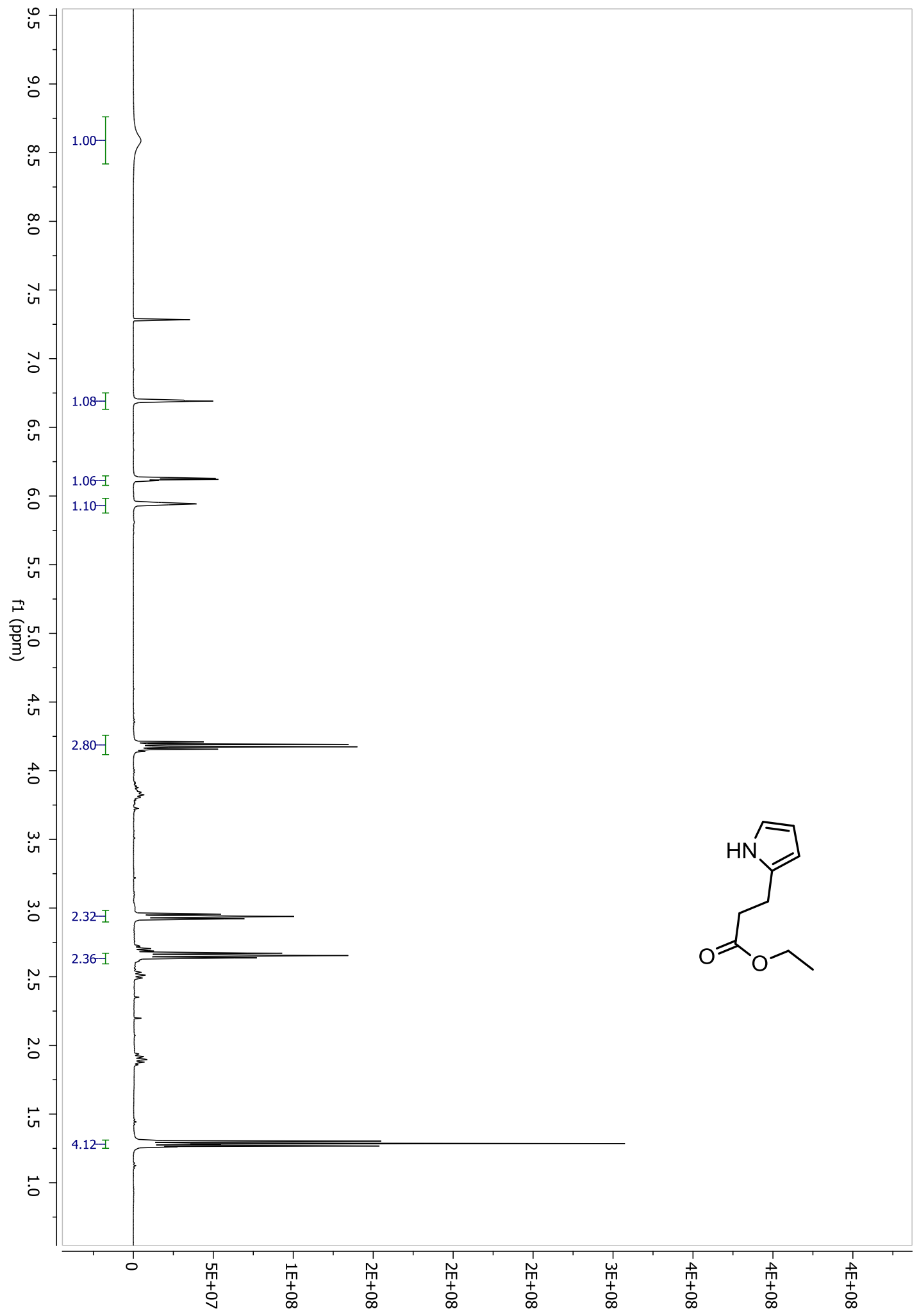

Figure A.138: ${ }^{1} \mathrm{H}$ NMR of 4.21 


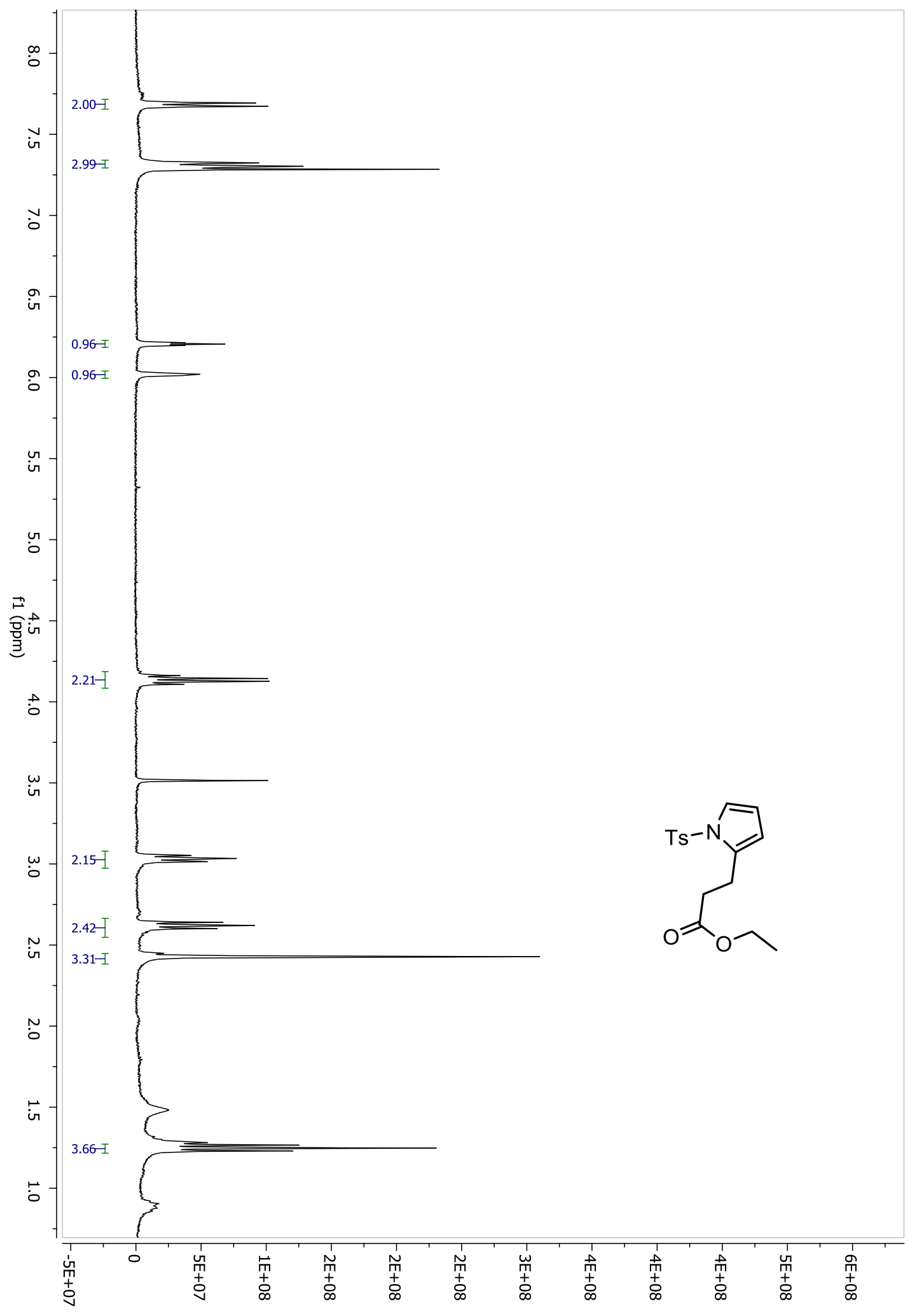

Figure A.140: ${ }^{1} \mathrm{H}$ NMR of 4.22 


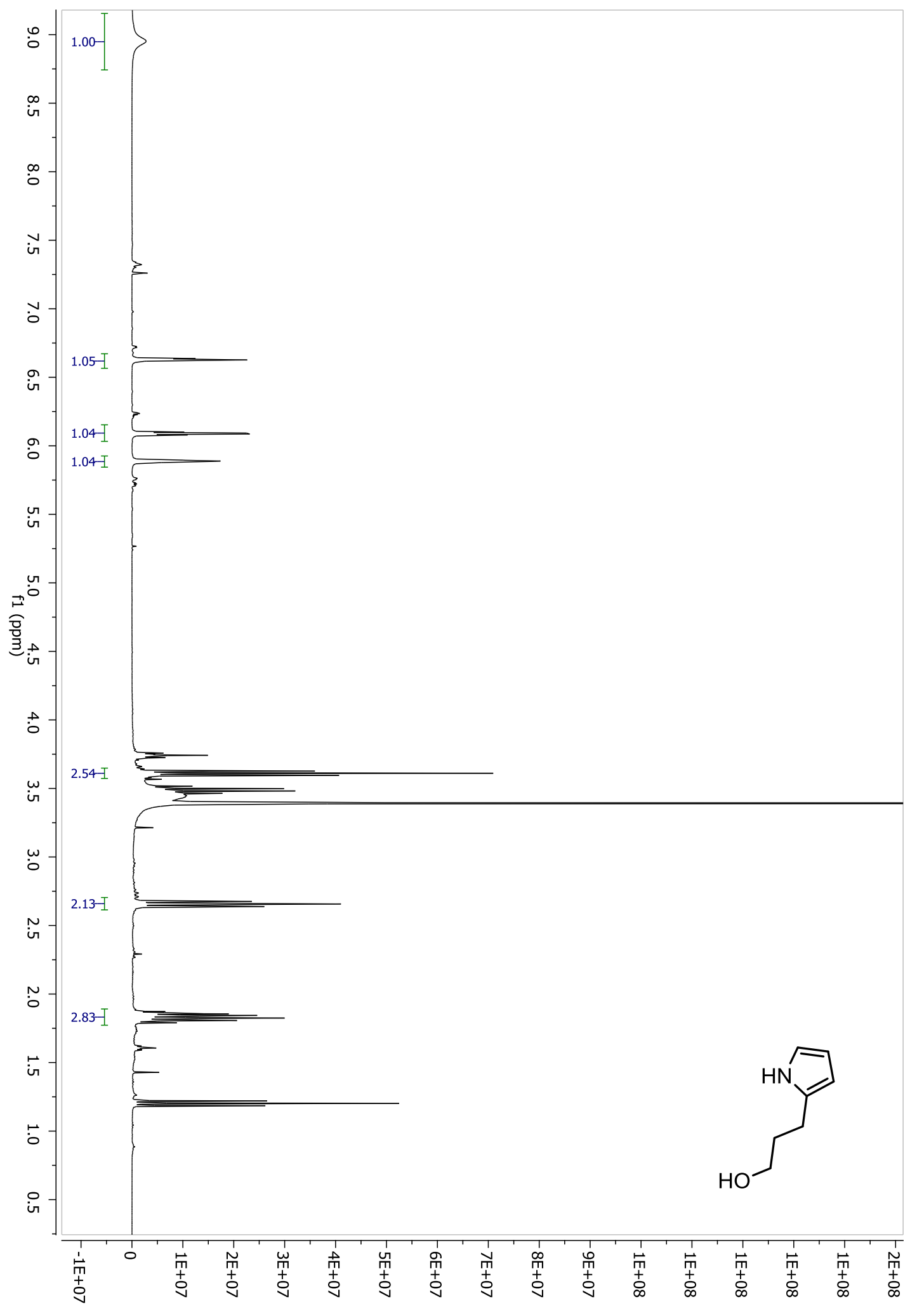

Figure A.141: ${ }^{1} \mathrm{H}$ NMR of 4.23 


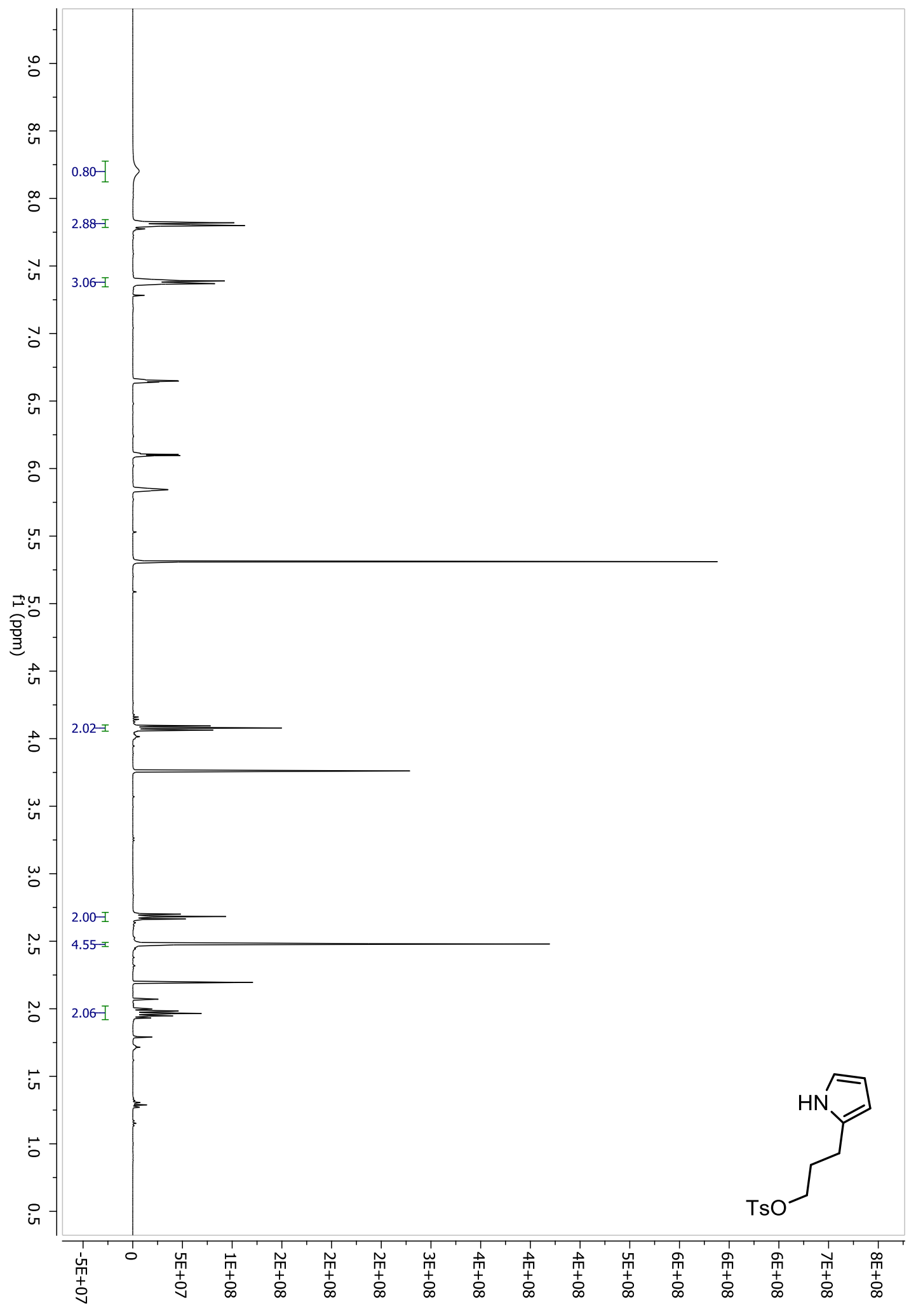

Figure A.142: ${ }^{1} \mathrm{H}$ NMR of 4.24 


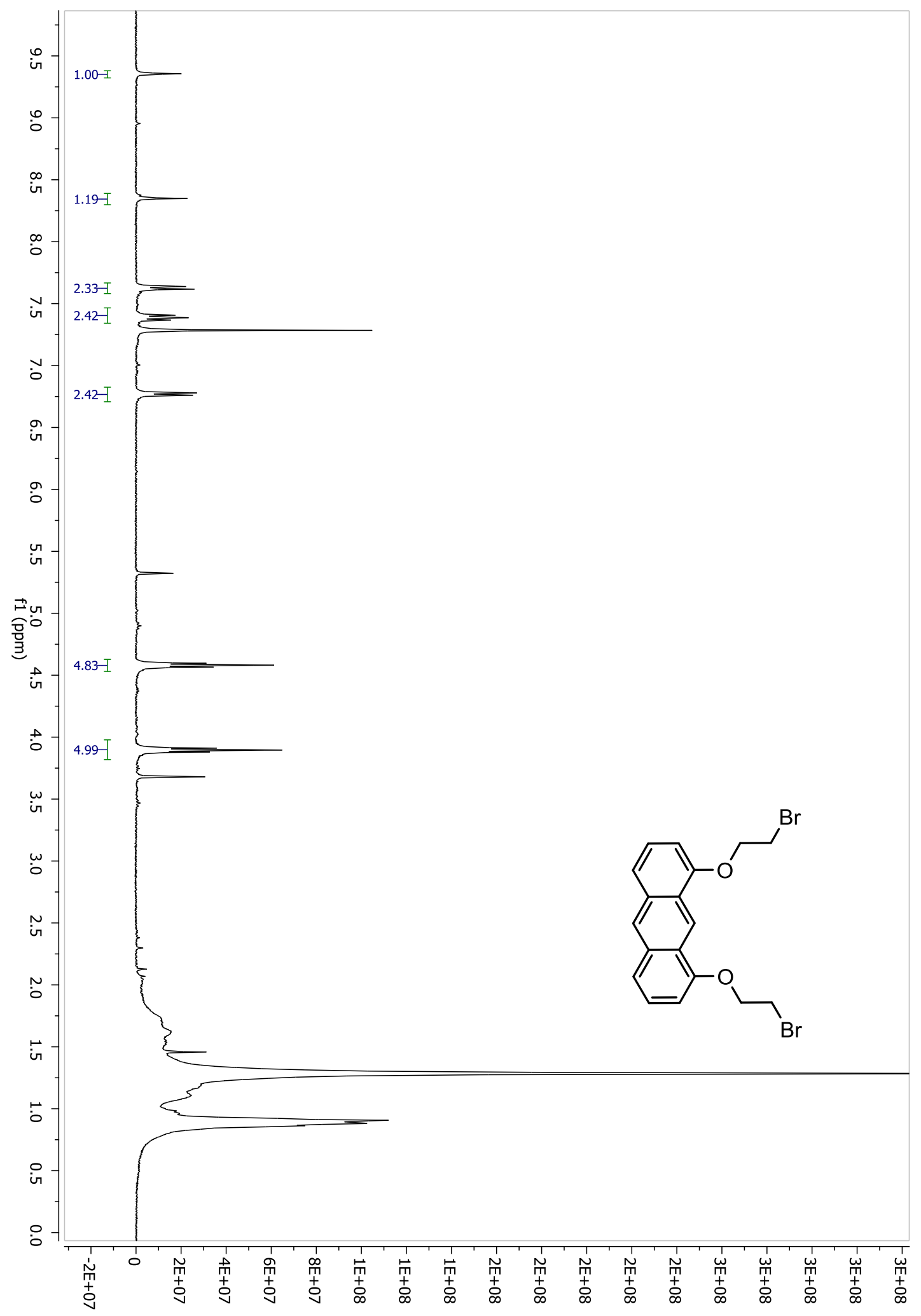

Figure A.143: ${ }^{1} \mathrm{H}$ NMR of 4.29 


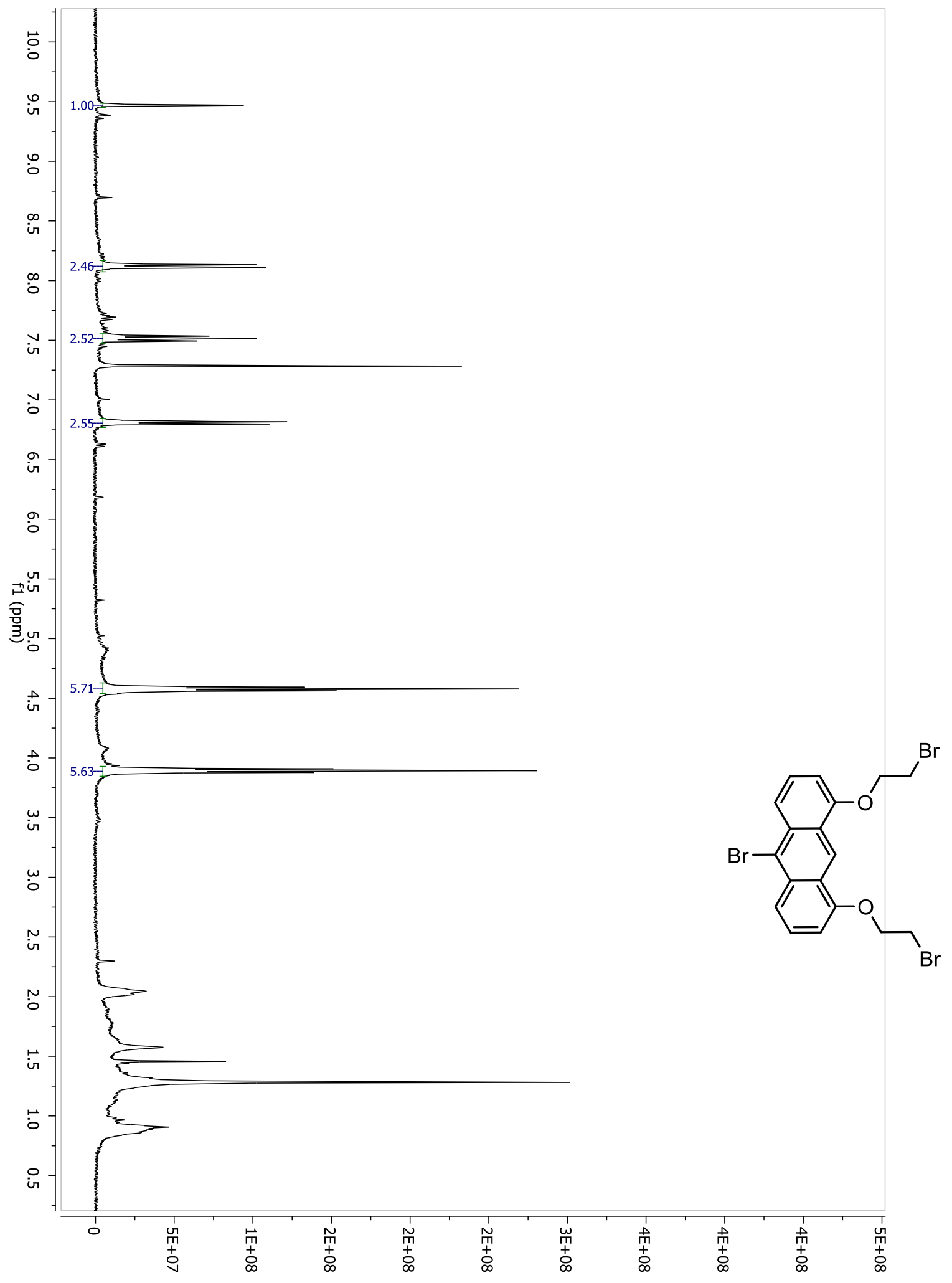

Figure A.144: ${ }^{1} \mathrm{H}$ NMR of 4.30 


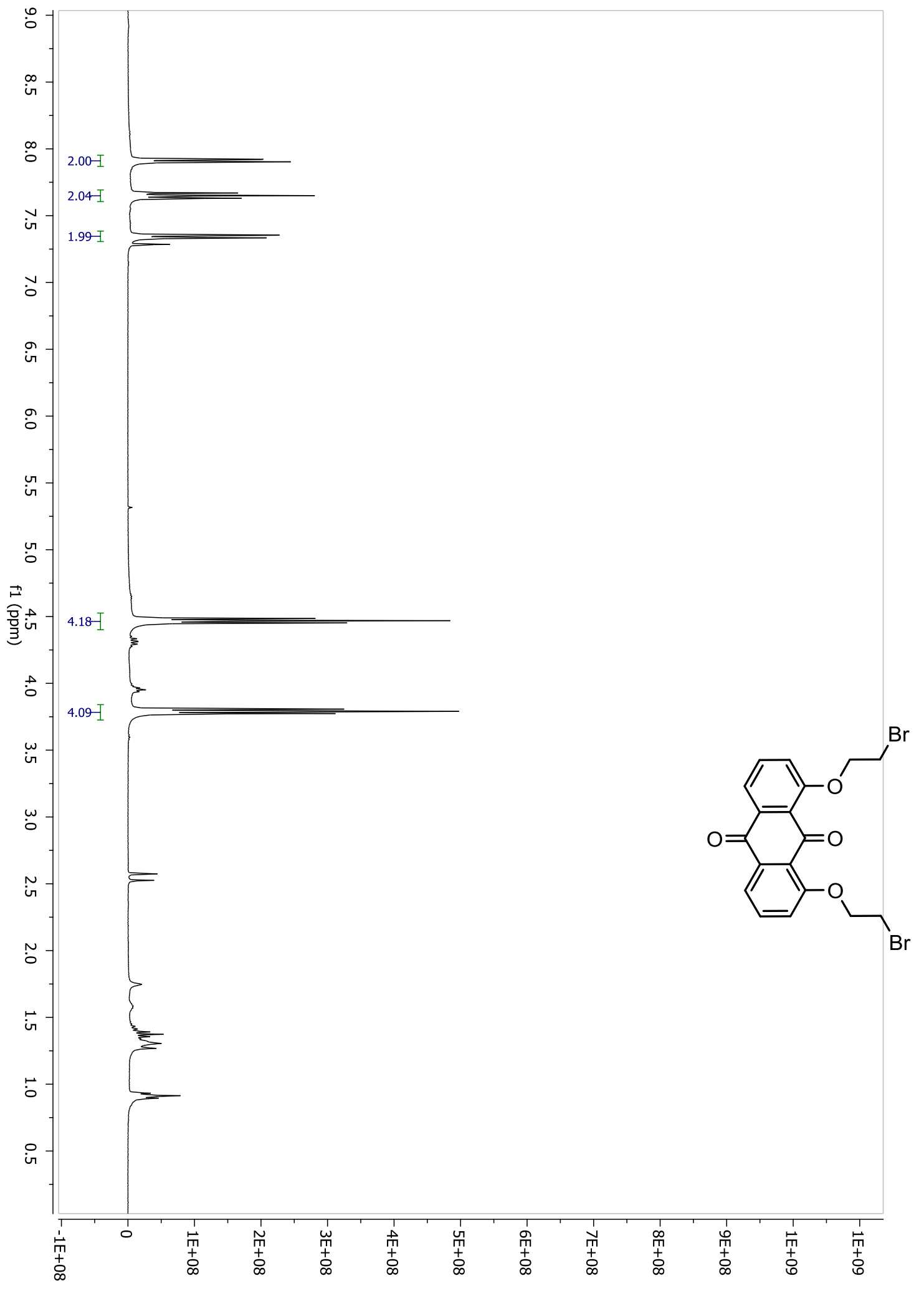

Figure A.1.45: ${ }^{1} \mathrm{H}$ NMR of 4.31 


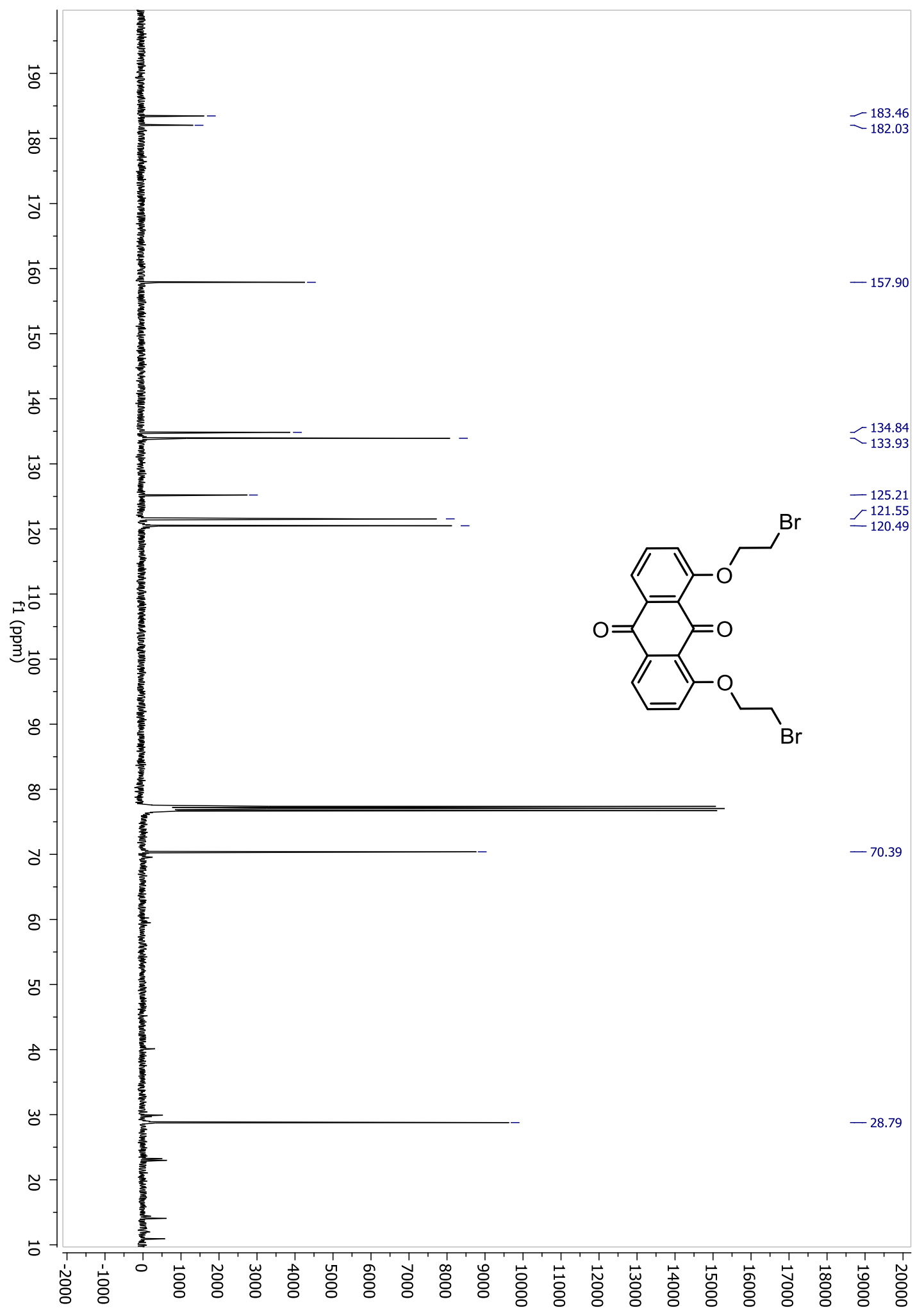

Figure A.146: ${ }^{13} \mathrm{C}$ NMR of 4.31 


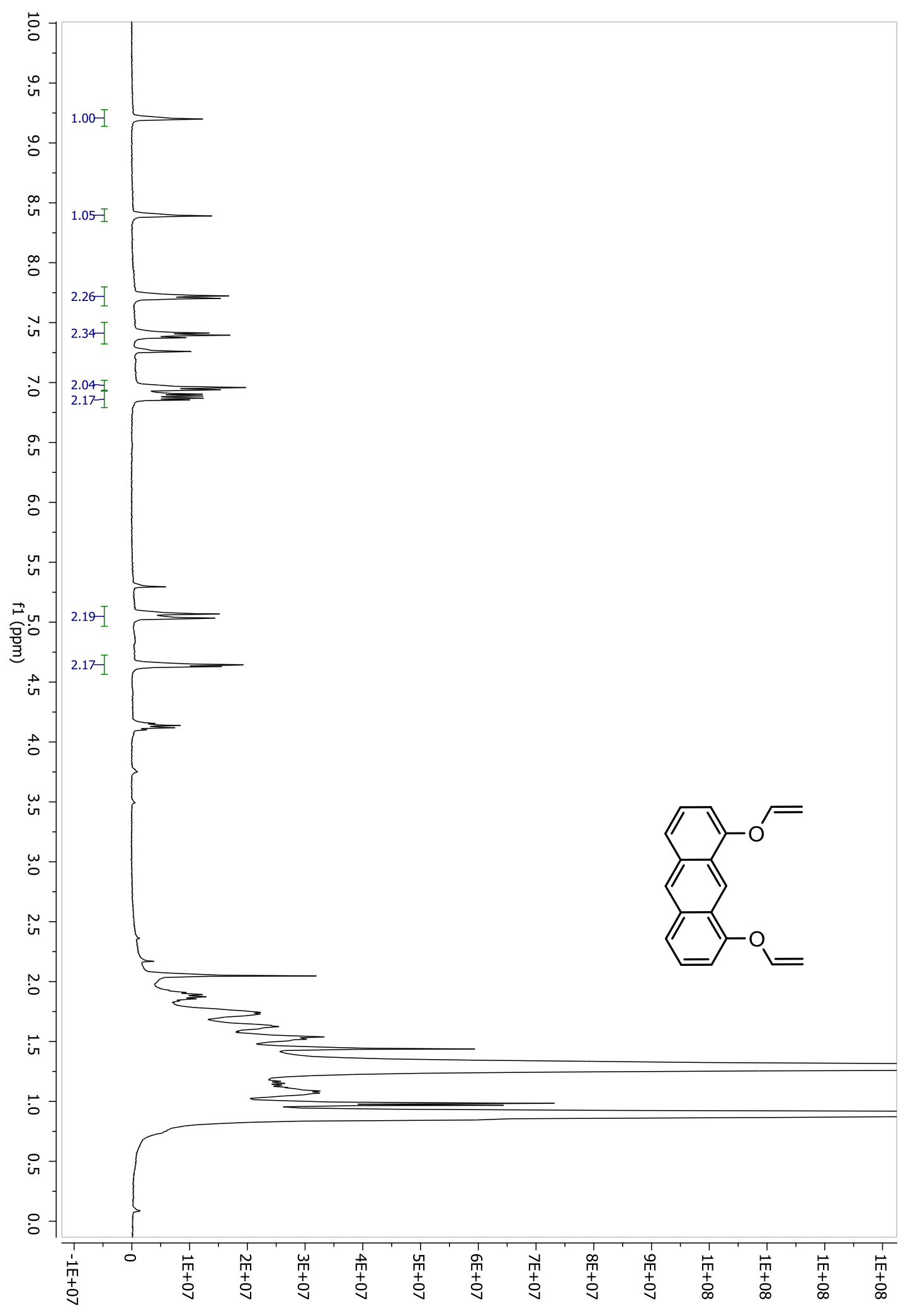

Figure A..147: ${ }^{1}$ H NMR of 4.33 


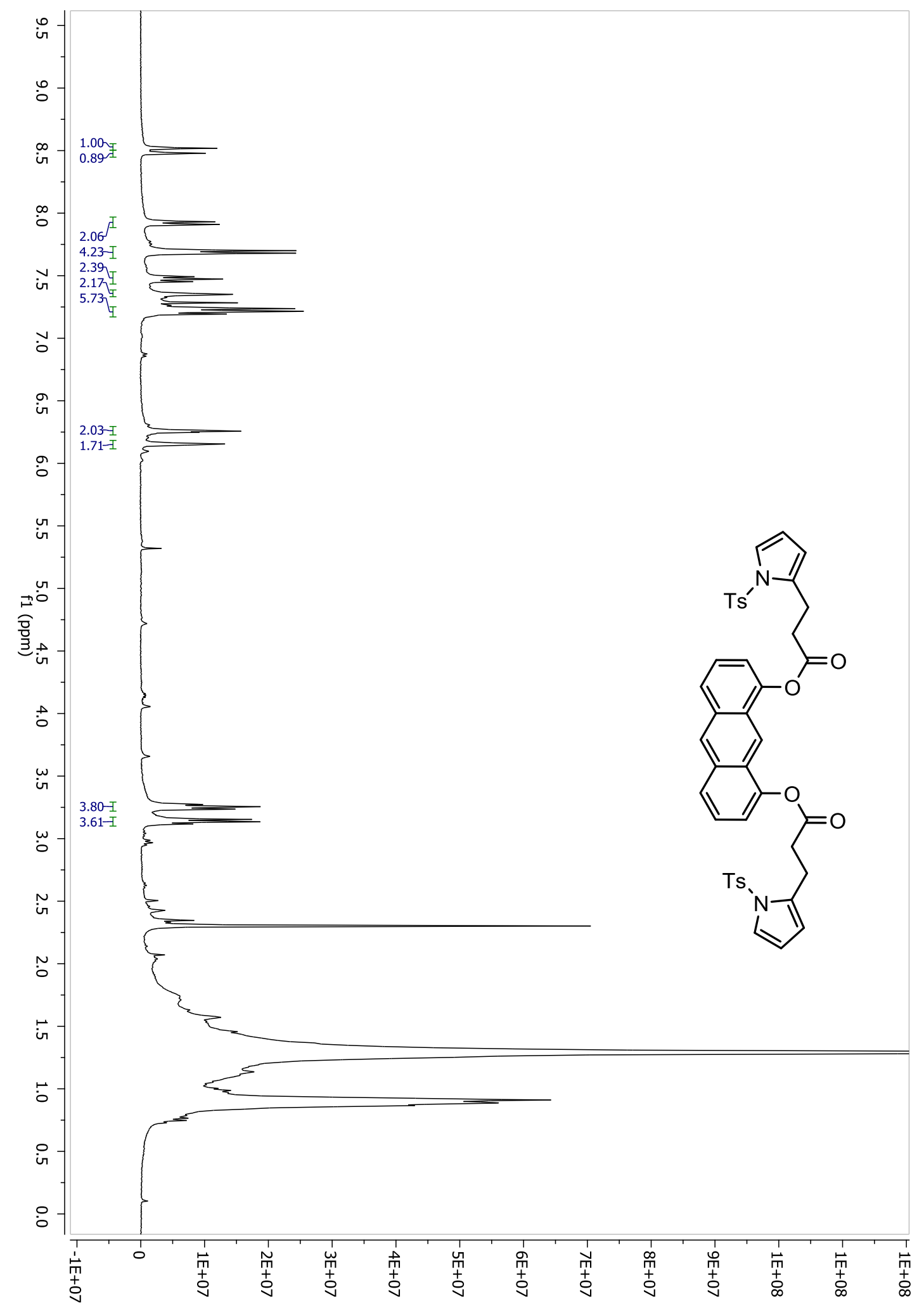

FigureA.148: ${ }^{1} \mathrm{H}$ NMR of 4.36 


\section{A4.3 Crystal structure data of compound $\mathbf{4 . 3 3}$}

Table 1. Crystal data and structure refinement for d1637.

Identification code

Empirical formula

Formula weight

Temperature

Wavelength

Crystal system

Space group

Unit cell dimensions

Volume

$\mathrm{Z}$

Density (calculated)

Absorption coefficient

$\mathrm{F}(000)$

Crystal size

Theta range for data collection

Index ranges

Reflections collected

Independent reflections

Completeness to theta $=67.146^{\circ}$

Absorption correction

Max. and min. transmission

Refinement method

Data / restraints / parameters

Goodness-of-fit on $\mathrm{F}^{2}$

Final $\mathrm{R}$ indices [I>2sigma(I)]

$\mathrm{R}$ indices (all data)

Extinction coefficient

Largest diff. peak and hole d1637

C18 H14 O2

262.29

147(2) K

$1.54178 \AA$

Monoclinic

$\mathrm{P} 21 / \mathrm{c}$

$\mathrm{a}=25.3737(7) \AA$

$\alpha=90^{\circ}$.

$\mathrm{b}=12.0863(3) \AA$

$\beta=96.8550(10)^{\circ}$.

$\mathrm{c}=13.0647(4) \AA$

$\gamma=90^{\circ}$.

12

$1.314 \mathrm{Mg} / \mathrm{m}^{3}$

$0.674 \mathrm{~mm}^{-1}$

1656

$0.120 \times 0.100 \times 0.060 \mathrm{~mm}^{3}$

1.754 to $67.146^{\circ}$.

$-30<=\mathrm{h}<=30,-14<=\mathrm{k}<=14,-15<=\mathrm{l}<=15$

84328

$7064[\mathrm{R}(\mathrm{int})=0.0452]$

$99.2 \%$

Semi-empirical from equivalents

0.7529 and 0.6848

Full-matrix least-squares on $\mathrm{F}^{2}$

7064 / 0 / 542

1.058

$\mathrm{R} 1=0.0390, \mathrm{wR} 2=0.1039$

$\mathrm{R} 1=0.0463, \mathrm{wR} 2=0.1104$

$\mathrm{n} / \mathrm{a}$

0.380 and -0.256 e. $\AA^{-3}$ 
Table 2. Atomic coordinates ( $\mathrm{x} 10^{4}$ ) and equivalent isotropic displacement parameters $\left(\AA^{2} \times 10^{3}\right)$ for $\mathrm{d} 1637$. $\mathrm{U}(\mathrm{eq})$ is defined as one third of the trace of the orthogonalized $\mathrm{U}^{\mathrm{ij}}$ tensor.

\begin{tabular}{|c|c|c|c|c|}
\hline & $\mathrm{x}$ & $\mathrm{y}$ & $\mathrm{z}$ & $\mathrm{U}(\mathrm{eq})$ \\
\hline $\mathrm{O}(1 \mathrm{~A})$ & 1996(1) & $6962(1)$ & 1189(1) & $34(1)$ \\
\hline $\mathrm{O}(2 \mathrm{~A})$ & 2205(1) & $4799(1)$ & $4300(1)$ & $39(1)$ \\
\hline$C(1 \mathrm{~A})$ & $1667(1)$ & $4742(1)$ & $4347(1)$ & $35(1)$ \\
\hline $\mathrm{C}(2 \mathrm{~A})$ & 1440(1) & $4214(1)$ & $5107(1)$ & $45(1)$ \\
\hline $\mathrm{C}(3 \mathrm{~A})$ & $881(1)$ & $4188(1)$ & $5077(1)$ & $50(1)$ \\
\hline $\mathrm{C}(4 \mathrm{~A})$ & $563(1)$ & $4703(1)$ & 4322(1) & $43(1)$ \\
\hline $\mathrm{C}(5 \mathrm{~A})$ & $785(1)$ & $5307(1)$ & $3542(1)$ & $34(1)$ \\
\hline$C(6 A)$ & $468(1)$ & $5886(1)$ & 2782(1) & $34(1)$ \\
\hline$C(7 A)$ & $680(1)$ & $6450(1)$ & 1993(1) & $30(1)$ \\
\hline $\mathrm{C}(8 \mathrm{~A})$ & $354(1)$ & 7013(1) & 1196(1) & $35(1)$ \\
\hline $\mathrm{C}(9 \mathrm{~A})$ & $571(1)$ & $7504(1)$ & $414(1)$ & $37(1)$ \\
\hline$C(10 A)$ & $1128(1)$ & $7496(1)$ & $379(1)$ & $34(1)$ \\
\hline$C(11 \mathrm{~A})$ & 1451(1) & $6999(1)$ & 1149(1) & $29(1)$ \\
\hline $\mathrm{C}(12 \mathrm{~A})$ & $1244(1)$ & $6442(1)$ & 1982(1) & $27(1)$ \\
\hline$C(13 A)$ & $1568(1)$ & $5887(1)$ & 2757(1) & $28(1)$ \\
\hline $\mathrm{C}(14 \mathrm{~A})$ & $1349(1)$ & $5321(1)$ & $3536(1)$ & $30(1)$ \\
\hline$C(15 A)$ & $2238(1)$ & 7701(1) & $594(1)$ & $37(1)$ \\
\hline$C(16 A)$ & $2680(1)$ & $7463(2)$ & $235(1)$ & $48(1)$ \\
\hline$C(17 A)$ & $2528(1)$ & $4006(1)$ & 4813(1) & $46(1)$ \\
\hline$C(18 A)$ & $3027(1)$ & $4174(2)$ & $5084(2)$ & $59(1)$ \\
\hline $\mathrm{O}(1 \mathrm{~B})$ & 3984(1) & $5260(1)$ & $3155(1)$ & $37(1)$ \\
\hline $\mathrm{O}(2 \mathrm{~B})$ & $4164(1)$ & $1960(1)$ & $5126(1)$ & $37(1)$ \\
\hline $\mathrm{C}(1 \mathrm{~B})$ & $4710(1)$ & $1967(1)$ & $5219(1)$ & $31(1)$ \\
\hline $\mathrm{C}(2 \mathrm{~B})$ & $5023(1)$ & $1172(1)$ & $5716(1)$ & $36(1)$ \\
\hline $\mathrm{C}(3 \mathrm{~B})$ & $5578(1)$ & $1226(1)$ & $5718(1)$ & $42(1)$ \\
\hline $\mathrm{C}(4 \mathrm{~B})$ & $5809(1)$ & 2046(1) & $5227(1)$ & $39(1)$ \\
\hline $\mathrm{C}(5 \mathrm{~B})$ & $5494(1)$ & 2903(1) & 4707(1) & $31(1)$ \\
\hline $\mathrm{C}(6 \mathrm{~B})$ & $5714(1)$ & $3765(1)$ & 4191(1) & $34(1)$ \\
\hline $\mathrm{C}(7 \mathrm{~B})$ & $5403(1)$ & $4593(1)$ & $3673(1)$ & $31(1)$ \\
\hline $\mathrm{C}(8 \mathrm{~B})$ & $5626(1)$ & $5484(1)$ & $3153(1)$ & $36(1)$ \\
\hline $\mathrm{C}(9 \mathrm{~B})$ & $5308(1)$ & $6258(1)$ & 2653(1) & $40(1)$ \\
\hline
\end{tabular}




\begin{tabular}{|c|c|c|c|c|}
\hline$C(10 B)$ & $4749(1)$ & $6214(1)$ & $2625(1)$ & $38(1)$ \\
\hline$C(11 B)$ & $4523(1)$ & $5392(1)$ & $3130(1)$ & $31(1)$ \\
\hline$C(12 B)$ & $4838(1)$ & $4548(1)$ & $3673(1)$ & $28(1)$ \\
\hline$C(13 B)$ & $4615(1)$ & $3690(1)$ & $4195(1)$ & $29(1)$ \\
\hline$C(14 B)$ & 4930(1) & $2868(1)$ & 4704(1) & $28(1)$ \\
\hline$C(15 B)$ & $3642(1)$ & $6107(1)$ & 2881(1) & $48(1)$ \\
\hline$C(16 B)$ & $3134(1)$ & $5978(1)$ & $2718(1)$ & $46(1)$ \\
\hline$C(17 B)$ & $3915(1)$ & $1297(1)$ & $5771(1)$ & $42(1)$ \\
\hline$C(18 B)$ & $3451(1)$ & $876(2)$ & $5496(1)$ & $50(1)$ \\
\hline $\mathrm{O}(1 \mathrm{C})$ & $2342(1)$ & $5150(1)$ & $-1276(1)$ & $36(1)$ \\
\hline $\mathrm{O}(2 \mathrm{C})$ & $2514(1)$ & $3123(1)$ & $1926(1)$ & $36(1)$ \\
\hline$C(1 C)$ & 1974(1) & $3071(1)$ & 1950(1) & $30(1)$ \\
\hline $\mathrm{C}(2 \mathrm{C})$ & $1743(1)$ & $2539(1)$ & $2700(1)$ & $36(1)$ \\
\hline$C(3 C)$ & 1184(1) & $2550(1)$ & $2677(1)$ & $38(1)$ \\
\hline$C(4 C)$ & $870(1)$ & $3088(1)$ & $1925(1)$ & $36(1)$ \\
\hline$C(5 C)$ & 1099(1) & $3649(1)$ & $1123(1)$ & $30(1)$ \\
\hline$C(6 C)$ & 792(1) & 4199(1) & $325(1)$ & $32(1)$ \\
\hline$C(7 C)$ & 1015(1) & $4720(1)$ & $-472(1)$ & $31(1)$ \\
\hline $\mathrm{C}(8 \mathrm{C})$ & $696(1)$ & $5251(1)$ & $-1305(1)$ & $38(1)$ \\
\hline $\mathrm{C}(9 \mathrm{C})$ & $920(1)$ & $5705(1)$ & $-2092(1)$ & $43(1)$ \\
\hline$C(10 C)$ & $1478(1)$ & $5683(1)$ & $-2108(1)$ & $41(1)$ \\
\hline$C(11 C)$ & $1795(1)$ & $5216(1)$ & $-1314(1)$ & $32(1)$ \\
\hline $\mathrm{C}(12 \mathrm{C})$ & $1580(1)$ & $4700(1)$ & $-467(1)$ & $28(1)$ \\
\hline$C(13 C)$ & 1893(1) & $4170(1)$ & $336(1)$ & $28(1)$ \\
\hline$C(14 C)$ & $1665(1)$ & $3642(1)$ & $1126(1)$ & $28(1)$ \\
\hline$C(15 C)$ & 2591(1) & $5897(2)$ & $-1850(1)$ & $47(1)$ \\
\hline$C(16 C)$ & $3049(1)$ & $5726(2)$ & $-2144(1)$ & $49(1)$ \\
\hline $\mathrm{C}(17 \mathrm{C})$ & $2825(1)$ & $2325(1)$ & $2452(1)$ & $39(1)$ \\
\hline$C(18 C)$ & $3302(1)$ & $2528(1)$ & 2879(1) & $44(1)$ \\
\hline
\end{tabular}


Table 3. Bond lengths $\left[\AA ̊ \AA\right.$ and angles $\left[{ }^{\circ}\right]$ for d 1637 .

\begin{tabular}{|c|c|}
\hline $\mathrm{O}(1 \mathrm{~A})-\mathrm{C}(15 \mathrm{~A})$ & $1.3767(17)$ \\
\hline $\mathrm{O}(1 \mathrm{~A})-\mathrm{C}(11 \mathrm{~A})$ & $1.3789(16)$ \\
\hline $\mathrm{O}(2 \mathrm{~A})-\mathrm{C}(1 \mathrm{~A})$ & $1.3771(18)$ \\
\hline $\mathrm{O}(2 \mathrm{~A})-\mathrm{C}(17 \mathrm{~A})$ & $1.3805(18)$ \\
\hline$C(1 \mathrm{~A})-\mathrm{C}(2 \mathrm{~A})$ & $1.364(2)$ \\
\hline$C(1 \mathrm{~A})-\mathrm{C}(14 \mathrm{~A})$ & $1.434(2)$ \\
\hline$C(2 A)-C(3 A)$ & $1.415(2)$ \\
\hline $\mathrm{C}(2 \mathrm{~A})-\mathrm{H}(2 \mathrm{AA})$ & 0.9500 \\
\hline$C(3 A)-C(4 A)$ & $1.349(2)$ \\
\hline $\mathrm{C}(3 \mathrm{~A})-\mathrm{H}(3 \mathrm{AA})$ & 0.9500 \\
\hline$C(4 A)-C(5 A)$ & $1.423(2)$ \\
\hline $\mathrm{C}(4 \mathrm{~A})-\mathrm{H}(4 \mathrm{AA})$ & 0.9500 \\
\hline$C(5 A)-C(6 A)$ & $1.388(2)$ \\
\hline $\mathrm{C}(5 \mathrm{~A})-\mathrm{C}(14 \mathrm{~A})$ & $1.432(2)$ \\
\hline$C(6 A)-C(7 A)$ & $1.397(2)$ \\
\hline $\mathrm{C}(6 \mathrm{~A})-\mathrm{H}(6 \mathrm{AA})$ & 0.9500 \\
\hline $\mathrm{C}(7 \mathrm{~A})-\mathrm{C}(8 \mathrm{~A})$ & $1.425(2)$ \\
\hline$C(7 A)-C(12 A)$ & $1.4316(19)$ \\
\hline $\mathrm{C}(8 \mathrm{~A})-\mathrm{C}(9 \mathrm{~A})$ & $1.354(2)$ \\
\hline $\mathrm{C}(8 \mathrm{~A})-\mathrm{H}(8 \mathrm{AA})$ & 0.9500 \\
\hline $\mathrm{C}(9 \mathrm{~A})-\mathrm{C}(10 \mathrm{~A})$ & $1.418(2)$ \\
\hline $\mathrm{C}(9 \mathrm{~A})-\mathrm{H}(9 \mathrm{AA})$ & 0.9500 \\
\hline$C(10 A)-C(11 A)$ & $1.360(2)$ \\
\hline $\mathrm{C}(10 \mathrm{~A})-\mathrm{H}(10 \mathrm{~A})$ & 0.9500 \\
\hline $\mathrm{C}(11 \mathrm{~A})-\mathrm{C}(12 \mathrm{~A})$ & $1.4314(19)$ \\
\hline $\mathrm{C}(12 \mathrm{~A})-\mathrm{C}(13 \mathrm{~A})$ & $1.3983(19)$ \\
\hline$C(13 A)-C(14 A)$ & $1.3955(19)$ \\
\hline $\mathrm{C}(13 \mathrm{~A})-\mathrm{H}(13 \mathrm{~A})$ & 0.9500 \\
\hline$C(15 A)-C(16 A)$ & $1.298(2)$ \\
\hline $\mathrm{C}(15 \mathrm{~A})-\mathrm{H}(15 \mathrm{~A})$ & 0.9500 \\
\hline $\mathrm{C}(16 \mathrm{~A})-\mathrm{H}(16 \mathrm{~A})$ & 0.9500 \\
\hline $\mathrm{C}(16 \mathrm{~A})-\mathrm{H}(16 \mathrm{~B})$ & 0.9500 \\
\hline $\mathrm{C}(17 \mathrm{~A})-\mathrm{C}(18 \mathrm{~A})$ & $1.290(3)$ \\
\hline $\mathrm{C}(17 \mathrm{~A})-\mathrm{H}(17 \mathrm{~A})$ & 0.9500 \\
\hline
\end{tabular}




\begin{tabular}{|c|c|}
\hline $\mathrm{C}(18 \mathrm{~A})-\mathrm{H}(18 \mathrm{~A})$ & 0.9500 \\
\hline $\mathrm{C}(18 \mathrm{~A})-\mathrm{H}(18 \mathrm{~B})$ & 0.9500 \\
\hline $\mathrm{O}(1 \mathrm{~B})-\mathrm{C}(15 \mathrm{~B})$ & $1.3628(18)$ \\
\hline $\mathrm{O}(1 \mathrm{~B})-\mathrm{C}(11 \mathrm{~B})$ & $1.3803(17)$ \\
\hline $\mathrm{O}(2 \mathrm{~B})-\mathrm{C}(17 \mathrm{~B})$ & $1.3710(18)$ \\
\hline $\mathrm{O}(2 \mathrm{~B})-\mathrm{C}(1 \mathrm{~B})$ & $1.3767(16)$ \\
\hline $\mathrm{C}(1 \mathrm{~B})-\mathrm{C}(2 \mathrm{~B})$ & $1.361(2)$ \\
\hline $\mathrm{C}(1 \mathrm{~B})-\mathrm{C}(14 \mathrm{~B})$ & $1.428(2)$ \\
\hline $\mathrm{C}(2 \mathrm{~B})-\mathrm{C}(3 \mathrm{~B})$ & $1.411(2)$ \\
\hline $\mathrm{C}(2 \mathrm{~B})-\mathrm{H}(2 \mathrm{BA})$ & 0.9500 \\
\hline $\mathrm{C}(3 \mathrm{~B})-\mathrm{C}(4 \mathrm{~B})$ & $1.351(2)$ \\
\hline $\mathrm{C}(3 \mathrm{~B})-\mathrm{H}(3 \mathrm{BA})$ & 0.9500 \\
\hline $\mathrm{C}(4 \mathrm{~B})-\mathrm{C}(5 \mathrm{~B})$ & $1.429(2)$ \\
\hline $\mathrm{C}(4 \mathrm{~B})-\mathrm{H}(4 \mathrm{BA})$ & 0.9500 \\
\hline$C(5 B)-C(6 B)$ & $1.394(2)$ \\
\hline $\mathrm{C}(5 \mathrm{~B})-\mathrm{C}(14 \mathrm{~B})$ & $1.4317(19)$ \\
\hline $\mathrm{C}(6 \mathrm{~B})-\mathrm{C}(7 \mathrm{~B})$ & $1.398(2)$ \\
\hline $\mathrm{C}(6 \mathrm{~B})-\mathrm{H}(6 \mathrm{BA})$ & 0.9500 \\
\hline$C(7 B)-C(8 B)$ & $1.426(2)$ \\
\hline $\mathrm{C}(7 \mathrm{~B})-\mathrm{C}(12 \mathrm{~B})$ & $1.4347(19)$ \\
\hline $\mathrm{C}(8 \mathrm{~B})-\mathrm{C}(9 \mathrm{~B})$ & $1.352(2)$ \\
\hline $\mathrm{C}(8 \mathrm{~B})-\mathrm{H}(8 \mathrm{BA})$ & 0.9500 \\
\hline $\mathrm{C}(9 \mathrm{~B})-\mathrm{C}(10 \mathrm{~B})$ & $1.416(2)$ \\
\hline $\mathrm{C}(9 \mathrm{~B})-\mathrm{H}(9 \mathrm{BA})$ & 0.9500 \\
\hline$C(10 B)-C(11 B)$ & $1.358(2)$ \\
\hline $\mathrm{C}(10 \mathrm{~B})-\mathrm{H}(10 \mathrm{~B})$ & 0.9500 \\
\hline$C(11 B)-C(12 B)$ & $1.4320(19)$ \\
\hline $\mathrm{C}(12 \mathrm{~B})-\mathrm{C}(13 \mathrm{~B})$ & 1.3971(19) \\
\hline$C(13 B)-C(14 B)$ & $1.3932(19)$ \\
\hline $\mathrm{C}(13 \mathrm{~B})-\mathrm{H}(13 \mathrm{~B})$ & 0.9500 \\
\hline$C(15 B)-C(16 B)$ & $1.290(2)$ \\
\hline $\mathrm{C}(15 \mathrm{~B})-\mathrm{H}(15 \mathrm{~B})$ & 0.9500 \\
\hline$C(16 B)-H(16 C)$ & 0.9500 \\
\hline$C(16 B)-H(16 D)$ & 0.9500 \\
\hline $\mathrm{C}(17 \mathrm{~B})-\mathrm{C}(18 \mathrm{~B})$ & $1.293(2)$ \\
\hline $\mathrm{C}(17 \mathrm{~B})-\mathrm{H}(17 \mathrm{~B})$ & 0.9500 \\
\hline
\end{tabular}




\begin{tabular}{|c|c|}
\hline $\mathrm{C}(18 \mathrm{~B})-\mathrm{H}(18 \mathrm{C})$ & 0.9500 \\
\hline $\mathrm{C}(18 \mathrm{~B})-\mathrm{H}(18 \mathrm{D})$ & 0.9500 \\
\hline $\mathrm{O}(1 \mathrm{C})-\mathrm{C}(15 \mathrm{C})$ & $1.3753(19)$ \\
\hline $\mathrm{O}(1 \mathrm{C})-\mathrm{C}(11 \mathrm{C})$ & $1.3838(17)$ \\
\hline $\mathrm{O}(2 \mathrm{C})-\mathrm{C}(1 \mathrm{C})$ & $1.3753(16)$ \\
\hline $\mathrm{O}(2 \mathrm{C})-\mathrm{C}(17 \mathrm{C})$ & $1.3765(17)$ \\
\hline$C(1 C)-C(2 C)$ & $1.363(2)$ \\
\hline$C(1 C)-C(14 C)$ & $1.4309(19)$ \\
\hline $\mathrm{C}(2 \mathrm{C})-\mathrm{C}(3 \mathrm{C})$ & $1.416(2)$ \\
\hline $\mathrm{C}(2 \mathrm{C})-\mathrm{H}(2 \mathrm{CA})$ & 0.9500 \\
\hline$C(3 C)-C(4 C)$ & $1.355(2)$ \\
\hline $\mathrm{C}(3 \mathrm{C})-\mathrm{H}(3 \mathrm{CA})$ & 0.9500 \\
\hline$C(4 C)-C(5 C)$ & $1.429(2)$ \\
\hline $\mathrm{C}(4 \mathrm{C})-\mathrm{H}(4 \mathrm{CA})$ & 0.9500 \\
\hline$C(5 C)-C(6 C)$ & $1.393(2)$ \\
\hline$C(5 C)-C(14 C)$ & $1.4340(19)$ \\
\hline $\mathrm{C}(6 \mathrm{C})-\mathrm{C}(7 \mathrm{C})$ & $1.392(2)$ \\
\hline $\mathrm{C}(6 \mathrm{C})-\mathrm{H}(6 \mathrm{CA})$ & 0.9500 \\
\hline $\mathrm{C}(7 \mathrm{C})-\mathrm{C}(8 \mathrm{C})$ & $1.429(2)$ \\
\hline$C(7 C)-C(12 C)$ & $1.4331(19)$ \\
\hline $\mathrm{C}(8 \mathrm{C})-\mathrm{C}(9 \mathrm{C})$ & $1.350(2)$ \\
\hline $\mathrm{C}(8 \mathrm{C})-\mathrm{H}(8 \mathrm{CA})$ & 0.9500 \\
\hline$C(9 C)-C(10 C)$ & $1.416(2)$ \\
\hline $\mathrm{C}(9 \mathrm{C})-\mathrm{H}(9 \mathrm{CA})$ & 0.9500 \\
\hline$C(10 C)-C(11 C)$ & $1.359(2)$ \\
\hline $\mathrm{C}(10 \mathrm{C})-\mathrm{H}(10 \mathrm{C})$ & 0.9500 \\
\hline$C(11 C)-C(12 C)$ & $1.434(2)$ \\
\hline$C(12 C)-C(13 C)$ & $1.3957(19)$ \\
\hline$C(13 C)-C(14 C)$ & $1.3971(19)$ \\
\hline $\mathrm{C}(13 \mathrm{C})-\mathrm{H}(13 \mathrm{C})$ & 0.9500 \\
\hline$C(15 C)-C(16 C)$ & $1.281(2)$ \\
\hline $\mathrm{C}(15 \mathrm{C})-\mathrm{H}(15 \mathrm{C})$ & 0.9500 \\
\hline$C(16 C)-H(16 E)$ & 0.9500 \\
\hline $\mathrm{C}(16 \mathrm{C})-\mathrm{H}(16 \mathrm{~F})$ & 0.9500 \\
\hline$C(17 C)-C(18 C)$ & $1.294(2)$ \\
\hline $\mathrm{C}(17 \mathrm{C})-\mathrm{H}(17 \mathrm{C})$ & 0.9500 \\
\hline
\end{tabular}




\begin{tabular}{|c|c|}
\hline $\mathrm{C}(18 \mathrm{C})-\mathrm{H}(18 \mathrm{E})$ & 0.9500 \\
\hline $\mathrm{C}(18 \mathrm{C})-\mathrm{H}(18 \mathrm{~F})$ & 0.9500 \\
\hline$C(15 A)-O(1 A)-C(11 A)$ & $118.09(11)$ \\
\hline $\mathrm{C}(1 \mathrm{~A})-\mathrm{O}(2 \mathrm{~A})-\mathrm{C}(17 \mathrm{~A})$ & $118.53(12)$ \\
\hline $\mathrm{C}(2 \mathrm{~A})-\mathrm{C}(1 \mathrm{~A})-\mathrm{O}(2 \mathrm{~A})$ & $124.22(14)$ \\
\hline $\mathrm{C}(2 \mathrm{~A})-\mathrm{C}(1 \mathrm{~A})-\mathrm{C}(14 \mathrm{~A})$ & $121.17(14)$ \\
\hline $\mathrm{O}(2 \mathrm{~A})-\mathrm{C}(1 \mathrm{~A})-\mathrm{C}(14 \mathrm{~A})$ & $114.59(12)$ \\
\hline$C(1 \mathrm{~A})-\mathrm{C}(2 \mathrm{~A})-\mathrm{C}(3 \mathrm{~A})$ & $119.90(15)$ \\
\hline $\mathrm{C}(1 \mathrm{~A})-\mathrm{C}(2 \mathrm{~A})-\mathrm{H}(2 \mathrm{AA})$ & 120.0 \\
\hline $\mathrm{C}(3 \mathrm{~A})-\mathrm{C}(2 \mathrm{~A})-\mathrm{H}(2 \mathrm{AA})$ & 120.0 \\
\hline $\mathrm{C}(4 \mathrm{~A})-\mathrm{C}(3 \mathrm{~A})-\mathrm{C}(2 \mathrm{~A})$ & $121.26(15)$ \\
\hline $\mathrm{C}(4 \mathrm{~A})-\mathrm{C}(3 \mathrm{~A})-\mathrm{H}(3 \mathrm{AA})$ & 119.4 \\
\hline $\mathrm{C}(2 \mathrm{~A})-\mathrm{C}(3 \mathrm{~A})-\mathrm{H}(3 \mathrm{AA})$ & 119.4 \\
\hline $\mathrm{C}(3 \mathrm{~A})-\mathrm{C}(4 \mathrm{~A})-\mathrm{C}(5 \mathrm{~A})$ & $120.57(15)$ \\
\hline$C(3 A)-C(4 A)-H(4 A A)$ & 119.7 \\
\hline $\mathrm{C}(5 \mathrm{~A})-\mathrm{C}(4 \mathrm{~A})-\mathrm{H}(4 \mathrm{AA})$ & 119.7 \\
\hline$C(6 A)-C(5 A)-C(4 A)$ & $121.71(14)$ \\
\hline$C(6 A)-C(5 A)-C(14 A)$ & $118.95(13)$ \\
\hline$C(4 A)-C(5 A)-C(14 A)$ & $119.33(14)$ \\
\hline$C(5 A)-C(6 A)-C(7 A)$ & $122.15(13)$ \\
\hline$C(5 A)-C(6 A)-H(6 A A)$ & 118.9 \\
\hline $\mathrm{C}(7 \mathrm{~A})-\mathrm{C}(6 \mathrm{~A})-\mathrm{H}(6 \mathrm{AA})$ & 118.9 \\
\hline$C(6 A)-C(7 A)-C(8 A)$ & $122.09(13)$ \\
\hline$C(6 A)-C(7 A)-C(12 A)$ & $118.54(13)$ \\
\hline$C(8 A)-C(7 A)-C(12 A)$ & $119.37(13)$ \\
\hline$C(9 A)-C(8 A)-C(7 A)$ & $120.44(13)$ \\
\hline $\mathrm{C}(9 \mathrm{~A})-\mathrm{C}(8 \mathrm{~A})-\mathrm{H}(8 \mathrm{AA})$ & 119.8 \\
\hline $\mathrm{C}(7 \mathrm{~A})-\mathrm{C}(8 \mathrm{~A})-\mathrm{H}(8 \mathrm{AA})$ & 119.8 \\
\hline$C(8 A)-C(9 A)-C(10 A)$ & $121.21(13)$ \\
\hline $\mathrm{C}(8 \mathrm{~A})-\mathrm{C}(9 \mathrm{~A})-\mathrm{H}(9 \mathrm{AA})$ & 119.4 \\
\hline $\mathrm{C}(10 \mathrm{~A})-\mathrm{C}(9 \mathrm{~A})-\mathrm{H}(9 \mathrm{AA})$ & 119.4 \\
\hline$C(11 A)-C(10 A)-C(9 A)$ & $119.67(13)$ \\
\hline$C(11 A)-C(10 A)-H(10 A)$ & 120.2 \\
\hline $\mathrm{C}(9 \mathrm{~A})-\mathrm{C}(10 \mathrm{~A})-\mathrm{H}(10 \mathrm{~A})$ & 120.2 \\
\hline$C(10 A)-C(11 A)-O(1 A)$ & $123.85(13)$ \\
\hline
\end{tabular}




\begin{tabular}{|c|c|}
\hline$C(10 A)-C(11 A)-C(12 A)$ & $121.70(13)$ \\
\hline $\mathrm{O}(1 \mathrm{~A})-\mathrm{C}(11 \mathrm{~A})-\mathrm{C}(12 \mathrm{~A})$ & $114.43(11)$ \\
\hline $\mathrm{C}(13 \mathrm{~A})-\mathrm{C}(12 \mathrm{~A})-\mathrm{C}(11 \mathrm{~A})$ & $122.58(12)$ \\
\hline$C(13 A)-C(12 A)-C(7 A)$ & $119.86(12)$ \\
\hline $\mathrm{C}(11 \mathrm{~A})-\mathrm{C}(12 \mathrm{~A})-\mathrm{C}(7 \mathrm{~A})$ & $117.55(12)$ \\
\hline $\mathrm{C}(14 \mathrm{~A})-\mathrm{C}(13 \mathrm{~A})-\mathrm{C}(12 \mathrm{~A})$ & $120.81(12)$ \\
\hline $\mathrm{C}(14 \mathrm{~A})-\mathrm{C}(13 \mathrm{~A})-\mathrm{H}(13 \mathrm{~A})$ & 119.6 \\
\hline $\mathrm{C}(12 \mathrm{~A})-\mathrm{C}(13 \mathrm{~A})-\mathrm{H}(13 \mathrm{~A})$ & 119.6 \\
\hline $\mathrm{C}(13 \mathrm{~A})-\mathrm{C}(14 \mathrm{~A})-\mathrm{C}(5 \mathrm{~A})$ & $119.65(13)$ \\
\hline $\mathrm{C}(13 \mathrm{~A})-\mathrm{C}(14 \mathrm{~A})-\mathrm{C}(1 \mathrm{~A})$ & $122.68(13)$ \\
\hline $\mathrm{C}(5 \mathrm{~A})-\mathrm{C}(14 \mathrm{~A})-\mathrm{C}(1 \mathrm{~A})$ & $117.66(13)$ \\
\hline $\mathrm{C}(16 \mathrm{~A})-\mathrm{C}(15 \mathrm{~A})-\mathrm{O}(1 \mathrm{~A})$ & $121.52(15)$ \\
\hline $\mathrm{C}(16 \mathrm{~A})-\mathrm{C}(15 \mathrm{~A})-\mathrm{H}(15 \mathrm{~A})$ & 119.2 \\
\hline $\mathrm{O}(1 \mathrm{~A})-\mathrm{C}(15 \mathrm{~A})-\mathrm{H}(15 \mathrm{~A})$ & 119.2 \\
\hline $\mathrm{C}(15 \mathrm{~A})-\mathrm{C}(16 \mathrm{~A})-\mathrm{H}(16 \mathrm{~A})$ & 120.0 \\
\hline $\mathrm{C}(15 \mathrm{~A})-\mathrm{C}(16 \mathrm{~A})-\mathrm{H}(16 \mathrm{~B})$ & 120.0 \\
\hline $\mathrm{H}(16 \mathrm{~A})-\mathrm{C}(16 \mathrm{~A})-\mathrm{H}(16 \mathrm{~B})$ & 120.0 \\
\hline $\mathrm{C}(18 \mathrm{~A})-\mathrm{C}(17 \mathrm{~A})-\mathrm{O}(2 \mathrm{~A})$ & $121.93(16)$ \\
\hline $\mathrm{C}(18 \mathrm{~A})-\mathrm{C}(17 \mathrm{~A})-\mathrm{H}(17 \mathrm{~A})$ & 119.0 \\
\hline $\mathrm{O}(2 \mathrm{~A})-\mathrm{C}(17 \mathrm{~A})-\mathrm{H}(17 \mathrm{~A})$ & 119.0 \\
\hline $\mathrm{C}(17 \mathrm{~A})-\mathrm{C}(18 \mathrm{~A})-\mathrm{H}(18 \mathrm{~A})$ & 120.0 \\
\hline C(17A)-C(18A)-H(18B) & 120.0 \\
\hline $\mathrm{H}(18 \mathrm{~A})-\mathrm{C}(18 \mathrm{~A})-\mathrm{H}(18 \mathrm{~B})$ & 120.0 \\
\hline $\mathrm{C}(15 \mathrm{~B})-\mathrm{O}(1 \mathrm{~B})-\mathrm{C}(11 \mathrm{~B})$ & $120.58(12)$ \\
\hline$C(17 B)-O(2 B)-C(1 B)$ & $118.82(11)$ \\
\hline $\mathrm{C}(2 \mathrm{~B})-\mathrm{C}(1 \mathrm{~B})-\mathrm{O}(2 \mathrm{~B})$ & $124.17(13)$ \\
\hline $\mathrm{C}(2 \mathrm{~B})-\mathrm{C}(1 \mathrm{~B})-\mathrm{C}(14 \mathrm{~B})$ & $121.62(13)$ \\
\hline $\mathrm{O}(2 \mathrm{~B})-\mathrm{C}(1 \mathrm{~B})-\mathrm{C}(14 \mathrm{~B})$ & $114.16(12)$ \\
\hline$C(1 B)-C(2 B)-C(3 B)$ & $119.63(14)$ \\
\hline $\mathrm{C}(1 \mathrm{~B})-\mathrm{C}(2 \mathrm{~B})-\mathrm{H}(2 \mathrm{BA})$ & 120.2 \\
\hline $\mathrm{C}(3 \mathrm{~B})-\mathrm{C}(2 \mathrm{~B})-\mathrm{H}(2 \mathrm{BA})$ & 120.2 \\
\hline$C(4 B)-C(3 B)-C(2 B)$ & $121.46(14)$ \\
\hline $\mathrm{C}(4 \mathrm{~B})-\mathrm{C}(3 \mathrm{~B})-\mathrm{H}(3 \mathrm{BA})$ & 119.3 \\
\hline $\mathrm{C}(2 \mathrm{~B})-\mathrm{C}(3 \mathrm{~B})-\mathrm{H}(3 \mathrm{BA})$ & 119.3 \\
\hline$C(3 B)-C(4 B)-C(5 B)$ & $120.55(14)$ \\
\hline $\mathrm{C}(3 \mathrm{~B})-\mathrm{C}(4 \mathrm{~B})-\mathrm{H}(4 \mathrm{BA})$ & 119.7 \\
\hline
\end{tabular}




\begin{tabular}{|c|c|}
\hline $\mathrm{C}(5 \mathrm{~B})-\mathrm{C}(4 \mathrm{~B})-\mathrm{H}(4 \mathrm{BA})$ & 119.7 \\
\hline$C(6 B)-C(5 B)-C(4 B)$ & $122.60(13)$ \\
\hline$C(6 B)-C(5 B)-C(14 B)$ & $118.60(12)$ \\
\hline $\mathrm{C}(4 \mathrm{~B})-\mathrm{C}(5 \mathrm{~B})-\mathrm{C}(14 \mathrm{~B})$ & $118.79(13)$ \\
\hline$C(5 B)-C(6 B)-C(7 B)$ & $122.26(13)$ \\
\hline $\mathrm{C}(5 \mathrm{~B})-\mathrm{C}(6 \mathrm{~B})-\mathrm{H}(6 \mathrm{BA})$ & 118.9 \\
\hline $\mathrm{C}(7 \mathrm{~B})-\mathrm{C}(6 \mathrm{~B})-\mathrm{H}(6 \mathrm{BA})$ & 118.9 \\
\hline $\mathrm{C}(6 \mathrm{~B})-\mathrm{C}(7 \mathrm{~B})-\mathrm{C}(8 \mathrm{~B})$ & $122.53(13)$ \\
\hline $\mathrm{C}(6 \mathrm{~B})-\mathrm{C}(7 \mathrm{~B})-\mathrm{C}(12 \mathrm{~B})$ & $118.64(13)$ \\
\hline$C(8 B)-C(7 B)-C(12 B)$ & $118.83(13)$ \\
\hline $\mathrm{C}(9 \mathrm{~B})-\mathrm{C}(8 \mathrm{~B})-\mathrm{C}(7 \mathrm{~B})$ & $120.33(14)$ \\
\hline $\mathrm{C}(9 \mathrm{~B})-\mathrm{C}(8 \mathrm{~B})-\mathrm{H}(8 \mathrm{BA})$ & 119.8 \\
\hline $\mathrm{C}(7 \mathrm{~B})-\mathrm{C}(8 \mathrm{~B})-\mathrm{H}(8 \mathrm{BA})$ & 119.8 \\
\hline $\mathrm{C}(8 \mathrm{~B})-\mathrm{C}(9 \mathrm{~B})-\mathrm{C}(10 \mathrm{~B})$ & $121.67(14)$ \\
\hline $\mathrm{C}(8 \mathrm{~B})-\mathrm{C}(9 \mathrm{~B})-\mathrm{H}(9 \mathrm{BA})$ & 119.2 \\
\hline $\mathrm{C}(10 \mathrm{~B})-\mathrm{C}(9 \mathrm{~B})-\mathrm{H}(9 \mathrm{BA})$ & 119.2 \\
\hline $\mathrm{C}(11 \mathrm{~B})-\mathrm{C}(10 \mathrm{~B})-\mathrm{C}(9 \mathrm{~B})$ & $119.72(14)$ \\
\hline $\mathrm{C}(11 \mathrm{~B})-\mathrm{C}(10 \mathrm{~B})-\mathrm{H}(10 \mathrm{~B})$ & 120.1 \\
\hline $\mathrm{C}(9 \mathrm{~B})-\mathrm{C}(10 \mathrm{~B})-\mathrm{H}(10 \mathrm{~B})$ & 120.1 \\
\hline $\mathrm{C}(10 \mathrm{~B})-\mathrm{C}(11 \mathrm{~B})-\mathrm{O}(1 \mathrm{~B})$ & $125.04(13)$ \\
\hline $\mathrm{C}(10 \mathrm{~B})-\mathrm{C}(11 \mathrm{~B})-\mathrm{C}(12 \mathrm{~B})$ & $121.23(13)$ \\
\hline $\mathrm{O}(1 \mathrm{~B})-\mathrm{C}(11 \mathrm{~B})-\mathrm{C}(12 \mathrm{~B})$ & $113.71(12)$ \\
\hline $\mathrm{C}(13 \mathrm{~B})-\mathrm{C}(12 \mathrm{~B})-\mathrm{C}(11 \mathrm{~B})$ & $122.41(12)$ \\
\hline$C(13 B)-C(12 B)-C(7 B)$ & $119.39(12)$ \\
\hline $\mathrm{C}(11 \mathrm{~B})-\mathrm{C}(12 \mathrm{~B})-\mathrm{C}(7 \mathrm{~B})$ & $118.19(12)$ \\
\hline $\mathrm{C}(14 \mathrm{~B})-\mathrm{C}(13 \mathrm{~B})-\mathrm{C}(12 \mathrm{~B})$ & $121.33(12)$ \\
\hline $\mathrm{C}(14 \mathrm{~B})-\mathrm{C}(13 \mathrm{~B})-\mathrm{H}(13 \mathrm{~B})$ & 119.3 \\
\hline $\mathrm{C}(12 \mathrm{~B})-\mathrm{C}(13 \mathrm{~B})-\mathrm{H}(13 \mathrm{~B})$ & 119.3 \\
\hline$C(13 B)-C(14 B)-C(1 B)$ & $122.30(12)$ \\
\hline $\mathrm{C}(13 \mathrm{~B})-\mathrm{C}(14 \mathrm{~B})-\mathrm{C}(5 \mathrm{~B})$ & $119.77(12)$ \\
\hline$C(1 B)-C(14 B)-C(5 B)$ & $117.92(12)$ \\
\hline $\mathrm{C}(16 \mathrm{~B})-\mathrm{C}(15 \mathrm{~B})-\mathrm{O}(1 \mathrm{~B})$ & $123.03(15)$ \\
\hline $\mathrm{C}(16 \mathrm{~B})-\mathrm{C}(15 \mathrm{~B})-\mathrm{H}(15 \mathrm{~B})$ & 118.5 \\
\hline $\mathrm{O}(1 \mathrm{~B})-\mathrm{C}(15 \mathrm{~B})-\mathrm{H}(15 \mathrm{~B})$ & 118.5 \\
\hline $\mathrm{C}(15 \mathrm{~B})-\mathrm{C}(16 \mathrm{~B})-\mathrm{H}(16 \mathrm{C})$ & 120.0 \\
\hline$C(15 B)-C(16 B)-H(16 D)$ & 120.0 \\
\hline
\end{tabular}




\begin{tabular}{|c|c|}
\hline $\mathrm{H}(16 \mathrm{C})-\mathrm{C}(16 \mathrm{~B})-\mathrm{H}(16 \mathrm{D})$ & 120.0 \\
\hline $\mathrm{C}(18 \mathrm{~B})-\mathrm{C}(17 \mathrm{~B})-\mathrm{O}(2 \mathrm{~B})$ & $121.96(15)$ \\
\hline $\mathrm{C}(18 \mathrm{~B})-\mathrm{C}(17 \mathrm{~B})-\mathrm{H}(17 \mathrm{~B})$ & 119.0 \\
\hline $\mathrm{O}(2 \mathrm{~B})-\mathrm{C}(17 \mathrm{~B})-\mathrm{H}(17 \mathrm{~B})$ & 119.0 \\
\hline $\mathrm{C}(17 \mathrm{~B})-\mathrm{C}(18 \mathrm{~B})-\mathrm{H}(18 \mathrm{C})$ & 120.0 \\
\hline C(17B)-C(18B)-H(18D) & 120.0 \\
\hline $\mathrm{H}(18 \mathrm{C})-\mathrm{C}(18 \mathrm{~B})-\mathrm{H}(18 \mathrm{D})$ & 120.0 \\
\hline $\mathrm{C}(15 \mathrm{C})-\mathrm{O}(1 \mathrm{C})-\mathrm{C}(11 \mathrm{C})$ & $117.90(12)$ \\
\hline $\mathrm{C}(1 \mathrm{C})-\mathrm{O}(2 \mathrm{C})-\mathrm{C}(17 \mathrm{C})$ & $118.03(11)$ \\
\hline $\mathrm{C}(2 \mathrm{C})-\mathrm{C}(1 \mathrm{C})-\mathrm{O}(2 \mathrm{C})$ & $123.62(13)$ \\
\hline$C(2 C)-C(1 C)-C(14 C)$ & $121.53(13)$ \\
\hline $\mathrm{O}(2 \mathrm{C})-\mathrm{C}(1 \mathrm{C})-\mathrm{C}(14 \mathrm{C})$ & $114.84(12)$ \\
\hline$C(1 C)-C(2 C)-C(3 C)$ & $119.82(14)$ \\
\hline $\mathrm{C}(1 \mathrm{C})-\mathrm{C}(2 \mathrm{C})-\mathrm{H}(2 \mathrm{CA})$ & 120.1 \\
\hline $\mathrm{C}(3 \mathrm{C})-\mathrm{C}(2 \mathrm{C})-\mathrm{H}(2 \mathrm{CA})$ & 120.1 \\
\hline$C(4 C)-C(3 C)-C(2 C)$ & $121.34(14)$ \\
\hline $\mathrm{C}(4 \mathrm{C})-\mathrm{C}(3 \mathrm{C})-\mathrm{H}(3 \mathrm{CA})$ & 119.3 \\
\hline $\mathrm{C}(2 \mathrm{C})-\mathrm{C}(3 \mathrm{C})-\mathrm{H}(3 \mathrm{CA})$ & 119.3 \\
\hline$C(3 C)-C(4 C)-C(5 C)$ & $120.31(13)$ \\
\hline $\mathrm{C}(3 \mathrm{C})-\mathrm{C}(4 \mathrm{C})-\mathrm{H}(4 \mathrm{CA})$ & 119.8 \\
\hline $\mathrm{C}(5 \mathrm{C})-\mathrm{C}(4 \mathrm{C})-\mathrm{H}(4 \mathrm{CA})$ & 119.8 \\
\hline $\mathrm{C}(6 \mathrm{C})-\mathrm{C}(5 \mathrm{C})-\mathrm{C}(4 \mathrm{C})$ & $122.32(13)$ \\
\hline $\mathrm{C}(6 \mathrm{C})-\mathrm{C}(5 \mathrm{C})-\mathrm{C}(14 \mathrm{C})$ & $118.39(13)$ \\
\hline$C(4 C)-C(5 C)-C(14 C)$ & $119.30(13)$ \\
\hline$C(7 C)-C(6 C)-C(5 C)$ & $122.34(13)$ \\
\hline $\mathrm{C}(7 \mathrm{C})-\mathrm{C}(6 \mathrm{C})-\mathrm{H}(6 \mathrm{CA})$ & 118.8 \\
\hline $\mathrm{C}(5 \mathrm{C})-\mathrm{C}(6 \mathrm{C})-\mathrm{H}(6 \mathrm{CA})$ & 118.8 \\
\hline$C(6 C)-C(7 C)-C(8 C)$ & $122.01(13)$ \\
\hline$C(6 C)-C(7 C)-C(12 C)$ & $118.93(12)$ \\
\hline$C(8 C)-C(7 C)-C(12 C)$ & $119.05(13)$ \\
\hline$C(9 C)-C(8 C)-C(7 C)$ & $120.69(14)$ \\
\hline $\mathrm{C}(9 \mathrm{C})-\mathrm{C}(8 \mathrm{C})-\mathrm{H}(8 \mathrm{CA})$ & 119.7 \\
\hline $\mathrm{C}(7 \mathrm{C})-\mathrm{C}(8 \mathrm{C})-\mathrm{H}(8 \mathrm{CA})$ & 119.7 \\
\hline$C(8 C)-C(9 C)-C(10 C)$ & $121.13(14)$ \\
\hline $\mathrm{C}(8 \mathrm{C})-\mathrm{C}(9 \mathrm{C})-\mathrm{H}(9 \mathrm{CA})$ & 119.4 \\
\hline $\mathrm{C}(10 \mathrm{C})-\mathrm{C}(9 \mathrm{C})-\mathrm{H}(9 \mathrm{CA})$ & 119.4 \\
\hline
\end{tabular}




$\begin{array}{ll}\mathrm{C}(11 \mathrm{C})-\mathrm{C}(10 \mathrm{C})-\mathrm{C}(9 \mathrm{C}) & 119.90(15) \\ \mathrm{C}(11 \mathrm{C})-\mathrm{C}(10 \mathrm{C})-\mathrm{H}(10 \mathrm{C}) & 120.0 \\ \mathrm{C}(9 \mathrm{C})-\mathrm{C}(10 \mathrm{C})-\mathrm{H}(10 \mathrm{C}) & 120.0 \\ \mathrm{C}(10 \mathrm{C})-\mathrm{C}(11 \mathrm{C})-\mathrm{O}(1 \mathrm{C}) & 123.48(13) \\ \mathrm{C}(10 \mathrm{C})-\mathrm{C}(11 \mathrm{C})-\mathrm{C}(12 \mathrm{C}) & 121.56(14) \\ \mathrm{O}(1 \mathrm{C})-\mathrm{C}(11 \mathrm{C})-\mathrm{C}(12 \mathrm{C}) & 114.89(12) \\ \mathrm{C}(13 \mathrm{C})-\mathrm{C}(12 \mathrm{C})-\mathrm{C}(7 \mathrm{C}) & 119.41(12) \\ \mathrm{C}(13 \mathrm{C})-\mathrm{C}(12 \mathrm{C})-\mathrm{C}(11 \mathrm{C}) & 122.95(13) \\ \mathrm{C}(7 \mathrm{C})-\mathrm{C}(12 \mathrm{C})-\mathrm{C}(11 \mathrm{C}) & 117.63(12) \\ \mathrm{C}(12 \mathrm{C})-\mathrm{C}(13 \mathrm{C})-\mathrm{C}(14 \mathrm{C}) & 121.08(12) \\ \mathrm{C}(12 \mathrm{C})-\mathrm{C}(13 \mathrm{C})-\mathrm{H}(13 \mathrm{C}) & 119.5 \\ \mathrm{C}(14 \mathrm{C})-\mathrm{C}(13 \mathrm{C})-\mathrm{H}(13 \mathrm{C}) & 119.5 \\ \mathrm{C}(13 \mathrm{C})-\mathrm{C}(14 \mathrm{C})-\mathrm{C}(1 \mathrm{C}) & 122.45(12) \\ \mathrm{C}(13 \mathrm{C})-\mathrm{C}(14 \mathrm{C})-\mathrm{C}(5 \mathrm{C}) & 119.85(12) \\ \mathrm{C}(1 \mathrm{C})-\mathrm{C}(14 \mathrm{C})-\mathrm{C}(5 \mathrm{C}) & 117.70(12) \\ \mathrm{C}(16 \mathrm{C})-\mathrm{C}(15 \mathrm{C})-\mathrm{O}(1 \mathrm{C}) & 123.37(16) \\ \mathrm{C}(16 \mathrm{C})-\mathrm{C}(15 \mathrm{C})-\mathrm{H}(15 \mathrm{C}) & 118.3 \\ \mathrm{O}(1 \mathrm{C})-\mathrm{C}(15 \mathrm{C})-\mathrm{H}(15 \mathrm{C}) & 118.3 \\ \mathrm{C}(15 \mathrm{C})-\mathrm{C}(16 \mathrm{C})-\mathrm{H}(16 \mathrm{E}) & 120.0 \\ \mathrm{C}(15 \mathrm{C})-\mathrm{C}(16 \mathrm{C})-\mathrm{H}(16 \mathrm{~F}) & 120.0 \\ \mathrm{H}(16 \mathrm{E})-\mathrm{C}(16 \mathrm{C})-\mathrm{H}(16 \mathrm{~F}) & 120.0 \\ \mathrm{C}(18 \mathrm{C})-\mathrm{C}(17 \mathrm{C})-\mathrm{O}(2 \mathrm{C}) & 122.21(15) \\ \mathrm{C}(18 \mathrm{C})-\mathrm{C}(17 \mathrm{C})-\mathrm{H}(17 \mathrm{C}) & 118.9 \\ \mathrm{O}(2 \mathrm{C})-\mathrm{C}(17 \mathrm{C})-\mathrm{H}(17 \mathrm{C}) & 118.9 \\ \mathrm{C}(17 \mathrm{C})-\mathrm{C}(18 \mathrm{C})-\mathrm{H}(18 \mathrm{E}) & 120.0 \\ \mathrm{C}(17 \mathrm{C})-\mathrm{C}(18 \mathrm{C})-\mathrm{H}(18 \mathrm{~F}) & 120.0 \\ \mathrm{H}(18 \mathrm{E})-\mathrm{C}(18 \mathrm{C})-\mathrm{H}(18 \mathrm{~F}) & 120.0 \\ & \end{array}$

Symmetry transformations used to generate equivalent atoms: 
Table 4. Anisotropic displacement parameters $\left(\AA^{2} \times 10^{3}\right)$ for d1637. The anisotropic displacement factor exponent takes the form: $-2 \pi^{2}\left[h^{2} a^{* 2} U^{11}+\ldots+2 h k a^{*} b^{*} U^{12}\right]$

\begin{tabular}{|c|c|c|c|c|c|c|}
\hline & $\mathrm{U}^{11}$ & $\mathrm{U}^{22}$ & $\mathrm{U}^{33}$ & $\mathrm{U}^{23}$ & $\mathrm{U}^{13}$ & $\mathrm{U}^{12}$ \\
\hline $\mathrm{O}(1 \mathrm{~A})$ & $29(1)$ & $36(1)$ & $37(1)$ & $8(1)$ & $6(1)$ & $-1(1)$ \\
\hline $\mathrm{O}(2 \mathrm{~A})$ & $44(1)$ & $34(1)$ & $37(1)$ & $6(1)$ & $-5(1)$ & $3(1)$ \\
\hline $\mathrm{C}(1 \mathrm{~A})$ & $45(1)$ & $27(1)$ & $33(1)$ & $-2(1)$ & $2(1)$ & $0(1)$ \\
\hline $\mathrm{C}(2 \mathrm{~A})$ & $67(1)$ & $34(1)$ & $34(1)$ & $7(1)$ & $7(1)$ & $3(1)$ \\
\hline$C(3 A)$ & $67(1)$ & $42(1)$ & $44(1)$ & $7(1)$ & $23(1)$ & $-4(1)$ \\
\hline $\mathrm{C}(4 \mathrm{~A})$ & $49(1)$ & $39(1)$ & $44(1)$ & $0(1)$ & $19(1)$ & $-5(1)$ \\
\hline $\mathrm{C}(5 \mathrm{~A})$ & $41(1)$ & $29(1)$ & $32(1)$ & $-5(1)$ & $10(1)$ & $-5(1)$ \\
\hline$C(6 A)$ & $31(1)$ & $34(1)$ & $37(1)$ & $-7(1)$ & $8(1)$ & $-3(1)$ \\
\hline $\mathrm{C}(7 \mathrm{~A})$ & $32(1)$ & $26(1)$ & $32(1)$ & $-6(1)$ & $3(1)$ & $-2(1)$ \\
\hline $\mathrm{C}(8 \mathrm{~A})$ & $29(1)$ & $34(1)$ & $40(1)$ & $-6(1)$ & $-1(1)$ & 2(1) \\
\hline $\mathrm{C}(9 \mathrm{~A})$ & $38(1)$ & $32(1)$ & $37(1)$ & $1(1)$ & $-5(1)$ & $4(1)$ \\
\hline$C(10 A)$ & 41(1) & $30(1)$ & $31(1)$ & $3(1)$ & 2(1) & $-1(1)$ \\
\hline $\mathrm{C}(11 \mathrm{~A})$ & $29(1)$ & $26(1)$ & $32(1)$ & $-2(1)$ & $4(1)$ & $-1(1)$ \\
\hline $\mathrm{C}(12 \mathrm{~A})$ & $30(1)$ & $22(1)$ & $29(1)$ & $-4(1)$ & 2(1) & $-1(1)$ \\
\hline$C(13 A)$ & $29(1)$ & $25(1)$ & $30(1)$ & $-3(1)$ & $3(1)$ & $-2(1)$ \\
\hline $\mathrm{C}(14 \mathrm{~A})$ & $39(1)$ & $23(1)$ & $28(1)$ & $-3(1)$ & $4(1)$ & $-3(1)$ \\
\hline$C(15 A)$ & $38(1)$ & $36(1)$ & $37(1)$ & $6(1)$ & $6(1)$ & $-4(1)$ \\
\hline$C(16 A)$ & $43(1)$ & $54(1)$ & $48(1)$ & $5(1)$ & $14(1)$ & $-6(1)$ \\
\hline $\mathrm{C}(17 \mathrm{~A})$ & $57(1)$ & $38(1)$ & $39(1)$ & $6(1)$ & $-4(1)$ & $9(1)$ \\
\hline $\mathrm{C}(18 \mathrm{~A})$ & $62(1)$ & $52(1)$ & $57(1)$ & $6(1)$ & $-16(1)$ & $10(1)$ \\
\hline $\mathrm{O}(1 \mathrm{~B})$ & $33(1)$ & $34(1)$ & $42(1)$ & $6(1)$ & $0(1)$ & $4(1)$ \\
\hline $\mathrm{O}(2 \mathrm{~B})$ & $28(1)$ & $40(1)$ & $42(1)$ & $10(1)$ & 2(1) & $-2(1)$ \\
\hline $\mathrm{C}(1 \mathrm{~B})$ & $29(1)$ & $34(1)$ & $29(1)$ & $-2(1)$ & 1(1) & $-1(1)$ \\
\hline $\mathrm{C}(2 \mathrm{~B})$ & $39(1)$ & $31(1)$ & $38(1)$ & $3(1)$ & $2(1)$ & 1(1) \\
\hline $\mathrm{C}(3 \mathrm{~B})$ & $38(1)$ & $39(1)$ & $47(1)$ & $5(1)$ & $0(1)$ & $9(1)$ \\
\hline $\mathrm{C}(4 \mathrm{~B})$ & $29(1)$ & $43(1)$ & $45(1)$ & $2(1)$ & 2(1) & $6(1)$ \\
\hline $\mathrm{C}(5 \mathrm{~B})$ & $30(1)$ & $34(1)$ & $28(1)$ & $-4(1)$ & $2(1)$ & 1(1) \\
\hline $\mathrm{C}(6 \mathrm{~B})$ & $27(1)$ & $40(1)$ & $34(1)$ & $-5(1)$ & $4(1)$ & $0(1)$ \\
\hline $\mathrm{C}(7 \mathrm{~B})$ & $33(1)$ & $36(1)$ & $24(1)$ & $-4(1)$ & $3(1)$ & $-3(1)$ \\
\hline $\mathrm{C}(8 \mathrm{~B})$ & $36(1)$ & $43(1)$ & $31(1)$ & $-1(1)$ & $7(1)$ & $-7(1)$ \\
\hline $\mathrm{C}(9 \mathrm{~B})$ & $50(1)$ & $38(1)$ & $33(1)$ & $3(1)$ & $10(1)$ & $-8(1)$ \\
\hline
\end{tabular}




\begin{tabular}{|c|c|c|c|c|c|c|}
\hline$C(10 B)$ & $48(1)$ & $36(1)$ & $31(1)$ & $5(1)$ & $3(1)$ & $2(1)$ \\
\hline $\mathrm{C}(11 \mathrm{~B})$ & $34(1)$ & $32(1)$ & $27(1)$ & $-3(1)$ & $2(1)$ & $0(1)$ \\
\hline$C(12 B)$ & $32(1)$ & $31(1)$ & $22(1)$ & $-4(1)$ & 1(1) & $-1(1)$ \\
\hline$C(13 B)$ & $26(1)$ & $32(1)$ & $27(1)$ & $-4(1)$ & $1(1)$ & $0(1)$ \\
\hline$C(14 B)$ & $30(1)$ & $30(1)$ & $24(1)$ & $-4(1)$ & $0(1)$ & 1(1) \\
\hline$C(15 B)$ & $42(1)$ & $34(1)$ & $66(1)$ & $9(1)$ & $-3(1)$ & $5(1)$ \\
\hline$C(16 B)$ & $42(1)$ & $40(1)$ & $54(1)$ & $3(1)$ & $0(1)$ & $10(1)$ \\
\hline$C(17 B)$ & $38(1)$ & $46(1)$ & $44(1)$ & 12(1) & $8(1)$ & $1(1)$ \\
\hline$C(18 B)$ & $42(1)$ & $60(1)$ & $51(1)$ & $4(1)$ & $12(1)$ & $-11(1)$ \\
\hline $\mathrm{O}(1 \mathrm{C})$ & $35(1)$ & $36(1)$ & $39(1)$ & $9(1)$ & $6(1)$ & $-1(1)$ \\
\hline $\mathrm{O}(2 \mathrm{C})$ & $31(1)$ & $36(1)$ & $41(1)$ & $10(1)$ & $-1(1)$ & $2(1)$ \\
\hline $\mathrm{C}(1 \mathrm{C})$ & $33(1)$ & $24(1)$ & $32(1)$ & $-2(1)$ & $2(1)$ & $-1(1)$ \\
\hline$C(2 C)$ & $44(1)$ & $30(1)$ & $31(1)$ & $2(1)$ & $2(1)$ & $-1(1)$ \\
\hline $\mathrm{C}(3 \mathrm{C})$ & $48(1)$ & $33(1)$ & $36(1)$ & $0(1)$ & $13(1)$ & $-7(1)$ \\
\hline$C(4 C)$ & $35(1)$ & $34(1)$ & $39(1)$ & $-7(1)$ & $10(1)$ & $-5(1)$ \\
\hline $\mathrm{C}(5 \mathrm{C})$ & $32(1)$ & $24(1)$ & $34(1)$ & $-7(1)$ & $5(1)$ & $-2(1)$ \\
\hline$C(6 C)$ & $27(1)$ & $31(1)$ & $38(1)$ & $-8(1)$ & $3(1)$ & $0(1)$ \\
\hline$C(7 C)$ & $32(1)$ & $26(1)$ & $35(1)$ & $-5(1)$ & $-1(1)$ & $2(1)$ \\
\hline $\mathrm{C}(8 \mathrm{C})$ & $34(1)$ & $35(1)$ & $42(1)$ & $-3(1)$ & $-5(1)$ & $7(1)$ \\
\hline $\mathrm{C}(9 \mathrm{C})$ & $46(1)$ & $38(1)$ & $41(1)$ & $5(1)$ & $-9(1)$ & $7(1)$ \\
\hline$C(10 C)$ & $50(1)$ & $36(1)$ & $37(1)$ & $8(1)$ & $2(1)$ & $0(1)$ \\
\hline$C(11 C)$ & $34(1)$ & $26(1)$ & $37(1)$ & $1(1)$ & $2(1)$ & $1(1)$ \\
\hline $\mathrm{C}(12 \mathrm{C})$ & $32(1)$ & $22(1)$ & $30(1)$ & $-2(1)$ & $2(1)$ & $1(1)$ \\
\hline$C(13 C)$ & $28(1)$ & $25(1)$ & $32(1)$ & $-2(1)$ & $2(1)$ & $0(1)$ \\
\hline$C(14 C)$ & $31(1)$ & $21(1)$ & $30(1)$ & $-4(1)$ & $3(1)$ & $-1(1)$ \\
\hline$C(15 C)$ & $49(1)$ & $47(1)$ & $47(1)$ & $15(1)$ & $10(1)$ & $-2(1)$ \\
\hline$C(16 C)$ & $49(1)$ & $42(1)$ & $58(1)$ & $3(1)$ & $17(1)$ & $-8(1)$ \\
\hline $\mathrm{C}(17 \mathrm{C})$ & $41(1)$ & $38(1)$ & $38(1)$ & $8(1)$ & 1(1) & $4(1)$ \\
\hline$C(18 C)$ & $40(1)$ & $45(1)$ & $43(1)$ & $9(1)$ & $-3(1)$ & $4(1)$ \\
\hline
\end{tabular}


Table 5. Hydrogen coordinates ( x 104) and isotropic displacement parameters $\left(\AA^{2} \times 10^{3}\right)$ for d 1637 .

\begin{tabular}{|c|c|c|c|c|}
\hline & $\mathrm{x}$ & $\mathrm{y}$ & $\mathrm{z}$ & $\mathrm{U}(\mathrm{eq})$ \\
\hline $\mathrm{H}(2 \mathrm{AA})$ & 1657 & 3864 & 5656 & 54 \\
\hline $\mathrm{H}(3 \mathrm{AA})$ & 726 & 3801 & 5599 & 60 \\
\hline $\mathrm{H}(4 \mathrm{AA})$ & 189 & 4662 & 4311 & 51 \\
\hline H(6AA) & 95 & 5898 & 2800 & 40 \\
\hline $\mathrm{H}(8 \mathrm{AA})$ & -19 & 7045 & 1212 & 42 \\
\hline H(9AA) & 347 & 7861 & -120 & 44 \\
\hline $\mathrm{H}(10 \mathrm{~A})$ & 1273 & 7835 & -179 & 41 \\
\hline $\mathrm{H}(13 \mathrm{~A})$ & 1943 & 5896 & 2755 & 33 \\
\hline $\mathrm{H}(15 \mathrm{~A})$ & 2079 & 8402 & 442 & 44 \\
\hline $\mathrm{H}(16 \mathrm{~A})$ & 2844 & 6764 & 382 & 57 \\
\hline $\mathrm{H}(16 \mathrm{~B})$ & 2840 & 7986 & -175 & 57 \\
\hline $\mathrm{H}(17 \mathrm{~A})$ & 2377 & 3315 & 4971 & 55 \\
\hline $\mathrm{H}(18 \mathrm{~A})$ & 3183 & 4860 & 4931 & 71 \\
\hline H(18B) & 3239 & 3612 & 5436 & 71 \\
\hline $\mathrm{H}(2 \mathrm{BA})$ & 4868 & 585 & 6060 & 44 \\
\hline $\mathrm{H}(3 \mathrm{BA})$ & 5795 & 674 & 6073 & 50 \\
\hline $\mathrm{H}(4 \mathrm{BA})$ & 6183 & 2053 & 5227 & 47 \\
\hline $\mathrm{H}(6 \mathrm{BA})$ & 6088 & 3791 & 4191 & 40 \\
\hline $\mathrm{H}(8 \mathrm{BA})$ & 6000 & 5533 & 3157 & 44 \\
\hline H(9BA) & 5464 & 6847 & 2313 & 48 \\
\hline $\mathrm{H}(10 \mathrm{~B})$ & 4533 & 6758 & 2254 & 46 \\
\hline $\mathrm{H}(13 \mathrm{~B})$ & 4242 & 3667 & 4204 & 34 \\
\hline $\mathrm{H}(15 \mathrm{~B})$ & 3783 & 6826 & 2806 & 58 \\
\hline $\mathrm{H}(16 \mathrm{C})$ & 2983 & 5267 & 2788 & 55 \\
\hline H(16D) & 2911 & 6594 & 2527 & 55 \\
\hline $\mathrm{H}(17 \mathrm{~B})$ & 4088 & 1141 & 6441 & 51 \\
\hline $\mathrm{H}(18 \mathrm{C})$ & 3273 & 1024 & 4829 & 61 \\
\hline H(18D) & 3289 & 419 & 5960 & 61 \\
\hline $\mathrm{H}(2 \mathrm{CA})$ & 1956 & 2162 & 3238 & 43 \\
\hline $\mathrm{H}(3 \mathrm{CA})$ & 1025 & 2172 & 3200 & 46 \\
\hline
\end{tabular}




\begin{tabular}{lrrrr}
$\mathrm{H}(4 \mathrm{CA})$ & 496 & 3093 & 1931 & 43 \\
$\mathrm{H}(6 \mathrm{CA})$ & 418 & 4220 & 326 & 39 \\
$\mathrm{H}(8 \mathrm{CA})$ & 322 & 5286 & -1306 & 45 \\
$\mathrm{H}(9 \mathrm{CA})$ & 701 & 6045 & -2644 & 52 \\
$\mathrm{H}(10 \mathrm{C})$ & 1629 & 5995 & -2674 & 49 \\
$\mathrm{H}(13 \mathrm{C})$ & 2268 & 4167 & 346 & 34 \\
$\mathrm{H}(15 \mathrm{C})$ & 2416 & 6574 & -2039 & 57 \\
$\mathrm{H}(16 \mathrm{E})$ & 3233 & 5056 & -1967 & 59 \\
$\mathrm{H}(16 \mathrm{~F})$ & 3204 & 6270 & -2540 & 59 \\
$\mathrm{H}(17 \mathrm{C})$ & 2685 & 1601 & 2503 & 47 \\
$\mathrm{H}(18 \mathrm{E})$ & 3448 & 3247 & 2835 & 52 \\
$\mathrm{H}(18 \mathrm{~F})$ & 3506 & 1958 & 3236 & 52 \\
& & & & \\
\hline
\end{tabular}


Table 6. Torsion angles $\left[{ }^{\circ}\right]$ for $\mathrm{d} 1637$.

\begin{tabular}{|c|c|}
\hline $\mathrm{C}(17 \mathrm{~A})-\mathrm{O}(2 \mathrm{~A})-\mathrm{C}(1 \mathrm{~A})-\mathrm{C}(2 \mathrm{~A})$ & $22.8(2)$ \\
\hline $\mathrm{C}(17 \mathrm{~A})-\mathrm{O}(2 \mathrm{~A})-\mathrm{C}(1 \mathrm{~A})-\mathrm{C}(14 \mathrm{~A})$ & $-159.12(13)$ \\
\hline $\mathrm{O}(2 \mathrm{~A})-\mathrm{C}(1 \mathrm{~A})-\mathrm{C}(2 \mathrm{~A})-\mathrm{C}(3 \mathrm{~A})$ & $-179.08(14)$ \\
\hline$C(14 \mathrm{~A})-\mathrm{C}(1 \mathrm{~A})-\mathrm{C}(2 \mathrm{~A})-\mathrm{C}(3 \mathrm{~A})$ & $2.9(2)$ \\
\hline$C(1 \mathrm{~A})-\mathrm{C}(2 \mathrm{~A})-\mathrm{C}(3 \mathrm{~A})-\mathrm{C}(4 \mathrm{~A})$ & $-1.8(3)$ \\
\hline$C(2 A)-C(3 A)-C(4 A)-C(5 A)$ & $-1.2(3)$ \\
\hline$C(3 A)-C(4 A)-C(5 A)-C(6 A)$ & $-177.07(15)$ \\
\hline$C(3 A)-C(4 A)-C(5 A)-C(14 A)$ & $3.0(2)$ \\
\hline $\mathrm{C}(4 \mathrm{~A})-\mathrm{C}(5 \mathrm{~A})-\mathrm{C}(6 \mathrm{~A})-\mathrm{C}(7 \mathrm{~A})$ & $-177.78(14)$ \\
\hline $\mathrm{C}(14 \mathrm{~A})-\mathrm{C}(5 \mathrm{~A})-\mathrm{C}(6 \mathrm{~A})-\mathrm{C}(7 \mathrm{~A})$ & $2.2(2)$ \\
\hline $\mathrm{C}(5 \mathrm{~A})-\mathrm{C}(6 \mathrm{~A})-\mathrm{C}(7 \mathrm{~A})-\mathrm{C}(8 \mathrm{~A})$ & $177.94(13)$ \\
\hline$C(5 A)-C(6 A)-C(7 A)-C(12 A)$ & $-1.0(2)$ \\
\hline $\mathrm{C}(6 \mathrm{~A})-\mathrm{C}(7 \mathrm{~A})-\mathrm{C}(8 \mathrm{~A})-\mathrm{C}(9 \mathrm{~A})$ & $-176.66(14)$ \\
\hline $\mathrm{C}(12 \mathrm{~A})-\mathrm{C}(7 \mathrm{~A})-\mathrm{C}(8 \mathrm{~A})-\mathrm{C}(9 \mathrm{~A})$ & $2.3(2)$ \\
\hline $\mathrm{C}(7 \mathrm{~A})-\mathrm{C}(8 \mathrm{~A})-\mathrm{C}(9 \mathrm{~A})-\mathrm{C}(10 \mathrm{~A})$ & $-1.5(2)$ \\
\hline $\mathrm{C}(8 \mathrm{~A})-\mathrm{C}(9 \mathrm{~A})-\mathrm{C}(10 \mathrm{~A})-\mathrm{C}(11 \mathrm{~A})$ & $-0.9(2)$ \\
\hline $\mathrm{C}(9 \mathrm{~A})-\mathrm{C}(10 \mathrm{~A})-\mathrm{C}(11 \mathrm{~A})-\mathrm{O}(1 \mathrm{~A})$ & $-179.07(12)$ \\
\hline $\mathrm{C}(9 \mathrm{~A})-\mathrm{C}(10 \mathrm{~A})-\mathrm{C}(11 \mathrm{~A})-\mathrm{C}(12 \mathrm{~A})$ & $2.5(2)$ \\
\hline$C(15 A)-O(1 A)-C(11 A)-C(10 A)$ & $18.18(19)$ \\
\hline$C(15 A)-O(1 A)-C(11 A)-C(12 A)$ & $-163.27(12)$ \\
\hline $\mathrm{C}(10 \mathrm{~A})-\mathrm{C}(11 \mathrm{~A})-\mathrm{C}(12 \mathrm{~A})-\mathrm{C}(13 \mathrm{~A})$ & $177.29(13)$ \\
\hline$O(1 \mathrm{~A})-\mathrm{C}(11 \mathrm{~A})-\mathrm{C}(12 \mathrm{~A})-\mathrm{C}(13 \mathrm{~A})$ & $-1.28(18)$ \\
\hline $\mathrm{C}(10 \mathrm{~A})-\mathrm{C}(11 \mathrm{~A})-\mathrm{C}(12 \mathrm{~A})-\mathrm{C}(7 \mathrm{~A})$ & $-1.69(19)$ \\
\hline $\mathrm{O}(1 \mathrm{~A})-\mathrm{C}(11 \mathrm{~A})-\mathrm{C}(12 \mathrm{~A})-\mathrm{C}(7 \mathrm{~A})$ & $179.73(11)$ \\
\hline$C(6 A)-C(7 A)-C(12 A)-C(13 A)$ & $-0.73(19)$ \\
\hline $\mathrm{C}(8 \mathrm{~A})-\mathrm{C}(7 \mathrm{~A})-\mathrm{C}(12 \mathrm{~A})-\mathrm{C}(13 \mathrm{~A})$ & $-179.70(12)$ \\
\hline$C(6 A)-C(7 A)-C(12 A)-C(11 A)$ & $178.28(12)$ \\
\hline $\mathrm{C}(8 \mathrm{~A})-\mathrm{C}(7 \mathrm{~A})-\mathrm{C}(12 \mathrm{~A})-\mathrm{C}(11 \mathrm{~A})$ & $-0.69(18)$ \\
\hline $\mathrm{C}(11 \mathrm{~A})-\mathrm{C}(12 \mathrm{~A})-\mathrm{C}(13 \mathrm{~A})-\mathrm{C}(14 \mathrm{~A})$ & $-177.72(12)$ \\
\hline$C(7 A)-C(12 A)-C(13 A)-C(14 A)$ & $1.24(19)$ \\
\hline$C(12 A)-C(13 A)-C(14 A)-C(5 A)$ & $-0.06(19)$ \\
\hline $\mathrm{C}(12 \mathrm{~A})-\mathrm{C}(13 \mathrm{~A})-\mathrm{C}(14 \mathrm{~A})-\mathrm{C}(1 \mathrm{~A})$ & $-179.94(12)$ \\
\hline $\mathrm{C}(6 \mathrm{~A})-\mathrm{C}(5 \mathrm{~A})-\mathrm{C}(14 \mathrm{~A})-\mathrm{C}(13 \mathrm{~A})$ & $-1.63(19)$ \\
\hline$C(4 A)-C(5 A)-C(14 A)-C(13 A)$ & $178.32(13)$ \\
\hline
\end{tabular}




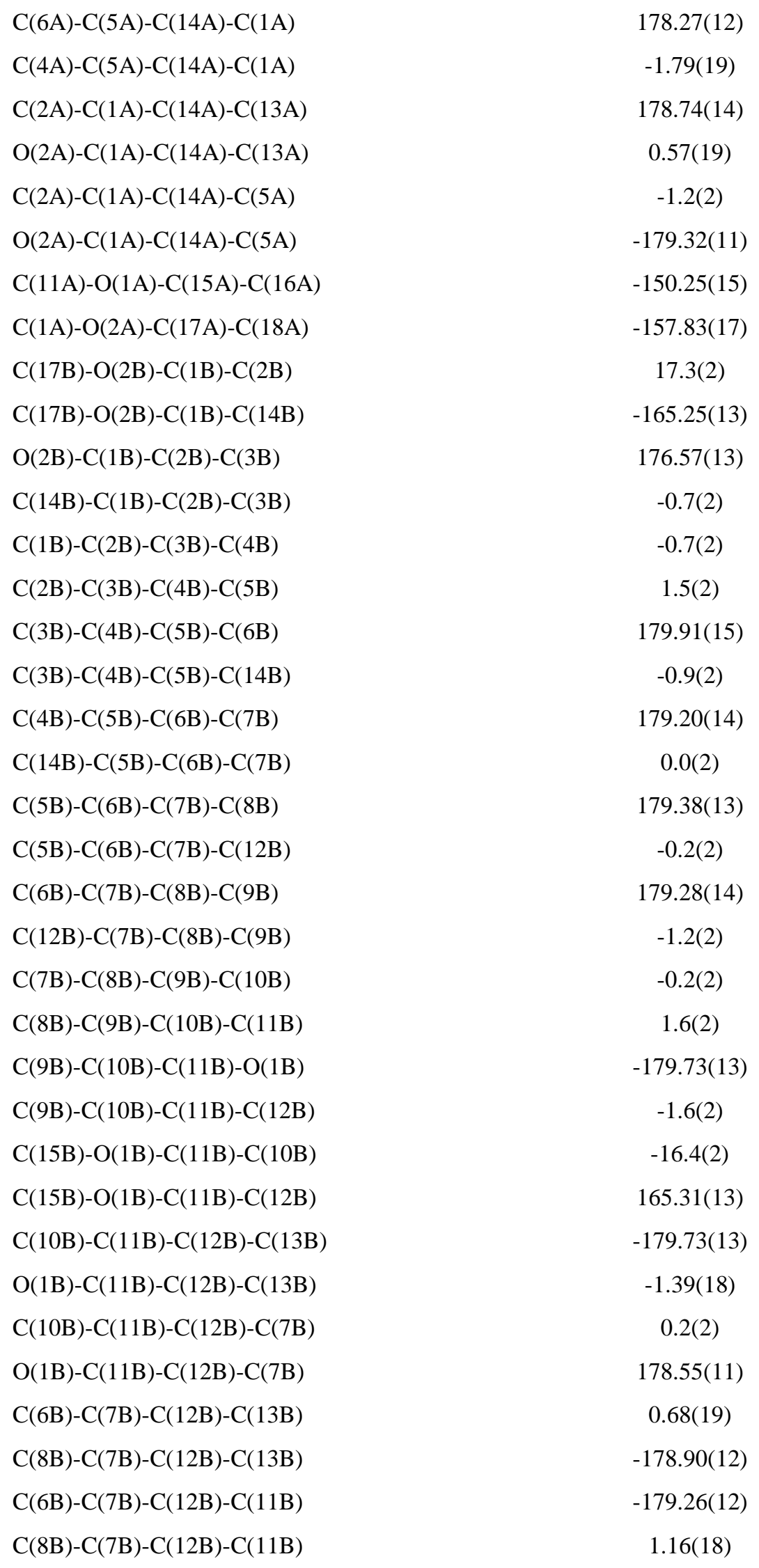




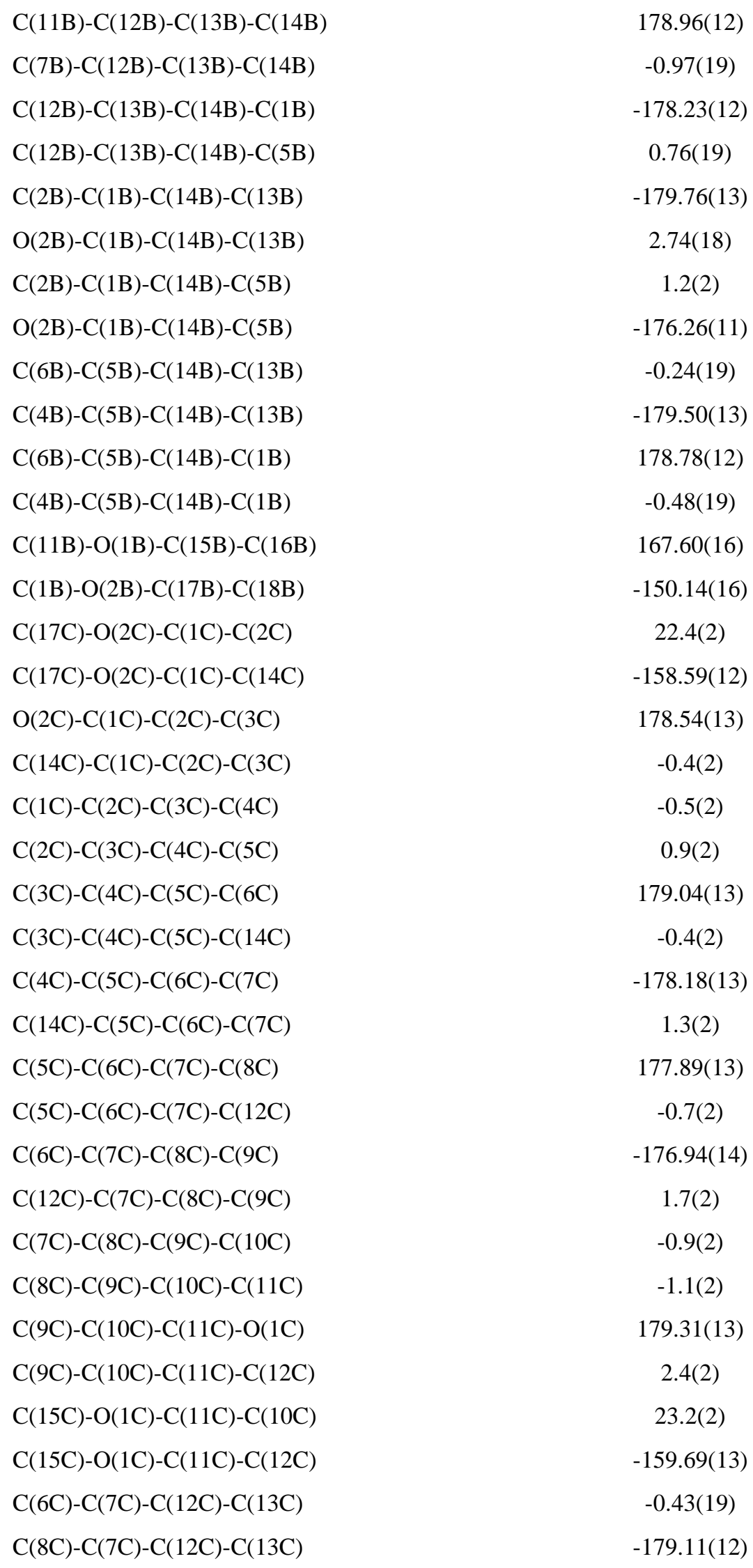




$\begin{array}{lc}\mathrm{C}(6 \mathrm{C})-\mathrm{C}(7 \mathrm{C})-\mathrm{C}(12 \mathrm{C})-\mathrm{C}(11 \mathrm{C}) & 178.24(12) \\ \mathrm{C}(8 \mathrm{C})-\mathrm{C}(7 \mathrm{C})-\mathrm{C}(12 \mathrm{C})-\mathrm{C}(11 \mathrm{C}) & -0.44(19) \\ \mathrm{C}(10 \mathrm{C})-\mathrm{C}(11 \mathrm{C})-\mathrm{C}(12 \mathrm{C})-\mathrm{C}(13 \mathrm{C}) & 177.02(14) \\ \mathrm{O}(1 \mathrm{C})-\mathrm{C}(11 \mathrm{C})-\mathrm{C}(12 \mathrm{C})-\mathrm{C}(13 \mathrm{C}) & -0.14(19) \\ \mathrm{C}(10 \mathrm{C})-\mathrm{C}(11 \mathrm{C})-\mathrm{C}(12 \mathrm{C})-\mathrm{C}(7 \mathrm{C}) & -1.6(2) \\ \mathrm{O}(1 \mathrm{C})-\mathrm{C}(11 \mathrm{C})-\mathrm{C}(12 \mathrm{C})-\mathrm{C}(7 \mathrm{C}) & -178.76(11) \\ \mathrm{C}(7 \mathrm{C})-\mathrm{C}(12 \mathrm{C})-\mathrm{C}(13 \mathrm{C})-\mathrm{C}(14 \mathrm{C}) & 1.06(19) \\ \mathrm{C}(11 \mathrm{C})-\mathrm{C}(12 \mathrm{C})-\mathrm{C}(13 \mathrm{C})-\mathrm{C}(14 \mathrm{C}) & -177.53(12) \\ \mathrm{C}(12 \mathrm{C})-\mathrm{C}(13 \mathrm{C})-\mathrm{C}(14 \mathrm{C})-\mathrm{C}(1 \mathrm{C}) & 178.73(12) \\ \mathrm{C}(12 \mathrm{C})-\mathrm{C}(13 \mathrm{C})-\mathrm{C}(14 \mathrm{C})-\mathrm{C}(5 \mathrm{C}) & -0.54(19) \\ \mathrm{C}(2 \mathrm{C})-\mathrm{C}(1 \mathrm{C})-\mathrm{C}(14 \mathrm{C})-\mathrm{C}(13 \mathrm{C}) & -178.44(13) \\ \mathrm{O}(2 \mathrm{C})-\mathrm{C}(1 \mathrm{C})-\mathrm{C}(14 \mathrm{C})-\mathrm{C}(13 \mathrm{C}) & 2.55(18) \\ \mathrm{C}(2 \mathrm{C})-\mathrm{C}(1 \mathrm{C})-\mathrm{C}(14 \mathrm{C})-\mathrm{C}(5 \mathrm{C}) & 0.85(19) \\ \mathrm{O}(2 \mathrm{C})-\mathrm{C}(1 \mathrm{C})-\mathrm{C}(14 \mathrm{C})-\mathrm{C}(5 \mathrm{C}) & -178.16(11) \\ \mathrm{C}(6 \mathrm{C})-\mathrm{C}(5 \mathrm{C})-\mathrm{C}(14 \mathrm{C})-\mathrm{C}(13 \mathrm{C}) & -0.62(19) \\ \mathrm{C}(4 \mathrm{C})-\mathrm{C}(5 \mathrm{C})-\mathrm{C}(14 \mathrm{C})-\mathrm{C}(13 \mathrm{C}) & 178.85(12) \\ \mathrm{C}(6 \mathrm{C})-\mathrm{C}(5 \mathrm{C})-\mathrm{C}(14 \mathrm{C})-\mathrm{C}(1 \mathrm{C}) & -179.92(12) \\ \mathrm{C}(4 \mathrm{C})-\mathrm{C}(5 \mathrm{C})-\mathrm{C}(14 \mathrm{C})-\mathrm{C}(1 \mathrm{C}) & -0.45(18) \\ \mathrm{C}(11 \mathrm{C})-\mathrm{O}(1 \mathrm{C})-\mathrm{C}(15 \mathrm{C})-\mathrm{C}(16 \mathrm{C}) & -157.14(17) \\ \mathrm{C}(1 \mathrm{C})-\mathrm{O}(2 \mathrm{C})-\mathrm{C}(17 \mathrm{C})-\mathrm{C}(18 \mathrm{C}) & -147.65(15) \\ & \\ & \end{array}$

Symmetry transformations used to generate equivalent atoms: 


\section{References}

(1) McQuarrie, D. A.; Simon, J. D. Physical chemistry: a molecular approach; 1997.

(2) Hussain, S. A. Energy 2009, 132 (6), 4.

(3) Balzani, V. Electron Transfer in Chemistry; 2008; Vol. 1.

(4) Kippelen, B.; Brédas, J.-L. Energy Environ. Sci. 2009, 2 (3), 251.

(5) Mao, M.; Zhang, X. L.; Fang, X. Q.; Wu, G. H.; Ding, Y.; Liu, X. L.; Dai, S. Y.; Song, Q. H. Org. Electron. physics, Mater. Appl. 2014, 15 (9), 2079.

(6) O’Regan, B.; Grätzel, M. Nature 1991, 353 (6346), 737.

(7) Hagfeldt, A.; Boschloo, G.; Sun, L.; Kloo, L.; Pettersson, H. Chem. Rev. 2010, 110 (11), 6595.

(8) Grätzel, M. Journal of Photochemistry and Photobiology C: Photochemistry Reviews. 2003, pp 145-153.

(9) Nguyen, T. V.; Lee, H. C.; Alam Khan, M.; Yang, O. B. Sol. Energy 2007, 81 (4), 529.

(10) Bonnier, C.; Machin, D. D.; Abdi, O.; Koivisto, B. D. Org. Biomol. Chem. 2013, 11 (22), 3756.

(11) Loudet, A.; Burgess, K. Chem. Rev. 2007, 107 (11), 4891.

(12) Boens, N.; Leen, V.; Dehaen, W. Chem. Soc. Rev. 2012, 41 (3), 1130.

(13) Hayashi, Y.; Yamaguchi, S.; Cha, W. Y.; Kim, D.; Shinokubo, H. Org. Lett. 2011, 13 (12), 2992.

(14) Ulrich, G.; Ziessel, R.; Harriman, A. Angewandte Chemie - International Edition. 2008, pp 11841201.

(15) Rousseau, T.; Cravino, A.; Bura, T.; Ulrich, G.; Ziessel, R.; Roncali, J. Chem. Commun. 2009, No. 13, 1673.

(16) Leen, V.; Yuan, P.; Wang, L.; Boens, N.; Dehaen, W. Org. Lett. 2012, 14 (24), 6150.

(17) Bessette, a; Hanan, G. S. Chem Soc Rev 2014, 43 (10), 3342.

(18) Ni, Y. Org. Biomol. Chem. 2014, 12 (23), 3774.

(19) Bonardi, L.; Ulrich, G.; Ziessel, R. Org. Lett. 2008, 10 (11), 2183.

(20) Cartrette, D. P.; Miller, M. L. J. Chem. Educ. 2013, 90 (2), 171. 
(21) Staniforth, M.; Young, J. D.; Cole, D. R.; Karsili, T. N. V; Ashfold, M. N. R.; Stavros, V. G. J. Phys. Chem. A 2014, 118 (46), 10909.

(22) Yuwei He, Maohui Lin, Zhongmin Li, Xinting Liang, Guilong Li, and J. C. A. Org. Electron. physics, Mater. Appl. 2011, 51 (30), 4490.

(23) Wang, J. B.; Fang, X. Q.; Pan, X.; Dai, S. Y.; Song, Q. H. Chem. - An Asian J. 2012, 7 (4), 696.

(24) M. J. Frisch, G. W. Trucks, H. B. Schlegel, G. E. Scuseria, M. A. Robb, J. R. Cheeseman, J. A. Montgomery Jr., T. Vreven, K. N. Kudin, J. C. Burant, J.M.Millam, S. S. Iyengar, J. Tomasi, V. Barone, B. Mennucci, M. Cossi, G. Scalmani, N. Rega, G. A. Peters, C. G. and J. A. P. Gaussian, Inc., Wallingford CT 2004.

(25) Zhu, S.; Bi, J.; Vegesna, G.; Zhang, J.; Luo, F.-T.; Valenzano, L.; Liu, H. RSC Adv. 2013, 3 (14), 4793.

(26) Jia, Y.; Li, J. Chem. Rev. 2015, 115 (3), 1597.

(27) Wu, W.; Cui, X.; Zhao, J. Chem. Commun. 2013, 49, 9009.

(28) Lin, H.-Y.; Huang, W.-C.; Chen, Y.-C.; Chou, H.-H.; Hsu, C.-Y.; Lin, J. T.; Lin, H.-W. Chem. Commun. 2012, 48 (71), 8913.

(29) Yin, X.; Li, Y.; Zhu, Y.; Jing, X.; Li, Y.; Zhu, D. Dalton Trans. 2010, 39 (41), 9929.

(30) Wu, X. . b; Wu, W. .; Cui, X. .; Zhao, J. .; Wu, M. . J. Mater. Chem. C 2016, 4 (14), 2843.

(31) Kaur, N.; Kaur, P.; Singh, K. Sensors Actuators, B Chem. 2016, 229, 499.

(32) Chen, S.; Chen, W.; Shi, W.; Ma, H. Chem. - A Eur. J. 2012, 18 (3), 925.

(33) Rao, M. R.; Kumar, K. V. P.; Ravikanth, M. J. Organomet. Chem. 2010, 695 (6), 863.

(34) Misra, R.; Dhokale, B.; Jadhav, T.; Mobin, S. M. Organometallics 2014, 33 (7), 1867.

(35) Misra, R.; Dhokale, B.; Jadhav, T.; Mobin, S. M. Dalt. Trans. 2013, 42 (37), 13658.

(36) Khan, T. K.; Pissurlenkar, R. R. S.; Shaikh, M. S.; Ravikanth, M. J. Organomet. Chem. 2012, 697 (1), 65.

(37) Ganapathi, E.; Madhu, S.; Ravikanth, M. Tetrahedron 2014, 70 (3), 664.

(38) Zatsikha, Y. V.; Maligaspe, E.; Purchel, A. A.; Didukh, N. O.; Wang, Y.; Kovtun, Y. P.; Blank, D. A.; Nemykin, V. N. Inorg. Chem. 2015, 54 (16), 7915.

(39) Bura, T.; Hablot, D.; Ziessel, R. Tetrahedron Lett. 2011, 52 (18), 2370.

(40) Yin, X.; Li, Y.; Li, Y.; Zhu, Y.; Tang, X.; Zheng, H.; Zhu, D. Tetrahedron 2009, 65 (40), 8373. 
(41) Galangau, O.; Fabre-Francke, I.; Munteanu, S.; Dumas-Verdes, C.; Clavier, G.; Méallet-Renault, R.; Pansu, R. B.; Hartl, F.; Miomandre, F. Electrochim. Acta 2013, 87, 809.

(42) Dhokale, B.; Gautam, P.; Mobin, S. M.; Misra, R. Dalt. Trans. 2013, 42 (5), 1512.

(43) Dyadchenko, V. P.; Dyadchenko, M. A.; Okulov, V. N.; Lemenovskii, D. A. J. Organomet. Chem. 2011, $696(2), 468$.

(44) Pan, Z.-H.; Luo, G.-G.; Zhou, J.-W.; Xia, J.-X.; Fang, K.; Wu, R.-B. Dalton Trans. 2014, 43 (22), 8499.

(45) Meng, G.; Velayudham, S.; Smith, A.; Luck, R.; Liu, H. Macromolecules 2009, 42 (6), 1995.

(46) Jiao, L.; Yu, C.; Li, J.; Wang, Z.; Wu, M.; Hao, E. J. Org. Chem. 2009, 74 (19), 7525.

(47) Martinez, C. R.; Iverson, B. L. Chem. Sci. 2012, 3 (7), 2191.

(48) Hunter, C. a; Sanders, J. K. M. J. Am. Chem. Soc. 1990, 112 (14), 5525.

(49) Cozzi, F.; Cinquini, M.; Annunziata, R.; Dwyer, T.; Siegel, J. S. J. Am. Chem. Soc. 1992, 114 (ii), 5729.

(50) Williams, J. H.; Cockcroft, J. K.; Fitch, A. N. Angew. Chemie Int. Ed. English 1992, 31 (12), 1655.

(51) Fukushima, K.; Funatsu, K.; Ichimura, A.; Sasaki, Y.; Suzuki, M.; Fujihara, T.; Tsuge, K.; Imamura, T. Inorg. Chem. 2003, 42 (10), 3187.

(52) Harvey, P. D.; Stern, C.; Gros, C. P.; Guilard, R. Coord. Chem. Rev. 2007, 251 (3-4), 401.

(53) Takai, A.; Gros, C. P.; Barbe, J. M.; Guilard, R.; Fukuzumi, S. Chem. - A Eur. J. 2009, 15 (13), 3110.

(54) Ferreira, K. N.; Iverson, T. M.; Maghlaoui, K.; Barber, J.; Iwata, S. Science 2004, 303 (5665), 1831.

(55) Reimers, J. R.; Hush, N. S. J. Am. Chem. Soc. 2004, 126 (13), 4132.

(56) Ottiger, P.; Köppel, H.; Leutwyler, S. Chem. Sci. 2015, 6 (11), 6059.

(57) Benniston, A. C.; Copley, G.; Harriman, A.; Howgego, D.; Harrington, R. W.; Clegg, W. J. Org. Chem. 2010, 75 (6), 2018.

(58) Dodziuk, H.; Vetokhina, V.; Hopf, H.; Luboradzki, R.; Gawe, P.; Waluk, J. J. Chem. Phys. 2012, 136 (7).

(59) Spanget-Larsen, J. Theor. Chim. Acta 1983, 64 (3).

(60) Srinivasan, M.; Sankararaman, S.; Hopf, H.; Dix, I.; Jones, P. G. J. Org. Chem. 2001, 66 (12), 4299.

(61) Caramori, G. F.; Galembeck, S. E.; Laali, K. K. J. Org. Chem. 2005, 70 (8), 3242. 
(62) Radaram, B.; Potvin, J.; Levine, M. Chem. Commun. (Camb). 2013, 49 (74), 8259.

(63) Rambo, B. M.; Sessler, J. L. Chem. - A Eur. J. 2011, 17 (18), 4946.

(64) Ajami, D.; Rebek, J. Acc. Chem. Res. 2013, 46 (4), 990.

(65) Yashima, E.; Maeda, K.; Okamoto, Y. Nature 1999, 449.

(66) Spectus, C. O. N. Acc. Chem. Res. 2009, 42 (8), 1117.

(67) Maeda, H.; Nishimura, T.; Akuta, R.; Takaishi, K.; Uchiyama, M.; Muranaka, A. Chem. Sci. 2013, 4 (3), 1204.

(68) Inokuma, Y.; Osuka, A. Chem. Eur. J. 2009, 15 (28), 6863.

(69) Inokuma, Y.; Osuka, A. Chem. Eur. J. 2009, 15 (28), 6863.

(70) Kissel, P.; Breitier, S.; Reinmüller, V.; Lanz, P.; Federer, L.; Schlüter, A. D.; Sakamoto, J. European J. Org. Chem. 2009, No. 18, 2953.

(71) Davis, N. K. S.; Thompson, A. L.; Anderson, H. L. Org. Lett. 2010, 12 (9), 2124.

(72) Davis, N. K. S.; Thompson, A. L.; Anderson, H. L. J. Am. Chem. Soc. 2011, 133 (1), 30.

(73) Wagner, R. W.; Lindsey, J. S. Pure Appl. Chem. 1996, 68 (7), 1373.

(74) Zhang, J.; Yang, Q.; Cross, J. B.; Romero, J. A. C.; Poutsiaka, K. M.; Epie, F.; Bevan, D.; Wang, B.; Zhang, Y.; Chavan, A.; Zhang, X.; Moy, T.; Daniel, A.; Nguyen, K.; Chamberlain, B.; Carter, N.; Shotwell, J.; Silverman, J.; Metcalf, C. A.; Ryan, D.; Lippa, B.; Dolle, R. E. J. Med. Chem. 2015, 58 (21), 8503.

(75) Hashmi, a. S. K.; Rudolph, M.; Huck, J.; Frey, W.; Bats, J. W.; Hamzić, M. Angew. Chemie - Int. Ed. 2009, 48 (32), 5848.

(76) Hansen, A. M.; Sewell, A. L.; Pedersen, R. H.; Long, D. L.; Gadegaard, N.; Marquez, R. Tetrahedron 2013, 69 (39), 8527.

(77) Lebel, H.; Davi, M. Adv. Synth. Catal. 2008, 350 (14-15), 2352. 